Proceedings of the

\title{
$55^{\text {th }}$ Annual Meeting
}

of the

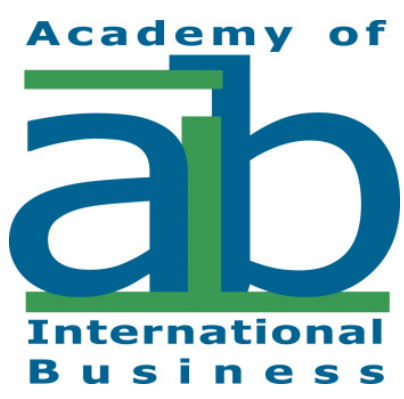

\section{Academy of International Business}

"Bridging the Divide: Linking IB to Complementary Disciplines and Practice"

Istanbul, Turkey

July 3-6, 2013

\section{Editors}

Patricia McDougall-Covin, Program Chair

Tunga Kiyak, AIB Managing Director

(c) 2013 Academy of International Business 
Proceedings of the

$55^{\text {th }}$ Annual Meeting

of the

Academy of International Business

"Bridging the Divide: Linking IB to Complementary Disciplines and Practice" Istanbul, Turkey

July 3-6, 2013

ISSN: 2078-0435

(c) 2013 Academy of International Business

For more information, please contact:

AIB Executive Secretariat

G. Tomas M. Hult, Executive Director, or

Tunga Kiyak, Managing Director

Eppley Center

465 N. Shaw Ln Rm 7

Michigan State University

East Lansing, MI 48824, USA

Phone: +1 (517) 432-1452 - Fax: +1 (517) 432-1009

E-mail: aib@aib.msu.edu•Web: http://aib.msu.edu/ 


\section{TABLE OF CONTENTS}

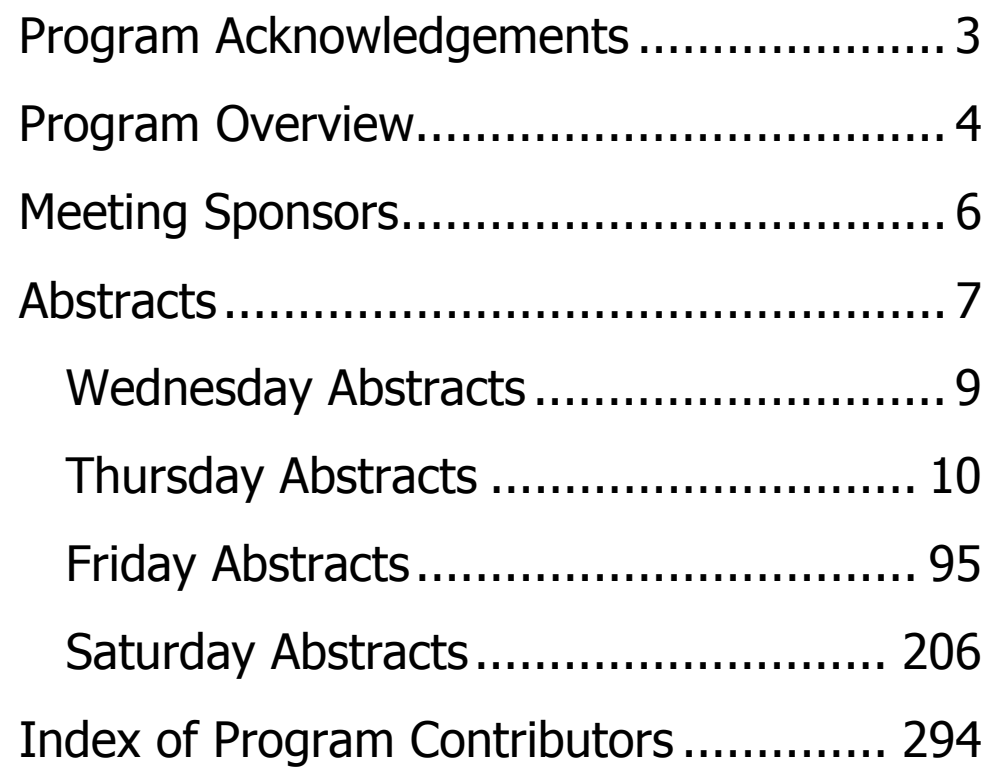


(This page is intentionally blank) 


\title{
2013 PROGRAM ACKNOWLEDGEMENTS
}

\author{
PROGRAM CHAIR \\ Patricia McDougall-Covin - Indiana University \\ PROGRAM CHAIR'S ASSISTANT \\ Sara Reeves, Indiana University \\ LOCAL HOST CHAIR \\ Nakiye Boyacıgiller - Sabanci University
}

LOCAL HOST CHAIR'S ASSISTANT

Eda Aksoy - Sabanci University

Sushil Vachani, Boston University

Susan Forquer Gupta, Monmouth University

Jane Lu, National University of Singapore

Dan Li, Indiana University

Danchi Tan, National Chengchi University

Kent Eriksson, KTH The Royal Institute of Technology

Seung Ho "Sam" Park, Skolkovo
TRACK CHAIRS

Rodrigo Bandeira-de-Mello, Fundação Getulio Vargas Mila Lazarova, Simon Fraser University Hussain Rammal, University of South Australia Manuel Serapio, University of Colorado Denver

Ram Mudambi, Temple University

Melodena Balakrishnan, University of Wollongong, Dubai Pavlos Dimitratos, University of Glasgow

\section{PRE-CONFERENCE PROGRAM COORDINATOR Klaus Meyer - CEIBS \\ DOCTORAL CONSORTIUM CHAIR \\ Grazia Santangelo, University of Catania \\ JUNIOR FACULTY CONSORTIUM CHAIR \\ Udo Zander, Stockholm School of Economics \\ MID-CAREER CONSORTIUM CHAIR \\ John Mezias, University of Miami \\ AIB/JIBS PAPER DEVELOPMENT WORKSHOP \\ Petra Christmann, Rutgers University \\ John Cantwell, Rutgers University \\ Pallavi Shukla, Rutgers University \\ PLACEMENT SERVICES DIRECTOR \\ Hadi Alhorr, Saint Louis University}

BUCKLEY AND CASSON AIB DISSERTATION AWARD SELECTION COMMITTEE

Alan Rugman, University of Reading (Chair) Anupama Phene, George Washington University

Peter Buckley, University of Leeds

Kevin Zhou, University of Hong Kong

\section{TEMPLE/AIB BEST PAPER SELECTION COMMITTEE}

Esra Gencturk, Ozyegin University (Chair)

Elizabeth Rose, University of Otago

Kazuhiro Asakawa, Keio University

Peter Liesch, University of Queensland

\section{HAYNES PRIZE SELECTION COMMITTEE}

K. Galen Kroeck - Florida International University (Chair) Jane Lu Wenzhen - University of Melbourne

Rudolf Sinkovics - University of Manchester

Olli Kuivalainen - Lappeenranta University of Technology

\author{
HOST INSTITUTIONS \\ Sabancı University \\ Koç University \\ Özyeğin University
}


AIB 2013 Program Overview

Istanbul, Turkey - July 3-6, 2013

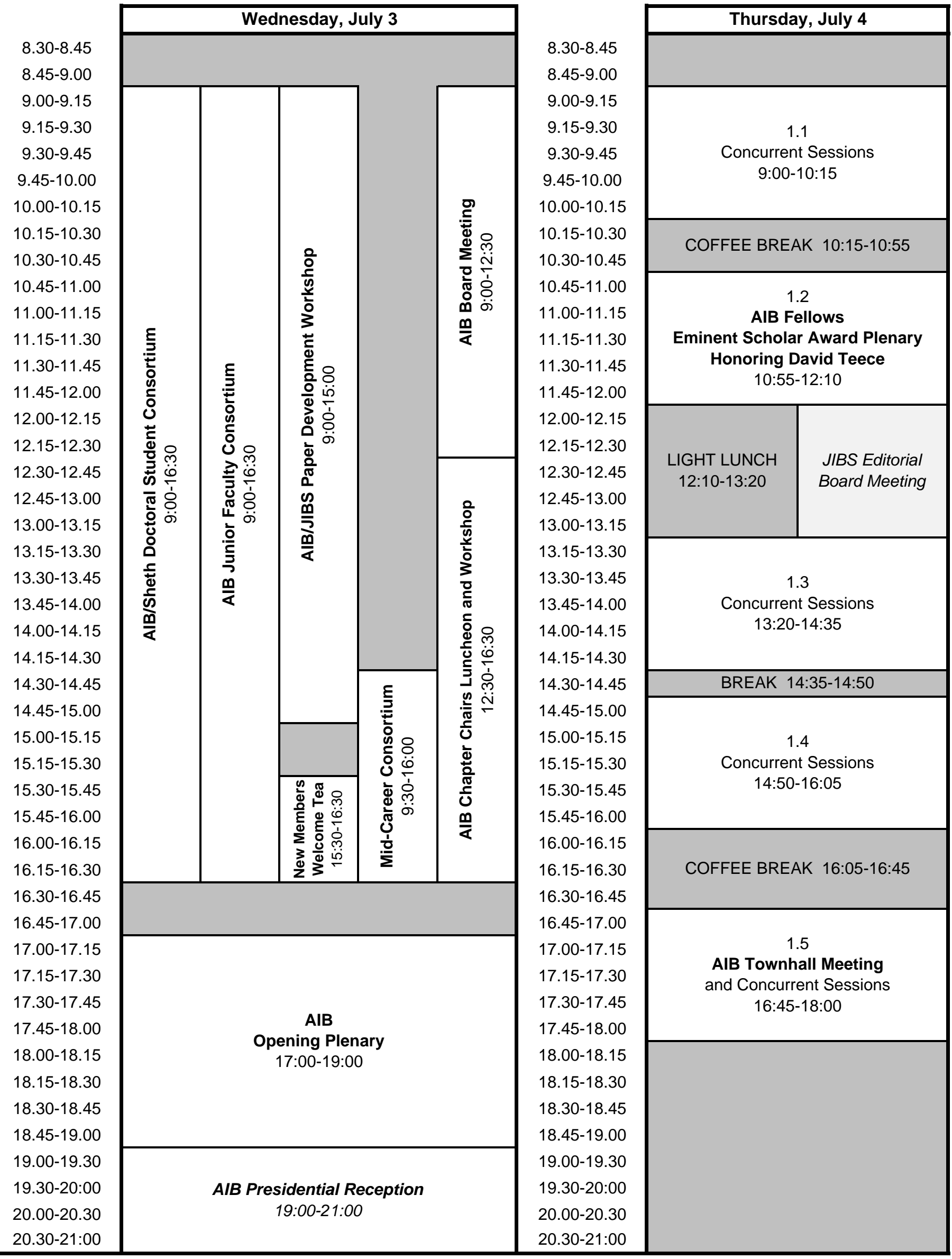

AIB 2013 Conference Proceedings 
AIB 2013 Program Overview

Istanbul, Turkey - July 3-6, 2013

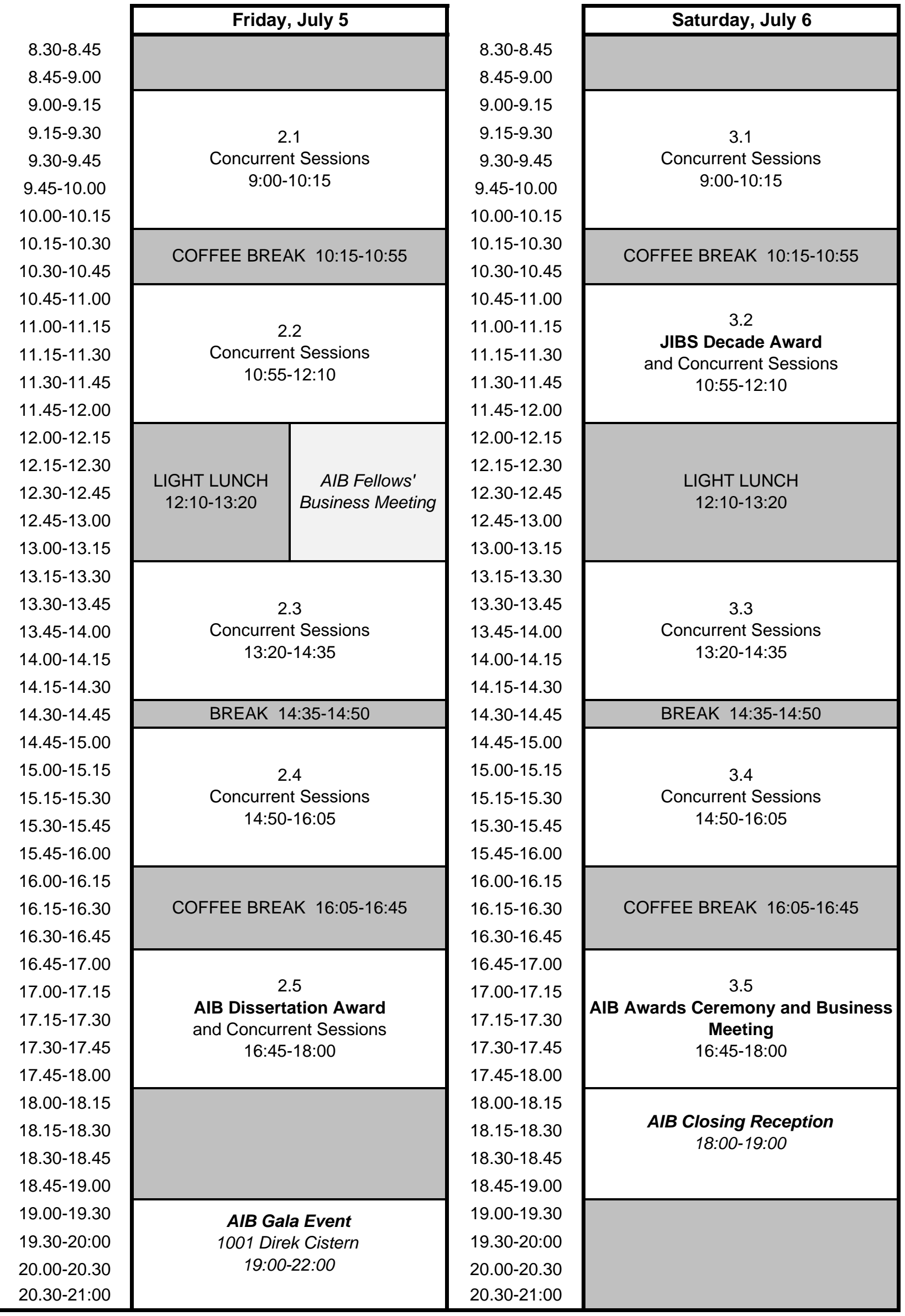

AIB 2013 Conference Proceedings 


\section{AIB 2013 CONFERENCE SPONSORS}

The Academy of International Business thanks the following sponsors for making the 2013 IstanbulConference possible through their generous contributions.
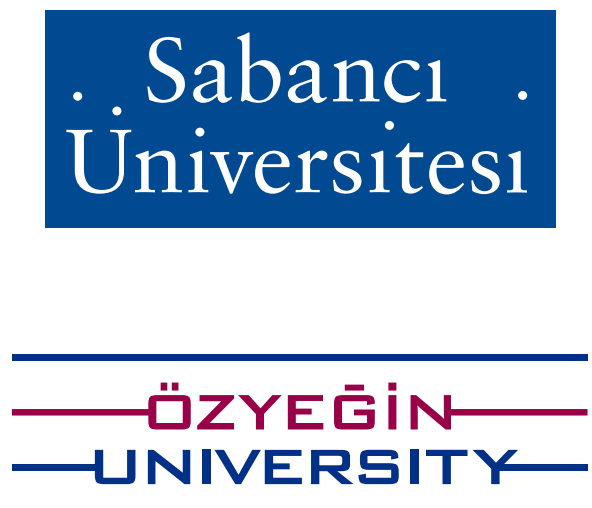
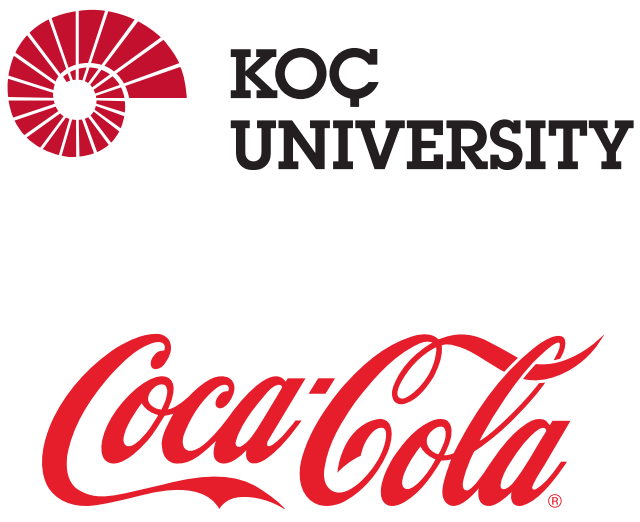

$\Psi$

KELLEY SCHOOL OF BUSINESS

INDIANA UNIVERSITY Center for International Business

Education \& Reasearch

П] Fox School of Business TEMPLE UNIVERSITY ${ }^{\circledast}$

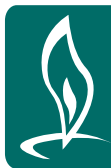

THE SHETH FOUNDATION palgrave macmillan
School of Business

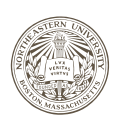

D'Amore-McKim

Northeastern University

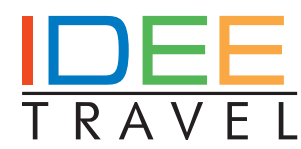

$\frac{\text { MICHIGAN STATE }}{\text { U N I V E R S I T Y }}$

Broad College of Business International Business Center

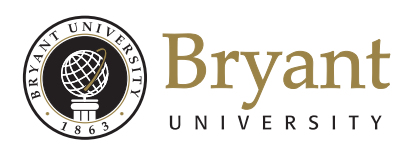

UNIVERSITY OF LEEDS 


\section{ABSTRACTS}


(This page is intentionally blank) 


\section{ABSTRACTS FOR WEDNESDAY, JULY 3, 2013}

Session: 0.7 - Plenary

\section{Opening Plenary: Recognizing IB Leaders}

Presented On: July 3, $2013-17: 00-19: 00$

Chair: Robert Grosse, George Mason University

Welcome Remarks

International Educator of the Year

Bernard Ramanantsoa, Dean, HEC Paris

International Executive of the Year Award

Muhtar Kent, Chairman and CEO of The Coca-Cola Company

\section{AIB Fellows Plenary: How Much Does Distance Still Matter in International Business?}

Presented On: July 3, $2013-17: 00-19: 00$

Chair: Alan M. Rugman, University of Reading

Keynote Speech: "Measuring and Bridging Distance In International Business"

Pankaj Ghemawat, IESE

Commentary: "Implications for Emerging Economies and Emerging Economy MNEs"

Bernard Yeung, National University of Singapore

Commentary: "Implications for High Quality International Business Education"

Dan Leclair, AACSB International 


\section{ABSTRACTS FOR THURSDAY, JULY 4, 2013}

\section{Session: 1.1.1 - Panel}

\section{AACSB Report on the Globalization of Business Education}

Presented On: July 4, 2013 - 09:00-10:15

Chair: Tomas Hult, Michigan State University

Panelists:

Tomas Hult, Michigan State University

Dan Leclair, AACSB International

Stefanie Lenway, Michigan State University

Hildy Teegen, University of South Carolina

Sri Zaheer, University of Minnesota

Rarely, if ever, have business schools experienced change as far-reaching and powerful as during the current wave of globalization. Understanding these changes, and their implications, was the charge given to an AACSB Task Force on Globalization of Management Education. The special session highlights and discusses the AACSB globalization report from a business school dean's perspective, from AACSB's perspective, and from an AIB perspective. (For more information, please contact: Tomas Hult, Michigan State University, USA: hult@msu.edu)

\section{Session: 1.1.2 - Panel}

Track: Track: 9 - Cross-Cultural Management and HRM

\section{Paternalistic Leadership: Conceptualization, Measurement, and Employee-Related Outcomes}

Presented On: July 4, $2013-09: 00-10: 15$

Chair: Zeynep Aycan, Koc University

Panelists:

Zeynep Aycan, Koc University

Guneri Tuncer, Koc University

Petek Demirer, Koc University

Zeynep Cemalcilar, Koc University

Debates on paternalism date back to the times of Aristotle and Confucius when family was considered to be the model for organizing and governing the state. Systematic research on paternalism in the context of leadership has only started around the turn of the century. Paternalistic leadership (PL) refers to a hierarchical relationship in which the leader guides employees in matters concerning their professional and personal lives in a manner resembling a parent, and, in return, expects loyalty and deference (Gelfand, Erez, \& Aycan, 2007). PL is commonly practiced in Asian, Middle-Eastern, Latin American, and African organizations. As such, it represents the leadership style of the 'majority world' (Kagitcibasi, 1996). Pellegrini and Scandura's (2008) literature review of PL revealed that "paternalistic leadership is an emerging and fascinating new area for research." The panel presents 4 papers on conceptualization of PL and cross-cultural validation of its measurement; developmental perspectives to formation of attitudes towards $\mathrm{PL}$; and positive (i.e., psychological empowerment) and negative (i.e., mobbing) employee-related outcomes associated with PL. (For more information, please contact: Zeynep Aycan, Koc University, Turkey: zaycan@ku.edu.tr) 
Session: 1.1.3 - Panel

Track: Track: 8 - Developing Country MNCs

\section{The Competitive Advantage of Emerging Market Multinationals}

Presented On: July 4, 2013 - 09:00-10:15

Chair: Afonso Fleury, University of São Paulo

Discussant: Ayse Karaevli, Sabanci University

Panelists:

Afonso Fleury, University of São Paulo

Peter Williamson, University of Cambridge

Ravi Ramamurti, Northeastern University

Maria Tereza Fleury, Fundacao Getulio Vargas

There is increasing evidence that as emerging country multinationals (EMNEs) mature, their advantages shift from CSAs to FSAs. This panel will look into three mechanisms by which EMNEs develop FSAs: through innovation; by the use of M\&As to access new capabilities that can be combined with existing resources to create new FSAs; and by developing novel ways of configuring their international value chains and managing foreign subsidiaries. The experience of EMNEs from Brazil, Russia, India and China expanding into global markets will be analyzed in relation to each one of those three sources competitive advantage thus explaining how they build FSAs, which may be underpinned by CSAs. The issue of whether and how theories of internationalization require adjustments in order to accommodate the evolution of competitive advantage within EMNEs will be addressed by the presenters and then open to discussion. (For more information, please contact: Afonso Fleury, University of São Paulo, Brazil: acfleury@usp.br)

\section{Session: 1.1.4 - Competitive}

Track: Track: 4 - Strategy, Alliances, and Competitiveness

\section{Multimarket Competition, Country Risk and M\&A}

Presented On: July 4, 2013 - 09:00-10:15

Chair: Sanjay Patnaik, George Washington University

\section{Expansion of Foreign Subsidiaries as a Response to Inward Foreign Competition}

Jan Hendrik Fisch, University of Augsburg

Wolfgang M. Gleich, University of Augsburg

This study investigates rivalry between foreign investors in each other's home markets. Multipoint competition theory suggests that a domestic firm under attack by foreign competitors' inroads into its home market benefits from expanding its subsidiaries in the attackers' home markets. We show that domestic firms retaliate by expanding their subsidiaries but carefully dimension their counterstrikes. Powerful subsidiaries in the attackers' home markets enable stronger reactions, whereas long-tenured subsidiaries mitigate the reactions. When the domestic firm's home market is heavily penetrated by rivals' subsidiaries or the firm is highly dependent on this market, the response is also more cautious. A panel analysis of 1,868 foreign subsidiaries owned by 912 service firms supports the hypotheses. The findings show the importance of foreign subsidiaries for competitive strategy and underscore the relevance of competition to international investment decisions. (For more information, please contact: Jan Hendrik Fisch, University of Augsburg, Germany: fisch@wiwi.uni-augsburg.de) 
Country Risk, International Multimarket Contacts and MNEs' Competitive Action

Yu-Ching Chiao, National Chung Hsing University

Kuo-Pin Yang, National Dong Hwa University

Chun Ju Huang, National Chung Hsing University

In light of the lack of empirical evidence on discussing international business issue by integrating MNEs competitive dynamics. This study investigates the moderating role that international multimarket contact play in MNEs' country risk and competitive action. Examining a sample of top 20 container shipping MNEs in terms of collecting 2,117 competitive actions from Shipping Digest, we find that when MNEs face higher country risks, they undertake fewer competitive actions. On the other hand, we find that MNEs with higher degrees of international multimarket contact between MNEs weaken the negative relationship between country risk and a focal firm's competitive actions. This study extends mutual forbearance theory to international context by investigating how international multimarket contact impacts the relationship between country risk and a focal firm's competitive actions. The implications of these findings for research and practice are discussed. (For more information, please contact: Chun Ju Huang, National Chung Hsing University, Taiwan: scar133@gmail.com)

The Dark Side of Network Embeddedness-Connections versus Expertise of Legal Advisors, and Acquirers' Failure to Learn: Evidence from the Market for Legal Services in Cross-Border M\&As, 1998-2010

Katrin Muehlfeld, Utrecht University

Utz Weitzel, Radboud Universiteit Nijmegen

Bastian Westbrock, Utrecht University

Drawing on theories of social networks and organizational learning, we theorize about the importance that acquirers attribute to network-related and expertise-related factors in selecting legal advisors in international M\&As. We also assess the outcome implications of these network-related and expertise-related factors with respect to two under-explored variables in cross-border acquisition processes: completion likelihood and duration of the public takeover phase. Finally, we investigate whether acquirers learn to rebalance the weights attached to these criteria with increasing M\&A experience. An analysis of 12,249 acquisition attempts in the time period 1998-2010 where the acquirer and the target come from a country in one of the regions North America, Western Europe, or East Asia supports our theory. General and country-specific expertise of legal advisors are key drivers of completion likelihood and time to completion, but receive relatively little weight as selection criteria. Network embeddedness, in turn, drives acquirers' selection of legal advisors but has comparatively little, and not unambiguously positive influence on pre-completion performance. Further, we find little evidence of organizational learning in terms of acquirers adapting their selection criteria with increasing M\&A experience to improve alignment between drivers of selection and pre-completion outcomes. (For more information, please contact: Katrin Muehlfeld, Utrecht University, Netherlands: k.muehlfeld@uu.nl)

Cross-Border Mergers and Acquisitions: Links between Acquiring Firm Capabilities and Resources and Target Country Location

Keith James Kelley, Florida International University

Ronaldo Couto Parente, Florida International University

Marcelo J. Alvarado-Vargas, Florida International University

Yannick Thams, Florida International University

Using a sample of cross-border mergers and acquisitions by US manufacturing firms between 1993-2010, the authors explore how acquiring firm capabilities and resources impact target firm country selection. Results show that US manufacturing firms with greater international management experience are less likely to select target firm country locations among OECD member nations with their cross-border M\&A strategy, whereas; firms with superior R\&D and innovation capabilities, reputation, and financial leveraging capabilities are more likely to do so. Hypotheses were tested using probit regression with a 2-year weighted average for independent variables. A discussion of results, contributions, limitations, directions for research, alongside conclusions are provided. (For more information, please contact: Keith James Kelley, Florida International University, USA: kkelley@fiu.edu) 


\section{Session: 1.1.5 - Competitive}

Track: Track: 9 - Cross-Cultural Management and HRM

\section{Cultural and Emotional Intelligence}

Presented On: July 4, $2013-09: 00-10: 15$

Chair: Ali Dastmalchian, University of Victoria

Cultural Intelligence and International Careers

Stefan Remhof, University of Magdeburg

Marjaana Gunkel, Leuphana University Lueneburg

Using social cognitive theory, we examine the influence of cultural intelligence on the intent to work abroad which has so far remained as a rather unexplored field. Previous research shows that subsequent migration behavior can be predicted with high accuracy by mobility intentions. By extending the conceptual framework, we measure the influence of language skills, international experience, and networks abroad on cultural intelligence. Our model is based on a sample of more than 500 German business students. The results show that cultural exposure positively influences cultural intelligence. Networks abroad have a direct influence on emigration intent, whereas the motivational facet of cultural intelligence mediates the relationship between language skills and emigration intent. The results for the moderating role of cultural distance were mixed. (For more information, please contact: Marjaana Gunkel, Leuphana University Lueneburg, Germany:

mgunkel@inkubator.leuphana.de)

\section{Culture and Emotional Intelligence}

Marjaana Gunkel, Leuphana University Lueneburg

Christopher Schlaegel, Otto von Guericke University Magdeburg

Robert L. Engle, Quinnipiac University

Abstract While a large body of research has examined the outcomes of emotional intelligence, relatively little is known about the antecedents of emotional intelligence. In particular, prior research suggests that emotional intelligence has different effects on management outcomes, such as task performance and leadership behavior, in different cultural contexts. Using a sample of 2,067 individuals in nine countries the present study explores the influence of cultural dimensions on emotional intelligence. Our results show that especially collectivism, uncertainty avoidance, and long-term orientation have a positive influence on the different dimensions of emotional intelligence. Theoretical and practical implications of these findings are discussed and future research directions are provided. (For more information, please contact: Marjaana Gunkel, Leuphana University

Lueneburg, Germany: mgunkel@inkubator.leuphana.de)

\section{International Exposure and Cultural Intelligence: A Five Country Comparison}

Christopher Schlaegel, Otto von Guericke University Magdeburg

Robert L. Engle, Quinnipiac University

Nikolay Dimitriadi, Rostov State Economic University (RINH)

Ekrem Tatoglu, Bahcesehir University

Jasenko Ljubica, University of Split

While a growing body of theoretical and empirical research has provided valuable insights into the outcomes of cultural intelligence, little empirical research has investigated the antecedents of this multidimensional construct. In this study we examine experiential antecedents of cultural intelligence facets. Analyzing 896 business students from five countries, we found that the total duration of stays abroad, an individual's orientation towards interaction with foreign cultures, and the number of languages spoken by an individual positively affect 
the different dimensions of cultural intelligence. Further, the results suggest that while the influence of duration and the number of languages on cultural intelligence differs across countries, the effect of orientation towards interaction is generalizable across countries. The results and their implications are particularly relevant to researchers as well as to human resource practitioners selecting managers and management trainees suitable for appointment to foreign assignments and positions that require intercultural competencies. (For more information, please contact: Christopher Schlaegel, Otto von Guericke University Magdeburg, Germany: cschlaeg@ovgu.de)

\section{Merging Approaches and Weighting Dimensions of Cultural Intelligence Monica J. Semeniuk, Simon Fraser University}

Since the introduction of the term "cultural intelligence" in 2002, two approaches to the concept have emerged. This paper examines each of these approaches for strengths, drawbacks, and alignment with the base concept, intelligence, as a foundation for a proposed merger of the two. An additional comparison is made between the assessment methods that were developed from the two concepts. The result is a set of propositions for a weighting schema that would calculate an overall score for cultural intelligence (CQ) as a composite of the underlying dimensions, using both self- and peer reports. These recommendations are made within the context of the nomological network of CQ, which is also reviewed. (For more information, please contact: Monica J. Semeniuk, Simon Fraser University, Canada: monica_semeniuk@sfu.ca)

\section{Session: 1.1.6 - Competitive}

Track: Track: 12 - Economic Geography and Value Chains

\section{Global Value Chains}

Presented On: July 4, $2013-09: 00-10: 15$

Chair: Aurelia Durand, HEC Montreal

The Hidden Hub: Coordinating Global Value Chains with Limited Formal Authority Randi Lunnan, Norwegian Business School Sara McGaughey, Griffith University

The value chains underlying most goods and services are becoming increasingly disaggregated across firms and globally dispersed. Through a review of the literature and empirical case study of a Norwegian shipbuilder, we explore the network orchestration capabilities and related routines required by a MNE to coordinate, direct, influence and manage other members of the value chain in order to create and extract value. Due to a changing business model, our focal firm has limited formal authority derived from ownership or explicit contracts, and is thus a 'hidden hub' in the global value chain. We categorise the routines of orchestration found in the case study according to two social mechanisms for network governance (restricted access and macroculture development), and link them to coordination and safeguarding, then to innovation and quality. (For more information, please contact: Randi Lunnan, Norwegian Business School, Norway: randi.lunnan@bi.no)

\section{Understanding Influences of Global Value Chains on Manufacturing Employment: A Time Series Study Min (Megan) Zhang, University of Western Ontario}

This study purports to advance the theoretical and empirical analysis of a controversial topic: manufacturing employment and off/backshoring. After reviewing the present OLI paradigm and its limited applications on the topic, the paper incorporates a coevolution perspective to propose that the institutional environments of the home country and the host country may become increasingly coupled over time. Moreover, it draws upon the 
economic sociology literature to explore how global value chain might act as a supranational system that shapes country-level institutions in a feedback loop. Using data from the United Nation and U.S. government websites, the study applies a vector error correction model (VECM) and relevant time series techniques to test the proposed coevolution processes. The results revealed a much more complex dynamics than what has been thought in the extant literature. The study contributes to the IB field as one of the first empirical paper that examines the logic of backshoring and the coevolutionary impact of global value chain. (For more information, please contact: Min (Megan) Zhang, University of Western Ontario, Canada: mzhang.phd@ivey.ca)

Global Delivery Models: The Role of Speed and Time Zones in Global Business Services

Stephan Manning, University of Massachusetts Boston

Marcus M. Larsen, Copenhagen Business School

Pratyush Bharati, University of Massachusetts Boston

This article examines antecedents and performance implications of global delivery models (GDMs) in global business services. GDMs require geographically distributed operations to exploit both proximity to clients and time-zone spread for efficient service delivery. We propose and empirically show that service providers who differentiate based on speed of service delivery are likely to set up GDM structures, and that these structures positively affect deal renewal rates if speed is important for clients in selecting vendors. Findings imply that, as co-location becomes less necessary for providing digitalized services, time zones increasingly affect coordination, performance and internationalization processes. (For more information, please contact: Stephan Manning, University of Massachusetts Boston, USA: stephan.manning@umb.edu)

\section{Session: 1.1.7 - Competitive \\ Track: Track: 10 - Economics, Finance and Accounting \\ Influence of Regulatory, Cultural and Institutional Environments}

Presented On: July 4, 2013 - 09:00-10:15

Chair: Martyn Lawrence, Emerald Group Publishing

\section{Systemic Banking Crises, Liberalisation and Governance}

Basma Majerbi, University of Victoria

Houssem Rachdi, University of Jendouba

We use multivariate logit models to estimate the probability of systemic banking crisis for a sample of 54 countries. In particular, we investigate the interaction between financial liberalization and banking governance and institutional variables in an Early Warning System framework. We find that most of the institutional and governance variables retained in this study have a positive and significant impact in terms of reducing the probability of systemic banking crises despite the potential negative effects of financial liberalization. We also find that the types of institutional variables that may be most effective in mitigating the liberalization effect on crises vary across countries/regions. (For more information, please contact: Basma Majerbi, University of Victoria, Canada: majerbi@uvic.ca)

The Influence of National Culture on Customers' Cross-Buying Intentions on Bancassurance You-il Lee, University of South Australia Jung-Kee Hong, University of South Australia

The paper examines the determinants of customers' cross buying intentions on bancassurance in Korea and Taiwan, and tests the influence of collectivism as a proxy of national culture to the relationships between these 
determinants and customers' cross buying intentions. A triangulation approach was employed in which data of structured surveys to 700 banking customers and 13 interviews with professional senior managers engaged in customers' cross buying activities of bancassurance in Korea and Taiwan was collected in 2011 and 2012. Confirmatory Factor Analysis and Hierarchal Multiple Regression were conducted on these data using AMOS 10.1 and SPSS 12.0. 'Perceived Value', 'Trust', 'Image', and 'Satisfaction' were found to be the determinants of customers' cross buying intentions on bancassurance in Korea and Taiwan. Out of which 'Trust' and 'Satisfaction' were significantly influenced by 'Collectivism'. The paper confirms that, even between countries with similar level of 'Collectivism', the influence of 'Collectivism' can differ due to the determinants of cross buying intentions on bancassurance. (For more information, please contact: You-il Lee, University of South Australia, Australia: you-il.lee@unisa.edu.au)

\title{
Cross-National Differences in Access to Finance: Influence of Culture and Institutional Environments
} Raj Aggarwal, University of Akron John W. Goodell, University of Akron

In spite of rising interest, there is little prior research on the degree to which national differences in access to finance are determined by national culture. Using World Economic Forum survey data for over eighty countries, this paper examines the determinants of 1) access to equity financing, 2) access to loan financing 3) access to venture capital and 4) overall access to capital. We document that less access to financing is associated with the cultural dimensions of uncertainty avoidance and masculinity. But, greater access to financing is positively associated with greater national wealth and better investor protection. Consistent with earlier literature we also find that greater access to finance is associated with greater government favoritism toward selected firms. These results should be of much interest to policy makers, scholars, bankers and managers of multinational firms. (For more information, please contact: John W. Goodell, University of Akron, USA: johngoo@uakron.edu)

\author{
Has East Met West? The Effect of Laws on Accounting Quality in the European Union \\ Frederick Lindahl, George Washington University \\ Hannu Schadewitz, University of Turku
}

The EU continues to converge: increasing use of the euro, harmonization of laws, increasing mobility of labor and expanding coordination of monetary and fiscal policy. One important mechanism to form a more solid union has been the adoption of IFRS. A single set of high quality accounting standards is intended to produce accounting reports of high quality. While this is the aim, it is by no means certain that this will be the result. If firms do not follow the standards, and if auditors do not enforce strict compliance, then the goal will not be met. The first objective of this paper is to evaluate the uniformity of compliance with IFRS among EU countries. The paper also investigates the causes for less-than-complete compliance. One factor that is often discussed is the legal system. This study investigates the effect of the law on financial reporting. It uses the "natural experiment" of the EU, where ten countries share a past tradition of socialist law, and the other fifteen Continental European countries share a tradition of Roman law. We find that the forces of convergence within the EU have not brought the legal quality in the eastern countries up to the level of the western neighbors. We hypothesize that the weaker legal force in the east is associated with poorer accounting quality. An effect, though not strong, is detectable in two tests of accounting quality. The lower cost of capital that results from good reporting may curb earnings management. (For more information, please contact: Frederick Lindahl, George Washington University, USA: lindahl@gwu.edu) 


\section{Session: 1.1.8 - Competitive \\ Track: Track: 3 - IB Theory, FDI, and Entry Mode}

\section{Political Risk and Political Strategies}

Presented On: July 4, $2013-09: 00-10: 15$

Chair: Brent Burmester, Auckland University

Assessing a Politically Hazardous FDI Environment: The Role of Heuristics and Experience Elizabeth Maitland, University of New South Wales

Andre Sammartino, University of Melbourne

How decision-makers incorporate assessments of political risk into internationalization decisions is a black box in international business (IB) strategy. Studies on the interplay between states and multinational enterprises (MNEs) almost exclusively use data aggregated at national, industry and firm levels. Key individual decisionmakers responsible for such strategic assessments are typically absent from IB studies. Utilizing a behavioral strategy lens, we introduce and explore the cognitive aspects of such decisions, presenting proposals on the role of heuristics, expertise and experience. We use the example of an inexperienced MNE's expansion into Africa to investigate these key microfoundations for possible firm capabilities in hazard assessment and internationalization. We find a crucial role for executives' prior experiences in shaping expertise. (For more information, please contact: Andre Sammartino, University of Melbourne, Australia: samma@unimelb.edu.au)

\section{Subjective Perceptions as Cause of Foreigners' Liability}

Jörg Zimmermann, European Commission - Joint Research Center

Focusing on the causes of the relative performance disadvantage of foreign-owned companies within host market settings, I argue on the basis of the comparative ignorance hypothesis that foreign decision-makers tend to perceive economic settings under uncertainty as more ambiguous than locals, which increases the perceived level of risk. As a result, they are more likely to avoid new opportunities or to opt for opportunities under ambiguity, and these choices result in a relative performance disadvantage. Empirical findings for business owners' decisions to invest in their companies' assets, the decision to change industrial sectors, and the subsequent performance implications support the hypotheses. The framework demonstrates that foreign decision-makers' strategic choices have a negative effect on their relative host market performance, as they are more likely to perceive strategic decisions under uncertainty as decisions under ambiguity instead of risk (For more information, please contact: Jörg Zimmermann, European Commission - Joint Research Center, Spain: jzi@gmx.de)

\section{Foreign Direct Investment Promotion: Using Advertising to Change Attitudes and Behaviors \\ Rick T. Wilson, Hofstra University \\ Daniel W. Baack, University of Denver \\ Donald E. Baack, Pittsburg State University}

Countries compete intensely for foreign direct investments (FDI) and perform a wide range of activities to attract them. Advertising is an area of FDI promotion that has not often been studied, and some questions remain regarding its efficacy. This research examines the effectiveness of FDI advertising using a survey to assess corporate site selection managers' responses to FDI ads. To guide the analysis, Dunning's (1993; 1998) FDI location determinants framework were used. The findings suggest that FDI advertisements do influence managerial attitudes and behaviors, even with a single exposure. (For more information, please contact: Rick T. Wilson, Hofstra University, USA: rick.t.wilson@hofstra.edu) 
Mitigating the Liabilities of Foreignness: Evidence from Newly Industrialized Economies

Chia-Wen Hsu, National Chung Cheng University

Homin Chen, National Taiwan University

Yin-Ru Chen, National Taiwan University

The purpose of this paper is to address the question of how to mitigate the liability of foreignness for MNEs from newly industrialized economies. Basing on a balanced panel data of 207 Taiwanese firms spanning six year (2000-2005), the empirical findings support that international ambidexterity can significantly mitigate the liability of foreignness. In addition, this paper also finds that resource commitment and learning engagement positively moderates the relationship between the international ambidexterity and the liability of foreignness. Theoretical and managerial implications of these findings and suggestions for future research are also discussed. (For more information, please contact: Chia-Wen Hsu, National Chung Cheng University, Taiwan: cwhsu@ccu.edu.tw)

\section{Session: 1.1.9 - Competitive}

\section{Track: Track: 11 - SMEs and Entrepreneurship}

\section{International Strategic Entrepreneurship and Performance of INVs}

Presented On: July 4, 2013 - 09:00-10:15

Chair: Jim Wills, University of Hawai'i at Manoa

Entrepreneurial Orientation and Performance: Evidence from China

Muammer Ozer, City University of Hong Kong

John E. Ettlie, Rochester Institute of Technology

Wen Zhang, City University of Hong Kong

Past research has long shown the positive impact of entrepreneurship on firm performance. It has also shown that this relationship is not always uniform and thus called for future research that examines how different environmental and strategic factors might moderate it. Responding to these calls for future research, our study showed that this relationship was positive in the Chinese context. Furthermore, it suggested that market turbulence hindered it. More interestingly, the results confirmed a three-way interaction model and showed that at high levels of market turbulence, the relationship was stronger when market orientation was high rather than low. (For more information, please contact: Muammer Ozer, City University of Hong Kong, Hong Kong, SAR-PRC: mgozer@cityu.edu.hk)

Integrating International New Ventures and Staged Theory: Age at Entry, Survival and Growth Alex Tai Loong Tan, University of Western Australia Terence Ping-Ching Fan, Singapore Management University

The literature on international new ventures (INVs) has shown how this new breed of firms is fundamentally different from their more traditional, staged-internationalizing counterparts. This study integrates these two opposing perspectives by considering the timing of international entry as a strategic choice for young firms. We hypothesize that the effect of a firm's age at international entry on survival and growth is more complicated than is argued in either of these two opposing schools. Utilizing panel data of small- and medium-sized Australian manufacturers, from which the original 'born-global' label was coined, we show that the failure probability at first decreases and then increases ('U-shape') as a function of firms' age at international entry, while the short-term sales growth at first increases and then decreases ('inverted U-shape'). This highlights the importance of the liabilities of newness and aging even after taking into account the potential learning advantage of newness as advocated by INV scholars. Interestingly, after accounting for the likelihood of internationalization based on a firm's characteristics, a stay-domestic strategy incurs a higher failure rate than 
an internationalizing one, regardless of the age at international entry. (For more information, please contact: Alex Tai Loong Tan, University of Western Australia, Australia: a.tan@uwa.edu.au)

Entrepreneurial Leapfrogging in the Context of ISE: The Salience of Disruptive Innovation by Emerging Multinationals

Peter Li, Copenhagen Business School

We know little regarding the underlying contexts and mechanisms for disruptive innovation initiated by the entrepreneurial firms in the emerging economies. Further, there is limited knowledge about the contexts and mechanisms for global latecomers to catch up with and leapfrog global early-movers. The cross-fertilization between such two research streams provides a great opportunity to shed light on their link toward an interdisciplinary domain of international strategic entrepreneurship (ISE). This article will develop an integrative typology of global innovations as well as a dynamic model of entrepreneurial leapfrogging as the initial building blocks of ISE. (For more information, please contact: Peter Li, Copenhagen Business School, Denmark: pli.int@cbs.dk)

The Mediating Role of Alliances in the International Market Orientation-Performance Relationship George Nakos, Clayton State University Pavlos Dimitratos, University of Glasgow Said Elbanna, UAEU

Increasing international performance is a critical issue for internationalizing SMEs. In the recent past researchers have suggested that companies need to increase their international market orientation in order to increase performance. In this paper we examined whether alliances may play a mediating role in the international market orientation and performance relationship. We investigated our hypothesis on a sample of 261 companies based in two developed and one emerging market. We discovered that alliances mediate the IMO performance relationship. (For more information, please contact: George Nakos, Clayton State University, USA: georgenakos@clayton.edu)

\section{Session: 1.1.10 - Special Session}

\section{The Global Entrepreneurship Monitor (GEM) in its 15th Year: What Do We Now Know and What Can We Still Learn About Global Entrepreneurship?}

Presented On: July 4, $2013-09: 00-10: 15$

Chair: Donna Kelley, Babson College

GEM Background

Donna Kelley, Babson College

GEM Research and Future Research Opportunities Jose Ernesto Amoros, Universidad del Desarrollo

Case Study of Turkey: Entrepreneurship Indicators

Esra Karadeniz, Yeditepe University

Case Study of Turkey: Entrepreneurship Ecosystem Factors

Ussal Sahbaz, The Economic Policy Research Foundation of Turkey (TEPAV) 
As the Global Entrepreneurship Monitor (GEM) enters its 15th year of data collection on entrepreneurship, over 100 economies have participated in the survey since its start, representing every corner of the world and a wide range of economic development levels. Since its first survey of just ten developed economies in 1999, GEM has grown to become the largest and most comprehensive study of entrepreneurs around the globe. The purpose of this session is to inform participants about what we have learned from research involving GEM data, and to consider what lies ahead in the form of future research opportunities in the area of global entrepreneurship.

GEM takes the pulse of societies about their attitudes about entrepreneurship, identifying potential entrepreneurs expressing intentions, and measuring participation in multiple phases of the business startup and ownership process. GEM provides data on the profile of entrepreneurs: for example, their age, gender, income levels, education, industry sector participation, and other characteristics. The research also measures growth ambitions, international sales, and levels of innovativeness-recognizing that not all entrepreneurs have the same impact on their societies. An additional key measure encompasses the motives that lead people to start businesses: whether they were primarily motivated by necessity or opportunity.

The session will open with background on GEM, reviewing its methodology, the most recent findings, and key indicators. The presentation will then turn to a review of key academic implications from studies using GEM data, and then identify some future research directions. A case study of Turkey will be presented to show an indepth example of ecosystem factors and entrepreneurship indicators that can lead to relevant research questions in global entrepreneurship. The audience will then join in the discussion about current and future research opportunities in this field of inquiry, particularly those using large databases like GEM. (For more information, please contact: Donna Kelley, Babson College, USA: dkelley@babson.edu)

\section{Session: 1.1.11 - Competitive \\ Track: Track: 1 - Institutions, Governance, and CSR}

\section{Governance, Institutions and Their Impact}

Presented On: July 4, $2013-09: 00-10: 15$

Chair: Lei Li, Nottingham University

Is "Contingent Reciprocity" a Fourth Governance Mode Applicable to Market Entry and Operation? Jean Boddewyn, Baruch College

Several recent publications have identified a fourth means of getting the intermediate products a firm needs when entering a market - namely, by plying a supplier with gifts or exercising power over him/her so that donations and political influence by themselves can achieve what is expected from the traditional governance modes of market contracting, alliance and internalization. This discovery of a fourth governance mode, which we named "contingent reciprocity," was submitted to theoretical testing which confirmed that it: (1) constitutes a distinct governance mode; (2) challenges the current assumption of transaction-cost economics that governance modes have to lie within a "continuum" between the price system and hierarchy, and (3) adds "nonmarket" mechanisms - namely donations and political influence - to the traditional "market" ones associated with "make, buy or ally." The theoretical nature and implications of this major discovery are further developed in terms of resource-dependence and institutional theories. Theoretical and managerial research implications are developed from this analysis. (For more information, please contact: Jean Boddewyn, Baruch College, USA: jean.boddewyn@baruch.cuny.edu) 


\section{Institutional Uncertainty, Managerial Cognition, and Internationalization Strategy}

Tolga Ulusemre, University of South Carolina

This study focuses on the cognitive processes that underlie strategic responses to institutional processes. More specifically, it examines firm's internationalization strategy as a strategic response to institutional uncertainty in a host country, and argues that managerial attention is the cognitive underpinning of this response. I suggest that firms whose managers focus their attention on institutional environment are more likely to take advantage of institutional uncertainty by expanding into emerging countries, whereas firms whose managers focus their attention on task environment are more likely to avoid it by expanding into developed countries. I study internationalization strategies of US manufacturing firms competing in global industries to support the arguments. (For more information, please contact: Tolga Ulusemre, University of South Carolina, USA: tolga.ulusemre@grad.moore.sc.edu)

\section{Seeing Hybrid Organizations in Context: Institutions and Mission Drift in Commercial Microfinance Joshua K Ault, University of Victoria}

I use multilevel modeling on 1,109 microfinance lenders in 95 countries to examine the impact of national institutions on "mission drift" in hybrid organizations (organizations that combine diverse institutional logics). A recent split of the microfinance industry into two models, a hybrid commercial model and a "pure" non-profit model, provides a naturally-occurring experiment to isolate the effects of hybridization on organizations. Results show that hybrid commercial lenders are more likely to drift from their core mission to serve the poor than nonprofit lenders. However, national institutions moderate this effect: commercial lenders in countries with capable state institutions are less prone to mission drift than those in countries with weak institutions. Hybrid commercial lenders perform more like non-profit lenders when the local context provides complementary institutions. (For more information, please contact: Joshua K Ault, University of Victoria, Canada: jault@uvic.ca)

\section{National Institutional Diversity and its Role in the Vertical Transformation of Science into Technology Tariq Hussain Malik, Dongbei University of Finance \& Economics}

Why some national innovation systems (NISs) are effective in transforming science into technology more than others? This article is an attempt to answer this question. Based on institutional framework (culture and legal system), it is proposed that the integration of upstream science and downstream technology can induce a better performance in innovation. The effectiveness of this integration depends on flexibility legal system and social vision in culture. To support this proposition, the evidence is drawn on 70 countries (1994-2010). The analysis based on panel data reveals some interesting insights in the context of national cultures and legal systems. In cultural context, long-term orientation (social vision) can have positive role in the transformation. Power distance and individualism can have positive role in the transformation. Uncertainty avoidance appears to have a negative role the transformation. In legal context, flexible law (common law) appears to have a stronger role in transformation science to technology. The article provides some implications and suggestions on mixed findings. (For more information, please contact: Tariq Hussain Malik, Dongbei University of Finance \& Economics, China: tmalik@dufe.edu.cn) 


\section{Session: 1.1.12 - Interactive \\ Track: Track: 1 - Institutions, Governance, and CSR}

\section{Institutions and Strategy}

Presented On: July 4, 2013 - 09:00-10:15

Chair: Michael A. Sartor, University of Western Ontario

\section{Double-Layered Institutional Environment in Offshoring Location and Governance Decisions} Julien Gooris, Université libre de Bruxelles

To exploit country-specific advantages, firms that source activities from abroad are forced to integrate the institutional environment into the choice not only of host-country, but also of governance model for their offshore activities. Considering inefficient institutions as drivers of transaction costs, this conceptual paper explores the impact of the host-country regulative environment in the interdependent decisions of country selection and governance model (captive or outsourcing) in firms' global sourcing strategies. We consider two classes of assets: transferred assets for knowledge/information flows, and local assets sourced from the host location. We show that each class involves specific institutional risks for offshoring practices. In turn, because of the different institutional exposures of the captive model and the outsourced one, the institutional risks associated with transferred and local assets have different implications for the choice of governance model. Firms react to institutional risks relative to transferred assets by internalizing their activity, but they bypass inefficient institutions for local assets using outsourcing. Based on the interaction of the institutional risks relative to each class of assets, we then obtain sufficient conditions that give the firm-optimal combinations of country selection and governance model. (For more information, please contact: Julien Gooris, Université libre de Bruxelles, Belgium: jgooris@ulb.ac.be)

\section{Not All Institutions Are Created Equal: The Institutionalization of Private International Business Regulations Nathan Emil Betancourt, University of Lugano \\ Johannes Meuer, University of Zurich \\ J. (Hans) van Oosterhout, Erasmus University}

Over the last few decades, the international business arena has shown a widening gap between the increasing globalization of business and the public laws and institutions that regulate it. As a response to this 'governance gap', there has been a proliferation of privately generated international business regulations (PIBRs) that have had varying degrees of success in terms of institutionalization. Using a comparative cases study of 64 cases of PIBR that are well documented in the academic and professional literatures, this study uses set theoretic methods (fsQCA) to build theory and develop a typology that will help us to understand why attempts at PIBR either fail or succeed in terms of two necessary conditions for successful institutionalization in the international arena: diffusion and resilience. The theory developed highlights the distinctive challenges that private regulatory institutions face in the international arena and will help future research to make sense of institutionalization processes across the many different fields in the international arena in which PIBRs have emerged (For more information, please contact: Nathan Emil Betancourt, University of Lugano, Switzerland: nathanbetancourt@gmail.com)

\section{National and Supranational Institutional Pressures and Nonmarket Actions of Firms in a Host Environment Cosmina Lelia Voinea, Radboud Universiteit Nijmegen Hans van Kranenburg, Radboud Universiteit Nijmegen}

This paper explores the relationships and sources of variation in the strength of institutional pressures and nonmarket actions of foreign firms in a host country that is member of a regionally integrated economy. Overaching the national institutions, a central role is attributed to supranational institutions which promulgate and enforce an important part of the national legislation and regulation. Therefore, we explore institutional 
factors explaining pressures for different types of institutions and the interplay between national and supranational instittions:, on the intensity of relational and transactional nonmarket actions undertaken by foreign firms to deal with these pressures in an open regionally integrated economy. Evidence is provided by a survey sample of 160 foreign firms operating in the Netherlands. Results show that four nonmarket institutions mainly explain the frequency of nonmarket action. National regulators, standards agencies, interest groups, and the media along with their specific institutional factors have the highest explanatory value for the increase in nonmarket actions of foreign firms. Empirical outcome also indicates a dependency of national level institutions on the supranational institutional echelon. (For more information, please contact: Cosmina Lelia Voinea, Radboud Universiteit Nijmegen, Netherlands: c.voinea@fm.ru.nl)

\section{Beyond Institutional Voids: Institutional Dynamics and Firm Strategies \\ Charles Dhanaraj, Indiana University \\ W.G. Douglas Fernandez, Florida International University \\ Sumit Kundu, Florida International University}

How do we capture the dynamics and diversity of the institutional contexts and their impact on firm level strategies? We present a critical appraisal of institutional voids (IV) approach (Khanna and Palepu, 1997; 2000) that has significantly influenced research on emerging markets in the last decade. We discuss the weaknesses of the IV approach, particularly its inability to address the dynamics, diversity, and equifinality in institutions across the globe. We integrate insights from political economy literature to develop a conceptual framework for a dynamic view of strategy in emerging markets. We present a framework incorporating two broad enabling mechnisms, information exchange and trust building, and two broad enforcing mechanisms, Behavior monitoring, and We discuss implications for empirical work by applying the framework to three critical phenomena in strategic management: the role of business groups, the differential evolution of industries, the locus of innovation and intellectual property rights. (For more information, please contact: Charles Dhanaraj, Indiana University, USA: dhanaraj@iupui.edu)

\section{The Effect of International Board Capital on Internationalization Strategy \\ W.G. Douglas Fernandez, Florida International University \\ Sumit Kundu, Florida International University}

Internationalization continues to be critical issue for firms competing in today's global marketplace. This study investigates the effect of board capital on firm internationalization process. Using a panel of Fortune 500 manufacturing firms, we predict and find that firms leverage board capital in the internationalization process, such that breadth and depth of international board capital corresponds with breadth and depth of firm internationalization respectively. (For more information, please contact: W.G. Douglas Fernandez, Florida International University, USA: wdoug002@fiu.edu)

\section{The Control of Foreign Operations: Is it Strategic, Economic or Politically Driven?}

Francisco Figueira-de-Lemos, Uppsala University

This paper studies the active role of governments in the internationalization of the multinational within the scope of management, economic and institutional theories. The model examines the influence of informal, formal and political differences of the institutional environment on contractual and ownership modes of foreign operations. Following a previous call for research from Henisz (2000), it also integrates a variable to infer how political ties can moderate political differences. The empirical test applies Structural Equation Modeling and path analysis to a sample of 249 diplomatic missions up to 94 different countries. The findings depict that political differences have an effect on ownership mode of foreign operations, and that these differences are indeed moderated by political ties. The most substantial contribution is the disclosure of diplomatic network intensity as a new measure for gravity models on international trade and foreign investment. (For more information, please contact: Francisco Figueira-de-Lemos, Uppsala University, Sweden: francisco.lemos@fek.uu.se) 
Session: 1.1.13 - Interactive

Track: Track: 10 - Economics, Finance and Accounting

\section{International Trade and Investment: Corporate Networks and Intellectual Capital}

Presented On: July 4, 2013 - 09:00-10:15

Chair: Mohamed Belkhir, UAE University

Comparing China and India: National Intellectual Capital Perspective

Carol Yeh-Yun Lin, National Chengchi University

Ahmed Bounfour, Université de Paris Sud 11

With longitudinal data from 1995 to 2010, this study attempts to assess national intellectual capital (NIC) of China and India, explore the relationship between their respective economic development and NIC, and identify the NIC strength of each country. Trend analysis, multivariate analysis of variance and regression analysis are employed to detect the variances between the two countries. We found that NIC starts to show higher influence on economic growth when the country is developed to around USD6000 GDP per capita (ppp). Market capital is the strength of both China and India. Human capital (market capital and process capital) is (are) highly correlated with GDP sustaining effect, boosting effect, and per capita (ppp) for China (India). The best predictors of GDP per capita (ppp) are market capital, and human capital co-developed with renewal capital. Internal resources substitution is observed in India. Without good human capital, India developed its market capital and process capital to sustain and boost its growth. This paper is original in expanding resources-based view and core competence theory to national level study. In addition, it extends general China and India economic report to the intangible NIC comparison. (For more information, please contact: Carol Yeh-Yun Lin, National Chengchi University, Taiwan: yehyunIn@nccu.edu.tw)

\section{Developments Are Accumulated Choices}

Jay Wu, Thammasat University

We developed a framework of accumulated choices (AC) to help integrate thoughts from economists and culturalists on national developments. AC is conditional choices expanded with conditional expectations or foresights and draws from three sources of other conditional choice models - path dependence theory (PDT), social choices theory (SCT) and economics of human skills formation (EHS). Instead of calling Total Factor Productivity (TFP) as the key for growth, like the economists do; or as state of mind, as culturalists do, the formula "Developments $=A C$ " serves us better. $\mathrm{AC}$ has the capacity to cover both institutions and accumulated preferences (AP). Built on AC/AP we then strive to answer three developmental questions: why conditional convergence failed to materialize; why Washington Consensus failed while Beijing Consensus is never a consensus and how national developments are related to human (child) developments - in addition the billion dollar question of why some countries are so rich while others so poor. Simply put, (a) developments feature varying, rather than constant, rate of changes; (b) Washington (Beijing) Consensus failed because it ignored AP by exclusively focusing on institutions; (c) each individual's preferences over both cognitive and social emotional domains are shaped by consistently repeated frequency of exposure to preferences. Overall, individual preferences converge into personality, which then merges into nationality that ultimately determines institutional efficiency and rate of growth. (For more information, please contact: Jay Wu, Thammasat University, Thailand: jwuinbkk@gmail.com) 


\section{Corporate Elite Networks and their Effects on Board Performance \\ Daniel Braun, University of Goettingen \\ Jana Oehmichen, Georg-August-University Goettingen \\ Michael Wolff, Georg-August-University Goettingen \\ Toru Yoshikawa, Singapore Management University}

Prior research revealed conceptual and empirical evidence for both positive and negative effects of multiple directorships on firm performance. This study shows how these inconclusive results can be explained by the institutional environment. Positive effects, such as resource provision increase with the number of directorates. Negative effects include busyness and the exploitation of social power. A large number of directorates leave directors less time for the tasks related to their board position and at the same time their elitist status in the social network gives raise to situations in which they decide in favor of the members of their social network and in disfavor of the focal company. The extent of this elitist exploitation of social power depends on characteristics of a country's corporate elite circle. We present evidence that the institutional setting (i.e., the density of a country's corporate elite network) influences the effect of multiple directorships on firm performance. Using a sample of European and North-American companies from 17 different countries covering the years from 2005 to 2010 we show that the overall effect of multiple directorates on company performance is more negative in countries with a dense elite network. We eventually discuss implications for researchers and regulators. (For more information, please contact: Daniel Braun, University of Goettingen, Germany: daniel.braun@wiwi.unigoettingen.de)

\section{Joint Evolution of Product and Destination Portfolios: Evidence from U.S. Exporters Wenjie Chen, George Washington University Fariha Kamal, US Census}

This paper presents a set of empirical results that characterize the dynamic paths exporters choose to expand or contract their exporting activities - along the product or destination margin or both - from the time of entry into the export market until they exit. Using detailed export transactions data of firms operating in the United States between 1992 and 2009 we categorize firms into four mutually exclusive categories of those selling a single product to a single destination, a single product to multiple destinations, multiple products to a single destination, or multiple products to multiple destinations. We uncover five key patterns in the data. First, exporters are more likely to enter as well as exit the export market selling a single product to a single destination. Second, selling multiple products to a single destination is the least popular mode of entry into or exit out of export markets. Third, exporters selling either one or multiple products but to multiple destinations account for about a third and half of total export value respectively. Fourth, of those exporters entering the export market selling a single product to a single destination that expand their product-destination portfolio do so by adding destinations rather than new products. Fifth, adding a destination increases export growth rates by almost as much as adding both products and destinations. (For more information, please contact: Wenjie Chen, George Washington University, USA: chenw@gwu.edu)

\section{A Framework for Cross-Border Investment and Currency Hedging Decisions \\ Kirt C. Butler, Michigan State University \\ Gwinyai Utete, Louisiana State University}

Conventional valuation of cross-border capital budgeting projects is set in a stylized world that assumes the international parity relations hold in integrated financial markets. The financial manager's exchange rate view and the project's operating exposure to currency risk are ignored. In such a world, the value of a cross-border project is the same whether viewed from the parent's or the project's perspective. Complications in the real world include disequilibria in the international parity relations, managerial exchange rate forecasts that differ from parity, operating exposures to currency risk, and the parent firm's currency hedging strategy. Managers' exchange rate forecasts in particular can have a profound impact on estimated project values and the currency 
hedging decision. The authors include these real-world complications in a comprehensive valuation framework, and show how the relative magnitudes of these effects influence managers' behaviors and the multinational corporation's currency risk management decisions. (For more information, please contact: Kirt C. Butler, Michigan State University, USA: butler@msu.edu)

\section{Fiscal Consolidation and the Cost of Credit: Evidence from Syndicated Loans Senay Agca, George Washington University \\ Deniz Igan, International Monetary Fund}

Using loan-level panel data and a new dataset on fiscal consolidations constructed for 16 advanced economies over the period 1990-2011, we examine how the cost of corporate credit varies around fiscal consolidations aimed at reducing government debt. We find that loan spreads increase with fiscal consolidations, especially for small firms, domestic firms, and for firms with limited alternative financing sources. These adverse effects are mitigated substantially if consolidations are large, and can be avoided if consolidations are also accompanied with accommodating monetary policies and implemented by a stable government. These findings suggest that lenders price the short-term recessionary effects in loans but large consolidations can reduce or undo the increase in spreads, especially under favorable country conditions, by signaling credibility and creating expansionary expectations. (For more information, please contact: Senay Agca, George Washington University, USA: sagca@gwu.edu)

\section{What Triggers IPOs. A Cross Country Comparison \\ Jose Luis Rivas, ITAM \\ Mercedes Adamuz, ITAM}

Using a sample of 88 countries we test the variables investor protection, uncertainty avoidance and venture capital on a relative measure of IPO activity: IPOs/GDP. We find that all three variables are significantly related to the level of IPO activity. We contribute to the entrepreneurship, management and international business literatures by bringing together a more holistic model of IPO determinants across countries. (For more information, please contact: Jose Luis Rivas, ITAM, Mexico: jose.rivas@itam.mx)

\section{Session: 1.1.14 - Interactive}

Track: Track: 11 - SMEs and Entrepreneurship

\section{Cultural, Social, and Institutional Contexts of International Entrepreneurship}

Presented On: July 4, 2013 - 09:00-10:15

Chair: Niina Nummela, Turku School of Economics

\section{In Search of Greener Pastures SMEs'Internationalization from Conflict-Prone Countries Ramzi Fathallah, University of Western Ontario Michael Carney, Concordia University}

We advance and illustrate a conceptual framework to describe the search and escape behaviors of SMEs located in conflict-prone countries. Conflict stimulates search and escape responses but also offers SME with opportunities that may go unperceived by SMEs resident in more peaceful domains. We reason that SME internationalization processes from conflict prone counties will also differ from those found in stable emerging markets such as China and India, where jurisdictional search \& escape is motivated by the international pursuit of scarce resources needed for growth. Accordingly, we expect the escape stimulus to engender a more heterogeneous range of internationalization processes than is found in extant theory. Drawing upon the 
experience of internationalizing Lebanese SMEs we use a comparative qualitative case study approach to uncover distinctive features of internationalizing processes from conflict-prone countries. (For more information, please contact: Ramzi Fathallah, University of Western Ontario, Canada: ifathallah.phd@ivey.ca)

\section{The Rise of the Global Social Entrepreneur: A Conceptual Framework \\ Melodena Balakrishnan, University of Wollongong in Dubai \\ Val Lindsay, University of Wollongong in Dubai}

With global turbulence and increased risk in areas of economics, natural environment, political stability \& security, resource challenges and integration challenges, governments and NGOs are less able to address social and civil challenges, making it necessary for the private sector to contribute. Besides MNCs, in sheer number of firms, potential to contribute to GDP, ability to weather environmental shocks and ability to employ, SMEs are an area of future focus. SMEs not only have the opportunity to embed CSR and sustainability practices but their initiators could do so at the start-up stage by becoming social entrepreneurs. Social Entrepreneurship (SE) research is still in the nascent stage (Zahra, Gedajlovic, Neubaum and Shulman 2009; Hervieux, Gedajlovic and Turcotte, 2010; Light 2008). A review by Jones, Coviello and Tang (2011) on International Entrepreneurship research from 1989-2009 finds no specific mention of social entrepreneurship. An important question is, how does SE theory relate to internationalization? International new ventures (INVs) and born globals are entrepreneurial firms, and also the subject of recent research interest in international business. This paper attempts to construct a conceptual model of the various typologies of social, building on previous models, and also providing tentative links with internationalization theory. (For more information, please contact: Melodena Balakrishnan, University of Wollongong in Dubai, United Arab Emirates: melodenabalakrishnan@uowdubai.ac.ae)

\section{Internationalization Process of For-Profit Social Entrepreneurs: Between Gradualist Models and Born Globals} Igor Kalinic, University of Groningen

Max Pater, University of Groningen

Social entrepreneurship is often described as a process whereby the creation of a new firm leads to social wealth enhancement. Despite the emerging interest for social entrepreneurship as solution of international social issues, previous literature did not regard sufficiently to the internationalization process of social entrepreneurs. The present paper analyzes in depth the internationalization process of For-profit Social Entrepreneurs and compares it to the existing literature. The empirical evidence of five firms suggests that the entrepreneurs follow a specific path between gradualist models and born globals. The internationalization choices are considerably influenced by the social mission of the entrepreneur rather than by economical evaluations. (For more information, please contact: Igor Kalinic, University of Groningen, Netherlands: i.kalinic@rug.nl)

\section{Variation in Antecedents of Entrepreneurial Intent across Cultures: A Multiple Group Moderated Mediation Analysis in SEM}

Ellen A. Drost, California State University Los Angeles

We examined the moderated mediating effect of entrepreneurial self-efficacy in the relationship between entrepreneurship education, experience, and entrepreneurial intent across the countries, namely China, Finland and the US. Results indicated that self-efficacy mediated the relationship between education, experience and entrepreneurial intent, but these relationships were moderated by culture. Bootstrapping and multiple group structural equation modelling techniques were used to analyze the data. Implications for entrepreneurship research are discussed. (For more information, please contact: Ellen A. Drost, California State University Los Angeles, USA: edrost@calstatela.edu) 
The Entrepreneurial Behavior and New Product Developments of Family Firms: Does Stewardship Orientation Manager Matter?

Chia-Jung Lee, National Taiwan University

Cheng-Yu Lee, Southern Taiwan University

Hsueh Liang Wu, National Taiwan University

There is much debate regarding entrepreneurial behavior of family firms. While some view family businesses as particularly supportive of corporate entrepreneurship, others see family businesses as being conservative and resistant to change. Drawing on stewardship perspective, this study aims to argue stewardship orientation, in terms of decision comprehensiveness and long-term orientation differentiate the most entrepreneurial family firms. Based on a sample of 290 Taiwanese family firms, the result shows that stewardship orientation drives managers to initiate explorative activities which attribute to the outcome of new product development. Additionally, our finding suggests stewardship orientation influences new product development performance through explorative behavior. Our study extends entrepreneurship literature by highlighting the importance of managers' stewardship orientation in driving the entrepreneurship outcome of family business. (For more information, please contact: Chia-Jung Lee, National Taiwan University, Taiwan: Icj73828@gmail.com)

\author{
Internalizing Legitimacy: The Indirect Link between Perceptions of Entrepreneurship Legitimacy and \\ Entrepreneurs' Innovative Behavior \\ Mihaela Dimitrova, University of Wisconsin-Milwaukee \\ Dilek Gulistan Yunlu, University of Wisconsin-Milwaukee \\ Victor P. Lau, Hang Seng Management College \\ Margaret Shaffer, University of Wisconsin-Milwaukee \\ Tzvetan Davidkov, Sofia University "St. Kliment Ohridski" \\ Desislava I. Yordanova, Sofia University "St. Kliment Ohridski"
}

We develop a model of entrepreneurs' internalization of legitimacy. We suggest that the more entrepreneurs perceive that society values entrepreneurship and has legitimized its existence (i.e., perceived social status of entrepreneurship) the more they will exhibit innovative behaviors as a way to preserve their own legitimacy. However, based on social cognition theories, we propose that this relationship is indirect and that the internalization mechanism includes perceptions of personal legitimacy (i.e., reputation and access to resources) and personal growth. We test the proposed model on multi-source pair-matched samples of Bulgarian and Chinese entrepreneurs and their subordinates. We find general support for our hypothesized relationships. While we do propose a universal model, we also explore possible differences between Bulgarian and Chinese entrepreneurs. (For more information, please contact: Mihaela Dimitrova, University of Wisconsin-Milwaukee, USA: mihaela@uwm.edu)

\title{
International Entrepreneurship and the Theory of Structuration: When is Entrepreneurship Truly Transformative? \\ Shazia Karmali, University of Victoria
}

This paper explores comparative entrepreneurship through Giddens' theory of structuration in order to provide an alternative approach to comparing entrepreneurship across national contexts, within the broad umbrella of international entrepreneurship research. By introducing the notion of the transformative capacity of entrepreneurship, this study challenges the existing outcomes of entrepreneurship to include social change, thereby broadening the range of activities that may be compared across borders, yet still be considered 'entrepreneurship'. An illustrative comparison of female entrepreneurs in the contexts of the United States and Uzbekistan is provided. A model for the transformative capacity of entrepreneurship is proposed. (For more information, please contact: Shazia Karmali, University of Victoria, Canada: skarmali@uvic.ca) 
Session: 1.1.15 - Interactive

Track: Track: 7 - Emerging Economies

\section{Corporate Governance and Sustainable Development in Emerging Markets}

Presented On: July 4, 2013 - 09:00-10:15

Chair: Xiaohui Liu, Loughborough University

Funding Social Sector Activity in Brazil: A Case Study Analysis of Agency Risk Incidence and Mitigation Katharina Agnes Dragon, Fundacao Getulio Vargas

Luís Henrique Pereira, Fundação Getulio Vargas

Recently, two innovative funding models have entered the Brazilian market in order to finance social enterprises (SEs) and social sector agents: an impact-investing venture capital (VC) fund and an independent social fund (SF). This study analyses both funds by linking the problem of financing social sector activity to traditional business theory. By using a case study approach, it is found that the agency risk explains the practices and the contractual design employed by both funding entities independent from their own, and the legal structure of funding recipients. (For more information, please contact: Katharina Agnes Dragon, Fundacao Getulio Vargas, Brazil: katha_dragon@gmx.de)

Are Foreign Governance at Work to Guard Against Corporate Financial Fraud in Transition Economies: Evidence from China

Daphne W. Yiu, Chinese University of Hong Kong

William P. Wan, City University of Hong Kong

Yuehua Xu, Sun Yat-Sen University

Using bivariate probit analysis of 658 observations on Chinese listed firms during 2000 to 2005, we examine if foreign governance mechanisms, newly emerged in transition economies, are at work to guard against corporate financial fraud. We identify three types of foreign governance mechanisms -foreign ownership, foreign partnership, and use of foreign auditor - and found that they are negatively related to the likelihood of committing corporate financial fraud. In addition, we found that such relationships are moderated by two institutional factors that are salient in the context of China, namely, central government control and provincial law development. Our findings provide insightful implications to corporate governance research and policy makers in transition economies. (For more information, please contact: Daphne W. Yiu, Chinese University of Hong Kong, Hong Kong, SAR-PRC: dyiu@cuhk.edu.hk)

\section{Chinese Enterprises in Africa: Transferring 'Yellow River Capitalism' to Africa and Its Implications} Connie Shao-mei Zheng, Deakin University

The paper discusses the extent of Chinese business involvement in Africa in recent years. Economic, social and political implications are analysed from a macro perspective. At micro level, international managerial challenges for Chinese enterprises operating in Africa are outlined. To build successful and sustainable businesses in the broader African society, Chinese enterprises need to focus on host country nationals' skill training and development and closely adhere to local employment laws and labour practices. They also need to adopt a new dimension of cross-cultural management which blends the Confucian values and Ubuntu ethos. The aim of such a crossvergent managerial approach is to build an ideal type of international organization that would help balance local African employees' work and life whilst achieving sustainable international businesses. (For more information, please contact: Connie Shao-mei Zheng, Deakin University, Australia:

connie.zheng@deakin.edu.au) 


\section{Ethical Decision-Making of Purchasing Professionals in Taiwan and China \\ Yi-Hui Ho, Chang Jung Christian University \\ Chieh-Yu Lin, Chang Jung Christian University}

Purchasing professionals are recognized for their key role in keeping long-term relationships with suppliers. In the running of purchasing practices, ethics have been considered to be an essential requirement in maintaining a good buyer-supplier relationship. Considerable attention has been paid on ethical issues in purchasing management. Nevertheless, only a few studies analyzed ethical decision-making of purchasing professionals, and none of them provided a thorough analysis on purchasing professionals' ethical decision-making. Most of them merely focused on a specific component of the ethical decision-making process. This paper attempts to explore the ethical decision-making of purchasing professionals in Taiwan and China. The study used a questionnaire survey to explore the differences in ethical decision-making between purchasing professionals in Taiwan and China, and analyze the associations between ethical decision-making of purchasing professionals and some individual and organizational factors. The Multidimensional Ethics Scales with purchasing-specific scenarios were used to measure purchasing professionals' ethical decision-making. Research findings reveal significant differences in ethical decision-making between purchasing professionals in Taiwan and China, and significant associations between ethical decision-making of purchasing professionals and individual and organizational factors. (For more information, please contact: Chieh-Yu Lin, Chang Jung Christian University, Taiwan: jylin@mail.cjcu.edu.tw)

\section{Corporate Social Responsibility and CEO Compensation: Evidence from China \\ Jie Zhang, Peking University \& China HuaRong Asset Management Co., Ltd. Chang Song, Renmin University of China \\ Xiyou Liu, Peking University}

Corporate social responsibility (CSR) is a dramatically expanding area of activity for managers and academics. The article explores the question of whether CEO remuneration is properly structured to provide incentive to the CEO to improve firm CSR. We also examine the extent to which the interaction of firm CSR and corporate governance determines remuneration. Our sample consists of firms from China firms from 2007 to 2008. Our results suggest that firm CSR is relevant to remuneration schemes. Specifically, we found a significant, positive main effect of firm CSR on CEO remuneration. Further, we found that state control and director shareholding typically strengthen the rewards CEOs may get for increasing levels of CSR performance. However, we do not find the effects of independent director on the relationship between CEO remuneration and firm CSR. (For more information, please contact: Jie Zhang, Peking University \& China HuaRong Asset Management Co., Ltd., China: jacqueline35@sina.com)

\section{It Takes Two to Tango: Exploring the Principal-Agent and Principal-Principal Problems among Public Family Businesses \\ Qian Gu, Georgia State University \\ Lin-Hua Lu, National Cheng Kung University}

Despite the increased attention to professional management in family businesses, current research drawing on agency theory has largely focused on the principal-principal conflicts (P-P conflicts) in the study of family business performance. The influence of principal-agent conflicts (P-A conflicts) that arise from the involvement of professional management stays unclear. In this study, we apply a multi-agency perspective and propose that both agency problems are at play in family businesses. P-A conflicts will predominate when a firm has a low level of family ownership, and P-P conflicts will prevail in firms with dominant family ownership. As a result, there is an inverted U-shaped relationship between family ownership and firm performance. In addition, we investigate two major contingencies that moderate the curvilinear relationship: interest alignment mechanism and control mechanism. We propose that the inverted U-shaped relationship will be more salient with a higher 
level of professional manager ownership and a lower level of professional manager control. Our argument has received overall support with a sample of 487 public listed family firms in Taiwan from 1996 to 2005. (For more information, please contact: Qian Gu, Georgia State University, USA: qgu@gsu.edu)

\author{
Does Environmental Management Help Foreign-Invested Firms Outperform in an Emerging Economy? \\ Nahyun Kim, Korea University \\ Jon Jungbien Moon, Korea University \\ Haitao Yin, Shanghai Jiao Tong University
}

Does environmental management help foreign-invested firms outperform local firms in emerging economies? Existing research suggests that environmental management may or may not benefit firm performance, but the question is particularly under investigated in emerging economies. This study explores whether foreign-invested local firms that can access better environmental management systems outperform comparable local firms in an emerging economy. In making this comparison, we use the propensity score matching along with difference-indifferences approach to handle the endogeneity problem inherent in foreign firms' investment decisions. By using financial and environmental data from China for the period 1998-2009, we find evidence that foreigninvested firms perform better than local firms when firms are under high environmental pressure. (For more information, please contact: Jon Jungbien Moon, Korea University, Korea, South: jonjmoon@korea.ac.kr)

\title{
Session: 1.2 - Plenary
}

\section{AIB Fellows Eminent Scholar Award Session: Honoring David Teece}

Presented On: July 4, 2013 - 10:55-12:10

Chair: Pankaj Ghemawat, IESE

Keynote Speech: "Dynamic Capabilities and Entrepreneurship in the Multinational Firm"

David Teece, University of California - Berkeley

Commentary: "Dynamic Capabilities and the Future of the Global Factory"

Peter ] Buckley, University of Leeds

Commentary: "Dynamic Capabilities in International Cooperative Agreements " Stephen Tallman, University of Richmond

Commentary: "Mechanisms of Governance and Dynamic Capabilities in the Multinational Enterprise" Alain Verbeke, University of Calgary

Presentation of the AIB Eminent Scholar Award

Alan M. Rugman, University of Reading

(For more information, please contact: Alain Verbeke, University of Calgary, Canada:

alain.verbeke@haskayne.ucalgary.ca) 
Session: 1.3.1 - Panel

Track: Track: 1 - Institutions, Governance, and CSR

\section{Transnational Regulatory Integration and Development: The Role of Business, Government, and NGOS}

Presented On: July 4, $2013-13: 20-14: 35$

Chairs: Gerald Andrew McDermott, University of South Carolina; Jonathan Doh, Villanova University

Discussant: Rajneesh Narula, University of Reading

Panelists:

Gerald Andrew McDermott, University of South Carolina

Jonathan Doh, Villanova University

Rafael Lucea, George Washington University

Yi Jia Kathleen Low, WU Vienna

Institutional perspectives have been adopted by international business scholars as a primarily lens through which to view cross-border phenomenon (Kostova \& Roth, 2002). At the same time, scholars in political science, sociology, and economics have focused attention on the ways in which the integration of nations, and particularly via the transnationalization of markets, create both new forms of transnational public and private regulation and reshape domestic paths of institutional change and firm behavior. In this panel, we seek to leverage institutional perspectives as adopted by IB scholars, and integrate them with those of political scientists and sociologists, to inform - and gain insight into - the process of transnational regulatory integration and upgrading. The panel will examine the emerging patterns of transnational public and private regulation, the role of business, government and NGOs in the process of regulatory diffusion and upgrading, with special emphasis on the impact of this process on paths of institutional and economic development in emerging market countries. (For more information, please contact: Gerald Andrew McDermott, University of South Carolina, USA: gerald.mcdermott@moore.sc.edu)

\section{Session: 1.3.2 - Panel}

Track: Track: 3 - IB Theory, FDI, and Entry Mode

\section{Regionalization and the Multinational Enterprise: The Emerging Issues}

Presented On: July 4, $2013-13: 20-14: 35$

Chairs: Ricardo Flores, University of New South Wales; Jin Uk Kim, University of Illinois at Urbana-Champaign Discussant: Alan M. Rugman, University of Reading

Panelists:

Ruth Aguilera, University of Illinois at Urbana-Champaign

Jin Uk Kim, University of Illinois at Urbana-Champaign

Jean-Luc Arregle, EMLyon Business School

Christian Asmussen, Copenhagen Business School

Bo Bernhard Nielsen, Copenhagen Business School

Tom Osegowitsch, University of Melbourne

Andre Sammartino, University of Melbourne

Rian Drogendijk, Uppsala University

Research on regionalization has advanced substantially since its initiation in the early 2000s and the current panel aims to add steam to this momentum by providing a platform for scholars to discuss the emerging issues 
and conspicuous caveats. Specific questions that will be addressed include: i) how do firms conceptualize regions and how does this affect their multinational activities; ii) does regionalization provide opportunities for new theorizing or is it merely a new context for old theories; iii) what is the relationship between homeregionalization and firm internationalization in general? The five presentations in the proposed panel will address these questions and stimulate insightful discussions that will take research stream to the next level. (For more information, please contact: Jin Uk Kim, University of Illinois at Urbana-Champaign, USA: jkim198@illinois.edu)

\section{Session: 1.3.3 - Panel \\ Track: Track: 3 - IB Theory, FDI, and Entry Mode}

\section{About Time: Putting Process Back into Firm Internationalisation Research}

Presented On: July 4, $2013-13: 20-14: 35$

Chairs: Niina Nummela, Turku School of Economics and Catherine Welch, University of Sydney

Panelists:

Sascha Fuerst, Universidad EAFIT

Thomas Hutzschenreuter, WHU Otto Beisheim School of Management

Marian Vanessa Jones, University of Glasgow

Peter Wayne Liesch, University of Queensland

Jan-Erik Vahlne, University of Gothenburg

Our aim with this panel is to advance understanding of how to study internationalisation as a process. While the internationalisation process of the firm is a well-established focus of inquiry in IB, we argue that the majority of past research has in fact not studied the phenomenon as a process. The panel takes a critical view of existing research on the internationalisation process of the firm and provides novel insights to how the process evolves over time, how it can be studied as a process, and which methodological and theoretical innovations should be introduced to increase our understanding. In order to encourage diverse viewpoints in the panel discussion, our panellists include both well-known and early-career scholars, as well as experts in different methodological approaches to internationalisation process research. (For more information, please contact: Niina Nummela, Turku School of Economics, Finland: niina.nummela@utu.fi)

\section{Session: 1.3.4 - Panel}

\section{Track: Track: 9 - Cross-Cultural Management and HRM}

\section{Academics on the Move: Advice for Junior Academics Developing Boundaryless Careers}

Presented On: July 4, $2013-13: 20-14: 35$

Chair: Majid Ghorbani, Renmin University of China

Panelists:

Sinan Caykoylu, Simon Fraser University

Masud Chand, Wichita University

Yuan Liao, University of New South Wales

Masoud Shadnam, Rouen Business School

Despite scholarly expertise in the area, international business academics still experience culture shocks and other surprises after moving across borders. Academics who take positions across national boundaries face 
additional challenges, such as difficulty forming mentorship relationships with colleagues or students (Richardson \& Zikic, 2007), indicating that scholars considering an international move would benefit from better preparation. Just as institutional pressures push universities to hire internationally-oriented faculty, faculty members themselves may also be experiencing increasing pressure to develop boundaryless academic careers. In fact, academics are an example of the broader shift in careers from traditional to boundaryless, with increased emphasis on personal development, over institutional loyalty (Arthur, 1994). In this panel, we will discuss challenges and coping strategies that junior academics experience when starting their academic careers in foreign countries. Participants will receive both general and region-specific tips about managing the early stages of this process, starting from applying to positions, and ending with the first year experience settling into the new position. The panel will combine a full-panel discussion with region-specific roundtables. (For more information, please contact: Stacey R. Fitzsimmons, Western Michigan University, USA: stacey.fitzsimmons@wmich.edu)

\title{
Session: 1.3.5 - Competitive
}

\section{Track: Track: 2 - Marketing and Supply Chain}

\section{Country of Origin (COO) Broad Reach into Strategic Brand Decisions}

Presented On: July 4, $2013-13: 20-14: 35$

Chair: Ahmet H Kirca, Michigan State University

Country of Origin Effect: What We Know and What We Need to Know Ahmet Bayraktar, Rutgers University

This paper aims at evaluating the three-decades of country of origin research, discussing the robustness of previous findings and uncovering the issues that have not been addressed by the researchers. For this purpose, it offers a holistic view of country of origin effect, proposing a model that includes the antecedents, moderators and dependent measures appeared in the extant COO literature. Furthermore, it presents new terms to combine the dispersed and abundant definitions of COO. In addition, it introduces the research issues and questions that the future research should address. (For more information, please contact: Ahmet Bayraktar, Rutgers

University, USA: ahmetbay@pegasus.rutgers.edu)

\author{
Global Brands and "Made-In" Labels \\ Chol Lee, Sogang University \\ Johny K. Johansson, Georgetown University
}

Building on previous research on global brands and country-of-origin effects, this study is concerned with how brand globalness and manufacturing location combined affect consumer evaluations and intentions. In particular this study explores the question of the extent to which a brand's globalness can overcome the negative effect from an inferior manufacturing location. The research hypothesizes that consumers make a trade-off between brand globalness and off-shored manufacturing, penalizing global brands less than local brands for moving manufacturing to a less desirable country. Using data from consumers from Korea and USA, the results show that brand globalness effect can be as strong as country-of-origin effect. Also, brand globalness effect is found to be more powerful in developing countries with less favorable image than in developed countries. However, the country-of-manufacture effect is less strong in more global brand than in local brand. These findings show that strong global brand attenuates the negative impact from an inferior production location, making it less damaging for a more global brand to shift production to a country with a negative image. (For more information, please contact: Chol Lee, Sogang University, Korea, South: chollee@sogang.ac.kr) 
Responsible Sourcing and Country of Origin of the Source: Influence on Brand and Country Image

Srdan Zdravkovic, Bryant University

In this empirical study, authors utilize experimental design and a student sample to investigate whether promoting responsible (sweat-free) sourcing works and if the country where (i)responsible sourcing happens matters. Results provide evidence that doing good could also contribute to favorable financial performance. Attitudes toward brand practices, the brand itself, and the intent to purchase the brand is significantly higher for brands that exhibit sweat-free behavior than for brands that exhibit sweatshop behavior. Subjects in our study rewarded high level of care for the employees and punished corporate behavior that creates a working environment considered to be unacceptably difficult or dangerous. Our findings suggest the origin of the foreign brand practices does not contribute to the subject's evaluation of brand practices. Additionally, results show the cosmopolitan consumer is quick to punish the brands if what they have to offer is perceived as unfair or endangering foreign workers. Somewhat unexpectedly, we find that subjects exposed to sweat-free brand practice, who were highly ethnocentric, had a significantly better attitude toward brand practice than subjects who were less ethnocentric. Finally, public policy makers and brand managers who plan to exploit the "made-in" label might be interested in findings that news regarding worker-related practices help build, not only brand, but also country image. (For more information, please contact: Srdan Zdravkovic, Bryant University, USA: szdravko@bryant.edu)

The Blame Game Post an Organisational Crisis: A Cross-National Study on the Role of Country of Origin Roberta Veale, University of Adelaide Vinh Nhat Lu, Australian National University Ke Chen, University of International Business and Economics

Product crises are increasingly more common, often related to imported products. Nevertheless, the influence of the country image associated with the foreign manufacturer in a crisis management context is significantly under researched. This study investigates the ability of home country image of an organization to influence attribution of blame and trust, both antecedents of consumer future purchase intentions, post a crisis. We utilized a two (country) $x$ three (crisis type) experimental design and collected data from 852 consumers in Australia and China. We found the organization originating from the country with the more favorable country image was held significantly less accountable than that with a significantly less favorable country image for two of the three crisis types tested, enjoying higher levels of trust and purchase intentions. These results reveal the potential benefits for organizations with positive country image, and the increased risks for organizations from countries with negative country image associations. (For more information, please contact: Vinh Nhat Lu, Australian National University, Australia: vinh.lu@anu.edu.au)

\section{Session: 1.3.6 - Competitive}

Track: Track: 6 - Innovation and Knowledge Mgmt.

\section{Learning Innovative Knowledge Management}

Presented On: July 4, $2013-13: 20-14: 35$

Chair: Gary Knight, Willamette University

MNEs' Learning from FDI Exit Experience - If and When It May Happen: Evidence from Korean MNEs Yuping Zeng, Southern Illinois University Edwardsville

Sangcheol Song, Saint Joseph's University

Soonkyoo Choe, Yonsei University 
This study is intended to fill the research gap of a lack of understanding of whether and under what conditions MNEs learn from their prior FDI exits. Drawing on literature on learning from failures, we argue that unintended prior FDI exits are effective stimulants of learning and this learning reduces subsequent subsidiary mortality. Furthermore, we identify four contingency factors that may moderate the effect of an MNE's FDI exit experience by influencing the magnitude of learning barriers the MNE faces and/or the MNE's ability to overcome these barriers. We test our hypotheses using a dataset containing the life history of FDIs by Korean MNEs during 1990 and 2006. We find a negative relationship between an MNE's FDI exit experience and subsidiary mortality, but this relationship only holds true when the cultural distance between the host countries of the exited FDIs and the MNE's home country is small, the percentage of JVs among the exited FDIs is low, the MNE's geographic scope is high, and peer MNEs' exit experience is low. (For more information, please contact: Yuping Zeng, Southern Illinois University Edwardsville, USA: yzeng@siue.edu)

\section{Exploring the Type and Locus of Embeddedness for Global Innovation \\ Kazuhiro Asakawa, Keio University \\ Bjoern Ambos, University St. Gallen}

This papers aims to shed light on the question how embeddedness impacts the potential of international R\&D subsidiaries to create innovations for the global - rather than the local - market. We examine the kinds (business vs. research) and the locus (local vs. global) of external ties that international R\&D subsidiaries maintain and test their relationship with the unit's global innovation potential drawing on a sample of 99 overseas R\&D subsidiaries belonging to Japanese multinational corporations. Our results reveal that certain ties enable overseas R\&D subsidiaries to pursue innovation for global markets more than others: Collaborating with overseas business organizations is positively related to subsidiary innovation for global markets; whereas ties with local business organizations turns out to be negatively associated with subsidiary innovation for global markets. While collaborating with research institutions is not directly associated with subsidiary innovation for global markets, such association becomes salient for subsidiaries with absorptive capacity. Our findings partly reconfirm the established wisdom on subsidiary embeddedness and innovation but also challenge the value of host market embeddedness vis-à-vis other forms of ties. (For more information, please contact: Kazuhiro Asakawa, Keio University, Japan: asakawa@kbs.keio.ac.jp)

\section{Learning Diversity and Product Innovation in an Emerging Economy Zhenzhen Xie, Hong Kong University of Science \& Technology Jiatao Li, HKUST}

Prior research has shown mixed findings on the relationship between international market diversification and a firm's innovation performance. Drawing on the learning theory and organizational diversity literature, we argue that one reason for explaining this mixed finding could be the confusion of combining a positive effect of "knowledge variety" and a negative effect of "knowledge separation" in international market diversification. Firms that compete in both domestic and overseas markets can have a better opportunity to build a diverse set of knowledge, the novel combination giving rise to innovation. However, when the two markets are highly separated from each other, there tends to be substantial difficulties in knowledge transfer, sharing and integration. We expect that firms that compete in less separated domestic and overseas markets benefit most in innovation performance. Data on 6,805 Chinese auto manufacturers generally supported our arguments. Our research contributed to the growing literature on organizational learning, innovation of emerging economy firms, and global strategy. (For more information, please contact: Zhenzhen Xie, Hong Kong University of Science \& Technology, Hong Kong, SAR-PRC: xiezz@ust.hk) 


\section{Session: 1.3.7 - Competitive}

\section{Track: Track: 5 - MNC Management and Organization}

\section{Communication Inside and Outside MNEs}

Presented On: July 4, $2013-13: 20-14: 35$

Chair: Felipe Monteiro, INSEAD

How MNCS May Improve the Efficiency of Language Communications and its Global Competitiveness: Implications from a Laboratory Study

Minyoung Kim, University of Kansas

Midam Kim, University of Kansas and Northwestern University

Ann Bradlow, Northwestern University

Tailan Chi, University of Kansas

When a firm conducts business across borders to exploit location, scale or scope economies, it inevitably encounters nontrivial costs of language communication that can dissipate the value that it attempts to create through its international activities. As such, mitigating the detrimental influences of language distance has important performance implications for any multinational corporation (MNC). Applying recent theories in psycholinguistics, this paper investigates the relationship between language distance and communicative efficiency and, more importantly, the moderating role of linguistic convergence on the relationship. Empirical findings corroborate the main thesis of the paper that language distance is detrimental to communicative efficiency but linguistic convergence between talkers can mitigate this detrimental effect. Implications on the MNC as a superior vehicle for the transfer of knowledge are also discussed. (For more information, please contact: Tailan Chi, University of Kansas, USA: chi@ku.edu)

Friend or Foe? The Effects of English as Lingua Franca on Cooperative versus Competitive Choices - An Experimental Study

Katrin Muehlfeld, Utrecht University

Diemo Urbig, University of Wuppertal

Arjen van Witteloostuijn, Tilburg University

Vivien Procher, University of Wuppertal

This study develops and tests theory about the multifaceted causal effect of foreign language on individuals' cooperative behavior and confidence therein. Based on mediation and moderation arguments, we disentangle two mechanisms: Language-induced information barrier and language-related cultural accommodation/alienation effect. While language proficiency mitigates the information barrier effect identified through the mediating lack of understanding, a larger 'cultural accommodation gap' ? a novel, individual-level concept ? enhances the direct culture-related language effect. Results of a randomized experiment with 238 Dutch participants support our predictions: Using a foreign language (here, English) affects cooperation through both mechanisms, and moderators display the predicted signs. (For more information, please contact: Katrin Muehlfeld, Utrecht University, Netherlands: k.muehlfeld@uu.nl)

Perceived Global Mindset: The Effect of National Social Identities and Demographic Characteristics

Orly Levy, IMD Business School

Sully Taylor, Portland State University

Schon Beechler, INSEAD

Nakiye A. Boyacigiller, Sabanci University

Maury Peiperl, IMD 
Drawing on social identity theory, this study examines the influence of national social identity on perceived global mindset, defined as the judgment that the multinational company (MNC) offers all employees the same career and promotion opportunities irrespective of nationality or location. Using survey data from 2,039 employees in seven MNCs, our analysis reveals two significant findings. First, perceived global mindset is significantly lower among host and third country nationals compare with parent country nationals. Second, these perception gaps increase with three moderating factors: gender, tenure, and education, although these results are inconsistent. These findings indicate that the national social identity of employees is salient in the context of the MNC, thereby affecting sense-making and perceptions of global mindset (For more information, please contact: Orly Levy, IMD Business School, Switzerland: orly.levy@imd.ch)

\title{
Business Diplomacy in MNCs: An Exploratory Study \\ Huub Ruel, Windesheim University of Applied Sciences/University of Twente \\ Tim Wolters, University of Twente \\ Raymond Loohuis, University of Twente
}

In order to survive, multinational corporations (MNCs) need the ability to manage complex interactions with governments, while at the same time they should be sensitive to the wishes and demands of the increasing number of local and international NGOs. Although the relevance of business diplomacy is evident, it is not clear how MNCs actually conduct business diplomacy. This research contributes to the existing literature by answering the question: How is business diplomacy enacted by and embedded in MNCs? Based on a multidimensional operationalization of business diplomacy semi-structured interviews were carried out with eight MNCs. The findings showed that seven of the eight MNCs conduct business diplomacy intensively. None of them applied a clear and organization-wide business diplomacy policy. Instead, general guidelines existed in five MNCs. In all MNCs, the responsibility for business diplomacy was largely decentralized to the foreign subsidiaries. All MNCs deployed a wide range of business diplomacy means: direct stakeholder dialogues, events, forums, meetings, industry associations, social projects and social partnerships. The findings further suggest that industry-specific factors affect business diplomacy intensity of MNCs and that companies operating in countries with weak institutions recognize the importance of business diplomacy more and hence implement it more intensively. (For more information, please contact: Huub Ruel, Windesheim University of Applied Sciences/University of Twente, Netherlands: huubruel@hotmail.com)

\section{Session: 1.3.8 - Competitive}

\section{Track: Track: 9 - Cross-Cultural Management and HRM}

\section{HR Issues in Transnational and MNC Contexts}

Presented On: July 4, $2013-13: 20-14: 35$

Chair: Elaine Farndale, Pennsylvania State University / Tilburg University

\author{
Developments in Knowledge Transfer Activities of Expatriates and Inpatriates \\ Anne-Wil Harzing, Melbourne University \\ Markus Pudelko, Tuebingen University \\ Sebastian Reiche, IESE
}

Drawing on the knowledge-based view of the firm, this paper provides the first empirical study that explicitly investigates the relationship between different categories of international assignees and knowledge transfer in MNCs. Specifically, we examine the changes in patterns of international staffing over time as a means to support MNC knowledge flows; the relative prevalence of one particular group of international staff, parent country national (PCN) expatriates, across a variety of functional areas; and the relative extent to which different categories of international assignees contribute to knowledge transfer from and to headquarters. We 
base our investigation on a large scale survey, encompassing data from more than 800 subsidiaries of MNCs in thirteen countries. We find that the proportion of PCNs has declined over time, whereas the proportion of third country nationals (TCNs) and former inpatriates has increased; demands for knowledge transfer differ according to the functional area and international staffing responds to these differing demands; the relevance of PCNs and former inpatriates varies for knowledge flows from and to headquarters. (For more information, please contact: Markus Pudelko, Tuebingen University, Germany: markus.pudelko@uni-tuebingen.de)

\section{Financial Incentivization in Transnational Contexts \\ Chris Brewster, University of Reading \\ James Walker, University of Reading \\ Geoffrey Wood, University of Warwick \\ Elena Beleska-Spasova, University of Reading}

We study the relative utilization of share ownership schemes and individual performance related pay paying particular attention to context and whether or not a firm is a multinational subsidiary. The international business literature on institutions has focused more on the implications for firms of entering and operating in a particular context, rather than the nature of national institutional frameworks and which specific sets of organizational practices are clustered where. We show that firms operating in differing capitalist economies exhibit large and significant differences in how they incentivise their employees financially. We also show that MNC subsidiaries are more likely to use incentives. The findings indicate that a more nuanced understanding of transnational contexts in general, and of capitalist varieties in particular, would expand scholars' understanding of international business. (For more information, please contact: Chris Brewster, University of Reading, United Kingdom: c.j.brewster@reading.ac.uk)

The Role of Parent-Country Nationals in Reducing the Adverse Effect of Perceived Value Inconsistency on the Commitment of Foreign Subsidiary Employees

Dana Minbaeva, Copenhagen Business School

Larissa Rabbiosi, Copenhagen Business School

Guenter K. Stahl, WU Vienna and INSEAD

Use of the parent country nationals as work group leaders is an important tool for creating alignment within the MNC network. We argue that the parent country expatriates have a key role in mitigating the adverse effects of perceived values' inconsistency on affective commitment of employees in foreign subsidiaries, and ultimately MNC performance. Drawing on data from a global survey of 1567 work groups in a large European MNC, we demonstrate the adverse effects of perceived gaps in corporate core values and demonstrate that by acting as culture carriers and translators of corporate messages, parent country expatriates can create alignment around core espoused values and limit the negative consequences of values' inconsistency at subsidiary level. (For more information, please contact: Dana Minbaeva, Copenhagen Business School, Denmark: dm.smg@cbs.dk)

Are We There Yet? Comparing Adoption, Internalization, and Effectiveness of High-Investment HR Values among Local Firms and MNC Subsidiaries of U.S. Firms in South Asia

Saba Colakoglu, Berry College and Koc University

Matt Allen, Babson College

Khasro Miah, North South University

Allan Bird, Northeastern University

Grounded in institutional theory, this study investigates the differential adoption, internalization, and effectiveness of high-investment HR values by indigenous companies and by subsidiaries of U.S. firms located throughout South Asia. Results suggest that MNC subsidiaries have a higher rate of adoption of highinvestment HR values compared to indigenous firms and that both types of firms are similar in the level of 
internalization of their respective HR values. While high-investment HR values are related to company performance across the board, this relationship tends to be stronger for MNC subsidiaries compared to local South Asian firms. Theoretical and practical implications for the successful transfer, implementation, and diffusion of high-investment HR values, policies, and practices in institutionally distant contexts are discussed. (For more information, please contact: Saba Colakoglu, Berry College and Koc University, USA: scolakoglu@berry.edu)

\section{Session: 1.3.9 - Competitive \\ Track: Track: 8 - Developing Country MNCs}

\section{Understanding the Dynamics of Emerging Markets}

Presented On: July 4, $2013-13: 20-14: 35$

Chair: James M. Hagen, Hamline University

Experiential and Vicarious Learning of Emerging Market Multinational Enterprises: An Investigation of CrossBorder M\&As

Sophia Hamblin Wang, Australian National University

Yi Li, Australian National University

This paper proposes a conceptual model that looks to explore emerging market multinational enterprises' (EMNEs) engagement in cross-border mergers and acquisitions (M\&As) using an organizational learning lens. In light of the apparent disconnect between the capabilities generated by EMNEs through experience and learning and the engagement in high risk and high commitment internationalization behavior, this paper addresses the research question, 'when do experiential and vicarious learning sources affect cross-border M\&A behavior by emerging market firms?' Under organizational learning literature, firms can learn from internal sources through experiential learning from past behaviors or external sources to the firm through vicarious learning and mimicry of others. Such learning sources are proposed to impact on the M\&A behavior of firm. This paper provides institutional and industry moderators that influence the relative effectiveness of each learning source, which is the main theoretical contribution of the study. According to our model, home institutional development and industry change in globalization will alter the relative effectiveness of experiential learning sources and vicarious learning sources on the decision to engage in a cross-border M\&A. (For more information, please contact: Sophia Hamblin Wang, Australian National University, Australia: sophia.hamblinwang@anu.edu.au)

\section{Emerging Market MNEs, Foreign Establishment Mode and Strategic Asset Seeking John Anderson, University of Northern Iowa and Durham University Dylan Sutherland, Durham University}

The growing theoretical literature on emerging markets MNEs highlights their use of aggressive acquisitions, often to psychically distant, highly developed host countries, to obtain the strategic assets that they themselves lack. These acquisitions, it is suggested, have also intensified in the wake of the global financial crisis. There is, however, still no systematic empirical analysis of why different entry modes are used by EM MNEs, or of the impacts of the financial crisis. We address these gaps by exploring the motivations for the use of entry modes by EM MNEs, with a specific focus on Chinese FDI to the US. Our results show that China's private sector MNEs do indeed use acquisitions to acquire strategic assets and that this activity intensified in the post-financial crisis period. (For more information, please contact: John Anderson, University of Northern Iowa and Durham University, United Kingdom: j.r.anderson@durham.ac.uk) 
Financial Depth of the BRIC: The Russian Dimension

Andrei Kuznetsov, Lancashire Business School

Olga Kuznetsova, Manchester Metropolitan University

Yakov Mirkin, Institute of World Economy and International Relations

The purpose of the paper is to assess the financial depth of the Russian economy in the BRIC context. Russian financial market is evaluated by a set of key indicators that characterize the level of maturity of the national financial system in respect to international standards. This task is implemented through descriptive analysis of extensive international data generated from a time series covering the period of 1995-2010. The paper demonstrates that in comparison to other BRIC countries, the financial depth of the Russian economy may be characterised as inadequate. In the Russian financial market potential for growth is combined with exceptionally high risks. Insufficient depth undermines its long-term competitiveness and exacerbates its exposure to shocks in the international market. (For more information, please contact: Andrei Kuznetsov, Lancashire Business School, United Kingdom: akuznetsov@uclan.ac.uk)

Informality, Institutions, and MNEs in Russia, 1995-2009

Natalya Smith, University of Liverpool, ULMS

Ekaterina Thomas, Staffordshire University

Despite the increasing number of contributions in the literature on determinants of informality, the informal economy at the regional level especially in emerging markets has been hardly studied. This paper studies the determinants of informality in the context of international business taking as an example one of the largest transition economies, namely Russia. This article derives the size of the informal sector and exploits its variation across Russia in 1995-2009. We find institutions to play a crucial role in determining the size of the informality. Whilst a negative relationship is observed between labour market participation rate, taxes, openness to trade and rate of crimes committed in a sphere of the economic activity; state capture, privatisation, the level of unemployment and the presence of both national and multinational firms in a region are observed to drive informality. (For more information, please contact: Natalya Smith, University of Liverpool, ULMS, United Kingdom: natalya.smith@liv.ac.uk)

\section{Session: 1.3.11 - Competitive}

Track: Track: 11 - SMEs and Entrepreneurship

\section{International New Ventures: Focusing on the Entrepreneur}

Presented On: July 4, $2013-13: 20-14: 35$

Chair: Sui Sui, Ryerson University

The "Outsider" Entrepreneurs: The Role of Founders' Immigrant Status in the Interna-tionalization and Performance of High Technology New Ventures

R. Isil Yavuz, Ozyegin University

Harry Sapienza, University of Minnesota

Sri Zaheer, University of Minnesota

While it is well-established that founders influence strategies and performance in young companies, little is known about the influence of founders' immigrant status on the early internationalization or the survival and growth of new high technology ventures. Traditionally, it has been assumed that immigrant entrepreneurs are disadvantaged relative to local entrepreneurs because they have limited access to resources and markets in their adopted country. In this paper, we draw on the concept of the outsider, which has both positive and negative connotations, to assess the advantages and disadvantages faced by immigrant entrepreneurs. By 
drawing on the behavioral theory of the firm, we argue that although the outsider status may convey some disadvantages domestically, this very status may serve as a spur to encourage international efforts. We test our hypotheses using data from the Kauffman Firm Surveys on newly founded high-tech ventures in the U.S. from 2004 to 2008. Our results show that new high-tech ventures with outsider founders are more likely to internationalize early, but somewhat surprisingly, these international ventures are less likely to survive and grow relative to their native-founded counterparts that have internationalized. This suggests that immigrant entrepreneurs tend to be overconfident in their own ability to internationalize successfully. (For more information, please contact: R. Isil Yavuz, Ozyegin University, Turkey: isil.yavuz@ozyegin.edu.tr)

\title{
International New Ventures, Founder Gender, and Export Performance In Hyeock (Ian) Lee, Loyola University Chicago Yongsun Paik, Loyola Marymount University
}

With a sample of 4,240 Korean new and young ventures, this study explores the gender-international performance relationship of new ventures by investigating upstream and downstream firm-specific advantages (FSAs) and country-specific advantages (CSAs) as three mediators. Results show that male-owned new ventures, compared to female-owned firms, are more likely to maintain higher levels of both innovation and marketing capabilities. Further, innovation and marketing capabilities are positively associated with the international performance of new ventures represented by exports per employee from foreign markets. As a result, we find evidence that upstream and downstream FSAs mediate the relationship between gender and international performance of Korean new ventures. The country destination of exports by new ventures in their home region, on the other hand, is shown to positively affect the international performance, but gender is not directly associated with the home region destination of exports in foreign markets. (For more information, please contact: In Hyeock (Ian) Lee, Loyola University Chicago, USA: ilee1@luc.edu)

\author{
Desirability and Feasibility in Internationalization Intentions: The Impact of Psychological Distance \\ Daniela Bolzani, University of Bologna \\ Riccardo Fini, University of Bologna \\ Gian Luca Marzocchi, University of Bologna
}

In this paper, we model entrepreneur's intentions to internationalize based on their perceptions of desirability and feasibility of such opportunities and we test how psychological distance moderate these relationships based on insights of Construal Level Theory. Recognizing that the impact of entrepreneurs' intentions and perceptions characterize strategic choices especially in new and small firms, we test our model on a sample of 169 entrepreneurs, owners of 140 newly established, independent, technological firms. Results show that internaldriven elements of desirability and feasibility, such as attitudes and skills, rather than external-driven ones, such as social norms and external supports, drive internationalization intentions. Evaluation of internationalization under different conditions of temporal distance, i.e. in the long run vs. in the short run, modifies the relative importance of perceived desirability and feasibility on intention. We discuss potential implications for researchers, practitioners and policy makers interested in entrepreneurial internationalization. (For more information, please contact: Daniela Bolzani, University of Bologna, Italy: daniela.bolzani2@unibo.it) 


\section{Session: 1.3.12 - Interactive}

\section{Diving into Deep Learning! Some Classroom Creativity \& Insights}

Presented On: July 4, $2013-13: 20-14: 35$

Chair: Amanda E.K. Budde-Sung, University of Sydney

Understanding a Firm's Internationalization Process - An Abductive Inspired Learning Approach

Roger Schweizer, University of Gothenburg

At the AIB 2013 conference, we would like to present a novel teaching approach that we have tested with two classes, enabling students a better understanding for the challenges experienced by managers during a firm's internationalization. The teaching approach is inspired by the abductive research approach that combines the deductive and inductive models of proposition development and theory construction. The idea of the course is to start with letting the students make empirical observations, i.e. to study a firm's internationalization process. This will be done without any theoretical pre-understanding. Once, students have made the observations, we then try to make sense of the discovered with the help of the existing literature. The purpose of the approach is to overcome a classical dilemma in teaching; i.e. to bring in reality into the class room and to allow students to grasp the complexity involved in firms' internationalization on one hand and to work with various theoretical conceptualizations on internationalization on the other hand. (For more information, please contact: Roger Schweizer, University of Gothenburg, Sweden: roger.schweizer@handels.gu.se)

\section{Developing Global Mindsets via the China Study Tour Connie Shao-mei Zheng, Deakin University}

The purpose of this paper is to explore whether the 21-day China Study Tour as a part of the MBA course and related assessment tasks we have developed have helped students gain insights in developing global mindsets and cultural intelligence for doing business cross-culturally. It is believed, or mostly assumed that the ongoing China Study Tour run by the Deakin Graduate School of Business (Australia) would provide a distinct advantage for business students to develop relevant global management skills and equip them with knowledge and skills for future work challenges at the global stage. So far, however, it is unclear whether students indeed have developed global mindsets and cultural intelligence, which are two most important elements of skill sets for effective global managers operating in a range of cultural environments (Adler ad Bartholomew, 1992; Henthorne et al., 2001; Javidan et al, 2006; Hitt et al., 2007). Have students obtained these skills after completing the tour? What triggers the change of their behavior? How could we better instill those triggers that could help facilitate positive behavioral changes and enable future students to better prepare for their global assignments? These are some specific questions this paper is intended to explore. (For more information, please contact: Connie Shao-mei Zheng, Deakin University, Australia: connie.zheng@deakin.edu.au)

\section{Human Factors in Virtual Team Pedagogies: ICT based Learning in Multi--Campus IB Education Gabriele Suder, SKEMA}

This presentation aims to address the benefits but also limits of IT-based e-and m-learning in international business teaching, and the specific need to blend pedagogies and learning tools. The main experience referred to a pedagogical innovation, in worldwide pre-introduction of Office 365, Microsoft's cloud-solution, and its pretest for academic application, support by Microsoft Corp./International and the EU through the Jean Monnet Chair attributed to SKEMA Business School. It was based on two main drivers: (1) the need to test multicampus teaching on international level for SKEMA Business School in a field perfectly suitable, i.e., international business; (2) the access to leading technology used by international companies (Sharepoint/ LiveMeeting by Office 365), for distance projects through the SKEMA- Microsoft partnership. Students on the three sites were 
shortly taught face-to-face on each site and then, with limited onsite- support on each campus, asked to work across three continents on a semester-long final project using cloud-communication only. (For more information, please contact: Gabriele Suder, SKEMA, France: gabriele.suder@skema.edu)

\author{
Problem Based Teaching in International Management: A Political/Economic Risk Assessment Exercise \\ Paula S. Daly, James Madison University \\ Marion Owyar-Hosseini White, James Madison University \\ Daniel S. Zisk, James Madison University \\ David E. Cavazos, University of New Mexico
}

This article draws from the current literature to examine problem based learning (PBL) as a management education tool, and provides an example of how to incorporate PBL into an undergraduate international management course. Also included are an explanation of, and specific guidelines for, a PBL exercise focused on the analysis of "country risk" (political/economic risks) for potential foreign direct investment. Additionally, the benefits and unique challenges of successfully implementing this type of exercise are discussed. (For more information, please contact: Paula S. Daly, James Madison University, USA: dalyps@jmu.edu)

\title{
Encouraging and Assessing Chinese Students' Class Participation \\ Dirk C. Moosmayer, Nottingham University
}

Understanding stereotypically silent Chinese students is an important prerequisite for integrating them into interactive learning environments in business schools around the globe. I present experiences from introducing the assessment of students' class participation and discuss challenges and good practices with the audience. (For more information, please contact: Dirk C. Moosmayer, Nottingham University, China:

dirk.moosmayer@nottingham.edu.cn)

Segmenting College Students by Demographic, Psychological, and Situational Variables as Predictors of Their Intentions to Travel Abroad for a Volunteer Project

Sarath A. Nonis, Arkansas State University

Clint Relyea, Arkansas State University

Using a sample of 193 undergraduate students, this study attempts to develop profiles of students that are "not likely" "maybe," and "most likely" to participate on a travel abroad volunteer project. Those who were "mostly likely" to participate had travelled abroad before, demonstrated high levels of self-congruity and diversity seeking, and stated financial costs to be less of a constrain in participating in such a project. Discussion of findings and direction for future research is also provided. (For more information, please contact: Sarath $A$. Nonis, Arkansas State University, USA: snonis@astate.edu)

\section{Session: 1.3.13 - Interactive}

\section{Culture and Research Methodology}

Presented On: July 4, $2013-13: 20-14: 35$

Chair: Pavlos Dimitratos, University of Glasgow

On a New Approach to Measure Regional Culture Differences: Food Style and Macro Indicators Linghui Tang, College of New Jersey 
This paper develops a new model to measure regional cultural differences inside a country by combining macro indicators with local food style. Based on a survey conducted in China in 2006, we validate our new approach by comparing job satisfaction of high-skilled labor across regions. The empirical results indicate that a local food consumption pattern is a stronger indicator of regional groups than commonly used factors such as income, geography, race, and political ideology. (For more information, please contact: Linghui Tang, College of New Jersey, USA: tang@tcnj.edu)

\section{Global Entrepreneurship Monitor: General Framework and Methodology \\ Jose Ernesto Amoros, Universidad del Desarrollo \\ Niels Bosma, Utrecht University \\ Alicia Coduras, Universidad Antonio de Nebrija/ Global Entrepreneurship Research Association Jonathan Levie, Strathclyde University \\ Yana Litovsky, Global Entrepreneurship Research Association}

This document is an introduction to the Global Entrepreneurship Monitor (GEM) project and its conceptual framework and methodologies. The objectives of this work are to provide details on how GEM emerged, what is the theoretical foundation of its model and, the main project indicators. We explain what are the main sources of data and how GEM's methodology produces indicators related to entrepreneurship dynamics across countries. Additionally we make a comparative analysis with other databases related to new business creation and entrepreneurship indicators. Finally we highlight how GEM is helping scholars from multidisciplinary and international comparative studies that employ its data in their work. (For more information, please contact: Jose Ernesto Amoros, Universidad del Desarrollo, Chile: eamoros@udd.cl)

\section{In-depth Interviews in International Business Research: Multiculturality, Power \& Language \\ David Sapto Adi Guttormsen, Coventry University \\ Ling Eleanor Zhang, Hanken School of Economics}

Although qualitative method has become more accepted in international business research, it remains as marginalised and under researched. Very little is known about how researchers should conduct cross-national field studies, particularly when both researchers and informants exhibit multiculturality, i.e. having multicultural backgrounds, which has become more and more common in the globalised world. This paper analyses the language, culture, social categorisation and power issues involved in in-depth interviewing with expatriates and host country employees. We, two multicultural researchers with very different backgrounds, draw upon our personal interview experiences during four separate intercultural and cross-national field research projects in Mainland China, Hong Kong and South Korea. Through conducting a self-reflexive analysis, we uncover how language, culture, social categorisation influences the power relationship between the interviewer and interviewee and how such influence might influence researchers' interpretation of the interview data and even the theorising process afterwards. Methodological and theoretical implications are discussed. (For more information, please contact: David Sapto Adi Guttormsen, Coventry University, United Kingdom: david.guttormsen@coventry.ac.uk)

\section{Students as Subjects Revisited: Establishing Measurement Invariance in a Cross-Cultural Context Valentina V. Kuskova, NRU Higher School of Economics Irina O. Volkova, NRU Higher School of Economics Liudmila M. Cheglakova, NRU Higher School of Economics}

Theoretical and empirical examination of "students as subjects" topic has been generating discussion for over fifty years, with empirical support found for both proponents and opponents. With management research expanding internationally, the issue of cross-cultural adaptation of developed and validated scales becomes increasingly important. As student subjects provide convenient samples for cultural validation of newly 
translated scales, "students as subjects" becomes a topic of discussion again, now in international context. Given the theoretical development in values research, it appears that values could play an important role in explaining the differences between students and non-students, and perhaps reconciling the previously observed differences between the samples. Anchoring the comparison of students to non-students in the study of values, we conducted a multi-sample comparison of 21 scales using samples of working adults in US and Russia, and a student sample in Russia. All tested scales performed as expected in all three groups, manifesting excellent model fit and factor loadings. Differences between students and non-students were observed as expected theoretically, and conditions for measurement invariance were established for conducting cross-cultural scale validation. (For more information, please contact: Valentina V. Kuskova, NRU Higher School of Economics, Russia: vkuskova@hotmail.com)

\section{A Cross-Cultural Examination of Consumer Wait-Perception Bias: Russia and the U.S.A. Kristie Kay Seawright, Brigham Young University \\ Scott E. Sampson, Brigham Young University \\ David C. Howe, University of Utah \\ Loren Haynes Rich, Brigham Young University}

Despite the pressures for convergence in the global economy, cultures remain distinctive. While attention has been given to the need to adapt global operations to local contexts (Bartlett and Ghoshal, 2003), many organizations with multi-national operations tend to retain significant portions of operations systems developed in their country of origin (Ferner, Quintanilla, and Varul, 2001). Cross-cultural service operations- particularly those high in customer contact-are especially vulnerable to customer dissatisfaction. Time is an element of cultural differentiation that has been studied from multiple perspectives. The difference in perception of the passage of time and the experience of waiting are potentially discerning cross-cultural factors that can impact consumer perceptions of service quality. This study indicates the existence of cross-cultural differences in perceived wait time between U.S. and Russian consumers. Yet, the primary contribution of this research is the development of video-based experimental methodology that allowed for the study of cross-cultural differences in wait perception. Presentation of standardized video treatments allowed respondents to experience the exact same waiting activity in distant locations while providing control for elements that could pose potential alternative explanations. We have demonstrated methodology to overcome limitations of many traditional empirical and experimental methods in cross-cultural research. (For more information, please contact: Kristie Kay Seawright, Brigham Young University, USA: kseawright@byu.edu)

\section{Session: 1.3.14 - Interactive}

\section{Track: Track: 1 - Institutions, Governance, and CSR}

\section{Corruption and Legitimacy}

Presented On: July 4, 2013 - 13:20-14:35

Chair: Jean Boddewyn, Baruch College

Corruption, Multinational Enterprises and Institutions in Emerging Markets: An African Perspective John Manuel Luiz, University of Cape Town

Callum Stewart, University of Cape Town

The paper examines the responses of emerging market multinational enterprises (MNEs) to corruption in African markets in the context of institutional voids. Corruption is a source of uncertainty and additional transactional costs for MNEs internationalising into emerging markets and it necessitates a strategic response. While these responses are intended to mitigate the costs of corruption, existing research suggests that they may also have an effect on the institutional frameworks in host countries. The research employs a qualitative study of a sample 
of MNEs with experience in internationalising into Africa. The results of the research indicate that corruption in African emerging markets is pervasive and the result of institutional voids in these countries. MNEs believe that strategic responses to corruption in African markets are important to address corruption operationally in the host country. (For more information, please contact: John Manuel Luiz, University of Cape Town, South Africa: john.luiz@gsb.uct.ac.za)

\title{
Grease or Dust in the Wheels \\ Canan Mutlu, University of Texas at Dallas \\ Seung-Hyun Lee, University of Texas at Dallas
}

Through the lens of institutional theory, this paper examines the effect of firm-level bribery on firm performance. We shift the focus of the opposing arguments of "grease in the wheels" and "dust in the wheels" from macro level to micro level. We later argue that the effect of firm bribery on firm performance depends on the efficiency of the institutions, more specifically the prevalence of corruption in an economy. (For more information, please contact: Canan Mutlu, University of Texas at Dallas, USA: cxm101020@utdallas.edu)

\author{
Culture, Wealth, and Corruption: Economic Development's Mediating Role in Predicting Political Corruption \\ Nicholas D. Rhew, University of Memphis \\ Frances Fabian, University of Memphis \\ Robert Steinbauer, University of Memphis \\ Nolan Gaffney, University of North Texas
}

Corruption is a millennia old problem; however, researchers and international governing bodies have only recently shown serious concern. Since the 1990s, many studies have examined the antecedents and outcomes of corruption. This study builds on previous work, notably Husted (1999), by updating the discussion of the role of culture and wealth in predicting corruption. Rather than viewing wealth and culture as unrelated antecedents, this paper proposes and tests a model where economic development mediates culture's influence. Using data from 63 countries, mediation is tested using both the traditionally applied causal steps approach, and the more recently suggested bootstrapping method. Our findings suggest that both economic development and several cultural dimensions significantly predict corruption. Moreover, the effects of the individualism vs. collectivism, power distance, long-term vs. short-term orientation, and indulgence vs. restraint dimensions are significantly accounted for by economic development. (For more information, please contact: Nicholas D. Rhew, University of Memphis, USA: ndrhew@memphis.edu)

\section{Corruption in Multinational Enterprises: The Confluence of Corruption Culture Distance and Firm Core Values Vijay Sai Sampath, Pace University \\ Noushi Rahman, Pace University \\ Robert Vambery, Pace University}

This paper examines the effects of cultural distance between MNE home and host country, and organizational distance between MNE entry modes and MNE headquarters' core values, on corporate corruption. Institutional and agency theories help to explain why cultural and organizational distances will affect MNE corruption. The empirical analysis used data from 221 cross-border transactions by 85 MNEs that were sanctioned by the United States Securities Exchange Commission and the Department of Justice under the Foreign Corrupt Practices Act during 1978 through 2011. When home country corruption culture is lower than that of host country, we find strong statistical support for all hypotheses capturing main and moderating effects. (For more information, please contact: Vijay Sai Sampath, Pace University, USA: vs31371n@pace.edu) 
Unethical Behavior: The Interaction of Hofstede's Cultural Dimensions

Robert Steinbauer, University of Memphis

Nicholas D. Rhew, University of Memphis

Nolan Gaffney, University of North Texas

Many of Hofstede's cultural dimensions have been associated with unethical conduct, but studies deliver contradicting results due to isolated analysis or focus on specific unethical conduct. This paper provides a detailed overview of ethical decision making relevant to cross cultural research. It is proposed that each cultural dimension contributes to but does not determine unethical conduct. Hofstede's (2010) index values for 76 countries are analyzed to identify cultures that are more likely to act unethical out of self-interest or group pressure. (For more information, please contact: Robert Steinbauer, University of Memphis, USA: rstnbuer@memphis.edu)

\section{Sources of Moral Legitimacy for Multinational Enterprises: Hypernorms and Human Rights Denis Arnold, University of North Carolina, Charlotte}

Many MNEs and their subsidiaries confront legitimacy concerns grounded in the perception that they violate ethical norms. Integrative Social Contracts Theory (ISTC) was developed by Donaldson and Dunfee to provide a theoretical response to concerns regarding the possibility of ethical "hypernorms" for business. This develops a critique of ISCT by providing a critical assessment of each of its three classes of hypernorms. An alternative grounding for the moral legitimacy of MNEs is then provided by appealing to human rights theory and the institutionalization of a new international coporatate human rights regime. The paper concludes with a research agenda regarding the prospects for human rights policies and practices being utilized to enhance the moral legitimacy of MNEs. (For more information, please contact: Denis Arnold, University of North Carolina, Charlotte, USA: denisarnold@uncc.edu)

\section{Corruption Distance and its Effect on FDI to Latin America: A New Perspective Jose Godinez, University of Edinburgh \\ Ling Liu, University of Edinburgh}

Studies of corruption and its relationship with Foreign Direct Investment (FDI) have yielded mixed results; some have found that corruption deters FDI but others having found the opposite. This paper seeks to advance our knowledge of how corruption affects FDI by analysing corruption distance and its relationship with FDI into Latin America. The results show that FDI from countries with high corruption levels is not affected negatively by perceptions of corruption in host countries. On the other hand, firms based on countries with low levels of corruption are negatively affected by corruption in the host countries. Here we argue that firms used to operating in a corrupt home environment acquired the necessary tools to cope with this problem abroad or do not face pressure from stakeholders. Conversely, firms without such knowledge or with higher pressure to behave ethically restrict the amount of FDI devoted to highly corrupt locations abroad. We develop the concept of "corruption distance" to handle these distinctions. (For more information, please contact: Jose Godinez, University of Edinburgh, United Kingdom: j.r.godinez@sms.ed.ac.uk)

\section{Greasing the Wheels of Change: The Impact of Corruption on Firms' Innovation in Transition Economies Sorin M.S. Krammer, Groningen University}

Conventional wisdom indicates a negative relationship between corruption and economic activities. However, recent studies suggest heterogeneous consequences on individual firm performance, given differences in organizational structures, strategies, industry regulations and surrounding institutions. Using responses from 7,000 firms in 30 transition economies, this study examines the impact of bribes on firm innovation. Due to numerous and rapid changes experienced over the past decades, these countries exhibit anomic conditions 
propitious for mass propagation of corruption. I argue that in these anomic settings, bribes present as a more efficient alternative to introduce innovative products to markets. Secondly, this effect is accentuated by the existence of "organized" corruption, which reduces the overall financial burden and informational asymmetry that firms face in their local environments. Finally, the efficiency of bribes is mitigated by the quality of existing formal (control of corruption) and informal (trust) institutions. (For more information, please contact: Sorin M.S. Krammer, Groningen University, Netherlands: m.s.s.krammer@rug.nI)

\section{Session: 1.3.15 - Interactive \\ Track: Track: 10 - Economics, Finance and Accounting \\ Cross-Border Acquisition Strategies}

Presented On: July 4, $2013-13: 20-14: 35$

Chair: Olivier Bertrand, SKEMA

Transfer Pricing Manipulation and Profit Transfers of Multinational Firms

Necla V. Geyikdagi, Yeditepe University

Filiz Karaman, Yildiz Technical University

Turkish businessmen, politicians and most academicians tend to see foreign direct investment (FDI) as a remedy for the chronic lack of capital accumulation in their country. The meager FDI inflows which followed the Customs Union Agreement with the European Union, in 1995, created a deep disapointment among these persons. Efforts to attract foreign capital intensified since 2005 and inflows soared. However, a large part of the increase is the result of the Turkish government's privatization program of publicly owned companies, and the acquisition of private firms by large multinational firms rather than greenfield investments. This research investigates FDI inflows to Turkey and tries to analyze the transfer of profits earned by FDI with the possible presence of transfer pricing manipulation. (For more information, please contact: Necla V. Geyikdagi, Yeditepe University, Turkey: geyikdagi@superonline.com)

How has the International Harmonization of Financial Reporting Standards Affected Merger Premiums within the European Union?

Konstantinos Bozos, Leeds University

Yasanji Ratnaike, Leeds University

Malek Alsharairi, German Jordanian University

We examine the impact of IFRS adoption on merger premiums. Using a comprehensive database of M\&A deals within the EU during 2000-2012 we investigate the impact of overall IFRS adoption, the differences between voluntary and mandatory adopters and the role of the target country's pre IFRS accounting infrastructure and framework (absence of IFRS and IAS). We find that IFRS adoption by targets is generally associated with lower merger premiums. This decline is more pronounced in mandatory adopters and does not apply to voluntary adopters. In addition, we find that the further away the target's country standards are from IFRS, the stronger the negative impact of IFRS adoption on merger premiums. The results are robust to an exhaustive number of control variables and alternative model specifications, as well as across different subsamples. (For more information, please contact: Konstantinos Bozos, Leeds University, United Kingdom: kb@lubs.leeds.ac.uk)

Cross-border Acquisitions (CBAs) vs. Domestic Acquisitions: How do they Affect CEO Compensation? Omer Faruk Genc, Temple University 
Based on Agency Theory, CEOs are criticized for using acquisitions to increase their earnings through empirebuilding. On the other hand, stewardship theory argues that CEOs don't necessarily behave on their interests at the expense of shareholders. We examined how CEO compensation is affected from acquisitions by using 19,168 firms/acquisitions between 1995 and 2007. We find that the increase in CEO compensation is lower following the acquisitions compared to pre-acquisition period. However, companies with acquisitions still have a higher growth rate compared to non-acquisition companies. On the other hand, the incentive based compensation increases following the acquisitions. Different from the prior literature, we distinguished CBAs from domestic acquisitions and looked at whether the impact of them is the same. Our results suggest that CEO compensation increases less after CBAs compared to domestic acquisitions. Finally, the relatedness of acquirer and target does not have a significant influence on post-acquisition compensation. Our study provide fresh insights to CEO compensation literature, as we showed the impact of acquisitions can vary based on acquisition characteristics such as cross-border vs. domestic. (For more information, please contact: Omer Faruk Genc, Temple University, USA: omer.genc@temple.edu)

\title{
Market Reaction to Cross-border Mergers and Acquisitions Announcements of Chinese Multinationals Bo Fan, Southern New Hampshire University Lingling Wang, Southern New Hampshire University \\ Aysun Ficici, Southern New Hampshire University C. Bulent Aybar, Southern New Hampshire University
}

The paper examines market reaction to cross-border merger and acquisitions (M\&As) announcement of Chinese Multinationals (CHMNEs) during 2000 and 2010. The merger data was extracted from SDC Platinum data base and each of CHMNEs' stock prices and market index was extracted from Data Stream. By applying event study methodology, the paper examines $46 \mathrm{M} \& \mathrm{~A}$ announcements by CHMNEs cross 10 years from 2000 to 2010. We employ Event-Study methodology in order to explore market reaction to the announcements. Shanghai stock exchange is utilized in observing market reaction. The results suggest mostly negative market reaction to CHMNE M\&A announcements expect the intervals of $(-5,-3),(-5,0)$, and $(-5,+1)$, which illustrate significant positive market reaction. (For more information, please contact: Bo Fan, Southern New Hampshire University, USA: bo.fan@snhu.edu)

\author{
Cross-Border Acquisitions and Value Creation of Chinese Firms: Where Is the Beef? \\ Lei Li, Nottingham University \\ Agyenim Boateng, University of Nottingham \\ Xiaogang Bi, Nottingham University \\ Winnie Tu, Nottingham University \\ Zhenning Wang, Nottingham University
}

The recent surge of cross-border acquisitions of Chinese firms seems to challenge the explanatory power of the established theories. This article seeks to take stock of the relevant literature in management and finance in the recent decade. We argue that several key factors such as the pursuit of resource accumulation, the leverage of social capital and the impact of home country institutional environment are overlooked in the existing research but are essential for understanding Chinese firms' cross-border acquisition strategy. We develop a cogent conceptual framework to guide future research. (For more information, please contact: Lei Li, Nottingham University, China: lei.li@nottingham.edu.cn)

When Do Chinese Firms Acquire Companies in Advanced Economies? Industry- and Firm- level Evidence from Cross-border M\&As in Japan

Yuan-yuan Gong, Kyoto University

Balazs Fazekas, Kyoto University 
The aim of the paper is twofold, first it attempts to provide additional explanation for what triggers M\&As in developed countries by firms from emerging market. Secondly it attempts to explain influence factors on emerging market firms' choice of entry-mode into developed markets (M\&A or greenfield) by looking at emerging market firm specifics. Existing literature explains that emerging market firms internationalize in order to gain access to strategic resources, know-how and brand names and that in the case of China the government actively promote outward FDI flows since 1999. We pointed out that it is also important to consider host country's industry- and firm-level conditions and whether Chinese firms had inward foreign experience prior to their entry to developed markets. We collected data on Chinese firms M\&A activity targeting Japanese firms inside Japan in the period between 2002 and 2011. We tested our hypotheses on a sample of 75 cases of investment by Chinese firms and found that lower industry growth rates and higher inward international experience coincided with a higher likelihood of M\&A. (For more information, please contact: Yuan-yuan Gong, Kyoto University, Japan: lillian.gong@gmail.com)

\section{Emerging Market Firms' Acquisitions in Developed Countries: An Investigation of Consumer Trust Cher-Min Fong, National Sun Yat-Sen University Chun-Ling Lee, National Sun Yat-Sen University Hui-Wen Wang, National Sun Yat-Sen University}

This study develops an interdisciplinary model, which integrates research streams of conventional and new internationalization perspectives, $\mathrm{COO}$, MNE legitimacy, and consumer behavior, to address the largely unexplored issue regarding emerging market (EM) acquisitions in developed countries (DCs). The EM to DC acquisition is an expansion strategy that contradicts conventional internationalization theories. In this article, given that a cross-border acquisition is a cross-country firm strategy, the research model involve country-level, firm-level, and consumer behavior constructs that are interrelated. Through a three-phase experimental study, we evaluate consumer trust in EM firms' acquisition of DC firms: consumers exhibit lower trust in EM firms' acquisitions of DC firms because of EM firms with inferior reputation associated with their home countries-oforigin (COOs). However, consumer trust in EM to DC acquisitions improves when EM firms enter DCs through an acquisition joint venture or locate their R\&D activities in DCs prior to the acquisitions. (For more information, please contact: Chun-Ling Lee, National Sun Yat-Sen University, Taiwan: d934010005@student.nsysu.edu.tw)

\section{Session: 1.4.1 - Panel \\ Track: Track: 3 - IB Theory, FDI, and Entry Mode \\ Why Do Multinational Firms Exist? The Search for Theory or Paradigms for the Multinationality-Performance Relationship}

Presented On: July 4, $2013-14: 50-16: 05$

Chair: Farok J. Contractor, Rutgers University

Panelists:

Farok J. Contractor, Rutgers University

Alain Verbeke, University of Calgary

Chang Hoon Oh, Simon Fraser University

Harry P. Bowen, Queens University of Charlotte

Torben Pedersen, Copenhagen Business School

Multinational firms exist because internationalization augments profits over a considerable range and scope of international expansion. The IB field still apparently lacks a consensus on a unifying paradigm that would explain under what exact circumstances incremental international expansion would add to a firm's performance. A few scholars (e.g., Verbeke et al., 2009) have taken so agnostic a position as to assert that contingent firm- 
specific factors -- such as research or advertising intensity, product diversification, size of home nation, the pattern of geographic dispersal of the MNE and the outsourcing and offshoring of pieces of the value chain (Wiersema \& Bowen, 2010) - overwhelm any a priori theory or paradigm that tries to explain the multinationality $(M)$ - performance(P) relationship. Others, however (Contractor, 2012) argue that multiple contingent or firm-specific factors should not prevent the theorist from proposing M-P paradigms together with moderating variables. The search for appropriate paradigms has been accompanied by a search for appropriate methodologies (Verbeke \& Forootan, 2012) since past empirical studies have produced seemingly contradictory results (Kirca, et al., 2011). A panel of scholars who have extensively researched the subject will engage in a "debate" as to the existence of an underlying paradigm, the search for appropriate models and methodological conundrums. (For more information, please contact: Farok J. Contractor, Rutgers University, USA: farok@andromeda.rutgers.edu)

\section{Session: 1.4.2 - Panel}

Track: Track: 2 - Marketing and Supply Chain

\section{Contemporary and Future Research in International Marketing}

Presented On: July 4, $2013-14: 50-16: 05$

Chair: Saeed Samiee, University of Tulsa

Panelists:

Saeed Samiee, University of Tulsa

Daniel Bello, Georgia State University

Constantine Katsikeas, Leeds University

Bodo B. Schlegelmilch, Wirtschaftsuniversität-Wien

This panel is designed to feature contemporary thought in IM via five research presentations by several key international marketing (IM) scholars. Concurrently, these projects chart the course for future IM research in their respective domains. Specifically, four contributions to this panel cover two of the most widely pursued themes in IM, i.e., export-related issues and country of origin (CO), and are complemented by an overarching study aimed at exploring methodology-related knowledge structure in comparative marketing within IM. The first two research domains are essential and recurring themes and remain among the most popular topics for IM/IB researchers. Yet conceptual, methodological, and strategy-based progress in these domains has been slow. With a view to the future, the panel is aimed at shedding light on these important topics. The exporting projects address export-related strategy, channel and exporter-importer relationships, and performance. With CO's extensive literature, the CO contribution affords us the opportunity for a closer look at the essence of the concept and reconceptualize $\mathrm{CO}$ so that more relevant and meaningful research might emerge. The crosscultural/cross-national methodology knowledge structure research topic is aimed at assessing past approaches in the IM literature with an eye on strengthening methods used in comparative IM studies. (For more information, please contact: Saeed Samiee, University of Tulsa, USA: samiee@utulsa.edu) 
Session: 1.4.3 - Panel

Track: Track: 7 - Emerging Economies

\section{Turkey as a Place to do Business: Comparative Perspectives}

Presented On: July 4, $2013-14: 50-16: 05$

Chair: Ahmet H Kirca, Michigan State University

Panelists:

Nakiye A. Boyacigiller, Sabanci University

Tamer Cavusgil, Georgia State University

Pervez N. Ghauri, King's College London

Cuneyt Evirgen, Sabanci University

Liesl Riddle, George Washington University

The purpose of this panel is to unveil and discuss the practical and theoretical issues pertaining to doing business in Turkey with a focus on complex political, economic and cultural forces that shape the Turkish institutional environment in which local and foreign firms do business. In particular, our five panelists who have extensive knowledge of the Turkish business environment and/or they have made substantial contributions pertaining to our understanding of Turkish business environment and how firms operate in this context will discuss the country level, industry level, as well as firm level factors that affect how firms do business in Turkey. The panel will follow a roundtable discussion format to encourage discussion among panelists and with the audience. (For more information, please contact: Ahmet H Kirca, Michigan State University, USA:

kirca@msu.edu)

\section{Session: 1.4.4 - Panel}

Track: Track: 6 - Innovation and Knowledge Mgmt.

\section{Advancing Research on International Aspects of Innovation Ecosystems, Consumer Benefits, and Other Demand-Side Perspectives}

Presented On: July 4, 2013 - 14:50-16:05

Chair: Ronaldo Couto Parente, Florida International University

Discussant: Ana Siqueira, Duquesne University

Panelists:

Felipe Monteiro, INSEAD

Ram Mudambi, Temple University

Peter Williamson, University of Cambridge

Ulf Andersson, Copenhagen Business School

Tina Ambos, University of Sussex

Demand-side approaches to value creation represent a new, bourgeoning area in the fields of technology innovation, entrepreneurship, and strategic management, examining such topics as "innovation ecosystems" and "consumer benefits." Nonetheless, demand-side studies are scarce in the international business field. Important questions that remain include: What conditions facilitate the transfer of user-innovation knowledge in multinational organizations? How do multinational organizations drive cross-border innovation ecosystems? How do global nonprofit organizations and social enterprises support innovation ecosystems with cross-border collaborations? To what extent does collaboration within an innovation ecosystem enhance the internationalization prospects of emerging market multinationals? How might demand-based approaches help 
extend international business theories? This Panel starts with an overview of critical questions for research on the international aspects of demand-side perspectives. Panelists will offer insights into this emerging field of research. Participants will then break up into small groups facilitated by panelists and organizers. We will conclude with a collective discussion of insights gained and next steps for future research. (For more information, please contact: Ronaldo Couto Parente, Florida International University, USA: rcparent@fiu.edu)

\section{Session: 1.4.5 - Competitive}

\section{Track: Track: 4 - Strategy, Alliances, and Competitiveness}

\section{Knowledge Management in International Alliances}

Presented On: July 4, 2013 - 14:50-16:05

Chair: Klaus E. Meyer, China Europe International Business School

\section{International Expansion of Emerging Economy Firms: Coping with Exploration across Institutional and Product Boundaries}

Majid Abdi, University of Melbourne

Preet S. Aulakh, York University

International expansion entails boundary-spanning search behaviors to draw upon new knowledge in institutional and product domains. To effectively confront with challenges of exploration in these two domains, a firm may span organizational boundaries by accessing relational resources of foreign partners. Our paper examines the role of relational resources in facilitating (undermining) the institutional and product exploration strategies in the course of international expansion. We argue that due to disparities in the nature of institutional and product explorations, reliance on relational resources has asymmetric impact on performance consequences of these two strategies. Whereas reliance on relational resources furthers the benefits conferred from exploration in the institutional domain, benefiting from product exploration in foreign markets stands on reducing the dependence on relational resources and internalization of international activities. Empirical results based on 234 exporting firms in three emerging economies support our theorized relationships. (For more information, please contact: Majid Abdi, University of Melbourne, Australia: majid.abdi@gmail.com)

Governance Structure and the Creation and Protection of Technological Competencies: International R\&D Joint Ventures in China

Jiatao Li, HKUST

Zhenzhen Xie, Hong Kong University of Science \& Technology

International research and development (R\&D) joint ventures have been widely used by multinational enterprises (MNEs) to enhance knowledge creation in a global network of foreign subsidiaries while at the same time, managing the critical task of protecting the knowledge created. In emerging economies where institutions for protecting intellectual properties are likely to be weak, equity joint ventures (EJVs) are often used to meet the needs for knowledge protection by aligning the interests of partners in cohabitation. However, the advantages of using EJVs come at a price-higher organizational cost and lack of flexibility. This study suggests alternative ways of creating and protecting technological competencies, i.e. through the choice of appropriate scope of R\&D activities and JV partners. We propose that MNEs can reduce their use of EJVs in creating and protecting their technological competencies when the scope of R\&D activities are limited to research-oriented ones or when academic institutions are chosen as local partners. Furthermore, a large cultural distance between the host and home countries of MNEs tends to strengthen these effects. Data on international R\&D JVs established in China during 1995-2002 largely support these hypotheses. (For more information, please contact: Jiatao Li, HKUST, Hong Kong, SAR-PRC: mnjtli@ust.hk) 
Antecedents of MNE Performance: Managing Firm-Specific and Country-Specific Advantages

Lars Matysiak, Justus Liebig University Giessen

The performance of MNEs is determined by top management teams' (TMTs') dynamic capabilities to achieve the complementary utilization of country-specific advantages (CSAs) and firm-specific advantages (FSAs) or recombinations of the two in order to achieve new advantages. We theoretically explain these relationships by integrating internalization theory with the resource-based view and the dynamic capabilities approach. The results of fixed effects regression analyses of a panel data set of German MNEs from 2005 to 2010 empirically support key assertions of our theoretical model. Specifically, we find that our hypothesized three-way interaction effect between investments into FSAs in technology, potential access to CSAs, and TMT dynamic capabilities is positive and significant. Our study thus contributes to research on antecedents of MNE performance by overcoming recent criticism of the large number of studies that disregards internalization theory as the dominant theory of the MNE, by offering results from an underrepresented non-Anglo-American research setting, and by focusing managerial attention on FSAs and CSAs as the true antecedents of MNE performance. (For more information, please contact: Lars Matysiak, Justus Liebig University Giessen, Germany: research@matysiak.com)

Reverse and Conventional Knowledge Transfers in International Joint Ventures: Evidence from South Korea Chansoo Park, Memorial University of Newfoundland Ilan Vertinsky, University of British Columbia Teresita Ireneo-Manalo, Acsenda School of Management

The purpose of this paper is to develop and test a theoretical model to examine the dynamics of knowledge transfers from foreign parent firms to their IJVs (conventional transfers) and knowledge transfers from IJVs to their foreign parent firms (reverse knowledge transfers). We estimated a structural equation model (AMOS 20.0) using survey data from 199 Korean IJVs. The key findings highlight the importance of the interaction between conventional and reverse knowledge transfer processes. We find that shared vision does not directly affect reverse knowledge transfer; rather, shared vision positively impacts reverse knowledge transfer indirectly through the full mediating effect of conventional knowledge transfer. We also find negative relationships between IJV performance and reverse knowledge flows, reflecting lower levels of engagement between parents and IJVs when IJVs perform well. This results in a lower level of learning by parents. The study confirms that learning intents and capability to absorb knowledge of IJVs positively affect conventional knowledge transfers. (For more information, please contact: Chansoo Park, Memorial University of Newfoundland, Canada: chansoo.park@ubc.ca)

\section{Session: 1.4.6 - Competitive \\ Track: Track: 8 - Developing Country MNCs}

\section{Cross-Border Acquisitions and Subsidiary Relationship in Developing Economy MNEs}

Presented On: July 4, $2013-14: 50-16: 05$

Chair: Afonso Fleury, University of São Paulo

Reverse Knowledge Transfer: A Survey of Acquisitions by Indian MNEs

Smitha Nair, University of Sheffield

Mehmet Demirbag, University of Sheffield

Kamel Mellahi, Warwick University 
This study examines the association between subsidiary level factors and reverse knowledge transfer in emerging markets multinationals. We develop a set of hypotheses and test them using data from a sample of Indian firms that acquired subsidiaries in Western economies. The ordinary least squares (OLS) regressions results show that subsidiaries that perform the role of specialised contributors contribute more towards RKT, higher levels of collaboration facilitate RKT to the parent firm, and knowledge outflow to the parent firm from subsidiaries from host countries with a higher competitiveness index is higher than from those with low competitive index. (For more information, please contact: Mehmet Demirbag, University of Sheffield, United Kingdom: m.demirbag@sheffield.ac.uk)

\author{
Institutional Distance and Headquarters-Subsidiary Relationship Quality: The Moderating Role of \\ Institutionalization of Headquarters' Practice in Subsidiaries \\ Jizhong Li, Curtin University \\ Fuming Jiang, Curtin University
}

We developed and tested a contingent conceptual framework linking multinational enterprises' external and internal institutions and quality of headquarters-subsidiary relationship. The data was collected from both headquarters and respective subsidiaries of 304 Chinese multinational enterprises. Our findings suggest that the institutional distance has a positive impact on headquarters-subsidiary relationship quality, and this relationship is stronger when the level of institutionalization of headquarters' practice in the subsidiary is high. We discuss scholarly and practical implications for understanding how firms' formal and informal institutions impact on quality of headquarters-subsidiary relationship and when the relationship is altered by the subsidiary's institutionalization of headquarters' practice. (For more information, please contact: Jizhong Li, Curtin University, Australia: forestapache@hotmail.com)

\title{
Cross-border Acquisitions by Emerging-Economy Firms: Institutional Gap as a Source of Value Creation Hamid Akbari, UOIT \\ Ellen R. Auster, York University
}

Emerging-Economy (EE) firms that pursue cross-border acquisitions in advanced economies challenge traditional perspectives of internationalization suggesting that increased institutional distance leads to decreased performance. Yet, empirical performance results are mixed with some studies showing positive performance effects and others revealing a negative impact. The purpose of this study is to extend previous research by empirically testing the relationship of institutional distance and performance using longitudinal data on 9,935 firms from 27 countries spanning 78 different industry groups. More specifically, this study examines the magnitude and direction of institutional distance, and the role of organizational slack over time on the performance of these EE firms' internationalizing through cross-border acquisitions in advanced economies. Our findings reveal that EE firms' acquisitions in advanced economies is associated with negative performance effects in the short-run as internationalization theories might predict. However, over the long-run, EE firms expanding to advanced economies experience positive performance outcomes highlighting the role of embeddedness in advanced economy institutional environments as a source of value creation for EE firms. A wider institutional distance and greater access to slack resources further improves the performance gains. (For more information, please contact: Hamid Akbari, UOIT, Canada: hamid.akbari@uoit.ca)

\section{The Location Choice of Cross-Border Acquisitions by Emerging Market Multinationals \\ Barclay E. James, Louisiana State University \\ Rajeev J. Sawant, Baruch College \\ Joshua Bendickson, Louisiana State University}

In the context of high-tech industries, we develop and test a "location attractiveness" theoretical framework proposing that emerging market firms that are seeking to exploit existing capabilities are more likely to make 
similar acquisitions (i.e., acquisitions in developing country markets) while EMNEs seeking to explore new opportunities are more likely to make acquisitions in highly developed countries. Our theoretical framework also suggests that the tendency of EMNE exploitative acquisitions in developing countries is strengthened when EMNEs possess firm-specific advantages (FSAs) over other EMNEs, because EMNEs can better exploit their unique capability sets in other developing countries rather than in highly developed countries. For EMNE exploratory acquisitions, we also examine whether an EMNE's ability to recognize and integrate new technological knowledge strengthens the likelihood of EMNE exploratory acquisitions in highly developed countries, an examination which allows us to tease out whether highly developed countries are attractive because of greater technological innovation or because of better developed, more transparent markets for corporate control and intellectual property legal protections. Overall, our study provides some of the first theoretical explanation and empirical evidence regarding how exploratory and exploitative motivations by EMNEs and EMNEs' firm-specific capabilities affect the geographic location choice of EMNE cross-border acquisitions. (For more information, please contact: Barclay E. James, Louisiana State University, USA: bjames@lsu.edu)

\section{Session: 1.4.7 - Competitive}

Track: Track: 1 - Institutions, Governance, and CSR

\section{Environmental Sustainability, Climate Change and Strategy}

Presented On: July 4, $2013-14: 50-16: 05$

Chair: Shankar Chelliah, Universiti Sains Malaysia

Clean Energy to "Dirty" Countries: The Effect of Host-Country Corruption and Home-Country Legal System on Transfer of Technologies

Neli Loewenthal, George Washington University

Goran Vojvodic, George Washington University

This study explores the effect of institutional factors, and particularly of corruption, on the amount of FDI to the renewables and conventional energy industries of emerging and developing countries. The focus is on the energy sector and different types of investments are considered. Some support is found for the hypothesis that investment in renewables decreases faster than investment in conventional energy sources as corruption distance increases. Home-country effects, such as legal system, are also explored. As opposed to the previous literature on related topics, a Bayesian analysis approach is used here. The benefit of Bayesian analysis is that we can express our uncertainty about random variables probabilistically - in this case, the probability that home-host country corruption distance will have more negative impact on renewables than on conventional energy investment is $\mathbf{8 5 . 9 4}$ percent. The results indicate that the probability that the FDI for MNCs from developed civil law countries which invest in emerging and developing countries' energy sectors is lower than the FDI for MNCs from developed common law countries which invest in emerging and developing countries' energy sectors is 99.9 percent. (For more information, please contact: Neli Loewenthal, George Washington University, USA: nelik@gwmail.gwu.edu)

\section{Geography, Institutions, and Managerial Disclosure: Oily Evidence Anthony Cannizzaro, George Washington University Robert Weiner, George Washington University}

Information has long been a contentious issue between MNEs and host countries, yet IB literature has not examined MNE disclosure of FDI-related information; indeed, we do not even know if MNES are less transparent abroad than at home. This paper conceptualizes and tests MNE managers' strategic choices about to voluntarily information disclosure regarding firm investments. Understanding the decision of what to reveal, which we term investment transparency, involves both institutional and agency-theoretic considerations. We model the decision 
to disclose as a function of the benefits bestowed by investors and stakeholders demanding transparency, as well as the liabilities of foreignness, corruption and political risks to which the MNE is exposed. Using a unique transaction-level dataset of reserve investments by oil industry MNEs, we are able to test this theory by examining managerial decisions to reveal or redact information about investment decisions. Our results indicate that cross-border transactions indeed tend to be less transparent than domestic transactions, and that larger firms and firms in need of financing disclose more in cross-border transactions. Consistent with theory, better home country institutions are related to greater disclosure; however, counter-intuitively, firms investing in countries with stronger institutions actually disclose less. (For more information, please contact: Anthony Cannizzaro, George Washington University, USA: tony_c@gwmail.gwu.edu)

\section{The Political Economy of Climate Change Regulation - Rent-Seeking in the European Union Emissions Trading Scheme (EU ETS) \\ Sanjay Patnaik, George Washington University}

In this paper, I analyze rent-seeking within the context of the European Union Emissions Trading Scheme (EU ETS), the largest cap-and-trade program in the world. The goal of this study is to advance our knowledge of how the institutional environment in different countries affects the outcome of rent-seeking processes. Using a panel dataset (2005-2009) on industrial sectors in 24 EU member states, I examine the distribution of emissions allowance rents by government regulators to those sectors. I find that certain industries are able to capture higher rents than others and industries with a smaller number of plants are more successful in their rentseeking efforts. My results also show that country-specific political and institutional characteristics have an effect on the allocation of allowance rents. Left-leaning governments distribute larger allowance rents to their industries than centrist or right-leaning governments. Countries with a legal tradition rooted in German Law are less susceptible to rent-seeking efforts than countries with a French Civil, Scandinavian or Common legal tradition. I also find that countries with more stable political institutions seem to be more insulated from rentseeking efforts. This paper provides novel insights into rent-seeking by industries within the context of climate change regulation. (For more information, please contact: Sanjay Patnaik, George Washington University, USA: spatnaik@gwu.edu)

\section{Session: 1.4.8 - Competitive \\ Track: Track: 1 - Institutions, Governance, and CSR}

\section{Institutions and Multinational Strategy}

Presented On: July 4, $2013-14: 50-16: 05$

Chair: Jennifer Oetzel, American University

\section{Multinational Corporations Participation in Global Governance: An Institutional Perspective M. Abrahim Soleimani, Eastern Washington University \\ William D. Schneper, Franklin \& Marshall College \\ Wendy Eager, Eastern Washington University}

The aim of this study is to investigate factors at organizational and national level that may affect the likelihood that corporations will engage in global governance. Drawing on institutional theory, we examine whether importance of global business and ownership structure of multinational corporations (MNCs) impact their participation in the United Nations Global Compact (UNGC). Further, we investigate how national institutions can empower the society to place formal and informal pressures on corporations and intensify the impact of organization-level drivers to join the UNGC. Using a sample of 271 MNCs from six countries, we find that relative size of global business as well as the level of embeddedness in the global market increase the likelihood of corporations to join the UNGC. Results also show that bank-controlled MNCs are more likely to participation in the UNGC while the likelihood for MNCs with dispersed ownership is less. In addition, we find that the impact of 
importance of MNCs' global business on the UNGC membership is greater in countries with a stronger rule of law and higher level of democracy. (For more information, please contact: M. Abrahim Soleimani, Eastern Washington University, USA: absoleimani@gmail.com)

The Effect of CEO Compensation on their Responses to Institutional Pressures: Evidence from the Choice between Cross-Border Alliances and Acquisitions

Mirko H. Benischke, University of Auckland

Drawing on neoinstitutional theory and the behavioral agency model, this paper offers an alternative explanation of multinational corporations' responses to local institutional pressures by argue that CEOs selfinterest explains how they respond to institutional pressures. Employing a sample of 1672 cross-border alliances and acquisitions conducted US manufacturing firms between 2003 and 2010, we find that the response to institutional pressures associated with the behavior of other organizations is contingent upon stock option pay and cash compensation. Our empirical results support our theoretical predictions, which emphasize importance of individual agency when explaining MNCs resistance to institutional pressures. (For more information, please contact: Mirko H. Benischke, University of Auckland, New Zealand: m.benischke@auckland.ac.nz)

\section{Institutions and Strategy: Corporate Social Responsibility as Stakeholder Management in Multinational Companies \\ Nikolas Rathert, Freie Universitaet Berlin}

What accounts for variations in CSR adoption patterns of multinational corporations? While extant research has established that companies manage stakeholder pressure and expectations through various "impression management" tactics, the MNC presents a challenge for students of institutional theory to understand diverse sources of institutional pressure. CSR is suggested as a response to stakeholder expectations by reflecting societal demands in corporate practices. At the same time, the management literature emphasizes companies' strategic ability to choose from an array of responses to such demands. The article brings together institutional and management literature by focusing on the role of CSR as a means for companies to actively and selectively manage stakeholder expectations. We develop hypotheses about CSR responses under diverse stakeholder expectations for a sample of 151MNCs from the UK for the issue area of labor rights. We operationalize a prominent assumption of the CSR literature that has rarely been tested - that CSR strategy is strongly driven by presence in foreign markets - by spatially weighting the influence of different investment locations of over 10.000 subsidiaries. Results confirm expectations that MNCs adopt CSR in a strategic manner: Stakeholder pressure induces CSR to different degrees, depending on the nature of the institutional pressure source. (For more information, please contact: Nikolas Rathert, Freie Universitaet Berlin, Germany: nikolas.rathert@fuberlin.de)

\section{Board Industry Expertise and Strategic Change: The Impact of Institutional Differences \\ Sebastian Schrapp, University of Goettingen \\ Jana Oehmichen, Georg-August-University Goettingen \\ Michael Wolff, Georg-August-University Goettingen}

In this paper we strive to answer the question whether and when board expertise is needed to induce corporate strategic change. Building on existing studies that combine considerations of both resource dependence and agency theory we aim to integrate country-level contingency factors that influence the effectiveness to which the board fulfills its functions - i.e. (1) the provision of counsel on organizational matters and (2) monitoring executives' actions. To test these ideas, we use a multinational dataset of 2,995 firm year observations across 17 European and U.S. countries. Generally, our results suggest that industry experience is an important driver of strategic change across countries. The strength of the effect, however, is subject to the quality of institutional aspects: country-specific differences mitigate experts' effect on strategic change by (1) providing alternative sources of information and (2) shifting monitoring power to entities other than the board. We derive 
two institutional levers accordingly: information provision and motivational stimulation. Overall, the results suggest that national mechanisms of information provision are a substitute to directors' advice provision function whereas motivational stimulation is a substitute to the monitoring function of boards in the context of strategic change. (For more information, please contact: Sebastian Schrapp, University of Goettingen, Germany: sebastian.schrapp@wiwi.uni-goettingen.de)

\section{Session: 1.4.9 - Competitive}

\section{Track: Track: 12 - Economic Geography and Value Chains}

\section{Innovation and Externalities}

Presented On: July 4, 2013 - 14:50-16:05

Chair: Carine Peeters, Universite libre de Bruxelles

Offshore and Domestic Subsidiary R\&D Sourcing: Its Antecedents and Innovation Outcomes Michael J Mol, University of Warwick

Olivier Bertrand, SKEMA

Other than through outsourcing and internal R\&D businesses often rely on sourcing R\&D inputs from other subsidiaries. We seek to understand under what conditions such subsidiary sourcing takes place and how it is related to innovation outcomes. We argue that subsidiary sourcing, especially when undertaken offshore, adds to the heterogeneity of knowledge inputs a business has available because of a larger cognitive distance. Furthermore, what constitutes a heterogeneous knowledge inputs depends on the recipient: For host subsidiaries offshore R\&D sourcing provides less heterogeneity than for domestic units. More cognitively distant inputs add to a firm's propensity to innovate, and therefore offshore subsidiary sourcing is most beneficial for domestic firms. But due to myopic search patterns foreign subsidiaries engage in offshore subsidiary R\&D sourcing more, as do export intensive firms and firms near the border. This framework is only partially supported empirically in tests on a large number of businesses in France, as we find no differences between foreign and domestic firms. (For more information, please contact: Michael J Mol, University of Warwick, United Kingdom: michael.mol@wbs.ac.uk)

Evolution of Innovation Networks across Geographical and Organizational Boundaries: $A$ Study of R\&D Subsidiaries in the Bangalore IT Cluster

Florian Taeube, EBS Business School

Amit Karna, EBS Business School

Petra Sonderegger,

In this paper, we investigate the evolution of MNC subsidiaries through development of innovation networks. Literature on innovation networks has seen them to develop in several different settings. These networks, during their evolution, span firm boundaries and geographic proximity. However, the evolution of innovation networks within and outside MNC subsidiaries has not been investigated from the perspective of these two dimensions: geographical and organizational distance. In order to find out a pattern, we chose the Information Technology cluster in Bangalore, India, where we find there are MNC subsidiaries that operate and innovate within and outside organizations, and have strong links with firms within and outside of Bangalore cluster. The globalized nature of the cluster helps us infer the evolution of innovation networks by taking a knowledge flow perspective. We identify four distinct phases based on what type of knowledge flows and where and how it flows (innovations networks). We find that the innovation networks of these MNC subsidiaries first develop as intra-organizational networks and then extend across organizations. Within the first part, the networks start with a non-local nature (phase-1) and develop into local networks (phase-2). However, within the latter part of the evolution, networks develop from local (phase-3) towards a non-local (phase-4) nature. (For more information, please contact: Florian Taeube, EBS Business School, Germany: florian.taeube@ebs.edu) 
The Role of Subnational Externalities and Industrial Heterogeneity in Driving Multinational Firms' Entry Strategy

Stefano Elia, Politecnico di Milano

Lucia Piscitello, Politecnico di Milano

Sergio Mariotti, Politecnico di Milano

This paper assesses the influence of spatial heterogeneity on the choice of entry modes by multinational enterprises (MNEs). We claim that the location of the target firm influences the choice of partial ownership, i.e., an MNE's choice to maintain a local partner. MNEs normally execute partial acquisitions to reduce their liability of foreignness and to preserve their target's inherent competencies, particularly in highly innovative and internationally competitive sectors. However, this phenomenon occurs less frequently if target firms are located in areas that are characterized by relevant externalities, such as core cities and industrial districts. In particular, core cities allow foreign MNEs to access not only a variety of information and knowledge, but also other externalities that are associated with international interconnectedness; industrial districts provide MNEs with easier access to industry-specific agglomeration economies (a local pool of skilled labor, local input-output linkages, and local knowledge spillovers). Thus, both types of locations reduce an MNE's need to maintain a local partner, although these locations provide substitutes for different aspects of the target firm's competences. Empirical evidence from foreign acquisitions of local manufacturing firms that occurred in Italy during the 20012010 period confirms these expectations. (For more information, please contact: Stefano Elia, Politecnico di Milano, Italy: stefano.elia@polimi.it)

\section{Institutions and Geography of innovation: A Study of Innovative Outputs of Multinational Enterprises and Domestic firms in China \\ Xuesong Geng, Singapore Management University \\ Kenneth Huang, Singapore Management University \\ Heli Wang, Singapore Management University}

Integrating the insights from both institutional theory and economic geography, we develop a new conceptual framework to explain how formal and informal institutions may influence geographically localized knowledge spillovers and thereby firms' innovative outputs. In particular, we argue that both the advancement of formal institutions and the prevalence of informal institutions mitigate the limitation of geographic proximity on knowledge spillovers. Empirical analysis using transnational patents that have been applied by and granted to the same firms in both China and the U.S. yields results that are consistent with our arguments. We find that patents generally garner greater future citations for firms operating in more clustered regions in China, especially for Western multinational enterprises relative to domestic Chinese firms. However, such differences across regions and across firms attenuate after the implementation of the major Chinese patent law reform in 2001. (For more information, please contact: Xuesong Geng, Singapore Management University, Singapore: xsgeng@smu.edu.sg) 


\section{Session: 1.4.10 - Special Session}

\section{Special Session of AJBS Best Papers}

Presented On: July 4, $2013-14: 50-16: 05$

Chair: Gary Knight, Willamette University

The Effects of Corporate-Level R\&D Policies and Laboratory-Level Collaborations on Laboratory R\&D

Performance

Naohiro Sawada, Aoyama Gakuin University

Hiroshi Nakamura, Keio University

Kazuhiro Asakawa, Keio University

No abstract available. (For more information, please contact: Kazuhiro Asakawa, Keio University, Japan: asakawa@kbs.keio.ac.jp)

MNE Dynamics, Distance and the Role of Space and Place in the Organization of MNE's Locations: Evidence from Japanese MNES

Majid Eghbali-Zarch, University of Western Ontario

No abstract available. (For more information, please contact: Majid Eghbali-Zarch, University of Western Ontario, Canada: meghbali-zarch.phd@ivey.ca)

Inter-Organizational Linkages and Partner Switching: A Look at the Japanese Automobile Industry Kunal Banerji, Eastern Michigan University

Rakesh Sambharya, Rutgers University

No abstract available. (For more information, please contact: Rakesh Sambharya, Rutgers University, USA: sambhary@camden.rutgers.edu)

\section{Session: 1.4.11 - Competitive}

\section{Track: Track: 6 - Innovation and Knowledge Mgmt.}

\section{Innovation and Knowledge Capabilities}

Presented On: July 4, $2013-14: 50-16: 05$

Chair: Michael C. Nippa, Freiberg University

Subsidiary Development of New, Complementary and Distant Technologies: The Influence of MNC and Host Country Technological Dynamism

Anupama Phene, George Washington University

Stephen Tallman, University of Richmond

This study integrates insights from the dynamic capabilities perspective and the multinational subsidiary literature to examine the drivers of development of new technologies by foreign subsidiaries of multinational. Subsidiary development of new technologies is evaluated along two dimensions, technologies that are complementary to and those that are distant from existing subsidiary technologies. We hypothesize that technological dynamism in the multinational firm (MNC) and the host country positively influence subsidiary 
development of new and complementary technologies. We further posit that subsidiary characteristics, innovative capability relative to the MNC and experience in the host country, moderate the effects of technological dynamism. We find support for the positive effects of technological dynamism in the MNC and host country on the development of new and complementary technologies by the subsidiary. Interestingly, the moderator effects of subsidiary characteristics operate in a manner contrary to our hypotheses. In the case of more innovative and experienced subsidiaries, we observe positive effects of technological dynamism on the development of new and distant technologies and negative effects on the development of new and complementary technologies. (For more information, please contact: Anupama Phene, George Washington University, USA: anuphene@gwu.edu)

\section{Decision-Making Autonomy and Subsidiary Innovation \\ Dut Van Vo, University of Groningen \\ Sjoerd Beugelsdijk, University of Groningen \\ Gjalt de Jong, University of Groningen \\ Björn Jindra, Halle Institute for Economic Research}

This paper investigates how decision-making autonomy affects the possibility and intensity of innovation in subsidiaries of multinational enterprises (MNEs). Subsidiaries are increasingly identified as sources of innovation and as vehicles for cross-border transfer of new competences. The question of how much decision-making autonomy subsidiaries should have is a core issue in the management of headquarters-subsidiary relationships. Using two complementary theoretical perspectives, we hypothesize a non-linear relationship between subsidiary's decision-making autonomy and innovation. We test our hypothesis in a multi-country and multiindustry database based on survey evidence of 134 subsidiaries located in five Central and Eastern European countries from 23 home countries. The empirical results provide support for a non-linear U shaped relationship between subsidiary decision-making autonomy and innovation intensity. (For more information, please contact: Dut Van Vo, University of Groningen, Netherlands: d.v.vo@rug.nI)

\section{Dynamic Capabilities for Successful Strategic Renewal: Internationalization and Sourcing Capabilities Monica Ileana Riviere, SKEMA Gabriele Suder, SKEMA}

Though strategic renewal has recently become central to the strategy field analyzed by organization and IB/learning literature, the capabilities needed by firms to achieve such renewal are less understood. This study aims to advance this understanding and to set a conceptual basis for further research. In particular, we are interested in how large multinational organizations renew their knowledge and capabilities through internationalization. By reviewing strategic management and IB literature, we determine that firm's ability to create new strategic alternatives for growth is largely contingent upon their internationalization and sourcing capabilities. Internationalization increases the ability to sense and evaluate strategic opportunities. Sourcing capability reflects in the faculty to seize opportunities, or the routine to select the mode of sourcing, that have the potential to affect firm's long-term prospects. Our contribution lies in clarifying to what extent and how internationalization and sourcing capabilities have the potential to explain the development of renewal capabilities. (For more information, please contact: Monica Ileana Riviere, SKEMA, France: monicaileana.rogoz@skema.edu)

\section{The Impact of Parent Firm Knowledge Transfers on Foreign Subsidiary Innovation Heather Berry, George Washington University}

In this paper, I examine how three knowledge transfer mechanisms, including managerial knowledge transfers, product transfers and technology transfers, impact the innovation output of foreign subsidiary operations. I argue that the combination of tacit and explicit transfers of parent firm knowledge through expatriate managers 
and product and technology transfers respectively will result in conditions that are more conducive to new knowledge generation in foreign subsidiaries. Empirical results from a comprehensive panel of US MNCs show that conditional on foreign operations doing R\&D, the combined effect of knowledge transfers through parent products and expatriate managers significantly impacts foreign subsidiary knowledge generation. By exploring how a combination of tacit and explicit knowledge transfer mechanisms can encourage knowledge extension and generation, this paper contributes to the knowledge management literature while also showing how a comprehensive database of US MNCs link their operations to extend their knowledge assets. (For more information, please contact: Heather Berry, George Washington University, USA: berryh@gwu.edu)

\title{
Session: 1.4.12 - Interactive Track: Track: 5 - MNC Management and Organization \\ Understanding Subsidiary Role and Performance
}

Presented On: July 4, $2013-14: 50-16: 05$

Chair: Pooja Thakur, Virginia Tech

Roles of Strategic Embeddedness and Specialized Resources in Multinational Subsidiary Autonomy and Performance in an Emerging Economy

Xiaoying Li, Brunel University

Xiaming Liu, University of London

Following the resource-based view and the network approach this paper investigates the relationships between strategic embeddedness, specialized resources and subsidiary autonomy and performance in an emerging economy. A conceptual framework is developed and tested on a data set of 336 multinational subsidiaries in China. Our results indicate that external strategic embeddedness is positively associated with a subsidiary's specialized resources which are in turn positively associated with the subsidiary's autonomy and profitability. The effects of specialized resources on both autonomy and performance are moderated by a subsidiary's experience and as well as its parent firm's overall strategy, reflecting some unique features of an emerging economy. Managerial and policy implications are discussed. (For more information, please contact: Xiaoying Li, Brunel University, United Kingdom: xiaoying.li@brunel.ac.uk)

\author{
Restructuring the MNC: Capability and Organizational Co-evolution in Functionally Differentiated Subsidiaries \\ Carlos Adrian Rodriguez, McGill University \\ Omar Toulan, McGill University
}

This paper analyzes the co-evolution of capabilities and organizational development in subsidiaries established to support Multinational Corporations (MNC) transition from decentralized to globally distributed models. Existing literature has described the increased responsibilities given to functionally differentiated subsidiaries (FDS) as a case of learning-by-doing, in which the adoption of more complex responsibilities is triggered as FDS develop capabilities. By drawing on the subsidiary evolutionary and capabilities literatures, we propose that proactive organizational development is instrumental in the development of globally distributed networks. Furthermore, we highlight the co-evolutionary process of capability and organizational development within the subsidiary as well as its impact on the parents as a whole. The model is illustrated by drawing on the historical development of McKC India, the largest knowledge center of McKinsey and Company. (For more information, please contact: Carlos Adrian Rodriguez, McGill University, Costa Rica: carlos.rodriguez@mail.mcgill.ca) 


\section{The Effect of Isomorphic Behavior on Subsidiary Performance \\ Naoki Ando, Hosei University}

This study examines whether isomorphic behavior by multinational corporations positively or negatively affect the performance of foreign subsidiaries. Although previous studies find that multinational corporations have a tendency to mimic practices adopted by other firms, the financial consequences of mimetic behavior remains uncovered. This study addresses multinational corporations' isomorphic behavior regarding foreign subsidiary staffing and hypothesizes the relationship between the imitation of the dominant practice for subsidiary staffing and the subsidiary performance. The panel dataset consisting of 3,284 foreign subsidiaries of multinational corporations are used to test the hypotheses. The results obtained from a fixed effect model indicate that the imitation of the dominant practice adopted in the host country negatively affects the subsidiary performance. This study also finds that the negative relationship between mimetic behavior and subsidiary performance becomes greater as the institutional distance between the host country and the home country increases. The results of this study suggest that isomorphic behavior in pursuit of legitimacy is accompanied by the loss of efficiency. (For more information, please contact: Naoki Ando, Hosei University, Japan: nando@hosei.ac.jp)

\section{A Neglected Level of Analysis in MNE's Strategy, Structure and Subsidiary Research Shu Yu, National University of Singapore \\ Andrew Delios, National University of Singapore}

This study systematically reviews the literature on the MNE (multinational enterprise) strategy and structure and MNE subsidiary with a focus on six major research streams: strategy-structure, headquarters-subsidiary relationships, MNE process, subsidiary role, subsidiary evolution, and subsidiary networks. Based on this systematic review, we propose a new classification: a headquarters (HQ) perspective versus a subsidiary perspective. We further propose that there is a neglected level of analysis---intermediate-level perspective based on this new classification on MNE's strategy, structure and subsidiary research. More importantly, this study discusses the new research opportunities which included the bridges to theory (foreign direct investment theory and institutional theory) and context (emerging economies) in this area. (For more information, please contact: Shu Yu, National University of Singapore, China: yushu@nus.edu.sg)

\section{Identification \\ Eva Andrea Alfoldi, University of Manchester \\ Jeremy Clegg, University of Leeds \\ Sara McGaughey, Griffith University}

The Co-Construction of MNE Subsidiary Mandates: The Role of Subsidiary Identity and Organisational

In this study, we examine the process by which MNE subsidiary mandates assigned by headquarters are enacted by subsidiaries in concert with other units within the MNE - a process which we term mandate coconstruction. We argue that headquarters-assigned mandates are inherently ambiguous, and therefore require interpretation to be given substance and enacted at the subsidiary level, where they are also influenced by other subsidiaries involved. We theorise that subsidiary identity and organisational identification play a major role in these processes and note that to date, there has been very little integration between the literature on subsidiary mandates and the literature on subsidiary identity and organisational identification. As such, we investigate how the interplay of identities and organisational identification across subsidiaries affect how headquarter-assigned mandates are actually interpreted, constructed and enacted by subsidiaries over time. We conduct a qualitative case study of the assignment and enactment of a regional management mandate (RMM) in Unilever, which involves the exercise of power and influence over other units, and thus challenges subsidiaries' existing identities and identifications within the organisation. The major contribution of our work is to develop a framework exploring the linkages between subsidiary identity, organisational identification and the mandate co-construction process. (For more information, please contact: Eva Andrea Alfoldi, University of Manchester, United Kingdom: eva.alfoldi@mbs.ac.uk) 


\section{Subsidiary-Level Firm-Specific Advantages, Governance and Subsidiary Performance in South East Asia Quyen Nguyen, University of Reading}

We examine the effects of subsidiary-level firm-specific advantages (FSAs) and governance on a subsidiary's performance. This is new work at subsidiary level, in contrast to most of the literature on FSAs at parent-firm level. From a sample of 101 British multinational enterprise (MNE) subsidiaries in six out of ten ASEAN countries over five-year period (2003-2007) and two-step analytical procedures, the analysis reveals four significant findings. First, subsidiary-level FSAs can be operationalized as multi-item and multi-dimensional constructs, including sources of capital, general management and marketing capabilities. Second, a multiple regression test confirms that subsidiary-level FSAs have statistically significant positive impact on sales growth, profit growth, return on capital employed (ROCE) and market share growth of subsidiaries. We find that over $90 \%$ of financing sources in the British MNE subsidiaries come from internal funds. This reflects a centralized financing policy, which is fully consistent with the "pecking order theory" in finance literature. Third, the impact of governance in terms of subsidiary autonomy in decision making, subsidiary's level of integration and national responsiveness through offerings of global standardized versus regional and local customized products and services, ownership and control through wholly owned foreign subsidiary (WOFS) versus joint venture (JV) on its performance is less clear. Fourth, from a Kruskal Wallis test, we find that there are no significant differences in FSAs and governance characteristics between WOFSs and JVs and the two groups perform equally. (For more information, please contact: Quyen Nguyen, University of Reading, United Kingdom:

\section{t.k.q.nguyen@henley.ac.uk)}

\section{Antecedents and Consequences of Relational Capabilities: A Study of MNE Subsidiaries in Thai Manufacturing Sector}

Rapeeporn Rungsithong, University of Bath

Anthony Roath, University of Bath

The relational capabilities approach is an emerging theory explaining firms' capabilities to deploy and create value from relational resources and capabilities. However, we lack understanding of the conditions under which relational capabilities enhance the alliance performance, especially in the context of MNEs subsidiaries in an emerging economy. To fill this literature gap, we develop a theoretical framework that shows how that the effectiveness of relational capabilities in post alliance management. Our framework integrates the relational capabilities concept with social capital theory and the cultural distance concept to explain the antecedents of firms' relational capabilities in strategic alliances. We test our hypothesis with a cross-sectional sample of 156 strategic alliance projects between MNE subsidiaries and their local suppliers in Thai manufacturing sector. Our results provide support for these antecedents, and show that relational capabilities enhance operational and strategic performance. Cultural distance, in contrast has no significant impact on relational capabilities. (For more information, please contact: Rapeeporn Rungsithong, University of Bath, United Kingdom: rr257@bath.ac.uk) 
Session: 1.4.13 - Interactive

Track: Track: 11 - SMEs and Entrepreneurship

\section{Emerging Domains in International Entrepreneurship Research}

Presented On: July 4, $2013-14: 50-16: 05$

Chair: Donna Kelley, Babson College

New Venture Internationalization as a Rite of Passage: Unpacking Transitional Vulnerabilities

Shameen Prashantham, Nottingham University

Steven W. Floyd, University of Massachusetts Amherst

As a complement to more economic explanations of why some international new ventures (INVs) survive while others don't, we offer an account grounded in social anthropology. We suggest new venture internationalization is akin to a rite of passage i.e. a transitional process of social actors to a new status, involving a between-andbetwixt "liminal" phase characterized by both ambiguity and liberation. Thus liminality is a double-edged sword, holding the potential danger of transitional vulnerabilities: premature entry, liminal overreach and prolonged liminality. These vulnerabilities could thwart liminal learning outcomes that are vital for the successful accomplishment of internationalization and threaten INV survival. (For more information, please contact: Shameen Prashantham, Nottingham University, China: shameen.prashantham@nottingham.edu.cn)

\section{The Framing of Internationalisation Decisions: Examining the Construction of Prospects in the Selection of a Course of Action \\ Lucrezia Casulli, University of Strathclyde \\ Marian Vanessa Jones, University of Glasgow}

In asking how decision-makers in internationalising firms reason in conditions of uncertainty, we aim to advance understanding of the internationalisation process of firms. In this study we follow an abductive process moving iteratively between the field and the literature to build theory on how principle decision makers (PDMs) in internationalising firms manage the uncertainty associated with unknown decisional outcomes. Using a causal mapping technique with PDMs, we reconstruct and explore their experiential cognitions and reasoning relative to the decisions made. Applying, an analytical framework, derived from Kahneman and Tversky's (1984) theory of heuristic reasoning a posteriori, we identify the experiential cues and retrieval processes with which PDMs frame internationalisation decisions. We advance a model of reasoning under uncertainty in making internationalisation decisions in the firm. The core contribution of our paper is to provide an insight into the reasoning process of the principal decision makers wherein sense-making from past experiences informs the construction of prospects in the current decision. In turn, the attractiveness or unattractiveness of those prospects determines the selection of a course of action. (For more information, please contact: Lucrezia Casulli, University of Strathclyde, United Kingdom: eziacasulli@hotmail.com)

\section{Born Global or Born to Run? The Long-Term Growth of Born Global Firms \\ Birgit Hagen, University of Pavia \\ Antonella Zucchella, University of Pavia}

Born globals have been studied and discussed broadly as regards their start-up, mainly trying to define and examine some key dimensions of their internationalisation process, namely intensity or degree, speed, and scope of internationalisation. The factors triggering this phenomenon have also been investigated but have rarely been related to the above key dimensions and tracked through time, along the born global life cycle. In other words, little research is available into the continued internationalisation of these firms (Liesch et al., 2007). This contribution aims at extending the analysis from the firm inception and childhood to its growth and 
maturity and to understand if born global firms are also "born to run" companies (Hagen et al., 2012) and thus to reconcile in the international entrepreneurship studies the approaches based on fast growing firms and gazelles (Autio et al. 2000; Acs, Mueller, 2008; Storey, 1994; Parker et al.2010) with approaches based on the analysis of international new ventures. We briefly present the most recent advances in modelling accelerated internationalisation over time; secondly, we discuss and develop an appropriate conceptual framework and a model to analyse along time the different variables informing about the born global firm evolution in an holistic perspective; finally, we match case study analysis with the framework and discuss our findings. Our findings show two groups of firm with significantly different long-term internationalisation paths and highlight the role of entrepreneurial/management teams in the differentiation of growth patterns, irrespectively of the industry (Rumelt, 1991). Strategic partnerships and the entry in complex markets also characterize long-term internationalisation success. (For more information, please contact: Birgit Hagen, University of Pavia, Italy: bhagen@eco.unipv.it)

\section{Beyond Entry Mode - SME's Escalation in Emerging Markets: A Conceptual Framework Guido Bortoluzzi, University of Trieste Yusaf Akbar, Central European University Andrea Tracogna, University of Trieste}

Drawing on core theories of International Business studies, this conceptual paper moves from a focus on the firms' "entry mode" decisions towards a very interesting aspect of small firms' international behavior: their escalation of commitment to emerging markets. Escalation is the dynamic aspect of the entry strategy (and international form of presence) and refers to the change in the mode of presence that firms experience in a market. We investigate this issue by building a conceptual framework that links the escalation of a firm's commitment in an emerging market to a firm's endowment of resources and capabilities, as well as to the feedback it receives from the market and to the moderating impact of Institutional Voids. (For more information, please contact: Guido Bortoluzzi, University of Trieste, Italy: guido.bortoluzzi@econ.units.it)

\section{Effectuation and Causation: Two Decision-Making Logics of INVs at the Early Stage of Growth and Internationalization?}

Aleksandra Wasowska, University of Warsaw

Mariola Ciszewska-Milinaric, Kozminski Academy

Krzysztof Obloj, Kozminski University and University of Warsaw

Michal Zdziarski, Warsaw University

The purpose of this study is to explore what decision making logic (effectual vs casual) is dominant in the new venture internationalization process and what influences its changes over time. With this ends we present a case study of a Polish INV, operating in an audiobook industry. The study findings reveal that decision making logic at the early stage of company growth and internationalization cannot be assigned to one type, but it may shift from effectuation to causation and vice versa. Moreover, it is argued that INVs may apply two logics simultaneously, depending on the characteristics of the problem space (uncertainty), but when making decisions on internationalization, entrepreneurs are more likely to follow effectual logic, especially if they do not possess prior international experience. The decision making logic is further mitigated by network partners who act as 'effectual stakeholders', reducing the level of uncertainty faced by an INV. Entry of a VC triggers the shift of the decision-making logic from effectuation to causation, especially in the well-recognized fields (such as domestic markets). However, VC may allow also the effectual logic, seeing this as asset when going international, i.e. venturing into a new field, characterized by high uncertainty. (For more information, please contact: Aleksandra Wasowska, University of Warsaw, Poland: awasowska@mail.wz.uw.edu.pl) 


\section{Causation, Effectuation and Opportunities during Internationalization}

Sylvie K. Chetty, University of Otago

Arto Ojala, University of Jyvaskyla

Tanja Kontinen, University of Jyvaskyla

We examine how entrepreneurial firms choose and operate in different foreign markets and how and why they entered these foreign markets. To understand the phenomenon, we combine two central discussions in entrepreneurship; opportunity recognition and creation perspectives with causation (goal oriented) and effectuation (means driven) logics. The causation and effectuation logics are subsequently integrated with internationalization theory to form the theoretical framework. The study involves the foreign market selection and entries of software firms from Finland and New Zealand. The findings imply that Finnish software firms used more opportunity recognition and causation logic in their foreign market selection. New Zealand firms, however, were more effectual by working jointly with their partners to create opportunities. In their foreign market entries software firms from both countries followed effectuation logic. New Zealand firms used effectuation logic in their foreign market selection to enter psychically close countries, such as Australia, UK and USA. Whereas, Finnish firms that followed causation logic entered psychically distant countries that were diverse. It is not conclusive whether one of these logics is more effective than the other. (For more information, please contact: Sy/vie K. Chetty, University of Otago, New Zealand: sy/vie.chetty@otago.ac.nz)

\section{Session: 1.4.14 - Interactive}

\section{Track: Track: 7 - Emerging Economies}

\section{Political Connections and Ties in Emerging Markets}

Presented On: July 4, 2013 - 14:50-16:05

Chair: Gul Berna Ozcan, University of London

\section{The Double-Edged Effect of Political Connections in a Regulated Industry: The Role of Political Rivalry} Zheng Yan, National University of Singapore

This study attempts to advance current research on firms' political connections, by examining both positive and negative impacts arising from political connections simultaneously during the consolidation of China's steel industry. Drawing upon resource dependence theory and political science research on China's political system, I found provincial governments assist acquirers connected to them in acquiring local firms, but resist against acquirers connected with rival governments. Further, the interpersonal relationships among provincial government officials and the central government officials moderate the effects on acquisition success from political connections with the central government, the provincial government, and the rival provincial government. (For more information, please contact: Zheng Yan, National University of Singapore, Singapore: zhengyan@nus.edu.sg)

\section{Does Kinship with Government Make Firm Immune to Institutional Pressure in Transition Economy? Shiye Wang, Peking University Guochen Du, Peking University}

The current inquiry examines how ownership types characterize the degree of firms' resource dependence on stakeholders and its responses toward institutional requirement in the context of China's transition economy. We contend that the kinship with government makes SOEs less dependent on relevant actors and thus immune to institutional pressure. Besides, this study also takes into account the tension between resource independence and visibility to audiences, and finds that though central government controlled SOEs enjoy the resource 
independence, their greater visibility to external audiences forces them to attend the conforming activity, leaving regional government controlled SOEs the only ones that ultimately chose to resist. (For more information, please contact: Shiye Wang, Peking University, China: wangshiye@pku.edu.cn)

\author{
Strange Bedfellows? Investigating the Survival of International Public-Private Equity Alliances in Emerging \\ Economies \\ Alex Mohr, University of Kent \\ Chengang Wang, University of Bradford \\ Fernando Fastoso, Bradford University
}

The current reemergence of governments as economic actors has increased the importance of public-private alliances not only on the national but also on the international level. We use resource dependence theory to analyze the effect that the participation of state-controlled actors has on the survival of equity alliances. We test our hypotheses using a newly created data set consisting of 711 international equity alliances in the People's Republic of China, of which 338 involved a local state-controlled actor as a partner. Our results show that the effect of state participation on the survival of such alliances varies with the type of state-controlled actor involved and that the strength of this effect depends on foreign investors' host country experience. (For more information, please contact: Alex Mohr, University of Kent, United Kingdom: a.t.mohr@kent.ac.uk)

\title{
The Effects of Human Capital and Corruption on the Global Integration of Emerging Economies Chuck Bryant, Cleveland State University Rajshekhar (Raj) G. Javalgi, Cleveland State University
}

This paper offers an empirical investigation of the impact of human capital investment and corruption on global integration. To support the assertion that higher levels of global integration lead to stronger economic growth, we argue that higher levels of corruption have negative direct effects on global integration and that higher levels of human capital investment have positive direct effects on global integration. Additionally, we argue that the presence of corruption has a significant moderating effect on the relationship between Human Capital investment and Global Integration. (For more information, please contact: Chuck Bryant, Cleveland State University, USA: chuckbryant@mac.com)

\author{
Business-Government Relations and Radical Innovation: Evidence of the Moderating Effects of Trust in \\ Emerging Markets \\ Juhee Kim, Korea University \\ Daeil Nam, Korea University
}

Emerging economies are characterized by an increasing market orientation and an expanding economic foundation. Because innovation increasingly shapes international competitiveness, radical innovation plays a key role in economic development. Yet, little is known about radical innovations in emerging economies. This paper examines the relationship between business-government relations and innovation. We categorize a firm's political strategies into two parts: 1) official and 2) unofficial relations with governments. We also present the moderating effects of trust that explain why firms differ in radical innovation. Based on data comprising 40,462 firms from 58 emerging economies, we found general support for the moderating effects of trust in official relations with governments, but not in unofficial relations. (For more information, please contact: Juhee Kim, Korea University, Korea, South: juhee421@gmail.com)

\section{An Empirical Investigation on Firms' Proactive and Passive Motivation for Bribery in China \\ Rui Wang, Peking University \\ Xiaoyu Zhou, Peking University \\ Yi Han, Peking University}


This research investigates firms' bribery motivations in China. Based on resource dependence theory and anomie theory, we identify resource conditions as firms' proactive motivation to bribe and firms' perceived institutional environment as their passive motivation to bribe. We use the data from 2002 World Business Environment Survey, collected by the World Bank, to investigate firms' bribery in the world's largest emerging market, China. We employ a multi-level logistic model to test our hypotheses. The results show that unsatisfactory general and task environmental conditions may trigger firms to bribe in order to compete for better resources and opportunities; institutional conditions such as the security expenditure and anomie climate may make firms perceive bribery as a common phenomenon and thus induce firms to bribe. This research provides some insights to understand business bribery behaviors in emerging market. It also discusses some managerial implications and guidelines for policy-making from the findings. (For more information, please contact: Rui Wang, Peking University, China: rwang@gsm.pku.edu.cn)

\title{
Session: 1.4.15 - Interactive
}

\section{Track: Track: 9 - Cross-Cultural Management and HRM}

\section{Global Leaders and Mentors}

Presented On: July 4, $2013-14: 50-16: 05$

Chair: Fida Afiouni, American University of Beirut

\author{
n-Culturals, the Next Cross-Cultural Dilemma: A Mentoring Perspective \\ Andre Anugerah Pekerti, University of Queensland \\ Miriam Moeller, University of Queensland \\ David Clinton Thomas, University of New South Wales \\ Nancy K Napier, Boise State University
}

This manuscript presupposes that multiculturalism is a fact of life in many parts of the world. We extend current conceptualizations of bi- and/or multi-culturalism by mapping necessary elements of knowledge, identification, commitment and internalization as necessary but are independently insufficient components of multiculturalism. An integral component of this conceptualization proposes that multicultural identity may go beyond two cultures and include both internal cognitive and sociocultural dimensions of culture alike, which are maintained by actively balancing saliences of multiple cultures. We use social identity theory as a lens to discuss the revamped multiculturalism phenomenon (n-culturalism). The manuscript suggests that $n$-culturals operate at the junctive of two or more cultures and integrate values, knowledge, feelings associated with, including commitment to each of these identities. We propose that managing multiple identities is a task likely to elevate on-going cognitive stress. To address how organizations manage and leverage such identity creation processes of multiculturals to achieve desired workplace outcomes, we propose that organizations implement a Multicultural Mentor Modeling (Triple-M) program to help address n-culturals' cognitive processes and subsequent behavioral choices. The Triple-M program primarily serves as a modeling mechanism, while making benefits accessible to individuals and organizations alike. (For more information, please contact: Andre Anugerah Pekerti, University of Queensland, Australia: a.pekerti@business.uq.edu.au)

Semi-regularity, Discontinuity, and Variety: Why Managers Do What They Do?

Jing Yu (Gracy) Yang, University of Sydney

Song Chang, Chinese University of Hong Kong

Fiona Kun Yao, University of California, Berkeley

This study aims to examine if the managerial work patterns primarily developed from western context are applicable to Chinese context. If the work patterns are still applicable, how the patterns vary across managerial 
ranks as well as firms with different degrees of formalization. We find that formalization 1) helps managers save time on routine tasks, and allow them spend more time on non-routine tasks; and 2) increases their work related interruptions but discourages the non-work interruptions. We also find that 1 ) executive and middle level managers spend a higher proportion of time on non-routine activities over routine activities than the lower level managers; 2) lower level managers receive the least work-related interruptions during their work time, whilst executive managers receive the most non-work interruptions; and 3) they also demonstrate the greatest diversity in work. (For more information, please contact: Jing Yu (Gracy) Yang, University of Sydney, Australia: gracy.yang@sydney.edu.au)

\section{Conceptual Confusion, Term Clarification and Reflections on Global Leadership- a Narrative Synthesis Jörg Hruby, Hochschule für Unternehmensführung Sebastian Noack, Quadriga Deutschland GmbH}

Global leadership as research has won prominence within the last two decades. Due to its young history and the work conducted by scholars entering from different research themes a common understanding of global leadership has not yet been established. This work develops a meta-framework that enables to catalogue the existing publications independent of the definitions used in their original and therefore helps to decrease the conceptual confusion. By employing a deductive narrative synthesis a meta-framework is developed in order to win a holistic understanding of global leadership. 181 documents evaluated from which 141 can be catalogued and - in a first round - 14 are taken for in-depth synthesis. The synthesis itself is still ongoing as the actual number does not deliver sufficient results. The preliminary conclusion delivers first advices on the metaframework. (For more information, please contact: Jörg Hruby, Hochschule für Unternehmensführung, Germany: hruby@bwl-studieren-ohne-grenzen.de)

\section{Confucius as Asian Business Leader: A Comparison of Paternalistic Leadership in China, Japan and South Korea} Pingping Fu, Chinese University of Hong Kong Sophia Jeong, Chinese University of Hong Kong Junichi Yamanoi, Chuo University

In this study, we compare the effect of paternalistic leadership in the three Asian countries that have been heavily influenced by Confucianism: China, Japan, and South Korea. We map paternalistic leadership onto other established leadership styles examined by the Global Leadership and Organizational Behavioral Effectiveness (GLOBE, House et al., 2004). We believe the results will not only enrich the leadership literature but also provide useful insights to practitioners in the three countries, or organizations and managers that strive to build relationships with companies based in these regions. This paper is part of a larger study and we present the propositions in the current paper that we plan to empirically test by the time the meeting starts. (For more information, please contact: Pingping Fu, Chinese University of Hong Kong, Hong Kong, SAR-PRC: ppfu@cuhk.edu.hk)

\section{Leader Reinforcement Behavior and Leader-Member Exchange: The Moderating Effect of Cultural Orientation Mahfooz A. Ansari, University of Lethbridge Rehana Aafaqi, University of Lethbridge}

Drawing on Reinforcement (Skinner, 1969) and Social Exchange (Blau, 1964) theories, we hypothesized that the relationship between leader reinforcement behavior and leader-member exchange (LMX) is contingent on individual cultural orientation. We conceptualized reinforcement behavior in terms of four behaviors: contingent reward (of good performance), contingent punishment (of bad performance), omission (non-reinforcement to good performance), and extinction (non-reinforcement to poor performance). We included four exchange currencies--contribution, affect, loyalty, and respect-in conceptualizing LMX. Finally, we used four cultural orientations: vertical individualism, vertical collectivism, horizontal individualism, and horizontal collectivism. 
Data were obtained from 820 employees and their 291 supervisors representing diverse organizations in Malaysia. Leader behavior and cultural orientations were reported by the subordinates and LMX was rated by their respective supervisors. The analysis indicated that, controlling for supervisor and subordinate gender and the duration of their work relationships, contingent reward had significant positive impact on all LMX currencies, but contingent punishment was positively related to just one currency of exchange. Interestingly, leader omission and extinction behaviors were unrelated to LMX currencies. More importantly, the relationship between leader behavior and LMX was found to be significantly moderated by the cultural orientation of the subordinates. (For more information, please contact: Mahfooz A. Ansari, University of Lethbridge, Canada: mahfooz.ansari@uleth.ca)

\section{Session: 1.5.1 - Special Session}

\section{Defining the Domain of IB: An AIB Town Hall Meeting}

Presented On: July 4, $2013-16: 45-18: 00$

Chair: Robert Grosse, George Mason University

Speakers (listed alphabetically):

Melodena Balakrishnan, University of Wollongong in Dubai

Peter J Buckley, University of Leeds

John Cantwell, Rutgers University

Kendall Roth, University of South Carolina

Gabriele Suder, SKEMA

What is the scope/turf of international business? What really are the dimensions of our field? Come and discuss/debate these issues at the AIB Domain of IB special session. This town hall meeting will try to build on the articles published in 2013 Q1 issue of AIB Insights. (For more information, please contact: Robert Grosse, George Mason University, USA: rgrosse@gmu.edu)

\section{Session: 1.5.2 - Panel}

\section{Developing Global Leaders through Programmatic and Experiential Teaching Methods}

Presented On: July 4, 2013 - 16:45-18:00

Chair: Davina Vora, SUNY New Paltz

Panelists:

Joyce Osland, San Jose State University

Davina Vora, SUNY New Paltz

Martha Maznevski, IMD

In today's increasingly globalized world, it is critical for tomorrow's business leaders to be able to navigate the various complexities of working in a culturally, economically, socially, and politically diverse world. As international business instructors, we must try to prepare our students to thrive in such an environment. One way to do this is by developing global leaders. The purpose of this panel is to provide an overview of cuttingedge methods to develop global leaders in undergraduate and graduate students. This panel will provide a brief overview of what is meant by global leadership, and then will delve into a variety of approaches for developing global leaders. A full-fledged program for developing leaders will be discussed, as well as specific projects that 
can be used to develop global leaders, including assessment of global leadership competencies and a personal development plan to improve such competencies, and team-based action learning projects during short-term trips abroad. Thus, this session will provide educators with multiple ways to develop global leaders, including broad-based curricular programs, individual student projects within the context of an international businessoriented course, and team-based activities that can be conducted in short-term trips abroad. (For more information, please contact: Davina Vora, SUNY New Paltz, USA: vorad@newpaltz.edu)

\section{Session: 1.5.3 - Panel Track: Track: 8 - Developing Country MNCs}

\section{Developing Country Multinationals: Black Cats, White Cats or Models of CSR?}

Presented On: July 4, $2013-16: 45-18: 00$

Chairs: Marilyn Taylor, University of Missouri at Kansas City ; Xiaohua Yang, University of San Francisco Discussant: Monika Hudson, University of San Francisco

Panelists:

Andreas Schotter, Western University

Loong Wong, Murdoch University

Gleb Nikitenko, University of San Francisco

Romie Frederick Littrell, Auckland University of Technology

Mary Boyden Teagarden, Thunderbird School of Global Management

Corporate social responsibility (CSR) has become a global movement that has been reshaping the global competitive landscape. As more MNEs are operating around the globe under the auspices of Global Compact and the like, we need to gain a better understanding of the interface between CSR and MNEs and how MNEs approach CSR in foreign markets and the challenges they face in aligning their CSR practices with the local environment to gain legitimacy (Yang \& Rivers, 2009). Most of the research on corporate social responsibility has focused on the strategies of companies in the developed world (Quazi \& O'Brien, 2000). The literature on multinational enterprises (MNEs) and CSR is still embryonic (Rodriguez, Siegel, Hillman, \& Eden, 2006). Literature on CSR and MNEs from emerging markets is even more limited. With the economic trajectory of emerging markets such as China becoming the world's second largest economy and is likely to become the world's largest economy in the foreseeable future (Hitt, Franklin, \& Zhu, 2006), understanding the role of CSR in globalization of business and how it might shape MNE strategy has implications not only for research, but also for practitioners and policy makers. (For more information, please contact: Xiaohua Yang, University of San Francisco, USA: xyang14@usfca.edu) 
Session: 1.5.4 - Panel

Track: Track: 12 - Economic Geography and Value Chains

\section{Process Perspectives on Offshoring}

Presented On: July 4, $2013-16: 45-18: 00$

Chair: Kristin Brandl, Copenhagen Business School

Panelists:

Kristin Brandl, Copenhagen Business School

Michael J Mol, University of Warwick

Carine Peeters, Universite libre de Bruxelles

Bent Petersen, Copenhagen Business School

Stephen Tallman, University of Richmond

There has been a lot of research interest in offshoring in recent years, as the offshoring phenomenon has exploded in popularity in practice. Some of this work has been descriptive, but most of it has focused on the antecedents or (performance) consequences of offshoring. It is only recently that scholars have started to explore processes in and around offshoring. These process perspectives on offshoring focus on decision making as a process and / or production as a process, including the offshoring of business and knowledge processes. This panel aims to ask and begin to answer some fundamental questions around such process perspectives on offshoring, including the following. What processes are involved in offshoring? What additional advantages does it bring to study offshoring as a decision-making process? What additional advantages does it bring to study the production of offshored services (and perhaps goods) as a process? How do process perspectives explain offshoring? What should be the objectives of process perspectives on offshoring? In doing this, the panel will seek to draw on existing offshoring research, but also on what we can learn in terms of theories and methods applied in process work in other areas, such as strategy process. (For more information, please contact: Kristin Brandl, Copenhagen Business School, Denmark: kbr.int@cbs.dk)

\section{Session: 1.5.5 - Competitive}

Track: Track: 11 - SMEs and Entrepreneurship

\section{Networks and Collaboration in International New Ventures and SMES}

Presented On: July 4, $2013-16: 45-18: 00$

Chair: Tarek Hatem , American University in Cairo

Lost in Translation: Relationship-Building Challenges of the Inexperienced INV in a High-Distance Market Carole Couper, University of Glasgow

Shameen Prashantham, Nottingham University

Stephen Young, University of Glasgow

This study extends international entrepreneurship research by addressing the question: Why are an inexperienced INV's relationship-building efforts in a high-distance market challenging? Based on an in-depth study of an inexperienced Western INV that unsuccessfully attempted to build a key relationship in the Chinese market, we argue that inappropriate behaviors, such as overcompensating on the affective aspects of a crosscultural relationship, results in an incomplete formation of trust i.e. of benevolent trust, but not calculative trust. Counterintuitively, the benevolent trust may not only be insufficient, but actually detrimental to calculative trust by suppressing sensemaking and timely actions to confront competence-related concerns. (For more information, please contact: Shameen Prashantham, Nottingham University, China: shameen.prashantham@nottingham.edu.cn) 
Entrepreneurial Network Strategy Conventions in China, Taiwan, and the US

Xin Yao, University of Colorado

Josh Keller, Nanyang Technological University

Renhong Zhu, Sun Yat-Sen University

Ru-Mei Hsieh, National Pingtung University of Science and Technology

In a two-stage study combining interviews of 72 entrepreneurs and a survey of 266 entrepreneurs, we examined the content and strength of conventions for entrepreneurial network strategies in Mainland China, Taiwan and the US. We also assessed the relationship between network strategies and networking practices. The interview data revealed 72 network strategies concerning network structure, content of ties, sources of ties, and the maintenance of ties. Our survey data revealed both consistent and distinct conventions. In addition, entrepreneurs in Mainland China who agreed with Mainland Chinese conventional strategies are more likely to engage in both broadening and deepening networking practices. (For more information, please contact: Xin Yao, University of Colorado, USA: xinevayao@gmail.com)

\section{Collaboration Networks as Resources for International New Ventures \\ Frances Y.M. Chang, Macquarie University \\ Cynthia M. Webster, Macquarie University}

This paper explores the characteristics of collaboration networks and examines the resources that these networks provide for international new ventures (INVs). The context of this study is the Australian healthcare industry and data were collected through in-depth face interviews. Our results suggest that developing collaborative networks is a strategic process by INVs who view collaboration as key to generating resources. Consistent with previous studies, INVs with diverse collaborative networks are more likely to succeed. Collaboration networks of knowledge and R\&D tend to be larger and contain many interconnected strong ties while networks that provide access and connection to international markets tend to be large with many members but sparse as members tend not to be connected with each. (For more information, please contact: Frances Y.M. Chang, Macquarie University, Australia: frances.chang@mq.edu.au)

\section{Session: 1.5.6 - Competitive \\ Track: Track: 3 - IB Theory, FDI, and Entry Mode}

\section{Cross-Border M\&As}

Presented On: July 4, $2013-16: 45-18: 00$

Chair: Andre Sammartino, University of Melbourne

Who Jumps from Spring Board First? Firm Level Determinants in Cross Border Mergers \& Acquisition Wave Manish Popli, Indian Institute of Management Lucknow

Ashutosh Kumar Sinha, IIM Lucknow

Emerging economy firms companies are using higher entry modes of internationalization to acquire strategic assets. This indeed has received immense attention by scholars across the globe; however not much is known about their timing aspects in this inorganic mode of international expansion. To that end, we seek to investigate firm level determinants which differentiate early movers from followers in the 'spring-board' approach. Integrating temporal perspectives of strategy with the motives of emerging market cross-border acquisitions, we center our research on cross-border mergers \& acquisition waves. We tested our hypotheses using data of Indian context in a span of eleven years (2001-2011) and our empirical results suggest that firms possessing prior international experience, market power and affiliation with business group are more likely to be first movers in a cross border M\&A wave. (For more information, please contact: Manish Popli, Indian Institute of Management Lucknow, India: fpm12004@iiml.ac.in) 
Risk Preferences in Cross-Border Acquisitions by Emerging Market Firms: The Influence of Acquirer and Target Ownership Structures

Yingdan Cai, University of Groningen

Hans van Ees, University of Groningen

Kees van Veen, University of Groningen

Sathyajit Gubbi, University of Groningen

When an MNE uses cross-border acquisitions to enter foreign markets, it can do so by either fully acquiring the target or by acquiring a partial equity stake in the target. The decision of full versus partial acquisition is determined upon the willingness of the acquirer to take risk and the target to sell. The objective of this paper is to develop a theoretical framework that examines the effects of acquirer and target ownership structures on the likelihood of partial acquisition. We argue that this decision is determined by the ownership concentration of the acquirer and public status of the target under contingencies such as host-home country differences and industry relatedness between the acquirer and the target. An empirical test of a unique sample of Brazilian cross-border acquisitions confirm the conjecture that there is a positive relationship between acquirer ownership concentration and the likelihood of partial acquisitions. (For more information, please contact: Yingdan Cai, University of Groningen, Netherlands: y.cai@rug.nl)

\section{The Effect of Language Distance on Cross-Border Acquisitions \\ Ilya Cuypers, Singapore Management University \\ Gokhan Ertug, Singapore Management University \\ Jean-Francois Hennart, Singapore Management University and University of Pavia}

We study the effects of language distance on the level of equity taken by acquirers from 67 countries in 60,889 acquisition targets in 69 host countries. Controlling for cultural distance and many other factors, language distance has a significant negative direct effect on the equity taken by acquirers. Moreover, this negative direct effect is amplified when the acquirer and the target are unrelated and when the target operates in an R\&D intensive industry. Our results clearly demonstrate that governance research and international business studies in general can benefit from incorporating language into their explanatory models. (For more information, please contact: Gokhan Ertug, Singapore Management University, Singapore: gokhanertug@smu.edu.sg)

\section{Cross-Border Acquisition Completion and Duration: The Moderating Role of Historical Ties Ben Kedia, University of Memphis Tsvetomira Vladimirova Bilgili, University of Memphis}

Recent research in cross-border M\&As has focused on the pre-completion stage of cross-border acquisitions as scholars have explored the reasons behind the abandonment of deals and the time taken for a deal to be completed after its announcement. We argue that these studies have omitted an important contextual variable. We believe that understanding the role of historical ties between the acquirer and the target firms' nations can provide us with important insights into the dynamics of cross-border M\&As and enrich our knowledge of dealbreakers in the pre-completion stage of the acquisition process. This paper contributes to the literature by adopting a historical perspective and exploring the role that historical ties play in explaining cross-border M\&A completion and duration. We advance propositions for the study of historical ties as a moderator of the relationships between select industry-, firm-, and deal-specific predictors and acquisition completion and duration. We propose that history of negative versus positive ties between pairs of countries will influence the strength of the aforementioned relationships. (For more information, please contact: Tsvetomira Vladimirova Bilgili, University of Memphis, USA: tvkaneva@memphis.edu) 


\section{Session: 1.5.7 - Competitive}

Track: Track: 5 - MNC Management and Organization

\section{Managing MNE Capability and Knowledge}

Presented On: July 4, $2013-16: 45-18: 00$

Chair: Irina Mihailova, Aalto University

Multinational Companies and Their International Experience: How Dynamic Capabilities Are Developed Sergio Janczak, Kings University College at Western University

This paper proposes an approach to explain the effects of MNCs knowledge transfer and the development of dynamic capabilities. Diversity of experience is assumed to reflect MNC's dynamic capabilities, which help MNCs better utilize their experiences in a more discerning way. It is argued in this study that diversity of experience enables an MNC to develop enabling processes, and ultimately, dynamic capability, which in turn moderates the various relationships between international experience and subsidiary performance. (For more information, please contact: Sergio Janczak, Kings University College at Western University, Canada: sjanczak@uwo.ca)

Headquarters Deployment of Capabilities in MNCs: Strategic Fit and the Impact of Capability Microfoundations for Performance

Olof Lindahl, Uppsala University

Henrik Dellestrand, Uppsala University

This paper explores determinants of the performance of headquarters deployment of capabilities across its subunits. We contrast six high- and low-performing cases of headquarters deployment of the same "core" capability to innovation projects within a multinational corporation. We suggest a model that elucidates determinants of the performance of headquarters capability deployment. Our results indicate that the performance of capability deployment can be explained through the analytical lens of capability microfoundations. We contribute to research on strategic fit by identifying the dimensions of activity and concept fit, and specify the implications of this finding for headquarters value creation. More specifically, headquarters deployment performance is found to be dependent on the "fit" between the two dimensions of the a priori capability and the same dimensions of the capability that is being deployed. (For more information, please contact: Olof Lindahl, Uppsala University, Sweden: olof.lindahl@fek.uu.se)

\section{The Firm as a Coordination System: Evidence from Software Services Offshoring Kannan Srikanth, Indian School of Business \\ Phanish Puranam, INSEAD}

To examine what, if any, are the differences in how activities are coordinated within-firms vs. between-firms we conducted interviews with 32 project managers regarding 60 projects in the offshore software services industry. Our evidence suggests that in co-located projects the same broad categories of coordination mechanisms are used both within- and between-firms. However, there is a qualitative difference in how geographically distributed projects are coordinated within- vs. between-firms. Distributed projects conducted within-firms rely extensively on tacit coordination mechanisms; such mechanisms are not readily available in between-firm projects conducted across geographies, leading to coordination difficulties. It is suggested that MNC's exist because of their advantage in recombining knowledge across geographies. Our work contributes to understanding the micro-foundations that underlie this MNC advantage. (For more information, please contact: Kannan Srikanth, Indian School of Business, India: kannan_srikanth@isb.edu) 
Foreign Partners' Disseminative Capacities and their Impact on Knowledge Transfers to International Joint Ventures

Chansoo Park, Memorial University of Newfoundland

Ilan Vertinsky, University of British Columbia

Dana Minbaeva, Copenhagen Business School

This paper attempts to fill this gap in the literature by focusing on the influence of knowledge senders' willingness to share knowledge, their disseminative capacities and the knowledge-transfer opportunities they create on the effectiveness of knowledge transfer. We develop a theoretical framework that examines the impacts of key knowledge-senders' abilities and behaviors. We test our theory using survey data collected from 199 South Korean IJVs. We find that the willingness of foreign parent firms to share knowledge is manifested in their efforts to increase their abilities to articulate and codify knowledge, and to apply those skills to the codification of knowledge relevant to their IJVs. A willingness to share knowledge also plays a role in increasing the opportunities for two-way interactions, especially face-to-face interactions between the employees of the parents and those of the IJVs. The impact of the abilities of foreign parents to articulate and codify knowledge for transfer is mediated by the efficacy of their organizational communication systems. We also find that the opportunities created for the transfer of explicit knowledge have a significant impact on such transfers.

However, opportunities for transfer of tacit knowledge only have an impact when senders and receivers have similar products and technologies. (For more information, please contact: Chansoo Park, Memorial University of Newfoundland, Canada: chansoo.park@ubc.ca)

\section{Session: 1.5.8 - Competitive}

Track: Track: 2 - Marketing and Supply Chain

\section{Indicators of Export Market Performance and Strategy}

Presented On: July 4, $2013-16: 45-18: 00$

Chair: Fatima Wang, King's College London

Export Market Orientation and Export Performance in SMEs: The Role of International Experience Eunmi Kim, Korea University Insik Jeong, Korea University

This study examines the issue of how export market orientation (EMO) influence export performance (EP) in SMEs context. Indeed drawing from contingency theory and the organizational learning perspective, the study tests a model of the effects of different forms of international experience - duration, scope, and intensity - on the EP of EMO. Using data from 144 exporting SMEs, the finding shows that export market oriented SMEs might enhance their export performance. In particular, when export intensity of SMEs is high, the positive relationship of EMO-EP is stronger. This study suggests that EMO can be powerful in export success, but it is important that the international marketing decisions are aligned with relevant experiential contexts. (For more information, please contact: Eunmi Kim, Korea University, Korea, South: giveme428@korea.ac.kr) Marketing Mix Adaptation and Export Performance: Examining the Moderating Effect of Export Manager Cultural
Intelligence

Peter Magnusson, Florida International University

Alexey V. Semenov, Saint Louis University

Arilova A Randrianasolo, Saint Louis University

This study examines how export manager cultural intelligence affects the relationship between marketing mix adaptation and export performance. From a resource-advantage theory perspective, the authors posit that 
export manager's motivational and meta-cognitive cultural intelligence are intangible, yet valuable resources influencing export performance. Using survey data from 153 U.S. exporting firms, we find that the export managers meta-cognitive cultural intelligence positively moderates the relationship between marketing mix adaptations and export performance. Further, the export manager's motivational cultural intelligence positively moderates the relationship between environmental differences and marketing mix adaptations. The findings have important theoretical and managerial implications. (For more information, please contact: Peter Magnusson, Florida International University, USA: peter.magnusson@fiu.edu)

\section{Cultural Values and Marketing Strategy: An Examination of "Theories in Use" Glenn Richey, University of Alabama \\ Simon Harris, University of Edinburgh}

The formulation of international marketing strategy is recognized to include elements of the market orientation, resource advantage, and transactions efficiency strategic perspectives. These well accepted marketing perspectives examine the management of market competition and customers; the acquisition and deployment of resources; and the cost efficient governance of relationships -- respectively. While these perspectives are all relevant to marketing and are each employed by all international managers, it remains unclear which perspective is actually emphasized most readily by managers in the formulation of marketing strategy. Moreover, in an increasingly global environment, no study has examined if a marketing manager's use of the three most common marketing perspectives differs across national cultures. This study uses a native qualitative method encompassing five years of field study to fill this gap in the literature. To emphasize that the differences are significant to marketing strategy research and practice, we examine three Western European nationalities (France, Netherlands, and United Kingdom) whose geographic and institutional proximity sometimes leads them to be grouped as one region (Western Europe) in discussions of international cultural differences. (For more information, please contact: Glenn Richey, University of Alabama, USA: grichey@cba.ua.edu)

\section{Relational Governance and Export Performance: The Moderating Roles of Institutional Distance and Domestic Industry Uncertainty}

Min Ju, University of Missouri - St Louis

Hongxin Zhao, Saint Louis University

Tiedong Wang, University of International Business \& Economics

Manufacturers often utilize relational governance in managing interfirm relationship with their distributors. Yet recent literature cautions that relational governance may only offer conditional benefits. In this study, we examine whether there is a limit to the positive export performance effect of relational governance. Drawing on the logic of institutional and industrial organization theories, we further examine the two conditioning factors: cross-country institutional distance and industry uncertainty. Using a survey of 184 export ventures from China, we find that relational governance has an inverted U-shaped relationship with performance. In addition, the findings show that cross-country institutional distance enhances the value of relational governance but the export performance effect of relational governance becomes less effective in uncertain industry environment. The results also show that manufacturers can combine output control with relational governance as a solution of the potentially declining effect. (For more information, please contact: Min Ju, University of Missouri - St Louis, USA: jum@umsl.edu) 


\section{Session: 1.5.9 - Competitive}

Track: Track: 9 - Cross-Cultural Management and HRM

\section{The Role of Language in MNCs}

Presented On: July 4, $2013-16: 45-18: 00$

Chair: Mary Yoko Brannen, University of Victoria

Language Differences and Operation Mode: The Role of Communicative Requirements

Angels Dasi, Universitat de Valencia

Torben Pedersen, Copenhagen Business School

Language serves different purposes depending on the international activity in question. Language has many dimensions and firms' communicative requirements vary by operational platform. We argue that different dimensions of language vary in their importance depending on the operation mode chosen for a foreign market, so that language distance matters in the case of a home-based sales force, while language incidence is key when operating through a local agent. The hypotheses are tested on a large data set encompassing 462 multinational corporations headquartered in Finland, South Korea, New Zealand, and Sweden that have undertaken a business operation in a foreign country. (For more information, please contact: Angels Dasi, Universitat de Valencia, Spain: angels.dasi@uv.es)

\section{Emotionally Intelligent Leadership in Multilingual Workplaces \\ Helene Tenzer, Tübingen University \\ Markus Pudelko, Tuebingen University}

This study is the first to systematically investigate how emotionally intelligent leaders can manage negative emotions induced by language barriers in an MNC's workforce. Based on semi-structured interviews with 8 senior managers, 15 team leaders and 67 of their subordinates in three automotive multinationals, we demonstrate the importance of leaders recognizing negative language-induced emotions among their subordinates. On this basis we show how leaders can actively regulate these disruptive emotions by improving cross-lingual communication, refocusing subordinates' attention and encouraging them to positively reappraise their situation. Our study contributes to language-sensitive international business research by confirming the obsolescence of instrumental views on language. It advances research on emotions in organizations by highlighting that emotions need to be effectively managed rather than suppressed. It contributes to leadership research by offering guidelines for multilingual bridge-making, people-oriented leadership and leveraging diversity. In terms of practical implications, our study outlines a set of concrete measures enabling leaders to address the specific emotional challenges of multilingual work environments. It demonstrates the value of constantly reflecting on emotion management practices and highlights the importance of emotional intelligence for effective leadership in multilingual environments. (For more information, please contact: Helene Tenzer, Tübingen University, Germany: helene.tenzer@uni-tuebingen.de)

The Impact of Foreign Subsidiary's Language Competence on the MNEs' Foreign Subsidiary Staffing Strategy Yong Suhk Pak, Yonsei University Jongkun Jun, Hankuk University of Foreign Studies Jongmin Lee, Yonsei University

Previous research suggests that multinational enterprises (MNE) are multilingual and their employees' language competence plays a critical role in foreign subsidiary control and management. Despite its importance, language competence has been rarely touched in past international business literature. We examined the impact of foreign subsidiary's language competence on MNEs' subsidiary staffing strategy with a sample of 9,623 Korean 
firms operating across 55 countries using multi-level modeling method. Our results clearly demonstrated that the language competence of foreign subsidiaries significantly affect the MNEs' staffing strategy: Foreign subsidiaries with low language competence are more likely to employ parent country nationals (PCN) expatriates from the headquarters and the effect of foreign subsidiary's language competence on subsidiary staffing varies with subsidiary experiences. (For more information, please contact: Jongmin Lee, Yonsei University, Korea, South: jongmin731@hotmail.com)

\section{Psychological Career Mobility and Person-Language Misfit in Multinational Corporations Maria Järlström, University of Vaasa Rebecca Piekkari, Aalto University Tiina Jokinen, University of Vaasa}

This conceptual paper offers a novel, language-centered perspective on career mobility. From an individual perspective, previous research commonly views career mobility as advantage for professional development and career success. However, from an organizational perspective, the risk of losing key talent due to staff turnover may have serious implications for the performance and long-term competitiveness of the firm. Previous research on the boundaryless career has emphasized physical rather than psychological career mobility. We focus on the latter as it is one of the antecedents of physical mobility with high predictable power. Neither career mobility nor turnover research has, however, considered language used at the workplace a factor that would influence perceived career opportunities and alternatives. Previous research shows how the introduction of a new corporate language creates a glass ceiling preventing some individuals, whose language proficiency is insufficient, from pursuing vertical career opportunities within the organization. Yet, these effects have not been systematically conceptualized. Based on an interdisciplinary literature review of careers, person-environment fit and language-centered work in international business we develop a conceptual model which explains psychological career mobility as an outcome of misfit between the individual and language considerations at the workplace. We apply the person-environment fit theory and distinguish between language misfit at four analytical levels: the organization, group, supervisor, and the job. We hypothesize that from an organizational perspective, language misfit steers, shapes and redirects psychological mobility in unintended and unexpected ways. (For more information, please contact: Rebecca Piekkari, Aalto University, Finland: rebecca.piekkari@aalto.fi)

\section{Session: 1.5.10 - Competitive}

Track: Track: 1 - Institutions, Governance, and CSR

\section{Effects of Emerging Market Institutions}

Presented On: July 4, 2013 - 16:45-18:00

Chair: Elizabeth Rose, Aalto University and University of Otago

The Effect of Resource Tangibility, Unrelated Diversification and Business Ties on MNE-Subsidiaries'Non-Market Approaches in Emerging Economies

Vikrant Shirodkar, University of Sussex

Firms can use various 'non-market' strategies to not only survive but also further their competitive advantage in domestic and international markets. An important decision for firms to take in this context is to choose between a 'transactional' and 'relational' approach to non-market strategy. Whereas transactional relates to a one-time, issue-based approach to interact with government officials, the relational approach involves developing personal relationships over time, so that when issues arise, the contacts are already in place to affect these issues. Prior research into this issue in emerging economies has predominantly focussed on the relational approach, in particular, on the creation and management of political ties between foreign firms' managers and government 
officials (particularly in China). However, as emerging economies develop their institutions, an interesting question is whether firms use a transactional approach, and what the factors are that influence the degree to which they use the transactional approach (instead/alongside to) the relational approach. This paper, using the resource dependence theory, argues that resource tangibility, unrelated diversification and business ties affect foreign firms' non-market approach (transactional vs. relational) in emerging economies. The hypotheses are tested using a sample of 105 foreign firms' subsidiaries operating in India. The empirical evidence supports the hypotheses that foreign firms that are characterized by greater dependence on intangible resources, lower unrelated diversification, and having more business ties, are more likely to use relational approaches to nonmarket action. (For more information, please contact: Vikrant Shirodkar, University of Sussex, United Kingdom: v.shirodkar@sussex.ac.uk)

\section{MNC Strategy and Social Adaptation in Emerging Markets Meng Zhao, Moscow School of Management SKOLKOVO Nan Zhou, Moscow School of Management SKOLKOVO Seung Ho Park, Moscow School of Management SKOLKOVO}

Multinational corporations (MNCs) have encountered increasing public crises in emerging markets due to stakeholders accusing them of social misdeeds. Existing research tends to focus on MNCs' economic adaptation while under-addressing how they can handle social transition. This paper fills this gap by combining the views of corporate social responsibility, stakeholder theory and MNC research to develop a social adaptation view. We test this view in the context of consumer rights in China. The results show that MNCs' entry timing and employee growth speed have a positive implication on their public crises in China while social adaptation mitigates the crisis risk. (For more information, please contact: Meng Zhao, Moscow School of Management SKOLKOVO, Russia: meng_zhao@skolkovo.ru)

\section{When Opposites Attract: Learning from Cross-Sector Alliances in Emerging Markets Aline Gatignon, INSEAD}

A growing body of literature suggests that firms in emerging markets can benefit from partnering with nonprofit organizations, local communities, or governmental bodies. These cross-sector alliances should give partners access to a broader knowledge base and promote social innovation in emerging markets. Yet this notion runs counter to conventional wisdom on private sector alliances, according to which firms should seek out partners that are as similar to themselves as possible. We seek to understand how alliances between very different organizations can enable partners to learn from one another in emerging market contexts. In particular, we ask how these cross-sector alliances can be managed in such a way as to reduce frictions and maximize learning, and through what mechanisms firms can stand to benefit from these relationships. We base our statistical analysis on a combination of interview, archival and survey data on 103 alliance projects in 57 countries, developed between global logistics provider TNT and the United Nations World Food Programme from 2002-2011. We find that the level of structural integration between partners is a crucial determinant of employee learning from the alliance experience. Employee learning is also affected by the extent to which employees are able to identify with the partner organization-despite high levels of organizational differences with the employing firms. Surprisingly, we also find that employees with more professional experience are less able to learn from the cross-sector alliance experience, most likely because it presents a less novel experience or because they are not as open to experiencing these differences. (For more information, please contact: Aline Gatignon, INSEAD, France: aline.gatignon@insead.edu)

Internationalization Challenges of Rural Area Expansions in Emerging Economies -Business-NGO Relationship Processes

Anna Ljung, Uppsala University 
Rural areas are becoming increasingly important for MNCs alongside the traditional urban areas in emerging economies, owing to the Base of the Pyramid opportunities. New areas of MNC internationalization are thereby gaining importance which calls for adaption as well as creation of new internationalization concepts. Institutional voids make relationships central in rural settings as well as NGOs given their networks, local knowledge and legitimacy. The objective then is to contribute to an analytical framework for the rural expansion process through the study of a focal business-NGO relationship of an MNC expansion to the Brazilian Amazon. Knowledge and legitimacy are applied as tools to analyze the relationship, with basis in internationalization process and business-NGO relationship literature. The results show differences from the traditional internationalization process to consider when expanding to rural contexts; findings interpreted as "intersectoralization". The intersectoralization process was driven back- and forward by the business-NGO relationship. Several studies underline the centrality of business-NGO relationships in rural expansion. However, as shown by these results, business-NGO relationships also need to be problematized per se in order to understand and manage rural expansion processes. (For more information, please contact: Anna Ljung, Uppsala University, Sweden: anna.ljung@fek.uu.se)

\section{Session: 1.5.11 - Competitive}

\section{Track: Track: 3 - IB Theory, FDI, and Entry Mode}

\section{FDIs from Emerging Economies}

Presented On: July 4, 2013 - 16:45-18:00

Chair: Mo Yamin, University of Manchester

Internationalize to Escape? Evidence from Family Firms in China

Kimberly Eddleston, Northeastern University

Ruihua Joy Jiang, Oakland University

Michael D. Santoro, Lehigh University

Elitsa Banalieva, Northeastern University

We apply institution-based view and behavior agency theory model to study the internationalization of family firms from transition economies. Specifically, we analyze how the speed of pro-market reforms (the rate of overall structural adjustment) affects the internationalization (the foreign market penetration) of family firms from China. We also suggest that this baseline relationship is further modified by the degree of family ownership (the cash flow stake of the family) of the Chinese family firms. We test our framework on 123 publicly listed family firms from 10 Chinese provinces with varying speeds of pro-market reforms during 20042009 and find overall support for our theory. (For more information, please contact: Ruihua Joy Jiang, Oakland University, USA: jiang@oakland.edu)

\section{Strategic Entry or Strategic Exit? International Presence by Emerging Economy Enterprises Stephanie Wang, University of Miami Yadong Luo, University of Miami Vladislav Maksimov, University of Miami}

By proposing an integrated strategic choice framework, we aim to systematically theorize the distinctive dynamics of international expansion by emerging market enterprises. Specifically, we theorize how these firms build international presence based on combined strategic entry (i.e., prompted by internal capabilities such as innovation and diversification) and strategic exit (i.e., pushed out by external handicaps at home such as institutional obstacles and market competition). Further, a firm's cooperative ties with foreign multinationals at home fortify the strategic entry intent, while ties with home government weaken the strategic exit intent. We also confirms that building an international presence helps bolster a firm's performance, highlighting the 
economic catch-up consequence of international expansion. Analyses of a two-year imbalanced panel data of 2,136 firms statistically support our hypotheses. (For more information, please contact: Stephanie Wang, University of Miami, USA: slu@bus.miami.edu)

\author{
Performance Implications of Internationalization Market Timing Strategy: Evidence from China \\ Lin Yuan, University of Macau \\ Xiaolin Qian, University of Macau \\ Nitin Pangarkar, National University of Singapore
}

We integrate market timing theory, which argues that firms opportunistically raise external capital (e.g., debt or equity) when they enjoy high market value, and international business literature to investigate firms' timing of international investments. We also examine the implications of investment timing for firm performance. Based on an analysis of data about listed Chinese multinational corporations between 1992 and 2010, we find that though market value in the immediately preceding time period is not a significant predictor of the likelihood of international investment, consistently high market values in the past can predict this likelihood well. We also find that alignment between firms' international investment timing and market timing leads to better performance in both the short and long runs. Our findings point to the importance of waiting for good market timing rather than pioneering in international expansion. (For more information, please contact: Lin Yuan, University of Macau, Macau: linyuan@umac.mo)

Session: 1.5.12 - Interactive

Track: Track: 1 - Institutions, Governance, and CSR

\title{
Bottom of the Pyramid and NGOs
}

Presented On: July 4, $2013-16: 45-18: 00$

Chair: Carlos Rufin, Suffolk University

Revisiting the Stakeholder's Salience Model in Non Governmental Organizations

Florent Pestre, University Paris Sud

Shahzad Khurram, University Paris Sud

We propose to build on Mitchell et al.'s (1997) theory of stakeholder salience and extend it from mere presence or absence of attributes to the domain of prioritization of attributes and their types. This paper will try to establish on the basis of institutional theory (Powell \& DiMaggio, 1991; Powell, 1991), that various stakeholder groups depending on the type of their institutional logic extract salience from different salience attributes differentially - such that the prioritization of salience attributes is different for various stakeholder groups. NGOs as stakeholders are of particular interest to us as we suggest that, unlike other stakeholders, NGOs carry unique institutional logic and extract salience differently when compared to other stakeholders. Utilizing the robust theoretical foundations provided by stakeholder and institutional theories, this paper examines the relationship between MNEs and NGOs to see how institutional logic affects the managerial perception which in turn makes managers attend various stakeholders differentially. (For more information, please contact: Florent Pestre, University Paris Sud, France: florent.pestre@u-psud.fr)

Women's Empowerment: Involving Local Actors and an International Non-Governmental Organization in a Fishing Village in the Northeast of Brazil

Regina Yoshie Matsue, University of Fortaleza

Mario Henrique Ogasavara, University of Fortaleza

Fatima Regina Ney Matos, University of Fortaleza 
This study explores the role of local actors and an international non-governmental organization (NGO) in the process of women's empowerment in a fishing village on the coast of a northeastern part of Brazil. The theoretical framework is based on concepts of gender and empowerment. The study has the anthropological perspective of field work research. The results show how the external influences brought about by tourism had a negative impact on the community's traditional way of life. Otherwise, these influence also brought about the first stimulus to the women's movement. Additionally, an international NGO headed by a Japanese woman acts as a mediator between the interests of the community and other partner institutions. Supported by the local people, this NGO achieves a crucial role on the process of empowering the community. Despite gender inequalities, women are the main protagonists in the struggle for the empowerment of the individuals and the community. (For more information, please contact: Regina Yoshie Matsue, University of Fortaleza, Brazil: rymatsue08@unifor.br)

\section{Multiple Institutional Logics and Inter-Organizational Partnerships: The Dynamics of Cooperation between Social Enterprises \\ Imran Chowdhury, Pace University}

This paper presents a study on the evolution of partnerships between social enterprises, organizations that are embedded in competing social and economic logics. Through longitudinal case studies of the interaction between four pairs of social enterprises operating in emerging economy settings, I analyze the factors which influence the evolution of knowledge transfer partnerships. Evidence from these case studies suggests that a variety of logics, not simply social and economic logics, were guides for organizational action, and that the extent to which these logics were aligned between firms determined the how firms managed the knowledge transfer process. (For more information, please contact: Imran Chowdhury, Pace University, USA:

ichowdhury@pace.edu)

\section{Sourcing from the Base of the Pyramid: Pricing Innovations when Markets do not Exist Dirk Michael Boehe, University Of Adelaide Leandro Pongeluppe, Insper Institute of Education and Research Sergio Giovanetti Lazzarini, Insper Institute of Education and Research}

This study addresses the problem of how private companies can source new supplies from the base of the pyramid despite inherent market failures. We argue and empirically illustrate that innovative pricing strategies should take account of externalities and imperfect information in order to generate new markets for supplies from the Base of the Pyramid (BoP). An in-depth case study of Latin American's largest cosmetics firm, Natura, reveals how such innovative pricing strategies work in practice and under what contextual conditions they may succeed. Our results suggest non-market pricing strategies where conventional micro-economic assumptions are not valid. Thus, this study intends to advance the scholarly and practitioner conversation on how upstream BoP markets can be further developed. (For more information, please contact: Dirk Michael Boehe, University Of Adelaide, Australia: dirkmichael.boehe@adelaide.edu.au)

\section{Innovation for Base of the Pyramid: A Study from the Perspective of Required Firm Capabilities Rifat Sharmelly, University of New South Wales \\ Sangeeta Ray, University of Sydney \\ Pradeep Kanta Ray, University of New South Wales}

Innovation strategies of multinational companies (MNCs) have been increasingly influenced by new approaches as companies from emerging economies (EEs) are developing low cost disruptive innovations. The base of the pyramid (BoP) in EEs is considered to be the largest untapped market on earth. Companies from developed and EEs are becoming increasingly eager to prosper in these markets. However, companies face the challenge to 
identify what organizational capabilities are required to serve mass market customers to meet their unique demand and price-performance conditions. In depth empirical studies in this context are largely unexplored in the academic literature. Focusing on the product innovation of two emerging market firms, our analysis reveal that combinative capabilities are required to achieve innovations with required affordability and acceptability criteria. Furthermore, alliance formation capabilities are required to enhance the innovative performance. In addition, capability to modularise is vital for modularity to meet evolving needs of BoP customers. Using case study design, our study aspires to contribute to the innovation literature on mass markets and provide managerial implications for MNEs as well as for academia. (For more information, please contact: Rifat Sharmelly, University of New South Wales, Australia: rifat.sharmelly@unsw.edu.au)

\section{Session: 1.5.13 - Interactive \\ Track: Track: 7 - Emerging Economies}

\section{Managing Institutional Environments in Emerging Markets}

Presented On: July 4, $2013-16: 45-18: 00$

Chair: Andrew Spicer, University of South Carolina

Entrepreneurship and the Face of Janus of Institutions: Stimulus Policies for High-Impact Entrepreneurs in Brazil and Russia

Gilberto Sarfati, FGV-EAESP

Institutional theory has been widely applied to the study of entrepreneurship. Based on the current understanding of the institutional gap, we suggest that the relationship between institutions and entrepreneurship in emerging economies is reminiscent of the Face of Janus. Janus is a mythological figure with two faces, one looking backward and the other looking forward. Therefore, he is associated with transition and the chaos connected with the ambiguous relationship between the past and the future. This ambiguity may be seen as characteristic of entrepreneurship in emerging economies. In other words, the Face of Janus that looks backward corresponds to the institutional void, and the face that looks forward corresponds to stimulus policies that promote high-impact entrepreneurs. In this article, we comparatively discuss two case studies in Brazil and Russia. In Brazil, the Agency for Innovation's (Financiadora de Estudos e Projetos - FINEP) INOVAR program was explored along with its relationship with the entrepreneur Daniel Heise of Grupo Direct. In Russia, the relationship between the Skolkovo Foundation and the entrepreneur Yuri Deigin of Pharma Bio was explored. This article contributes to the entrepreneurship literature by advancing the concept of the institutional void in the context of emerging economies. (For more information, please contact: Gilberto Sarfati, FGV-EAESP, Brazil: gilberto.sarfati@fgv.br)

\section{Good Intentions, Fragile Legitimacy: Evidence from the Airline Industry in Africa Joseph Amankwah-Amoah, Bristol University \\ Yaw A. Debrah, Swansea University}

Existing literature on the use of strategic alliances to achieve organisational legitimacy has largely focused on firms in developed countries, overlooking the experiences of firms in emerging economies in changing institutional environments. This article presents evidence of an study of eight African airlines' attempts to gain legitimacy through alliance formation. The study revealed that the firms are driven into alliances with established or well-known carriers not only to overcome resource and capability constraints, but crucially for signalling to acquire legitimacy and, hence, greater credibility in dealing with key external constituents such as customers, suppliers and investors. The findings also indicate that firms' attempts to overcome legitimacy deficits are hampered by deviant acts by similar firms in the same environment. The broader theoretical, managerial and policy implications of these findings are then discussed. (For more information, please contact: Joseph Amankwah-Amoah, Bristol University, United Kingdom: joseph.amankwah-amoah@bristol.ac.uk) 
Institutional Environment and Multinational Enterprises' Post Entry Choices-an Institutional Perspective

Chang Liu, University of International Business and Economics

Zijie Li, University of International Business and Economics

Yi Li, Australian National University

Yuting Liang, University of International Business and Economics

This study investigates the post entry ownership decisions of multinational enterprises (MNEs) when they enter emerging economies. Based on institutional perspective, we propose a decision-making framework of MNEs? post entry ownership and discuss determinants of the decisions. We argue that local government influence on firms is positively related to the international joint ventures? (IJVs?) conversion to wholly-owned subsidiaries. And the positive relationship will be moderated by local parents? contribution to legitimacy and home-host countries cultural distance. We test our hypotheses using a sample of 977 Sino-foreign IJVs over a three-year period (2006-2008) using Cox hazard models. The majority of our hypotheses have been supported by empirical results. (For more information, please contact: Chang Liu, University of International Business and Economics, China: szs_/c1992@163.com)

\section{Emerging markets, informal institutions and strategy formulation Michael A. Sartor, University of Western Ontario}

This paper investigates how differences in the informal institutions prevailing in the home and host countries influence the foreign entry strategies of multinational enterprises (MNEs). While the relationship between formal institutions and MNE strategy has been the subject of considerable academic scrutiny, less is known about the role of informal institutions. Our theory contends that the type of uncertainty precipitated by informal institutions is critical to understanding the strategic behavior of foreign-investing MNEs. More specifically, we disaggregate the informal institutions construct and develop two new, more explicit, latent constructs behaviorally-oriented informal institutions and technology-oriented informal institutions. Our theory posits that increased behavioral informal institutional distance will precipitate a preference for full ownership (WOS) and a greater proportion of expatriates in subsidiary investments. Conversely, heightened technological informal institutional distance will predict the opposite outcome - a preference for shared ownership (JV), host country partners rather than home country partners and a smaller proportion of expatriate employees. We test our theory using a longitudinal sample of subsidiary investments that were established in emerging markets for the purpose of engaging in innovation-related activities. The results provide support for our conceptualization of informal institutions and the construct's relationship with the entry strategies of foreign-investing MNEs. (For more information, please contact: Michael A. Sartor, University of Western Ontario, Canada: msartor.phd@ivey.ca)

\section{The Domain of Emerging Markets: An Exploratory Examination into the Nature and Characteristics of this Concept}

Francesca Spigarelli, University of Macerata

Daniel Rottig, Florida Gulf Coast University

The existing literature does not converge on a common and unique description of the characteristics of emerging economies (EMs), besides the significant importance and relevance of this term from an economic, academic and political perspective. The paper is a first attempt to explore a broad, comprensive, and dynamic definition of EMs, embracing not only economic parameters but involving social, institutional, and cultural aspects. We compare and analyse the main country classifications used within international institutions explore commonalities and critical issues. We then focus on the interest on EMs for international business studies, examining trends in publications related to EMs. We finally look at several open issues related to EMs in term of economic development, political changes, people attitude and behaviour, technologies, firm strategies and international market participations. (For more information, please contact: Daniel Rottig, Florida Gulf Coast University, USA: drottig@fgcu.edu) 
Internal Market Failure in Emerging Economy Firms

Michael N. Young, Hong Kong Baptist University

Mike W. Peng, University of Texas at Dallas

Omer Nesim Gokalp, University of Texas at Dallas

Xinran Wang, Hong Kong Baptist University

Although emerging economies $(\mathrm{EE})$ are now responsible for a much larger share of world GDP, there are still few examples of EE-based firms that excel in high value-added activities requiring specialized human capital investment. We argue that this situation is partly due to the institutional conditions in EE. Previous research has pointed out that the institutional structure of EE leads to higher costs for arm's-length exchanges, which provide less support for market transactions leading to a higher incidence of market failure. However, much less attention has been paid to the institutional structure inside of firms. Applying the logic of the Coase Theorem, we argue that poorly-specified organizational property rights make for a series of firm inefficiencies that collectively are termed internal market failures. This makes it difficult for EE firms to compete in global markets. This is particularly true for high value-added, knowledge-based activities. After reviewing and synthesizing research on the relationship between the institutional environment and internal market failures, we discuss the implications for management research and practice and provide several possible extensions for further research. (For more information, please contact: Omer Nesim Gokalp, University of Texas at Dallas, USA:

omer.gokalp@utdallas.edu)

\section{Session: 1.5.14 - Interactive}

Track: Track: 9 - Cross-Cultural Management and HRM

\section{Top Management Teams, Leader Succession \& Organizational Culture}

Presented On: July 4, 2013 - 16:45-18:00

Chair: Karsten Jonsen, IMD

\section{Post-Succession Predecessor-Successor Interactions and Their Relational and Organizational Outcomes Sinan Caykoylu, Simon Fraser University}

The psychological transition from one role to another that the actors have to go through during a succession process, carries significant risks and opportunities for family businesses. The general assumption about the succession process is that incumbents gradually decrease their involvement in organizational activities and the successors increase theirs. In the end, once the transfer of managerial responsibility and authority is complete, predecessors quickly and quietly fades away from the picture. Although this view of succession may be true for some organizations, findings of this qualitative exploratory research indicate that this is not the case for the majority of family firms where there are on-going and overlapping business and kinship relations. Data collected through interviews with twenty-six participants, predecessors and successors, from nineteen Turkish family businesses led to the development of a model that shows post-succession predecessor and successor interactions do not end with the transfer of title, authority and/or responsibility from one to the other. Instead, it continues in various forms and shapes affecting their relationships with each other and affecting the business. This continued predecessor-successor interaction during the post-succession period carries significant theoretical and practical implications. (For more information, please contact: Sinan Caykoylu, Simon Fraser University, Canada: scaykoyl@sfu.ca)

The Effect of CEOs Expertise on the Performance of Microfinance Institutions: A Global Survey Neema Mori, University of Dar es Salaam Daudi Pascal, University of Agder Roy Mersland, University of Agder 
This study examines the extent to which the Chief Executive Officers' (CEOs) expertise is associated with the performance of Microfinance institutions (MFIs). We are guided by upper echelons and resource based theories and use a global dataset of 403 rated MFIs located in 74 countries for the period of 2001 to 2009. The study uses random effects panel data estimations to analyses the effect of CEOs with business education and business experience on return on assets, operational costs, and portfolio at risk of 30 days, which measures MFIs' financial performance; and average loan size, women clients and number of clients, which capture, outreach performance. The results show that MFIs whose CEOs have business experience are associated with better returns, lower costs, fewer defaults, smaller loans and higher number of clients. While MFIs whose CEOs have formal business education are associated with higher percentage of women clients, credit clients' growth and smaller loans compared to MFIs whose CEOs do not have formal business education. These findings suggest that the microfinance industry can benefit from non-microfinance experienced CEOs. (For more information, please contact: Neema Mori, University of Dar es Salaam, Tanzania: neema@imedtz.org)

\section{Top Management Teams' Competencies and its Influence on a Firm's Results: Internationalization Perspective Analysis}

Marina Latukha, Saint-Petersburg State University

Andrei Panibratov, Saint-Petersburg State University

Firms nowadays face numerous challenges when operating abroad. Among others, an important factor influencing on companies' successful operations in international markets is the competencies of the top management team (TMT), which significantly influence an organization's results. This paper explores how TMT's competencies are associated with the realization of a firm's internationalization strategy. The findings indicate the strong correlation between top management competencies and the firm's international results. Moreover, we found that the most significant competencies for internationalization strategy are rational exactingness, ability to create and to use foreign contacts, project aptitude, and ability to create multicultural interaction. (For more information, please contact: Marina Latukha, Saint-Petersburg State University, Russia:

marina.latuha@gsom.pu.ru)

Involvement Work Systems, Climate of Participation, and Facility Performance: Exploring the Moderating Effect of National Power Distance

Yuan Jiang, Indiana University - Purdue University Fort Wayne

Saba Colakoglu, Berry College and Koc University

Dave Lepak, Rutgers University

Joseph Blasi, Rutgers University

Doug Kruse, Rutgers University

We develop a model of a system of involvement-oriented work practices and its effects on organizational climate of participation and facility performance. We also incorporate the influence of national cultural differences in power distance. Analysis of multi-source, multi-level data from 260 facilities of a multinational company operating in 22 countries indicates that the relationship between involvement work systems and performance is mediated by a participatory climate. Furthermore, involvement work systems create a stronger organizational climate and participatory climate is a stronger predictor of performance in facilities from lower power distance countries. On the other hand, the direct influence of the work system on performance is stronger in high power distance societies. Results point to cross-cultural differences in reactions to the symbolic versus instrumental aspects of involvement work systems and the importance of the role that shared employee perceptions play in the involvement and performance relationship. (For more information, please contact: Saba Colakoglu, Berry College and Koc University, USA: scolakoglu@berry.edu) 
Internationalization of Top Managers in Germany and the UK - Cross-national Differences or Similarities?

Tobias Dauth, Rouen Business School

Stefan Schmid, ESCP Europe

Dennis Wurster, ESCP Europe

Many management scholars and practitioners expect an increasing convergence of corporate governance practices across countries. The question if and how (converging) corporate governance systems affect top management team composition and top management internationalization in particular is one important topic within this debate that is still underexplored. Building on human capital theory we explain why international firms can benefit from employing international top managers. Furthermore, we draw on literature assuming a convergence of governance practices to argue that many firms across European countries face the demand to increase the internationalization of their top management. In our empirical analysis we shed light on the internationalization of top managers in German and UK firms. Our findings show that the de jure convergence of corporate governance practices has not yet led to a de facto convergence in top management internationalization. Based on a multiple construct of 'internationalization', our study illustrates that the way how top managers of German and UK firms internationalize during various stages of their life and career varies considerably depending on their national context. Institutional theory helps us to explain which country-specific economic, political, legal and socio-cultural factors are associated with top management internationalization in Germany and the UK acting as impediments to convergence in corporate governance practice. (For more information, please contact: Tobias Dauth, Rouen Business School, France: tdt@rouenbs.fr)

\section{Are Culturally Robust Japanese and US Companies Built to Last? The Effect of Organizational Culture on Organizational Mortality \\ Takashi Sakikawa, Niigata University}

In this research, by drawing on evidence from Japanese and US companies, I explore the effect of organizational culture on organizational mortality or, conversely, organizational survival, which is a significant, but unproved claim in early organizational studies. The results of survival analysis revealed that culturally strong companies from Japan as well as the US have highly likely survived beyond the turn of the century into the present. They also showed that, even after controlling for the country effect or irrespective of difference in national environments, strong culture can sustain organizations over a period of long years. (For more information, please contact: Takashi Sakikawa, Niigata University, Japan: saki@mot.niigata-u.ac.jp)

\section{across the Taiwan Strait \\ Yi-Hui Ho, Chang Jung Christian University \\ Chieh-Yu Lin, Chang Jung Christian University}

The Association between Leader Moral Development and Follower Moral Development: A Comparative Study

Several business scandals have highlighted ethical leadership. The ethical concerns of leaders may influence ethical decision-making of followers. The purpose of this paper is to explore the relationship between leader moral development and follower moral development. Furthermore, both China and Taiwan are apparently influenced by the Chinese culture; however, they have been separated for nearly 60 years, and each of them has developed its own sociocultural system. Still, business activities across the Taiwan Strait were grown significantly for the past decades. A comparison on the leader-follower moral development relationship between these two areas is also conducted in the study. A sample of purchasing professionals in Taiwan and China was assessed in terms of their moral development measured by the Defining Issues Test. The findings indicate that leaders have higher overall scores on moral development than the followers. There is a positive relationship between leader moral development and follower moral development. The followers exhibit higher moral development while their leaders have higher moral development. The leader-follower moral development relationship is stronger for Chinese purchasing professionals than their Taiwanese counterparts. Implications of research findings are also discussed. (For more information, please contact: Chieh-Yu Lin, Chang Jung Christian University, Taiwan: jylin@mail.cjcu.edu.tw) 


\section{Factors Affecting the Staffing of General Managers of Foreign Subsidiaries \\ Hsiao Wen Liu, National Chengchi University \\ Hui Yun Chiu, National Chengchi University \\ Chow Ming Yu, National Chengchi University}

Following the path of multinational corporations (MNCs) from developed countries (DCs), more and more firms from developing countries (LDCs) have engaged in foreign direct investment (FDI), especially in DCs (i.e., upstream FDI). However, only a few researchers have examined the issues related to upstream FDI. Furthermore, in examining firms' activities in host countries, most researchers have taken manufacturing subsidiaries as taken-for-granted research samples. Only a few studies put sales subsidiaries into consideration. Using a case study approach, this study addresses the following two research questions: (1) what is the process of setting up sales subsidiaries in DCs by Taiwanese high-tech firms and how these subsidiaries operate? , and (2) how do Taiwanese high-tech firms select the first country managers for sales subsidiaries in DCs? The research findings would enhance our understanding of upstream investment and offer managerial implications for LDCMNCs when operating sales subsidiaries in DCs. (For more information, please contact: Hui Yun Chiu, National Chengchi University, Taiwan: 99355503@nccu.edu.tw)

\section{Session: 1.5.15 - Interactive Track: Track: 3 - IB Theory, FDI, and Entry Mode}

\section{FDI and Location}

Presented On: July 4, 2013 - 16:45-18:00

Chair: Mohammad Akbar, Indian Institute of Management Lucknow

The World is Spiky: Firm Internationalization in a Semi-Globalized World Jin Uk Kim, University of Illinois at Urbana-Champaign

Recent works in international business indicate that majority of multinational enterprises operate on a regional rather than a global basis due to the partially integrated or semi-globalized nature of the world economy. To understand the dynamics of firm internationalization in a semi-globalized world, the nexus between firm and countries need to be supplemented with that between firm and regions. We propose a conceptual framework that captures how the regional dimension influences the process of firm internationalization. Integrating key ideas from the research on regionalization, Uppsala internationalization process model and organizational learning literature, we argue that cross-border expansion in a semi-globalized world unfolds through three distinct phases: i) intra-regional exploitation; ii) regional-reconfiguration; iii) inter-regional exploration. After elaborating on the mechanism that drives each phase, we explicate the link between each phase and derive testable propositions. We further theorize how firm and industry level contingencies may moderate the predictions of our model and derive testable propositions, providing a comprehensive model that captures firm internationalization in a semi-globalized world. (For more information, please contact: Jin Uk Kim, University of Illinois at Urbana-Champaign, USA: jkim198@illinois.edu)

The Regionalization Strategy Hypothesis: Evidence from the Australian Internationalization Experience Youngok Kim, University of New South Wales

Sidney John Gray, University of Sydney

In the context of the regionalization versus globalization strategy debate, this study examines the relevance of Rugman et al.'s home-regionalization claim in a unique empirical setting i.e., Australian internationalization. Using longitudinal two-wave data for 2001 and 2007 from 170 Australian listed MNEs, we find that while home regionalization is evident, its degree is less pronounced when modified classification thresholds are used. Furthermore, home-regionalization is found to decrease over the 2001-2007 period. A more fine-grained 
measure of internationalization newly introduced in our study supports regionalization, albeit not necessarily home-regionalization. Unlike the largest firms included in prior studies, however, Australian MNEs demonstrate substantial host-regionalization as well. (For more information, please contact: Youngok Kim, University of New South Wales, Australia: y.kim@unsw.edu.au)

\author{
Foreign Market Selection of Established MNEs' New Business Units \\ Thomas Hutzschenreuter, WHU Otto Beisheim School of Management \\ Ingo Kleindienst, WHU \\ Christina Guenther, WHU \\ Martin Hammes, WHU
}

This study takes a business unit level perspective to explore foreign market selection of established MNEs' new business units (NBUs). Entering foreign markets in which their parent MNEs are already active in with other business units, NBUs encounter conditions that are significantly different from those encountered by MNEs entering a foreign market for the first time. Most notably, NBUs may engage in knowledge transfer processes in order to obtain foreign market specific business knowledge and institutional knowledge. We hypothesize that both relatedness to existing business units in the foreign market as well as size of the presence of the parent MNE in the respective market increase NBUs' foreign market entry likelihood. Conversely, we hypothesize that geographic distance to NBUs' market of inception has a negative effect on foreign market entry. Studying 482 target country entries by 788 new business units of 90 German MNEs between 1985 and 2007 we find strong support for our hypotheses. (For more information, please contact: Thomas Hutzschenreuter, WHU Otto Beisheim School of Management, Germany: th@whu.edu)

\title{
Geographic Concentration and Institutional Diversification: The Location Strategy for Multinational Banks I Han, Feng Chia University Hsin-Yu Liang, Feng Chia University
}

This paper demonstrates how FDI location strategy can enhance multinational enterprise (MNE) performance. Based on the eclectic paradigm, we argue that the FDI location strategy of geographic concentration can enhance the MNE's monopolistic power to reduce costs of using location-specific endowments in the host country. Meanwhile, the location strategy of institutional diversification can internalize the MNE's differential market imperfections across dissimilar institutions to reduce risks across countries. We use the data from the top 100 global banks during 2002 to 2007 to support our arguments. Our findings provide the evidence that geographic concentration and institutional diversification location strategies can enhance MNE performance independently, interactively, and simultaneously. Instead of merely examining geographic scope and scale of FDIs, we make contributions to theoretical and practical implications by suggesting that the FDI decision demands a fundamental evaluation by incorporating the geographic location strategy. (For more information, please contact: Hsin-Yu Liang, Feng Chia University, Taiwan: lianghy@fcu.edu.tw)

\section{Substitutes or Complements? Performance Implication of Joint Decision on FDI Location and Entry Mode Min Jung Kim, Korea University Jon Jungbien Moon, Korea University}

This study examines how entry mode and location choice with respect to agglomeration influence each other when firms make a FDI using a large panel data from China and how these interrelated choices would affect the performance of foreign invested firms. In order to address endogeneity issue in comparing the performance, we employ the propensity score matching method and difference-in-differences approach. We find that firms consider high agglomeration and joint venture as substitutes in gaining local knowledge and the benefits from $\mathrm{JV}$ and benefits from agglomeration economies can be different in terms of getting access to local knowledge. (For more information, please contact: Jon Jungbien Moon, Korea University, Korea, South: jonjmoon@korea.ac.kr) 
China's Outward Foreign Direct Investment (oFDI) from the Private Sector: Explaining the Differences of the Locational Determinants between Privatized State-Associated Enterprises (PSES) and Privately Owned

Enterprises (POEs)

Wonchan Ra, Hankuk University of Foreign Studies

Chunxiang Qian, Hankuk University of Foreign Studies

This study examines the determinants of foreign direct investment (FDI) location choices by Chinese firms from a unique perspective. Based on research into the privatization process in China, we categorize Chinese private enterprises into two types: privatized state-associated enterprises (PSEs) and enterprises that have been privately owned from their founding (POEs). To our knowledge, this has not been done before in the international business literature. We establish four hypotheses regarding the differences between PSEs and POEs in how they select outward FDI (oFDI) locations and empirically test these by estimating a regression model, using the host country variables of natural resources, strategic assets, risk and Chinese networks. The results indicate that PSEs are more attracted to host countries with rich natural resources and strategic assets than are POEs. However PSEs are less concerned with local Chinese networks. This study is the first attempt to investigate how privatized state enterprises and purely private firms differ in their FDI location choices. It recognizes the profound impact of both the Chinese privatization process and the intra-group heterogeneity of private firms on their FDI behavior by a comparison between PSEs and POEs that has not previously been explored in the extant literature. (For more information, please contact: Wonchan Ra, Hankuk University of Foreign Studies, Korea, South: wonra1@gmail.com) 


\section{ABSTRACTS FOR FRIDAY, JULY 5, 2013}

\section{Session: 2.1.1 - Panel}

\section{Turkey Today: Three Contemporary Views on the Political and Economic Context of Turkey}

Presented On: July 5, $2013-09: 00-10: 15$

Chair: Nakiye A. Boyacigiller, Sabanci University

Turkey's Place in the World: Relations with Europe, Middle East and the US Soli Ozel, Kadir Has University

Turkey's Economy: Strengths and Weaknesses as it Moves Toward EU Membership or Regional Leadership Izak Atiyas, Director, TUSIAD-Sabanci University Competitiveness Forum

The State of Turkey's Democracy: Constitutional Reforms and the Recent Peace Initiative

E. Fuat Keyman, Director, International Policy Center

(For more information, please contact: Nakiye A. Boyacigiller, Sabanci University, Turkey: nakiye@sabanciuniv.edu)

\section{Session: 2.1.2 - Panel \\ Track: Track: 1 - Institutions, Governance, and CSR \\ Corporate Social Responsibility: Defining the Domain}

Presented On: July 5, $2013-09: 00-10: 15$

Chair: Sushil Vachani, Boston University

Panelists:

Sushil Vachani, Boston University

Hildy Teegen, University of South Carolina

Jennifer Oetzel, American University

Nalin Kulatilaka, Boston University

Sarianna Lundan, University of Bremen

As multinationals increase reliance on global value chains, venture further into unstable and risky institutional environments, delve deeper into bottom-of-the-pyramid markets, and are faced with environmental changes such as climate change, expectations about corporate social responsibility (CSR) are evolving. This roundtable will focus on three broad areas that are relevant for defining CSR's domain. First, risk, which varies across international contexts in nature and extent. It discourages funding of social initiatives, such as "green projects", and deters multinationals from tapping into lucrative business opportunities. Second, transparency, the lack of which presents a challenge in the evaluation of responsibility for infringement of human rights in global value chains and the market's willingness to fund social initiatives. Third, organizational capabilities, which, if appropriately developed, can generate social value as well as create competitive advantage. (For more information, please contact: Sushil Vachani, Boston University, USA: sushilvachani@gmail.com) 
Session: 2.1.3 - Panel

Track: Track: 8 - Developing Country MNCs

\section{Regional Aspects of the Multinationality-Performance Research: Does the Context Matter for the M-P Relationship in Emerging Markets?}

Presented On: July 5, $2013-09: 00-10: 15$

Chair: Sumit Kundu, Florida International University

Panelists:

Jean-Francois Hennart, Singapore Management University and University of Pavia

Alan M. Rugman, University of Reading

Alvaro Cuervo-Cazurra, Northeastern University

Ahmet H Kirca, Michigan State University

This panel proposes that the current debate on the M-P relationship should focus more heavily on the regional aspects of multinationality-performance relationship because contextual considerations are as critical as, if not more than, the methodological refinements in the multinationality research. Thus, the purpose of this panel is to unveil and discuss the theoretical challenges and opportunities in the next decade in this line of research and practice with a special focus on the regional aspects of M-P research. In particular, our four panelists who have made substantial contributions pertaining to the contextual issues in the MP literature will discuss how and why context matters (or not) for the M-P relationship in emerging country markets. After four decades of extensive research on $M-P$, it appears to be the right time to take stock and reflect on what we know so far and assess the current state of the M-P research with a regional focus and to provide a roadmap for future research, practice, and education. (For more information, please contact: Ahmet H Kirca, Michigan State University, USA: kirca@msu.edu)

\section{Session: 2.1.4 - Panel}

Track: Track: 6 - Innovation and Knowledge Mgmt.

\section{Cross-Border R\&D Collaborations and Innovation Performance}

Presented On: July 5, $2013-09: 00-10: 15$

Chair: Annique Un, Northeastern University

Discussant: Ulf Andersson, Copenhagen Business School

Panelists:

Annique Un, Northeastern University

Kazuhiro Asakawa, Keio University

Anupama Phene, George Washington University

Mariko Sakakibara, UCLA

In this panel we will analyze how firms establish cross-border R\&D collaborations and the impact of these on innovation performance. We will establish a bridge between two strands of the literature that have operated mostly independently: the literature analyzing R\&D collaborations, which has mostly studied collaborations within one country, and the literature studying cross-border R\&D investments, which has mostly focused on the decision to subcontract or internally invest in R\&D. We will argue that linking these two streams can enrich both by identifying new areas of study, particularly the identification of the challenges of establishing and benefitting from R\&D collaborations. The panelists will go deep into the analysis of different types of R\&D collaboration (with universities, suppliers, customers, and competitors), review what we know about the topic, and identify 
new avenues for research that the cross-border dimension creates. Some of these are, the added challenges of establishing connections with local universities when facing national security concerns, the integration of conflicting insights from customers across countries, the reluctance to transferring technologies jointly developed with suppliers in one country to suppliers in other countries, or the added complexity to coopetitive interactions across countries. (For more information, please contact: Annique Un, Northeastern University, USA: a.un@neu.edu)

\section{Session: 2.1.5 - Competitive \\ Track: Track: 6 - Innovation and Knowledge Mgmt.}

\section{Knowledge Development}

Presented On: July 5, 2013 - 09:00-10:15

Chair: Sylvie K. Chetty, University of Otago

\section{Managing Competency Creating R\&D Subsidiaries in Multinationals: Not too much autonomy or too much control}

Kazuyuki Motohashi, University of Tokyo

When an overseas subsidiary is given strategically important role in world-wide activities of multinationals, a managerial tension between headquarter and its subsidiary arises. In this paper, we found that either too much control of headquarter or autonomy of subsidiary does not work for effective competency creation at multinational's local site. In addition, it is important for a headquarter to accumulate experiences at host country operation to manage competence creating overseas subsidiary, particularly in emerging economies such as China, where a local business context is much different from home country. (For more information, please contact: Kazuyuki Motohashi, University of Tokyo, Japan: motohashi@tmi.t.u-tokyo.ac.jp)

\section{Institutional Regime Shift in Intellectual Property Rights and Patenting Strategies of Firms in China Kenneth Huang, Singapore Management University \\ Xuesong Geng, Singapore Management University Heli Wang, Singapore Management University}

This study develops a new conceptual framework to understand the differential impact of formal institutional regime shift in intellectual property rights on the innovation and patenting strategies of Chinese and Western firms operating in China. We argue that compared with Western firms, Chinese firms will more likely resort to alternative informal practices that are deeply rooted in the traditional Chinese philosophies to safeguard their intellectual assets from expropriation. Moreover, the complex and ambiguous interdependence between institutional forces in China makes it more feasible for Chinese firms to adopt such informal approaches. Using the major China patent law reform of 2001 as a natural experiment, we find results consistent with our key arguments: With the strengthening of (a previously weak form of) patent protection, Chinese firms do not increase the adoption of such protection for their internationally valuable innovations as much as Western firms do. However, the difference becomes less salient in regions with more robust legal institutions that foster R\&D and innovation, and when Western firms operate longer in China. This study advances our understanding of how institutions can shape the strategic innovative behaviors of Chinese and Western firms in the transitional economy of China. (For more information, please contact: Kenneth Huang, Singapore Management University, Singapore: kennethhuang@smu.edu.sg) 
The Knowledge Broker. A Strategic Orientation and an Organizational Capability for Competing in Global Markets

Stefano Denicolai, University of Pavia

Antonella Zucchella, University of Pavia

Roger Strange, University of Sussex

This study analyses the knowledge broker, defined as a knowledge intensive type of intermediation among dispersed expertise at the global level - upstream - and client's needs or latent opportunities - downstream -. Hargadon in 1998 introduced this concept setting a promising research agenda, but then focused mainly on the technology brokering activity. After 15 years this model calls for further investigation. The aim of this contribution is to provide a better understanding about knowledge brokering: which are the differences from other strategic orientations, the key capabilities needed to support effectively this activity and the business practices in the actual scenario, its impact on the competitive advantage. The study proposes a case study survey concerning three companies: Accenture, Alessi, ENI. Evidences support knowledge broker as a 'creator' and not purely carrier of knowledge between disconnected domains. The geographic dimension emerges in all the analyzed cases. The knowledge broker is characterized by a global orientation of upstream (technology, design, ... ) and downstream (customers, distributors, ... ) markets and by a multilocal understanding of where and how the needed specific knowledge can be found. Literature review and empirical evidences also permit to clarify analogies and differences compared to related constructs. (For more information, please contact: Stefano Denicolai, University of Pavia, Italy: stefano.denicolai@unipv.it)

\section{Learning, Design and Internationalization of Distributed Work: The Case of Clinical Trials} Metin Onal Vural, IE Business School

I examine the effects of design, resources and international dispersion of project experience on the performance of distributed work projects in clinical trials. Whereas outsourcing complex projects have become the norm in new product development and testing, there is a commensurate cost associated in designing and managing such types of project work. I use clinical trials as a setting to examine the effect of project design choices relating to both complexities and resource constraints and learning from joint work experiences on the performance of distributed work projects. My data include 5446 clinical trials spanning five disease areas between 1981 and 2009. I find that, while the dispersion of R\&D projects across multiple sites in international locations hinder project performance, learning from past hybrid-projects enhances organizational capability of undertaking such projects. Moreover, the negative effect of international dispersion is counterbalanced by an increased ability to access critical resources that exist across multiple countries. I elaborate on these findings for theories of learning and project design, internationalization or R\&D and its implications for innovation management. (For more information, please contact: Metin Onal Vural, IE Business School, Spain: onalv@yahoo.com)

\section{Session: 2.1.6 - Competitive \\ Track: Track: 11 - SMEs and Entrepreneurship}

\section{SMEs Exporting and International Market Expansion}

Presented On: July 5, $2013-09: 00-10: 15$

Chair: Arpita Joardar, Clark University

Stages and Patterns of the Internationalization of Chinese-Owned Firms: Market-Seeking versus ResourceSeeking Firms

Roberto Curci, Butler University

Ling-Yee (Esther) Li, Lingnan University 
This study compares and contrasts Chinese firms with internationally active value chains that started their internationalization efforts by engaging in international revenue generation or downstream value chain activities (defined as market-seeking firms) versus those that began through international sourcing or upstream value chain activities (defined as resource-seeking firms). Face-to-face survey interviews conducted with firm managers during the autumn 2011 yielded complete data for 308 Chinese firms. Our findings suggest firms that start their internationalization process by engaging in "market-seeking" behavior showcase better performance than those that begin by engaging in "resource-seeking" activities. In addition, financial indicators are found to be strong factors that discriminate between market-seeking and resource-seeking Chinese firms. (For more information, please contact: Roberto Curci, Butler University, USA: rcurci@butler.edu)

\section{Internationalization Strategy, Dynamic Capabilities, and the Survival of SMEs in the Export Market} Sui Sui, Ryerson University Matthias Baum, University of Giessen

Does born global internationalization enhance or endanger a firm's survival in the export market? Despite the ongoing scholarly debate on born global firms, we know little about what drives their survival in the export market. In particular, different theories yield conflicting predictions regarding whether born global internationalization is superior or inferior to born regional and gradual internationalization. In this study, we use the resource- and dynamic capability-based view to resolve this conflict. Analyzing a longitudinal dataset (19972005) of 1959 Canadian small and medium-sized enterprises (SMEs), we find that in a conventional analysis, born global firms and born regional firms have a lower probability of survival in the export market than do firms that gradually internationalize. However, after controlling for the endogeneity of firms' strategic choices, we no longer find a causal link between internationalization strategy and export market survival. SMEs pursue internationalization strategies that match their resource base; their export market survival is determined by their international experience and innovative capabilities. These findings support the resource- and dynamic capability-based view by showing that the development of resources and capabilities is more important than the choice of internationalization strategy in ensuring the survival of SMEs in the export market. (For more information, please contact: Sui Sui, Ryerson University, Canada: sui.sui.66@gmail.com)

\section{Selection Forces and Mimetic Isomorphism - International Market Entry Mode and Region Choices of Small and Medium Sized Firms in the German Biotech Industry 1996-2011 \\ Marie Oehme, University of Mannheim \\ Suleika Bort, University of Mannheim}

Investigating the phenomenon of small and medium sized firm (SME) internationalization and its patterns, the international entrepreneurship literature challenged our understanding of internationalization patterns derived from extensive studies of large multinational companies. Although progress in refining our knowledge on SMEs propensity to internationalize has been made there is yet only little understanding on their market entry mode and host country choice. Drawing on organizational ecology, institutional theory, the network literature, and the internationalization process model we contribute to the literature on SME internationalization by showing how population level selection forces, network level induced mimetic isomorphism and experiential knowledge impact and interact with regard to SMEs internationalization patterns beyond general internationalization propensity and speed. We test our hypotheses on the complete population of German biotechnology firms from 1996 to 2011. Our findings indicate positive effects of population level selection forces and experiential knowledge on SMEs' market entry mode and region choices, while effects of network level induced mimetic isomorphism can only be observed for entry mode choices. Considering firm level experiential knowledge, our findings furthermore shed new light on the persistence of selection forces and mimetic isomorphism and thus challenge our understanding of SMEs' internationalization processes. (For more information, please contact: Marie Oehme, University of Mannheim, Germany: oehme@bwl.uni-mannheim.de) 
Entry Speed of SMEs into Export Markets and Survival: The Moderating Roles of Industrial Competition and Foreign Ownership

Ziliang Deng, Renmin University of China

Ruey-Jer Bryan Jean, National Chengchi University

This paper examines whether and how SMEs can survive after rapid internationalization and whether the internationalization of born-global firms is more likely to result in survival than that of firms internationalizing in a paced and sequential mode. A survival analysis model is developed and tested that examines the impacts of entry speed on SMEs' survival, using a large panel dataset of 88,902 Chinese SMEs for the period 1998-2008. In line with time-compression diseconomies and absorptive capacity perspectives, the findings alert us to the potentially hazardous consequences of rapid internationalization. Foreign ownership may increase the survival probability for firms internationalizing early and thus have a risk-reducing function. Industrial competition in export markets may further aggravate the failure hazard of early internationalization, indicating that early internationalization may be a relatively viable strategy for firms in less competitive industries. (For more information, please contact: Ziliang Deng, Renmin University of China, China: dengziliang@rbs.org.cn)

\title{
Session: 2.1.7 - Competitive
}

\section{Track: Track: 4 - Strategy, Alliances, and Competitiveness}

\section{Management of International Alliances}

Presented On: July 5, $2013-09: 00-10: 15$

Chair: Manuel Portugal Ferreira, Uninove - Universidade Nove de Julho

\author{
International Joint Venture Survival: The Role of Partner Diversity \\ Anthony Goerzen, Queen's University \\ Alex Mohr, University of Kent \\ Chengang Wang, University of Bradford
}

Multi-party international joint ventures (MPIJVs) are prevalent organizational forms that are qualitatively different than dyadic joint ventures due to the existence of multiple partners. Yet, little research has considered the key aspects of MPIJVs to provide insight to scholars and guidance to managers. We combine the Resourcebased View of the Firm and Transaction Cost Economics to investigate the effects of partner diversity on MPIJV survival by distinguishing between the key facets of variety, balance, and degree of difference. Our hypotheses are tested using data on 840 firms within in 248 MPIJVs in China. We find empirical support for an inverted Ucurve shaped effect of variety and a positive linear effect of balance on MPIJV survival, although no support for our hypothesized effect of the degree of differences in partner firms' institutional backgrounds. (For more information, please contact: Anthony Goerzen, Queen's University, Canada: agoerzen@business.queensu.ca)

\section{Reducing Cultural Uncertainty Abroad through Experience Gained in the Domestic Market Sungjin J. Hong, Queen's University Belfast \\ Seung-Hyun Lee, University of Texas at Dallas}

This paper proposes the boundary conditions of when and why some prior experiences gained outside the focal host country are able to increase the likelihood of platform investment in that country. This paper extends one of the core assumptions of the international strategy literature: the transfer of experiential knowledge would be bound within a firm's home country national cultural boundary. However, this paper also argues that some experienced firms would be able to transfer experiential knowledge, gained in either their home market or other host countries, to the focal host country beyond a firm's home country cultural boundary in its initial 
investment. This paper proposes the boundary conditions that how a multinational corporation (MNC) with experiential knowledge gained in its domestic market through joint venturing with foreign partners can benefit in its platform investment. (For more information, please contact: Sungjin J. Hong, Queen's University Belfast, United Kingdom: s.hong@qub.ac.uk)

\section{Board Composition in International Joint Ventures \\ Ilya Cuypers, Singapore Management University \\ Gokhan Ertug, Singapore Management University \\ Jeffrey Reuer, Purdue Universtiy \\ Ben Bensaou, INSEAD}

A considerable literature on the composition of corporate boards has developed in recent years. However, relatively little attention has been paid to boards in international joint ventures (IJVs), and the composition of these boards in particular. To advance our knowledge in these areas, we combine the corporate governance and IJV literatures to examine the determinants of foreign partners' representation on IJV boards. We hypothesize that a foreign partner's representation on the IJV board is related to the equity distribution of the IJV. Furthermore, we argue that both environmental and behavioral uncertainty moderate the strength of this relationship, albeit in opposite ways. These results provide insights into the conditions under which a partner might wish to secure greater board representation for its level of equity, or utilize less board representation than might be suggested by its equity level alone. (For more information, please contact: Gokhan Ertug, Singapore Management University, Singapore: gokhanertug@smu.edu.sg)

\section{Session: 2.1.8 - Competitive \\ Track: Track: 9 - Cross-Cultural Management and HRM}

\section{Challenging Cultural/Psychic Distance; What is Wrong and How can We Fix It?}

Presented On: July 5, 2013 - 09:00-10:15

Chair: Jing Betty Feng, Georgia State University

Differences in Work Values: Understanding the Role of Intra- versus Inter-Country Variation André van Hoorn, University of Groningen

A growing literature emphasizes the need for research taking a contingency perspective to international HRM to move beyond mean country differences in work values and start considering intra-country variation (ICV). We use individual-level data on Hofstedeian values-not hitherto available-to infuse this literature with systematic quantitative evidence on the importance of ICV vis-à-vis inter- or between-country variation (BCV). We start by estimating various random effects models, finding that ICV accounts for the bulk of total variation in work values, some $85 \%$. To add a much-lacking comparative dimension and because ICV only has real-life relevance if we know its sources and can observe them, a three-level hierarchical linear analysis provides a novel disentanglement of the importance of country relative to region and socio-economic stratum as readily observable within-country sources of variation in values. Results show the value for practitioners and scholars of not focusing on country differences strictly but to also consider sub-national categorizations when seeking to understand differences in work values. Key contribution of this paper is to take the debate on ICV out of the theoretical into the practical realm. Implications of our findings are discussed. (For more information, please contact: André van Hoorn, University of Groningen, Netherlands: vanhoorn@gmail.com) 
On the Symmetry and Discordance of Cultural Distance in Cross-border M\&As: A Two-Country Study

Harun Emre Yildiz, Stockholm School of Economics

Carl Felix Fey, Nottingham University

Cultural distance occupies a central role in international business studies. Notwithstanding its prevalence as a key explanatory factor for myriad of questions studied in the field, the concept of cultural distance entails several entrenched assumptions that have not been put to sufficient systematic scrutiny. Situated within the specific context of cross-border mergers and acquisitions, we address this gap and investigate assumptions of symmetry and discordance. Data collected through decision simulations with professionals in Sweden and China reveals that distance perceptions are asymmetric and create contrasting attitudes among Swedish and Chinese respondents. We also detect mixed empirical evidence regarding discordance assumption, which suggests that perceptions and reactions towards a foreign acquirer at different ends of a given country pairing do not necessarily mirror each other. Findings reported in this study pinpoint important theoretical implications regarding cultural distance, which could contribute not only to cross-border M\&A literature but also to general IB research concerned with cross-cultural phenomena. (For more information, please contact: Harun Emre Yildiz, Stockholm School of Economics, Sweden: emre.yildiz@hhs.se)

\section{Cultural Distance in International Business and Management: From Mean-based to Variance-based Measures Marjolijn Onrust, University of Groningen \\ Sjoerd Beugelsdijk, University of Groningen \\ Robbert Maseland, University of Groningen \\ Arjen Slangen, Rotterdam School of Management}

Extant practice in international management is to measure cultural distance as a nation-to-nation comparison of country means on cultural values, thereby ignoring the cultural variation that exists within countries. We argue that these traditional mean-based measures of cultural distance should take within-country cultural variation into account. Therefore, we propose the use of variance-based measures of cultural distance. To illustrate our argument, we examine total US foreign affiliate sales in more than 40 host countries over the 1983-2008 period, complemented with data from the World Values Survey. We analyze the effects of three cultural distance measures: the Kogut and Singh (1988) mean-based index of cultural distance, the Kogut and Singh (1988) index conditioned by host-country cultural variation, and a variance-based measure that takes into account both home and host-country cultural variation. Our findings indicate that, when within-country cultural variation is taken into account, the explanatory power of the Kogut and Singh (1988) index is substantially decreased. In addition, our variance-based measure of cultural distance outperforms the Kogut and Singh (1988) measure in the explanation of foreign US sales. We therefore suggest to move from mean-based to variance-based measures of cultural distance, thereby taking the cultural variation within countries into account. (For more information, please contact: Marjolijn Onrust, University of Groningen, Netherlands: m.onrust@rug.nI)

\section{Understanding Psychic Distance and Its Asymmetry - Distance Judgments as Social Comparisons Anja Maria Schuster, University of St. Gallen \\ Bjoern Ambos, University St. Gallen}

One of the central concepts in today's international management research is the concept of psychic distance. Whereas the concept enjoys unabated popularity, the literature still reveals considerable weaknesses regarding its theoretical foundation as well as appropriate operationalization. In particular, empirical evidence of asymmetric psychic distances calls into question the prevalent unidirectional conceptual approaches and urges us to reconsider prevailing measures. Building on insights from social psychology literature, we scrutinize the concept's most common operationalization as a judgment of proximity or similarity between two countries. We use social identity as well as social comparison theory, to suggest that a nation constitutes a social group to which an individual belongs and that inquiries on the similarity to other nations trigger a social comparison process. As these are influenced by self-esteem and self-evaluation needs, we expect the relative country 
statuses to cause assimilation or contrast effects, increasing or decreasing perceived similarity with the comparison target. We use published psychic distance data combined with secondary country-level data to test our hypotheses for which we find support. Our results reveal that psychic distance judgments are subject to cognitive influences which can lead to perception gaps. The findings deepen our understanding of the psychic distance concept and enable future research to use it more consciously. (For more information, please contact: Anja Maria Schuster, University of St. Gallen, Switzerland: anja.schuster@unisg.ch)

\section{Session: 2.1.9 - Competitive \\ Track: Track: 3 - IB Theory, FDI, and Entry Mode}

\section{Host Country Environment and FDI}

Presented On: July 5, 2013 - 09:00-10:15

Chair: Elena Beleska-Spasova, University of Reading

Multinational Sensitivity to Human Rights: The Influence of Nationality in American and Chinese Foreign Direct Investment

Brent Burmester, Auckland University

Ben Simons, University of Auckland

Institutional idiosyncrasies of the home environment may impinge on the sensitivity of outward investors to host-nation characteristics. This study compares the relative importance of human rights to American and Chinese foreign direct investment (FDI) in 51 developing countries, between 2007 and 2010. Contrary to expectations, Chinese FDI, unlike its American counterpart, is found to gravitate toward host countries in which human rights are protected. Moreover, a separate analysis of Chinese non-financial FDI for a sample of 66 developing countries between 2004 and 2006 further reveals a significant positive relationship with host-nation respect for human rights. The results of this study suggest that national origin does influence the role given to host country human rights in multinationals' location decisions, but not necessarily in conformity with expectations. (For more information, please contact: Brent Burmester, Auckland University, New Zealand: b.burmester@auckland.ac.nz)

\section{FDI Communities and Foreign Subsidiary Performance Jing'an Tang, Sacred Heart University}

Though Foreign Direct Investment Communities (FDIC) are considered an important source of legitimating influence for foreign subsidiaries in the host country (Li, Yang and Yue, 2007), less known is how the development of FDIC affects subsidiary performance. Drawing on multiple theoretical lenses, this study investigates the process of FDIC development and its impact on foreign subsidiary performance. We argue that driven by the various institutional, social and economic forces, a typical FDIC develops through several distinctive stages, which in turn, partly explain variations in foreign subsidiary performance. Our analysis of 1,685 FDICs worldwide confirmed our argument and found that the development stage at which a foreign subsidiary entered an FDIC has an S-shaped relationship with subsidiary survival and a U-shaped relationship with subsidiary profitability. (For more information, please contact: Jing'an Tang, Sacred Heart University, USA: tangj3@sacredheart.edu)

Institutional Distance, Direction and Complementarity: Impacts on the Mode of Entry Decision

Kai Xu, Texas A\&M University

Lorraine Eden, Texas A\&M University 
This study adds to the institutional distance literature by introducing two new constructs, institutional complementarity and institutional direction, and investigating their impact on the MNE's mode of entry decision. First, we separate institutions into two types, formal and informal. Formal institutions are explicit and neutral between local and foreign firms; informal institutions are tacit and non-neutral. Because both affect MNE strategies, their combined effects, which we call institutional complementarity, can be offsetting or reinforcing. We introduce a second construct, institutional direction, by assessing the relative strength/weakness of host country compared with home country institutions. We examine the separate and combined effects of institutional complementarity and institutional direction on the MNE's entry mode choice, testing our hypotheses on a sample of 2,424 MNEs from 48 home countries that invested in 23,673 foreign subsidiaries in 51 host countries between 2007 and 2010. Our empirical results suggest formal and informal institutions are complementary when we take into account institutional direction. Specifically, when formal or informal institutions are higher/stronger in the host country relative to the home country, MNEs choose wholly owned entry modes; when they are lower/weaker, MNEs prefer joint ventures. We also examine the interactive effects of formal and informal institutions, finding that formal institutions are more influential on MNE entry mode choices than informal institutions. (For more information, please contact: Lorraine Eden, Texas A\&M University, USA: leden@tamu.edu)

\section{Session: 2.1.10 - Special Session}

\section{Special Session of BALAS Best Papers}

Presented On: July 5, 2013 - 09:00-10:15

Chair: Robert Grosse, George Mason University

In celebration of the contributions of Harvey Arbelaez to AIB and the field of IB.

Exporting from Latin America - Insights from Chilean Entrepreneurial Firms

Luciano Ciravegna, University of London

No abstract available. (For more information, please contact: Luciano Ciravegna, University of London, United Kingdom: luciano.ciravegna@rhul.ac.uk)

Latin America in the Wine Market:The Country of Origin Effect on Consumers 'Preferences Christian Felzensztein, Universidad Adolfo Ibanez

No abstract available. (For more information, please contact: Christian Felzensztein, Universidad Adolfo Ibanez, Chile: c.felzensztein@uai.cl)

Causal Recipes for Internationalization Speed - an Exploratory Study Luis E. Lopez, INCAE Business School

No abstract available. (For more information, please contact: Luis E. Lopez, INCAE Business School, Costa Rica: luis.lopez@incae.edu) 


\section{Session: 2.1.11 - Interactive}

Track: Track: 2 - Marketing and Supply Chain

\section{Ins and Outs, Supply Chain and Exports}

Presented On: July 5, $2013-09: 00-10: 15$

Chair: William Murphy, University of Saskatchewan

Understanding the Determinants of Export Performance: A Meta-Analysis

Christopher Schlaegel, Otto von Guericke University Magdeburg

There is a considerable body of research examining the factors that influence a firm's export performance. While the evidence for those factors that determine export performance is accumulating, there is a lack of clarity on the determinants that are most impactful on export performance. This meta-analytic review identifies a total of 17 determinants which have been most widely researched in the export performance literature in the past 15 years. Results from 100 studies (108 independent samples, $\mathrm{N}=23,369$ ) were aggregated to estimate the effect sizes of the identified variables and provide a better understanding of the factors that affect export performance. The results show that management characteristics, firm characteristics, and firm strategy have the strongest influence on export performance. Moreover, the results of the moderator analyses suggest that contextual and methodological characteristics, such as national context, firm unit of analysis, and the operationalization of variables, moderate the relationships between export performance and its antecedents. The paper concludes with a discussion of the results and their implications for research and practice, as well as limitations and suggestions for future research. (For more information, please contact: Christopher Schlaegel, Otto von Guericke University Magdeburg, Germany: cschlaeg@ovgu.de)

\section{An Empirical Investigation of the Antecedents and Performance Outcomes of Export Innovativeness Katerina Makri, Athens University of Economics and Business Marios Theodosiou, University of Cyprus Evangelia Katsikea, Athens University of Economics and Business George J. Avlonitis, Athens University of Economics and Business}

The positive impact of innovativeness on performance is widely acknowledged in the marketing literature. However, most research attention has been devoted on specific innovations rather than on the broader role of innovativeness as part of organizational culture. Furthermore, the role of innovativeness within the context of exporting operations has been largely ignored. In response to these research gaps, this paper develops and empirically tests a comprehensive conceptual model of the antecedents (organizational, strategic, and environmental factors) and performance outcomes (export market and financial performance) of export innovativeness. The findings indicate a significant positive relationship between export innovativeness and export performance. (For more information, please contact: Katerina Makri, Athens University of Economics and Business, Greece: katerinamakri83@gmail.com)

\section{Marketing Strategies-Performance Relationship: An Investigation on Micro and Small Brazilian Enterprises Reynaldo Dannecker Cunha, ESPM \\ Thelma Valeria Rocha, ESPM}

The relationship between marketing strategy and performance has been well documented in developed economies. However, empirical work in the context of export marketing in emerging economies has been fragmented. The authors investigate the marketing strategy-performance relationship in the context of micro and small enterprises (MSE) in Brazil. The study differs from previous export marketing studies in that (1) the dimension entrepreneurial marketing as potential determinant of export market performance is considered; (2) 
the unit of analysis is the individual product-market export venture in an emerging economy; and (3) the conceptual framework of export marketing strategy and performance is based on Cavusgil and Zou (1994) and adapted to MSE. This framework is tested by a survey with 173 Brazilian MSE from different sectors and the data analysis was performed using structural equation modeling. The results support the importance of marketing activities to the export performance of MSE, mainly by adapting the price to the targeted market, improving products' competitiveness. The study also highlights the company's international competence (expertise) and entrepreneurial marketing as influencing the export performance. As contributions, the results provide support to the management development of MSE, so important for the Brazilian economy and the other emerging countries. (For more information, please contact: Reynaldo Dannecker Cunha, ESPM, Brazil: rcunha@espm.br)

\title{
Effect of Brazilian Public Export Assistance Programs: Implications for Export Performance in a Longitudinal Approach
}

Flavia de Holanda Schmidt, IPEA

Jorge Ferreira da Silva, PUC-Rio

Jorge Carneiro, Pontifical Catholic University of Rio de Janeiro

The value of world exports increased $130 \%$ over the last decade. In this scenario, Brazil currently faces complex challenges regarding its external insertion, regarding issues like its relative small share in world exports, the concentration of exports in a few companies and the increasing presence of commodities in the export basket. Despite the fact that all factors of this process cannot be controlled by government, literature shows the impact of government policies on the export performance of firms. In this sense, Brazil has strengthened its commitment to export assistance. The purpose of this paper is to evaluate the impact on the export performance of Brazilian manufacturing exporters from participation in export assistance programs. The research strategy was based on the estimation of the causal effects of treatment using propensity score matching for the three programs evaluated. Panel models were applied. Results showed that participation in the programs had an impact on the export performance of the firms supported, but this impact manifested differently among the programs and the indicators of export performance adopted. (For more information, please contact: Flavia de Holanda Schmidt, IPEA, Brazil: flhschmidt@yahoo.com.br)

\author{
Objectively Measured Factors Affecting the Price of Exported Australian Wines \\ Elyse Renee Shane, University of Adelaide \\ Susan Freeman, University of Adelaide \\ Wahid Murad, University of Adelaide
}

This study seeks to determine the effect of objectively measured factors on the price of Australian wines exported to the UK and to analyse the impact of store type on these factors. In doing so a number of new theories are introduced to the Hedonic Pricing literature and previous findings from economic and consumer behaviour approaches are compared. This study compares sales in supermarkets and small stores and differences in attribute valuation between the two sales channels are identified. These trends identified here in the UK market are not consistent with those obtained in similar studies conducted in Italy. Hence the study highlights the importance of context in research related to consumer behaviour particularly regarding wine. (For more information, please contact: Elyse Renee Shane, University of Adelaide, Australia:

elyse.shane@adelaide.edu.au)

Marketing and Supply Chain Capabilities' Role in Responding to Institutional Pressure and Internationalization of Emerging Economy Firms to Developed Economies

Ismail Golgeci, University of Vaasa

Ahmad Arslan, University of Vaasa 
Despite extensive research on internationalization, little attention has been paid on marketing supply chain issues, thus leaving international marketing scholars and practitioners with an incomplete understanding of the marketing and supply chain side of this important phenomenon. To address this gap, this paper proposes to examine internationalization from marketing and supply chain perspectives, adopting dynamic capabilities and institutional theories. In specific, the interaction between marketing and supply chain capabilities and institutional pressures are analyzed to develop a framework for the internationalization of emerging economy firms to developed economies. Marketing and supply chain capabilities are suggested to positively influence both supply chain learning and conformance to institutional pressures emerging economy firms face in developed economies. This paper contributes to internationalization literature by offering a novel perspective to explain somewhat overlooked phenomenon of emerging economy firms' internationalization to developed economies. (For more information, please contact: Ismail Golgeci, University of Vaasa, Finland: igolgeci@uva.fi)

\section{Microfoundations of Supply Chain Agility and Resilience and their Role in International Performance Ismail Golgeci, University of Vaasa \\ Ausrine Silenskyte, University of Vaasa}

This paper examines organizational capabilities which currently are of a key interest in both theory and practice because of their importance to organizational performance and long-term survival in highly turbulent international business environment. The scant knowledge on behavioral antecedents of dynamic capabilities justifies the reasoning for this research. Hence, the purpose of this paper is to examine the relationship between individual capabilities and supply chain capabilities in interaction with human resource management related factors that affect this relationship; and to uncover the nature of the connection between the supply chain capabilities and long-term international performance. As a result, completely new theorization, which incorporates aspects of supply chain management, organizational behavior and human resource management, is offered to explain how individual factors within a supply chain system drive supply chain capabilities and longterm international performance in interaction with human resource management related factors. Paper contributes to dynamic capabilities theory by shedding light on individual-level antecedents of dynamic capabilities of supply chain agility and resilience as well as to international business literature by providing example of how multidisciplinarity and incorporation of human dimension can result in highly explanatory and insightful theoretical model. (For more information, please contact: Ismail Golgeci, University of Vaasa, Finland: igolgeci@uva.fi)

\section{Session: 2.1.12 - Interactive \\ Track: Track: 1 - Institutions, Governance, and CSR}

\section{Institutions, CSR and Performance}

Presented On: July 5, $2013-09: 00-10: 15$

Chair: Jeannine Comma, University of the West Indies

The Effect of the Regulatory Environment on Firm Performance: Explaining Industry Differences Nicholas James Bailey, University of South Carolina

This study suggests that the extent of the effect of the regulatory environment on firm performance depends on the industry-specific context in which each firm operates. We argue that firms' susceptibility to changes in institutional environments is unequal across industries because the significance of changes in the regulatory environment varies depending on the industry. Using a random coefficient growth model, analysis of 752 firms in manufacturing, services, and natural resource extraction industries in 25 countries suggests that the performance of firms in natural resource extraction industries are the most susceptible to regulatory policy, followed by services, and then manufacturing firms. (For more information, please contact: Nicholas James Bailey, University of South Carolina, USA: nicholas.bailey@grad.moore.sc.edu) 
Creating Organizational Resilience: What We Can Learn from Financial Services Firms That Have Successfully

Braved the Economic Crisis-and from Those That Did Not

Markus Venzin, Università Bocconi

Why are some firms more resilient-better able to successfully adjust and thrive in conditions of adversity-than others? We define organizational resilience as the capacity to deliver predictably strong and stable performance in the face of adversity. We develop a measure for organizational resilience that allows us to identify and compare firms from six industries with a variety of resilience levels. We find that highly resilient firms (1) have a strong core business that can be protected, (2) serve customers before shareholders, and (3) are able to make fast strategic decisions based on the social controls of key actors. (For more information, please contact: Markus Venzin, Università Bocconi, Italy: markus.venzin@unibocconi.it)

\section{Need Some Privacy? Consequences of Being Public vs. Private on Firms' Investments \\ Aude Le Cottier, IE Business School \\ Juan Santalo, IE Business School}

We investigate the drivers of differences in firm investment between listed and non-listed companies across countries. We argue that public companies will invest more than their private counterparts, the better the institutional framework protects minority shareholders of public companies from the risk of rent appropriation. This protection decreases the relative cost of capital for public companies, hence facilitating the undertaking of larger investment levels. However, when regulations limit managerial discretion in listed companies, public firms' investments diminish. Besides, public ownership moderates the effect of two other factors on firms' investments. Being listed, and hence subject to stock market short-term pressure, downplays the positive effect of society long-term orientation on firms' investments. On the contrary, when intangible assets are important, the extra regulations and disclosures imposed on public firms have a positive effect on investors' confidence and ultimately on public firms' investments. Our results, based on 2005-2010 panel data of 6550 matched listed and unlisted firms across the world support our hypotheses. (For more information, please contact: Aude Le Cottier, IE Business School, Spain: ale.phd2013@student.ie.edu)

\section{A Vocabulary Structure Approach to Analyze a Struggle Around Global Supply Chain Responsibility \\ Dirk C. Moosmayer, Nottingham University \\ Susannah M. Davis, Nottingham University}

This paper utilizes a vocabulary structure approach to investigate developing norms of supply chain responsibility (SCR). We analyze an NGO coalition's normative delegitimation campaign against Apple, studying the vocabularies and rhetoric used by the organizations in order to understand their underlying institutional logics. Our paper demonstrates the value of linguistic approaches to investigate the developing norms and practices of SCR in the Chinese context as well as the role of civil society and corporations in their construction. Our study has implications for MNCs with supply chains in China as well as for NGOs and policymakers with an interest in SCR. (For more information, please contact: Dirk C. Moosmayer, Nottingham University, China: dirk.moosmayer@nottingham.edu.cn)

\section{The Role of Governments in Designing Global Players \\ Lucia Piscitello, Politecnico di Milano \\ Sergio Mariotti, Politecnico di Milano \\ Andrea Colli, Bocconi University}

Traditional international business literature has widely-acknowledged the role of governments in promoting multinational companies and foreign direct investment. However, while the role of host-country governments has attracted significant research, less attention has been devoted to the role of home country, and even less to 
the interaction between companies and governments, which is instead often critical for the formation of global players. This paper aims to offer a contribution to fill this gap. Adopting an institution-based view, we model the international growth of firms as stemming from their strategic interaction with governments, and highlight that successful internationalization stems from cooperative solutions through which companies and governments share both goals and strategies. Specifically, new world-class multinational corporations are more likely to emerge in coordinated market economies and politically sensitive industries, where governments act as strategists and maintain a high involvement through either the ownership of the relevant companies and/or the use of a panoply of mechanisms that create favorable conditions towards internationalization. Our conjectures have been empirically supported by a comparative analysis of the international diversification and growth of previously-national champions in European energy and telecommunications industries, during the last decade. (For more information, please contact: Lucia Piscitello, Politecnico di Milano, Italy: lucia.piscitello@polimi.it)

\section{Session: 2.1.13 - Interactive}

\section{Track: Track: 7 - Emerging Economies}

\section{Knowledge Transfer, R\&D, and Innovation}

Presented On: July 5, $2013-09: 00-10: 15$

Chair: Jaeyong Song, Seoul National University

Language as a Barrier to Understanding: Professional Discourse and Business Sensemaking in Transition Economies

Olga Kuznetsova, Manchester Metropolitan University

Andrei Kuznetsov, Lancashire Business School

The paper examines a relatively new and mainly unobserved in the IB literature phenomenon of communication disengagement in transitional and emerging markets resulted from the insufficiency of the local professional business discourse rooted in language limitations. This creates a barrier to understanding, in particular between representatives of different cultures, as certain instances of experience do not find adequate linguistic expression. This may have a profound impact on the exchange of information and expertise development. For international companies this may complicate cross-cultural adjustments and produce financial losses. We recommend that linguistic hazard is included in risk evaluation of international projects. (For more information, please contact: Olga Kuznetsova, Manchester Metropolitan University, United Kingdom:

o.kuznetsova@mmu.ac.uk)

Could you repeat that, please, in our language? The Role of Corporate Language in Knowledge Transfer to MNE Subsidiaries in China

Audra I. Mockaitis, Monash University

Jinwei Zhu, Jiangnan University

Dayna Simpson, Monash University

Cherrie Jiuhua Zhu, Monash University

Multinational enterprises (MNEs) that operate across institutions, cultures and languages encounter barriers to the smooth transfer of knowledge especially between distant organizational units. We explore relationships between language and knowledge transfer from expatriate to subsidiary employees. We test our propositions with 132 MNE subsidiaries operating in China. Results indicated significant, negative effects when language is used as power on both communication quality and knowledge transfer but a non-significant effect from language standardization. Communication quality was significant and positively related to knowledge transfer. Socialization and shared vision significantly strengthened these relationships. We contribute to an important and 
growing body of work on the role of language in international management. (For more information, please contact: Audra I. Mockaitis, Monash University, Australia: audra.mockaitis@monash.edu)

\author{
Resource Constrained Innovation at the Base of the Pyramid: Towards a Theoretical Framework \\ Manas Puri, University of Rome Tor Vergata \\ Ernesto Tavoletti, University of Macerata
}

The traditional concept of innovation involves substantial financial investments, resource usage and existence of supporting institutions. However, in emerging economies that lack such resources and institutions the concept of innovation has to be modified accordingly. Thus, the focus is on understanding how innovation can be pursued with the existence of such constraints. The extant literature on this subject stands divided and fragmented, with no clear theoretical framework that coherently explains the concept of resource constrained innovation. This paper systematically reviews the literature on the existing research work and leverages on two relevant case studies to propose a model of resource constrained innovation in emerging economies. (For more information, please contact: Manas Puri, University of Rome Tor Vergata, Italy: puri@economia.uniroma2.it)

\title{
Relating Export Growth Strategy to Technological Capability Development: The Case of China Jason Z. Yin, Seton Hall University
}

It remains a challenge to empirically verify whether the large scale of exports as the result of China's exportoriented growth strategy has transformed into its endogenous technological capability. In the current literature of export performance study, however, there is a methodological gap in quantifying the domestic contents of exports from total exports. Therefore the domestic technological change cannot be clearly identified and analyzed. This paper bridged the gap by employing a unique Input-Output model to derive the domestic contents from total exports and to analyze the nature of related technological change. Our data analysis of China's exports of manufacturing products for 1992-2009 reveals: (1) China's participation in the global vertical specialization (VS) in high-tech sector grew faster and faster chronically; (2) the VS participation was more aggressive in high-tech than in the medium-tech, low-tech and resources based sectors; and (3) the net domestic contents in China's exports in high-tech sectors grew much faster than the other sectors. The findings indicate that China's export-oriented growth strategy, instead of creating dependence on low-tech and resource-based exports, had led to a rapid growth in sophisticated high-tech manufacturing with its endogenous capability. (For more information, please contact: Jason Z. Yin, Seton Hall University, USA: yinjason@shu.edu)

\section{MNC Subunit Knowledge Sourcing - Patterns and Impacts Xiaoyu Pu, SUNY Oswego \\ John Cantwell, Rutgers University}

This research examines the patterns of MNC subunits' knowledge sourcing in terms of the technological and geographical dispersion of knowledge sources. It proposes a dynamic model in which the extent to which a subunit diversifies its innovative activity is influenced by the technological and geographical dispersion of that subunit's knowledge sourcing. The results show a consistently positive relationship between the technological and geographical dispersions of knowledge sourcing, an effect that is moderated by the extent of subunit specialization in general purpose technology (GPT) fields, and the geographical proximity between dispersed knowledge sources. While both technological and geographical dispersions of knowledge sources are positively related to subunit's technological diversification, the concentration of GPT fields and the geographic proximity of sources might make a difference. (For more information, please contact: Xiaoyu Pu, SUNY Oswego, USA: xiaoyu.pu@oswego.edu) 
Session: 2.1.14 - Interactive

Track: Track: 7 - Emerging Economies

\section{Firm Growth and Performance in Emerging Markets}

Presented On: July 5, $2013-09: 00-10: 15$

Chair: Wlamir Goncalves Xavier, FGV/EAESP e UNISUL

Contract Specificity and Supplier Performance in China: The Contingent Roles of Institutional Environments Qiyuan Zhang, University of Hong Kong

Kevin Zheng Zhou, University of Hong Kong

How does contracting function in emerging economies in which legal institutions are underdeveloped but guanxi norms are highly pervasive? Building on transaction cost economics and institutional theory, this study untangles two distinct facets of contracting, task specificity and contingency specificity and examines their contingent effects on exchange performance given different levels of legal inadequacy and guanxi importance. The results from a survey of 307 manufacturer-supplier dyads in China show that task specificity is overall more effective than contingency specificity at promoting exchange performance. However, its role declines when legal support is inadequate and when guanxi becomes prevalent within transactional environment. Contingency specificity is only useful when guanxi is important; it is not helpful when legal is inadequate. (For more information, please contact: Qiyuan Zhang, University of Hong Kong, Hong Kong, SAR-PRC: bessieqy@hku.hk)

The Influence of Business Group Heterogeneity on Corporate Diversification and Firm Performance: An Examination across Manufacturing and Service Sectors

Saptarshi Purkayastha, Indian Institute of Management Kozhikode

Somnath Lahiri, Illinois State University

This study examines how the influence of key heterogeneous business group features on the corporate diversification-firm performance relationship varies across industry sectors. Using a sample of Indian firms the study finds evidence that the influence of business group size, diversity and share ownership on the diversification-firm performance relationship varies significantly depending on whether the focal firm belongs to the manufacturing or service sector. Results reveal that corporate diversification has a negative impact on the performance of group-affiliated manufacturing firms but has a positive impact for group-affiliated service firms. Results further reveal that group diversity does not exert any influence on the diversification-performance relationship for manufacturing firms but does so for service firms. Influence of different share ownership forms differs to some extent, but not completely, across manufacturing and service sectors. The paper concludes with a discussion of the findings and providing suggestions for future research. (For more information, please contact: Somnath Lahiri, Illinois State University, USA: slahiri@ilstu.edu)

Rethinking Medical Tourism Research: A Proposed Conceptual Framework Using Competitive, Supportive and Emerging (CSE) Factors in Achieving Competitiveness in Healthcare Industry

Shankar Chelliah, Universiti Sains Malaysia

Thilagavathi Krishnan, Universiti Sains Malaysia

Malaysia is in the progress of diversifying its economic activities towards a service-based economy. At the same time, the world had recognized healthcare sector as a highly potential global industry. Therefore, based on the available resources in the country and the changing trend of the world economy, Malaysia is noticeably recognizing healthcare travel as one of the new source of sustainable economic activity. Due to that, academic research is needed to device strategies to gain competitive advantage in medical tourism industry. Therefore, the purpose of the paper is to propose a conceptual framework examining the factors that contribute to the 
growth of the medical tourism industry in the emerging economy. Medical cost, quality healthcare services and specialist medical services are identified as competitive factors. Government regulations, contribution of medical tourism agents and tourism are defined as the supportive factors and ethical issues are identified as emerging factor. The proposed framework is designed to integrate the relationship between competitive, supportive and emerging factors that may have effects on medical tourists' satisfaction. Predominantly, the framework is using resource based view (RBV) theory. The practical implication of the study is focused to the management of the international healthcare providers in adopting strategies in achieving greater medical tourists' satisfaction. (For more information, please contact: Shankar Chelliah, Universiti Sains Malaysia, Malaysia: shankar@usm.my)

\section{Performance of MNEs in an Emerging Economy during Crisis: What Makes the Difference between Survival and Bankruptcy? \\ Cosmina Lelia Voinea, Radboud Universiteit Nijmegen}

The Great depression (2008-2009) followed the period of relatively rapid growth of economies, foreign inward investments, etc. (Roberts, 2010). However, many firms, even those which were relatively successful before the crisis disappeared. In transition economies the impact was even harder and the survival circumstances for the foreign firms operating in these environment were extremely difficult. This study focuses on the types of foreign firms located in the emerging and transition economy of Romania that manage to survive (remain in business) before, dring, and after the crisis. More specifically we analyse the relationship between financial condition, size of tangible resources, intangible resources, country of origin, while controlling for type of industry; and survival of the foreign companies operating in 36 industries in Romania. Empirical findings show that the type of the industry, country of origin, differences in industrial development and political systems between the home and the host countries have a significant relationship with survival of the companies. These findings indicate the increasing intricacy of the relationships between among various host economies, between players of economic origins but also political institutions, as well as a global interconectedness and home-host dynamics wave effects. (For more information, please contact: Cosmina Lelia Voinea, Radboud Universiteit Nijmegen, Netherlands: c.voinea@fm.ru.nl)

\section{Managerial Resources and the Penrose effect: Evidence of an Emerging Market Min-Ping Kang, National Taiwan Normal University Wein-Hong Chen, National Dong Hwa University Po-Yuan Chen, National Chengchi University}

Penrose effect is known as the impact of managerial constraint on a firm's growth when the firm's internal managerial resources are not sufficient to handle more complicated organization tasks associated with rapid expansion. We assert that firms are less likely to experience the Penrose effect when they can economize the utilization of managerial capacities or enhance managerial learning. Specifically, we propose that firms with well-developed programs and shared firm-specific experiences are more likely to economize the utilization of managerial capacities in making authoritative communications. Additionally, firms that develop higher-level learning capabilities are more likely to change manager's perception of uncertainties and risks associated with the expansion decision. We find partial empirical supports for our hypotheses in the context of Taiwanese manufacturers (For more information, please contact: Min-Ping Kang, National Taiwan Normal University, Taiwan: mpkang@ntnu.edu.tw)

\section{Financial Reforms and their Effect on Domestic and Foreign Investment in China and India Suma Athreye, Brunel University Abubakr Saeed, Brunel University}

Given the mixed evidence on the firm specific competences driving Indian and Chinese foreign investments, we explore the possibility that the spurt of outward foreign investment from India and China may have been in 
large part due to a reversal of past financial constraints and a response to buoyant demand conditions. We adapt the Euler model of the financial determinants of investment, to estimate the effect of financial reform on domestic and foreign investment from India and China. Based on a cross-industry panel of 508 non-financial Indian and Chinese firms in the period 2000-2009 we find that these firms still face considerable financial constraints to domestic and foreign investment, although these have eased gradually since the financial reform. We find also that business houses in India and state-owned Chinese enterprises have benefited more from financial reform to their domestic and foreign investments. Chinese domestic and foreign investments and Indian firms in fast growing sectors have also benefited from foreign listing. (For more information, please contact: Abubakr Saeed, Brunel University, United Kingdom: abubakr.sd@gmail.com)

\section{Session: 2.1.15 - Interactive}

Track: Track: 9 - Cross-Cultural Management and HRM

\section{Understanding Global Employees: Multiculturals, Cosmopolitans \& Global Leaders}

Presented On: July 5, 2013 - 09:00-10:15

Chair: Brian Toppin, University of the West Indies

Implications of Gender Differences in Global Leadership Style: Examining the Global Mindset Inventory Amanda Bullough, Thunderbird School of Global Management Mansour Javidan, Thunderbird School of Global Management Rebekah Dibble, University of San Fransisco

Top management teams (TMTs), with their expertise, power, and relationships, are at the apex of any organization. Functional top management teams have executives with different skill sets, made possible by assigning people to teams from both genders. This paper applies upper echelon and strategic leadership theories to the top management teams of global organizations and examines how men and women differ in their strategic leadership styles. We propose that men and women differ in their leadership characteristics and it is this diversity that strengthens the collective global competency of the TMT. We test these assertions with data from the Global Mindset Inventory on a sample of 1,187 managers from 97 companies in 46 countries. Implications for business leaders highlight the importance of including women on top management teams because of the unique competencies they contribute. (For more information, please contact: Amanda Bullough, Thunderbird School of Global Management, USA: amanda.bullough@thunderbird.edu)

\section{Exploring Expatriates' Cultural Identity Reconstruction: A Relational and Network Approach Yan Shen, University of Victoria Jina Mao, Skidmore College}

We explore the social mechanisms that help maintain or facilitate change in expatriates' cultural identity adaptation during expatriation. We highlight network's culture base as an important construct, measured by cultural diversity, cultural density, and cultural embeddedness within a social network, in examining expatriates' cultural identity reconstruction. We propose that expatriates adopt one of the four types of cultural identity: home culture identity, host culture identity, bicultural identity, and global identity. For each cultural identity type, we describe the prototypical cultural characteristics of an expatriate's social network, and discuss how the network's culture base helps or hinders the reconstruction of that cultural identity. The model contributes to the studies of expatriates and offers some important practical implications to global companies and individual expatriates. (For more information, please contact: Yan Shen, University of Victoria, Canada: yanshen@uvic.ca) 


\section{A Cultural Experience-based Typology of Internationally Skilled Mobile Employees}

Julia Ines Raupp, WU Vienna

Jonas F. Puck, WU Vienna

As a consequence of internationalization, firms' exposure to geographically distant, culturally unfamiliar and hence uncertain environments increases. In order to limit uncertainty, firms often prioritize employing staff according to their specific international experience as such employees may contribute with their complex knowledge of specific-cultures and/or their general and cross-cultural competence. Yet, hitherto literature has been fragmented and failed to consistently delineate employees endowed with cultural competence. Hence, this paper intends to review and clarify the differentiation of the plethora of classifications of internationally experienced and qualified employees. For this purpose, the term internationally skilled mobile employee (ISME) is introduced and literature on different types of internationally skilled mobile employees is reviewed. We introduce a novel typology for comprehensively structuring and assessing the differing cultural competencies of ISME according to the two dimensions 'depth of culture-specific knowledge' and 'breadth of culture-general competence' and discuss implications for theory and practice. (For more information, please contact: Julia Ines Raupp, WU Vienna, Austria: julia.raupp@wu.ac.at)

\section{Cosmopolitanism in a Globalized World: A Multidisciplinary Approach Levy Orly, IMD \\ Maury Peiperl, IMD \\ Karsten Jonsen, IMD}

We offer a new perspective on cosmopolitanism by drawing on multiple academic disciplines and integrating these to advance our fundamental understanding of what cosmopolitanism means in a contemporary setting. First, we put forward a conceptual framework that maps out the cosmopolitan literature and delineates its core dimensions. Second, we focus attention on different types of cosmopolitans-the elite, the professional and the ordinary - and examine the relations between macro-level dynamics and cosmopolitanism as an individual disposition. Third, we explore how cosmopolitans can be an asset to the MNC in a globalized world. (For more information, please contact: Karsten Jonsen, IMD, Switzerland: karsten.jonsen@imd.org)

\section{Multiculturals As Cultural Brokers: Their Roles in New Product Development Teams Hae-Jung Hong, Rouen Business School}

Through a 10-month ethnographic field study of multicultural workforce in global new product development teams, I build theory about cultural brokerage role. Specifically, I examine how multiculturals influence team work processes and outcomes by managing knowledge processes and handling conflicts. Multiculturals' cultural brokerage in global teams was identified through brining new local product and market knowledge, translating cultural nuances, and connecting geographically diverse knowledge and skills for a team's knowledge processes; reducing inter-regional misunderstanding and displaying flexible behavior to do with people from diverse regions and cultures for a conflict management. Implications of my findings for both theory and practice are discussed. (For more information, please contact: Hae-Jung Hong, Rouen Business School, France: hjh@rouenbs.fr)

\section{The Internationalism Career Anchor: A Validation Study \\ Mila Lazarova, Simon Fraser University \\ Jean Luc Cerdin, ESSEC \\ Yuan Liao, University of New South Wales}

International mobility, the volatile individual career trajectories it produces, and its implication for human resource management in organizations are not well understood. This paper addresses this deficiency and introduces empirical evidence for an internationalism career anchor, a construct that can enhance our 
understanding of individual predisposition to undertake international mobility. We present a conceptualization and a measurement scale based on Schein's (1990) original career anchors framework and Suutari and Taka's (2004) extension that suggested the presence of an internationalism career anchor. Our analyses, conducted using two samples, one of students and one of business professionals in France, provide evidence for construct validity and reliability of the proposed construct. (For more information, please contact: Mila Lazarova, Simon Fraser University, Canada: mbl@sfu.ca)

\section{Corporate Global Mindset - Antecedents, Measurements and Interventions \\ Jörg Hruby, Hochschule für Unternehmensführung \\ Bjoern Peiter, Hochschule für Unternehmensführung}

This paper deals with corporate global mindset and how to cultivate it in companies. The literature on organizational cognition and measures of corporate global mindset are reviewed. The influencing factors or antecedents in order to cultivate a global mindset in companies are explored and organizational measures in order to develop a global mindset are identified. Our study employs 30 in-depth interviews with Austrian international and global managers responsible for international strategies. The results show that there are four categories identified which correspond with existing literature. Our study reveals that there is still a lack on conceptual models of how to develop an organizational global mindset. Therefore an organizational developmental model which has to be empirically tested is proposed. (For more information, please contact: Jörg Hruby, Hochschule für Unternehmensführung, Germany: hruby@bwl-studieren-ohne-grenzen.de)

\section{Session: 2.2.1 - Panel \\ Track: Track: 7 - Emerging Economies}

\section{Innovation in the TRIC Emerging Economies}

Presented On: July 5, $2013-10: 55-12: 10$

Chair: Bruce McKern, China Europe International Business School

Panelists:

Tamer Cavusgil, Georgia State University

Sergey Filippov, Delft University of Technology

Ravi Ramamurti, Northeastern University

George Yip, China Europe International Business School

This panel brings together researchers from four of the most important emerging economies-Turkey, Russia, India and China. Each will focus on the opportunities and challenges of innovation in their respective countries. Each will discuss innovation by both local and foreign companies. A key feature of this panel will be comparison among these four countries, which will be the focus of the panel debate following the individual presentations on each country. Another key feature will be the comparison of innovation and knowledge management strategies followed by local companies with those used by foreign MNCs. Hence this panel will contribute to the ongoing debate about the managerial capabilities of emerging economy firms and how these might differ from developed economy firms. Each of the four countries is at a different stage of its development, and innovation plays different roles in each country, along with that of foreign direct investment. These differences will make for simulating comparison and debate in this panel. China has been shifting to a new phase, from exportoriented manufacturing towards domestic consumption and sophisticated innovation-intensive businesses. (For more information, please contact: George Yip, China Europe International Business School, China: gyip@ceibs.edu) 
Session: 2.2.2 - Panel

Track: Track: 1 - Institutions, Governance, and CSR

\title{
Research Frontiers in Institutions and Strategy
}

Presented On: July 5, $2013-10: 55-12: 10$

Chairs: Aya Chacar, Florida International University and Gurneeta Vasudeva, University of Minnesota

\author{
Research Frontiers in Institutions and Strategy \\ Lorraine Eden, Texas A\&M University \\ Pankaj Ghemawat, IESE \\ Tatiana Kostova, University of South Carolina \\ Klaus E. Meyer, China Europe International Business School \\ Jennfier Spencer, George Washington University
}

Formal and informal institutions are both obstacles and enablers to the functioning of businesses within and across national institutional boundaries. There has been an increasing awareness of the importance of such institutions in international business research marked by special journal issues and conference panels on the topic. This panel asks: what are the next research frontiers, to a panel of seasoned researchers who are doing research and reviewing on different aspects of institutional contexts and institutional theory based research. The panel will be organized in an interactive format wherein each panelist will address a set of questions on the state of the field and research frontiers. The organizers will moderate and facilitate the discussion and foster debate among the panelists and the participants. (For more information, please contact: Gurneeta Vasudeva, University of Minnesota, USA: gurneeta@umn.edu)

\section{Session: 2.2.3 - Panel \\ Track: Track: 9 - Cross-Cultural Management and HRM \\ The Yin Yang Perspective on Culture and Cross-Cultural Management: Pilot Studies from Diverse National and Organizational Contexts}

Presented On: July 5, $2013-10: 55-12: 10$

Chairs: Tony Fang, Stockholm University and Ayse Burcin Erarslan, Koc University

The Yin Yang Perspective on Culture and Cross-Cultural Management: Pilot Studies from Diverse National and Organizational Contexts

Tony Fang, Stockholm University

Ayse Burcin Erarslan, Koc University

Rubens Pauluzzo, University of Udine

Reimara Valk, Avans University of Applied Sciences

Siegrid Guillaumon, Catholic University of Brasilia

Cheryl Cordeiro-Nilsson, University of Gothenburg

Mikhail Volkov, National Research University Higher School of Economics

The aim of this panel is to discuss the emergent Yin Yang perspective on culture (Fang, 2012) and its applications in cross-cultural management in the age of globalization through case studies in diverse national and organizational contexts. An approach that is deeply rooted in Chinese philosophy, a Yin Yang perspective posits that all cultures (e.g., national culture, regional culture, organizational culture) are dynamic, and that apparently paradoxical value orientations are constantly shaped and re-shaped depending on situation, context and time. Through seven presentations, this panel will examine the potential of the Yin Yang thinking to address 
the challenges and benefits associated with managing people in diverse intra- and international business environments, as well as to gain deeper understanding of national cultures and intra-national diversity. Furthermore, the panel will address the relationship between the Yin Yang perspective and other dynamic approaches to the study of culture and cross-cultural management, thus building connections with some of the prevailing theoretical perspectives in these areas. In addition, the panel serves to stimulate discussion on the application of the Yin Yang thinking in the study of international human resources management, multicultural work teams and many other HR practices in a globalized context. Professor Rosalie L. Tung has accepted our invitation to serve as the discussant for this panel session. (For more information, please contact: Ayse Burcin Erarslan, Koc University, Turkey: aerarslan@ku.edu.tr)

\section{Session: 2.2.4 - Competitive}

\section{Alternative IB Research Methodologies}

Presented On: July 5, $2013-10: 55-12: 10$

Chair: James Agarwal, University of Calgary

The Use of Historical Research Methods in International Business Peter J Buckley, University of Leeds

In response to problems in international business research including endogeneity issues, historical research methods can improve understanding. A critical analysis of all "texts" (sources), time series analyses, comparative methods (across time periods and space), counterfactual analysis and the examination of outliers are all shown to have the potential to improve current international business research practices. (For more information, please contact: Peter J Buckley, University of Leeds, United Kingdom: pjb@lubs.leeds.ac.uk)

\section{Do IB Researchers Account for Language Issues? State of the Art and Lessons for the Future Emmanuella Plakoyiannaki, Aristotle University of Thessaloniki Agnieszka Chidlow, Manchester Metropolitan University Catherine Welch, University of Sydney}

In this paper, we problematize the way language has been treated in international business (IB) research. We conduct a hermeneutic content analysis of both qualitative and quantitative cross-language studies that have been published in four core IB journals over the course of a decade. We find that of those articles that do discuss language, most restrict themselves to a narrow lexical view of translation equivalence. In this paper, we offer an alternative approach that is grounded in interpretivist approaches and informed by translation studies. This approach entails consideration of equivalence of meaning, response equivalence, difference and context. (For more information, please contact: Emmanuella Plakoyiannaki, Aristotle University of Thessaloniki, Greece: emplak@econ.auth.gr)

\section{Generations and Shifts in International Values}

André van Hoorn, University of Groningen

Whilst the idea of cultures converging and the development of a global business culture has settled in the minds of many, surprisingly, empirical evidence on this issue has hardly developed beyond static bi-country comparisons of managerial values. We extend the critique that the convergence literature has applied flawed research designs and develop a theory-driven empirical approach that revolves around generational cohorts. This way we can move forward from suspect empirical evidence and use the power of longitudinal data to distinguish between genuine cultural convergence (or divergence) and previously neglected confounders. Other 
key novelties of our study are that it covers the complete spectrum of human motivations and that we assess convergence for a large and highly diverse group of countries. Data cover over 37,000 managers from 29 culturally highly diverse countries and were collected at four different points in time. Results show systematic (generational) shifts in managerial values towards a steady waning of cultural dissimilarities between managers. Although cultural convergence is not universal across values domains, nationality is increasingly becoming a less relevant factor in managerial values. We now have strong empirical reason to move beyond thinking in simple country dichotomies when considering managerial values and business culture. (For more information, please contact: André van Hoorn, University of Groningen, Netherlands: vanhoorn@gmail.com)

The Disciplining Forces of Disciplines and Their Effects on Interdisciplinarity: The Case of International Business Studies

Sierk Horn, University of Leeds

Adam Cross, University of Leeds

Malcolm Kenneth Chapman, University of Leeds

Although commonly portrayed as unconscious and arbitrary, disciplinary boundaries provide guidance for academic legitimacy, and resultant social identification determines intergroup relations. In this study, we develop a quadrimodal framework to investigate the dynamics of interdisciplinarity. We consider factors underpinning the receptiveness to sojourning scholarship and test our model and its hypothesized antecedents in the context of international business academia. Variations of interdisciplinary engagement are revealed. Routes to interdisciplinary engagement are moderated by individual dispositions and disciplinary culture prevalent amongst international business scholars. With respect to disciplinary capacity building our study offers insights into the barriers and carriers of outside expertise. (For more information, please contact: Sierk Horn, University of Leeds, United Kingdom: s.horn@leeds.ac.uk)

\section{Session: 2.2.5 - Competitive}

\section{Track: Track: 10 - Economics, Finance and Accounting}

\section{Trends and Determinants of M\&As and Financing Patterns}

Presented On: July 5, $2013-10: 55-12: 10$

Chair: Sylvia Kim, Fresno Pacific University

Cross-border Financial Intermediation and Domestic Acquisitions: The Role of Host Country Experience Sofie De Prijcker, Ghent University

Mike Wright, Imperial College

Sophie Manigart, Vlerick Business School

This paper studies the effect of host country experience of cross-border financial intermediaries on the probability of a domestic versus an international acquisition. Using a unique dataset of 296 Continental European acquisitions guided by cross-border private equity investors, this paper shows how host country experience enhances local information transfer through local embeddedness. This reduces the information costs of domestic acquirers which increases the probability of a domestic acquisition. The positive effect of crossborder private equity investors' host country experience on the probability of a domestic acquisition is higher for financial than for strategic acquisitions. This is the result of increasing network connections between private equity investors and financial acquirers compared to strategic acquirers. Finally, host country experience is less positively associated with a domestic acquisition under higher levels of host country financial market development. The increased accessibility of local information within more developed financial markets substitutes for benefits of local information transfer by cross-border intermediaries with higher levels of host country experience. (For more information, please contact: Sofie De Prijcker, Ghent University, Belgium: sofie.deprijcker@ugent.be) 
In the Eye of the Beholder: The Asymmetric Impact of National Cultural Distance on Target Premiums for U.S. and Foreign Firms in Cross-Border M\&As

Jongha Lim, University of Missouri

Anil K. Makhija, Ohio State University

We find that greater cultural distance significantly lowers the premium paid by U.S. bidders for foreign targets but not premiums paid by foreigners for U.S. targets. Prior literature routinely assumes the same impact of a given cultural distance on target premiums irrespective of switching the national cultures of the bidder and target. But, foreigners may not similarly apply a cultural discount for U.S. targets if there is greater familiarity with U.S. culture abroad than the other way around. Using flow of travelers and exchange of students between the U.S. and foreign countries as proxies for cross-cultural familiarity, we affirm the asymmetric impact of cultural distance on premiums for foreign versus U.S. targets. (For more information, please contact: Jongha Lim, University of Missouri, USA: limjong@missouri.edu)

\section{Financing Patterns, Multinationals and Performance: Firm-Level Evidence from 47 Countries \\ Sushanta Mallick, Queen Mary University of London \\ Yong Yang, University of Sussex}

The main purpose of this paper is to provide new empirical evidence on the effects of financing patterns by firms, focusing on two related questions: (i) Do sources of financing matter for firm performance and whether leverage boosts or harms performance, even in times of crisis? and (ii) whether multinationals' performance and leverage get transmitted to their overseas affiliates? This paper draws on firm-level data covering over 10,000 firms from 47 countries to answer these questions. We find that while retained earnings and equities have a positive effect on productivity, bank and non-bank loans tend to negatively influence productivity. We also find a negative relationship between leverage and productivity, but for large firms leverage has positive effect on performance. Further, while the multinational parents' performance significantly influences the subsidiaries' performance, higher debt ratios of parents lead to lower productivity of subsidiaries only in developing countries perhaps due to their financial dependence on parents. (For more information, please contact: Yong Yang, University of Sussex, United Kingdom: yong.yang@sussex.ac.uk)

\section{Session: 2.2.6 - Competitive \\ Track: Track: 3 - IB Theory, FDI, and Entry Mode \\ Interactions Between Foreign-Owned and Indigenous Firms}

Presented On: July 5, $2013-10: 55-12: 10$

Chair: Jörg Zimmermann, European Commission - Joint Research Center

Learning or Competition? The Impacts of Domestic Enterprises on Foreign Direct Investment Productivity in a Transition Economy

Yi Tang, Hong Kong Polytechnic University

Cuili Qiang, City University of Hong Kong

Haibin Yang, City University of Hong Kong

This study investigates the impacts of domestic enterprises on the productivity of foreign invested enterprises (FIEs) in the transition economy of China. Specifically, domestic enterprises that differ by ownership type are expected to affect their foreign counterparts' productivity differently. We propose that the presence of stateowned enterprises (SOEs) exerts a negative impact on the productivity of FIEs both within and across industries. In contrast, the presence of private enterprises is likely to exert a negative impact on FIEs' productivity within the same industry, but a positive impact across industries. We further argue that the above 
relationships are moderated by FIEs' age in the host country market. Empirical analyses using a large panel dataset in China from 1998 to 2007 render strong support to our theoretical predictions. (For more information, please contact: Yi Tang, Hong Kong Polytechnic University, Hong Kong, SAR-PRC: msytang@polyu.edu.hk)

The Sectoral and Spatial Impacts of Inward Foreign Direct Investment on Indigenous New Firm Creation in the Republic of Korea

Eunsuk Hong, SOAS, University of London

Laixiang Sun, SOAS, University of London

In Hyeock (Ian) Lee, Loyola University Chicago

This paper examines the impact of inward foreign direct investment (FDI) on the variation in the creation of new firms across high- versus low-technology industrial sectors and geographic space. Using a dataset of 44,434 newly created small firms in 234 regions of South Korea in 2000-2004, this study finds that while the spillover impacts of FDI in the low-tech industry are positive and significant across almost all four possible combinations of the intra-/inter-regional and intra-/inter-sectoral channels, the impacts in the high-tech industry are largely intra-sectoral within the host region and across neighboring regions. Moreover, all statistically significant spillover effects follow an inverted ' $U$ '-shaped curvilinear trend. (For more information, please contact: Eunsuk Hong, SOAS, University of London, United Kingdom: e.hong@soas.ac.uk)

\section{MNC Subsidiary Closure: What Stays When the MNC Leaves? \\ Pedro de Faria, University of Groningen \\ Miguel Torres Preto, Technical University of Lisbon and Center for Innovation, Technology and Policy Research Wolfgang Sofka, Copenhagen Business School}

We investigate the consequences of MNC subsidiary closures for employees who lose their jobs. We ask to what degree the foreign knowledge that they were exposed to is valued in their new job. We argue theoretically that this foreign knowledge is both valuable and not readily available in the host country but is also distant and therefore difficult to absorb. We predict an inverse u-shaped relationship between the exposure to foreign knowledge and the salary in the new job. We empirically support our predictions for a sample of almost 140,000 affected employees in Portugal from 2002 to 2009. (For more information, please contact: Pedro de Faria, University of Groningen, Netherlands: p.m.m.de.faria@rug.nI)

\section{The Impact of Foreign Direct Investment on Local Venture Founding: Evidence from China Junichi Yamanoi, Chuo University \\ Xufei Ma, Chinese University of Hong Kong}

Although previous studies have investigated the foreign direct investment (FDI) spillover effects on incumbent local firms' productivity and survival, they have virtually ignored the impact of FDI on local venture founding. Using the theoretical framework of entrepreneurial and institutional processes, we argue that FDI in an industry in a geographic region has a nonlinear impact on the founding rate of new ventures; it initially enhances entrepreneurial opportunities for potential entrepreneurs due to learning from foreign entrants, but gradually depreciates them through intense competition with competitive foreign entrants and improved local incumbents. However, FDI in related industries would promote the founding rate, because potential entrepreneurs discovering entrepreneurial opportunities in a focal industry can avoid direct competition with the foreign entrants and improved local incumbents by exploiting the opportunities in its related industries. Additionally, we argue that, as aspects of the institutional environment, governmental hands-off policy on market economy and intellectual property rights protection will encourage potential entrepreneurs to exploit opportunities brought from FDI. Using data from Chinese manufacturers from 1998 to 2009, we found general support to our hypotheses. (For more information, please contact: Junichi Yamanoi, Chuo University, Japan:

yamanoi@fps.chuo-u.ac.jp) 


\section{Session: 2.2.7 - Competitive}

\section{Track: Track: 1 - Institutions, Governance, and CSR}

\section{Politics and Strategy}

Presented On: July 5, $2013-10: 55-12: 10$

Chair: Charles Hermann, Texas A\&M University

How Corruption Matters on FDI Flows: Home and Host Country Effects

Manuel Portugal Ferreira, Uninove - Universidade Nove de Julho

Helder Costa Carreira, Instituto Politecnico de Leiria \& globADVANTAGE

Dan Li, Indiana University

Fernando Ribeiro Serra, Uninove - Universidade Nove de Julho \& globADVANTAGE

Prior research has identified a negative impact of corruption on countries' ability to attract Foreign Direct Investment (FDI). Only a few scholars have argued that corruption can act as "grease", perhaps attracting FDI. We extend prior research by, first, distinguishing the pervasiveness and arbitrariness of host country corruption and their effects on FDI inflows, and second, testing whether the level of corruption of the investors' home country affects FDI decisions. Our results show that the pervasive corruption negatively drives FDI but not the arbitrariness component. Our results reveal that home country corruption negatively impacts the overall FDI outflows, but investors from countries with high levels of corruption do not seem to be deterred by a high level of corruption in the host country. (For more information, please contact: Manuel Portugal Ferreira, Uninove Universidade Nove de Julho, Brazil: manuel.portugal.ferreira@gmail.com)

\section{Political Geography and Foreign Direct Investment Performance: Is Spatial Proximity to Central Government Politicians and Bureaucrats of Capital Value? \\ Marie-Ann Betschinger, NRU Higher School of Economics}

This paper investigates if spatial proximity to central government politicians and bureaucrats matters for foreign direct investment performance. We argue that proximity to national government politicians and bureaucrats, and the formal and informal communities they are part of, permit firms to obtain wider and quicker access to information and gain weight in influencing political or bureaucratic outcomes. Analyzing a sample of 6,698 foreign affiliates of 1,339 Japanese parent firms in 76 countries, we find that a capital city location increases foreign affiliate performance. A country's institutional environment, parent firm, and affiliate-level characteristics moderate the effect. (For more information, please contact: Marie-Ann Betschinger, NRU Higher School of Economics, Russia: mbetschinger@hse.ru)

\section{Multilevel Normative Institutions: Host Market Bribery and Multinational Enterprise Strategy Michael A. Sartor, University of Western Ontario}

This study investigates whether petty corruption and grand corruption differ in their impact upon the entry strategies of foreign-investing MNEs. While extant theory suggests that the pervasiveness of host market corruption will influence the equity ownership decisions of these MNEs, subsequent research has not found a statistically significant relationship that empirically validates these propositions. These results seem counterintuitive, particularly given the substantial evidence that has been garnered which suggests that host market corruption influences the global location of foreign direct investment. We synthesize insights from institutional theory and integrative social contracts theory to argue that host market normative institutions, such as public sector corruption, should be conceptualized as multi-level phenomena. We theoretically distinguish between petty corruption and grand corruption in order to develop hypotheses pertaining to the relationship 
between each type of corruption and three foreign entry decisions undertaken by MNEs (entry mode, partner choice and expatriate staffing). We test the hypotheses with a longitudinal sample of 961 subsidiaries established in 35 countries. The results reveal that the normative institution of corruption is a multilevel phenomenon and that each of the two types of public sector corruption exerts a different impact upon the three foreign entry decisions. (For more information, please contact: Michael A. Sartor, University of Western Ontario, Canada: msartor.phd@ivey.ca)

\author{
Transparency and Corruption: MNCs Relationship with Political Actors \\ Amjad Hadjikhani, Uppsala University \\ Cecilia Pahlberg, Uppsala University \\ Pervez N. Ghauri, King's College London
}

Globalization and increasing availability of information frequently face international firms with the serious issue of transparent business activities and avoidance of corruptive behavior. The main question in this paper is how international firms handle these issues. Standing on business network theory the paper presents an analytical view on business and political actors' relationship with emphasis on transparency and corruptive behavior. We build on the relationship elements of knowledge and legitimate/illegitimate commitment and illustrate with cases showing two European MNCs and their behaviors in foreign markets. The main contribution is that deeper understanding is obtained by including the concept of trust/distrust in the relationship. Further, while transparent behavior means that the actors keep their ground for legitimacy, corruptive behavior means the opposite. (For more information, please contact: Amjad Hadjikhani, Uppsala University, Sweden: amjad.hadjikhani@fek.uu.se)

\title{
Session: 2.2.8 - Competitive
}

\section{Track: Track: 6 - Innovation and Knowledge Mgmt.}

\section{Knowledge Utilization}

Presented On: July 5, $2013-10: 55-12: 10$

Chair: Angelika Lindstrand, Stockholm School of Economics

\section{Exploring Licensing Performance through a Capabilities Approach: Evidence from Spanish Manufacturing Firms Mattia Bianchi, Stockholm School of Economics Jose Lejarraga, IE University}

Firms increasingly pursue technology licensing for appropriating economic returns from their R\&D investments. Despite this tendency, extracting revenues from licensing remains a challenge for most firms, as highlighted by the substantial variability among firms' licensing performance found in recent studies. Because the complexities posed by technology licensing, in terms of identifying suitable partners and in the marketing of an intangible and idiosyncratic good, require specific knowledge and learning structures which may be independent of the technological assets possessed by the firm, we explore the determinants of licensing performance from a capabilities perspective. To address this issue, we build two different conceptualizations of a licensing capability, based on task-related experience and on workforce's skills. Using longitudinal data of Spanish manufacturing firms, we find that the licensing revenues increase with specialized experience and advanced employees' skills. (For more information, please contact: Mattia Bianchi, Stockholm School of Economics, Sweden: mattia.bianchi@hhs.se) 
External and Internal Tacit Knowledge, Within-Strategy Accord and Tension in Open Action Strategies, and Knowledge Generation of International Project Teams

Taewon Suh, Texas State University

Omar J. Khan, Morgan State University

In this paper, we explore the within-strategy accord and tension caused by transferred tacit knowledge in order to better manage the creation of innovation through international project teams. We postulate that team knowledge generation is related in opposing directions to external tacit knowledge and internal tacit knowledge. We further explore the within strategy accord and tension between external tacit knowledge and team knowledge generation. We also posit the moderating role of internal tacit knowledge diversity between internal tacit knowledge and knowledge generation. We test our hypotheses through hierarchical regressions with data from 162 firms engaged in international marketing projects. We, thus, propose a paradoxical resolution of tacit knowledge management in order to effectively manage innovation generation of international project teams. Managerial implications are discussed. (For more information, please contact: Omar J. Khan, Morgan State University, USA: omar.khan@morgan.edu)

\author{
Reverse Knowledge Transfer and Globalization of Chinese Enterprises \\ Cindy Qin, University of San Francisco \\ Xiaohua Yang, University of San Francisco \\ Roger Chen, University of San Francisco
}

Drawing on knowledge transfer, international strategy and MNC literature, we propose a model linking strategic assets seeking motives, headquarters control, subsidiary age and reverse knowledge transfer in emerging economy multinationals (EEMs). The model is tested in the context of Chinese enterprises in the US. Our exploratory study provides initial evidence that strategic assets seeking motives and headquarters control are significantly and positively related to reverse knowledge transfer. Furthermore, our empirical evidence shows negative relationship between subsidiary age and reverse knowledge transfer. We discuss the implications of our analysis on the EEMs theory development, draw out inferences for knowledge management within EEMs and suggest avenues for future research. (For more information, please contact: Cindy Qin, University of San Francisco, USA: dqin3@usfca.edu)

\author{
Internationalization of R\&D and Innovation Performance in Small Emerging Economies \\ Chia-Wen Hsu, National Chung Cheng University \\ Yung-Chih Lien, National Taiwan University \\ Homin Chen, National Taiwan University \\ Yin-Ru Chen, National Taiwan University
}

This paper examines the effects of R\&D internationalization on firm's innovation performance. Based on a longitudinal data of Taiwanese high-tech firms, we respectively test the effects of R\&D internationalization in terms of intensity and scope. Both the examination results reveal an inverse U-shaped curvilinear relationship, suggesting the costs of R\&D internationalization may outweigh its benefit after critical intensity and scope. Additionally, we found that firm's experience and pace in foreign expansion may have different impacts on the relationship between R\&D internationalization and innovation performance, suggesting the effect is contingent on firm's capability in dealing with the complexity and uncertainty of international business. (For more information, please contact: Chia-Wen Hsu, National Chung Cheng University, Taiwan: cwhsu@ccu.edu.tw) 


\title{
Session: 2.2.9 - Competitive
}

\section{Track: Track: 6 - Innovation and Knowledge Mgmt.}

\section{Entrepreneurial Knowledge Management}

\author{
Presented On: July 5, $2013-10: 55-12: 10$
}

Chair: Rian Drogendijk, Uppsala University

Generating Entrepreneurial Strategies for Building Horizontal Knowledge Network Embeddedness Stoyan Petrov Stoyanov, University of Edinburgh

Richard Woodward, University of Edinburgh

The study concentrates on exploring how balancing networks within ethnic Diasporas empowers transnational entrepreneurs to capitalize on external processes, among which gaining new knowledge and information, the capitalization on which requires extending to other parties through reputation channels. Starting with an exploration of the embeddedness' paramount role for companies, as perceived by the transnational actors, the research then leads us to the strategic practices that transnational entrepreneurs (TEs) engage in. The study follows the mentioned order as only after knowing the underpinnings of the motivation, can we build full understanding of the way embeddedness occurs and the steps that low-power actors, such as immigrant entrepreneurs, take when determined to build higher positions that maximize network outcomes. To achieve these objectives, the study has relied on ethnographic fieldwork, which has attempted to convey a more comprehensive account and therefore more reflexivity, higher personification and closer embodiment of the researched phenomenon. The conducted ethnography has facilitated typology construction and ultimately, theory on transnational entrepreneurs' inter-organizational strategies. (For more information, please contact: Stoyan Petrov Stoyanov, University of Edinburgh, United Kingdom: s.p.stoyanov@sms.ed.ac.uk)

\section{How Cultures Influence National Rate Of Innovation? - A Longitudinal Analysis of 52 Countries Jing Betty Feng, Georgia State University Leigh Anne Liu, Georgia State University}

We investigate how culture interacts with institutional factors such as R\&D investment to influence national rate of innovation over time. We propose a framework that clusters cultural values into innovation encouraging, enforcing, constraining, and injunctive norms. Using longitudinal data during 1995-2010, based on four sources of culture measurements (Hofstede, 2001; Schwartz, 2005; House et al., 2004; Gelfand et al., 2011), we found that the innovation encouraging and enforcing norms positively strengthen the relationship between R\&D investment and national rate of innovation over time while the innovation constraining and injunctive norms negatively weaken such relationship. Our findings challenge some previous results and conventional wisdom on culture and innovation at the country level and provide new insights on the dynamic and sophisticated role culture plays to influence innovation. (For more information, please contact: Jing Betty Feng, Georgia State University, USA: jfeng4@gsu.edu)

Knowledge Creation and Sharing and the Integration-Responsiveness Framework: The Role of Functional Subunits of the MNC

Paz Estrella Tolentino, University of London

Odile E.M. Janne, University of London

Pi-Chi Chen, University of London

We theoretically and empirically examine the role of functional subunits in knowledge creation and sharing in the differentiated multinational company (MNC). Specifically, we look at the extent to which functional subunits acquire knowledge from their host country environment which they then share with other parts of the MNC 
network. Inspired by the influential integration-responsiveness framework in strategic management, we posit a model to show how the strategy dimensions of functional subunits (i.e. integration and responsiveness) act in a complementary way to determine their knowledge creation and sharing capacities. We test this empirically using data from close to 100 functional activities of 16 American wholly owned subsidiaries in Taiwan's IT industry. The results confirm our theoretical proposition while exerting significant implications for our research topic more generally as well as for the theoretical approach we adopted. First, knowledge creation and sharing in a differentiated MNC emanate from all functional activities and all national subsidiaries and not just in specific functions or in subsidiaries with particular mandates or strategy type. Secondly, it is necessary to consider both demand-side and supply-side factors and to assess their relative importance in the knowledge creation and sharing of MNC units and subunits. (For more information, please contact: Paz Estrella Tolentino, University of London, United Kingdom: p.tolentino@bbk.ac.uk)

\title{
First-Mover Advantages Before and After TRIPS: Evidence from the Indian Pharmaceutical Industry Chirantan Chatterjee, Indian Institute of Management Bangalore \\ Ajay Bhaskarabhatla, Erasmus University
}

Scholars argue that the presence of persistent first-mover advantages obviates the need for relatively long-lived patents as incentives for innovations. What then is the impact of the strengthening of patent protection, particularly in developing economies, on non-patent-based first-mover advantages? We investigate this question by estimating the extent of erosion of first-mover advantages in the Indian pharmaceutical industry due to the introduction of a stronger product-patent regime in January 2005. We distinguish between newly created and pre-existing markets, in which the strength of product patent protection differs systematically. Our analyses account for the endogeneity of entry order. We find evidence of erosion-as high as 50-percent-that is robust to alternative estimation strategies and definitions of dependent variables. We extend the Suarez-Lanzolla framework and highlight the role of regulatory factors on the relationship between first-mover advantages and intellectual property rights. We conclude by discussing managerial and policy implications. (For more information, please contact: Chirantan Chatterjee, Indian Institute of Management Bangalore, India: chirantan.chatterjee@iimb.ernet.in)

\section{Session: 2.2.10 - Competitive}

\section{Track: Track: 9 - Cross-Cultural Management and HRM}

\section{Supporting Organizational and Self-Initiated Expatriates}

Presented On: July 5, $2013-10: 55-12: 10$

Chair: Vesa Peltokorpi, Japan Advanced Institute of Science and Technology

\author{
Expatriate Performance in High-risk Countries: Influence of Family-Related Stress \\ Benjamin Bader, University of Hamburg \\ Nicola Berg, University of Hamburg \\ Dirk Holtbruegge, University of Erlangen-Nuernberg
}

Expatriates of foreign firms are not only sent to industrialized countries with stable environmental conditions, but also to countries that bear high political, social, and even terrorist risk. Despite its practical relevance, the role of expatriate's families on assignments in high-risk countries has not been addressed yet. Applying hierarchical regression analysis we investigate the family-related performance antecedents of 121 married (or living in a relationship) expatriate managers assigned to a terrorism-endangered country. We find evidence that intra-family tension as well as a high global stress level significantly impede expatriates' work performance. Perceived organizational support can help to diminish this influence. We discuss our results and conclude with further implications for theory and practice. (For more information, please contact: Benjamin Bader, University of Hamburg, Germany: benjamin.bader@uni-hamburg.de) 
Effectiveness of Perceived Organizational support for SIEs: Career Networks as Moderators

Lan Cao, Leuphana University Lueneburg

Andreas Hirschi, University of Lausanne

Juergen Deller, Leuphana University Lueneburg

Previous literature in SIE (self-initiated expatriation) has been mostly focused on an individual perspective. Studies on SIEs in organizational context are scarce. The current paper sought to examine the effect of perceived organizational support (POS) on SIE employees' intention to stay in the host country, mediated by career satisfaction. Furthermore, we examined the moderating roles of career-related social networks with host and home country nationals on the effectiveness of POS. Data from 112 SIE employees in Germany were collected and analyzed. Empirical results partially supported our proposed model: there were significant negative indirect effect between POS and intention to stay, when career network size with home country nationals was high. The direct effect between POS and intention to stay was positive. For HR practice, our paper gave insight to understand SIE employees' needs for support and mobility preferences, which can help organizations to develop more targeted HR development measures and assignment strategies for them. (For more information, please contact: Lan Cao, Leuphana University Lueneburg, Germany: lan.cao@leuphana.de)

\section{Thriving and Retention of Expatriates: A Conservation of Resources Perspective Hong Ren, University of Wisconsin-Milwaukee Dilek Gulistan Yunlu, University of Wisconsin-Milwaukee}

Taking a 'positive psychology' perspective and drawing on Conservation of Resources theory, we apply the concept of "thriving" to explain expatriate retention. Using longitudinal data from 103 international teachers in the United States, we found that organizational embeddedness directly influenced thriving, which in turn led to expatriate actual retention. In addition, organizational embeddedness's positive effect on thriving was more pronounced when cultural intelligence was high. Applying the concept of thriving to explicate expatriate experiences, we make several contributions to the global employment literature. (For more information, please contact: Dilek Gulistan Yunlu, University of Wisconsin-Milwaukee, USA: dgyunlu@uwm.edu)

\section{Work Stress, Support Perceptions, and Expatriate Performance: An Interactive Perspective Norifumi Kawai, University of Sussex \\ Alex Mohr, University of Kent \\ Marv Khammash, University of Sussex}

The main objectives of this paper are: (1) To explore the relationship between different facets of role stressors and expatriates' job satisfaction and (2) to test the contingency effects of perceived organizational support and perceived supervisor support on this relationship. An attempt to meet these objectives was made through moderated regression analysis based on a sample of 126 Japanese expatriates in Germany. Three meaningful results were found. First, the empirical evidence from Japanese expatriates offers a solid proof that role novelty positively determines both job satisfaction and task performance while role ambiguity has a detrimental influence over job satisfaction. Second, the quantitative evidence reveals that perceived organizational support buffers the negative impact of role ambiguity on task performance. Third, this study further demonstrates that perceived supervisor support tends to strengthen the positive effect of role novelty on both job satisfaction and task performance. Managerial implications were highlighted at the end. (For more information, please contact: Norifumi Kawai, University of Sussex, United Kingdom: n.kawai@sussex.ac.uk) 


\section{Session: 2.2.11 - Interactive \\ Track: Track: 10 - Economics, Finance and Accounting \\ Exchange Rates and the Effects of Culture and Liability of Foreignness on Financial Management}

Presented On: July 5, $2013-10: 55-12: 10$

Chair: C. Bulent Aybar, Southern New Hampshire University

Forecasting the Exchange Rate as a Group Experiment: The Wisdom of Crowds and the Social Influence Effect Haruo Hagiwara Horaguchi, Hosei University

Social psychologists, cognitive scientists, economists and managerial scientists recognize the existence of the wisdom of crowds' effect: estimations that are made by a large number of people coincide with the true values. It has also been pointed out that the estimations by the crowds tend to have certain biases. These biases are called social influence effect. This article explains why the wisdom of crowds' effect exist, inquires to what extent crowds can foresee the future and assesses whether the social influence effect really exist. To answer these questions, I show simulation results, give mathematical proofs of them and use the data from forecasted foreign exchange rates. The data are provided by Nikkei, which holds a forecasting competition by a group of students. The participants consist of 3,657 groups over a period of eight years in Japan. The participants recorded 1.43 percent as the average absolute deviation during an eight-year period. They show some evidences that their forecasts are affected by the real exchange rate at the time of their forecasts. These results suggest that the social influence effect exists in this data set. (For more information, please contact: Haruo Hagiwara Horaguchi, Hosei University, Japan: horaguch@hosei.ac.jp)

\section{The Usefulness of Factor Models in Forecasting the Exchange Rate: Results from the Brazilian Case Jose Luiz Rossi Junior, Insper \\ Wilson Felicio, Insper}

This paper studies the usefulness of factors embedded on the common movements of exchange rates in forecasting the exchange rate Real/Dollar. The results show that when considering the entire period of the sample, from January 1999 to August 2011, no one model containing the factors is able to beat the random walk benchmark. However, when the period directly following the adoption of the floating exchange rate regime is discarded, there is evidence that several models containing these factors beat the random walk. Lastly, the paper shows that the addition of factors improves the predictive power of the models comprising only macroeconomic variables commonly used in the literature to forecast the exchange rate. (For more information, please contact: Jose Luiz Rossi Junior, Insper, Brazil: joselrj1@insper.edu.br)

\section{The Mediating Impact of National Culture on Real Options Exercise: Potential for Suboptimal Time Delays and Value Destruction \\ Derek G. Lehmberg, North Dakota State University \\ Matt Davison, University of Western Ontario}

Real options (RO) valuation has been suggested as a way to evaluate investment opportunities and make investment decisions that takes into account the value of managerial flexibility in the face of uncertainty. Although RO enjoys a substantial body of literature considering its application and applicability in different situations, the impact of national culture on the application of real options has received little attention. National culture plays a role in how managers frame information and make decisions; it can also influence how individuals inside organizations communicate and interact. Culture, therefore, can systematically impact the way in which real options are applied, potentially leading to sub-optimal outcomes. In this paper, we develop a 
process model of real options application, hypothesize how culture affects RO exercise along specific steps of the model, we estimate the magnitude of sub-optimality resulting from these impacts. Our estimates suggest real options may not be a superior valuation approach to net present value for national cultures with certain characteristics. (For more information, please contact: Derek G. Lehmberg, North Dakota State University, USA: derek.lehmberg@ndsu.edu)

\section{Auditors' Assessment of the Liability of Foreignness: Evidence from ADRs Kimberly Gleason, University of Pittsburgh \\ Yezen Kannan, Duquesne University}

The Liability of Foreignness paradigm has been applied to capital markets participants who exhibit a "home country bias" when they make and evaluate their investments. In this paper we use the Capital Markets Liability Of Foreignness (CMLOF) framework and a sample of foreign firms cross-listed on U.S. exchanges to investigate auditor pricing behavior. Drawing on agency theory, this paper is the first to apply the CMLOF to examine whether auditors price aspects of foreignness of their non-U.S. headquartered clients, and the factors that mitigate perceived agency costs to the shareholders of these firms. Our results indicate that auditors price the liability of foreignness when they consider excess cash holdings of ADRs, and that the costs are higher to shareholders of firms with a dissimilar legal system, low disclosure regulations, and weak enforcement of law. Furthermore, the agency costs of excess cash are mitigated by a stronger institutional framework. (For more information, please contact: Kimberly Gleason, University of Pittsburgh, USA: gleasonk@pitt.edu)

\section{Session: 2.2.12 - Interactive \\ Track: Track: 5 - MNC Management and Organization}

\section{MNE, Subsidiaries, and Knowledge Management}

Presented On: July 5, $2013-10: 55-12: 10$

Chair: Danchi Tan, National Chengchi University

Psychic Distance, Control Mechanism and Subsidiary Performance

Manfred Fuchs, University of Graz

Beatrix Joebstl, University of Graz

This study analyses how psychic distance affects control mechanism and how they relate to the performance of subsidiaries in psychically close and psychically distant countries. It is based on an analysis of 75 Austrian MNE Headquarters and two of its subsidiaries, each located in one as distant and one as close perceived locations $(\mathrm{N}=150)$. Effects between formatively and reflectively measured latent variables psychic distance, control mechanisms, and subsidiary performance have been tested using SmartPLS. Results suggest that psychic distance affects the selection of control mechanisms and it can be shown that more autonomy in psychically distant subsidiaries shows a robust effect on better performance compared to close locations. Results suggest that psychic distance affects the selection of control mechanisms and it can be shown that the appliance of uniform accounting practices and control through socialization are influenced by psychic distance. However, it is individual control which positively affects performance of subsidiaries located in countries perceived as close and control through socialization having a negative effect on subsidiary performance. In psychically distant countries, centralized decision-making in financial aspects positively affects subsidiary performance while control through uniform accounting practices and direct control have a negative effect. (For more information, please contact: Manfred Fuchs, University of Graz, Austria: manfred.fuchs@uni-graz.at) 


\title{
Which Factors Influence the Level of Control over the Foreign R\&D Subsidiaries? Kazuhiro Asakawa, Keio University
}

We investigate the extent to which the headquarters' perceived knowledge about overseas labs influences the level of control over the R\&D subsidiaries. We confirm that the headquarters' knowledge about the local would lower the level of control over the R\&D subsidiaries. But surprisingly legitimacy granted to the R\&D subsidiaries does not necessarily lead to the reduced headquarters' control over them. Nor does legitimacy of the R\&D subsidiaries influence the effect of headquarters' knowledge about them on the level of control. While the headquarters' knowledge about the R\&D subsidiaries tends to grant legitimacy to them, the effect of legitimacy of the R\&D subsidiaries seems rather minimal. Such findings imply that the headquarters rests more assured to reduce control over the subsidiaries by having updated knowledge about their current situation than by the established positive image of the R\&D subsidiaries. (For more information, please contact: Kazuhiro Asakawa, Keio University, Japan: asakawa@kbs.keio.ac.jp)

\section{Control in Subsidiary Networks: The Case of French MNCs in Asia Johannes Schaaper, Bordeaux Management School Jacques Jaussaud, University of Pau UPPA Bruno Amann, University of Toulouse}

This research considers two research questions. First, do new forms of control mechanisms, appeared in the last decade thanks to the NTICs (ERP, increased travel and the reinforcement of regional headquarters), fit the "centralisation-socialisation-formalisation" framework. Second, how MNCs articulate dimensions of an enriched C-F-S framework to keep control over their networks of subsidiaries? We conducted 77 interviews with expatriate managers of subsidiaries in Asia established by 47 French MNCs. By combining factor analysis and qualitative content analysis, we reveal four dimensions of control: (1) centralisation of decision making at $\mathrm{HQ}$; (2) formalisation; (3) informal, through short-term missions and visits, facilitated by regional headquarters; (4) expatriation. Training of local employees constitutes a shared control mechanism across dimensions. Our results are consistent with the C-F-S framework. We identify five groups of MNCs exercising different degrees of control through implementing different mixes of the control dimensions, outlined by the enriched C-F-S framework. This research offer three contributions: it updates the classical C-F-S framework, it shows that combining qualitative and quantitative research offers perspectives for international management research and it helps MNCs improve the design of their international control systems. (For more information, please contact: Johannes Schaaper, Bordeaux Management School, France: jan.schaaper@bem.edu)

\author{
Gone are the Creatures of Yesteryear? On the Diffusion of Technological Capabilities in the Modern MNC \\ Katarina Blomkvist, Uppsala University \\ Philip Kappen, Uppsala University \\ Ivo Zander, Uppsala University
}

This paper probes the notion that the emergence of the 'modern' multinational corporation (MNC) has resulted in the increasingly rapid diffusion of technological capabilities across units of the multinational network. It draws upon a database containing the complete U.S. patenting history of 24 Swedish multinationals over the 19462008 period, which allows us to trace both the emergence and subsequent intra-firm diffusion of technological capabilities throughout the post-war period. The findings reveal that the pace of diffusion has indeed become faster over the observed time period, and that the main period of change has occurred over the past two decades. In line recent observations about skewed patterns of innovation activity in larger MNCs, the findings nevertheless indicate substantial variation in the firms' adoption of modern ways of organizing and managing technology within the MNC network. (For more information, please contact: Katarina Blomkvist, Uppsala University, Sweden: katarina.blomkvist@fek.uu.se) 
This research synthesizes international and comparative management perspectives to develop a framework for analyzing the transfer and management of organizational practices in multinational corporation (MNC) crossborder affiliates. The key subjects for an examination of the framework and the issues it raises are five Hong Kong international firms operating in three cities in China, including Shenzhen, Shanghai, and Ningbo, through the medium of joint ventures or wholly-owned affiliates. The framework suggests that there are four main kinds of accommodation that MNCs seek and achieve between their standard international practices and those of local national partners or staffs. The study provides further empirical evidences that MNC practices cannot be homogeneously applied to all firms. Instead, there can be different and clearly discernable practices which reflect each party's cognitive and behavioural preferences under the different accommodation processes. The study offers new insights into (1) the processes that lead to convergence and divergences of organizational practices, and (2) the need for managers to understand the underlying connection between behavioural and cognitive accommodation in responses to both the contextual and contingency requirements. (For more information, please contact: Leanne Chung, Cardiff University, United Kingdom: chungl1@cf.ac.uk)

\section{Advantages to Creativity in a Multinational Manufacturing Firm - Perspective from Knowledge Diversification and Social Ties \\ Chih-Hsing Sam Liu, Ming Chuan University \\ Bernard Gan, University of New South Wales \\ Sheng-Fang Chou, National Taiwan Normal University}

This study aims to investigate the relationship between the social ties of employees, their access to heterogeneous knowledge and the generation of creativity. We tested our hypotheses in a sample of 350 employees from a large, multinational manufacturing firm. Our findings suggest that, while social ties matter, access to heterogeneous knowledge is of equal importance in the generation of employee creativity, and receiving help and support from colleagues has a positive influence on social ties, heterogeneous knowledge and creativity. (For more information, please contact: Chih-Hsing Sam Liu, Ming Chuan University, Taiwan: phd20110909@gmail.com)

\section{Matrix Veneer: Quantitative Models of Information-Processing by Matrix in Multinational Corporations Jane Qiu, University of New South Wales Lex Donaldson, University of New South Wales}

Why multinational corporations (MNC) use matrix structures instead of elementary structures in spite of their high cost and conflict-prone nature? How much extra advantage do matrix structures confer in comparison to elementary structures? Can these advantages be quantitatively studied? In this paper we seek to provide new thoughts to these questions by considering the amount of advantage conferred by matrix structures used by MNC in information-processing. Based on two different views about the structure of MNC matrix, two quantitative models are developed to predict the amount of extra advantage in information-processing brought by matrix to an MNC. The models are empirically explored in a secondary analysis using data from a study of German MNCs. (For more information, please contact: Jane Qiu, University of New South Wales, Australia: janeq@unsw.edu.au)

Foreign Subsidiary Performance and Home Country Alliance Experience: $A$ Theoretical Investigation Linda Rademaker, Tilburg University

Xavier Martin, Tilburg University 
The growing presence of emerging economy firms in global markets has led scholars to become increasingly interested in understanding how these firms expand internationally. This paper addresses the ways in which home country alliances with foreign multinational enterprises allow local firms to gain access to knowledge that can be used as the foundation on which to build future internationalization. Through the identification of the types of knowledge that can be transferred between multinational enterprises and local firms we develop a theoretical framework for learning from alliances with foreign multinational enterprises. In addition, our study reveals a number of contingencies that affect the extent to which local firms can benefit from alliances with foreign firms in their home country. We advance propositions about the ways in which this learning can increase foreign subsidiary performance and use fieldwork conducted in China to support our theoretical arguments. This study seeks to contribute to our understanding of learning from strategic alliances, the liability of foreignness, and the internationalization of emerging economy firms and as such open up new avenues for research. (For more information, please contact: Linda Rademaker, Tilburg University, Netherlands:

c.h.a.rademaker@tilburguniversity.edu)

\section{Session: 2.2.13 - Interactive}

\section{Track: Track: 8 - Developing Country MNCs}

\section{Location Choices}

Presented On: July 5, $2013-10: 55-12: 10$

Chair: Luis Alfonso Dau, Northeastern University

The Influence of Firm Resources and Internationalization Motivations on the Location Choice of Emerging Market MNEs

Naveen Kumar Jain, University of Akron

Somnath Lahiri, Illinois State University

The purpose of this paper is to develop a research framework that elucidates the role of firm-specific resources and internationalization motivations on the location choice of emerging market firms (EMFs). Location choice made by EMFs constitutes an important yet somewhat neglected topic in international business (IB) research. To address this gap we first review the literature pertaining to firm-specific resources and internationalization motivations as determinants of location choice. This is followed by the development of a research framework grounded in the emerging market context. Our framework illustrates how location choice of EMFs is determined by the interplay of various resources (relationship-based, home experiences-based and country created assetsbased) and internationalization motivations (market-seeking, asset-seeking, and resource-seeking). Through our discussion and research propositions we contribute to the location choice literature in particular and IB scholarship in general. (For more information, please contact: Somnath Lahiri, Illinois State University, USA: slahiri@ilstu.edu)

\section{Location Choices by Multinational Enterprises from Emerging Markets: Legitimizing versus Economizing Logic Ali Taleb, MacEwan University \\ Louis Hebert, HEC Montreal}

This paper investigates the dominant logics underlying foreign location choices by multinational enterprises from emerging markets (EM-MNEs). The economic view of host country selection suggests that MNEs in general tend to focus their activities in nearby countries for efficiency reasons. In contrast, recent studies with institutional view posit that EM-MNEs in particular tend to prevail in developing markets for legitimacy imperatives. We mobilize and contrast economic efficiency and institutional legitimacy rationalities to study the logic underlying location choices by EM-MNEs. We analyze the configurations of the location networks of 203 EM-MNEs and show general empirical support for our theoretical predictions. In particular, EM-MNEs are found to mobilize different logics depending on whether location choices are made within their home regions or elsewhere. 
Specifically, they follow economizing logic only in their home regions. In other regions, they seem to arbitrate legitimizing logic and economizing logic depending on the degree of their international experience. Our findings make important contributions to the current debates on the regional nature of international strategy and on the internationalization process of EM-MNEs. They also have important theoretical and managerial implications. (For more information, please contact: Ali Taleb, MacEwan University, Canada: taleba@macewan.ca)

The Effect of Host Institutional Quality on the Location Choice of Emerging Market Multinationals: Evidence from China

Geng Cui, Lingnan University

Xiaolin Li, Guangdong University of Business Studies

Tsang-Sing Chan, Lingnan University

This study examines the effect of the institutional quality of host country on the location choice of emerging market multinational corporations (EMMNCs). Drawing from the work of Dixit (2012) and given the interplay between institutional experience and transaction cost, we propose a curvilinear relationship between host institutional quality and country location choice, which is moderated by the bilateral relationship in both trade and investment. Based on the data from 342 Chinese firms, the authors find that the relationship between host institutional quality and location choice exhibits an inverted U-shape and that bilateral investment positively moderates the impact of host institutional quality, while bilateral trade does not. These findings furnish meaningful insight into the location choice of EMMNCs and their internationalization behavior. (For more information, please contact: Geng Cui, Lingnan University, Hong Kong, SAR-PRC: gcui@In.edu.hk)

\section{Globalization of Indian Multinationals: Case Study on Cultural Strategies in Developed Countries Roli Nigam, Laval University \\ Zhan Su, Laval University}

The objective of this study is to focus on the cultural adaptation of Indian multinationals in developed North America. An extensive review of the literature shows that although multinationals from emerging countries are climbing up the global ladder rapidly, there is still a serious lack of studies on the subject, especially empirical studies. Our paper fills the gap in the literature through case study of three Indian multinationals and their six subsidiaries present in developed countries. The results indicate that Indian multinationals make use of a mixed strategy to attain successful cultural adaptation. With the use of this strategy they are also able to maintain high level control over the subsidiary on one hand and able to learn from their international experience on the other hand. The paper advances our understanding on the deployment of adaptation strategies by multinationals from emerging countries in general and India in particular. (For more information, please contact: Roli Nigam, Laval University, Canada: rolinigam@gmail.com)

\section{Country Institutions Behind Firms' Offshoring Decisions and Location Choices David H. Weng, City University of Hong Kong}

How do country institutions influence firms' offshore outsourcing decisions and location choices? While prior offshoring research has shown that product- and firm-level factors are crucial determinants, the role of country institutions has not been fully explored. Drawing on the institution-based view, we propose three arguments in this study. First, home country institutions influence firms' offshore outsourcing decisions. In particular, marketsupporting institutions and social trust in the home countries increase firms' tendencies to conduct offshoring. Second, firms' offshoring location choices are affected by the distance of market-supporting institutions and that of social trust between home country and an offshoring location - the greater the distance, the lower the likelihood that a given location will be chosen. Finally, compared with local firms, multinational enterprises (MNEs) are more likely to be influenced by the distance of market-supporting institutions and that of trust in their offshoring location choices. Amassing a sample of 1,519 firms' offshoring decisions and location choices, we find support for these arguments. (For more information, please contact: David H. Weng, City University of Hong Kong, Hong Kong, SAR-PRC: d.weng@cityu.edu.hk) 
Time to Move On? The Impact of Institutions and Economic Crises on Headquarter Mobility

Alfredo Valentino, Luiss Guido Carli University

Phillip Nell, Copenhagen Business School

Jasper Hotho, Copenhagen Business School

Matteo Giuliano Caroli, Luiss Guido Carli University

Building on prior research, we examine theoretically the institutional factors that push top managers to relocate $\mathrm{HQ}$ units overseas. To this end, we first discuss how coercive, isomorphic, and normative pressures may differentially affect HQ relocation decisions. Subsequently, we consider how economic crises influence the effects of the institutional factors in our model. We argue that economic crises have a dual effect on the international configuration of $\mathrm{HQ}$ activities. First, during times of crisis, we expect firms to react stronger to coercive, isomorphic, and normative pressures. Second, we expect economic crises to decrease the overall institutional quality in a given country context, pushing firms to move out. Our theoretical framework contributes to an improved understanding of how institutional factors influence firm behaviour under varying environmental conditions. Furthermore, we contribute to the literature on the role of economic crises in shaping economic organization. (For more information, please contact: Alfredo Valentino, Luiss Guido Carli University, Italy: valentinoa@luiss.it)

\section{Session: 2.2.14 - Interactive}

Track: Track: 4 - Strategy, Alliances, and Competitiveness

\section{Ownership Strategies and Structures}

Presented On: July 5, $2013-10: 55-12: 10$

Chair: Liena Kano, University of Calgary

Shareholder Value Creation in Cross-Border Acquisitions: Does Ownership Structure Matter?

Dynah A Basuil, Asian Institute of Management

Deepak K Datta, University of Texas at Arlington

Drawing on agency theory, corporate governance, and the merger and acquisitions literatures, we develop arguments relating ownership structures to shareholder value creation in the context of cross-border acquisitions. The study is based on a sample of 440 foreign acquisitions undertaken by 385 U.S. acquiring firms between 1991 and 2006. Our findings indicate that greater equity ownership by dedicated institutional investors, outside directors and insiders are all positively associated with shareholder value creation in foreign acquisitions. In addition, our analysis indicates that ownership by transient institutional investors has a negative impact on shareholder value creation. Implications of our study findings from the standpoint of research and practice are discussed. (For more information, please contact: Dynah A Basuil, Asian Institute of Management, Philippines: dbasuil@aim.edu)

\section{Ownership Categories and Internationalization \\ Xavier Castañer, Université de Lausanne \\ Nikolaos Kavadis, Erasmus University}

We advance that different categories of owners will have heterogeneous effects on a firm's internationalization. In a panel of large, French publicly-traded firms, we find that domestic state ownership and ownership by domestic corporations and foreign states, including foreign sovereign funds, promote commercial internationalization. Domestic state ownership also increases international diversification, whereas family ownership and domestic corporate ownership reduce it. We also find that foreign corporate owners prevent riskreduction strategies across the countries where a firm commercializes its goods. We also observe that CEO 
ownership has no effect on three internationalization dimensions we examine. (For more information, please contact: Xavier Castañer, Université de Lausanne, Switzerland: xavier.castaner@unil.ch)

\author{
The International Ownership Strategy of Chinese MNEs: The Role of Economic and Institutional Factors \\ Martin Hemmert, Korea University \\ Youngwoo Lee, Korea University \\ Jongsoo Kim, University of Illinois at Urbana-Champaign
}

We study the determining factors of the ownership strategies of Chinese multinational enterprises (MNEs) by introducing a framework based on transaction cost theory and institutional theory. Analyzing firm-level data on outward foreign direct investment (OFDI) of Chinese MNEs during 2005-2011, we find that Chinese firms are more likely to take a higher equity share when they are operating in a high-tech industry. Contrary to our expectations, Chinese firms take lower equity shares when investing in geographically proximate countries, suggesting they may have different risk perceptions from developed country MNEs. Chinese firms' international ownership decisions are also influenced by their home- and host-country institutional environments. Our results indicate that a high cultural distance between home and host country induces Chinese firms to take lower equity positions in their OFDI. Furthermore, investing firms' state ownership is related to lower equity investments, suggesting that state-owned firms respond to institutional pressures by their risk-averse home country government. Taken together, our results indicate that the ownership strategies of Chinese MNEs are partially deviating from predictions which are based on existing theories of foreign direct investment. (For more information, please contact: Martin Hemmert, Korea University, Korea, South: mhemmert@korea.ac.kr)

\title{
How Dispersed is Ownership of the Crown Jewels of the Multinational? Risk Management and the Internal Ownership of Firm-Specific Advantages
}

Catherine Magelssen, Rutgers University

Drawing on property rights theory to theorize internal FSA ownership, this study examines whether multinational firms (MNCs) choose FSA ownership structures based on the risks faced. Firm-specific advantages (FSAs) play a critical role in the theory of the multinational firm. Firms establish foreign operations to capitalize on the ownership of FSAs. Although FSAs are an "internal public good" which the parent and affiliates may use, the entities within the MNC that own the FSAs are entitled to the profits. The MNC entities that own the FSAs (FSA owners) internally license the FSAs and contract other MNC entities (FSA users) to perform activities on their behalf. While many researchers have studied FSAs at the MNC-level and the external licensing and contracting relationships of the firm, affiliate-level FSA ownership and the internal licensing and contracting relationships have remained unexplored. MNCs vary in the extent to which FSA ownership is centralized into one entity or dispersed across many entities. FSA ownership has implications on the risks borne by the entities, incentives, resource allocation, and power distribution within the firm. Using a unique, confidential dataset, this paper contributes to the literature by examining the determinants of the dispersion of FSA ownership within the MNC. (For more information, please contact: Catherine Magelssen, Rutgers University, USA:

catiem@pegasus.rutgers.edu)

\section{The Effect of Subnational Institutions on the Ownership Strategies and Local Partner Selections of MNCS: Evidence from China Shu Yu, National University of Singapore}

In this paper, we examine the following important issues in international business. How the subnational institutions in emerging economies affect foreign firms' ownership strategies, and further affect the local partner selections for the joint ventures? We are trying to introduce subnational institutions as an important factor influencing the ownership strategies and local partner selections of MNCs. Following the key tenets of institutional theory, we argue that foreign firms will form joint ventures with local firms in the host provinces where the institution is less developed. Specifically, foreign firms that come from the less developed countries 
are more likely to choose joint venture as their ownership strategy when they face less developed institutional environment. We further propose that foreign firms are more likely to choose non-state local firms as their partner when they entering less developed institutional environment. Based on the empirical analysis of MNCs in China, our hypotheses are supported. (For more information, please contact: Shu Yu, National University of Singapore, China: yushu@nus.edu.sg)

\title{
State Ownership, Institutional Effects and Value Creation in Cross-border Mergers \& Acquisitions by Chinese Firms
}

Min Du, University of Nottingham

Agyenim Boateng, University of Nottingham

This paper considers the effects of state ownership and institutional influences on value creation through crossborder mergers and acquisitions by Chinese firms during the period using a sample of 468 firms. The findings indicate that Chinese bidders experience wealth gains ranging from $0.4771 \%-1.5210 \%$ over a 10 -day event window. The cross-sectional analysis indicates that state ownership, formal institutional distance, reforms in the foreign currency approval system exert significant impact on shareholder value. By considering the state ownership and institutions, this study provides evidence that government and institutions play a huge role in value creation of emerging market firm internationalisation through cross-border mergers \& acquisitions (CBM\&A). (For more information, please contact: Agyenim Boateng, University of Nottingham, United Kingdom: agyenim.boateng@nottingham.edu.cn)

\section{Dealing with Uncertainty through the Ownership Entry Strategy: Do Emerging and Advanced Multinational Firms Differ?}

\author{
Stefano Elia, Politecnico di Milano \\ Lucia Piscitello, Politecnico di Milano \\ Filip De Beule, University of Leuven
}

This article examines the acquisition behavior of emerging market firms (EMFs) compared to advanced market firms (AMFs). Specifically, we relate the governance mode (i.e. the degree of commitment) to exogenous and endogenous uncertainty. As a result of endogenous uncertainty due to their liability of origin, EMFs are likely to acquire less control, which is exacerbated by exogenous uncertainty when acquiring targets in high-tech sectors. Furthermore, EMFs experience a higher propensity to control the local partner the higher the institutional distance with the host country, since they enjoy a better institutional environment when they invest in advanced countries and, hence, they are less likely to need a local partner. To test our hypotheses, we develop an econometric analysis applied to foreign acquisitions in Italy between 2001 and 2010 and we study the control of AMFs as compared to EMFs. Our results confirm that EMFs acquire less control than AMFs, especially in high-tech industries, while institutional distance in trade and investment freedom effectively increase the probability to undertake full acquisition for EMFs as opposed to AMFs. (For more information, please contact: Stefano Elia, Politecnico di Milano, Italy: stefano.elia@polimi.it)

\section{Foreign Ownership Structure, Technology Upgrading and Exports: Evidence from Chinese Firms Yundan Gong, Aston University Sourafel Girma, University of Nottingham Holger Görg, University of Kiel and CEPR Sandra Lancheros, Aston University}

We examine the role of foreign ownership structure in stimulating technology and skill upgrading, and exporting in Chinese manufacturing firms that were taken over by foreign owners. The analysis considers the period 2001 to 2007. We use a propensity score reweighted least squares estimation to control for the possible endogeneity of the acquisition decision. Our results indicate that there are strong effects on export activity post-acquisition 
for all types of ownership share. We also find that targets that are taken over with a less than 100 per cent foreign ownership share experience increases in new product development and R\&D upgrading due to the acquisition. We find positive effects on skill upgrading but only amongst fully acquired firms located in export processing zones (EPZs). Overall, our results suggest that joint ventures between foreign owners and Chinese firms can contribute positively to China's "science and technology take-off". (For more information, please contact: Yundan Gong, Aston University, United Kingdom: y.gong1@aston.ac.uk)

\section{Session: 2.2.15 - Interactive \\ Track: Track: 12 - Economic Geography and Value Chains}

\section{Spatial Considerations}

Presented On: July 5, $2013-10: 55-12: 10$

Chair: Hao Tan, University of Newcastle

\section{A Study on the Effect of Trade and Investment Liberalization on Spatial Concentration of Economic Activity Yener Kandogan, University of Michigan-Flint}

I examine the heterogeneous responses of countries to trade and investment liberalization while taking into consideration some external factors such as country size, level of economic development, and political structure and stability. I argue that liberalization increases the concentration but high population, large geographic size, and high income levels can disperse economic activity. Politically stable federal countries are also expected to have dispersed economic activity. Using data from 168 countries for the period of time starting in 1980s, and I found support for all hypotheses except liberalization having a more concentrating effect on developing countries than developed countries. (For more information, please contact: Yener Kandogan, University of Michigan-Flint, USA: yener@umflint.edu)

\section{New Insights to Offshore Outsourcing: A Typology to Enhance the Understanding of Value Creation Debmalya Mukherjee, University of Akron \\ Steven Ash, University of Akron}

The phenomenon of offshore outsourcing and its value creation potential has attracted a lot attention in the recent years. However, scant attention has been given to the value creation aspect of this strategic tool. In this paper we develop and extend a typology of modes of value creation by considering two relevant strategic dimensions driving this phenomenon--relationship and knowledge motives of offshore outsourcing. While doing so we discuss how transactional, strategic and transformational forms of value are created when we combine the aforementioned dimensions. The paper ends with a discussion on theoretical as well as practical ramifications of our conceptual model. (For more information, please contact: Debmalya Mukherjee, University of Akron, USA: dmukher@uakron.edu)

\section{Global Sourcing Flexibility: Promoting Process Standardization, Location Capacity and Mobility of Resources}

Peter D. Oerberg Jensen, Copenhagen Business School

Bent Petersen, Copenhagen Business School

Recent studies show that flexibility is a key concern for firms that engage in the global sourcing of services. In this conceptual paper, we seek to explore two central aspects of global sourcing flexibility: In the first part of the paper, we provide a definition of the construct of global sourcing flexibility. Here we draw on prior research in the fields of organizational flexibility, international business and global sourcing as well as case examples and secondary studies. In the second part of the paper, we discuss the implications of global sourcing flexibility for 
firm strategy and operations against the backdrop of the theory-based definition of the construct. We discuss in particular the importance of global sourcing flexibility for operational performance stability, and the trade-off between specialization benefits, emerging from location and service provider specialization, versus the higher costs (but decreased risk for value chain disruption) embedded in a more flexible global sourcing model that allows the firm to replicate and/or relocate activities across multiple locations. We develop a model and propositions on facilitating and constraining conditions of global sourcing flexibility. We finally discuss implications for management and international business research, within and beyond the domain of global services sourcing. (For more information, please contact: Peter D. Oerberg Jensen, Copenhagen Business School, Denmark: poe.smg@cbs.dk)

\section{International Sourcing - A Competitive Perspective \\ Bjoern R. Schmeisser, University of Augsburg \\ Wolfgang M. Gleich, University of Augsburg}

Literature on global value chain strategy suggests that international sourcing of inputs offers promising avenues for firms to benefit from multinationality. We introduce a domestic firm's international sourcing strategy as an effective response to increased competition. We hypothesize that foreign and domestic competition increase a domestic firm's magnitude of international sourcing. Moreover we argue that such competitive forces drive the modification of a domestic firm's sourcing pattern, i.e. the composition of sourced activities from high and lowcost destinations. We differentiate foreign competition into high and low-cost competition and find different effects on a domestic firm's sourcing magnitude and pattern. We test our hypotheses on a set of 771 German service firms. (For more information, please contact: Bjoern R. Schmeisser, University of Augsburg, Germany: bjoern.schmeisser@wiwi.uni-augsburg.de)

\section{Distance, Trust and the Role of Intermediaries in Global Value Chains Alessandro Perri, University of South Carolina}

I set forth a model of trade intermediation in global value chains in which the intermediaries acts as institutional arbitrageurs, reducing cultural and institutional distance between international buyers and local suppliers, and profiting from the resulting reduction in transaction costs. I propose that intermediaries reduce distance in the global value chain by means of distinct effective mechanism of trust development in their dyadic relations with international buyers and local suppliers. (For more information, please contact: Alessandro Perri, University of South Carolina, USA: alessandro.perri@grad.moore.sc.edu)

\section{Session: 2.3.1 - Special Session}

\section{AIB Fellows Special Panel: Celebrating 25 years of JF Hennart's 'A Transaction Costs Theory of Equity Joint Ventures'}

Presented On: July 5, $2013-13: 20-14: 35$

Chair: Paul W. Beamish, University of Western Ontario

Keynote Speech: "The Transaction Costs Theory of International Joint Ventures - 25 Years Later"

Jean-Francois Hennart, Singapore Management University and University of Pavia

Commentary: "The Past and Present Significance of JF Hennart's JV Model for Managerial Practice"

Tamer Cavusgil, Georgia State University 
Commentary: "Alternative Perspectives on Joint Ventures in International Business"

Farok J. Contractor, Rutgers University

Commentary: "Reevaluating the Use of Transaction Costs Analysis for International Joint Ventures in Emerging Economies"

Klaus E. Meyer, China Europe International Business School

(For more information, please contact: Alain Verbeke, University of Calgary, Canada:

alain.verbeke@haskayne.ucalgary.ca)

\section{Session: 2.3.2 - Panel}

\section{Integrating International Business Curriculum Delivery}

Presented On: July 5, $2013-13: 20-14: 35$

Chair: Srdan Zdravkovic, Bryant University

Panelists:

Srdan Zdravkovic, Bryant University

Madan Annavarjula, Bryant University

Andres Ramirez, Bryant University

The panel will discuss best practices related to delivery of integrated International Business curriculum at Bryant University, USA. International Business program at Bryant University is internationally recognized and panelists will share curriculum delivery in which four classes (International Accounting, International Finance, International Management, and International Marketing) are integrated into one cohesive program. Integration is done by using consulting activities, integrated simulation, globally integrated class project, integrated cases, speaker series, and other. (For more information, please contact: Srdan Zdravkovic, Bryant University, USA: szdravko@bryant.edu)

\section{Session: 2.3.3 - Panel}

\section{Alternative Research Methods for Understanding Management in Under Researched Locations}

Presented On: July 5, $2013-13: 20-14: 35$

Chair: Betty Jane Punnett, University of the West Indies

Discussant: Donald Wood, Eureka Mgmt. Consultants

Panelists:

Betty Jane Punnett, University of the West Indies

Terri Lituchy, University of the West Indies

Bella Galperin, University of Tampa

Margaret Phillips, Pepperdine University

Andrea Scott, Pepperdine University

Joyce Osland, San Jose State University 
This panel addresses the need to find alternative research methods that can enable AIB scholars to begin to understand business and management issues in under-researched countries. The panel participants argue that our knowledge of business and management is currently biased towards western concepts and practices, because academics have focused on the triad countries of Western Europe, Japan, and North America. The world of business is increasingly encompassing the wider world, as developing countries and emerging market become more involved in international trade and investment. AIB researchers need to be part of this changing world and include under-researched locations in their research. The panel presents alternative research methods that can make the investigation of under-researched locations more effective. The panel will discuss under-researched locations as well as specific research methods. It will also draw on the wider IB community to present a larger array of methods. A discussant will consider the presentations from a practical management perspective, and lead an interactive discussion with audience participants. (For more information, please contact: Betty Jane Punnett, University of the West Indies, Barbados: eureka@caribsurf.com)

\section{Session: 2.3.4 - Panel}

\section{Track: Track: 8 - Developing Country MNCs}

\section{Markets for Executives and Non-Executives in Eastern Europe}

Presented On: July 5, $2013-13: 20-14: 35$

Chair: Peder Greve, University of St. Gallen

Panelists:

Winfried Ruigrok, University of St. Gallen

Liviu Voinea, Academy of Economic Studies Bucharest

Gjergji Filipi, AGENDA Institute

Dumitru Slonovschi, Magenta Consulting

Lucia Casap, Magenta Consulting

This roundtable panel reports from a pioneering and recently completed research project investigating the markets for top managers and board members at the largest companies in three Eastern European developing economies. The project team leaders, who will all join this panel, will outline the key findings of the research project, explain how conceptual and methodological challenges were overcome in the research process, and discuss the vast and mutually beneficial research opportunities available through extensive investments in research collaborations between partners from developed and developing economies. The objective of this panel session is essentially twofold. On the one hand, the panel participants will discuss the research insights that have been gained from the project, i.e. an in-depth understanding of the markets for top business leaders and their services in the target countries, including several original findings that are likely to be of interest to a broad audience including IB scholars, practitioners, and policymakers. On the other hand, the panel session should motivate AIB members to engage more actively in collaborations between researchers from developed and developing countries, building on the experiences that have been made in this project, while also learning to avoid some of the potential challenges and pitfalls. (For more information, please contact: Peder Greve, University of St. Gallen, Switzerland: peder-mathias.greve@unisg.ch) 
Session: 2.3.5 - Competitive

Track: Track: 7 - Emerging Economies

\section{Capability Building in Emerging Markets}

Presented On: July 5, $2013-13: 20-14: 35$

Chair: Bruce McKern, China Europe International Business School

The Effects of OFDI on Home Employment and Skill Upgrading: The Roles of Technology Intensity and Location Wen Chung Hsu, National Chi Nan University

This research examines empirically the relationship between outward foreign direct investment (OFDI) and home employment. It is marked out from existing studies in the following respects. First, instead of advanced economies, it focuses on the home country employment effect in a newly industrialized economy. Second, it not only addresses the general issue of whether employment or production abroad complements or substitutes for employment in parent companies but also examines how OFDI contributes to skill upgrading in the home country. Third, this paper allows the results to vary between labour-intensive industries and technologyintensive industries. Finally, we also explore how the home country employment effect, if any, varies between different locations of investment. The results demonstrate that the location of investment and industry characteristics matter for explaining the effect of OFDI on home employment. Specifically, we find that while OFDI by Taiwanese multinationals in China depresses employment in Taiwan in both labour- and technologyintensive industries, OFDI in other countries only produces a negative employment effect in technologyintensive sectors. An interesting finding of this study is the evidence of positive effects of OFDI on skill upgrading in Taiwan. This paper supports the argument that relocation of productivity abroad axes low skilled workers at home. Moreover, OFDI in China has a particular positive impact on skill upgrading in the home industry, lending support to the view that MNEs outsource labour-intensive goods from their affiliates in lowincome countries. (For more information, please contact: Wen Chung Hsu, National Chi Nan University, Taiwan: wchsu25@gmail.com)

\section{How IJVs Build Differentiation Capability in China? To Be Technology-Oriented or Customer-Oriented? Alex Xin Chen, University of Hong Kong Kevin Zheng Zhou, University of Hong Kong Xiaoyun Chen, University of Macau}

Should international joint ventures (IJVs) be technology-oriented or customer-oriented when attempting to achieve differentiation advantage in emerging economies such as China? To answer this question, we examine the roles of technology and customer orientations in building differentiation capability in emerging market-based IJVs and compare their relative effects. Findings from a survey of 156 IJVs in China indicate that technology orientation has a stronger positive effect on IJV differentiation capability than customer orientation and both effects depend on cultural distance and three key components of foreign parent control. Technology orientation leads to stronger differentiation capability when foreign ownership is higher and the level of foreign parent's operational control is higher, whereas customer orientation is more beneficial when the level of foreign parent's operational control is lower and communication with headquarter is more frequently and effective. (For more information, please contact: Alex Xin Chen, University of Hong Kong, Hong Kong, SAR-PRC: alexchen@hku.hk)

\section{Comparing Exporters in Advanced and Emerging Markets: A Resource Based Approach}

Maria Chiarvesio, University of Udine

Guido Bortoluzzi, University of Trieste

Eleonora Di Maria, University of Padova

Raffaella Tabacco, University of Udine 
The aim of this paper is to contribute to the international business literature focusing on the characteristics of firms exporting in emerging markets. Having adopted a resource based theoretical perspective, our results show that companies addressing emerging markets have greater international experience, managerial and marketing capabilities than those operating only in developed markets. A firm's size, its experience in the business and industry are not determining factors in those processes. (For more information, please contact: Maria Chiarvesio, University of Udine, Italy: chiarvesio@uniud.it)

\section{Imitation to Innovation: Technological Catch-up Strategy of Firms from Emerging Economies \\ Sungyong Chang, Columbia University \\ Hyunseob Kim, Ohio State University \\ Jaeyong Song, Seoul National University}

To catch up with firms in developed economies, striking a fine balance between imitation and innovation is critical for firms from emerging economies. However, few researches have investigated the firms from emerging economies' optimal resource allocation between innovation and imitation in the catch-up process. Drawing on evolutionary economics, we built a computer simulation model on technological catch-up strategy of firms from emerging economies. The model suggests that one-sided dependency upon either imitation or innovation deters firms from emerging economies' technological catch-up in the long run. In addition, the model shows that, in the initial stages of catch-up, firms from emerging economies should focus on imitation and begin to build technological capabilities and absorptive capacity. Then, in the later stages, when the technological gap has narrowed, they should increase its investment in innovation and attempt technological leapfrogging. We also test this model under a range of technological regime variables including appropriability, cumulativeness, and technological opportunity. Our model shows that although technological regimes influence the probability of catch-up, our original finding is resilient across a wide range of variance in technological regime variables. (For more information, please contact: Jaeyong Song, Seoul National University, Korea, South: jsong@snu.ac.kr)

\section{Session: 2.3.6 - Competitive}

\section{Track: Track: 3 - IB Theory, FDI, and Entry Mode}

\section{Interstate Relations and FDI}

Presented On: July 5, $2013-13: 20-14: 35$

Chair: Peter Wayne Liesch, University of Queensland

Role of Country Alliances in Reducing Transaction Costs in Internationalisation of Indian Multinational Enterprise Firms

Peter J Buckley, University of Leeds

Peter Enderwick, Auckland Technical university

Nicolas Forsans, University of Leeds

Surender Munjal, University of Leeds

This paper presents a country-level study of the role of home-host country alliances in reducing transaction costs in internationalisation of the Multinational Enterprises (MNEs). Country alliances inhere are social, economic, and political alliances between home and host countries; and, transaction costs are external transaction costs that emerge outside the firm in the host country in which firms aims to internationalise, not the internal transaction costs arising within the firm. We test this framework and its hypotheses about the role of country-level alliances and firm internationalisation with comprehensive longitudinal multi-industry data on 315 Indian firm's acquisitions between 2000 and 2007 on a panel of 82 host countries. Results show that homehost country's alliances reduce the transaction costs which positively influence the internationalisation of Indian MNEs. However, the extent to which such internationalisation of Indian firms takes place depends upon the nature of the country-level alliances. (For more information, please contact: Surender Munjal, University of Leeds, United Kingdom: smu@lubs.leeds.ac.uk) 


\section{Interstate Political Relations and Multinational Investment \\ Tatiana Lukoianova, University of Western Ontario \\ Quan Li, Texas A\&M University}

Understanding how political risk influences multinational enterprises' (MNEs) behaviors has long attracted scholarly attention in three fields: international business (IB), international economics, and international political economy (IPE). Scholars in the three fields have almost exclusively focused on country-specific political risks, ignoring the importance of bilateral political relation-specific risks to MNE investment. The few exceptional studies that do analyze such risk produce conflicting theoretical expectations, diffuse explanations, and mixed evidence. Extending the "new" new trade theory, we offer a coherent theoretical framework that identifies precisely the conditions under which bilateral risks do and do not influence MNE investment. Bilateral risks change MNE behaviors only if they change the FDI productivity cutoff above which MNEs operate profitably in a host. Lower bilateral risks lower the FDI productivity cutoff and consequently, increase the amount of bilateral FDI. Firm- and dyad-level tests substantiate our argument. Our research makes several contributions and offers practical lessons for governments and business managers. (For more information, please contact: Tatiana Lukoianova, University of Western Ontario, Canada: vshchlk@gmail.com)

\section{The Bilateral Relation and Foreign Subsidiary Survival \\ Tianyou Hu, National University of Singapore}

This study examined the relationship between bilateral relation between the home and host countries, and the survival of the foreign subsidiaries. Integrating the institutional theory and legitimacy perspectives, this study established the theoretical linkage of the fluctuation of bilateral relation and the failure of FDI, followed by an empirical study that examined the extent to which bilateral relation could affect subsidiary survival and how foreign firms could mitigate such risks. The survival analysis of 5248 Japanese subsidiaries in China during 1991-2010 showed that the exit likelihood of the subsidiaries was positively associated with the deterioration of Sino-Japan bilateral relation. Such a relation hazard could be alleviated through accumulating local experience, locating in regions with advanced market development and implementing localization strategies. (For more information, please contact: Tianyou Hu, National University of Singapore, Singapore: ht@nus.edu.sg)

\section{Spatial Dependence of Country Relatedness: The Role of Host-Country Connectedness Sokol Celo, Suffolk University}

The conventional determinants of FDI location decisions are limited to the characteristics of the host country or the home-host dyad. Borrowing from research in spatial dependence and social network analysis, we argue that the decision of home country firms to invest in a host country depends also on the ability of the host country to serve as a stepping-stone to invest in third countries. Consequently, on average the indirect route connecting a home with a host country via an intermediate country will be more effective than the direct one. By examining the action of surviving firms' international investments, we are able to capture the home-host country relatedness for international investments and, building on it, the host-country connectedness, and examine their relationship. Our empirical tests provide evidence for the positive effect of host-country connectedness on home-host country relatedness and also support our prediction that the indirect route might be more effective. Finally we propose a way to measure the betweenness centrality for the countries and provide several illustrations. (For more information, please contact: Sokol Celo, Suffolk University, USA: scelo@suffolk.edu) 


\section{Session: 2.3.7 - Special Session}

\section{Global Value Chains, the Trade-Investment Nexus and Development (UNCTAD WIR Panel)}

Presented On: July 5, $2013-13: 20-14: 35$

Chair: James Zhan, Division on Investment and Enterprise, UNCTAD

Discusssants: Ram Mudambi, Temple University and Peter J. Buckley, University of Leeds

Panelists:

Hafiz Mirza, Division on Investment and Enterprise, UNCTAD

Axele Giroud, UNCTAD

Christos Pitelis, Cambridge University

Lorraine Eden, Texas A\&M University

This session focuses on the burning issue of global value chains (GVCs) and how host countries can most effectively utilize them for building productive capacity and economic and social upgrading. With the rise of GVCs, a trade-investment nexus permeates and defines today's global economy. The vast preponderance of global trade is ultimately governed by transnational corporations, which orchestrate and coordinate GVCs through FDI, non-equity modes (such as services outsourcing, contract farming and contract manufacturing) and cross-border transactions with independent firms. There are both opportunities and risks associated with GVCs for host countries which need to be addressed, especially by developing countries. At the same time, developing countries are highly diverse, so a differentiated approach towards GVCs is needed by policymakers, reflective of the interests of each country. (For more information, please contact: Hafiz Mirza, Division on Investment and Enterprise, UNCTAD, Switzerland: Hafiz.Mirza@unctad.org)

\section{Session: 2.3.8 - Competitive \\ Track: Track: 11 - SMEs and Entrepreneurship \\ The Expansion of International New Ventures: The Role of Dynamic and Complementary Capabilities}

Presented On: July 5, $2013-13: 20-14: 35$

Chair: Peter Li, Copenhagen Business School

The Interplay between Dynamic Capabilities and Entrepreneurial Processes and their Impact on the SME Exporters' Performance Improvement during Recession

Dario Miocevic, University of Split

From marketing logic context, this article investigates the interplay between dynamic capabilities and entrepreneurial processes and their overall impact on exporting firm's performance improvement during the recent economic downturn (2008-2011). The baseline model inspects the role of entrepreneurial cognition (opportunity recognition), and behavior (opportunity exploitation) on performance improvement and is extended by analyzing the indirect effects through the mediating role of dynamic capabilities (DCs) and the moderating effects of exporting resources. In line with the main research problem, the theoretical framework along with a set of research hypotheses has been developed. The study was carried out on the sample of 118 SMEs in Croatia that have continuous export activity. With the survey data and objective indicators (e.g., performance indicators, export diversity, export intensity, level of diversification, etc.), the findings show that DCs mediate the relationship among the entrepreneurial processes and performance improvement. Also, the findings from the post-hoc moderated-mediation analysis suggest that export resources are significant moderator in the 
mediating relationship between opportunity exploitation, adaptive capabilities, and performance improvement. In the end, this study offers valuable theoretical and practical implications within the constituent fields of international business. (For more information, please contact: Dario Miocevic, University of Split, Croatia: dmiocevi@efst.hr)

\section{Competing on Service Advantage based on Service Capabilities: An Empirical Study of the Antecedents of Performance in International New Ventures}

Silvia Martin, Lynchburg College

Rajshekhar (Raj) G. Javalgi, Cleveland State University

In the context of high-technology international new ventures (INVs) the literature on has failed to consider informational resources and its impact on service capabilities within the strategy interplay in approaching new markets overseas. Building upon the resource based view of the firm (RBV) this study examines empirically the relationships among informational resources on service capabilities and service advantage. The study sampling frame consisted on 260 INV firms from Mexico. Structural equation modeling was used to test measurement veracity and proposed relationships between the constructs constrained in the measurement model. Findings show that informational resources play a central role in the process of attaining superior performance when developing a service advantage. These results indicate that the rapid environment that INVs find themselves produces a need to develop new philosophies and strategies from which to attain service advantage. Informational resources help INVs to focus on a service advantage based on marketing differentiation and delivery differentiation to achieve superior performance. The study findings have important implications for research on new venture decision-making and international marketing. (For more information, please contact: Silvia Martin, Lynchburg College, USA: sy/vie.Izn@gmail.com)

\section{Dynamic Capabilities for Internationalization: from Market Entry to Sustainable Internationalization Margaret Fletcher, University of Glasgow \\ Simon Harris, University of Edinburgh Volker Mahnke, University of Edinburgh}

Internationalization process research historically has focused on market entry, without considering the longer term consequences for firms. This paper investigates the dynamic capabilities firms may need for sustainable internationalization. We draw on Teece's (2007) framework which distinguishes sensing, seizing and reconfiguring dynamic capabilities, and from dynamic capabilities that have been implicit in internationalization process and international entrepreneurship research to identify the dynamic capabilities for internationalization. We study the development of these in a single longitudinal case study of a firm as it developed from being an international new venture to its evolution as a successful international enterprise. We find sensing and seizing capabilities for market entry and for network relationships are most important at early and growth stages of internationalization and reconfiguration capabilities are imperative for sustainability and profitability. Teece's framework is a useful perspective for extending internationalization process research to consider sustainable internationalization. Managerial relevant research would show managers the dynamic capabilities they need as they internationalize their firms, and help them to develop sustainably and avoid costly mistakes. (For more information, please contact: Margaret Fletcher, University of Glasgow, United Kingdom:

margaret.fletcher@glasgow.ac.uk)

The Speed of Internationalization and the Capabilities Development: From a Collection of Individual-level Capabilities to a Bundle of Firm-level Organizational Routines Masahiro Kotosaka, Ritsumeikan University

This research investigates the process in which smaller, younger internationalizing firms explore and exploit the required knowledge and capabilities. This research adopted an exploratory, case-based theorising approach, 
and conducted a longitudinal empirical case study of four firms, each of which took a different internationalization path. The analysis focuses on how the four firms developed the firm-level organizational routines for exploring and exploiting external knowledge and capabilities, by investigating and comparing the level of routines development at the time of initial internationalization and these two years after this. As a result, this research finds that the level of the development of the firm-level organizational routines for internationalization is low, regardless of the timing and pace of internationalization, at the time of internationalization. Further, by analyzing the complete list of knowledge sources and knowledge types, this research argues the advantages of grafting for developing a wider variety of firm-level organizational routines to accelerate the pace of internationalization. Moreover, the distinction between ordinal and change routines has enabled this study to investigate the exploitation aspect of capabilities, and discusses the value of analyzing the higher-level routines that foster the exploitation, which distinguish rapidly internationalizing firms from slowly internationalizing firms. (For more information, please contact: Masahiro Kotosaka, Ritsumeikan University, Japan: kotosaka@gmail.com)

\title{
Session: 2.3.9 - Competitive
}

\section{Track: Track: 9 - Cross-Cultural Management and HRM}

\section{Expatriate Adjustment}

Presented On: July 5, $2013-13: 20-14: 35$

Chair: Benjamin Bader, University of Hamburg

\section{Expatriate Skills for Cross-Cultural Competence \\ Dan Wang, Monash University \\ Taiwen Feng, Northwestern Polytechnical University \\ Susan Freeman, University of Adelaide \\ Di Fan, Victoria University \\ Cherrie Jiuhua Zhu, Monash University}

International management literature identifies several important skill sets that are important for expatriates' cross-cultural management. However, the direct and indirect effects of these skills on cross-cultural competence (CCC) and the interrelationships of skill clusters are not well examined. Drawing on social learning theory, we test the relationships among important skill clusters, and the mechanisms through which they contribute to CCC of expatriate managers based on a survey of Chinese expatriate managers in their overseas assignments. Using structure equation modeling, we demonstrate that self-maintenance skills and communication skills are significantly and directly related to CCC, while perceptual skills and interpersonal skills relate to CCC indirectly. Principally, perceptual skills contribute to CCC via interpersonal skills and communication skills, while interpersonal skills are conducive to CCC through communication skills. (For more information, please contact: Dan Wang, Monash University, Australia: stella8497@gmail.com)

\author{
Expatriate Work and Family Role Adjustment: Scale Development and Validation \\ Margaret Shaffer, University of Wisconsin-Milwaukee \\ Sebastian Reiche, IESE \\ Mihaela Dimitrova, University of Wisconsin-Milwaukee \\ Mila Lazarova, Simon Fraser University \\ Shoshi Chen, Tel Aviv University \\ Mina Westman, Tel Aviv University
}

Expatriate adjustment is viewed as one of the most important determinants of successful global assignments. While there are several conceptualizations and instruments created to capture adjustment to the foreign 
environment, the majority of past research has taken a stress perspective. In contrast, it has not examined how expatriates adjust to their new and redefined work and family roles. Using role theory we develop a scale of work and family role adjustment and validate it through two interlocking studies. We find general support for the psychometric properties of the scale and for its positioning within the nomological network of demands faced by expatriates and resources available to them. The development of the work and family role adjustment scale has important implications for both expatriate research and practice. (For more information, please contact: Mihaela Dimitrova, University of Wisconsin-Milwaukee, USA: mihaela@uwm.edu)

Seniority as an Asset? The Moderating Effect of Age on the Relation between Emotional Intelligence and CrossCultural Adjustment of Expatriates

Heidi Marie Wechtler, Macquarie University

Alexei Koveshnikov, Hanken School of Economics

Cecile Dejoux, CNAM Paris

This paper focuses on the moderating effects of age on the relationship between emotional intelligence (EI) and cross-cultural adjustment (CCA) of expatriates. Grounded in lifespan development theory and more particularly socioemotional selectivity theory, our research shed additional light on one of this under-researched areas in expatriation research. Our hypotheses are tested through hierarchical models using data from 254 expatriate managers. Our findings show that age is a facilitator of regulation and utilization of emotions on general living adjustment and of regulation of emotions on interactional adjustment. Complementary analyses show that previous expatriates' experience is not a substitute of age: the moderating effect of experience on the relationship between EI and CCA appears to be less prominent than that of age. (For more information, please contact: Heidi Marie Wechtler, Macquarie University, Australia: heidi_wechtler@yahoo.fr)

\author{
Multidimensionality and Subjectivity of Culture Shock: A Contingent Approach of Cross-Cultural Dynamic \\ Processes \\ Heidi Marie Wechtler, Macquarie University \\ Olivier Wurtz, Vaasa University
}

This study examines the trajectory of cross-cultural adjustment based on the multidimensional and subjective aspects of culture shock. We focus on six intrapersonal diversity factors that affect international adjustment in order to build a taxonomy of expatriates. On the basis of a sample of 584 observations, we show that these different groups of expatriates follow different patterns of adjustment. In some specific cases a U-curve is observed. (For more information, please contact: Heidi Marie Wechtler, Macquarie University, Australia: heidi_wechtler@yahoo.fr)

\title{
Session: 2.3.10 - Competitive Track: Track: 5 - MNC Management and Organization
}

\section{Structuring Multinationals and Multinationality}

Presented On: July 5, $2013-13: 20-14: 35$

Chair: Birgitte Grogaard, University of Calgary

Conformity or Differentiation in Multinationality? The Case of the Italian Ceramic tile Manufacturers Claudio Giachetti, Ca' Foscari University of Venice Ettore Spadafora, University of South Carolina 
Many studies in international management have addressed the issue of whether and how multinationality influences firms' economic performance. However, no general consensus about the multinationalityperformance relationship has been reached by scholars In order to address the multinationality-performance relationship, we introduce the construct of strategic deviation in multinationality, which expresses the extent to which a firm's multinationality differs from the multinationality of its peers at a particular point in time, and test a set of hypotheses concerning its effect on firm performance. We find that a higher profitability results from either low or high levels of strategic deviation in multinationality. By contrast, lower profitability is associated with moderate levels of strategic deviation in multinationality. Hypotheses are tested with data on 57 Italian ceramic tile manufacturers over the 2005-2009 time period. (For more information, please contact: Claudio Giachetti, Ca' Foscari University of Venice, Italy: claudio.giachetti@unive.it)

\section{How Should MNCS Control the Breath and Depth of their International Diversification?}

Gongming Qian, Chinese University of Hong Kong

Lee Li, York University

This study explores how the breadth and depth of international diversification interact with each other to affect the performance of multinational enterprises. Evidence collected in this study suggests that the interaction effect is positive and significant when the level of both breadth and depth is moderate. When either dimension increases further, the interaction effect is still positive and grows even more significant. However, the positive and significant effect reverses and becomes negative (although non-significant) when a high level of both dimensions is reached. These relationships indicate that the adoption of international diversification strategy should take into consideration breadth and depth simultaneously as they affect each other mutually in determining firm performance. Findings of this study provide important guidelines for managers to manage the breadth and the depth of their international business in their efforts to maximize profits. (For more information, please contact: Gongming Qian, Chinese University of Hong Kong, Hong Kong, SAR-PRC: qian@baf.msmail.cuhk.edu.hk)

\section{An Evaluation of Matrix Structures in Today's MNCS William G. Egelhoff, Fordham University Joachim Wolf, University of Kiel}

As international strategies become more complex, there is a growing interest on the part of MNC managers in matrix structures. Recent exploratory research on matrix structures in 15 German MNCs reveals that a new form of matrix, the rule-based matrix, appears to be emerging. The paper contrasts the information-processing capabilities of the new rule-based matrix against those of the classical balanced matrix. They are different and appear to address different situations. The paper suggests that MNCs may be able to employ both types of matrix structure on a contingency basis. (For more information, please contact: William G. Egelhoff, Fordham University, USA: egelhoff@fordham.edu)

\section{Multinational Structure and Legal Systems \\ Elizabeth Rose, Aalto University and University of Otago \\ Kiyohiko Ito, University of Hawaii at Manoa \\ Amanda E.K. Budde-Sung, University of Sydney \\ Tanya Peacock, University of Hawaii at Manoa}

While past research has emphasized control issues between headquarters and subsidiaries, we consider a different aspect of firm structure and foreign direct investment, building on law and finance theory, the resource-based view of the firm, and models derived from the governance mode literature. Our findings suggest that U.S. multinational corporations (MNCs) tend to have fewer foreign subsidiaries in countries whose legal systems are not based on common law and that a MNC's total number of foreign subsidiaries are positively 
related to the size of the parent, but negatively related to employee productivity and experience. Country-level subsidiary numbers of MNCs are related to host country attributes that include political risk, corporate tax rates, market size, and geographic and cultural distances. This research contributes to the corporate governance literature and extends the eclectic paradigm by incorporating a consideration of MNCs' legal structures and hostcountry legal environments into the explanation of foreign direct investment. (For more information, please contact: Amanda E.K. Budde-Sung, University of Sydney, Australia: amanda.budde-sung@sydney.edu.au)

\section{Session: 2.3.11 - Interactive \\ Track: Track: 1 - Institutions, Governance, and CSR}

\section{Corporate Social Responsibility}

Presented On: July 5, $2013-13: 20-14: 35$

Chair: Susan Elizabeth Feinberg, Temple University

\section{CSR Strategy within the FSA/CSA Matrix: an Institutional and Resource-based View Arilova A Randrianasolo, Saint Louis University}

This research takes on the perspective that corporate social responsibility (CSR) is an organizational process that internationalizing firms partake in to concurrently boost firm-specific advantages (FSA) as well as decrease the liability of foreignness. In this research, MNE CSR activity is viewed as part of the firm's internationalization strategy. A model of how FSA and country-specific advantages (CSA) affect CSR activity is conceptualized. Furthermore, CSR activity is examined within Rugman's $(1981,2007)$ FSA/CSA matrix in order to fully analyze how CSR is embedded within internationalization strategy. The relationships proposed leads to theoretical propositions made within the paper. Methodology for future research is proposed in order to empirically test the proposed relationships. A 2-study analysis is proposed. First, a hierarchical multiple regression analysis is proposed to test the relationships proposed within the conceptual model. Second, an analysis of covariance (ANCOVA) is proposed to test the relationships within the FSA/CSA matrix. Contributions and implications are discussed. (For more information, please contact: Arilova A Randrianasolo, Saint Louis University, USA: randriaa@slu.edu)

\section{Lagging Behind? The CSR Character of Nestle Ulf Henning Richter, Nottingham University}

In this in-depth case study, I analyze and discuss the CSR character of Nestlé, applying the theoretical framework developed by Basu and Palazzo (2008). I conducted thirteen in-depth, semi-structured interviews in two rounds with senior managers from Nestlé which were analyzed using qualitative (multiple coding) and quantitative (ANOVA, ?2 analysis, correspondence analysis) techniques. The findings indicate that Nestlé's CSR approach can be characterized as that of a "laggard" which is only slowly closing the gap to other world-leading companies facing similar serious environmental and social challenges. The study shows that understanding the cognitive processes underlying the CSR decision making process is of fundamental value of analyzing and changing the CSR approach of a firm. (For more information, please contact: Ulf Henning Richter, Nottingham University, China: ulf.richter@nottingham.edu.cn)

Corporate Social Responsibility, Smoking Bans and Market Seeking FDI in the Tobacco Industry.

Chris Jones, Aston University

Nigel Driffield, Aston University

Jo Crotty, University of Salford Manchester 
This paper seeks to examine the relationship between smoking bans and the propensity of tobacco firms to engage in FDI. Using a firm level database of all tobacco firms, we examine the FDI decision in this sector through the combined lenses of CSR and institution theory to show that smoking bans at home are an important institutional intervention, as they appear to reduce the propensity of FDI in developing countries. Our analysis also suggests that a higher proportion of the global tobacco industry will be dominated by firms from countries without a smoking ban. (For more information, please contact: Chris Jones, Aston University, United Kingdom: c.jones2@aston.ac.uk)

\section{Understanding the Words of Relationships: Language as an Essential Tool to Manage CSR Risk in Communities of Place}

W. Travis Selmier, II, Indiana University

Aloysius Newenham-Kahindi, University of Saskatchewan

Chang Hoon Oh, Simon Fraser University

Multinational enterprises (MNEs) encounter relentless stakeholder pressures operating across linguistic and cultural boundaries, particularly where communities of place at the base of the pyramid are involved. Using (micro)sociolinguistics - the study of how language influences society and business relations - we explore how language empowers some MNEs to successfully manage stakeholder relationships while other MNEs falter. Surveying corporate social responsibility strategies of 15 MNEs from Australia, Canada, China, South Africa, and the U.K. operating across four Swahili-speaking East African countries, our research examines how a regional lingua franca, like Swahili, may be employed to build sustainable forms of MNE-stakeholder interactions. (For more information, please contact: W. Travis Selmier, II, Indiana University, USA: wselmier@indiana.edu)

\section{The Drivers of Global Corporate Social Responsibility: The Community-Event-Firm Triangle Luis Ballesteros, University of Pennsylvania}

I contribute to the study of corporate social responsibility (CSR) at the international level with a theoretical model comprising three systems: the firm, the beneficiary community, and the event. I test the theoretical value of my approach by using a panel of 2012 multinational corporations and analyzing data of donations to the relief fund of natural catastrophes (corporate philanthropic catastrophe response or CPCR) that affected different countries in the last 10 years. I show that the institutional and socioeconomic development of the beneficiary community, in addition to measures of cross-national distance, exert a nontrivial influence in the variance of donation. Additionally, I demonstrate that the salience of the event for the global community is positively associated with the likelihood of a firm engaging in catastrophe response. Understanding the factors that affect CPCR behavior not only has relevant theoretical implications for the study of global CSR. Information of the firm-community linkages and the political and institutional arrangements that foster and help predict CPCR may have important public-policy implications given the increasing role that the corporate community is assuming in financing catastrophe relief and recovery around the world. (For more information, please contact: Luis Ballesteros, University of Pennsy/vania, USA: Iuisf@wharton.upenn.edu)

\section{Corporate Social Responsibility: Stakeholders Influence on MNEs'Activities Byung Il Park, Hankuk University of Foreign Studies Pervez N. Ghauri, King's College London}

This research attempts to examine how specific stakeholders groups influence multinational enterprises' corporate social responsibility practices in South Korea. Generally speaking, the results show that both primary and secondary stakeholders positively influence multinational company's corporate social responsibility. Contrary to previous research, this work also demonstrates that business collaborators have a negative and significant effect on multinational companies' corporate social responsibility. Based on the findings this paper wishes to offer a framework for multinational enterprises to thoroughly consider the impact of stakeholders when drawing 
a picture for their CSR strategy. Further, this work also hopes to contribute to current discussions in the area of corporate social responsibility by bringing a new stream of research into the international business field. In addition, this work wishes to provide useful and practical implications for multinational enterprises wanting to operate in the South Korea market. (For more information, please contact: Byung Il Park, Hankuk University of Foreign Studies, Korea, South: leedspark@hufs.ac.kr)

\section{Session: 2.3.12 - Interactive}

\section{Track: Track: 6 - Innovation and Knowledge Mgmt.}

\section{Innovation Management}

Presented On: July 5, $2013-13: 20-14: 35$

Chair: Patricia McDougall-Covin, Indiana University

How Private Equity Firms Make Cross-Border Investments: Learning to Internationalize

Yang Fan, Erasmus University

Hans Bruining, Erasmus University

This paper examines the main effects of domestic and international investment experience of private equity firms on the likelihood of doing a standalone cross-border investment. We distinguish between experiential learning and inter-organizational learning from previous domestic and international investments respectively in stand-alone investments and in syndicated investments. Hypotheses are developed for the likelihood of making stand-alone cross-border investment. Additionally, the moderating effects of industry specialization and prior experience with syndication were assessed on the chance of making standalone cross border investments. The hand-collected dataset comprises 15.522 deal observations from 2237 private equity investors located in 66 countries. Analyses indicate domestic experience has positive effects on standalone cross-border investments, but international experience has negative effects. Furthermore, a strong focus on service industry will promote the use of syndication, and reduce the likelihood of standalone cross-border investments. (For more information, please contact: Yang Fan, Erasmus University, Netherlands: yfan@rsm.nl)

\section{Birds of a Feather Flock Together - Technological Integration of Foreign Subsidiaries of the Multinational Corporation}

Katarina Blomkvist, Uppsala University

Philip Kappen, Uppsala University

This paper explores subsidiary technology integration within multinational corporations (MNCs). Specifically, it empirically investigates the degree of technological integration of competence-creating subsidiaries with headquarters and sister subsidiaries, while also incorporating the effects of inter-subsidiary differentiation. The complete U.S. patenting activity by 23 Swedish multinationals from 1893-2008 reveals significant differences in technological integration of greenfield and acquired competence-creating subsidiaries. In accordance to expectations, the results indicate that greenfield subsidiaries seem more integrated with headquarters and other greenfield subsidiaries than with acquired subsidiaries. The acquired subsidiaries, on the other hand, do not appear to be as easily integrated into the MNC, with evidence showing very little technological integration. Suggesting that the distinct pattern of subsidiary technological integration has been overlooked, the findings offer insights into the role of competence-creating subsidiaries as providers of technological capabilities to headquarters and other subsidiaries of the MNC. (For more information, please contact: Philip Kappen, Uppsala University, Sweden: philip.kappen@fek.uu.se) 
Technological Capability Building in MNE-Related Social Businesses of Less Developed Countries: The Experience of Grameen-Danone Foods Limited

Jahan Ara Peerally, HEC Montréal

Paulo N. Figueiredo, Brazilian School of Public and Business Administration (EBAPE)

Although there has been considerable research on firm-level technological capability building in the context of developing economies, there is a scarcity of studies which examine this issue in multinational enterprises' socially motivated businesses located in less developed economies. This paper examines the latter issue on the basis of first-hand empirical evidence derived from an extensive field research on Grameen-Danone Foods Limited (GDFL) in Bangladesh. The study found that GDFL generated relevant spillovers to the host economy by accumulating production capabilities in association with innovation capabilities at intermediate levels across four technological functions: project management, process and production organization, product centred and equipment-related. Apart from revealing the types of frugal and reverse innovations which emanates from such a business, our study also explores - unlike existing studies which only focus on the financial and social benefits - the technological benefits generated from a social business model. Understanding the nature and dynamics of technological activities in social businesses of less developed economies is relevant for the achievement of enhanced local, autonomous and sustainable economic and social development. (For more information, please contact: Jahan Ara Peerally, HEC Montréal, Canada: jahan.peerally@hec.ca)

\section{From Concept to Market: The Role of Knowledge-sharing in Facilitating Global Product Innovation Karina R. Jensen, Reims Management School}

A dynamic and evolving global marketplace demands an agile and adaptable organization that can respond to new market opportunities around the world. Organizations need to leverage cross-cultural collaboration in order to facilitate knowledge-sharing that produces successful market solutions. How can organizations facilitate local team collaboration when planning and executing new products for international markets? A qualitative study is used to examine this question through interviews with 60 senior managers responsible for global products and cross-cultural teams in 32 multinational enterprises with headquarters based in Europe, Asia, and the US. By examining project collaboration interactions that influence knowledge-sharing between the project leader and the geographically distributed team, this study contributes to the understanding of knowledge-sharing challenges and motivations in planning and executing new products for international markets. Furthermore, the findings uncover research needs concerning knowledge flow between $\mathrm{HQ}$ and subsidiary teams that have an impact on the MNEs international market performance. (For more information, please contact: Karina R. Jensen, Reims Management School, France: karina.jensen@reims-ms.fr)

\section{Does Tacit Knowledge Matter? The Impacts of Modularity and the Social Governance Mechanism on Technological Capability and Performance \\ Yong Kyu Lew, University of Manchester \\ Rudolf R. Sinkovics, University of Manchester}

This paper investigates the impacts of the governance mechanism (i.e. product modularity and social governance) on technological capability and firm and relationship performance. Drawing on modularity and social governance theories, we broadly view a form of international technology venture between heterogeneous firms as a modular and social structure. We empirically test the inter-relationships between the constructs, analyzing data from 110 software and hardware firms that have formed international technology ventures. The results indicate the positive effects of both product modularity and social governance on technological capability. The two relationships are significantly moderated by the venture partners' level of knowledge tacitness. In the end, technological capability is positively associated with both firm and relationship performance. Product modularity prompts a firm's internationalization and technological capability through technology ventures, and in turn affects firm and relationship performance. This study shows that product modularity can be a strategic choice for a firm that complements social governance, particularly when tacit knowledge transactions are involved in international technology ventures. (For more information, please contact: Yong Kyu Lew, University of Manchester, United Kingdom: yongkyu.lew@mbs.ac.uk) 
Session: 2.3.13 - Interactive

Track: Track: 6 - Innovation and Knowledge Mgmt.

\section{Knowledge and Innovation}

Presented On: July 5, $2013-13: 20-14: 35$

Chair: Kent Eriksson, Royal Institute of Technology

Knowledge Governance Mechanisms and Repatriate's Knowledge Sharing: The Mediating Roles of Motivation and Opportunity

Ming-Chang Huang, Providence Univesity

Yaping Chiu, Chung Yuan Christian University

Hsiang-Lin Cheng, National Chung-Cheng University

Ting-Chun Lu, National Chengchi University

This paper uses theoretical and empirical approaches to explore the relationships among knowledge governance mechanisms (KGMs), knowledge sharing motivations and opportunities, and knowledge sharing behavior by repatriates at multinational corporations (MNCs). The empirical evidence from 140 repatriates at 66 MNCs indicated that both formal and informal KGMs facilitated knowledge sharing behavior through motivation and opportunity building. Knowledge sharing motivations and opportunities simultaneously mediated the relationship between knowledge governance mechanisms and knowledge sharing behavior. (For more information, please contact: Ming-Chang Huang, Providence Univesity, Taiwan: mchuang@pu.edu.tw)

The Dynamics of the Parent-subsidiary Relationship in Building Exploitative and Explorative Capabilities in Foreign Subsidiaries

Jae-Eun Lee, Sunchon National University

Lian Zhang, Yonsei University

Soonkyoo Choe, Yonsei University

Building on the theories of organizational learning, parent-subsidiary relationship, and intrafirm knowledge transfer in multinational enterprises, this study investigated how the parent firm's administrative activities for integrating overseas operations into its corporate system and transfering its firm-specific advantages to overseas operations influence the exploitative and explorative capabilities in foreign subsidiaries. The results of our analysis suggest that MNEs could relatively easily develop a foreign subsidiary's exploitative capabilities based on the parent firm's supportive activities and socialization practices. But, we found evidence that it would be a difficult challenge to build explorative capabilities in foreign subsidiaries, since those mechanisms for enhancing exploitative capabilities and parent control may have unexpected negative and disrupting effects on them under certain conditions. (For more information, please contact: Soonkyoo Choe, Yonsei University, Korea, South: skychoe@yonsei.ac.kr)

\section{Knowledge Transfer in a Strategic Alliance Network - The Case of Caixa of Brazil \\ Iuri Castro, Caixa Economica Federal - Brazil \\ Josep Rialp, Autonomous University of Barcelona \\ Cristina Stoian, Brunel University}

This paper investigates the knowledge transfer process between the companies involved in a network of strategic alliances, specifically how the flow occurs from the parent company to its network of small and medium-sized enterprise (SME) correspondents and vice versa. Drawing on relevant literature on strategic alliances, knowledge management and the Resource-based View, a qualitative research pre-test was carried out. Based on this initial research, the present study argues that knowledge characteristics, management 
commitment and transfer intention play a key role in the knowledge transfer process in a network of strategic alliances. Our statistical results generally support our arguments. This paper brings insights to the knowledge transfer process by analysing this process not only from the parent company towards the SME network and vice-versa, but also by considering the network configuration as a whole. (For more information, please contact: Cristina Stoian, Brunel University, United Kingdom: cristina.stoian@brunel.ac.uk)

\section{How Dynamic Is Technological Change? An Empirical Study on Dynamic Capabilities \\ Shihmin Lo, National Chi Nan University \\ Hsiao-Chen Mei, National Chi Nan University \\ Peter J. Sher, National Chi Nan University}

This study investigates the impact of technological change on a firm's development of capabilities in the context of mobile telecommunications. We propose a new measure of technological change in terms of stability of technology scope - the diversity and ranking of patent classes of the industry over time. In a total of 21 firms and their 286,953 patents related in the field of mobile telecommunications, emprical results show that different sources of dynamic capabilities such as in-house R\&D, inter-firm collaboration or technology transfer contribute to differently concentrate or diversify technological portfolio in addressing to technological change. (For more information, please contact: Shihmin Lo, National Chi Nan University, Taiwan: smlo@ncnu.edu.tw)

\section{Internationalizing Emerging Market Organizations \\ Miriam Moeller, University of Queensland \\ Jane Maley, Jane Maley Consulting \\ Michael Harvey, University of Mississippi \\ Timothy Kiessling, Bond University}

As the internationalization process dictates the existence of cross-country networks it is essential to explore, from a marketing perspective, avenues that allow EMMNCs to share and receive knowledge that can benefit emerging market multinationals locally and globally. This manuscript addresses the significance of creating a global marketing mindset while articulating mechanisms that influence global marketing managers' abilities to engage effectively with other global marketing managers across borders. It is argued that global marketing managers engage in reciprocal learning processes to obtain new knowledge about how to develop new products, overcome branding challenges and so on. The manuscript suggests that the effectiveness of new marketing knowledge creation is dependent upon appropriate input, throughput, and output competencies. A proper feedback loop in which global marketing managers may elect to revise current standards or practices of global marketing is of equal importance. Propositions foreshadowing cultural and personal characteristics in the new marketing knowledge creation process are articulated. (For more information, please contact: Miriam Moeller, University of Queensland, Australia: m.moeller@uq.edu.au)

\section{How to Ensure Knowledge Sharing of Autonomous Subsidiaries - The Moderating Role of Global Mandates Barbara Brenner, Danube University Bjoern Ambos, University St. Gallen}

This study focuses on subsidiary autonomy and knowledge transfer within multinational corporations (MNEs). Drawing on resource dependency theory and the literature on global mandates we develop and empirically test a set of hypotheses aiming at exploring a) how autonomous subsidiaries can accumulate critical knowledge b) what mechanisms the headquarters can employ to ensure efficient knowledge flows within the group. Data collected from 193 Europe-based subsidiaries of American, European and Asian MNEs serve to test these hypotheses. Our results show that the relation between subsidiary autonomy and knowledge flows is mediated by knowledge criticality and that global mandates are positively associated with knowledge flows. (For more information, please contact: Barbara Brenner, Danube University, Austria: barbara.brenner@donau-uni.ac.at) 


\title{
Session: 2.3.14 - Interactive
}

Track: Track: 2 - Marketing and Supply Chain

\section{Mind the Gap-buyer Seller, Channels, and Technology}

\author{
Presented On: July 5, $2013-13: 20-14: 35$
}

Chair: Daniel Bello, Georgia State University

How Firm-Specific Marketing and Technology Capabilities Matter in Geographic Expansion of Emerging Market Firms?

Jie Wu, University of Macau

Nitin Pangarkar, National University of Singapore

This study links regional diversification to global diversification and examines the moderating role of firmspecific technological and marketing capabilities in this relationship. Drawing on the organizational learning theory and the resources/capabilities view, this study develops and tests hypotheses on a sample of 687 manufacturing firms across multiple industries in China. Our analysis reveals that: regional diversification has a positive impact on global diversification, both technological capability and marketing capability positively moderate the effect of regional diversification on global diversification, and, interestingly, this interactive effect is stronger for marketing capability than for technological capability. (For more information, please contact: Jie Wu, University of Macau, Macau: jiewu@umac.mo)

\section{Network Positions and Internationalization: A Panel Data Analysis of the Distribution Channels in Semiconductor Industry}

Joseph Shyh-Yeu Tzou, National Taiwan University

Y.L. Jaw, National Taiwan University

This study argues that firm's network positions play important roles in its internationalization, and intends to provide a network explanation for the purported relationship between firm's network positions and its internationalization in the context of semiconductor industry. This study adopts the quantitative methodology to explore the research questions. Drawing on thirty years of observations for each of the five years from 1980 to 2010 for distributor firms in the semiconductor industry, this study utilizes panel data regression to examine the effects of a firm's network positions on its internationalization. The sample of this study is the 113 franchised semiconductor distributor firms in Taiwan of the WSTS's (World Semiconductor Trade Statics) 63 member companies. WSTS's member companies represent more than $75 \%$ of the world semiconductor market. Worldwide top 20 semiconductor suppliers are WSTS members. This virtually includes all major semiconductor manufacturers from 4 major regions, namely America, EMEA(Europe, Middle East, and Africa), Japan and Asia Pacific. Results of this study support the positive relationship between firm's centrality of its network positions and its internationalization, the positive relationship between firm's structural holes of its network positions and its internationalization, and the positive relationship between firm's brokerage of its network positions and its internationalization. The moderator effects of firm's joining association and going initial public offering (IPO) in the structural holes-internationalization relationship are supported by this study, while the moderator effects of firm's joining association and going IPO in the centrality-internationalization relationship are not supported by this study. (For more information, please contact: Joseph Shyh-Yeu Tzou, National Taiwan University, Taiwan: joesytzou@gmail.com) 


\section{The Effects of the Salesperson Characteristics on Buyer-Seller Relationships \\ Yonghoon Choi, Doshisha University \\ Ying Huang, University of Massachusetts Lowell \\ Brenda Sternquist, Michigan State University}

This study examines the influence of the salesperson characteristics (organizational commitment and disposition to innovate) on buyer behaviors in buyer-supplier relationships. A model is proposed depicting the effects of the salesperson's organizational commitment and disposition to innovate on buyer long-term orientation and opportunism through partner-specific value to the buyer. Data were collected from 155 sales professionals of Japanese manufacturers. Structural equation modeling was used to analyze the data. As predicted, the salesperson's organizational commitment and disposition to innovate enhance buyer long-term orientation through providing partner-specific value to the buyer, and in turn, buyer long-term orientation mitigates opportunism. The salesperson plays an important role for developing and maintaining buyer-seller relationships. Based on our results, firms should promote salespeople's organizational commitment because a highly committed salesperson is likely to be more innovative when managing the relationship with the buyer, and in turn, increase the relationship specific value to the buyer. (For more information, please contact: Ying Huang, University of Massachusetts Lowell, USA: ying_huang1@uml.edu)

The Role Asset Specificity in Trust Buling Process: An Emperical Study of Strategic Alliances between MNE Subsidiaries and Local Suppliers in Thai Manufacturing Sector

Rapeeporn Rungsithong, University of Bath

Anthony Roath, University of Bath

This study investigates an under-researched topic: the role of asset specificity in inter-personal trust and interorganizational trust building process in strategic alliances. We combine the concept of relationship asset specificity and the social exchange perspective of intensity of the alliance partner relationships that transform key resources into the level of trust. Employing a sample of 156 alliance projects between MNE subsidiaries and local suppliers in Thai manufacturing sector, our investigation demonstrates intensity of inter-personal relationship and inter-organizational facilitate the level of trust in strategic alliances while asset specificity has negative impact on the level of inter-personal trust between alliance partners. However, our research shows that asset specificity enhances trust through the mediating effect of intensity of inter-personal and interorganizational relationships. Moreover, our research supports the link transference from inter-personal trust to inter-organizational trust. The implications of these findings for theoretical development and future research are discussed. (For more information, please contact: Rapeeporn Rungsithong, University of Bath, United Kingdom: rr257@bath.ac.uk)

The Influence of Non-Verbal Communication on B2B Negotiations: Some Preliminary Evidence from France and Germany

Laetitia Dreyer, ESC Rennes Business School

Mohammad Niamat Elahee, Quinnipiac University

The role of non-verbal communication in business negotiations is well documented. Based on empirical data collected from people engaged in face-to-face negotiations in business-to-business contexts in France and Germany, this paper examines how the importance of non-verbal behaviour is perceived by the French and German negotiators and how different aspects of non-verbal communication affects their negotiation style. The paper also examines the impact of gender and age on the use of non-verbal communication, and consequently, how they affect business-to-business negotiations. The paper also discusses the managerial implications of the findings and makes appropriate recommendations for further studies. (For more information, please contact: Mohammad Niamat Elahee, Quinnipiac University, USA: mohammad.elahee@quinnipiac.edu) 
Global Account Management: A Boundary Spanning Capabilities Approach

Linda Hui Shi, University of Victoria

Anthony Goerzen, Queen's University

Global Account Management (GAM), defined as the centralized selling activities to serve key global account customers, is an emerging research area in International Marketing. Given the importance of global key accounts, GAM research lies at heart of Customer Relationship Management (CRM). A major concern of global account management studies relates to the capabilities the global account managers need and how these capabilities can be cultivated and moderated in different cultural contexts. According to previous scholars, the capabilities possessed by managers can be examined in terms of the cognitive and affective systems at both the organizational and the individual level. Thus, this study will address the aforementioned key concern by applying boundary spanning capability framework and incorporating cognitive and affective systems at both levels as antecedents. (For more information, please contact: Linda Hui Shi, University of Victoria, Canada: Ishi@uvic.ca)

\section{Session: 2.3.15 - Interactive}

Track: Track: 4 - Strategy, Alliances, and Competitiveness

\section{Managing Alliance Partners and Portfolios}

Presented On: July 5, $2013-13: 20-14: 35$

Chair: Ali Taleb, MacEwan University

Unpacking Cross-border Knowledge Transfer and Learning Paradoxes: Contextual Differences Matter Mia Hsiao-Wen Ho, Soochow University

This research unpacks the paradoxes in knowledge transfer and learning processes in international strategic alliances by highlighting the contextual differences between partner firms. We distinguish country- from firmlevel differences and argue that although institutional distance facilitates knowledge transfer because it lessens the need of knowledge protection, it negatively influences the alliance performance because it restricts the firm's realized absorptive capacity to apply the acquired knowledge into the alliance context. To overcome such inherent, perplexing challenges resulting from contextual differences, our findings suggest that relational capital accumulated by partner firms during collaborations positively moderates the effects of institutional distance on cross-border knowledge transfer and learning processes. Theoretical and managerial implications are provided. (For more information, please contact: Mia Hsiao-Wen Ho, Soochow University, Taiwan: mia.hohw@gmail.com)

\section{Partner Selection in International Technological Alliances: The Role of Institutional Differences, Historical Ties and Size of the Focal Firm \\ Sorin M.S. Krammer, Groningen University}

This study posits that institutional distance has a negative influence on partner selection in international technological alliances. Empirical results based on a dataset of firms in the global tire industry confirm that firms prefer technological partners from closer cognitive, normative and regulatory environments. Moreover, the analysis indicates that formal (economic treaties) and informal (colonial past) historical ties, and focal firm size moderate positively this relationship through different institutional channels. (For more information, please contact: Sorin M.S. Krammer, Groningen University, Netherlands: m.s.s.krammer@rug.nI) 


\section{Modeling Strategic Partnership in International and Domestics Relations \\ Justyna Swiatowiec-Szczepanska, Poznan University of Economics}

The paper analyses research trends in the development of international relations based on strategic partnership. The literature review stresses the need to consider today's perceived partnership risk, including relational and performance risk. This aspect is included in a developed definition of partnership, where the key problem is relational governance. The paper presents a strategic partnership model including partnership risk and mechanisms to control it. Hypotheses are put forward and tested by means of a structural equation modelling (SEM) procedure. The study uses a sample of 215 large and medium-sized companies (with over 50 employees) operating in Poland. The research sample includes 98 companies making up international strategic partnerships and 117 enterprises in strategic relations with domestic partners. The aim of the research is to identify a universal strategic partnership model which could be applied in the global economy. The analysis demonstrates a great significance of behavioural aspects of cooperation - including relational norms and goodwill - in the reduction of partnership risk. Formal mechanisms of cooperation control play a considerably lesser role. (For more information, please contact: Justyna Swiatowiec-Szczepanska, Poznan University of Economics, Poland: justyna.swiatowiec-szczepanska@ue.poznan.pl)

\section{Learning Strategies as an Interfirm Ambidexterity in Reconciling Interorganizational Relational Outcomes Tension \\ Pei-Li Yu, National Cheng Kung University}

Achieving high interfirm adaptation and low ex-post opportunism enable superior interfirm relational outcomes, but raise challenging tensions. Combining both types of activities and achieving organizational ambidexterity, however, leads to the presence of multiple and often conflicting goals, and poses considerable challenges to develop the effective learning strategies in ambidextrous organizations. This study explores the role of learning strategies in reconciling conflicting interests in interfirms and achieving organizational ambidexterity. While there seems to be little doubt about the positive impact of learning strategies on interorganizational releationships (IORs), the author posits that the direct effect is mitigated by a variable specific to the interfirm context, namely cultural differences. This study finds partial empirical support for the developed theory in the context of high technology industry. The results show positive interaction effects between the exploitation of learning strategies and a lower cultural difference on a higher interfirm adaptation of interfirm ambidexterity. (For more information, please contact: Pei-Li Yu, National Cheng Kung University, Taiwan: h4584933@ms24.hinet.net)

The Effects of Asset Specificity in International Partnerships and in Domestic Partnerships: Comparisons and Research Propositions

Yanhui Zhao, Michigan State University

Thomas P. Murtha, Michigan State University

Stefanie Lenway, Michigan State University

Drawing on transaction cost economics (TCE) framework, we attempted to compare the effects of asset specificity in international partnerships with that in domestic partnerships. It was proposed in this study that the association between asset specificity and transaction hazards was stronger in international partnerships than in domestic partnerships. Nevertheless, our research did not discourage firms from dedicating specific investments to international partnerships, instead it was suggested that asset specificity in international partnerships generated higher positive outcomes than that in domestic partnerships - the positive impacts of asset specificity on collaborative performance and radical innovations were argued to be higher in international partnerships than in domestic partnerships. This study is an explorative study providing testable propositions for future research. (For more information, please contact: Yanhui Zhao, Michigan State University, USA:

zhaoyanh@msu.edu) 


\section{Alliance Portfolio Diversity Configuration and Multinational Firms' Continuing Foreign Expansion-Examination from a Real Options Perspective}

Wei He, Purdue University North Central

Jinlin Zhao, Florida International University

In this conceptual paper we propose a conceptual model to explain the determinants of multinational firms' strategic choices of continuing foreign expansion. This conceptual work advances the extant literature on the process of internationalization by bridging two lines of research-firms' external collaboration and firms' internationalization through a real options approach. Built from a real options perspective, the conceptual framework in this study identify some key exploratory factors across firm-, industry-, country- levels on multinational firms' propensities of continuing foreign expansion. In particular, we investigates the twodirectional continuing internationalization, which takes into account both the depth and breadth dimension of foreign expansion comparing to prior studies that mainly focuses on one-directional subsidiary growth or newcountry entry. This study especially highlights the strategic roles of existing alliance portfolio configuration in influencing a firm's strategic decision-making. By examining how firms' propensities of continuing foreign expansion are influenced by existing alliance portfolios' diversity configuration, this study extends the current international business and strategic management research by bridging the research gap between determinants of initial entry mode and driving forces of post-entry expansion in the foreign counties. (For more information, please contact: Wei He, Purdue University North Central, USA: weihe@pnc.edu)

\section{Match Making, Structural Holes, and Arranged Marriages: The Process of Small Multinational Enterprises International Alliance Formation Clifford Wymbs, Baruch College-CUNY}

I analyzed conceptual and practical issues related to the changing role third parties play in bringing together alliance partners for small businesses from two distant countries. I found that to share in the gains of globalizing economy, smaller multinational enterprises find surrogates outside their firm, third party enablers, to help bridge informational structural holes and build multi-nodal social networks that link cross-cultural alliances

partners. Propositions are developed that explain similarities and differences between large and small firms, the role third parties play with small firms, and then how these third-party behaviours co-evolve with the alliance partners. A qualitative case study of a successful international strategic alliance is used to develop propositions and additional cases in different industries are used to triangulate these findings. (For more information, please contact: Clifford Wymbs, Baruch College-CUNY, USA: clifford.wymbs@baruch.cuny.edu)

\section{Strategies for Making Global-Local Alliances Work in Emerging Markets Private Equity}

Markus D. Taussig, National University of Singapore

Pavel Zhelyazkov, Harvard Business School

We study the factors affecting whether US venture capital and private equity firms select partners with host country origins when they engage in syndicated deals outside of the US. Our sample covers the years 2005 to 2011 and covers deals in 46 host countries. We find evidence that host country origins increase the likelihood of co-investing with a diversifying US firm, especially if the host country contracting institutions are weak. We find evidence of that direct ties, overlap in geographic experience, and overlap in industry experience on the likelihood all have stronger positive influences on partner selection decisions involving a host country firm. US firms with host country experience are also more likely to partner with a host country firm. We discuss the implications for international alliance strategy. (For more information, please contact: Markus D. Taussig, National University of Singapore, Singapore: markus@nus.edu.sg) 
Session: 2.4.1 - Special Session

\section{AIB Fellows Special Panel: Celebrating 25 Years of Kogut and Singh's 'The Effect of National Culture on the Choice of Entry Mode'}

Presented On: July 5, $2013-14: 50-16: 05$

Chair: Eleanor Westney, York University

Keynote Speech: "The Effect of National Culture on the Choice of Entry Mode - 25 Years Later" Bruce Kogut, Columbia University

Commentary: "Cultural Distance and Institutional Distance in IB Studies: Complements or Substitutes? " Tatiana Kostova, University of South Carolina

Commentary: "What Matters in IB is 'Added Distance': A New Approach to Assess the Effects of Cultural Distance on International Expansion"

Thomas Hutzschenreuter, WHU Otto Beisheim School of Management

Commentary: "A Practitioner's Perspective on Cultural Distance and IB"

Sevkinaz Alemdar, Procurement Coordinator, Erdemir Group

\section{Session: 2.4.2 - Panel}

\section{Track: Track: 5 - MNC Management and Organization}

\section{The Multifaceted Role of Language in International Business: A Critical Challenge for MNC Theory}

Presented On: July 5, 2013 - 14:50-16:05

Chair: Mary Yoko Brannen, University of Victoria

Panelists:

Rebecca Piekkari, Aalto University

Susanne Tietze, Sheffield Hallam University

Terry Mughan, University of Victoria

Oded Shenkar, Ohio State University

Despite the dominance of English as a lingua franca, IB encounters have not become monolingual. On the contrary, given the growing importance of BRIC countries, languages such as Chinese, Russian, and Spanish are gaining prominence. Still the IB literature to date fails to take adequate account of the influence of language and its impact on realizing international business goals. This panel aims to discuss the role of language in the field of IB and how the discipline may be rendered more language-sensitive. It responds to the call of the conference to link IB more strongly with complementary disciplines. Our panellists and co-editors of a JIBS special issue on this topic will address the following questions: What is the role of language in IB research? How does a language-sensitive perspective that draws on interdisciplinary insights challenge established theories about the MNC? How can language-related decisions be made more transparent when studying IB phenomena in the field? What role should language play in future IB research? The session will be run according to a roundtable format. (For more information, please contact: Rebecca Piekkari, Aalto University, Finland: rebecca.piekkari@aalto.fi) 


\section{Session: 2.4.3 - Panel \\ Track: Track: 12 - Economic Geography and Value Chains \\ Knowledge Diffusion and Innovation at the Intersection of Global Value Chains and Local Clusters: Perspectives from Emerging Markets}

Presented On: July 5, $2013-14: 50-16: 05$

Chair: Gerald Andrew McDermott, University of South Carolina

Discussant: David Audretsch, Indiana University

Panelists:

Gerald Andrew McDermott, University of South Carolina

David Audretsch, Indiana University

Elisa Giuliani, University of Pisa

Eric Thun, Oxford University

This panel examines the ways in which firms learn and innovate, with particular focus on discerning the relative value of MNC subsidiaries, local firm clusters, and local non-market organizations in facilitating knowledge creation and diffusion. With an emphasis on emerging markets, the papers analyze the conditions under which foreign subsidiaries and local firms forge different patterns of inter-organizational networks and create distinct support institutions, which in turn shape different paths firm level product and process upgrading. The papers integrate concepts and methods from different disciplines to create novel approaches to understanding the evolution of GVCs and clusters in settings of weak institutions and resources. (For more information, please contact: Gerald Andrew McDermott, University of South Carolina, USA: gerald.mcdermott@moore.sc.edu)

\section{Session: 2.4.4 - Competitive \\ Track: Track: 5 - MNC Management and Organization \\ Understanding Subsidiary Development and Strategy}

Presented On: July 5, $2013-14: 50-16: 05$

Chair: Dan Li, Indiana University

Can Subsidiary Executives Influence Initiative Outcomes in Their Subsidiaries? Interaction Effect of Their Entrepreneurial Cognition and Contextual Perception

Hsiang-Lin Cheng, National Chung-Cheng University

Ming-Chang Huang, Providence Univesity

Wen-Ting Lin, National Chung-Cheng University

This study uses a cognitive approach to clarify the roles of subsidiary executives' mindsets in leading initiatives in the subsidiaries they manage. Empirical evidence was obtained for 185 subsidiaries of Taiwanese MNEs operating in China. Data were triangulated by connecting two data sources: a secondary dataset and a survey conducted firsthand. First, the results show a positive impact of subsidiary executives' individual entrepreneurial cognitions, with which these executives can identify several emerging opportunities, on firm-level subsidiary initiative outcomes. Although the perception of normative signals from local personal networks and that of commitment signals from the dependence on their headquarters positively moderate the positive impact of entrepreneurial cognitions on subsidiary initiatives, regulatory signals from the host country's legal restrictions negatively moderate this impact. Second, subsidiary executives' perception of attachment signals from the shared identities to the headquarters has a direct positive impact on subsidiary initiative outcomes. Third, the 
perception of credibility signals from intra-firm trust has a direct but negative impact on subsidiary initiative outcomes. (For more information, please contact: Hsiang-Lin Cheng, National Chung-Cheng University, Taiwan: cheng5689@gmail.com)

\author{
Anti-Competitive Regulations and Subsidiary Relational Embeddedness Strategies \\ Grazia Santangelo, University of Catania \\ Ulf Andersson, Copenhagen Business School
}

The institution-based view (IBV) of firm strategy argues that formal and informal institutions combine to govern firm behavior and suggests a predominant reliance on network-based informal relationships when formal institutions are absent or deficient. Our knowledge on how formal and informal institutions combine to govern firm behavior remains scant when formal institutions are present but show varying degrees of non-marketsupporting regulation. This issue gains great relevance for subsidiaries which in host locations have also to confront the liability of outsidership. To advance IBV, we draw on industrial organization and firm productivity literature. In particular, we propose and test a conceptual model illustrating a U-shaped relationship between the extent of anti-competitive regulation in relevant service supplying sectors and the degree to which subsidiaries embed with value chain partners. Our model also suggests that this U-shaped relationship is steeper for subsidiaries with a center of excellence role within the multinational enterprise, and that highly embedded subsidiaries are superior performers. (For more information, please contact: Grazia Santangelo, University of Catania, Italy: grsanta@unict.it)

\title{
Knowledge Sharing and Subsidiary R\&D Mandate Development: A Matter of Dual Embeddedness Fariza Achcaoucaou, University of Barcelona Paloma Miravitlles, University of Barcelona Fidel Leon-Darder, University of Valencia
}

Sharing knowledge across borders has proven to be especially relevant to multinational corporations (MNCs). Foreign subsidiaries have become active players in these knowledge flows. However, the network effects of interacting with multiple agents on the evolution of the R\&D role played by subsidiaries are still undeveloped. The present study focuses on changes in subsidiary capabilities and on the dynamic mechanisms by which their R\&D role might evolve, especially, as a consequence of their interaction with a variety of knowledge networks. We examine this issue by conducting four longitudinal case studies of subsidiaries operating in Spain. Using an inductive approach to theory building, we develop a general theoretical framework considering the subsidiary's embeddedness in the knowledge networks within the MNC (internal) and within the host country (external). We find that evolving towards a competence-creating mandate is characterised by the simultaneous growth of embeddedness in both internal and external networks; otherwise, a subsidiary may gravitate away from upgrading its $R \& D$ role. Thus, the contribution of this paper is to present a dynamic model that sheds light on how internal and external knowledge embeddedness interact in generating outcomes for subsidiary R\&D roles. (For more information, please contact: Fariza Achcaoucaou, University of Barcelona, Spain: farizaa@ub.edu)

\section{Managing Resource Flows within MNCS \\ Heather Berry, George Washington University}

Despite long-standing arguments about the benefits multinational corporations (MNCs) can achieve from sharing and transferring their resources across borders, there has been much less examination of how MNCs actually manage, control and coordinate resource flows across their worldwide operations. In this paper, I examine how MNCs use their home country expatriate managers to control and coordinate resource flows from their foreign subsidiaries and posit that the role of expatriates will be contingent on the knowledge autonomy of the foreign subsidiary. Using a comprehensive panel of US MNCs based on confidential data from the Bureau of Economic Analysis (BEA), I use propensity score matching methods to isolate the impact of home country 
expatriate managers on subsidiary resource flows and show that expatriates significantly increase resource flows to third country operations in subsidiaries that are dependent on parent firm knowledge while expatriates significantly increase resource flows to parent firm operations in subsidiaries that generate their own knowledge assets. Overall, this paper extends both the knowledge based view and expatriate literatures by showing how MNCs use their expatriates to extend and coordinate firm resources originating from both parent and foreign subsidiary operations. (For more information, please contact: Heather Berry, George Washington University, USA: berryh@gwu.edu)

\section{Session: 2.4.5 - Competitive Track: Track: 7 - Emerging Economies}

\section{Understanding Firm Performance in Emerging Markets}

Presented On: July 5, $2013-14: 50-16: 05$

Chair: William Newburry, Florida International University

Firm, Industry and Country Effects in Emerging and Developed Economies: Paradigm Shifts in the PostRecession Era

Vassiliki Bamiatzi, Leeds University

Konstantinos Bozos, Leeds University

Tamer Cavusgil, Georgia State University

Tomas Hult, Michigan State University

In this study we offer new insights on the influences of firm, industry and country effects on firm profitability. We consider the role of the global economic crisis and how this impacts on the underlying relationships between countries at different development stages, namely developed and emerging. Using a 3-Level HLM model, we examine 15,280 firms in 68 SIC2 industries within ten emerging and ten developed countries, and compare and contrast firm, industry and country effects on profitability during the period 2005-2011. We find that firmspecific effects are predominant determinants of profitability, while industry and country effects are also present. The latter two are more important in emerging than in developed countries. When country-industry interactions are accounted for, they overtake the main industry and country effects, especially for firms in emerging economies, suggesting that the capabilities, attributes and expertise a country shares in certain industries can be more important in emerging markets. Under conditions of economic adversity, firm effects are strengthened to the expense of industry and country effects, both across developed and emerging countries, thus rendering firms even more responsible for their own fate. (For more information, please contact: Vassiliki Bamiatzi, Leeds University, United Kingdom: vb@lubs.leeds.ac.uk)

\section{Branding and Performance Implications in Emerging Markets Katharina Maria Hofer, Johannes Kepler University Linz}

This study examines the impact of branding aspects on firm performance in the emerging markets of Central and Eastern Europe. Specifically, the question of standardization versus adaptation of brand promotion is the focus of attention. After literature review, a conceptual model suggests that the standardization of brand promotion as well as a long-term brand vision provided by management positively influence target market performance. Furthermore, the model considers external environmental factors. Data gathered from a survey with managers allow testing the hypotheses through structural equation modelling. The results of the quantitative study largely support the hypotheses. (For more information, please contact: Katharina Maria Hofer, Johannes Kepler University Linz, Austria: katharina.hofer@jku.at) 
The Effect of Product Innovativeness, Channel Networking and Competitive Intensity on Export Performance:

Evidence from Ghana

Nathaniel Boso, University of Leeds

Vicky M. Story, University of Nottingham

John W. Cadogan, Loughborough University

Emerging markets offer substantial growth opportunities for firms that are operating in, or out of, these countries. However, only few studies have examined product innovativeness and its relationship with export performance in emerging market firms, and none of these have investigated non-linear associations. The purpose of this study is, therefore, to develop a model of the export success outcomes of product innovativeness in emerging markets, focusing on the potential for curvilinear associations to be present, as well as to incorporate an understanding of the possibility that channel networking and competitive intensity may act to moderate any curvilinear relationships present. The model is tested using longitudinal data from exporting firms operating in Ghana. Results show that the impact of product innovativeness on export performance is Ushaped; and that this curvilinear relationship between product innovativeness and export success is strengthened as channel networking increases, and becomes invert U-shaped as competitive intensity increases. The paper concludes that, for emerging market firms seeking to compete in foreign markets, the product innovation activity undertaken should be planned with optimal levels of product innovativeness sought given firms' channel networking levels, and degree of competitive activities in their export markets. (For more information, please contact: Nathaniel Boso, University of Leeds, United Kingdom: n.boso@leeds.ac.uk)

\section{Auditor Quality, CEO Turnover and Firm Performance}

Jie Zhang, Peking University \& China HuaRong Asset Management Co., Ltd.

Xiyou Liu, Peking University

Jiawen Yang, George Washington University

This paper investigates whether boards are more likely to fire CEOs for poor performance or reward CEOs for good performance as the perceived earnings credibility signaled by audit quality in a sample of China's listed firms from year 2000 to 2007 The results show that audit quality enhance the sensitivity of chief executive officer (CEO) turnover to firm performance, which indicates that audit quality plays an important role in determinants of CEO turnover and do have a corporate governance role by alleviating the manager-shareholder conflicts. The results also show that the link between Audit quality and CEO turnover-Performance sensitivity is greater in the listed firms that have a non-state controlling shareholder. Since a potential issue with the research design we employ is that CEO turnover and auditor choice may be determined simultaneously, we also examine auditor switches in a sub-sample, which contains firms that have switched across auditor class groups, and the result is robust. (For more information, please contact: Jie Zhang, Peking University \& China HuaRong Asset Management Co., Ltd., China: jacqueline35@sina.com)

\section{Session: 2.4.6 - Competitive \\ Track: Track: 3 - IB Theory, FDI, and Entry Mode}

\section{Internationalization Theory}

Presented On: July 5, $2013-14: 50-16: 05$

Chair: Sokol Celo, Suffolk University

Resource-Capability Recombinations and Multinational Enterprise Theory

Lars Matysiak, Justus Liebig University Giessen

Alan M. Rugman, University of Reading

Andreas Bausch, Justus Liebig University Giessen 
According to the resource-based view (RBV), resources and capabilities (RCs) that exhibit value, rarity, inimitability and organizational appropriability are the foundations of competitive advantages. Dynamic capabilities which allow to continuously achieve recombinations of RCs (that serve to exploit evolving opportunities or neutralize surfacing threats) are the basis of sustaining competitive advantages. Yet, this explanation of sustainable competitive advantages of firms largely disregards the location/country aspects of multinational enterprises (MNEs). In contrast, internalization theory, the dominant theory of the MNE, incorporates location aspects but was not intended primarily to explain competitive advantages. Here we explicitly integrate RBV insights into internalization theory. This allows us to carefully reconceptualize and clarify key constructs of MNE theory. We develop the multinational advantages vector space (MAVS) framework as a new integrative theoretical model. This explains that competitive advantages of MNEs consist of three dimensions: (i) country-specific, (ii) non-location bound firm-specific, and (iii) location bound firm-specific advantages. We extend MNE theory by conceptually distinguishing between these endogenous advantages and their causal RCs. We find that, from a dynamic point of view, MNEs need higher-order firm-specific advantages, conveyed by dynamic capabilities to continuously attain new advantages via recombining RCs, in order to sustain competitive advantages. (For more information, please contact: Lars Matysiak, Justus Liebig University Giessen, Germany: research@matysiak.com)

\section{Dynamics of International Business Research: A Relational Cluster Perspective Werner Kunz, University of Massachusetts Boston Stephan Manning, University of Massachusetts Boston Torben Pedersen, Copenhagen Business School}

This study examines the evolution of the field of international business (IB) research based on a relational cluster analysis of co-citations in the Journal of International Business Studies (JIBS) from 1982 to 2011. Particularly, we examine the changing role of theory in relation to empirical IB research. Based on a factor and network analysis, we identify distinct clusters of IB research and their relations over time. We show that the initial focus of IB research on issues of international expansion has shifted towards issues of governance and implementation. In this process, debates on foreign entry mode and culture have served as important integrating themes. At the same time, theory debates in IB have expanded from a narrow focus on explaining international expansion, to a rather heterogeneous conglomerate of approaches which have assisted increasingly differentiated empirical research. Our findings have important implications for evaluating the present state and potential future of IB as a research field. (For more information, please contact: Stephan Manning, University of Massachusetts Boston, USA: stephan.manning@umb.edu)

\section{Internationalization of Emerging Market Firms: Capability Renewal through Competitive Dynamics Joon-Ho Shin, ESADE Business School Matthew Allen Hawkins, ESADE Business School Xavier Mendoza, ESADE Business School}

In this paper, we argue that FDI (Foreign Direct Investment) of emerging market firms is driven by the strategic interaction of firms operating within a competitive environment. Through the competitive dynamics approach, based on the action and reaction of competitors, a framework to discuss how emerging market firms react to foreign entrants at home and how this reaction motivates a firm to expand internationally in a fast and risky way is developed. The framework explains the outward FDI mechanisms emerging market firms pursue as they become emerging market multinational enterprises, supporting the springboard behavior of EM MNEs. Firms from emerging markets are not just passive reactors who become multinational enterprises by defending their positions with available resources, but who become MNEs by aggressively searching and acquiring the necessary resources to enhance their competences to secure their key markets and to pursue further expansion. This view helps explain the renewal process were initial competitive advantages mainly based on location advantages of home markets transition into advanced firm-specific advantages, which prior literature has been unable to fully explain. (For more information, please contact: Joon-Ho Shin, ESADE Business School, Spain: joonho.shin@esade.edu) 
Inertia and the Change of State in the Uppsala Internationalization Process Model

Douglas Dow, Melbourne Business School

Peter Wayne Liesch, University of Queensland

Lawrence Welch, Melbourne Business School

Even in its most recent formulations, the Uppsala model focuses on a narrow set of factors - uncertainty, risk, trust and lack of awareness of opportunities - to explain the mechanisms that delay a firm's internationalization. By introducing inertia to the Uppsala model, we propose that a broader range of mediating factors may be at work. This development has implications for the role of experiential learning in the internationalization process, re-introduces managerial intentionality to the internationalization debate and illustrates how changes in the external environment may trigger changes in a firm's commitment to a market or network. (For more information, please contact: Peter Wayne Liesch, University of Queensland, Australia: p.liesch@uq.edu.au)

Session: 2.4.7 - Competitive

Track: Track: 10 - Economics, Finance and Accounting

\section{Public and Private Enterprises: Government Influence, Legal Origins and Financial Constraints}

Presented On: July 5, 2013 - 14:50-16:05

Chair: Elizabeth Rose, Aalto University and University of Otago

Product Market Competition, Corporate Governance and Legal Origin

Dominic Chai, Seoul National University

Simon Deakin, University of Cambridge

Ajit Singh, University of Cambridge

Prabirjit Sarkar, Jadavpur University

Using the persistence of profits as a measure of the intensity of product market competition in 19 countries for the period 1995-2005, we find that civil law systems are more competitive, in this sense, than common law ones. The strengthening of shareholder protection through company law increases competition between firms in common law countries, but reduces it in civil law ones. We conclude that shareholder rights act as complements to product market competition in the common law world but not in the civil law world, and explain this result by reference to the common-law origins of shareholder-orientated corporate governance. (For more information, please contact: Dominic Chai, Seoul National University, Korea, South: dchai@snu.ac.kr)

\section{Cash Holdings in Private and Public Firms: Evidence from Europe \\ Serkan Akguc, Temple University \\ Jongmoo Jay Choi, Temple University}

In this paper, we examine the differences in cash holdings between publicly and privately held firms in 33 European countries during 2002-2011 and shed light on the cash holding behaviors of private firms in Europe. We find that European public firms on average hold more cash than private firms. This finding is robust in various model settings. We also show that during the recent European financial crisis, firms in Euro-zone countries increased their cash holdings, whereas firms in non-Euro countries decreased their cash holdings. This signifies the presence of Euro policy coordination risk. Furthermore, we find that public firms hold more cash than private firms in Euro-zone countries than in non-Euro countries, indicating greater precautionary demand for cash by public firms in Euro countries. We also find that firms in countries with better shareholder protection hold less cash. Moreover, we show that both public and private firms in Europe actively adjust to target levels of cash, and both public and private firms show significant cash flow sensitivity to cash holdings. (For more information, please contact: Serkan Akguc, Temple University, USA: serkan.akguc@temple.edu) 
Wenjie Chen, George Washington University

Dazhong Cheng, Fudan University

This paper evaluates the effects of government involvement in outward foreign direct investment (OFDI) on the performance of state-owned versus privately owned firms. Using a combined dataset on Chinese OFDI projects and firm-level characteristics, we find that firms with dominant private owners have higher profitability compared to firms with dominant state owners following OFDI. Furthermore, we find significant performance differences depending on the level of government affiliation. Firms with lower level of government affiliation are more likely to experience performance improvements post OFDI compared to those with higher level of government affiliation. (For more information, please contact: Wenjie Chen, George Washington University, USA: chenw@gwu.edu)

\section{Session: 2.4.8 - Panel}

\section{Experiential Learning in the IB Classroom: Simulations and Role-playing}

Presented On: July 5, 2013 - 14:50-16:05

Chair: Suresh George, Coventry University

Panelists:

Suresh George, Coventry University

Basil Janavaras, Minnesota State University

Maurice Hendrix, Coventry University

The panel will showcase our experiences of teaching International Business and Global Strategy using the an online teaching software - the GMMSO at Minnesota State University, Mankato, USA and Coventry University, UK. In addition, we will showcase the existing development work being undertaken by both Universities in developing the 4th version of the teaching system (For more information, please contact: Suresh George, Coventry University, United Kingdom: s.george@coventry.ac.uk)

\section{Session: 2.4.9 - Competitive}

Track: Track: 1 - Institutions, Governance, and CSR

\section{Impact of Ownership and Institutions}

Presented On: July 5, $2013-14: 50-16: 05$

Chair: Rakesh Sambharya, Rutgers University

Concentrated Owners in Asia: Contingent Performance Effects of Owner Types and Market - Supporting Institutions

Marc van Essen, University of South Carolina

Pursey P.M.A.R. Heugens, Erasmus University

J. (Hans) van Oosterhout, Erasmus University

Eric R. Gedajlovic, Simon Fraser University

Michael Carney, Concordia University

In this paper we develop a contingency theory of concentrated ownership, which details how specific combinations of owner type (relational versus transactional owners) and market-supporting institutions 
(enabling versus constraining institutions) drive firm performance. Our findings show that the relative performance of firms with relational owners is better where enabling institutions are underdeveloped, but poorer where constraining institutions are weak. In contrast, the relative performance of firms with transactional owners is better where these owners can compensate for underdeveloped constraining institutions, but transactional owners cannot make a positive difference when enabling institutions are weak. We test our predictions through a theory-building meta-analysis of 234 primary studies nested in 17 different Asian countries. (For more information, please contact: Marc van Essen, University of South Carolina, USA: marc.vanessen@moore.sc.edu)

The Role of State Ownership and Political Institutions in Stock Price Infomativeness: Evidence from Privatization Hamdi Bennasr, King Saud University

In this paper, we examine the relation between government ownership and stock price informativeness. Using a multinational sample of privatized firms from 41 countries between 1983 and 2007, we find strong and robust evidence that state ownership is associated with a lower firm-level stock price variation i.e., stock price informativeness. Furthermore, we find that the relation between state ownership and stock price informativeness depends on the political environment. Particularly, the adverse effects of state ownership on stock price informativeness are more pronounced in countries with lower political rights (i.e., lower political constraints on the government) and higher risk of government expropriation, respectively. We also find that earnings quality conditions the relation between state ownership and stock price informativeness. Specifically, we find that earnings quality magnifies the impact of state ownership on stock price informativeness. (For more information, please contact: Hamdi Bennasr, King Saud University, Saudi Arabia: hbennasr@ksu.edu.sa)

\section{Shareholder Activism in Europe: Evidence from Shareholder Dissent in France, Germany, and the United Kingdom \\ Marc van Essen, University of South Carolina \\ Steve Sauerwald, University of Texas at Dallas \\ J. (Hans) van Oosterhout, Erasmus University}

Grounded in an organizational approach to comparative corporate governance, we develop and test a theory on how cost/benefit considerations affect shareholders dissent-i.e., shareholder votes cast against the recommendations of management at shareholder meetings. Using a large sample of more than 11,000 voting results on shareholder and management proposals in France, Germany, and the United Kingdom in 2008 and 2009, we find that absolute cost/benefit considerations (i.e., returns of dissent) as well as relative cost/benefit considerations (i.e., availability of substitute governance mechanisms) affect dissent. From an absolute cost/benefit perspective, we find that shareholders economize on their limited attention by using external signals such as RiskMetrics voting recommendations, but struggle to interpret internal signals such as CEO entrenchment. From a relative cost/benefit perspective, we find that large shareholders substitute their dissent for alternative activism strategies, and find weak evidence that shareholders effectively defer control duties to independent boards. Finally, we explore country level differences from a comparative perspective and present preliminary evidence that national level institutions affect dissent. We conclude with implications for comparative corporate governance research and policy debates on the role of shareholders in Europe. (For more information, please contact: Marc van Essen, University of South Carolina, USA:

marc.vanessen@moore.sc.edu)

\section{Foreign Institutional Ownership and Board Independence in Taiwan}

Liang Guo, Rouen Business School

Lawrence King, Cambridge University

Medhi Nekhili, Rouen Business School 
Globalization results in a remarkable resurgence in the study of corporate governance. This study explores the impact of foreign institutional ownership on the board independence of firms in Taiwan. Given the poorlydeveloped institutional environment in Taiwan and the pervasiveness of tight family ownership, foreign institutional owners might seek to take advantage of information asymmetries favoring insiders and thus would not promote board independence. The study tests the hypotheses with data from the 150 largest Taiwanese companies listed publicly from 1998 to 2006 . The results show that foreign institutional ownership reduces the level of board independence. The research highlights the implications for the understanding of path-dependency theory. (For more information, please contact: Liang Guo, Rouen Business School, France: Igg@rouenbs.fr)

\section{Session: 2.4.10 - Competitive \\ Track: Track: 6 - Innovation and Knowledge Mgmt.}

\section{Institutions, Innovation and Knowledge}

Presented On: July 5, $2013-14: 50-16: 05$

Chair: Xiaolan Fu, Oxford University

Institutional Logics and Knowledge Transfers in Multiparty Collaboration: Evidence from the Mobile Handset Industry

Gurneeta Vasudeva, University of Minnesota

Elizabeth Alexander, University of West England

Stephen Jones, University of Minnesota

We extend research on multiparty arrangements by conceptualizing these as venues where firms imprinted with distinctive collaborative logics derived from their institutional backgrounds, coalesce to achieve common goals. We offer insights into the interplay between the dominant collaborative logic circumscribing the multiparty arrangement, and the logic at the dyadic level at which inter-organizational knowledge transfers occur. We empirically test our theory using longitudinal analysis of 181 dyads involving 26 firms participating in 17 standard setting organizations in the global mobile handset industry. Based on the well-established corporatistpluralist institutional typology of polities to which countries belong, we find, after accounting for alternative sources of heterogeneity, that distinctive collaborative norms enacted by pluralist versus corporatist firms exert a significant influence on inter-organizational knowledge transfers between pairs of participating firms. In particular, dominant competitive norms fostered by pluralism contribute to greater inter-organizational knowledge transfers; conversely, an emphasis on coordination and consensus-building fostered by corporatism reduces knowledge transfers. Importantly, the effect of the dominant collaborative norms is diffused when firms in a dyad are imprinted by contradictory logics. Further, knowledge transfers affected by the dominant collaborative norms are contingent on the technological distance but not relational embeddedness between firms in a dyad. (For more information, please contact: Gurneeta Vasudeva, University of Minnesota, USA: gurneeta@umn.edu)

\section{Institutional Embeddedness of Search Strategies and the Implications for Innovation Performance Christoph Grimpe, Copenhagen Business School Wolfgang Sofka, Copenhagen Business School}

The innovation performance of modern firms is increasingly determined by their ability to search and absorb external knowledge. However, after a certain threshold firms "oversearch" their environment and innovation performance declines. In this paper, we argue that prior literature has largely ignored the institutional context that provides or denies access to external knowledge at the country level. Combining institutional and knowledge search theory, we suggest that the market orientation of the institutional environment and the magnitude of institutional change influence when firms begin to experience the negative performance effects of 
oversearch. Based on a comprehensive sample of almost 8,000 firms from ten European countries, we find that institutions matter considerably for firms' search activity. Higher market orientation of institutions increases the effectiveness of firms' search for external knowledge while higher magnitudes of institutional change decrease it. Our results provide important insights for management on how to adapt search strategies to the institutional context. (For more information, please contact: Christoph Grimpe, Copenhagen Business School, Denmark: cg.ino@cbs.dk)

\section{Managing Institutional Impediments through Business Networks Angelika Lindstrand, Stockholm School of Economics Nurgul Ozbek, Stockholm School of Economics Kent Eriksson, Royal Institute of Technology}

Differences in institutional contexts create obstacles, which we in this paper label institutional impediments that affect firm performance on foreign markets. International expansion can be discussed in terms of developing international business relationships, thus we investigate these impediments on a relationship level as well as on a network level, as relationships are connected into networks. We argue that the negative effects of institutional impediments on international performance can be managed by making relationship specific investments and through network dependency. Two different parts of a network are investigated; the local institutional net and the local business net. To examine these effects we develop five hypotheses, which are tested on a sample of 244 internationalizing SMEs by using structural equation modeling and linear regression analysis. The results show that relation specific investments have a mediating positive effect on performance, whereas the institutional net only have an indirect mediating positive effect through the business network. Thus institutional impediments can be managed by investing in the business relationship that the firm is engaged in and by depending on the different parts of the network in combination. (For more information, please contact: Angelika Lindstrand, Stockholm School of Economics, Sweden: angelika.lindstrand@hhs.se)

\section{Isomorphism and the Development of Absorptive Capacity: The Role of Rationalization and Professionalism on Emerging Market Firms}

Yi Jia Kathleen Low, WU Vienna

Jonathan Doh, Villanova University

James Robins, WU Vienna

Institutional theory has been used widely to inform international business phenomenon. In this paper, we extend extant applications of institutional theory to explore how it may influence the process of knowledge transfer from foreign MNEs to local emerging market firms. We suggest that the three pillars of institutional theory affect the degree of rationalization and professionalization within emerging and transition economy firms. In so doing, those factors affect the capacity of local firms to absorb knowledge. We introduce and develop the concept of "foreign knowledge absorptive capacity" to characterize the degree to which domestic firms are able to capture and put to use the knowledge provided by their MNC counterparts. We suggest the rationalization and professionalization of those indigenous firms - and the environment in which they operate - are critical to the success of knowledge transfer from foreign to local sources. We discuss our insights for institutional perspective on IB, and an expanded understanding of absorptive capacity in international strategy. (For more information, please contact: Yi Jia Kathleen Low, WU Vienna, Austria: ylow@wu.ac.at) 
Session: 2.4.11 - Interactive

Track: Track: 11 - SMEs and Entrepreneurship

\section{Entrepreneurial Orientation (EO), International Entrepreneurial Orientation (IEO), Risk Tolerance, and the Internationalization Decision of New Ventures}

Presented On: July 5, $2013-14: 50-16: 05$

Chair: Antonella Zucchella, University of Pavia

From Resources to International Performance of New Ventures: Are Innovation and Flexibility Strategies the Puzzle's Missing Pieces?

Vitor Corado Simoes, Advance, ISEG, Technical University of Lisbon

Nuno Crespo, Advance, ISEG, Technical University of Lisbon

Margarida Fontes, UMOSE - Laboratório Nacional de Engenharia e Geologia and Dinamia

A major weakness in the international entrepreneurship (IE) field is the empirical focus on direct relationships between the antecedents of IE and the outcomes of the internationalization process. This provided the motivation for the present research, which is aimed at contributing to a better understanding of the strategic processes which mediate the conversion of firms' characteristics and resources into performance, more specifically international performance. Drawing on IE literature, a model is developed, introducing strategic approaches as mediators between entrepreneurial orientation and firm resources, on the one hand, and firm international performance, on the other. Two basic strategies were considered: innovation and flexibility. The empirical testing of the model, carried out on a sample of 416 international new ventures (INV), confirms that the firm strategies play an important role as determinants of international performance. A negative relationship was found between the availability of generic resources and the adoption of both innovation and flexibility strategies. This suggests that INVs may adopt those strategies to escape resource constraints. The research makes a three-fold contribution to the IE field. First, the results show that firm strategies (in this case, innovation and flexibility strategies) are relevant as mediators of the resources-performance relationship. Second, firm generic resources are negatively related to these strategies, suggesting that INV's strategic choices are influenced not just by existing but also by missing resources. Third, innovation strategy is based on a wider mix of resource requirements than flexibility strategy. These findings may pave the way towards further research dealing with the understanding of the role played by strategic approaches in explaining INV's international performance. (For more information, please contact: Vitor Corado Simoes, Advance, ISEG, Technical University of Lisbon, Portugal: vcs@doc.iseg.utl.pt)

\section{An Empirical Analysis of the Dual Effects of EO and Foreignness on Entrepreneurial Performance Arpita Joardar, Clark University \\ Sibin Wu, University of Texas-Pan American}

This paper draws from research on entrepreneurial orientations (EO) and liabilities of foreignness to examine their combined effects on entrepreneurial performance. We attempted to find out when foreignness can be liability and benefit. Data obtained from 697 domestic and foreign entrepreneurs operating in the U.S. was used in this study. A three-step regression analysis was conducted to test the proposed hypotheses. Results indicate that there is evidence of foreignness having moderating effect on the relationship between EO and performance. Implications of the findings are discussed. (For more information, please contact: Arpita Joardar, Clark University, USA: ajoardar@clarku.edu) 
This study examines what really accounts for the early adoption of internationalization by the Indian knowledgeintensive service firms and their continued success in international markets. On the basis of extant literature, a tentative frame of international entrepreneurial orientation, learning orientation is suggested and then used to analyse empirical material gleaned from multiple case studies of three Indian born global firms. Findings reveal that, despite the scarce resources, Indian born global firms leverage a distinctive mix of entrepreneurial orientations and strategies that allow them to succeed in diverse international markets. And their entrepreneurial orientation is nurtured through the learning processes. (For more information, please contact: Nishant Kumar, Stockholm University, Sweden: nku@fek.su.se)

\section{How Does Risk Preference Personality affect Decision-Making in Chinese Outward Direct Investment? A Conceptual Framework based on the Upper Echelons Theory}

Yue Xu, Hull University Business School

Hui Wang, Peking University

We argue past empirical studies regarding outward direct investment (ODI) of emerging market enterprises can be flawed from the risk neutral assumption. In reality top managers are not risk neutral in decision-making. To develop this argument, we propose a conceptual model by drawing insights from the upper echelons theory. Our model aims to examine the effect of executive orientation, namely, top managers' risk preference personality and experiences, on international venturing decision-making. We highlights indigenous characteristics specific to the context of Chinese ODI. Accordingly, three moderating effect is considered in the model, they are: corporate governance conditions, organisational conditions (mainly intangible resources) and industry conditions. Theoretical contribution and limitation of the research are also discussed. (For more information, please contact: Yue Xu, Hull University Business School, United Kingdom: tina.yuexu@gmail.com)

\section{Beyond Path Dependence: Explorative Orientation, Slack Resources and SMEs' Internationalization Angels Dasi, Universitat de Valencia Vicente Safon, Universitat de Valencia Maria Iborra, Universitat de Valencia}

Exploration and exploitation orientation of the firm represent two different learning processes. Applying this organizational learning perspective this study analyzes the influence that these two orientations have on the SMEs' managerial intentionality to internationalize. We hypothesize that exploitative orientation is going to reinforce the internationalization trajectories the firm already has, whereas explorative orientation has a direct and positive effect over manager's willingness to increase international activities. In addition, we study the effect that slack resources have on the managerial intentionality to internationalize. We test our hypothesis on a data set of 136 small and medium enterprises. Our findings support the role of an explorative orientation on management intentionality towards internationalization. We also confirm the moderator role of management discretionary resources on this relationship. (For more information, please contact: Angels Dasi, Universitat de Valencia, Spain: angels.dasi@uv.es) 
Conceptualizing the Dynamics of International Entrepreneurship: A model of International Entrepreneurial Orientation, Opportunity Recognition, Internationalization, and Performance

Rico Baldegger, University of Applied Sciences Western Switzerland

Patrick Schueffel, University of Applied Sciences Western Switzerland

This paper contributes to the existing research by integrating the notions of opportunity recognition and international entrepreneurial orientation into the body of the new venture theory of internationalization. It helps to explain and understand which factors compel SMEs into a rapid mode of internationalization or into a stage-

wise approach. A conceptual framework is developed that presents potential relationships between key concepts from the field of entrepreneurship on the one hand and the degree of internationalization and the performance of an internationalizing SME on the other. Our results suggest that internationalization may not just be the result of one or multiple push or pull factors, but that internationalization itself may significantly influence the international entrepreneurial orientation and the opportunity recognition capabilities of a firm. Consequently, internationalization may be seen as an independent variable, a fact that was largely neglected in past research on SME internationalization. (For more information, please contact: Rico Baldegger, University of Applied Sciences Western Switzerland, Switzerland: rico.baldegger@hefr.ch)

\title{
Entrepreneurial Orientation and Performance of SMEs: Moderating Role of Institutional Environment Ahreum Lee, Temple University Deeksha Singh, Temple University
}

This paper examines the relationship between entrepreneurial orientation (EO) and firm performance in the case of SMEs. Recognizing the resource scarcity that SMEs face, we argue for a "U" shaped relationship between EO and performance. Further, we propose that this relationship is contingent on the level of institutional support that SMEs receive in a given context. More specifically, in the presence of a higher level of political support and a higher level of social support, SMEs are able to derive greater benefits from their entrepreneurial orientation. Our empirical analysis, based on a sample of 284 SMEs in Germany largely supports our arguments. (For more information, please contact: Ahreum Lee, Temple University, USA: ahreum.lee@temple.edu)

\author{
Entrepreneurial Orientation and SME International Scope: The Differential Roles of Innovativeness, \\ Proactiveness, and Risk-Taking \\ Li Dai, Loyola Marymount University \\ Vladislav Maksimov, University of Miami \\ Brett Gilbert, Rutgers University \\ Stephanie A. Fernhaber, Butler University
}

This study advances research on small and medium-sized enterprises (SMEs) and entrepreneurial orientation by examining how the latter's sub-dimensions of innovativeness, proactiveness, and risk-taking individually shape the ability of SMEs to simultaneously pursue multiple opportunities in foreign markets. Using a unique data set of 500 SMEs spanning 10 industries, we found negative implications of adopting a "stuck-in-the-middle" strategy with respect to being innovative and proactive. Our theory on the costs and benefits of entrepreneurial orientation dimensions in capturing international opportunities also reveal the nuanced impact of risk-taking on SME international scope. Overall, the findings of non-uniform and non-linear relationships contribute to a better understanding of when the individual dimensions of entrepreneurial orientation represent an asset or a liability for entrepreneurial firms in the internationalization process. (For more information, please contact: Li Dai, Loyola Marymount University, USA: li.dai@Imu.edu) 
Session: 2.4.12 - Interactive

Track: Track: 1 - Institutions, Governance, and CSR

\title{
Impact of Owners and Other Stakeholders
}

Presented On: July 5, $2013-14: 50-16: 05$

Chair: Ruth Aguilera, University of Illinois at Urbana-Champaign

Corporate Ownership and Internationalization: The Effects of Family, Bank and Institutional Investor Ownership in the UK and in Continental Europe

Vincenza Odorici, Univesity of Bologna

Antonio Majocchi, University of Pavia

Manuela Presutti, Univesity of Bologna

While the role of corporate governance has been analysed, it is only recently that the effects of corporate governance features on firm international strategies have been considered. The objective of this quantitative paper is to analyze the potential role played by different kind of shareholders on firm internationalization level. First, using a European sample of listed firms, we intend to assess if and how different shareholders typologies affect the firms' degree of internationalization. Second, distinguishing between the firms quoted in the UK from those listed in Continental Europe's countries (France, Germany, Italy, Poland, Spain) we investigate if the different characteristics of the corporate governance systems in the two regions have any impact on firms' internationalization. Our results confirm that different kind of ownerships affect with different degree of intensity the overall level of firm's internationalization. First, we find that ownership matters. Second, we show that the effects of ownership over firm's international strategies depend also on the context of analysis. We suggest that the attitude towards internationalization of financial institutional investors differ in institutional contexts. While in the Continental Europe context, bank ownership negatively affects the level of internationalization, our result show that in an Anglo-Saxon context institutional investors promote internationalization. (For more information, please contact: Vincenza Odorici, Univesity of Bologna, Italy: vincenza.odorici@unibo.it)

\author{
Foreign Ownership and Corporate Governance Patterns: The Board-Auditor Relationship in Japan \\ Kurt Desender, University Carlos III, Madrid \\ Rafel Crespi, Universidad de las Islas Baleares \\ Monica Lopez-Puertas Lamy, Unicredit Banca - University of Bologna
}

Auditing is an important piece of the corporate governance puzzle because it is an instrument enhancing the protection of investors' rights. We draw on a contingency approach which conceptualizes corporate governance as a system of interrelated elements having strategic or institutional complementarities and claims that particular governance practices will only be effective in certain combinations which may lead to different corporate governance patterns. We claim that the relationship between boards and auditors is contingent on the ownership of the firm, and in particular on the degree of foreign ownership. To test this logic, we focus on the relationship between board characteristics and audit fees in Japan-an institutional setting which differs substantially from the U.S., and has witnessed a high increase in Anglo-American institutional investors. Our results show that board independence, as well as board of corporate auditors' independence and the external auditor become complementary when foreign ownership is high, while such relation is absent when foreign ownership is low. Our findings highlight the possibility of different patterns of corporate governance within one country, shaped by the weight of foreign owners. (For more information, please contact: Kurt Desender, University Carlos III, Madrid, Spain: kdesende@emp.uc3m.es) 


\section{Family and Institutional Ownership and Firm Performance: A Cross-National Analysis \\ Jean B. McGuire, Louisiana State University \\ Sandra Dow, Monterey Institute of International Studies}

Using a sample of firms from over 40 countries, we examine the relationship between family ownership and firm performance. We find a negative relationship between family ownership and firm performance. and a positive relationship between institutional investment and firm performance. (For more information, please contact: Jean B. McGuire, Louisiana State University, USA: mcguire@Isu.edu)

\section{How Much I am Going to Tell You? A Study of Differences in Ownership Strategic Behavior Kurt Desender, University Carlos III, Madrid Jun Ho Lee, University of Illinois at Urbana-Champaign}

Although current corporate governance studies have emphasized that institutions matter for the diffusion of corporate practices, less attention has been paid to how different institutions matter for the implementation of corporate practices, especially when different shareholders who have been embedded in different institutional environments coexist. To answer the question, from the institutional perspective, we develop a theoretical framework to explain how a corporate earnings disclosure practice is implemented by different owners (i.e. Relation-oriented vs. Transaction-oriented owners) who represent different institutional features (i.e.

Stakeholderism vs. Shareholderism). We also examine how a board of directors functions differently in earnings disclosure, depending on ownership structure in a corporation. We test our hypotheses with the analysis of 2,151 Japanese firms for the 2005-2011 period. (For more information, please contact: Jun Ho Lee, University of Illinois at Urbana-Champaign, USA: lee453@illinois.edu)

\section{First Impressions: Stakeholder Networks, Proactive Engagement and Stakeholder Opinions of Corporations Sinziana Dorobantu, New York University Lite Nartey, University of South Carolina Witold Henisz, University of Pennsylvania}

Stakeholders receive information about a firm's activities through the media, from other stakeholders, and from the company. We study the relative weight of four different mechanisms through which stakeholders form their opinion of corporations: (1) rational processing of media information; (2) sifting of information received through social networks; (3) following of opinion leaders; and (4) impression formation through interaction with the company. We design our analysis to assess the relative importance of the four mechanisms using data from 4,652 social, political and economic stakeholders (government representatives, communities, non-governmental organizations, unions, etc.) that have voiced a position in relation to one of 26 gold mining projects around the world. We show that stakeholders' first impressions of companies are shaped by the media and by direct interactions initiated by the company. These results highlight that through proactive stakeholder engagement companies can go a long way in terms of managing their reputations and building positive social capital with stakeholders. (For more information, please contact: Sinziana Dorobantu, New York University, USA: sdoroban@stern.nyu.edu)

\section{A Participatory Approach to Stakeholder Engagement: Defining a Hierarchy of Strategic Action Lite Nartey, University of South Carolina \\ Sinziana Dorobantu, New York University Witold Henisz, University of Pennsylvania}

Scholars of stakeholder engagement and corporate social responsibility have long grappled with the question of how to strategically engage stakeholders to obtain the strategic benefits of cooperation and reduce conflict with stakeholders. We use insights from the literatures on civic participation and participatory development to 
identify five strategic actions, and the underlying mechanisms of these actions, that firms can use to strategically engage stakeholders to increase cooperation and reduce conflict These strategic actions includeAnnouncements, Meetings, Payments, Activity, and Monitoring and Evaluation (M\&E). We find similar impact of payment and activity, similar but lower impact of both Announcements and Meetings, and lowest impact of M\&E. We further find that stakeholder-led strategic actions have a consistently greater impact than firm-led strategic actions, and that joint actions are not perceived as joint but as rather firm-led or possibly staged. Our context is an original dataset of roughly 52,000 media reported stakeholder events that we use to capture dyadic relations and engagement among stakeholders and firms associated with 19 gold mining companies operating 26 mines in 20 countries. (For more information, please contact: Lite Nartey, University of South Carolina, USA: lite.nartey@moore.sc.edu)

\author{
Business Groups' Ownership Structure in Emerging Countries \\ Wlamir Goncalves Xavier, FGV/EAESP e UNISUL \\ Silvio Parodi Camilo, UNESC \\ Rosilene Marcon, UNIVALI \\ Rodrigo Bandeira-de-Mello, Fundação Getúlio Vargas
}

Business Groups prevail in emerging countries as diverse organizational structures that aggregate various companies under the control of a family or of a reduced number of people (Khanna and Yafeh, 2007; Schneider, 2008). This economically relevant structure is responsible for a significant share of countries' Gross Domestic Product (GDP) and frequently congregates the largest private companies in their respective countries (Casanova, 2009; Ghosh, 2010). Business Groups exist partly due to the need to overcome the limitations of the market in which they exist, such as a lack of adequate infrastructure or of an effective financial system (Khanna and Palepu, 1997). Furthermore, governments leverage and enable BGs. Institutional reforms have been implemented in emerging economies in order to support the integration of other nations from a commercial perspective. Consequences of such reforms include opening internal markets and introducing common intermediation financial mechanisms. These reforms produce profound changes in the companies of such countries (Peng, 2003), and it is within this context that this study seeks to analyze the relationship between the ownership structure of private Business Groups and the institutional environment. (For more information, please contact: Wlamir Goncalves Xavier, FGV/EAESP e UNISUL, Brazil: wlamirxavier@gmail.com)

\title{
Session: 2.4.13 - Interactive \\ Track: Track: 8 - Developing Country MNCs \\ Internationalization of Emerging and Developing Economy MNEs
}

Presented On: July 5, $2013-14: 50-16: 05$

Chair: Jorge Carneiro, Pontifical Catholic University of Rio de Janeiro

\author{
Leveraging or Dissipating Resources: Virtuous and Vicious Internationalization Process of Emerging Market \\ Multinationals \\ Aleksandra Wasowska, University of Warsaw \\ Krzysztof Obloj, Kozminski University and University of Warsaw
}

In the view of traditional IB theories, the decision to internationalize operations, made by entrepreneurial SMEs from emerging markets seems almost to be a paradox. The objective of this paper is to explore this paradox, by studying internationalization from a processual perspective, and answering two major questions: how do entrepreneurial companies from an emerging economy make their choices and leverage initial country conditions and resources, when entering foreign markets?, and Do they learn to overcome resources deficiencies via FDI? Using case study method we show dynamic of vicious and virtuous cycles of 
internationalization depending upon initial conditions, motives, pacing and speed of internationalization and resource allocation, usage and leverage. The study suggests that these relations can be systemic (holistic) or non-systemic. The vicious process is at extreme disconnected and asymmetrical. Initial conditions do not prepare firm for challenges of internationalization, motives are idiosyncratic and opportunistic, internationalization is rapid and involve significant resource commitments without producing instant rewards. The process runs away and resources are not accumulated, integrated or created. The virtuous process is at extreme systemic and symmetrical. The internationalization process absorbs resources, but at the same time enhances their reorganization and reinforcement if initial conditions in company's environment facilitated motives of internationalization create right context. (For more information, please contact: Krzysztof Obloj, Kozminski University and University of Warsaw, Poland: kobloj@kozminski.edu.pl)

\section{How Do Emerging Market Firms Become "Champions" in Global Markets? Evidence from Turkey Türkan Yosun, Sabanci University \\ Dilek Cetindamar, Sabanci University}

In order to shed some light to the key pillars of global competitiveness for emerging economies, this paper analyzes internationally successful small and medium sized firms that are the so-called "hidden champions". All firms face various difficulties during internationalization; developing country firms, however, encounter disadvantages specific to their contexts. Acknowledging these challenges and observing successful practices might help to understand emerging market firms' internationalization. Based on the empirical observation of 10 Turkish hidden champions, we highlight innovative strategies of these companies, which we believe might serve as role models for others in their efforts to overcome the difficulties of internationalization, specifically for those companies from emerging market contexts. We conclude with some managerial and policy implications. (For more information, please contact: Türkan Yosun, Sabanci University, Turkey: turkanyosun@sabanciuniv.edu)

\section{Downstream Upgrading in the Canned Tuna Global Value Chain Pavida Pananond, Thammasat University}

This paper analyses how firms from developing economies upgrade their position in a global value chain to become global leaders in their industry. The analysis of a food manufacturer, the Thai Union Group, reveals three insights. First, developing country firms use international expansion as a means to upgrade their position in the global value chain of their industry. Starting as a low-cost supplier to global buyers, the Thai Union Group moved up the value chain by acquiring brands and distribution channels in developed economies. Second, developing country firms can enhance their strengths in the lower-value operations through strategic production management, whereby different production sites are used for different purposes. Third, emerging Thai multinationals internationalise in the direction of their global value chain governance to upgrade their position within the chain structure. By integrating the literature on emerging market multinationals (EMNES) and on the global value chain, the paper sheds more light on how internationalization can be used as a strategy for lowcost suppliers in developing countries to enhance their position within the global value chain. (For more information, please contact: Pavida Pananond, Thammasat University, Thailand: pavida@tbs.tu.ac.th)

\section{Strategy, Process and Philosophy behind Internationalization of Indian Multinationals - The View from the Bridge}

Mohan Thite, Griffith University

Adrian Wilkinson, Griffith University

Pawan Budhwar, Aston University

The multi-polar world demands re-examination and refinement of our understanding of the internationalization strategies and competitive advantages of multinational firms by incorporating the unique characteristics of firms from emerging economies. Based on a case study of five Indian multinationals and in-depth interviews, we 
present directly the "voices" of Indian corporate leaders to provide evidence that supports and develops extant literature on the internationalization process and the country and firm specific advantages of emerging multinationals. In the process, we identify the need for cross national transfer of emerging management models and practice to facilitate "travel of ideas" in multiple directions. (For more information, please contact: Mohan Thite, Griffith University, Australia: m.thite@griffith.edu.au)

\section{Globalization from East to West: Are Current Internationalization Theories Relevant to Emerging Market Firms? Brian Li, GP Electronics (Hong Kong) Ltd. \\ Maya Kumar, Western University \\ Mary Ann Von Glinow, Florida International University}

Uppsala Internationalization Model and Eclectic Paradigm, two seminal theories of firm globalization, were developed using data from firms originating in developed countries. This paper examines the applicability of these models of internationalization to firms from less industrially developed countries, such as Hong Kong was in 1990s. Using a historical analysis methodology, this retrospective longitudinal case study analyzes globalization of Gold Peak Electronics Limited (GPE), a Hong Kong electronics firm, from late 1980s to today. While the Eclectic Paradigm and Uppsala Internationalization Model can help in understanding GPE's globalization, using the GPE history we discovered issues not addressed by these theories. Based on these findings, this paper proposes an updated model for foreign market entry and development by emerging market firms that includes the acquisition of local market specific knowledge and excess management capacity, rather than its internal development. (For more information, please contact: Maya Kumar, Western University, Canada: mkumar@ivey.ca)

\section{Can Emerging Country Multinationals Be Categorized According To Global Mindedness? The Case Of Brazilian Multinationals}

Germano Glufke Reis, FGV/EAESP

Felipe Zambaldi, FGV/EAESP

Maria Tereza Fleury, Fundacao Getulio Vargas

Afonso Fleury, University of São Paulo

Despite the relevance of developing a global mindedness (GM) in order for firms to explore global opportunities, extant literature on GM has mostly focused on developed country multinationals, overlooking its relevance to emerging country multinationals which have unique features and internationalization patterns. In addition, the studies addressing the impacts of GM on internationalization have mostly relied on the assumption of homogeneity rather than differentiation of GM among multinationals of the same country. This study addresses this theme by exploring GM diversity among Brazilian multinationals. For that purpose, a previously developed multidimensional GM scale is validated followed by a cluster analysis. As a result, a GM taxonomy is identified: fully globally minded, cross-culturally skilled, international market-oriented, and domestic market-oriented multinationals. To illustrate the taxonomy, four cases involving major Brazilian multinationals are brought to the fore. Besides the validation of the GM scale and the identification of the taxonomy, another key conclusion points to the need to study GM in conjunction with both institutional and economic factors to explain the different internationalization trajectories of firms from a single country. (For more information, please contact: Germano Glufke Reis, FGV/EAESP, Brazil: germanoglufkereis@yahoo.com.br)

\section{Home Country Institutional Environments and Foreign Expansion of Emerging Market Firms \\ Jie Wu, University of Macau \\ Xiaoyun Chen, University of Macau}

We propose that home country institutional environment shapes emerging market firms' foreign expansion. We argue that better-developed home country institutional environment promotes emerging market firms' 
expansion to foreign markets more advanced than the home country, while institutional stability in the home country reduces this propensity. We further hypothesize that the effects of home country institutional environment are contingent on firm-specific government ownership. Data on the foreign expansion of 921 Chinese firms in the period of 1996-2000 provide strong support for the effects of home country's institutional development and institutional instability. We also find that a high degree of government ownership weakens the positive effect of home country's institutional development on emerging market firms' propensity to expansion to more advanced markets. (For more information, please contact: Jie Wu, University of Macau, Macau: jiewu@umac.mo)

\title{
Session: 2.4.14 - Interactive Track: Track: 3 - IB Theory, FDI, and Entry Mode
}

\section{Internationalization Processes}

Presented On: July 5, $2013-14: 50-16: 05$

Chair: Jin Uk Kim, University of Illinois at Urbana-Champaign

\author{
Experiential Knowledge and the Internationalization Process: The Case of the Australia and New Zealand \\ Banking Group \\ Sidney John Gray, University of Sydney \\ Caroline Takchi, PricewaterhouseCoopers
}

This study examines the internationalization process of the Australia and New Zealand Banking Group (ANZ) from 1980 to 2010. Using an exploratory case study methodology and drawing on the bank's archival documents, the role of the individual decision maker, namely the Chief Executive Officer (CEO), was examined within the context of the updated Uppsala model (Johanson and Vahlne 2009). Experiential knowledge was found to be an important factor in the internationalization process. The Board of Directors used the experiential knowledge of potential CEOs as a basis for appointing them to the top job. In this way, the influence of the CEO on international strategy was, to an extent, dictated by the Board. It was also found that psychic distance had relatively poor predictive power in explaining the internationalization process of ANZ. In contrast, the existing networks and past relationships of the CEO were significant drivers of the internationalization process. The internationalization of ANZ was not an incremental process, but rather an opportunistic process of internationalization, de-internationalization and re-internationalization. (For more information, please contact: Sidney John Gray, University of Sydney, Australia: sid.gray@sydney.edu.au)

Capabilities, Uncertainty, and Timing of Knowledge-Seeking FDI: Evidence from Overseas R\&D Labs of Japanese Pharmaceutical MNCS

Sang Ji Kim, Seoul National University

Yeonjin Park, Seoul National University

Jaeyong Song, Seoul National University

This study proposes a capability-based view and the lens of uncertainty to examine factors influencing the timing of knowledge-seeking FDI (foreign direct investment) of multinational corporations (MNCs). Using event history analysis, we investigate 205 R\&D subsidiaries of 16 Japanese pharmaceutical MNCs. Our findings show that strong technological capabilities of a MNC headquarter and favorable business environment of a host country reduce uncertainty of local knowledge sourcing, thereby promoting knowledge-seeking FDI. Our results also show that abundant technological resources of a host country have a positive impact on the timing of knowledge-seeking FDI and the impact is strengthened when business environment of a host country is favorable. (For more information, please contact: Jaeyong Song, Seoul National University, Korea, South: jsong@snu.ac.kr) 
Listing on Foreign Stock Markets as a Stage of Internationalization: Evidence from Chinese Firms

Xueji Liang, National University of Singapore

Jane Wenzhen Lu, University of Melbourne

The internationalization process (IP) model suggests that firms internationalize through incremental steps in global markets, with accumulated knowledge and enhanced commitment. This study extends the IP model by arguing that listing on foreign stock markets can be perceived as a stage of internationalization process. More specifically, firms gain international recognition and legitimacy through listing on major foreign stock exchanges, which affect their knowledge accumulation and perceived opportunities in the global markets. Greater knowledge and more opportunities enhance firm commitment and thus facilitate further internationalization process. Our empirical results, based on a panel data analysis of Chinese firms, suggest that global IPO increases the degree of internationalization and leads to better firm performance. Moreover, empirical evidence indicates that the strategic value of foreign listings is contingent on firm corporate governance structure, in which the better the corporate governance, the greater the performance of foreign listings. (For more information, please contact: Xueji Liang, National University of Singapore, Singapore: g0900955@nus.edu.sg)

\section{What Accelerates the Emerging-Market Internationalisation Process? - An Empirical Study in the Case of China Leo Li'HC, King's College London Emma L. Punton-Li, Department for Work and Pensions Fatima Wang, King's College London}

Our contribution is to identify two factors accelerating emerging-market foreign direct investment. Firstly, an accelerated path of intra-company knowledge acquired via business alliances between inward overseas investment and home country companies. Through this process, the emerging-market ventures may become internally international before the initial FDI, we name this internalisation of internationalisation (i2). Secondly, an exclusive ownership advantage generated from home country imperfection which can be used to leverage other competitive advantages, namely exclusive relational asset (ER). The ER may encourage investors to leverage the desired resource from an overseas location via a lower cost. Our research links these factors to emerging-market FDI research and enriches the classical FDI theories. (For more information, please contact: Leo Li'HC, King's College London, United Kingdom: leo.hc.li@gmail.com)

\section{Political Knowledge, Political Turbulence and Uncertainty in Emerging Market Internationalization Processes Mikael Hilmersson, Linnaeus University \\ Firouze Pourmand, Linnaeus University \\ Susanne Sandberg, Linnaeus University}

This paper is concerned with knowledge related to uncertainty management in the internationalization process of SMEs. Dramatic changes have been seen in the world economy during the last decades. Two major developments have put uncertainty management on top of most firms' agenda: First, we have seen an increased importance of institutionally underdeveloped emerging markets serving as growth engines of the world economy. Second, political initiatives as a response to economic drawbacks and challenges change the playground for international firms. In this paper, the authors identify political knowledge gaps and political turbulence as two key determinants of uncertainty in the SME internationalization process. A conceptual model is derived from extant literature that examines the individual, as well as interaction, effect of political knowledge gaps and political turbulence on uncertainty. The model is confronted with a dataset collected on site at 203 SMEs with experience of entries into the new Eastern European EU member-states, Russia and China. The analysis shows that experiential knowledge is important for reducing uncertainty if the environment is stable. If the environment is turbulent, however, previous experiences are less relevant as the perceived uncertainty is still high. The authors argue that, under such circumstances, experiential knowledge might even be misleading or obsolete. (For more information, please contact: Susanne Sandberg, Linnaeus University, Sweden: susanne.sandberg@Inu.se) 
Subsidiary-Specific Advantages in Multinational Enterprises: A Case Study of Unilever Subsidiaries in South East Asia

Quyen Nguyen, University of Reading

We conduct a case study of Unilever's subsidiaries in Indonesia and Vietnam to establish the extent to which they generate new subsidiary-specific advantages (SSAs). We find evidence that the subsidiary managers generate SSAs by recombining the firm-specific advantages (FSAs) of their parent firm with newly developed location-bound (LB) FSAs by subsidiaries obtained in interacting with local host country-specific advantages (CSAs). They do this principally through the development of "flagship" relationships with key partners in the value chains such as key suppliers, key distributors, selected key competitors and institutions in the nonbusiness infrastructure. We find that these flagship strategies are a type of network capabilities that can lead to non-location-bound (NLB) FSAs for the MNE. They also contribute to performance of the subsidiaries and help develop local networks of small and medium enterprises (SMEs) which enhance economic development. (For more information, please contact: Quyen Nguyen, University of Reading, United Kingdom:

t.k.q.nguyen@henley.ac.uk)

\section{Session: 2.4.15 - Interactive}

\section{Track: Track: 2 - Marketing and Supply Chain}

\section{Brand, COO and Consumer Behavior}

Presented On: July 5, $2013-14: 50-16: 05$

Chair: Sergio Carvalho, University of Manitoba

How Japan's Global Brands Were Built: Lesson's for Emerging Market Companies? Johny K. Johansson, Georgetown University

The need for a strong brand constitutes one of the highest entry barriers for companies from emerging markets entering mature economies. The strategic solutions adopted range from OEM manufacturing to the acquisition of existing leading brands. To gain perspective on the likelihood of success on such strategies, this paper examines how the Japanese companies managed to introduce their now well established and strong global brands into Western markets. Analyzing the successful Japanese companies' entries into the U.S. market, several features stand out. Their brands represented the identity of the corporation, and were never viewed as separate from the product or service offered. Their market success came from the product uniqueness and product quality, in-house manufacturing and assembly, and a coherent internal organizational culture with a strong customer-orientation. To help acceptance of their brands, the Japanese adopted a view of distribution channel members as first-line customers, and outsourced promotions to local ad agencies. Where management erred on one or more of these factors, the Japanese companies failed to establish a successful brand. The application of the lessons is necessarily constrained by the particular historical context, but the Japanese successes still suggest some fundamental requirements of global brand building by emerging companies. (For more information, please contact: Johny K. Johansson, Georgetown University, USA: johanssj@georgetown.edu)

\section{Evaluating "Made in America" a critique of the American Automotive Labeling Act and a proposed Alternative} Frank DuBois, American University

Advances in logistics and the increased globalization of the automotive industry have made it increasingly difficult to determine the country of origin of a vehicle. The purpose of this paper is to evaluate the American Automotive Labeling Act (AALA) of 1994 and its use as guidance in the evaluation of the domestic content of passenger vehicles and light trucks sold in the US market. We propose that given the deficiencies in using the 
AALA process to recognize the impact of the location of manufacture of the vehicle and the country of origin of its major components, there is an alternative formula that takes into account ancillary impacts of auto vehicle manufacturing and which provides a better indication of the true country of origin of the vehicle. This alternative paints a better picture of the actual economic impacts that the purchase of the vehicle has on the country where it is assembled and sold. (For more information, please contact: Frank DuBois, American University, USA: jetfrank@yahoo.com)

\section{Country of Origin in a Digital Context \\ Shane Mathews, Queensland University of Technology \\ Hsiu-Li Chen, Ming Chuan University}

We propose that due to a convergence in global consumer trends because of the Internet and the emergence of new major export markets there is a need to re-evaluate country of origin in this context. We argue that Internet technologies through Internet marketing activities have the potential capacity to dilute negative country of origin (COO) bias. That is, the Internet is making purchasing an international product easier and as a consequence international consumer behavior is more dynamic. Moreover, growing international country export markets such as China and India are plagued with a negative COO bias. Our proposition is that as the Internet evolves Internet marketing activities will give those emerging country brands with negative country of origin an opportunity for a more level playing field. We propose an experimental design to assess the potential impact of the Internet on negative $\mathrm{COO}$ bias. (For more information, please contact: Shane Mathews, Queensland University of Technology, Australia: sw.mathews@qut.edu.au)

\section{Strong Brand from Consumers' Perspective: A Cross-Cultural Study \\ Chenchen Li, Nanyang Technological University \\ Dongmei Li, Nanyang Technological University \\ Chi-yue Chiu, Nanyang Technological University \& The Chinese Academy of Social Sciences \\ Siqing Peng, Peking University}

The present research investigates cross-cultural differences in traits associated with brand strength evaluation and the mechanism underlying these cultural differences. Using data from US and China, we find that American consumers judge brands with personal traits to be stronger than those with relational traits, while Chinese consumes show a reverse pattern. Furthermore, cultural differences in brand strength evaluation are mediated by consumers' internalized preferences rather than what they perceived to be shared within the culture. Cultural differences in brand strength evaluation are salient only when consumers rate brands that are connected with their self-concept. (For more information, please contact: Chenchen Li, Nanyang Technological University, Singapore: lich0017@e.ntu.edu.sg)

\section{Antecedents of Brand Loyalty: A Cross-National Study in the Wine Sector Constanza C. Bianchi, Universidad Adolfo Ibanez \\ Christian Felzensztein, Universidad Adolfo Ibanez}

The wine industry has become fiercely competitive worldwide, and consumers are increasingly exposed to a wider range of wines in retail outlets. Thus, wineries need to develop and build consumer loyalty toward their brands. The authors empirically test a model of brand loyalty in two culturally different emerging wine producing countries: Australia and Chile. The conceptual model considers brand loyalty as the dependent variable and the following independent variables: brand satisfaction, brand image, wine knowledge and wine experience. Hypotheses are tested with structural equation modeling (SEM). Findings show that brand satisfaction is the most important driver of brand loyalty in the wine sector of both countries. The result also shows differences in the antecedents of brand satisfaction between Australia and Chile. (For more information, please contact: Constanza C. Bianchi, Universidad Adolfo Ibanez, Chile: constanza.bianchi@uai.cl) 
On Advancing the Conceptual and Methodological Foundations in Cross-Cultural Consumer Behavior: Where Have We Been and Where Do We Go From Here?

Attila Yaprak, Wayne State University

Hugh M. Cannon, Wayne State University

Daniel Friesen, Wayne State University

Cross-cultural consumer behavior has been a revered topic in international marketing. Yet, the stock of knowledge in this domain has lacked sufficient theoretical grounding and measurement and validation difficulties. Much of the earlier conceptual work lacked sufficient empirical support for research findings, and the methodological problems faced in conducting cross-cultural research have not been adequately addressed. Further, research issues embedded in newly developing contexts, such as those in emerging market settings, have not been investigated fully. This paper focuses on these issues and offers avenues for future research. (For more information, please contact: Attila Yaprak, Wayne State University, USA: attila.yaprak@wayne.edu)

\section{Exploring the Impact of Illicit Tobacco Trade on Smoking Demarketing Efforts \\ Alan S. Zimmerman, City University of New York-College of Staten Island \\ Peggy E. Chaudhry, Villanova University}

The concept of demarketing essentially means using the marketing mix elements to try to reduce demand on a permanent or temporary basis. Demarketing has been applied in attempts to reduce alcohol consumption, fight obesity or reduce smoking. In the latter area, studies have shown various levels of effectiveness for some of the marketing mix tools but product and price have produced limited effects. The most comprehensive study by Shiu et al concludes that product did not influence attitudes toward smoking. We propose that the results described may be caused by the availability of illicit, contraband or counterfeit cigarettes. The institutional environment makes these kinds of cigarettes more attractive to consumers and draws in illicit traders. This highly profitable market produces over 600 billion illicit cigarettes each year or more than $10 \%$ of the global cigarette market. This paper describes the specific institutional aspects contributing to consumer demand and attractiveness for traders. (For more information, please contact: Alan S. Zimmerman, City University of New York-College of Staten Island, USA: alan.zimmerman@csi.cuny.edu)

\section{Consumer-Driven Adaptation in International Markets: A Practices Perspective on Beauty Product Consumption Melissa Archpru Akaka, University of Denver \\ Daniel W. Baack, University of Denver \\ Susan M. Mudambi, Temple University}

The adaptation of marketing mix activities has been a growing concern in international marketing for the past several decades. Recent calls for stronger theoretical foundations highlight the fragmented nature of research on the topic, and raise issues with values-based models of culture and firm-centric views on consumer value creation. To address these issues, we introduce an alternative framework. The proposed framework reconsiders the nature of culture and focuses on a consumer-centric perspective on value. In particular, we introduce a practices approach as a more dynamic means of conceptualizing culture. This approach draws on consumer culture theory and sociology of culture, and centers on the way in which the enactment of consumption practices contribute to the co-creation of cultural meanings, as well as to value. We investigate research questions using a qualitative, empirical study of beauty product consumption. Interviews of consumers from two distinct countries and cultures, Taiwan and Indonesia, focus on beauty product practices and meaning. Our findings reveal that consumers across different cultures draw on varying repertoires of global, regional and local consumption practices to create value for themselves. Through the integration of these practices consumers then contribute to the co-creation of collective, but distinct, meanings of beauty. (For more information, please contact: Daniel W. Baack, University of Denver, USA: dbaack@du.edu) 


\section{Session: 2.5.1 - Special Session}

\section{Peter J. Buckley and Mark Casson AIB Dissertation Award Presentations}

Presented On: July 5, $2013-16: 45-18: 00$

Chair: Alan M. Rugman, University of Reading

Presentations by the 2013 Peter J. Buckley and Mark Casson AIB Dissertation Award Finalists. The finalists are listed alphabetically. The Peter J. Buckley and Mark Casson AIB Dissertation Award is being sponsored by Henley Business School, University of Reading and the Centre for International Business, University of Leeds (CIBUL).

Internationalization and Performance of Emerging Market Firms: Institutional Embeddedness in Advanced Economies (Ph.D. Awarded by York University)

Hamid Akbari, UOIT

No abstract available. (For more information, please contact: Hamid Akbari, UOIT, Canada:

hamid.akbari@uoit.ca)

Three Essays on Foreign Entrepreneurs (Ph.D. Awarded by University of Toronto)

Elena Kulchina, Duke University

No abstract available. (For more information, please contact: Elena Kulchina, Duke University, USA:

elena.kulchina@duke.edu)

Networks Of Influence: Implementing Politically Sustainable Multinational Stakeholder Strategies (Ph.D.

Awarded by University of Pennsy/vania)

Lite Nartey, University of South Carolina

No abstract available. (For more information, please contact: Lite Nartey, University of South Carolina, USA:

lite.nartey@moore.sc.edu)

British Multinationals in South East Asia: Strategy, Subsidiaries and Performance (Ph.D. Awarded by University of Reading)

Quyen Nguyen, University of Reading

No abstract available. (For more information, please contact: Quyen Nguyen, University of Reading, United Kingdom: t.k.q.nguyen@henley.ac.uk)

Essays on International Non-market Strategy and the Political Economy of Environmental Regulation (Ph.D. Awarded by Harvard University)

Sanjay Patnaik, George Washington University

No abstract available. (For more information, please contact: Sanjay Patnaik, George Washington University, USA: spatnaik@gwu.edu) 
Session: 2.5.2 - Panel

Track: Track: 9 - Cross-Cultural Management and HRM

\section{Women in Business in Turkey}

Presented On: July 5, $2013-16: 45-18: 00$

Chairs: Gabriele Suder, SKEMA and Janet Y. Murray, University of Missouri-St. Louis

Panel Sponsored by WAIB, Women in the Academy of International Business.

Panelists:

Gülden Türktan, President, KAGIDER

Hande Eskinazi, Director of Corporate Strategy and Business, Pfizer

Renin Varnali, Bogazici University

Melsa Ararat, Sabanci University

Aylin Kalafatoglu Kurtulus, Co-chairman, Ant Group Corporation

This panel provides professional guidance on the challenges and opportunities faced by women in business.

Three business executives and two academics in Turkey will bring their unique perspectives in discussing critical issues faced by women in the business world. (For more information, please contact: Janet $Y$. Murray,

University of Missouri-St. Louis, USA: murrayjan@umsl.edu)

\section{Session: 2.5.3 - Special Session}

Track: Track: 12 - Economic Geography and Value Chains

\section{Showcase Panel on Economic Geography}

Presented On: July 5, $2013-16: 45-18: 00$

Chair: Mark Lorenzen, Copenhagen Business School and Editor, Industry and Innovation

Discussant: Ram Mudambi, Temple University

Panelists:

Michael Storper, UCLA and London School of Economics

Ron Boschma, Utrecht University

Peter Maskell, Copenhagen Business School

Neil Wrigley, University of Southampton and Editor, Journal of Economic Geography

(For more information, please contact: Ram Mudambi, Temple University, USA: rmudambi@temple.edu) 
Session: 2.5.4 - Panel

Track: Track: 5 - MNC Management and Organization

\section{Bridging History (back) to Contemporary International Business: Hidden Answers in History to Today's Puzzles Facing the Multinationals}

Presented On: July 5, $2013-16: 45-18: 00$

Chair: Victor Zitian Chen, University of North Carolina, Charlotte

Discussant: Farok J. Contractor, Rutgers University

Panelists:

John Cantwell, Rutgers University

Geoffrey Jones, Harvard Business School

Karl Moore, McGill University

Oded Shenkar, Ohio State University

Mira Wilkins, Florida International University

We seek to link lessons and conclusions from business history to contemporary puzzles facing IB scholars and practitioners. This echoes Jones and Khanna's (2006: 453) JIBS article, which argued that "IB should evolve its rhetoric from 'history matters' to how it matters". The purpose of this panel is ambitious and theoretically- and practically relevant: we will attempt to explore how history can help to build resilient and evolutionary IB theories to explain contemporary happenings, and prepare contemporary IB practitioners for seeming surprises. The panel could trace the emergence of multinationals, starting with the Assyrian Kingdom shortly after 2000 B.C., and explore the differences and similarities between historical- and contemporary multinationals using theory gaps to explain repeated similarities as well as differences. Lastly, the panel can see how history can inform or alert us about surprises and discontinuities that appear to crop up from time to time in the world economy. (For more information, please contact: Victor Zitian Chen, University of North Carolina, Charlotte, USA: emgp.editor@gmail.com)

\section{Session: 2.5 .5 - Competitive}

Track: Track: 7 - Emerging Economies

\section{Doing Business in Latin America}

Presented On: July 5, $2013-16: 45-18: 00$

Chair: Alvaro Cuervo-Cazurra, Northeastern University

\section{Export Intensity, Scope, and Destinations: Evidence from Brazil \\ Dirk Michael Boehe, University Of Adelaide \\ Gongming Qian, Chinese University of Hong Kong}

How do distinct dimensions of geographic export diversification, i.e. export intensity, export scope and export destinations, interact in determining firm performance? Drawing on an institutional and resource-based conceptual lens and using panel data from Brazilian exporters, our results suggest that the negative relationship between export intensity and performance turns positive when exporters from emerging economies increase their exports to a limited number of other emerging economies. Our results imply that much of the controversy in the geographic diversification - performance literature could be clarified by adopting a three-dimensional geographic export diversification construct. (For more information, please contact: Dirk Michael Boehe, University Of Adelaide, Australia: dirkmichael.boehe@adelaide.edu.au) 


\title{
Exploring the Emerging Economy Context: SMEs and Social Capital in Brazil \\ Ruth Clarke, Nova Southeastern University \\ Ramdas Chandra, Nova Southeastern University \\ Marcilio Machado, Fucape - Fundação Capixaba de Pesquisa
}

This exploratory, empirical study reports on social capital in a commodity-based industry in an emerging market context. We examine whether Brazilian entrepreneurs and SMEs in the Stone industry experience contextual normative pressure sufficient to retard or promote the growth of social capital. Further we link social capital development to internationalization and performance. We find that there is a lack of social capital development and propose that this is related to the country context of Brazil and the stage of development along the industry value chain. We begin to answer the question, essentially, are the benefits of social capital universally implementable or does societal context for social capital retard usage? We suggest propositions for future study. (For more information, please contact: Ruth Clarke, Nova Southeastern University, USA: rclarke@nova.edu)

\section{A Cluster Analysis of FDI in Latin America \\ Rosa Portela Forte, University of Porto \\ Nancy Santos, University of Porto}

Over the past 30 years there has been a strong growth of Foreign Direct Investment (FDI) worldwide. Since 1982 the global FDI flows have increased almost 30 times, thus contributing to the increasing globalization of the world economy. In the context of Latin American FDI flows have also grown and there is an increase in the importance of this region as recipient of FDI. Individually, however, countries have different levels of performance. In the last five years (2007-2011, average values) Brazil was the largest recipient of FDI, followed by, Mexico, Chile, Panama and Colombia. Since there are few articles focusing on FDI in Latin America, this study analyzes empirically the FDI in this region. Using the cluster analysis, a technique rarely used in studies about FDI, we analyze the regional distribution of FDI in Latin America. Cluster analysis allows grouping countries according to their similarity regarding a data set of variables directly related to the determinants of FDI. The empirical findings of the analysis show a level of economic development quite different among Latin American countries, allowing for three clusters that show statistically significant differences in several variables related to FDI determinants, such as market size, economic stability, quality of infrastructure, human capital and political stability. (For more information, please contact: Rosa Portela Forte, University of Porto, Portugal: rforte@fep.up.pt)

\author{
Reverse Innovation as an Inducer of Centers of Excellence in Foreign Subsidiaries of Emerging Markets \\ Felipe Mendes Borini, ESPM/SP \\ Sidney Costa, USP \\ Maite Bezerra, ESPM/SP \\ Moacir de Miranda Oliveira Junior, USP
}

The novelty of this article lies in assessing the impact of autonomy and integration on reverse innovation of foreign multinational companies located in emerging markets, specifically, in Brazil. Furthermore, this article hypothesizes that reverse innovation is related to the establishment of centers of excellence for subsidiaries in emerging markets and that autonomy and integration are critical for centers of excellence, albeit indirectly, that is, intermediated by reverse innovation. Structural equation modeling is used for that purpose. The respondents were subsidiary CEOs or any board member designated by that executive of 172 companies (17\% response rate). The subsidiaries are from North America, Western Europe, and Japan. The current article shows the importance of reverse innovation for the establishment of centers of excellence in foreign subsidiaries in Brazil. Moreover, it indicates that subsidiaries must have autonomy for their initiatives conciliated with integration with the headquarters, which ensures a minimum strategic alignment so that reverse innovation occurs. The article shows that the rationale of centers of excellence in emerging markets is different than the rationale of centers 
of excellence in developed countries. Second, this study demonstrates the importance of the structure of multinational corporations for the advent of reverse innovation. (For more information, please contact: Felipe Mendes Borini, ESPM/SP, Brazil: fborini@espm.br)

\section{Session: 2.5.6 - Competitive}

\section{Track: Track: 2 - Marketing and Supply Chain}

\section{Supply Chain and Sales for Effective Customer Relationships and Growth}

Presented On: July 5, $2013-16: 45-18: 00$

Chair: Brenda Sternquist, Michigan State University

Responsiveness in Global Supply Chains

Ayman Omar, American University

Elizabeth Davis-Sramek, University of Louisville

Richard Germain, University of Louisville

The focus of this research is to investigate how supplier contextual factors, including risk and distance, can impact both a supplier's ability to be responsive to unexpected changes and to the manufacturer's ability to differentiate its offerings through operational service effectiveness. Findings indicate a negative association between cultural distances (national and corporate) and supplier's responsiveness. Perceived country risk was found to negatively impact operational service performance. Managerial and theoretical implications are discussed as well as area for future research. (For more information, please contact: Ayman Omar, American University, USA: omar@american.edu)

\section{Social Influence and Contextual Utilization of CRM Technology in an International Field Sales Organization} David S. Baker, University of Louisiana at Lafayette

This research addresses social normative issues related to institutional implementation of CRM technology within multinational sales organizations. Building from related inter-disciplinary theories, it identifies social influence related variables that are linked to contextual utilization of technology within an international business-to-business sales force. Four of seven primary hypotheses were supported, predicting over $65 \%$ of the variance in the research model. Effort expectancy, uncertainty avoidance, collective performance expectancy, and internal/organizational social influence all showed antecedent significance to contextual utilization. Technological self efficacy, external/customer social influence, and individual performance expectancy all showed bi-variate correlative significance with contextual utilization, but did not show incremental independent significance within the theorized model. Key Terms: Culture, Sales, Technology, Social Influence, Normative, Marketing, CRM, Sales Force Automation (For more information, please contact: David S. Baker, University of Louisiana at Lafayette, USA: dbaker@louisiana.edu)

\section{A Review of Literature Relevant to International Selling and Sales Management \\ Wyatt Austin Schrock, Michigan State University \\ Douglas E. Hughes, Michigan State University}

Although researchers have recently taken steps towards improving our understanding of international selling and sales management, what we know is limited. Accordingly, the authors review literature that helps to answer the following question: "What do we know that might apply to international selling and sales management?" A review of 90 articles revealed four research clusters or themes: sales force management, buyer-seller relationships, drivers of sales performance and global account management. In providing a somewhat fine- 
grained review of a wide range of recent research relevant to international selling and sales management, the literature review is the first of its kind. (For more information, please contact: Wyatt Austin Schrock, Michigan State University, USA: schrockw@msu.edu)

\author{
How to Increase the Effects of Logistics on International Trade? \\ Paloma Bernal Turnes, Georgetown University \\ Ricardo Ernst, Georgetown University
}

This article explores how investments in logistics infrastructures have a direct effect on international trade. We measure and test the association between logistics capacity and trade flows is also mediated by logistics efficiency, and by the time and the costs to reach market destination. These indirect effects represent $27.89 \%$ of the variability of international trade. On the other hand, the investment in logistics infrastructures has a strong direct effect $(62.11 \%)$ on international trade. Both direct and indirect effects increase international trade $33 \%$. This study refines existing theorical understanding by supporting the economic perspective of international trade, and the variables that better promote international goods flows. (For more information, please contact: Paloma Bernal Turnes, Georgetown University, USA: pb737@georgetown.edu)

\title{
Session: 2.5.7 - Competitive
}

\section{Track: Track: 8 - Developing Country MNCs}

\section{Internationalization and Performance of Developing Economy MNEs}

Presented On: July 5, $2013-16: 45-18: 00$

Chair: James P Johnson, Rollins College

Internationalization and Performance of Emerging Multinationals: The role of Clustering and Certification Rajesh Upadhyayula, Indian Institute of Management Kozhikode

Amit Karna, EBS Business School

Karthik Dhandapani, Indian Institute of Management Ahmedabad

Although emerging market multinational corporations (EMNCs) are growing at a rapid rate, little is known about their internationalization process and the impact it has on the firm performance. We adopt an institutional arrangement lens to investigate the internationalization drivers of EMNCs that act as a credible signaling mechanism to overcome the lack of credibility enhancing institutions within their home country environment. We examine cluster presence and quality certification as factors affecting EMNC internationalization, and their moderating effect on firm performance of internationalized firms. We analyze panel data from 174 Indian IT firms during 1997-2002 period to investigate how these EMNCs internationalized and what role did their cluster presence and quality certifications play in their internationalization. The results show that certification plays a positive role in internationalization, but negatively affects firm performance. Interestingly, it has a strong moderating effect on performance effects of internationalization. Further, although clustering is not associated with either internationalization or performance, it negatively moderates the internationalization effect on performance. Our findings advance the theory on internationalization of EMNC by proposing a larger role for certification as a signaling and an efficiency building mechanism. (For more information, please contact: Amit Karna, EBS Business School, Germany: amit.karna@ebs.edu) 
Addressing the Heterogeneity of Results in Internationalization-Performance Studies on Emerging Markets: $A$

Meta-Analytic Approach

Jose Mauricio Geleilate, Florida International University

Marcelo J. Alvarado-Vargas, Florida International University

Sumit Kundu, Florida International University

Ronaldo Couto Parente, Florida International University

The growing importance of emerging markets has promoted many studies assessing the relationship between internationalization and performance of firms from these countries. Not differently from the results of similar researches with firms from developed countries, studies involving emerging country firms have showed a varying number of results. Assessing this heterogeneity, we used a meta-analytic approach focused in a sample of 58 studies addressing emerging markets. Many different study-related characteristics and also countryrelated aspects were found to be responsible for this multitude of results. The overall effect of internationalization showed to be slightly positive, with the predominance of an inverted-U shape. (For more information, please contact: Jose Mauricio Geleilate, Florida International University, USA: jgele002@fiu.edu)

\section{The Effects of Internationalization on Composition of Debt \\ Vinicius Silva Pereira, Fundacao Getulio Vargas \\ Hsia Hua Sheng, FGV-EAESP}

This study aims to analyze the effects of internationalization on the types of debt of Latin American multinationals. Data from 16 types of debts with differences such as contract terms, maturity and financing agent were collected and analyzed between 2007 and 2011. The results indicate that the degree of internationalization: (i) increases the level of debt of almost all debts provided by financial agents (banks), as maintained by the Upstream-Downstream Hypothesis and reduces debts provided by non-financial agents (trade-credit ) as maintained by the theory of financial constraint; (ii) has no effect on the maturity of debt; (iii) differently from what was expected, does not increase debt via the national development bank (incentived loans), and (iv) was relevant to alter the composition of debt of multinationals compared to domestic companies. Finally, the form of entry into internationalization does not affect the composition of debt. (For more information, please contact: Vinicius Silva Pereira, Fundacao Getulio Vargas, Brazil: viniciuss56@gmail.com)

\section{BRIC Investor Targeting by European Investment Promotion Agencies: Comparative Analysis Sergey Filippov, Delft University of Technology}

MNEs from emerging economies are becoming significant players in the globalised world economy. As the volume of FDI from emerging economies is rising, and emerging multinationals increasingly seek access to markets and assets in advanced developed economies. The member states of the European Union are becoming attractive destinations for such outward FDI of investors from Brazil, Russia, India and China (BRIC). In this situation, the host governments feel the need to define their responses and policies, particularly, because the effects of outward FDI from emerging markets are often contradictory. The objective of this paper is to examine the strategic stance of national European investment promotion agencies (IPAs) towards emerging multinationals originating from BRIC economies. It inventorises promotion efforts of these IPAs tailored to the BRIC companies, such as specifically customised information provision on IPA websites and the presence of IPA representative offices in the BRIC region. On the basis of comparative analysis, it offers reflections on the challenges and opportunities presented by emerging multinationals to European host countries. (For more information, please contact: Sergey Filippov, Delft University of Technology, Netherlands: s.filippov@tudelft.nI) 


\title{
Session: 2.5 .8 - Competitive
}

\section{All the World is My Classroom - Effective Management Strategies}

Presented On: July 5, $2013-16: 45-18: 00$

Chair: Bernard Wolf, York University

Looking Liability in the Eye: Managing Risk in International Study Tours

James M. Hagen, Hamline University

David M. Berg, Hamline University

When risks and potential liabilities inherent in study abroad programs are not adequately addressed, they can diminish a university's study abroad offerings and success. For example, faculty members sometimes decline opportunities to lead study abroad tours when they are unclear of their personal exposure to legal and financial liability. We identify risks and potential liabilities and ways they can be be reduced, mitigated and/or insured against. The target audience for this presentation is individual faculty members who may be interested in leading international study tours, as well as international program directors. (For more information, please contact: James M. Hagen, Hamline University, USA: jhagen05@hamline.edu)

\author{
Managing Exposures to Currency Risk at Michigan State University (Currency Risk Management for an IB \\ Audience) \\ Kirt C. Butler, Michigan State University
}

Michigan State University is a world leader in study abroad, with nearly 3,000 students participating in 300 programs in more than 60 countries and on all seven continents. As a consequence, MSU has significant exposures to foreign exchange risk (particularly to the euro, the British pound, and Australian dollar) as it pays for its programs' expenses. The case takes the perspective of MSU's Director of Study Abroad as he considers his institution's exchange rate exposures and what to do about them. The case provides a non-technical review of the various types of currency risk exposure and develops the case for managing transaction exposures to currency risk. Topics covered include exchange rate volatility, value-at-risk, currency forward contracts, and currency risk management. (For more information, please contact: Kirt C. Butler, Michigan State University, USA: butler@msu.edu)

\section{Using Adobe Connect To Engage Globally Dispersed MBA Students in International Business Courses P. Roberto Garcia, Indiana University}

The reality of business education today is that many of us are facing the necessity to teach our courses online. Thus, business professors today need to be familiar with the tools and techniques available for online education. The session will feature a useful introduction to the use of Adobe Connect Web conferencing tool to stimulate engagement and interaction among online students. The current proposal describes an approach to teaching online MBA courses in a way that attempts to overcome many of the disadvantages of e-Learning. The particular issue to be addressed will be the lack of student interaction which might lead to a feeling of isolation and a perception of stale and boring content and materials. The approach described here has been developed over a 10 year span at a top-20 MBA program and has resulted in a high level of engagement among scores of online graduate students. Students will benefit by having more faculty using a more engaging and stimulating set of teaching techniques in their online courses. Instructor will benefit by the possibility of incorporating several tools and tips for a more effective and enjoyable online teaching experience. (For more information, please contact: P. Roberto Garcia, Indiana University, USA: prgarcia@indiana.edu) 
A Global Classroom? A Multi-Method Evaluation of Effectiveness of International Collaboration Exercises in International Management Education

Vas Taras, University of North Carolina, Greensboro

Alexander Bode, Technische Universität Darmstadt

Alfredo Jiménez, University of Burgos

Anja Maria Schuster, University of St. Gallen

Charles Wankel, St. John's University

Clara Lei, Institute for Tourism Studies

Dan Caprar, University of New South Wales

Daniel Rottig, Florida Gulf Coast University

Erika Vaiginiene, Vilnius University

Fabian Jintae Froese, Georg-August-University Goettingen

Fang Zhao, American University of Sharjah

Hanoku Bathula, AIS St. Helens

Michael Minor, University of Texas Pan American

Nilay Yajnik, Narsee Monjee Institute of Management Studies

Norhayati Zakaria, University of Wollongong in Dubai

Victor Zengyu Huang, Zayed University

Rico Baldegger, University of Applied Sciences Western Switzerland

Xavier Ordenana, ESPAE Graduate School of Management

Based on the X-Culture project, an international collaboration exercise involving over 4,000 students from over forty countries, the study evaluates the effects of experiential multi-country team-based projects in International Management education. Multi-source longitudinal data were used, including student and instructor feedback, course evaluations, assessment of changes in knowledge, attitudes and behaviors following the experiential project, and various indicators of individual and team performance. The effectiveness of this experiential learning approach was evaluated at the levels of reactions, learning, attitudes, behaviors, and results. The findings show positive outcomes on each level, but also reveal challenges and limitations of using international virtual collaboration projects. We conclude with guidelines for incorporating international collaboration exercises in the International Management curriculum. (For more information, please contact: Vas Taras, University of North Carolina, Greensboro, USA: v_taras@uncg.edu)

\section{Session: 2.5.9 - Competitive}

\section{Track: Track: 10 - Economics, Finance and Accounting}

\section{Financial Markets: Reforms and Foreign Exchange Exposure}

Presented On: July 5, $2013-16: 45-18: 00$

Chair: John W. Goodell, University of Akron

The Role of Labor in the Privatization Design: International Evidence from the Choice between Public and Private Capital Markets

Mohamed Belkhir, UAE University

Hamdi Bennasr, King Saud University

We argue that the extent to which the labor force is legally protected impacts not only the extent of privatization as shown by Subramaniam and Megginson (2011), but also its choice of whether to privatize through share issues in the public stock exchange (SIPs) or through asset sales to a small group of investors. In particular, we advance several arguments suggesting that strong legal protection of labor shapes the costs and benefits of workers, investors, and governments in a way that makes SIPs more likely than asset sale privatizations. We test this hypothesis on a sample of 4,400 privatization transactions, which occurred between 
1989 and 2008 in 55 countries. Using various measures of labor protection, and controlling for political, legal, and economic factors, we find that the stronger is the legal protection of labor and the more likely that a government uses SIPs. We also argue that the effect of labor protection on the privatization method depends on the extent to which investors are legally protected. Specifically, we test and find evidence in favour of the hypothesis that strong labor protection is not likely to lead to SIPs in institutional environments where investor protection is weak. (For more information, please contact: Mohamed Belkhir, UAE University, United Arab Emirates: m.belkhir@uaeu.ac.ae)

\title{
Foreign Exchange Exposure and its Determinants in Emerging Markets: Firm Level Evidence from India Ekta Sikarwar, Indian Institute of Management Indore Roopak Kumar Gupta, Indian Institute of Management Indore
}

We examine the unanticipated exchange rate exposure and its determinants for a sample of 361 Indian non financial firms over the period April 2006-March 2011 by using Multi-factor model. We extend the Multi-factor model with respect to the unique market factors of emerging economies having managed float. We use central bank intervention as one of the macro-economic factors influencing the exchange rate. Our results report that only 13 percent of firms are having significant exposure to exchange rate risk and overall firms incur loss from an appreciation of the Rupee which indicates that most of the Indian firms are exporters. We find that hedging and ownership structure are significant determinants of exchange rate exposure. The findings have important implications for managers to understand the impact of unexpected exchange rate changes on the value of firm and to frame their exchange rate risk and hedging policies accordingly. (For more information, please contact: Ekta Sikarwar, Indian Institute of Management Indore, India: f10ektas@iimidr.ac.in)

\author{
Liability of Foreignness on International Bond Markets: A "Nested Legitimacy" Framework \\ Gregor Binder, WU Vienna \\ Igor Filatotchev, City University London \\ Jonas F. Puck, WU Vienna
}

The accelerating pace of global capital market integration has provided new opportunities for firms to raise capital abroad through global debt issues. In this paper, we expand the Liability of Foreignness (LOF) research beyond the product market domain to include liabilities faced by firms attempting to secure resources in foreign bond markets. We identify key differences between product and bond markets and suggest that performance of the foreign firm's bond issue in the U.S. will defined, inter alia, by firm-, industry- and country-level factors that affect the firm's legitimacy among the U.S. investors. We suggest and empirically test a "nested legitimacy" framework that includes firm-level signaling, industry isomorphism, and institutional characteristics of the firm's home country as legitimization factors that jointly affect investor perceptions of the quality of bond issue. We also outline directions for further theoretical and empirical development of the capital market LOF research. (For more information, please contact: Gregor Binder, WU Vienna, Austria: gregor.binder@wu.ac.at)

\section{Session: 2.5.10 - Competitive}

Track: Track: 1 - Institutions, Governance, and CSR

\section{Performance Effects of Operating across Diverse Institutional Environments}

Presented On: July 5, $2013-16: 45-18: 00$

Chair: Lorraine Eden, Texas A\&M University

The Transferability of Political Risk Management Experience Across Borders Jennifer Oetzel, American University

Chang Hoon Oh, Simon Fraser University 
Research on experiential learning has increasingly emphasized the need to better understand how context affects the value of knowledge and when experiential learning can be transferred across borders. We address this gap by investigating when corporate-level risk management experience can be leveraged across borders and when experience needs to be country- (context-) specific to be valuable. We test our hypotheses using a unique multi-source panel dataset of 380 large multinational corporations (MNCs) from 29 home countries and their subsidiaries in 124 host countries, with a total of 41,923 observations over a ten-year period, 1999-2008. Results show that while both country- and corporate-level experience provide learning advantages, countryspecific experience was significantly more valuable than corporate-level experience in responding managerially to armed conflicts involving the host country government. (For more information, please contact: Jennifer Oetzel, American University, USA: oetzelj@american.edu)

\section{Does Foreign Investment Carry Baggage from Home? \\ Reid Click, George Washington University \\ Robert Weiner, George Washington University \\ Yujin Jeong, American University}

Literature on international investment often examines institutions in host countries, but seldom considers the role of institutions in the home country. Recent growth of MNEs from emerging-market countries has generated interest in viewing institutions in a broader perspective. We reconsider the new FDI paradigm in the literature, and propose a heterodox paradigm which offers a different perspective on the home country in crossborder investment. Using data on firms' acquisitions of petroleum reserves, we test whether foreign investment carries home-country institutions ("baggage from home") with it. We find that weak (strong) institutions in the home country reduce (increase) the amount that firms from that country are willing to pay for a petroleum reserve. (For more information, please contact: Reid Click, George Washington University, USA: rclick@gwu.edu)

\section{Product-Geographic Diversification and Firm Performance: The Moderating Effects of Home Country Institutions Brian Roy Tan, Nanyang Technological University Asda Chintakananda, Nanyang Technological University}

This paper investigates how country institutions of the home country influence the product and geographic diversification performances of firms. Specifically, we examine how political institutions, legal institutions, and social institutions shape the transaction costs and the development of the resources and capabilities of a firm to influence the firm's performance abroad. We propose that institutional dimensions such as political stability positively influences, while the effectiveness of the legal institutions and some aspects of social institutions negatively influence firms' diversification-performance relationships. Empirical analysis on over 12,000 firms across 22 countries provided support for the hypotheses. (For more information, please contact: Asda Chintakananda, Nanyang Technological University, Singapore: asda@ntu.edu.sg)

\section{Why Do Publicly Listed Family Firms Perform so Differently across Emerging Markets? An Institution-based Theory and Meta-Analytic Test \\ Patricio Duran, University of South Carolina \\ Pursey P.M.A.R. Heugens, Erasmus University \\ Tatiana Kostova, University of South Carolina}

We provide an institution-based theory explaining the substantial performance differences of publicly-listed family firms (PFFs) across emerging economies. We test this theory through a meta-analytic study, drawing on a dataset of 1,401 effect sizes harvested from 177 primary studies nested in 49 emerging economies. Our observation window spans the crucial period between 1973 and 2011, in which many emerging markets 
underwent rapid institutional changes. While our results show a small but significantly positive overall association between family control and firm performance, the cross-country variability in this relationship is very substantial. In order to explain this variability, we introduce a new typology for partitioning institutional effects, based on juxtaposing two salient dimensions of institutional development: formal versus informal, and constraining versus enabling institutions. This typology serves as the conceptual framework for our institutionbased theory, which integrates several substantive theories to explain the relative out- or underperformance of PFFs in specific emerging market contexts. Our findings show that the performance of PFFs is stronger where formal constraining institutions are underdeveloped, but poorer where formal and informal enabling institutions as well as informal constraining institutions are weak. Overall, results demonstrate that family firm profitability in emerging markets is predictably conditioned by institutional factors. (For more information, please contact: Patricio Duran, University of South Carolina, USA: patricio.duran@grad.moore.sc.edu)

\title{
Session: 2.5.11 - Interactive
}

\section{Track: Track: 3 - IB Theory, FDI, and Entry Mode}

\section{Theory Development on Internationalization and Multinational Management}

Presented On: July 5, $2013-16: 45-18: 00$

Chair: Anne Kristin Hoenen, WU Vienna

\author{
OLI 2.0: The OLI Is Dead. Long Live the OLI! \\ Xin Li, Copenhagen Business School \\ Jens Gammelgaard, Copenhagen Business School \\ Liyun Feng, A.P. Moller Maersk Group A/S
}

When it comes to the debate on whether the traditional theories of MNE and foreign direct investment (FDI) are adequate to account for the rise of emerging market (EM) MNEs, our stance is that although some EM MNEs do challenge the conventional wisdom, the existing theories are still valuable as they can explain the majority of the EM MNEs; and instead of rejecting them altogether, we should modify, extend and refine them by taking into account those challenging phenomena of the EM MNEs. In this paper, we take on Dunning's OLI paradigm and present our redefinition effort. In what we call the OLI 2.0 model, we transform Dunning's original model of ownership-location-internalization advantages into the new model of advantages of ownership, localization, and internalization. We argue this redefined model is more more coherent than Dunning's eclectice paradigm as a theory of FDI or MNE. We also explain that the various writings of Stephen Hymer, the intellectual father of the IB field, have covered, explicitly or implicitly, most of the ideas of the three elements of our OLI 2.0 model. Therefore, the new OLI 2.0 model can be treated as partly a rediscovery of Hymer's insights. (For more information, please contact: Xin Li, Copenhagen Business School, Denmark: xl.int@cbs.dk)

\section{On Borderless Management}

Fenghua Wang, Shanghai International Studies University

Zheng Fan, Shanghai International Studies University

Zhaoyi Ren, Shanghai International Studies University

Qingli Fan, St. Andrews University

Politics may have borders while information and economic globalization as well as regional economic integration bring about borderless corporations with borderless management that can surpass national borders. This paper examines the background against which borderless corporations exist, makes rectifications to the understanding of the concept of borderless management, and proposes its strategic path and implementation framework. The paper also analyses the case of borderless management in Motorola. (For more information, please contact: Fenghua Wang, Shanghai International Studies University, China: wangfenghua@shisu.edu.cn) 
The Variations of Liability of Foreignness: Examining the Effect of the Alignment between the Firm Resources and the Environmental Factors

Alexey V. Semenov, Saint Louis University

Hadi Alhorr, Saint Louis University

While liability of foreignness (LOF) associated with geographic diversification strategies has been examined as a source of disadvantages that foreign firms face relative to local firms when operating in host country, the extent, to which the alignment between the firm resources and the environmental factors within the host country affect the intensity of the LOF experienced by firms, has not gained adequate attention in the literature addressing multinational operations in host countries. Building on the literature that examines performance of multinational enterprises (MNEs) and the alignment between firm resources and the environment, we argue that the alignment between firm-specific advantages (FSAs) and host country-specific advantages (CSAs) can also aid MNEs in reconfiguring their international strategies and their choice of host countries to minimize the extent of their inherited disadvantage of foreignness. As such, in this paper, we utilize the FSA/CSA matrix to explain why the differences in the levels of liability of foreignness exist and how the MNE can potentially reduce the costs associated with their foreignness by making systematic decisions for its internationalization strategy. (For more information, please contact: Alexey V. Semenov, Saint Louis University, USA: asemenov@s/u.edu)

China's Value Stream Approach to the Internationalization Process: Early Stages, Attempts to Go Global, and Returnees Localizing a "Third Way"

Weidong Xia, Florida International University

Mary Ann Von Glinow, Florida International University

Yingxia Li, Beijing Union University

Yannick Thams, Florida International University

Jun Yang, Florida International University

Ying Liu, Florida International University

International Business research has developed based on various theoretical assumptions over the years with a main focus on the direction from the developed world to the developing world. Many emerging economies, particularly China, have recently challenged these theoretical assumptions about how firms in today's world internationalize. Using the case of China, we present one in-depth clinical case study on one IT outsourcing service company to illustrate this new phenomenon of the value stream approach to internationalization. We conducted interviews, focus groups, visited numerous IT offshoring providers in High Tech parks across China, as well as government ministries, and have culled through policy statements for the last 3 decades regarding the IT industry. This process has taken five years to gather. We review relevant IB literature, anchor it in the context of China to illustrate how significant these changes are and how they fundamentally challenge extant theory. We propose a three-stage value stream model of the internationalization process and discuss key characteristics related to each stage. Our clinical case vividly illustrate the unique characteristics of the different value stages, particularly the third stage, and it is this third part of our investigation that adds significant value theoretically to the internationalization process. (For more information, please contact: Yannick Thams, Florida International University, USA: thamsy@fiu.edu)

What are the Sources and Characteristics of the Advantages of Business Group Affiliated Multinationals? Roderick Bugador, University of Reading

Extant literature on business groups (BGs) deals rigorously with ethnocentric view or home country explanation of their existence and growth. Today, this home country explanation alone is inadequate because many BGs are now operating as multinationals. I propose that the starting point in understanding the multinationality of BGs is through their advantages. But, I argue that the sources and characteristics of the advantages of BGs are 
different from the traditional FSAs. I posit that the advantages of BGs are the internal bundle of resources and capabilities derived from their distinct coordinating mechanisms. Through sharing and exploitation, these advantages are decomposed to affiliate firms and define their individual firm advantages. I conclude that these advantages have implications on their future mode of operations, performance and multinationality overtime. (For more information, please contact: Roderick Bugador, University of Reading, United Kingdom: r.bugador@pgr.reading.ac.uk)

\section{Towards a Institutions-based Understanding of IJV Performance Hemant Merchant, University of South Florida - St. Pete Len Trevino, Loyola University New Orleans Hui Zhu, Cape Bretton University}

We broaden theoretical and empirical understanding of international joint ventures by employing a framework that explicitly considers Scott's (2005) three 'institutional pillars' (regulative, normative, cognitive) to explain their performance. We hypothesize that because strategic organizational practices are meaning and value based (Kostova, 1999), the success of their transfer and ultimately in enhanced IJV performance is more likely to be influenced by constructs that reside in the cognitive and normative pillars than by those associated with the regulative pillar. Our results were consistent with this theoretical reasoning. Theoretical and managerial implications are discussed (For more information, please contact: Hemant Merchant, University of South Florida - St. Pete, USA: hmerchant@usfsp.edu)

\section{Advancing Process-Oriented Theories of IJV Exit: Empirical Evidence from an In-depth Case Study Michael C. Nippa, Freiberg University \\ Alexander Nemeth, Freiberg University}

Extant research on success factors of International Joint Ventures and especially determinants of IJV exit has produced numerous findings. Reviews, however, reveal debates regarding appropriate conceptualizations, lack of theory building, reliability and validity of measures, and inconsistent results. Taking up calls for advanced approaches we apply resource dependence as well as learning theory to develop a process-related IJV exit framework. Corresponding propositions are empirically tested based on 22 in-depth case studies. Based on our findings IJV learning theory is extended among others by identifying moderating factors such as IJV performance that lead to other IJV exit decisions than previously predicted. (For more information, please contact: Michael C. Nippa, Freiberg University, Germany: nippa@bwl.tu-freiberg.de)

\section{Session: 2.5.12 - Interactive}

\section{Track: Track: 1 - Institutions, Governance, and CSR}

\section{Strategy, Resources and Government}

Presented On: July 5, $2013-16: 45-18: 00$

Chair: Timothy Michael Devinney, University of Technology, Sydney

\section{A Resource Environment Model of Competitive Advantage: The Paradox of Environmental Embeddedness Heechun Kim, Georgia State University \\ Robert Hoskisson, Rice University}

Our study proposes a resource environment model (REM) of competitive advantage by unpacking the environmental origins of a firm's competitive advantage, which are strategic factor markets and institutions. In particular, a resource environment-based competitive advantage framework presented in our study draws 
insights from the notion of "the paradox of environmental embeddedness" of competitive advantage. The paradox lies in the fact that the same country resource environments that maximize a firm's probability of creating a competitive advantage can paradoxically also minimize its probability of sustaining an advantage. Another important aspect of our study is that, based on the combined effects of strategic factor markets and institutions, it proposes a typology of environmental paradox management strategies: capability leadership, influence leadership, capability-driven hybrid strategy, and influence-driven hybrid strategy. This typology that will be a function of dynamic capabilities and political influence in the resource environments enhances a contextualized understanding of how firms create and sustain a competitive advantage by managing the paradox of environmental embeddedness. It indicates that firms in a given industry in different countries pursue distinct strategies of creating and sustaining a competitive advantage rather than simply applying the single best strategy across countries. (For more information, please contact: Heechun Kim, Georgia State University, USA: heechunkim@gsu.edu)

\section{Historical Anchors Over Resources in International Strategy}

Silvio Luis de Vasconcellos, IENH - Instituicao Evangelica de Novo Hamburgo

Ivan Lapuente Garrido, UNISINOS - Universidade do Vale do Rio dos Sinos

Cyntia Vilasboas Calixto, Fundação Getulio Vargas (FGV - EAESP)

Jefferson Marlon Monticelli, UNISINOS - Universidade do Vale do Rio dos Sinos

Previous theoretical findings in organizational studies assure that resources must be reconfigured constantly to sustain competitive advantages. Also, there is a commonsensical that on global competition there are more variables to consider before making-decision. A range of reflections from industrial, organizational and institutional orders must be considered. An relevant link among these approaches is path dependence. We developed two case studies to observe how resources were reconfigured over time, according to the focus of Resource-based View (RBV), and how they were able to contribute or respond to strategic changes in regarding to international market issues. These two case studies took place in Brazil. We analyzed footwear exporters observing how they redefined their resources during juncture events. The results indicated that changing strategies for international business involves companies' history. Path dependence may accelerate or retard resource reconfigurations. Additionally, a theoretical model is proposed in order to promote further research. (For more information, please contact: Silvio Luis de Vasconcellos, IENH - Instituicao Evangelica de Novo Hamburgo, Brazil: silvio.v@ienh.com.br)

\section{Unveiling the Antecedents of International Diversification: An Agency Theory Approach Giovanni Battista Dagnino, University of Catania Claudio Giachetti, Ca' Foscari University of Venice Maurizio La Rocca, University of Calabria Pasquale Massimo Picone, University of Catania}

While various studies have developed hypotheses about the antecedents of international diversification drawing mainly on the resource-based view, the behavioral theory of the firm, and the transaction costs literature, a few studies have developed arguments based on the agency theory. Assuming agency theory perspective, this paper aims to detect the extent to which and under which corporate governance conditions firms choose to internationally diversify. In particular, using a sample panel of 167 Italian firms longitudinally evaluated over a 31-year period from 1980 to 2010, this study tests whether the firm's choice to spread operations in multiple foreign countries is conditioned by past free cash flow, debt, ownership concentration and growth opportunities. (For more information, please contact: Claudio Giachetti, Ca' Foscari University of Venice, Italy: claudio.giachetti@unive.it)

Strategizing by the Government: Industrial Policy and Sustainable Competitive Advantage Sergio Giovanetti Lazzarini, Insper Institute of Education and Research 
Despite the prevalence of active governmental policy devised to foster firms and industries in various countries, the link between industrial policy (IP) engendered by local governments and competitive advantage has received scant attention in strategic management. I propose a model where IP influences the accumulation and churning of resources and capabilities which can be flexible or specialized. I also introduce the concept of support-adjusted sustainable competitive advantage (SASCA), which occurs if a firm's observed economic performance is superior to the expected performance of competitors had they received the same array of policies. In my framework, SASCA is created by the interplay of three factors: global integration, geographical specificity, and governmental capability. Thus, the model enhances our understanding of the determinants of competitive advantage in a context of governmental intervention. (For more information, please contact: Sergio Giovanetti Lazzarini, Insper Institute of Education and Research, Brazil: sergiogl1@insper.edu.br)

\section{Inter-Governmental Organizations and Innovation Srividya Jandhyala, George Washington University Anupama Phene, George Washington University}

Intergovernmental Organizations (IGOs) - with countries as members - are important organizations that enable the integration of economic actors across countries. As IGOs channel information and facilitate exchange of knowledge, we posit that they influence national innovative output. We propose that the extent of connectedness of a country in the IGO network and the diversity of its network have a positive influence on national innovation. Moreover, we hypothesize that these effects are contingent on a country's complementary opportunities - the extent to which existing domestic knowledge and capabilities can be leveraged in other, as yet unoccupied domestic sectors. We use a panel dataset of 45 countries observed over 1996-2004 to test our hypotheses. We find that both, the extent of connectedness to the IGO network, and the diversity of the IGO network enhance national innovation. Domestic complementary opportunities act as a moderator weakening the effects of IGO network connectedness and diversity on national innovation. (For more information, please contact: Srividya Jandhyala, George Washington University, USA: srividya@gwu.edu)

\section{Shifting Paradigms in the Postnational Constellation: A Deliberative View on the Role of the Firm Ulf Henning Richter, Nottingham University}

In this article, I analyze the debate on the role of corporation in a globalizing world. I discuss the implications of globalization, in particular, the increasing power of multinational corporations (MNCs) and the rise of civil society. I provide a comprehensive historical overview of the appearance of MNCs as political actors. Finally, I outline why the concept of corporate responsibility remains blurry and highly debated. (For more information, please contact: Ulf Henning Richter, Nottingham University, China: ulf.richter@nottingham.edu.cn)

\section{Session: 2.5.13 - Interactive}

\section{Track: Track: 9 - Cross-Cultural Management and HRM}

\section{HRM Issues}

Presented On: July 5, $2013-16: 45-18: 00$

Chair: Jacob Eisenberg, University College Dublin

Job Resources and Employee Engagement: A Cross-National Investigation

Elaine Farndale, Pennsylvania State University / Tilburg University

The purpose of this study is to explore the moderating effect of national culture on the relationship between job resources and employee engagement. Questionnaire responses from 21,154 employees of a large multinational financial services corporation in Mexico, the Netherlands, Taiwan, and the USA are analyzed. Regression 
analyses and a study of effect sizes show that certain job resources (financial rewards, team climate, and participation in decision-making) positively influence engagement in all four countries. However, the study also shows the (subtle) distinctions between the strength of relationships between job resources and engagement per country which are explained through cross-cultural reasoning. The study highlights the importance of firms' awareness of the equivalence of constructs and their relationships across countries. (For more information, please contact: Elaine Farndale, Pennsy/vania State University / Tilburg University, USA: euf3@psu.edu)

\section{Job Satisfaction and Confidence of Asian Managers in Japanese MNCS Yoshitaka Yamazaki, International University of Japan}

I examined how job satisfaction rests on confidence in Asia. A total of 914 employees who participated in this study consisted of Japanese, Chinese, Hong Kong, Malaysian, and Thai managers who work as parent or host country nationals for a Japanese multinational corporation expanding Asian markets. I initially confirmed that a level of each key psychological variable: job satisfaction and confidence, significantly differed in those countries. As the entire group of the participated Asian managers, by controlling age, gender, tenure, past work experience, and management positions, results of regression analysis showed that confidence powerfully increased job satisfaction. Further, with regard to the individual five different areas in Asia, results in each country also illustrated the great effect of confidence on job satisfaction. Accordingly, in this study, I have led to a conclusion that this relationship between the foregoing two psychological variables tends to be universalistic rather than culturally contingent. (For more information, please contact: Yoshitaka Yamazaki, International University of Japan, Japan: yyama@iuj.ac.jp)

\section{Speaking Up or Striking Back? Exploring why Employees Respond Differently to Psychological Contract Breach in a Chinese Context \\ T.K. Peng, I-Shou University \\ Cheng-Chen (Timothy) Lin, National Pingtung University of Science and Technology}

Research in psychological contract breach (PCB) has gained momentum over the past decade or so. It is evident that individuals take action to restore equity when they perceive obligations unfulfilled by their employers.

Although employees' reacting negatively to unpleasant event such as PCB is well understood, the possibility that they may also react positively to PCB to remedy inequity is much less studied. Moreover, it is unclear how Chinese employees react to disappointing encounters in the workplace. To fill the voids, we proposed employees' traditionality, a deep-rooted Chinese cultural belief, as a potential moderator in their reactions to unpleasant events. Our analysis of data from 152 employees and 26 supervisors in Taiwan, collected at two points in time, largely supports the hypothesized effects. Specifically, we found that unmet expectations were conducive to deviance and sometimes to voice behavior. Furthermore, employees high on traditionality were prone to display less deviance and more voice behavior. Implications for theory and practice are addressed. (For more information, please contact: T.K. Peng, I-Shou University, Taiwan: tkpeng@isu.edu.tw)

\section{Extension of Meyer and Allen's Three Component Model of Organizational Commitment in South Korea Mannsoo Shin, Korea University \\ Gahye Hong, Korea University \\ Youngsam Cho, Korea University \\ Boine Kim, Sung Kyun Kwan University}

Meyer and Allen's (1991) three component model of organizational commitment has received widespread use, but little attempt has been made to examine the extent to which the twenty four item model provides sufficient cross-cultural validity in the East Asian context. Two studies were conducted to extend the Meyer and Allen's three component model with samples from South Korea. Besides their original Affective Commitment Scale (ACS), the Continuance Commitment Scale (CCS), and the Normative Commitment Scale (NCS), both the 
Benefit-related Commitment Scale (BCS) and the Relational Commitment Scale (RCS) newly developed were incorporated in the extended Meyer and Allen's commitment model to reflect unique cultural and institutional characteristics of Korean labor market. Study 1, conducted with 280 employees from four manufacturing organizations, showed strong evidence for convergent and discriminant validity of newly developed BCS and RCS as well as ACS, CCS, and NCS. Study 2, conducted with 341 employees from fifteen service organizations also provided strong support for the validity of the two additional commitment scales. Based on multiple regression analyses, both BCS and RCS were found out to be better antecedents of job satisfaction and turnover intention than CCS and NCS, showing predictive validity of both scales. Practical managerial implications were also discussed. (For more information, please contact: Mannsoo Shin, Korea University, Korea, South: shinms@korea.ac.kr)

\section{English Language Ability and Employees' Commitment to Globalization of the Firm Sachiko Yamao, University of Melbourne Tomoki Sekiguchi, Osaka University}

Based on theories of self-efficacy and social exchange, this study suggests that employees' self-perceived foreign language ability and HR practices that induce their language learning will contribute to their commitment to firms' globalization. We also suggest that perceived English language ability and internal career opportunity are important moderating factors that improve the relationship between language learning inducement practices and employees' commitment to firms' globalization. Utilizing the survey data collected from 770 organizational employees in Japan, we tested our hypotheses and found support for hypotheses. Implications gained from the current study are relevant for employee management in firms that are extending their businesses into countries where knowledge of local language makes a huge difference in achieving business goals. (For more information, please contact: Sachiko Yamao, University of Melbourne, Australia: syamao@unimelb.edu.au)

\section{Antecedents of Citizenship Behavior in Arab Employees in Kuwait \\ Paula S. Daly, James Madison University \\ Marion Owyar-Hosseini White, James Madison University \\ Mohammad E. Alloughani, James Madison University}

This study identifies antecedents of two specific dimensions of organizational citizenship behavior (OCB), altruism and civic virtue, in a sample of Arab employees working in Kuwait. Results indicate that a collectivist orientation is a significant predictor of altruism; and that procedural justice and job satisfaction are significant predictors of civic virtue. Additionally, respondents indicate that altruism and civic virtue behaviors are more likely to be perceived as in-role than extra-role behaviors by study participants. Findings provide additional insight into the relevance of OCB and its antecedents in organizations with Arab employees, and resultant implications for managerial processes and decisions. (For more information, please contact: Paula S. Daly, James Madison University, USA: dalyps@jmu.edu)

The Influence of Organizational and Family Expectations, Cross-Domain Technology Usage, and Attachment Style on Work-Family Conflict: A Study of Employees in China, Hong Kong, Mexico, and the United States Anne Marie Francesco, Hong Kong Baptist University Janice R. W. Joplin, Southern Illinois University Edwardsville Margaret Shaffer, University of Wisconsin-Milwaukee Theresa Lau, Hong Kong Polytechnic University

Our study considered the direct and indirect influence of the domain-specific antecedents of organizational and family expectations and cross-domain technology usage (CDTU) attachment style on bi-directional work-family conflict (WFC) using a sample of 451 employees from four countries. Organizational and family expectations 
were associated with higher WFC, but only CDTU with business associates was related to work interference with family (WIF). Counterdependency had a direct positive association with WFC. For overdependents, WFC increased along with CDTU, while for interdependents, family interference with work (FIW) went up as CDTU with family increased. Country moderated the relationship between CDTU with business associates and WIF; unlike the other three countries, there was no relationship between levels of CDTU with business associates and WIF in the U.S. There was also a significant interaction of family expectations by country for FIW. In China, FIW remained at the same level, regardless of family expectations; whereas in the other three countries there was a positive relationship. (For more information, please contact: Anne Marie Francesco, Hong Kong Baptist University, Hong Kong, SAR-PRC: amfrancesco@yahoo.com)

\section{Session: 2.5.14 - Interactive \\ Track: Track: 3 - IB Theory, FDI, and Entry Mode}

\section{Human Aspects of FDI}

Presented On: July 5, $2013-16: 45-18: 00$

Chair: Naomi A. Gardberg, Baruch College-CUNY

The Impact of MNE's Institutional Complexity, TMT Turnover, and TMT Size on MNE Performance: A Strategic Contingency Perspective Yu-Kai Wang, Soochow University

Aya Chacar, Florida International University

We propose in this study that institutional complexity is an important characteristic of a multinational enterprise (MNE). This complexity results from a greater diversity and breadth of institutional environments face by the MNE. We then argue that greater institutional complexity facing the multinational both increases the complexity of the management task but also the opportunities for arbitrage across different institutional environment with mixed impact on MNE performance. However, MNEs with top management teams (TMT) with greater information processing capability will be better able to analyze and understand more profoundly the institutional environment in which they operate and will outperform other MNEs under high institutional complexity. This greater TMT information processing can be achieved by reducing TMT turnover to allow for TMT learning and increasing TMT size for greater specialization and increased individual information processing potential. Our empirical results support our hypotheses and are in line with the strategic contingency, institutional, and upper echelons perspectives. (For more information, please contact: Yu-Kai Wang, Soochow University, Taiwan: ywang012@scu.edu.tw)

\section{MNCs' Greening Human Capital Management in China-integrative perspective of Resource-Based and Institutional Theory \\ Bih-Shiaw Jaw, National Sun Yat-Sen University \\ Christina Yu-Ping Wang, National Sun Yat-Sen University \\ Meng-Hsiu Lee, National Sun Yat-Sen University}

The increased attention to corporate greening in MNCs has raised an important question of how MNCs build their competitive human capital for greening management. By integrating RBV and institutional theory, this research was conducted in an attempt to offering theoretical framework of MNCs' greening human capital management (HCM). Firstly, RBV asserts that green leadership and green HCM can result in an environmental competitive advantage. Secondary, according to institutional theory, MNCs need to realign subsidiary's greening $\mathrm{HCM}$ to different responding strategies to host country's institutional context. Conducting the results of preliminary qualitative studies of 8 MNC's cases in China market, this research finds out that: (1) HCM will mediate the relationship between green entrepreneurial leadership and green competitive HC. (2) Institutional 
response strategies will moderate the relationship between leadership, HCM and green competitive HC. That is to say, if the leader is possessed of higher level of green entrepreneurial leadership, with more proactive response strategy to institutional context, this firm will focus more on both leveraging and investing $\mathrm{HC}$, and in turn, the high level of green competitive $\mathrm{HC}$ will be shaped. This study is expected to make contribution to theoretical development of greening HCM in MNCs. (For more information, please contact: Meng-Hsiu Lee, National Sun Yat-Sen University, Taiwan: st873217@yahoo.com.tw)

\section{Top Management Team Influence and Discretion in Foreign Market Entry Mode Decisions Peder Greve, University of St. Gallen Mathias Imbach, University of St. Gallen Winfried Ruigrok, University of St. Gallen}

How firms expand beyond their home country is one of the most fundamental questions in international business. Drawing on agency theory and taking into account particularities of the Indian study sample and context, we find that board monitoring of the TMT, which impacts executives' latitude of action, moderates the effects of strategic decision-making (SDM) and rational choice (RC) models on firms' market entry mode decisions. In the SDM model, the link between TMT international experience and a preference for lower control entry modes is stronger in environments with low TMT monitoring. In the RC model, the association between prior firm multinational experience and preference for high control entry modes is stronger in environments with high TMT monitoring. We discuss theoretical and practical implications of these findings in the context of internationalizing multinational enterprises from emerging markets. (For more information, please contact: Peder Greve, University of St. Gallen, Switzerland: peder-mathias.greve@unisg.ch)

CEO Succession Events and Internationalization Processes: Does Change in a Firm's Leadership Mean Change in Internationalization Rhythm?

Michael-Jörg Oesterle, University of Stuttgart

Corinna Rupf, University of Stuttgart

Christina Marion Stein, University of Stuttgart

Top managers are strongly related to a firm's strategy. Different characteristics of the top managers have been identified as influencing factors on strategic change. The CEO is the most powerful decision-maker in the firm and therefore, has the power to cause changes in the internationalization strategy. This can be reflected in the regularity of the rhythm of the internationalization process of the firm. We try to operationalize the internationalization process adequately and investigate the influence of the CEO on the internationalization process. We want to test our hypotheses with panel regression. (For more information, please contact: MichaelJörg Oesterle, University of Stuttgart, Germany: michael-joerg.oesterle@bwi.uni-stuttgart.de)

\section{Effects of Outward Migration on Inward FDI: From Social Networks to Human Capital \\ Xiyan Yang, Shanghai University of International Business and Economics \\ Xiaohua Lin, Ryerson University}

We examine the link from outward migration to inward FDI in China by building a human capital component to the prevailing perspective of social networks. Several hypotheses are tested using complementary datasets. According to our findings, there is a positive relationship between outward migration and inward FDI, but the relationship exists only for skilled migrants. Further investigations suggest that the effect of skilled migration is contemporaneous and not lagged, and that the overall positive migration effects have been weakened over time. (For more information, please contact: Xiaohua Lin, Ryerson University, Canada: hlin@ryerson.ca) 
Executive Power and its Influence on the Success of Negotiation for Inter-organizational Alliance Formation

Tariq Hussain Malik, Dongbei University of Finance \& Economics

This article conceptualizes relationship between the executive power and its influence on the success of negotiation for an inter-organizational alliance formation. The argument of the paper is based on functional paradigm of power associate the Aristotelian view. The argument draws on two sources of executive power: position and expertise. It argues that the negotiating executive's power has a positive influence on the formation of the alliance. However, the relationship is mediated by national cultures. National cultures lead to psychic distance, which in turn can mediate the executive's bases. Based on the interaction between positive influence of the executive power and negative influence of cultural distance, this article proposes several testable propositions in the framework. Article also provides a detailed account of methodology for future empirical research. Thus, the article makes explicit contribution by linking the executive's position and knowledge power contingent upon cultural variation. Therefore, in broader contextual setting, perception, actions and influence are context dependent. (For more information, please contact: Tariq Hussain Malik, Dongbei University of Finance \& Economics, China: tmalik@dufe.edu.cn)

\section{Implications of Managerial Personality, Trust and Procedural Justice Perceptions on International Interorganizational Cooperation}

Furkan Amil Gur, Louisiana State University

Joshua Bendickson, Louisiana State University

International interorganizational cooperation (i.e., international strategic alliance) is among the most widely investigated streams of international business research. Even though previous research recognizes the vital role of key alliance managers on formation, continuity, and outcomes of alliances, there is limited research on managerial antecedents of international interorganizational cooperation. In the present paper we develop an integrative framework that encompasses managerial personality, cooperative tendencies, cultural distance, trust, and justice perceptions and their implications on international interorganizational cooperation outcomes. (For more information, please contact: Furkan Amil Gur, Louisiana State University, USA: fgur1@lsu.edu)

\section{Session: 2.5.15 - Interactive}

Track: Track: 6 - Innovation and Knowledge Mgmt.

\section{Institutions, Suppliers, and Knowledge}

Presented On: July 5, $2013-16: 45-18: 00$

Chair: Paul Brewer, University of Queensland

The Effects of Supplier Involvement and Knowledge Protection on Product Innovation in Customer-Supplier Relationships: A Study of Global Automotive Suppliers in China

Ruey-Jer Bryan Jean, National Chengchi University

Globalization drives firms to develop product innovation through their global supply chains. While innovations generated by supply channel members, as opposed to individual partners, are playing an increasingly important role in the success of all supply chain partners, there has been limited research on how supply chain relationships cultivate the process of such innovation generation, particularly in emerging markets. Correspondingly, this study explores how multinational suppliers can develop adaptive product innovation to create competitive advantage in emerging markets. Drawing on the knowledge-based view and transaction cost economics, this study investigates the influence of supplier involvement and other factors on supplier innovation and performance. The results of a survey of 170 multinational automobile suppliers in China provide support for most of the hypotheses. Specifically, supplier involvement in co-design has an inverted U-shaped relationship 
with product innovation. Furthermore, knowledge protection, trust and technological uncertainty are all found to drive greater product innovation. In addition, the institutional environment moderates the effect of product innovation on performance. Overall, this study enhances our understanding of how MNEs can acquire local knowledge and develop adaptive products in emerging markets. (For more information, please contact: RueyJer Bryan Jean, National Chengchi University, Taiwan: bryan@nccu.edu.tw)

\section{Determinants of Global Innovation Network Effectiveness: Strategic Talent Management Capabilities Mary Boyden Teagarden, Thunderbird School of Global Management Andreas Schotter, Western University}

This multi-level study examines the determinants of intra-firm innovation network effectiveness in MNCs with R\&D nodes in China and India, countries with location specific munificence of skilled high-tech talent pools. Innovation Network effectiveness depends on individual knowledge worker contributions that are captured and amplified through dynamic strategic talent management capabilities. These capabilities must address tensions between locally responsive and globally integrated practices; and also address idiosyncratic needs of individuals as determinants of innovation network effectiveness. A multi-level analysis of qualitative data from ten intrafirm networks finds a strong positive relationship between environmental, organizational and talent management effectiveness, and innovation network performance. We present this in an integrated framework. (For more information, please contact: Mary Boyden Teagarden, Thunderbird School of Global Management, USA: mary.teagarden@thunderbird.edu)

\section{Management Issues and Strategies for the Tourism Industry Areas in a Low-Carbon Society Ying Sheng Yao, National Chi Nan University}

The Taiwanese scholars explored how the tourism industry works in a low-carbon society using real cases based on the new policies of energy saving and carbon reduction. In Taiwan, P Tourism Pasture has been in the business for over 50 years. The features of the Pasture include explanation of milking, DIY workshop, milk industry culture exhibition, a place for BBQ, a camp site, and an outdoor place for activities. Assistant Profession Fu-Ci Huang and Mr. Ying-Sheng Yao studies how the concept of low-carbon society is applied in the tourism industry using the Delphi method. 51 indexes were derived from the 4 aspect of sustainable tourism development, environment, management, tourist, and resident to explore management issues and strategies of the leisure industry. (For more information, please contact: Ying Sheng Yao, National Chi Nan University, Taiwan: michelle_hsu@weichuan.com.tw)

\section{Two Faces of Business Groups on Innovation Performance: Internalization and Institutional Legitimacy Zhaojun Gao, Peking University Changqi Wu, Peking University}

Focusing on group-affiliated firms, our paper investigates the relationship between internalization and innovation performance, and examines the impact of institutional legitimacy on innovation performance. We propose the so-called two faces of business groups on innovation performance. On the positive side, the internalization of affiliated firms can constantly improve innovation performance. However, such positive impact is weaker than that of independent firm. Moreover, we argue that business groups negatively moderate the relationship between internalization and innovation performance. We further examine the influence of institutional legitimacy on the above factors and posit a three way moderation of institutional legitimacy on group-affiliated firms. The contributions include a synthesis of internalization and institutional perspectives and an exploration of innovation in the emerging markets. (For more information, please contact: Zhaojun Gao, Peking University, China: thinking4ever2009@pku.edu.cn) 


\section{Knowledge Acquisition and Application in International Buyer-Supplier Alliances}

Chia-Ling (Eunice) Liu, National Cheng Kung University

Rudolf R. Sinkovics, University of Manchester

Cross-border knowledge flows from partners contribute to the success of the firm. However, our knowledge of the outcomes of effective transfer mechanisms is still scarce. This study builds on the resource-based view, organizational learning, and network research to investigate the interplay between inter-firm and intra-firm learning and performance outcomes in cross-border buyer-supplier partnerships. In particular, the moderating effect of absorptive capacity is examined. Based on 160 local suppliers in relationships with their global buyers in the IT industry, the empirical results indicate that knowledge acquisition and knowledge application influence firms' capability enhancement positively. When firms are active in enhancing their capabilities, they are also capable of improving their network performance. (For more information, please contact: Chia-Ling (Eunice) Liu, National Cheng Kung University, Taiwan: clliu@mail.ncku.edu.tw) 


\title{
ABSTRACTS FOR SATURDAY, JULY 6, 2013
}

\author{
Session: 3.1.1 - Panel \\ Track: Track: 9 - Cross-Cultural Management and HRM \\ Geert Hofstede: "If I Was Going to Do It Again...., I Would Use the World Values Survey"
}

Presented On: July 6, 2013 - 09:00-10:15

Chair: Mikael Soendergaard, Aarhus University

Panelists:

Geert Hofstede, University of Maastricht

Michael Minkov, International University College

Sjoerd Beugelsdijk, University of Groningen

Mark F. Peterson, Florida Atlantic University

Maaja Vadi, University of Tartu

Aycan Kara, Florida Atlantic University

This panel aims to promote research within the AIB community using the World Value Survey (WVS) and the European Values Survey (EVS). Like tour guides in the WVS/EVS jungle, experienced researchers will lead you through the wilderness of the freely available WVS/EVS dataset. They will point out multiple methodological challenges, issues that need to be addressed and provide suggestions for new routes that researchers can take. Hofstede explains why he would use the WVS dataset if had he to start over again. Minkov discusses why WVS values for children contain some of the most meaningful information for cultural comparisons, and which dimensions can be extracted from the dataset, such as indulgence. Beugelsdijk demonstrates how the WVS/EVS dataset can be used to assess contemporary questions in International Business (IB), by addressing the temporal stability of the Hofstede scores, using a comparative cohort analysis of a replicated set of Hofstede's dimensions based on the WVS/EVS dataset. Peterson discusses the sensitive dependence of paradigms on initiation conditions in international organization studies and reflects on the consequences of Hofstede's work had it been based on WVS scores rather than IBM scores. Vadi reports from a study of regional cultural differences within European countries using data from the European Social Survey (ESS) and the EVS. Finally, Kara shares the experiences she gained whilst writing a dissertation using the WVS/EVS dataset. Kara points out un-researched IB issues together with the problems that IB researcher will have to tackle as they go along. (For more information, please contact: Mikael Soendergaard, Aarhus University, Denmark:

msoendergaard@econ.au.dk) 
Session: 3.1.2 - Panel

Track: Track: 1 - Institutions, Governance, and CSR

\section{The Role of CSR in the Growth and Success of Chinese Multinationals}

Presented On: July 6, $2013-09: 00-10: 15$

Chairs: Xiaohua Yang, University of San Francisco and Marilyn Taylor, University of Missouri at Kansas City Discussant: Pervez N. Ghauri, King's College London

Panelists:

Denis Arnold, University of North Carolina, Charlotte

Timothy Michael Devinney, University of Technology, Sydney

Lei Li, Nottingham University

Christof Miska, Vienna University of Economics and Business

Huaichuan Rui, University of London

Guenter K. Stahl, WU Vienna and INSEAD

The aim of this proposed symposium is two-fold: 1 ) explore the role of CSR in globalization of Chinese business 2) to analyze the firm and institutional contexts in which CSR is practiced 3) draws implications for future research on interplay between institutional environment and firms' sustainable competitive strategies. This symposium will explore the following questions: what is the role of CSR in the globalization of Chinese businesses? Do widely accepted CSR practices have any embodiments in Chinese multinational firms? Do Chinese firms implement different CSR policies in different regions, such as in developed countries vs developing countries? Is CSR practiced differently in state-owned enterprises than non-state-owned enterprises? How is CSR integrated in Chinese multinational firms' global strategy to create sustainable competitive advantages? The key contributions of this proposed panel are: 1) it will comprehensively examine the relationship between CSR and globalization of Chinese business; 2) it will identify research gaps for future studies and provide implications for augmenting existing theories and new theory building in the area of FDI; 3 ) it will stimulate further debates and conversations on globalization of Chinese firms. (For more information, please contact: Xiaohua Yang, University of San Francisco, USA: xyang14@usfca.edu)

\section{Session: 3.1.3 - Competitive}

\section{Re-Energizing Your Teaching Tools to Create Student Engagement}

Presented On: July 6, 2013 - 09:00-10:15

Chair: Melodena Balakrishnan, University of Wollongong in Dubai

The Full Circle: Case Teaching and Writing in International Business Courses Marina Apaydin, American University of Beirut

This paper describes a "full circle" system of case method implementation (including case teaching and case writing) in two American universities in the Middle East. Theoretical grounding is summarized first, followed by practical methods and examples of implementation, with student feedback on the process. Benefits for all stakeholders: students, instructors and companies are highlighted. A list of on-line tools and resources is provided at the end. More resources are available upon request. (For more information, please contact: Marina Apaydin, American University of Beirut, Lebanon: ma266@aub.edu.Ib) 
There is No Free Lunch But There Are Free Apps to Teach International Business

Tanvi H. Kothari, San Jose State University

Mobile learning also known as m-learning is thought to place Universities and institutions at the forefront of pedagogical excellence of practice, answering student requirements for flexibility and ubiquity: 'anywhere, anytime, and any device' access to information (Fetaji, Ebibi \& Fetaji, 2011). With the rise in penetration of the iPads, Tablets, iPhones and other mobile devices among students and instructors, there is a unique opportunity to engage students by using iPads in the classroom. The goal of this presentation is to provide an interactive demonstration of various 'Apps' that can be used as learning tools in business classroom at various levels including undergraduate, MBA or Executive education. Teachers with a love of technology and a passion for nurturing the minds of their students can easily discover creative ways to incorporate the iPad into the daily routine, and some of the educational applications to be presented are bound to help them get started.

Attendees at the conference are encouraged to bring Internet-accessible iPads or Mac computers to experience the demonstration of the various Apps. Benefits to students, instructors, administrators, and researchers will be discussed (For more information, please contact: Tanvi H. Kothari, San Jose State University, USA:

kothari.t@gmail.com)

\section{Born to Become Global \\ Michal Zdziarski, Warsaw University}

I present a prototype of the board game "Born to Become Global" designed to facilitate the experiential learning of international entrepreneurship. The simulation models decisions dynamics of an entrepreneur who starts a born global company with intention to expand into foreign markets in a novel, quickly developing industry. The learning experience may be enriched by multimedia materials that include the video interview with an entrepreneur who is leading a born global company in the industry simulated in the game. (For more information, please contact: Michal Zdziarski, Warsaw University, Poland: m.zdziarski@uw.edu.pl)

\section{Authentic Learning Experience: Using Live Industry Projects in an International Business Course Vinh Nhat Lu, Australian National University Brett David Scholz, University of Canberra}

Authenticity in learning experience and industry engagement are two of the most important issues in business education. In an international business course at the Australian National University, we reinforce the usefulness of industry engagement via the implementation of live business projects as an assessment component of curriculum design. We collected data from 22 focus groups and a longitudinal survey with two cohorts of students who had participated in an international business plan competition to reflect on their experience in 2011 and 2012. The focus group data indicated three main themes, including the ways in which students oriented to the live business project preparing them for the future (their future orientations), the ways in which the project motivated students to engage with and take pride in their work (their project orientations), and how the students linked the live business project to the wider world outside of the project or university context (their external orientations). The survey data also suggested a very high level of satisfaction with the integration of the live business projects in the course curriculum, driven by individual traits, group interaction, and successful task performance. (For more information, please contact: Vinh Nhat Lu, Australian National University, Australia: vinh.lu@anu.edu.au) 
Session: 3.1.4 - Panel

Track: Track: 8 - Developing Country MNCs

\section{Internationalization and Resilience of Business Groups in Developing Economies: $A$ Comparative and Evolutionary Perspective}

Presented On: July 6, 2013 - 09:00-10:15

Chairs: Asli M. Colpan, Harvard University and Geoffrey Jones, Harvard Business School

Panelists:

Maria Ines Barbero, Universidad de San Andrés and Universidad de Buenos Aires

Asli M. Colpan, Harvard University

Takashi Hikino, Kyoto University

Geoffrey Jones, Harvard Business School

Hicheon Kim, Korea University

Ravi Ramamurti, Northeastern University

Mira Wilkins, Florida International University

This panel session aims to examine the internationalization strategy adopted by business groups as a critical factor in their growth and resilience in developing economies from a comparative and evolutionary perspective. Business groups have long been identified as the dominant form of organization in the large-enterprise sector of many late-industrializing economies, and business group-affiliated firms often function as the core of what has become to be known as the "emerging market multinationals". It has been argued that the competitive and liberalized institutional environment in emerging markets shall be conducive to foreign MNCs and specialized stand-alone domestic firms, which suggests a declining role for the business groups in their home markets and, by extension, a possible decline in their relative importance in outward FDI. The evidence that business groups are in fact undergoing such diminishing roles is scant, however. This session examines the internationalization strategies exhibited by the emerging market multinationals that utilized that strategy as a critical instrument for their continuous growth and competitive resilience. In contrast to previous approaches to business groups, there will be a strong emphasis on historical and comparative explanations by panelists, who span disciplines including international business, strategy, economic development, political science and business history. (For more information, please contact: Asli M. Colpan, Harvard University, USA:

colpan@fas.harvard.edu)

\section{Session: 3.1 .5 - Competitive}

Track: Track: 11 - SMEs and Entrepreneurship

\section{Knowledge, Learning and New Venture Internationalization}

Presented On: July 6, 2013 - 09:00-10:15

Chair: R. Isil Yavuz, Ozyegin University

International Entrepreneurship Learning of Born Global

Fu-Mei Chuang, Loughborough University

Chueh-An Hsieh, National Sun Yat-Sen University

This study contributes to the international entrepreneurship literature by developing and testing the entrepreneurship learning and innovation model of international new ventures (born globals) and their outcomes in an important emerging economy setting. We propose conceptual arguments to establish relationships between entrepreneurship market-driven learning and entrepreneur generative learning with new 
product novelty and new product meaningfulness, respectively. Further, drawing from the organizational ambidexterity literature and emerging economy context, we theorize the effects of ambidextrous learning upon the new product creativity components. We then test the moderating effects of emerging international market venture type (i.e., independent or corporate) on the links between new product creativity and performance. Our model is empirically tested with data generated from a sample of 187 high-technology international new ventures in China. The findings show positive relationships between market-driven learning and new product meaningfulness and generative learning and new product novelty. A surprising finding is the insignificant relationship between ambidextrous learning and meaningfulness, whereas the positive link between ambidextrous learning and novelty is supported. New product meaningfulness outperforms new product novelty in explaining international new venture performance. Finally, emerging market venture type conditions the performance relevance of novelty, but not meaningfulness. (For more information, please contact: Fu-Mei Chuang, Loughborough University, United Kingdom: f.chuang@lboro.ac.uk)

\title{
Organizational Learning in Young Transnational Firms \\ Mareike Schmidt, University of Bremen \\ Jörg Freiling, University of Bremen
}

Despite competitive pressure in global markets and several liabilities several young firms internationalize their business activities right from inception. International new ventures are not at all new phenomenon. However, as yet we know little about circumstances and factors that account for a successful establishment of international new ventures in the global marketplace. This paper illuminates an underresearched type of born globals, namely the so-called 'born transnationals' as young transnational companies. It is argued that these born transnational firms are equipped with specific governance structures that could be favourable to get established in global competition. Due to the particular profile of these born transnationals this paper pinpoints the role of organizational learning as a core lever of successfully establishing this kind of international new ventures in the market. In this vein, the question arises how 'born transnationals' generate and institutionalize new knowledge and adapt their knowledge base to changing environmental conditions. Based on a particular model of organizational learning, the paper presents research propositions on the learning process of 'born transnationals' and tests them in real-life context. (For more information, please contact: Jörg Freiling, University of Bremen, Germany: freiling@uni-bremen.de)

\author{
Knowledge and New Venture Internationalization: A Review and Knowledge Components Framework \\ Sarah Park, EMLyon Business School \\ Joseph A. LiPuma, EMLyon Business School \\ Christiane Prange, EMLyon Business School
}

Explanations of internationalization focus on the accumulation of knowledge and experience over time. Research on international new ventures (INVs) suggests that young companies follow an accelerated expansion strategy, based on knowledge of the entrepreneur or that of associated outsiders such as venture capitalists. This paper offers a review of the key conceptual and empirical research on international knowledge related to new ventures, and provides a framework of different types of knowledge components that constitute international knowledge. We provide directions for future theoretical and empirical research based on our framework, and insights for managers and investors in the development of their internationalization strategy. (For more information, please contact: Sarah Park, EMLyon Business School, France: park@em-lyon.com)

Exploring Rapid International Growth of High Technology Ventures: From a Knowledge Perspective Fiona Xiaoying Ji, Ohio University

Catherine N. Axinn, Ohio University 
Research in international management has traditionally studied internationalization processes from an exploitation perspective and has focused on mature multinational corporations (MNCs) and their incremental process of international expansion. Increased prevalence of international new ventures calls into question the stages model and the incremental mode of growth. International new ventures might also exploit existing knowledge and use such knowledge to pursue rapid growth in less related foreign markets. In order to better understand the growth paths of these firms, we develop and test a conceptual model by explicating key drivers of rapid international growth among new ventures. By using a sample of 311 international high technology ventures, we found that technological knowledge drives rapid growth of new ventures and this relationship is moderated by both domestic and foreign alliances joined by the focal ventures. (For more information, please contact: Fiona Xiaoying Ji, Ohio University, USA: jix@ohio.edu)

\section{Session: 3.1.6 - Competitive}

\section{Track: Track: 9 - Cross-Cultural Management and HRM}

\section{Unpacking Cross-Cultural Interactions}

Presented On: July 6, $2013-09: 00-10: 15$

Chair: Davina Vora, SUNY New Paltz

Unpacking the Dynamics of the Post-Merger Integration Process: Interaction between Identities, Interpretations, Emotions and the Role of Language

Guldem Karamustafa, University of Geneva

Susan Carol Schneider, University of Geneva

Jeff Davis, Orabrush

Despite best intentions, mergers and acquisitions often do not live up to the expectations for performance. Cultural differences, especially those across borders, is among the many reasons. Rather than differences many scholars agree that it is the integration process that matters and have called for a process approach to unpack the "black box." We conducted an in-depth case study in the acquisition by a large American consumer goods MNC of a German family owned beauty business to explore the dynamics over time. Analysis reveals the interaction of multiple identities on sensemaking, and the consequent emotional and behavioral reactions. The role of language is highlighted. Implications for further research and practice are discussed. (For more information, please contact: Guldem Karamustafa, University of Geneva, Switzerland: guldem.karamustafa@unige.ch)

Voicing Across Cultures: The Role of Communication Style and Relationships in Employee Voice Anna-Katherine Ward, Virginia Tech

Elizabeth Ravlin, University of South Carolina

Brian Klaas, University of South Carolina

Nancy R. Buchan, University of South Carolina

Cultural diversity in an organization may increase performance due to the variety of perspectives it brings to decisions. However, expressed perspectives may not reflect the actual diversity of the workforce, as past research suggests that individuals from more relationally-oriented cultures are less likely to voice their input. The current study hypothesizes, based on High-/Low-Context Theory, that this culture/voice link is misconstrued. We explored the relationship between individual-level culturally-influenced communication style and two distinct types of prosocial voice (promotive and prohibitive) in a sample of 100 internationally-diverse employees of a multi-national firm. Contrary to our predictions, relationship-quality (leader-member exchange) did not moderate the relationship between communication style and promotive voice. However, as hypothesized, indirect (i.e., group-/relationship-oriented) communicators were less likely than others to practice 
prohibitive, but not promotive, voice, suggesting that related research should focus on how, rather than whether, individuals from more relationally-oriented societies voice. Implications are discussed. (For more information, please contact: Anna-Katherine Ward, Virginia Tech, USA: annakward@hotmail.com)

The Challenge of Nonverbal Context in International Business: A Model of Communication and Social Interaction Style

Nancy R. Buchan, University of South Carolina

Wendi L. Adair, University of Waterloo

Xiaoping Chen, University of Washington

Dong Liu, Georgia Institute of Technology

Culture is enacted through communication between individuals in specific organizational contexts. Therefore, we propose an interdisciplinary-based model and measurement tool to assess Communication and Social Interaction Styles (CSIS) with the understanding that the specific strategies people employ are the simultaneous function of both environmental cues and mental structures. In this model we identify four environmental cues in communication (the primarily nonverbal contexts of message, relationship, time, and space) and one mental structure (self-construal) in delineating individual and cultural differences in exchanging information and conveying meaning. The CSIS instrument is validated in three cultures, providing empirical evidence for the theoretical model. (For more information, please contact: Nancy R. Buchan, University of South Carolina, USA: nancy.buchan@moore.sc.edu)

\section{The Role of Energy in Intercultural Work Relationships \\ Dana M. McDaniel, California State University, Long Beach}

Intercultural work relationships are increasingly prevalent in global workplaces, yet are at risk and are tenuously maintained due to cross-cultural communication challenges. In this theory paper, energy is posed as a relational resource that can be leveraged in these relationships to effectuate interdependent, intercultural work. Drawing from existing research on energy and interpersonal interactions, we develop propositions which explain the role of energy in intercultural interactions as a process. Our theoretical development suggests that successfully navigating these interactions may be particularly crucial for building and maintaining effective work relationships and accomplishing interdependent work in multicultural settings. (For more information, please contact: Dana M. McDaniel, California State University, Long Beach, USA: dana.mcdaniel@csulb.edu)

\section{Session: 3.1.7 - Competitive}

\section{Track: Track: 10 - Economics, Finance and Accounting}

\section{Perspectives on Trade and Learning}

Presented On: July 6, 2013 - 09:00-10:15

Chair: Alan S. Zimmerman, City University of New York-College of Staten Island

The International Transmission of Liquidity Shocks: A Cross Country Study on Trade Credit and Financial Crises Rafael Schiozer, FGV - Brazil

Miyamoto Renata, FGV

This paper investigates the transmission of international liquidity shocks to the supply of trade credit by publicly traded firms in six different countries: France, Germany, Italy, Spain, the United Kingdom and Brazil, exploiting two distinct liquidity shocks: i) the global freeze that followed the demise of Lehman Brothers in 2008 and; ii) the sharp increase in the yields of sovereign debt of certain European countries in the last months of 2010. 
Recent international literature documents that, during the peak of a financial crisis, trade credit supply increases as constrained companies demand trade credit as a substitute to bank loans, although after the peak moment this supply decreases significantly because large, publicly traded firms (typical trade credit suppliers) face financing constraints.. Even though there is just punctual evidence that during the 2008 global financial crisis the trade credit supply increased, the post-crisis effect is noticeable during the 2011 European sovereign debt crisis, as the firms in the most affected European countries decreased their supply of trade credit, also evidencing that these companies were either finding difficulties to obtain funding or deliberately holding up to more liquid assets. (For more information, please contact: Rafael Schiozer, FGV - Brazil, Brazil: rafael.schiozer@fgv.br)

\section{Beyond Learning-by-Exporting: When Does Exporting Lead to Better Performance? \\ Jaiho Chung, Korea University \\ Jon Jungbien Moon, Korea University \\ Hyejin Cho, Korea University \\ Jiyoung Shin, Korea University}

This study examines when exporting firms outperform comparable non-exporting firms by evaluating the performance of newly exporting firms versus continuing non-exporting firms. We employ the propensity score matching method and difference-in-differences approach in order to control for the endogeneity problem inherent to firms' exporting decisions. This research design allows us to tease out the effect of exporting on firm performance. We find strong evidence that newly exporting firms outperform comparable non-exporting firms in China, especially when the newly exporting firms are larger, equipped with better absorptive capacity, and operating in a market with higher exporter/foreign-invested firm agglomeration. This improved performance can be attributable to increases in sales, labor productivity, and intangible assets. (For more information, please contact: Jon Jungbien Moon, Korea University, Korea, South: jonjmoon@korea.ac.kr)

\section{Poor Predictive Power and the Unrealism of International Trade Models: Proposing a More Realistic Behavioral Economics-Based Model \\ Hamid S. Hosseini, King's College}

Beginning with David Ricardo, if not Adam Smith, economists have developed numerous models to explain and predict trade among nations. As I will demonstrate, many of these models have had poor predictive powers. It can be argued that neither gravity model, nor different versions of the comparative advantage doctrine, nor even the more recent model developed by Paul Krugman, could explain international trade during the great recession that began in August 2007. For example, these models could not explain why between the first quarter of 2008 and the first quarter of 2009 global GDP fell by $4.5 \%$ while world exports declined as much as $17 \%$. The scale and speed of that trade collapse poses a challenge to various international trade models. As I will show, this problem stems largely from lack of realism on the part of the assumptions of those models, the inadequacy and incompleteness of the causes of specialization in those models, and the neglect of trade finance in all those models. After discussing the causes of specialization in existing models, I will develop a more realistic model that resolves the above-mentioned shortcomings. (For more information, please contact: Hamid S. Hosseini, King's College, USA: hamidhosseini@kings.edu) 


\section{Session: 3.1.8 - Competitive}

\section{Qualitative Methodology and IB}

Presented On: July 6, $2013-09: 00-10: 15$

Chair: Ilir Haxhi, University of Amsterdam

Critical Realism as a Third Lens to Distance in International Business and International Management Research Tiina Ritvala, Aalto University

Emmanuella Plakoyiannaki, Aristotle University of Thessaloniki

This paper proposes a third approach to distance in international business and international management research to complement the predominant positivist and social constructivist approaches. On the basis of reflection upon claimed weaknesses of positivist distance measures (neglect of agency), and social constructivist reduction of social reality to agency (neglect of structure), a critical realist approach is proposed. An explanatory case study of the unsuccessful transfer of a consumer concept from Finland to the USA is used to illustrate how critical realism can benefit the field by discovering hidden structures generating distance. The study is based mainly on 32 interviews with managers and scientists from Finland and the USA. The paper contributes to the field by providing a contextual explanation of distance missing from the current debate. It is argued that critical realism is well suited for multidisciplinary and multilevel research efforts tackling the increased dynamics and complexities of modern societies. (For more information, please contact: Tiina Ritvala, Aalto University, Finland: tiina.ritvala@aalto.fi)

We Want to Learn All About You: The Interview as Ethnographic Event in International Business Research Fiona Moore, University of London

Although there is an increasing amount of research on the impact of language on business, little has thus far been written on the role of language, or linguistic events, on the study of international business. In particular, although the unstructured or semi-structured interview is the dominant form of qualitative data-gathering in IB studies, and although there has been research on this in other disciplines, the sociolinguistics of interviews is a neglected area in IB. In this paper, I draw on Briggs' ethnographic research on the social dynamics inherent in the act of interviewing, to analyse the power relations and norm construction in a set of interviews conducted between 2002 and 2006 in a qualitative case study of BMW MINI. I conclude that considering interviews in this way reveals otherwise hidden data about the company, the researcher, and the place of researchers in the organisations, and that IB researchers consequently need to pay more attention to the ways in which we report and analyse interviews. (For more information, please contact: Fiona Moore, University of London, United Kingdom: fiona.moore@rhul.ac.uk)

\section{Causal Recipes for Internationalization Speed \\ Luis E. Lopez, INCAE Business School \\ Luciano Ciravegna, University of London}

This study explores the reasons of internationalization speed. It uses Fuzzy Set Qualitative Comparative Analysis to study a sample of small entrepreneurial software exporters. The study examines the configurations of motivations and perceptions that lead entrepreneurs to fast internationalization. The study finds three configurations that combine the insights of different theoretical approaches and lead to the same outcome. (For more information, please contact: Luis E. Lopez, INCAE Business School, Costa Rica: luis.lopez@incae.edu) 
A Methodological Approach for Bargaining Power Dynamics: Event Analysis, Event Sequencing, and Action Rates James Nebus, Suffolk University

Carlos Rufin, Suffolk University

Laura Albareda, Deusto Business School

IB studies of bargaining power can be criticized because they take a static, cross-sectional, view of bargaining. We begin to address this criticism by using qualitative event analysis methodology to analyze actors' (e.g. nation-state, MNE) sequences of actions and reactions during bargaining in a specific issue area. The questions we strive to answer are: what factors cause actor B to respond to actions from actor $A$ ? and what is the likelihood that actor B will respond to actor A within a given time interval? Our contributions to the analysis of bargaining power between MNEs and other actors are threefold. First, we identify "precipitating events," or the actions of one actor which elicit a response by another actor. Second, the method links interdependent actions occurring at multiple levels (e.g. nation state, institutional, and individual). Third, the method helps us to identify environmental factors which influence an actor's propensity to act, such as: the number of actors participating in bargaining, the geographic concentration of actions, and the temporal concentration of actions. Finally, the output from our method is a time-sequenced data set of bargaining events that constitutes the input to quantitative hazard rate analysis for prediction of future actions in bargaining settings. (For more information, please contact: James Nebus, Suffolk University, USA: jnebus@suffolk.edu)

\section{Session: 3.1.9 - Competitive}

\section{Track: Track: 2 - Marketing and Supply Chain}

\section{Market Entry and Subsidiaries: Structuring for Growth}

Presented On: July 6, 2013 - 09:00-10:15

Chair: Johny K. Johansson, Georgetown University

Marketing Adaptation and Foreign Market Entry-The Link to Foreign Subsidiary Establishment Desislava Dikova, Vienna University of Economics and Business

Arjen Slangen, Rotterdam School of Management

We extend the discussion on the adaptation/ standardization in foreign markets by introducing a new strategic link, the foreign subsidiary establishment mode choice of multinational firms. We suggest that the penetration of certain markets requires extensive adaptation achieved by assimilating local brands or developing insightful local promotion, while others can be served by offering relatively standard products. We examine how the perceived need for local adaptation affects the establishment mode choice. We argue that at high levels of adaptation, firms are likely to choose an acquisition establishment mode. This relationship however is contingent upon how challenging the local adaptation is for the firm. We hypothesize that realizing a given degree of local adaptation will be less challenging for firms with better worldwide marketing skills hence for such firms the positive impact of adaptation on the likelihood of choosing an acquisition entry will be weaker. Realizing a given degree of local adaptation will be more challenging for the firm if the foreign subsidiary sells different products or is based in a culturally more distant country. In this case, the positive impact of adaptation on the likelihood of choosing an acquisition entry will be stronger. We test our hypotheses on a sample of 248 foreign subsidiaries established by 159 Dutch multinational firms and find support for our hypotheses. (For more information, please contact: Desislava Dikova, Vienna University of Economics and Business, Austria: desislava.dikova@wu.ac.at)

Marketing Strategy in MNC Subsidiaries: Pure versus Hybrid Archetypes David Midgley, INSEAD

Sunil Venaik, University of Queensland 
Porter's (1980) distinction between the generic strategies of low cost and differentiation provides the theoretical foundation for the standardization versus adaptation debate in international business. Porter posits that firms must pursue one of these (pure) strategies to achieve above-average performance. In contrast, Miller (1993) argues that firms often pursue hybrid strategies for superior performance rather than the pure type, as suggested by Porter. Hybrid strategies are those that combine aspects of two or more pure strategies. And Bartlett and Ghoshal (1989) postulate a specific hybrid configuration for marketing mix standardizationadaptation in MNCs. We test these three competing theories in the context of marketing strategy in MNC subsidiaries using a novel archetypal analysis methodology. Our results show that $71 \%$ of MNC subsidiaries in our sample pursue hybrid strategies. Contrary to both Porter and Miller's arguments, we find no difference in performance between subsidiaries that pursue pure strategies (standardization or adaptation) and those that pursue hybrid strategies (standardization and adaptation). In addition to Bartlett and Ghoshal's configuration, we identify several new hybrid forms of strategy not previously recognized in the literature. Overall, some $58 \%$ of the subsidiaries in our sample pursue these new forms of hybrid strategies. (For more information, please contact: Sunil Venaik, University of Queensland, Australia: svenaik@business.uq.edu.au)

\title{
Concurrent Sourcing Strategy of Multinational Firms: Drivers and Performance Implications Gerald Yong Gao, University of Missouri-St. Louis Janet Y. Murray, University of Missouri-St. Louis Masaaki Kotabe, Temple University
}

As a prominent form of plural governance, concurrent sourcing-the simultaneous use of insourcing and strategic alliance-based sourcing-is critical to multinational firms' operations in emerging economies, and yet it has received limited attention in the literature. In this study, we investigate the driving factors of firms' concurrent sourcing strategy and further examine whether concurrent sourcing outperforms other types of sourcing strategies. Our findings suggest that firms use concurrent sourcing at high levels of institutional regulation and firms with a multi-market focus are more likely to employ concurrent sourcing strategy. The results also provide supportive evidence that the performance impact of concurrent sourcing strategy is contingent on demand uncertainty and institutional regulation. (For more information, please contact: Gerald Yong Gao, University of Missouri-St. Louis, USA: gaogy@umsl.edu)

\author{
The Role of Network Structure in Equity Based Partnerships: A Social Network Analysis of Domestic vs. \\ International Joint Venture Formations \\ Steven Carnovale, Rutgers University \\ Sengun Yeniyurt, Rutgers University
}

This paper develops a network theory based framework of manufacturing equity-based partnership formations and provides an empirical test in the context of the global automotive industry. Specific theoretic hypotheses are formulated regarding the implications of the network structure for a firm's partner selection in global manufacturing Joint Ventures. The role of network theory constructs such as network centrality, tie length, and structural embededness are explored. Additionally, the differences in the effects of these network variables on the likelihood of new JV formation between international and domestic joint venture formations are explored. A comprehensive dataset containing 1,158 automotive firms over the course of 19 years is utilized to test the hypotheses. Results provide empirical support for the role of network structure in mitigating the uncertainties of new equity based partnership decisions in global supply chain networks. (For more information, please contact: Steven Carnovale, Rutgers University, USA: scarnova@pegasus.rutgers.edu) 


\section{Session: 3.1.10 - Competitive}

Track: Track: 1 - Institutions, Governance, and CSR

\section{Institutions, CSR and Value Creation}

Presented On: July 6, 2013 - 09:00-10:15

Chair: S. Prakash Sethi, City University of New York

Generating Profit or Generating Value through Multinationality

Jan Hendrik Fisch, University of Augsburg

Michael-Jörg Oesterle, University of Stuttgart

Cigdem Polat, University of Augsburg

This article proposes a decreasingly positive impact of multinationality on the profit potential and a U-shaped impact on the social legitimacy of firms. Combining profit potential and social legitimacy as mutual demands of shareholders suggests that the effect of multinationality on value be inverted S-shaped, except of firms with institutional investors who place a strong emphasis on financial aspects. Panel regressions support the predictions. The study furthermore replicates earlier findings that the relationship between multinationality and profit is, contrary to the relationship between multinationality and value, S-shaped. Contradictory performance effects can prevent firms from reaching optimal levels of multinationality. (For more information, please contact: Jan Hendrik Fisch, University of Augsburg, Germany: fisch@wiwi.uni-augsburg.de)

The Impact of International Experiences on Corporate Social Performance: A Case of Korean MNCS Eun Woo Kim, Yonsei University Jooyoung Kwak, Yonsei University Soonkyoo Choe, Yonsei University

While corporate social performance has been discussed mainly in the context of internal organizational factors and of domestic markets, MNCs have developed corporate social performance, learning from international experiences. This study integrates stakeholder theory, institutional theory and global strategy, and suggests a relationship between MNCs' international experiences and corporate social performance. In the host markets, MNCs should comply with the norms, regulations and the expectations desired by varying types of social actors. Accordingly, having more international experiences is likely to lead MNCs to better corporate social performance. Further, entries into countries where the behavioral standards are set higher than others, may cause increases in corporate social performances. However, since foreign entries are costly, excessive international experiences may discourage MNCs from pursuing corporate social performance. Hence, we hypothesize an inverted U-shaped relationship between international experience and corporate social performance. With a FDI dataset of Korean MNCs, we conducted a truncated model analysis. The findings support our hypothesis. The results also suggest that, as entries into countries with a lower level of desired standards for corporate behavior increase, Korean MNCs are less likely to pursue corporate social performance. Our study demonstrates that international experiences constitute a major determinant of corporate social performance. (For more information, please contact: Soonkyoo Choe, Yonsei University, Korea, South: skychoe@yonsei.ac.kr)

Corporate Philanthropy and Corporate Financial Performance: An Institutional Comparison between China and the United States

Heli Wang, Singapore Management University

Jane Wenzhen Lu, University of Melbourne

Qian Wang, Zhejiang University 
We examine institutional influences on the relationship between corporate philanthropy (CP) and corporate financial performance (CFP). Comparing and contrasting US and China samples, we find that the CP-CFP relationship is stronger in US than in China. The differential moderating effects of sin stocks and advertising intensity on the CP-CFP relationship between the two samples point to stakeholder perception and information availability as the key mechanisms underpinning the CP-CFP relationship which are subject to institutional differences. (For more information, please contact: Qian Wang, Zhejiang University, China:

fionawq2010@gmail.com)

\section{Does Distance Matter? Cross-Border Acquisitions and Corporate Value for Multinationals in Greater China Meijui Sun, Ming Chuan University}

This study explores whether acquisition announcements generate stock price reactions and the sources of likely bidders' wealth gains. We also examine significant determinants of acquisitions, such as cultural distance, economic distance, and industry distance. This study investigates whether acquisitions in Hong Kong provide significantly positive stock price reactions and better long-term performance. We examine 3092 mergers and acquisitions (M\&As) announcements associated with 947 Hong Kong listed companies during the sample period from 2000 to 2011. For this empirical study, we use an event study to examine how M\&As announcements influence company performance in the short term. Furthermore, we employ ordinary least squares (OLS) regression model to examine the relationship between cultural distance, economic distance, industry distance, institutional distance and size distance with the control variable-size, debt ratio, political risk and holding ratio of the acquiring firm. The results show that the cultural distance and size distance are not significant and industry distance, institutional distance and economic distance are negatively related to company performance. (For more information, please contact: Meijui Sun, Ming Chuan University, Taiwan: mjsun@mail.mcu.edu.tw)

\section{Session: 3.1.12 - Interactive \\ Track: Track: 10 - Economics, Finance and Accounting}

\section{Ownership, Governance and Capital Structure}

Presented On: July 6, 2013 - 09:00-10:15

Chair: Jean-Claude Cosset, HEC Montreal

Market Liquidity and Bank-dominated Corporate Governance: Evidence from Japan Hideaki Sakawa, Nagoya City University

Masato Ubukata, Kushiro Public University

Naoki Watanabel, Ritsumeikan University

This paper investigates the relationship between market liquidity and bank-dominated corporate governance structure of Japanese listed firms, represented as main bank relationships and cross shareholdings. We find that (i) main bank lending relationships increase market liquidity and reduce asymmetric information in the Tokyo Stock Exchange and (ii) foreign shareholdings are positively related to market liquidity and negatively correlated with asymmetric information. This suggests that the bank monitoring mechanisms would take a substitute information production role for block holder monitoring role in the market-oriented corporate governance countries like US. (For more information, please contact: Hideaki Sakawa, Nagoya City University, Japan: sakawa@econ.nagoya-cu.ac.jp) 
The use of Financial Contracting Arrangements to Constrain Rent-Seeking Behaviour of Managers of Subsidiary Firms: Evidence from UK and US Multinational Subsidiary Firms

Robert Suban, University of Manchester

Michael Bowe, University of Manchester

Mo Yamin, University of Manchester

We propose and test two hypotheses on how multinational (MNC) parents can use different financial contracting arrangements when financing their subsidiaries in order to constrain the rent-seeking behaviour of subsidiary management. Furthermore, we also examine how the MNC parent can effectively enhance the monitoring of their subsidiaries by delegating this function to external financial institutions. One hypothesis analyses the use of short-term debt. The second hypothesis investigates the use of short-term external debt. Moreover, our analysis is enriched by investigating these two hypotheses in two different settings, namely by comparing between UK domestic and UK subsidiary firms and by comparing UK and US subsidiary firms. This enables us to measure the subsidiary effect and the location/distance effect. Using an unbalanced panel data of 11762 and 10096 firm-year observations we find that UK subsidiaries have more short-term debt and more short-term external debt compared to equivalent UK domestic firms and that US subsidiaries have more short-term debt and less short-term external debt compared to equivalent UK subsidiaries. Our results are both statistically and economically significant. We find these results in spite of our domestic firms being larger, being more profitable, having higher growth opportunities, and having higher tangibility, all factors which should translate in higher amounts of debt. Our results are robust, even after controlling for all the variables that have been found to affect leverage in the literature and when we use a matched sample approach to test our hypotheses. Furthermore, we also consider and rule out other possible explanations for our results. (For more information, please contact: Robert Suban, University of Manchester, United Kingdom: robert.suban@postgrad.mbs.ac.uk)

\section{Does Corporate Governance in Cash Management Adds Value to Multinational Firms? Daniel Gomes de Moraes, FGV-EESP \\ Hsia Hua Sheng, FGV-EAESP \\ Mayra Ivanoff Lora, FGV-EESP}

The aim of this work is to analyze whether the adoption of best in class corporate governance practices related to cash management adds value to multinational firms, as well as to define which the most effective practices are. The sample was comprised of 207 multinational firms from 32 countries which have answered a broad survey regarding their cash management practices in 2010. We concluded that some practices related to cash management governance do add value. Among such practices, we noticed that: (i) firms that present cash pooling policies have lower cost of debt and shorter cash conversion cycle; (ii) firms that with developed InHouse Banks are more profitable; and (iii) firms that present automatic payments, receivables and reconciliation processes have better operating margin. On the other hand, the data did not prove significant for practices related to cash visibility, intercompany netting and the use of Shared Services Center. Keywords: Cash Management, Treasury Governance, Liquidity, Working Capital, MNC. (For more information, please contact: Daniel Gomes de Moraes, FGV-EESP, Brazil: daniel.moraes@citi.com)

\section{The Impact of Crisis on Determinants of Leverage: European Evidence \\ Victoria Krivogorsky, San Diego State University \\ Gun-Ho Joh, San Diego State University \\ Andrei Filip, ESSEC}

This paper investigates the role of institutions and macroeconomic indicators of economic health in determining firms' leverage before and after the financial shocks in Europe in 2008. The importance of this investigation stems from the current state of the global economy, where market confidence has been severely impacted in virtually every area. In light of this, estimation of the risk associated with a firm is more challenging now than at any time in recent history. We go about our examination using cluster analysis and find a significant reduction 
in the use of debt from the pre- to post 2008 period. Among country specific variables, direct foreign investment into firms, maturity of credit laws, availability of bank funds and GDP growth ratio gain significance in their bearing on using both bank loans and bonds after 2008. The public deficit ratio and national variations in use of debt vs. equity significantly impact only the use of bonds after 2008 . We also document evidence that country risk and inflation impact companies' financing decisions. (For more information, please contact: Victoria Krivogorsky, San Diego State University, USA: vkrivogo@mail.sdsu.edu)

\section{Risk Management and Transaction Cost Theory: The Case of Project Finance Jakob Müllner, Vienna University of Economics and Business}

In this paper we attempt to integrate transaction cost theory (TCT) and financial risk management. We show how the exogenous nature of risks and the negligence of capital structure overemphasize the asset side of transactions, implying structural irrelevance of capital. We propose three extensions to TCT using project finance (PF) as an illustrative case. Overall, they form what we refer to as the "liabilities' side" of the transaction. First, we account for the fact that PFs commonly involve the cooperation of multiple participants making a joint investment. Second, we introduce capital structure and other managerial instruments as responses to risks. We complement Sawant's $(2012,2010)$ studies on risk mitigation in PF, adding two distinct RM mechanisms: risk diversification and transfer. Finally, we account for high up-front governance cost and increased internal uncertainty caused by RM in PF. Recognizing this trade-off allows us to theoretically position PF as a governance alternative in situations where external risks are less manageable than internal risks. (For more information, please contact: Jakob Müllner, Vienna University of Economics and Business, Austria: jakob.muellner@wu.ac.at)

\section{Hedging and the Failures of Corporate Governance: Lessons from the Financial Crisis Rodrigo Zeidan, Fundação Dom Cabral and Nottingham University}

The paper identifies which failures of corporate governance allowed non-financial companies around the world to develop hedging strategies that led to hefty losses in the aftermath of the financial crisis. The sample is comprised of 346 companies from 10 international markets, of which 49 companies (and a subsample of 13 distressed companies) lost a combined US $\$ 18.9$ billion. An event study shows that most companies that presented losses in derivatives experienced negative abnormal returns, including a number of companies in which the effect was persistent after a year. The results of a probit model indicate that the lack of a formal hedging policy, no monitoring to the CFOs, and considerations of hubris and remuneration contributed to the mismanagement of hedging policies. For heavily distressed companies, there is evidence that higher ownership concentration implies in a lower probability of designing speculative hedging positions. (For more information, please contact: Rodrigo Zeidan, Fundação Dom Cabral and Nottingham University, China: rodrigo.zeidan@fdc.org.br)

\section{Session: 3.1.13 - Interactive}

\section{Track: Track: 6 - Innovation and Knowledge Mgmt.}

\section{Management of Knowledge}

Presented On: July 6, 2013 - 09:00-10:15

Chair: Changsu Kim, Sogang University

How Teams Interact with Interfirm Learning for New Variety Tomohiro Machikita, Institute of Developing Economies Yasushi Ueki, Institute of Developing Economies 
This paper examines whether firms consider adopting cross-functional teams as a device of transforming external information to innovation. While addressing the difference between firms with and without crossfunctional teams, we examine the effects of complementarities between internal and external resources on product innovation and product-level creative destruction, using data on focal manufacturers and their suppliers in Indonesia, the Philippines, Thailand, and Vietnam. The firms with cross-functional teams are more likely to have higher impacts of exchanging engineers on product innovation and destruction. We also find that crossfunctional teams can enhance the impact of exchanging engineers to acquire information on past failures of other firms. But this is not true for the case of knowledge sharing within a single department through QC circles. Empirical results clearly suggest that product innovation and replacement need a wider or "global" sharing of outside knowledge across several departments within a firm. (For more information, please contact: Tomohiro Machikita, Institute of Developing Economies, Japan: tomohiro_machikita@ide.go.jp)

\author{
Leveraging Local Talent for Global Learning \\ Shad Morris, Ohio State University \\ Daniel Han Ming Chng, China Europe International Business School \\ Jian Han, China Europe International Business School \\ Bijuan Zhong, Ohio State University
}

A unique challenge of developed market multinational enterprises (MNEs) is the significant knowledge gap between corporate headquarters and their foreign subsidiaries in emerging markets. Given the limits of extant theory, we engaged in an abductive theory building study of eight MNEs operating in China. The result is a framework that describes the various stages of the knowledge transfer process by local talent within the foreign subsidiary. Specifically, we examined how a foreign subsidiary's local talent base first, imitates MNE-specific knowledge, second, combines MNE-specific knowledge with local market knowledge to achieve local adaptation, and third, shares local adaptations within the MNE to bring about global innovation. We identify specific types of human and social capital as well as specific HR practices that will differentially influence organizational capabilities related to knowledge imitation, local adaptation, and global sharing. Implications for managing global innovation through local talent are discussed. (For more information, please contact: Bijuan Zhong, Ohio State University, USA: zhong_40@fisher.osu.edu)

\title{
Extroversion, Team Helping, and the Performance of Demographically Diverse Teams Hsi-An Shih, National Cheng Kung University \\ Yun-Hwa Chiang, Ming Chuan University \\ Hsiao-Yun Liang, National Cheng Kung University
}

Team helping is a team-level phenomenon concerning team members' aggregated voluntary helping behaviors toward other members with work related activities. Based on the personality literature, we propose that team members' extroversion will moderate the effect of team diversities of age and gender with team helping. Moreover, based on the team helping literature, we propose that team helping will mediate the moderating effect of team aggregated extroversion on the relationship between team age and gender diversities with team performance. Collecting survey data with time lag from Taiwanese work teams, we find support for our hypotheses. Our study enriches scholarly understanding about how team members' personality may affect the relationship between team diversity and team performance. (For more information, please contact: Hsiao-Yun Liang, National Cheng Kung University, Taiwan: ireneyun0916@gmail.com)

\section{Top Management Team Experiences and the Growth Value of Corporate Venturing Yu-Chen Chen, National Taiwan Normal University \\ Shu-Jou Lin, National Taiwan Normal University}

The purpose of study is to investigate whether and how TMT diversity influences future growth value generated by corporate venture investments. Different from previous TMT studies mainly conducted in the context of firm 
exploitation, we use corporate venturing to examine the existing TMT theories in an exploratory setting. We found that TMT composition is a determinant of heterogeneous CVI contribution to firm growth value.

Specifically, among various refined measures of a TMT's industry experiences, we find that each team member's versatile experiences positively moderate the relationship between the magnitude of CV investments and firm growth value. (For more information, please contact: Yu-Chen Chen, National Taiwan Normal University, Taiwan:600560110@ntnu.edu.tw)

\section{Slips and Accommodations in MNC Subsidiary Strategizing Activities \\ Robert Demir, The Ratio Institute and Stockholm University}

The present paper addresses a phenomenon often neglected in IB research - coordinated strategizing in MNCs. In particular, the present study aims to identify the sources, mechanisms, and activities that underlie distributed coordinated strategy work within MNC subsidiaries. In drawing from the concept of boundary objects and computing anomalies or slips, the present study analyzes a large multinational packaging firm's strategizing activities in its North Europe Market cluster by analyzing the practice slips that the organizations have faced after the introduction of a large enterprise resource planning system (ERPS) and the accommodations made in response to those slips in order to restore strategy practice. Results are presented and implications are discussed on. (For more information, please contact: Robert Demir, The Ratio Institute and Stockholm University, Sweden: rd@fek.su.se)

\section{Overcoming Regulatory Hurdles: A Global Analysis of Venture Innovativeness and VC Experience Elisa Alvarez-Garrido, Georgia State University}

There is empirical evidence that Venture capital (VC) firms foster innovation in the U.S. As a result, many foreign governments have provided incentives to VC investments in the hope that this will translate into venture innovation. Yet, we know less about the effect of VCs on innovation abroad. Furthermore, the presence of weaker regulatory and enforcement systems may create obstacles for such effect, and these regulatory issues are among the top concerns of VCs globally. This paper argues that experienced VCs may overcome these legal hurdles, but where the experience was acquired - in the country of interest or abroad - is important, and so is whether the problems derive from weak business regulation, or from weak intellectual property enforcement. Using a sample of VC-backed biotech ventures, I find that VC experience nurtures venture innovation of U.S. ventures, hence replicating the findings of previous research. However, an analysis of this result in a sample of 329 foreign ventures from 21 countries uncovers the subtleties of this effect. I find that VC local experience compensates for a weaker private sector regulation. (For more information, please contact: Elisa AlvarezGarrido, Georgia State University, USA: ealvarezgarrido@gsu.edu)

\section{Between the Global and the Local? Knowledge Sharing and Neo-Colonial Relations in Professional Service Multinationals \\ Mehdi Boussebaa, University of Bath \\ Andrew Sturdy, University of Bristol \\ Glenn Morgan, University of Cardiff}

This paper explores the nature of the contemporary multinational corporation (MNC) through a study of the use of knowledge management systems (KMS) in the UK subsidiaries of four international management consultancies. In particular, we examine the extent to which horizontal (i.e. inter-subsidiary) knowledge sharing works in practice and compare this against the two dominant images of the MNC: as an integrated network where knowledge flow is multi-directional and as a fragmented space where knowledge is socially embedded and therefore difficult to transfer. Our analysis reveals the presence of horizontal knowledge flows within the case study firms, but flows that are constrained by inter-context differences and partly shaped by neo-colonial relations. Thus, our study gives partial support to both of the dominant approaches to knowledge flow in MNCs. 
However, and importantly, it also provides a critical development of both perspectives by drawing attention to the geopolitical dynamics of knowledge transfer. At the same time, it challenges the claims of consulting firms and other positive accounts that knowledge flows freely as well as of critics of KMS who question their potential to facilitate knowledge transfer. (For more information, please contact: Mehdi Boussebaa, University of Bath, United Kingdom: m.boussebaa@bath.ac.uk)

\section{Session: 3.1.14 - Interactive}

\section{Track: Track: 3 - IB Theory, FDI, and Entry Mode}

\section{Internationalization and Performance}

Presented On: July 6, $2013-09: 00-10: 15$

Chair: Peder Greve, University of St. Gallen

\section{Persistence of Superior Financial Performance: Impact of Internationalization and Its Entrainment with} Institutional Reforms

Manish Popli, Indian Institute of Management Lucknow

Mohammad Akbar, Indian Institute of Management Lucknow

Vikas Kumar, University of Sydney

This article proposes that the degree of internationalization and its entrainment with an external cycle of reforms constitutes an important strategic lever for value creation during institutional transition. Using longitudinal data of Indian automotive sector firms from 2001 to 2010, we find that firms' degree of internationalization (DOI) positively moderates the impact of reforms on superior financial performance. Furthermore, we theorize and find support for our hypotheses that the moderation impact of DOI with reforms is increasingly greater when firms entrain the pace and phase of their process of internationalization with the flux of institutional reforms. Our findings advance the role of strategic choice during institutional transition and offer implications for organizational practice to understand the source of competitiveness through a temporal lens. (For more information, please contact: Manish Popli, Indian Institute of Management Lucknow, India: fpm12004@iiml.ac.in)

The Relationship between Internationalization and Performance of Mid-Size Indian Firms - The Role of Family Control

Faisal M Ahsan, Indian Institute of Management Lucknow

Ajay Kumar Singal, Indian Institute of Management Lucknow

Arun Kumar Jain, Indian Institute of Management Lucknow

The focus of this study is on the relationship between internationalization and performance of mid-size firms from an emerging economy (India). Most of these mid-size firms continue to be predominantly familycontrolled. Grounded in the stage model of internationalization, findings of the present study suggest that these firms remain stuck in the stage I of internationalization and exhibit a downward sloping relationship between degree of internationalization and performance. Family ownership is found to have a negative impact on the degree of internationalization. (For more information, please contact: Faisal M Ahsan, Indian Institute of Management Lucknow, India: fpm12005@iiml.ac.in)

Multi-Nationality and Performance Effects of Internationalization: China OFDI from the Portfolio Perspective Hsin-Mei Lin, National Chi Nan University

Hinrich Voss, University of Leeds

I-Fan Yen, National Chi Nan University 
This paper develops a portfolio perspective on foreign affiliate investments of Chinese firms, which represent a new pattern---multiple simultaneous foreign investments as a portfolio---different from the extant pattern of developed countries. Extant pattern of multinational foreign investments is more likely to be a growing trend. However, in response to prospective economic development and political embeddedness, Chinese firms when they represent heterogeneity and uniqueness as newly being multinational enterprises (MNEs) under conditions of non-ergodic global uncertainty, some of they adopt a newly one-shot portfolio investment pattern. How does the new portfolio pattern benefit the performance of Chinese MNE from enjoying the diversified stream of revenue of the multinational firm's several foreign affiliates as a portfolio? How is the result of performance effect if the foreign affiliates are not ready to overcome the liabilities of foreignness, particular the role of payback is not realized role with other affiliates, or if political institutional coercive conditions of the home country are highly correlated with those of portfolio investments in which the multinational firm operates portfolio strategies. We wish to find result for these questions by testing on a comprehensive sample of 4833 observations of 324 Chinese publicly-list firms during 1991-2010. (For more information, please contact: I-Fan Yen, National Chi Nan University, Taiwan: ifan0715@gmail.com)

\section{Why Some Firms Succeed in Exporting While Others Do Not? The Impact of Firms' Resources and Capabilities on Export Performance}

Manuel Portugal Ferreira, Uninove - Universidade Nove de Julho

Luis Ganhao Simoes, ESTG - Instituto Politecnico de Leiria

Fernando Ribeiro Serra, Uninove - Universidade Nove de Julho \& globADVANTAGE

Why do some firms succeed in exporting while others do not? Export activities are a crucial area of international business but the drivers of superior export performance are not yet well understood. In this study, employing a sample of 52 Portuguese firms, we examine what are the distinctive resources and capabilities associated to superior export performance through the establishment of sustainable competitive advantages. Different combinations of export-related resources and capabilities are identified as sources of cost, product and servicetype advantages and then these are examined as to their impact on three types of export performance: economic, strategic and relational dimensions of export performance. The results show that resources and capabilities combine in complex manners to build competitive advantages with impact on export performance differences. The findings of this study have important implications for theory, managers and policymakers. (For more information, please contact: Manuel Portugal Ferreira, Uninove - Universidade Nove de Julho, Brazil: manuel.portugal.ferreira@gmail.com)

\section{Foreign Direct Divestment: Strategic Groups, Firm Capabilities and Market Exit Naomi A. Gardberg, Baruch College-CUNY Mehmet Erdem Genc, Montclair State University William Newburry, Florida International University}

For almost 20 years, retail executives and scholars have bemoaned the poor performance of international retailers. In this manuscript, we use this context to examine the antecedents of market exit or foreign direct divestment, building on internationalization theory, competitive dynamics and the resource based view (RBV) of the firm. We thus complement the rich research on market entry. Using a hazard model, we analyze the 274 market exits of the world's largest 200 retailers from 1997 to 2006. We find that corporate strategy and competitive dynamics rather than corporate performance affect market exits. Specifically, global strategy (multinationality), capabilities (formats), entry pace and market rivals influence market exit often with nonlinear relationships. In addition, firms are most likely to exit geographically and culturally distant markets even with growing GDP and large populations. (For more information, please contact: Naomi A. Gardberg, Baruch CollegeCUNY, USA: naomi.gardberg@baruch.cuny.edu) 


\author{
Institutional Diversification and Multinational Enterprise Performance \\ Yi Li, Australian National University \\ Lin Cui, Australian National University \\ Sophia Hamblin Wang, Australian National University
}

This paper extends the literature on the international diversification strategies of multinational enterprises (MNEs) by focusing on the performance benefits of institutional diversification along two different dimensions. Most of the existing studies have not explicitly taken into consideration the host-country institutional differences when examining the effect of international diversification. We seek to fill this research gap by developing a conceptual model of the effect of institutional diversification, an important dimension of international diversification, and deriving propositions about the relationship between different types of institutional diversification and the performance of MNEs. According to our model, the degree of institutional diversification will influence the performance of an MNE, while the scope of institutional diversification will negatively moderate this main effect. (For more information, please contact: Yi Li, Australian National University, Australia: yi.li@anu.edu.au)

\author{
How Industry Influences the Relationship between Firm Internationalization and Performance \\ B. Elango, Illinois State University \\ Mona Makhija, Ohio State University
}

The influence of industry context on the relationship between a firm's international strategy and its performance has been acknowledged but not carefully analyzed. Using a sample of 9050 firm-year observations over 12 years and five countries across four differing levels of industry globalization, we find that a multinational firm's international industry context strongly influences its internationalization-performance relationship. Specifically, we find a negative linear relationship for multidomestic industries, an inverted U-shaped relationship for simple global industries, a sigmoid relationship for complex global industries, and no relationship in the case of multidomestic-transitional industries. These findings indicate the importance of taking into account the level of industry globalization when considering the effect of a firm's internationalization on its performance. In doing so this research adds important insights to the debate on internationalization and performance. (For more information, please contact: B. Elango, Illinois State University, USA: elango@ilstu.edu)

\author{
Speed and Level of International Dispersion and Firm Performance \\ Pham Hoanh Son Nguyen, France Business School \\ Tugrul Atamer, EMLyon Business School \\ Zied Guedri, EMLyon Business School \\ Alain Charles Martinet, University of Lyon 3
}

This research shows that it is possible and necessary that we simultaneously bring together and study triple facets - degree, context and process - of internationalization in RIP research. Such an analysis approach, often seen as important, has been paradoxically very little adopted in past research. In observing this methodological gap, we attempt to examine not only the impact on performance of the sliding of diverse degrees of internationalization but also the context in which and the speed at which it occurs. By adopting this contextand-process-based approach, and leaning on a sample of 69 large international French firms over seven years, 2001 - 2007, we establish conceptually and empirically that (i) the relationship between the dispersion of internationalization and financial performance is of curvilinear and three-stage nature, taking the form of an inverted-S, and that (ii) whatever international dispersion pattern of the firm, a faster speed of dispersion of internationalization moderates negatively the relationship between the level of dispersion of internationalization and performance. (For more information, please contact: Pham Hoanh Son Nguyen, France Business School, France: pham.nguyen@esc-clermont.fr) 
Session: 3.1.15 - Interactive

Track: Track: 7 - Emerging Economies

\section{Marketing Strategy in Emerging Markets}

Presented On: July 6, $2013-09: 00-10: 15$

Chair: Constanza C. Bianchi, Universidad Adolfo Ibanez

Advertising in Emerging Economies: The Advertising Context of China

Marin Alexandrov Marinov, University of Gloucestershire

Svetla Trifonova Marinova, Aalborg University

Dan Alex Petrovici, University of Kent

John B. Ford, Old Dominion University

Emerging economies signify a large, expending, yet not well-studied market. Advertising in this context represents a serious challenge. Having studied the perceived advertising intrusiveness and the potential behavioural fallout of ad avoidance in the context of China this study tests a set of hypotheses. It has been found out that in forming their attitudes towards adverting the Chinese are profoundly affected by Confucian values, where the maintenance of harmony in personal relations is sought and protected. The findings confirmed that the role some predictors of ad avoidance (age) play is in contrast with that in developed economies. Respondents' level of education attainment reduces the likelihood to avoid ads both mechanically and behaviourally, which significantly differentiates China from other emerging economies. One the basis of the research findings recommendations are given to assist the development of advertising campaigns in the enormous Chinese marketplace. The paper provides evidence of the mediating role of ad annoyance in the relationship between perceived advertising intrusiveness and ad avoidance. It also specifies that while demographics play a significant role in predicting ad avoidance, some of these effects (e.g. education) may not be as linear as assumed in previous studies. (For more information, please contact: Marin Alexandrov Marinov, University of Gloucestershire, United Kingdom: m_a_marinov@hotmail.com)

Emerging Economies and Mega-Sporting Events: A Longitudinal Analysis of Advertising Patterns and Market Strategies of 2008 Beijing Olympic Games' Sponsoring Partners

Harald Dolles, Molde University College

Sten Soderman, Stockholm University

Sponsorship of mega-sporting events such as the Olympic Games the Football World Cup has become a multimillion dollar business. Established multinational enterprises and brands such as VW or Coca-Cola use such events as an international marketing platform. But sponsoring mega-sporting sporting event has also developed as a strategic way for local companies to enter new markets or to prove themselves as being ready for the world market. This is especially the case, when the event takes place in an emerging economy. Most sport sponsorship research has focused on the markets in North America, Europe or Australia, thus covering a different economic development and representing not the same market conditions. This research aims to fill the gap by describing and analysing the advertising behaviour of the sponsoring partners to the Beijing Olympic Games. A means-objective framework of sponsoring focussing on six factors was applied to analyse 739 advertisements, articles and press releases collected from Chinese newspapers and Chinese official web pages between 2001 and 2008. By applying a qualitative content analysis, we discovered eight dominant meansobjectives combinations leading to different sponsor advertising strategies depending on the lead time to the Olympic Games and the level of internationalization of the sponsoring company. (For more information, please contact: Harald Dolles, Molde University College, Norway: harald.dolles@himolde.no) 
The Implementation of Brand Management Practices in an Emerging Country Market: An Exploratory Study

Ekrem Tatoglu, Bahcesehir University

Daragh O'Reilly, University of Sheffield

Very little empirical work has been carried out on the sets of brand management practices (BMPs) employed by firms in emerging country (EC) markets. With the growth in economic power of those countries, and the strategic importance of brand competition generally, there is therefore a clear need to generate insight into specific BMPs in EC markets. Using Keller's Brand Report Card (BRC) as an analytical framework, a survey was carried out into the BMPs of a range of organizations in a key EC market, Turkey. A set of hypotheses were developed regarding the relative use of BRC-specified practices with reference to sample characteristics. The results of the survey indicate strong support for the hypothesis that the relative use of BMPs varies with the existence of a product/brand management department within the firm. There was also considerable support for the hypothesis that the relative use of BMPs varies with the ownership pattern of firm. A reasonable level of support was found for the size of firm and the availability of brand support affecting relative use of such practices. There is, however, no support for either the age of the firm or the industry sector affecting the relative use of BMPs. (For more information, please contact: Ekrem Tatoglu, Bahcesehir University, Turkey: ekrem.tatoglu@bahcesehir.edu.tr)

\section{Cultural Eco Map: Exploring the Interrelations of Heterogeneous Cultural Resources and Customer Value Proposition of Business Model}

Andrejs Cirjevskis, Riga International School of Economics and Business Administration (RISEBA)

The aim of this research paper is to identify new Customer Value Proposition (CVP) of Riga Central Market (RCM) of Latvia, a huge infrastructural establishment. The number of customers has been decreasing during the past four years due to economic crisis. Having answered first research question about what is current CVP of RCM, authors have conducted unstructured interview with RCM management and merchants operating at RCM. Answering the second research questions about what Latvian cultural resources can underpin of new CVP of RCM, a survey was conducted among foreign visitors of RCM. Summing up results Eco-map has been used in order to classify the Latvian cultural resources available for the new modified CVP of RCM and define their availability, applicability and interrelationships. Eco map facilitated to discover how to dispose national cultural resources innovatively and efficiently, and thus sustain competitive advantage by reinforcing CVP of organisation. (For more information, please contact: Andrejs Cirjevskis, Riga International School of Economics and Business Administration (RISEBA), Latvia: andrejs.cirjevskis@riseba.Iv)

\section{Export Credit Agencies, Cross Border Trade, Times of Crisis, and Increased Value Added in Emerging Economies Industrial Sectors \\ Hilmar Thor Hilmarsson, University of Akureyri \\ Trung Quang Dinh, University of Akureyri}

Abstract. Export Credit Agencies (ECAs) played an important role in cushioning the downturn in cross border trade to emerging market economies during the economic and financial crisis that started in the fall 2008. In addition to facilitating trade during times of crisis ECAs can also help companies in emerging countries access long term funding at lower interest rate than they could access locally. This can also help companies modernize their processing lines, especially those engaged in capital intensive activities, and enable economies in transition increase the value added of their industries. This article discusses the role of ECAs in facilitating cross border trade to emerging markets as well as the economic rationale for the existence of such agencies. It also demonstrates how selected risk mitigation instruments of ECAs, namely: (i) buyer credit guarantee, (ii) supplier credit guarantees and (iii) export loans have been applied in practice. Finally cases are presented that highlight how companies have used the service of ECAs, for example, to obtain better terms, including longer term loans and/or lower interest rates. (For more information, please contact: Hilmar Thor Hilmarsson, University of Akureyri, Iceland: hilmar@unak.is) 


\section{Session: 3.2.1 - Special Session}

\section{JIBS Decade Award}

Presented On: July 6, $2013-10: 55-12: 10$

Chair: John Cantwell, Rutgers University

The 2013 JIBS Decade Award, sponsored by Palgrave Macmillan, honors the most influential paper published in the 2003 volume of the Journal of International Business Studies

A Retrospective on their article by the winners of this year's JIBS Decade Award, reconsidering the issues raised in their 2003 JIBS paper on "MNC knowledge transfer, subsidiary absorptive capacity, and HRM"

Dana Minbaeva, Copenhagen Business School

Torben Pedersen, Copenhagen Business School

Ingmar Björkman, Aalto University

Carl Felix Fey, Nottingham University

Hyeon Park, Georgia State University

Discussants and Commentators:

Jaeyong Song, Seoul National University

Paula Caligiuri, Northeastern University

(For more information, please contact: John Cantwell, Rutgers University, USA: cantwell@business.rutgers.edu)

\section{Session: 3.2.2 - Panel \\ Track: Track: 11 - SMEs and Entrepreneurship \\ Role of International Entrepreneurship in Global Business: Present \& Future}

Presented On: July 6, $2013-10: 55-12: 10$

Chairs: Tugba Kalafatoglu, ESADE Business School and Yusuf Kurt, University of Manchester

Panelists:

Sylvie K. Chetty, University of Otago

Gary Knight, Willamette University

Olli Kuivalainen, Lappeenranta University of Technology

Pinar Ozcan, Warwick Business School

The panel will address the role of international entrepreneurship in global business. With the globalization of the world economy, interest in international entrepreneurship has increased rapidly over the years, especially in developing economies. For many firms, going global is the key to their business success. International markets present opportunities in all regions of the world, especially for entrepreneurs. However, the question of which firms are responding to worldwide opportunities remains. What factors play important role in international entrepreneurship? What models and other theoretical approaches have the greatest explanatory value in international entrepreneurship? What is the relevance of the models/theoretical approaches over time when internationally entrepreneurial firms develop further? Research in international entrepreneurship is still at an early stage. As indicated above, numerous issues remain to be investigated. Accordingly, the panelists will address these and many other issues related to international entrepreneurship. (For more information, please contact: Tugba Kalafatoglu, ESADE Business School, Spain: tugba@tugbakalafatoglu.com) 
Session: 3.2.3 - Panel

Track: Track: 1 - Institutions, Governance, and CSR

\section{Global Governance and International Strategies}

Presented On: July 6, $2013-10: 55-12: 10$

Chair: Srividya Jandhyala, George Washington University

Discussant: Steve Kobrin, University of Pennsylvania

Panelists:

Jonathan Doh, Villanova University

Eun-Hee Kim, George Washington University

Srividya Jandhyala, George Washington University

Robert Weiner, George Washington University

Noel Maurer, Harvard Business School

Daniel Blake, IE Business School

This panel addresses how MNCs respond to the challenges and opportunities presented by the spread of global governance institutions. Examining the different types of global institutions, including climate change treaties, international investment agreements, and regional trade agreements, this panel presents interdisciplinary research that provides insight on how MNCs interact with the global institutional environment. Limiting or boundary conditions where global regimes are ineffective are also identified. (For more information, please contact: Srividya Jandhyala, George Washington University, USA: srividya@gwu.edu)

\section{Session: 3.2.4 - Panel}

Track: Track: 9 - Cross-Cultural Management and HRM

\section{Women Navigating in Global Male Dominated Industries: Explaining the Factors for Success}

Presented On: July 6, 2013 - 10:55-12:10

Chairs: Joan P Mileski, Texas A\&M University at Galveston and Tugba Kalafatoglu, ESADE Business School

Panel Sponsored by WAIB, Women in the Academy of International Business.

Panelists:

Joan P Mileski, Texas A\&M University at Galveston

Aylin Alpustun, Turk Ekonomi Bankasi

Gulfem Toygar, Otto Group

A. Guldem Cerit, Dokuz Eylul University

Seval Ozcan, Blanchard International Turkey

This panel will address the art and mechanics women can use to successfully navigate male dominated global industries. The importance of women using the right tools to gain success in a man's world of global business is well documented. Key issues raised by women have been: How much should I be "one of the boys"? Should I even enter this industry? Should I avoid operations and look to finance, law, consulting or other technical skills career paths? Who should be my advisor/mentor? How do handle isolation or non-inclusion? This panel will address the skills, the tools and the temperament required of women to successfully navigate these global industries. The panelists bring their experiences and expertise to the roundtable format to begin a discussion of 
what they have learned and the keys to a successful business career in these industries. Thus, this panel hopes to "bridge the divide: linking IB theory to practice." (For more information, please contact: Joan P Mileski, Texas A\&M University at Galveston, USA: mileskij@tamug.edu)

\title{
Session: 3.2.5 - Competitive
}

\section{Track: Track: 9 - Cross-Cultural Management and HRM}

\section{MNCs and Host-Country Nationals}

Presented On: July 6, 2013 - 10:55-12:10

Chair: Winfried Ruigrok, University of St. Gallen

\author{
International Human Resource Management Practices and Host Country National Attitudes toward Expatriates \\ Haiying Kang, University of South Australia \\ Jie Shen, University of South Australia \\ Peter John Dowling, La Trobe University
}

This study explored the effects of socially responsible international human resource management (SRIHRM) on host-country national $(\mathrm{HCN})$ attitudes toward expatriates. Analysis of the data from South Korean MNEs operating in China indicate that external corporate social responsibility-supportive IHRM and employee-oriented IHRM practices directly and indirectly influence $\mathrm{HCN}$ attitudes toward expatriates through the mediation of organizational identification and perceived organizational support respectively. These findings indicate that in addition to personal factors, MNE policies and practices, such as IHRM are important antecedents to HCN attitudes toward expatriates. (For more information, please contact: Peter John Dowling, La Trobe University, Australia: p.dowling@latrobe.edu.au)

\section{Localization and the Performance of Foreign Subsidiaries \\ Naoki Ando, Hosei University \\ Yongsun Paik, Loyola Marymount University}

The primary purpose of this study is to examine whether localization of the foreign subsidiary positively or negatively affects the subsidiary performance. Hypotheses regarding the effect of localization on the subsidiary performance are developed based on the arguments about challenges that foreign firms confront in host countries and roles that host country nationals play in foreign subsidiaries. Using the panel dataset that consists of 4,880 foreign subsidiaries of multinational corporations, this study finds that localization is positively associated with subsidiary performance. This study also finds the significant role of moderators that influence the extent of the positive effect of localization on subsidiary performance. The results shows that the positive effect of localization on subsidiary performance strengthens as the ratio of parent country nationals to subsidiary employees increases. In comparison, the results reveal that the positive effect of localization declines as the parent firm accumulates international experience. The results also finds that the positive effect of localization decreases with country risk of the host country. (For more information, please contact: Naoki Ando, Hosei University, Japan: nando@hosei.ac.jp)

Influence of Localization on Host Country Nationals' Withdrawal Cognition in China: The Moderating Effect of Demographics

Azusa Hitotsuyanagi, Georg-August-Universitaet Goettingen

Fabian Jintae Froese, Georg-August-University Goettingen

Yong Suhk Pak, Yonsei University 
In the recent decades, an increasing number of foreign multinational enterprises (MNEs) have entered the Chinese market. However, the high turnover rate among host country nationals (HCNs) provokes great challenges in retaining talent in China. Therefore, this study focuses on how localization of foreign MNEs affects withdrawal cognitions among HCNs. Based on the Social Identity Theory, we have developed a model to explain the heterogeneous nature of this influence across diverse demographic groups of HCNs. Survey results from 243 Chinese employees show that localization increases organizational commitment and reduces turnover intentions among Chinese employees, in particular among the highly educated male employees. (For more information, please contact: Azusa Hitotsuyanagi, Georg-August-Universitaet Goettingen, Germany: azusa.hitotsuyanagi@wiwi.uni-goettingen.de)

\section{Performance Outcomes of Leadership Succession at Foreign Subsidiaries in Japan Fabian Jintae Froese, Georg-August-University Goettingen \\ Ralf Bebenroth, Kobe University}

Leadership succession has been an important topic in the general management literature but received little attention in international business research. This study integrates leadership succession and expatriate staffing literatures by investigating performance outcomes of leadership succession at foreign subsidiaries in Japan. Building on agency and institutional theory, we developed a model explaining the different inside and outside status of expatriate and local subsidiary managers and how these affect subsidiary performance. We distinguished four types of subsidiary CEO successors: expatriate followers (expatriate succeeds another expatriate), localizers (local manager succeeds an expatriate), local followers (local manager succeeds another local manager), and ambassadors (expatriate succeeds a local manager). Our theory and evidence from 2,113 firm-year observations, including 521 successions, suggests that successor types have direct and moderating effects with contextual firm-level factors (pre-performance and subsidiary age) on subsidiary performance. (For more information, please contact: Fabian Jintae Froese, Georg-August-University Goettingen, Germany: fabian.froese@gmail.com)

\section{Session: 3.2.6 - Competitive}

\section{Track: Track: 8 - Developing Country MNCs}

\section{Organizational Structure Effects on Internationalization and Performance of Developing Economy MNES}

Presented On: July 6, $2013-10: 55-12: 10$

Chair: Paz Estrella Tolentino, University of London

Internationalization of Emerging Multinationals: Role of Owner-CEOs

Raveendra Chittoor, Indian School of Business

Preet S. Aulakh, York University

Sougata Ray, Indian Institute of Management Calcutta

Deepak Jena, Indian School of Business

A growing body of work has highlighted that the internationalization paths, processes and positions of emerging multinationals (EMNEs) are distinct and differ from those of the traditional MNCs from the West. We are yet to understand, however, some of the underlining drivers that give rise to these differences. In this paper, we identify and theorize on one such key driver of EMNEs' internationalization namely, managerial intentionality specifically owners taking on the role of manager-CEOs. We propose that firms with owner-CEOs, aided by their long-term orientation and less-restricted decision making powers, are likely to attain higher degree of internationalization. Further, the effect of owner-CEOs on internationalization is higher for independent firms than for firms affiliated to business groups. We test our predictions using a proprietary, longitudinal panel data 
set of 368 Indian firms (that were a part of the BSE 500 index as on September 2012) over the ten-year period from 2002 to 2011 and find supporting evidence. (For more information, please contact: Raveendra Chittoor, Indian School of Business, India: raveendra_chittoor@isb.edu)

\section{Business Group Heterogeneity and the Internationalization-Performance Relationship: Evidence from Indian Business Groups}

Saptarshi Purkayastha, Indian Institute of Management Kozhikode

Vikas Kumar, University of Sydney

Jane Wenzhen Lu, University of Melbourne

Business groups constitute a dominant organizational form in many Asian markets, and many of these groups expand operations into international markets. Previous studies ignore the heterogeneity in business groups' ability to manage internationalization, based on their resources and capabilities. The present study builds on existing research to examine heterogeneity among business groups in terms of specific resources and capabilities that influence internationalization. In particular, this article characterizes business group heterogeneity by size, product diversification, and ownership, because these factors should moderate the relationship between internationalization and performance. Taking a sample of 107 Indian business groups for a period of 5 years (2004-2008), the study finds that at high levels of internationalization, larger, less diversified groups should enjoy superior performance, and ownership groups proxied by the extent of shareholding should exert differential impacts on group performance. (For more information, please contact: Saptarshi Purkayastha, Indian Institute of Management Kozhikode, India: spurkayastha1@gmail.com)

\section{Performance Persistence of Emerging Economy Firms: The Interaction Effects of Business Group, Home Institution and Internationalization \\ Lin Cui, Australian National University \\ Helen Hu, University of Melbourne \\ Preet S. Aulakh, York University}

Drawing on insights from the institutional perspective and resource-based view on business groups, we examine how and why institutional plurality in the forms of home-country institutional configurations and foreign institutional environments can affect performance persistence of business group firms in two large emerging economies - China and India. An analysis of 1,153 Chinese and Indian firms between 2005 and 2010 demonstrates that the affiliation with a business group positively influences the firm's performance persistence. However, this positive relationship is moderated by (1) the institutional environment of the firm's home country, and (2) the extent to which a firm escapes from its home institutional environment. In particular, this relationship is strengthened by a coordinated market institution of China compared with a liberal-market institution of India, and is weakened by the firm's degree of internationalization. These findings suggest that knowledge of the interactions between business groups and the institutional environments in which they operate is essential for understanding the performance outcomes of business group affiliates. (For more information, please contact: Lin Cui, Australian National University, Australia: lin.cui@anu.edu.au)

\section{Business Model Management in the Internationalization of Emerging Market Firms: The South African Experience}

Margarete Kalinowski, ESADE Business School

Luis Vives, ESADE Business School

The phenomenal surge in internationalization by emerging market multinationals (EMNEs) has gained momentum in academic research. This paper contributes to the growing stream of research by exploring the internationalization trajectories and business model management strategies of firms from emerging markets during their processes of global expansion. Inductively drawing from in-depth analysis of eighteen South African 
multinational firms, this paper exhibits four strategic groups classified by the differences in their business model management strategies during global expansion. We advance on the insights and propose a conceptual model of internationalization for emerging market firms through a combination of exploitation and exploration strategies along the dimensions of institutional difference and strategic initiatives in terms of leveraging their own business models or developing new business models. The model reveals four different business model management strategies EMNEs can pursue during their internationalization processes. Firms that are able to supplement their global expansion through single business model management, based on the conventional exploitation strategies, with exploration of new advantages through development of new business models could emerge as African contenders with capabilities that could challenge MNEs from the developed world. The paper concludes with theoretical and managerial implication and fruitful avenues for future research. (For more information, please contact: Margarete Kalinowski, ESADE Business School, Spain:

margarete.kalinowski@esade.edu)

\section{Session: 3.2.7 - Competitive}

Track: Track: 1 - Institutions, Governance, and CSR

\section{Role of the CEO and Board}

Presented On: July 6, $2013-10: 55-12: 10$

Chair: Maria Alejandra Gonzalez-Perez, Universidad EAFIT

The Impact of CEO Incentive and CEO Monitoring Mechanisms on Risky International Expansion Yu-Kai Wang, Soochow University

Chris Changwha Chung, Korea University

This study examines risky international expansion associated with both new products and new geographic markets. From the perspective of chief executive officers (CEOs), such expansion is associated with high risk and long-term payoff. Thus, most CEOs may not favor these activities, even though these activities could result in new capabilities that would serve not only the local markets but also the parent firm and sister subsidiaries. Drawing upon agency theory, this study argues that CEO incentive alignment and CEO monitoring mechanisms are remedies for the agency problem. The empirical results of the study support the arguments. (For more information, please contact: Yu-Kai Wang, Soochow University, Taiwan: ywang012@scu.edu.tw)

\section{An Investigation of the Relationships between Board Characteristics, CEO Influence, and the Performance of Cross-Border Acquisitions \\ Deepak K Datta, University of Texas at Arlington \\ Dynah A Basuil, Asian Institute of Management}

This study on the relationships between board characteristics, CEO influence and cross-border acquisition performance is based on a sample of 440 cross-border acquisitions undertaken between 1991 and 2006 by U.S. firms. Grounded in agency and resource dependence theories, our research reveals that U.S. acquiring firms with larger boards and outside directors with longer tenure are associated with greater shareholder gains from their acquisitions. However, contrary to our expectations, we found board independence to be negatively associated with shareholder value. Likewise, our findings indicate that the influence exerted by the CEO (proxied by duality and high CEO position tenure) is positively associated with shareholder gains. We discuss the implications and contributions of our findings from the standpoint of research and practice. (For more information, please contact: Deepak K Datta, University of Texas at Arlington, USA: ddatta@uta.edu) 
Exchange Rate and Macroeconomic Fluctuations as Sources of Luck in CEO-Compensation

Clas Wihlborg, Chapman University

Hsin Hui Chiu, California State University

Lars Oxelheim, Lund University

Jianhua Zhang, University of Gothenburg

Macroeconomic fluctuations reflected in exchange rates and other macroeconomic variables can be considered sources of good or bad "luck" for corporate performance. Incentive effects of performance-based compensation for management may be weakened or biased by macroeconomic influences on remuneration depending on inability of management to adjust operations within the time frame of macroeconomic events. We decompose the changes in CEO-compensation to distinguish between macroeconomic and "intrinsic" sources of compensation in order to evaluate the contribution of macroeconomic fluctuations over different time horizons. Total US CEO-compensation is measured both including options awarded and options exercised. Both measures depend strongly on variations in macro-factors but the time patterns differ. The estimated effects of macroeconomic factors on compensation change substantially when we allow for asymmetric effects on compensation. (For more information, please contact: Clas Wihlborg, Chapman University, USA: wihlborg@chapman.edu)

\section{Session: 3.2.8 - Competitive}

\section{Track: Track: 12 - Economic Geography and Value Chains}

\section{Sourcing and Offshoring}

Presented On: July 6, $2013-10: 55-12: 10$

Chair: Aks Zaheer, University of Minnesota

The Time Opportunity Cost of Cultural Differences in Global Services Sourcing

Carine Peeters, Universite libre de Bruxelles

Catherine Dehon, ULB - Solvay Brussels School of Economics and Management

Patricia Garcia-Prieto, ULB - Solvay Brussels School of Economics and Management

We show that cultural differences between home and host countries of offshored services delay the attainment of target service levels, but positively affect the realization of cost savings expectations. Hence, cultural differences would be best interpreted in terms of opportunity cost of time. For a fast transition to service levels, a culturally close host country may be preferred, provided sufficient effort is put in developing accurate cost savings expectations and controlling implementation costs. If a longer transition time is acceptable, firms may opt for a culturally distant country that gives access to location specific advantages not available otherwise. (For more information, please contact: Carine Peeters, Universite libre de Bruxelles, Belgium:

carine.peeters@ulb.ac.be)

\section{Hidden Costs and the Role of Modularity: A Study on Offshoring Process Performance}

Marcus M. Larsen, Copenhagen Business School

Abstract: This paper addresses estimation errors in strategic decision-making processes due to hidden costs. While previous research has investigated the antecedents of hidden costs, this paper investigates performance consequences. Using unique data on 221 offshoring implementations, it is argued that the inability to effectively estimate the costs of implementing an activity in a foreign location has a negative impact on the process performance of that activity. Performance is deterred as operations are likely to be disrupted by opportunity costs and managerial responses. However, this relationship is mitigated by the degree of modularity in the activity as it reduces the need for costly coordination in offshoring. This paper contributes to research on 
offshoring and strategic decision-making by emphasizing the importance of organizational design and of estimating the costs of internal organizational change. (For more information, please contact: Marcus M. Larsen, Copenhagen Business School, Denmark: mml.smg@cbs.dk)

\author{
Business Process Offshoring: Investigating the Role and Impact of International Strategy \\ Niccolo Pisani, IESE \\ Joan Enric Ricart, IESE
}

Business process offshoring, intended as the firm-level choice of relocating service and higher-skilled activities abroad, predominantly to lower-cost countries, has decidedly grown in the last decade and it is today envisaged as a crucial aspect in the reconfiguration of modern multinationals. International management scholars have increasingly investigated such phenomenon. Yet, a thorough examination of the offshoring literature noticeably indicates that little attention has been paid to appreciate the role and impact of international strategy in guiding offshoring implementations. We contend that such dearth is also caused by terminological incongruities in the literature that prevent an encompassing appraisal of the relation between these two constructs. Thus, building on a rigorous definition of international strategy and contextualizing the offshoring choice by distinguishing between strategic and tactical offshoring, we hypothesize that the formulation (versus non-formulation) of an international strategy for guiding an offshoring implementation increases the likelihood that such offshoring implementation is strategic (versus tactical). Additionally, we posit that the formulation (versus non-formulation) of an international strategy for guiding a strategic offshoring implementation has a positive effect on its outcome. We empirically validate our hypotheses testing them on a fine-grained dataset of 1,110 offshoring implementations executed by 324 multinationals offshoring to 76 countries. (For more information, please contact: Niccolo Pisani, IESE, Spain: npisani@iese.edu)

\title{
Sourcing from Multinational Suppliers to Overcome Weak Contracting Institutions and Gain Supply Chain Capabilities \\ Markus D. Taussig, National University of Singapore \\ Kjell Carlsson, Wilson Perumal \& Company
}

This paper examines how characteristics of global buyers of labor-intensive manufacturing goods and the institutions in the host countries where the goods they buy are produced influence the propensity for purchases to be made from multinational suppliers. We find that buyers are more likely to source from multinational firms when contracting institutions are weaker and buyer purchasing is more complex, but less likely to buy from multinationals when buyers are more experienced. Our study takes advantage of a unique proprietary dataset on the sourcing activities of ten large US-based companies and their 350 suppliers in sportswear, apparel, footwear, toy, and furniture industries and shipping from 21 countries during 2007-2009. (For more information, please contact: Markus D. Taussig, National University of Singapore, Singapore: markus@nus.edu.sg)

\section{Session: 3.2.9 - Competitive \\ Track: Track: 7 - Emerging Economies \\ Qualitative Research in Emerging Markets}

Presented On: July 6, $2013-10: 55-12: 10$

Chair: Seung Ho Park, Moscow School of Management SKOLKOVO 
The Professional Services Firm (PSF) in a Globalised Economy: A Case Study of the Performance of Foreign and Domestic Securities Firms in an Emerging Market

Mehmet Demirbag, University of Sheffield

Martina McGuinness, University of Sheffield

Ahmet Akin, Fatih University

Nizamettin Bayyurt, Fatih University

Eyup Basti, Fatih University

This study explores the performance of securities firms in Turkey and offers conceptual and managerial insights utilizing data envelopment analysis. Through a complete sample of local and foreign owned securities firms in Turkey, we examine the impact of liabilities of foreignness and localness upon knowledge intensive firm performance in an emerging market economy. We have extended this approach through our consideration of liability associated with market globalness. Our findings indicate the importance of size for firm efficiency with bank affiliation and foreign ownership also having positive effects on efficiency. For smaller firms, the impact of affiliation and ownership is ore mixed; impacting positively on medium-sized firms but negatively on small firms. Our study makes a contribution conceptually, methodologically and empirically to a growing literature on emerging economies. We also make a valuable addition to the limited empirical work conducted on the securities industry to date. Finally, through our contextualization of Turkish securities firms as professional services firms (PSFs), our research extends the narrow focus on law and accounting which currently dominates the burgeoning research strand on PSFs. (For more information, please contact: Mehmet Demirbag, University of Sheffield, United Kingdom: m.demirbag@sheffield.ac.uk)

\section{Building on Institutional Voids: A Case of Business Groups in an Emerging Economy Ashar Saleem, Lahore University of Management Sciences \\ Abdur Rahman, Lahore University of Management Sciences}

This paper studies the relationship a business group (BG) holds with the market institutions of its environment. The literature draws two different pictures of business groups in emerging economies; one in which BGs are filling missing market institutions through their specific structure; and the other in which they are exercising their power to shape their environment and hence market institutions. The problem seems methodological, however, as there is hardly any empirical work which compares how business group and independent firms are related to their market institutions in one given context. Through a unique case study which focuses on one business group firm and four non-group firms in the same sector ( i.e., home textile industry) in one geographical location in Pakistan, the research tries to find out an answer to our research question through formulating alternate sets of hypotheses. Our research question is that what is the relationship, firms from a Business group and firms working independently hold with the market institutions of their environment. Based primarily on interviews with managers and owners of these firms supplemented by secondary source data, the study concludes that Business Groups play a decisive role in perpetuating and expanding the existing and creating new institutional voids in their environment. Contributions is made to the theory in identifying specific types of institutional voids and processes of their emergence and to the methodology of studying Business Groups . (For more information, please contact: Ashar Saleem, Lahore University of Management Sciences, Pakistan: ashars/m@gmail.com)

\section{Diversification Strategies of Transition Economy Entrepreneurs: Qualitative Research as a Bridge to} Quantitative Analysis

Anna Lamin, Northeastern University

Sheila Puffer, Northeastern University

Daniel McCarthy, Northeastern University

The purpose of this article is to explore the nature of, and influences of entrepreneurial characteristics on, growth strategies of highly successful Russian entrepreneurs. Using content analysis of qualitative materials of 
finalists of the Ernst \& Young Russian Entrepreneur of the Year competition (2003 through 2008), we created a data set of quantitative variables to examine these relationships. Seriality, or the number of startups an entrepreneur had previously founded, was positively related to the extent of both vertical integration and horizontal diversification that was accomplished. Additonally, risk-averse entrepreneurs were more likely than risk-embracing entrepreneurs to engage in unrelated horizontal diversification strategies, suggesting that riskaverse entrepreneurs spread risk among multiple opportunities. As such, entrepreneurial characteristics in turbulent transition environments may lead to growth strategy decisions that may in some cases be similar to, and in others different from, those in more developed economies. The study may have implications for institutional entrepreneurship in emerging economies. This article demonstrates how a bridge can be built to translate the richness of qualitative research into the precision of empirical analysis to better shed light on underlying phenomena than strictly empirically based research. (For more information, please contact: Anna Lamin, Northeastern University, USA: a.lamin@neu.edu)

Service Integration as a Business Model for Firms from Emerging Countries: Insights from the Medical Tourism Industry

Pooja Thakur, Virginia Tech

Christian Wernz, Virginia Tech

Kongkiti Phusavat, Kasetsart University

This paper introduces service integration as a business model for emerging country firms. Service integration is the innovative combination of services to achieve higher customer satisfaction. Service integration, occurring at the firm level, is the enabler and precursor of service convergence, an industry level phenomenon. We analyzed the business model of Bumrungrad International Hospital (BIH), Thailand, an emerging country firm and a major player in the medical tourism industry. We identified nine key steps BIH has taken to stay ahead of their competitors. BIH followed a two-fold strategy: (1) low cost through operational efficiencies, and (2) differentiation through service integration. In particular, we discuss how BIH's business model led to the integration of medical and hospitality services. (For more information, please contact: Pooja Thakur, Virginia Tech, USA: pthakur@vt.edu)

\section{Session: 3.2.10 - Competitive}

Track: Track: 6 - Innovation and Knowledge Mgmt.

\section{Innovation, Knowledge and Performance}

Presented On: July 6, $2013-10: 55-12: 10$

Chair: Xiaobai Shen, University of Edinburgh

\section{Reverse International Knowledge Transfer in the MNE: (Where) Does Affiliate Performance Boost Parent} Performance?

Nigel Driffield, Aston University

James Love, Aston University

Yong Yang, University of Sussex

We examine the extent to which the knowledge or technological capability of foreign affiliates actually enhances the performance of their parent companies. Our results draw on a firm-level panel of more than 1,600 multinationals and more than 4,000 of their overseas affiliates, covering 46 home and host countries. We find considerable evidence of enhanced parent productivity as a result of their affiliates' performance, which we interpret as evidence of reverse knowledge transfer from affiliates to parents. This effect is robust to different tests including IV estimation and a falsification exercise based on unconnected 'matched' affiliates. We find that both physical and strategic location markedly affects the affiliate-parent relationship, and that distance reduces 
the positive impact that affiliate performance has on that of the parent. (For more information, please contact: Yong Yang, University of Sussex, United Kingdom: yong.yang@sussex.ac.uk)

\author{
International Technology Sourcing Strategies and Inventor Employment \\ Roger Smeets, Rutgers University
}

This study investigates the extent to which domestic and foreign R\&D workers (i.e. "inventors") employed by multinationals (MNEs) are complements or substitutes to each other. We argue that this relationship will be contingent on the strategy underlying MNEs' foreign R\&D activities, where we identify three such strategies: technological catch-up, technological diversification, and creating a R\&D springboard. In the case of catch-up, we don't expect to find complementarities, but instead either substitution or no relationship at all. Under diversification, we expect complementarities to arise at intermediate levels of technical (dis)similarity between the domestic and foreign operations. Finally, in the case of R\&D springboards, we also expect complementarities to arise. These expectations are largely corroborated in a sample of large and innovative European MNEs during the period 1995-2005. (For more information, please contact: Roger Smeets, Rutgers University, USA: rsmeets@business.rutgers.edu)

\title{
Trust, Transaction Cost and Knowledge Sharing in Strategic Alliances: A Study of Supplier Relationship in Thailand
}

Rapeeporn Rungsithong, University of Bath

Klaus E. Meyer, China Europe International Business School

Anthony Roath, University of Bath

This paper introduces a trust view of knowledge sharing in cross-cultural alliances and tests the contingency effect of transaction cost factors of the relationship between trust and knowledge sharing. In our model, the positive relationship between trust and knowledge sharing is moderated by transaction cost to which an alliance is exposed to, including tangible and intangible asset specificity, ownership modes and human resource distance between alliance partners. Using a sample of 156 strategic alliance projects between MNE subsidiaries and local suppliers in the Thai manufacturing sector, we find empirical support for most hypotheses derived. Implications are drawn for both trust and TCE to strategic alliance research. (For more information, please contact: Rapeeporn Rungsithong, University of Bath, United Kingdom: rr257@bath.ac.uk)

\section{Selective Attention and the Initiation of the External Knowledge Sourcing Process in Multinational Corporations Felipe Monteiro, INSEAD}

Multinational corporations (MNCs) frequently use subsidiaries around the globe to identify new opportunities to access external knowledge. Decision makers in MNCs, however, are selective in the issues they attend to at any time and it is bound that a number of opportunities to transfer external knowledge that subsidiaries identify will be screened out. This paper examines why some types of opportunities to transfer external knowledge are more likely to be screened out and the role of subsidiary managers in influencing decision makers' selective attention. I suggest that given the behavioral and cognitive mechanisms influencing selective attention, decision makers are more likely to screen out those opportunities which are not proven and which contradicts what the MNC already knows. The paper also suggests that the amount of effort subsidiary managers put not only in selling external opportunities internally but also in searching for internal needs and matching external opportunities to those needs, plays a critical role in decreasing the likelihood that decision makers will screen out an opportunity to transfer external knowledge. I test my hypotheses using a proprietary database on 137 external knowledge sourcing opportunities analyzed by one of the largest telecommunication services providers in the world. (For more information, please contact: Felipe Monteiro, INSEAD, France: felipe.monteiro@insead.edu) 


\section{Session: 3.2.11 - Interactive \\ Track: Track: 3 - IB Theory, FDI, and Entry Mode}

\section{Institutional Perspectives on FDI}

Presented On: July 6, $2013-10: 55-12: 10$

Chair: Jean-Francois Hennart, Singapore Management University and University of Pavia

The Impact of Institutional Imprinting on Inward Internationalization Diversity: Evidence from Chinese Private Sector

Hongxin Zhao, Saint Louis University

Jieqiong Ma, Saint Louis University

Long been neglected by the internationalization research, inward internationalization can be of significance to the outward internationalization process. We explore institutional determinants of inward internationalization combining the perspectives of organizational imprinting theory, theory of planned behavior, and organizational learning theory. Specifically, we propose that due to the imprinting effect of organizational learning orientation in turbulent transitional environment, firms founded in the experimental stage would be more inclined to participating in inward internationalization than firms founded in the implementation stage. We also consider the moderating roles of institutional development and corporate governance structure in the relations of institutional imprinting and inward internationalization. The empirical results show that experimental stage firms are more diverse in terms of inward internationalization than their implementation stage counterparts and that institutional development and corporate governance structure negatively moderate the relationship between institutional imprinting and inward internationalization diversity. (For more information, please contact: Jieqiong Ma, Saint Louis University, USA: jma17@slu.edu)

\section{The Effect of National Ethical Environment on Japanese FDI in Developing and Developed Countries} George Z. Peng, University of Regina

Jean-Marie Nkongolo-Bakenda, University of Regina

We examine the relationships between Japanese foreign direct investment (FDI) and the national ethical environment (NEE) in developed and developing countries by adopting the eclectic paradigm and institutional theory. Based on data from the Japanese Government's Ministry of Finance, an NEE index developed by Franke and Nadler (2008), and other sources, we find that the underlying host country development stage moderates the relationship between the level of NEE and Japanese FDI inflow: the relationship is positive for developing countries, but negative for developed countries. The results shed interesting light on FDI behavior in the context of business ethics research. Theoretical and practical implications of the findings are discussed. (For more information, please contact: George Z. Peng, University of Regina, Canada: george.peng@uregina.ca)

\section{Cross-Border Megamergers as Socio-Cognitive Hubs: A Dominance Analysis Approach Weilei (Stone) Shi, Baruch College-CUNY Ravi Madhavan, University of Pittsburgh EunJoo Yi, University of Pittsburgh}

Adopting a socio-cognitive lens, we propose that cross-border megamergers will have a disproportionate influence on participating nations' subsequent foreign direct investment (FDI) flows. Such megamergers, by virtue of their size and visibility, will attract FDI decision makers' attention and serve as initiating forces that shape subsequent FDI efforts. The hypothesis that nations participating in cross-border megamerger deals will experience higher FDI flows in subsequent years finds support in data on cross-border megamergers for the period 1996-2007. We employ Dominance Analysis to compare the effect of Megamergers with that of non- 
Megamergers, and conclude that Megamergers do have a greater impact. (For more information, please contact: Weilei (Stone) Shi, Baruch College-CUNY, USA: weilei.shi@baruch.cuny.edu)

\author{
The Determinants of China's Outward FDI: From the Home Country's Institutional Perspective \\ Mengmeng Shan, Fudan University \\ Yuanxu Li, Fudan University
}

Recently, outflows of FDI from emerging markets attract increasing shares in the total outflows of world economy. China leads the list of emerging markets in outward FDI with the implementation of "go global strategy". And we also note the variation in outward FDI from different regions across China, the typical country with large geographic size and various sub-national institutions. From a home country's institutional perspective, this paper examines the specific impact of market fragmentation and state-owned share of emerging markets, which are shaped by local institutions, on the decision of the firms to undertake outward FDI. Using provinciallevel panel data (30 sub-regions) from China throughout the year of 2002 to 2010, our results suggest that regions with higher level of market fragmentation and state-owned share are more likely to invest oversea, and that actually utilized foreign direct investment inflows to local regions facilitates outflow of FDI. Finally, conclusions are drawn for theoretical and practical implications. (For more information, please contact: Mengmeng Shan, Fudan University, China: tosmm1988@163.com)

\title{
Paradox of Cultural Distance in Determining the Investment Timing of Corporate Venture Capital Investment Shin Hyung Kang, KAIST Business School \\ Zong-Tae Bae, Kaist Business School
}

As CVC investors are potential imitators, CVC investment is materialized when entrepreneurs decide to take the imitation risk. Cultural distance lowers trust of entrepreneurs in CVC investors, enlarging the expected imitation risk. However, cultural disparity provides learning opportunities to entrepreneurs, increasing the expected payoff of the investment. If the hindering effect prevails, entrepreneurs will delay the investment timing as late as possible, whereas if the benefits are more salient, they will accept CVC investment as early as possible. This research suggests establishing a local entity in the host country moderates the effect of cultural distance. It is shown by ordered logistic regression analysis that in cross-border CVC investment, cultural distance is more critical than geographical distance and the major role of the local entity should be interaction facilitator rather than searching role. (For more information, please contact: Shin Hyung Kang, KAIST Business School, Korea, South: davidkang@business.kaist.ac.kr)

\section{China's Formal Institutions for Outward FDI \\ Victor Zitian Chen, University of North Carolina, Charlotte \\ Karl P. Sauvant, Columbia University}

China has become the top emerging market outward investor, with no sign of a slowdown. A growing body of literature suggests that Chinese formal institutions, notably government policies, are a major determinant of China's rising outward foreign direct investment (FDI). This paper undertakes a review of the major policies and regulations currently dealing with China's outward FDI, and discusses their implications for international business research and China. Overall, China has a regulatory framework in place that enables outward FDI (OFDI), but there are still strong elements of administrative control that make it cumbersome. China also has a sophisticated array of supportive instruments in place that facilitates and encourages such investment; stateowned enterprises (SOEs) tend to benefit particularly from these supportive instruments. Moreover, China's approach to OFDI is pursued in the framework of an overall development strategy, and this is likely to continue. (For more information, please contact: Victor Zitian Chen, University of North Carolina, Charlotte, USA: emgp.editor@gmail.com) 
Global Oligopolistic Competition and Multinational Corporations: A Strategic Interation Perspective on Foreign

Direct Investment

Michael W Hansen, Copenhagen Business School

Anne Kristin Hoenen, WU Vienna

The contemporary International Business (IB) literature has 'forgotten' a key insight of the early foreign direct investment (FDI) literature, namely that FDI often is driven by strategic interaction of multinational corporations (MNCs) in oligopolistic industries. Instead, the IB literature has focused, first on FDI as a way to generate efficiency in cross border transactions, and later on FDI as a way to leverage and build capabilities across borders. In recent decades we have however seen a profound concentration at the global level so that more and more industries can be characterized as global oligopolies. We argue that in this situation there is a pertinent need to 'rediscover' the strategic interaction perspective on FDI, but now from a global perspective. Based on early insights of the Industrial Organization FDI tradition as well as on more recent insights from Global Strategic Management, we position the strategic interaction perspective in relation to more conventional perspectives on FDI and develop a typology of generic FDI strategies in global oligopolistic industries. (For more information, please contact: Michael W Hansen, Copenhagen Business School, Denmark: mwh.ikl@cbs.dk)

\section{Session: 3.2.12 - Interactive}

\section{How can You Make Your Program Design More Effective to Achieve IB Learning Outcomes?}

Presented On: July 6, $2013-10: 55-12: 10$

Chair: Paloma Bernal Turnes, Georgetown University

Cross-Cultural Formative Feedback: An Exploratory Framework for Business Schools

Mark Tayar, Swinburne University of Technology

Yuqian Zhang, Macquarie University

As well as internationalizing the Business School curriculum itself, there are other aspects of our teaching which may need internationalizing. In culturally diverse university classrooms, the effectiveness of feedback may vary due to different attitudes and intercultural interpretations of teacher comments. This paper proposes a model to assist university teachers in understanding how students from different cultures may interpret and react to different types of formative feedback. Types of feedback investigated include error-corrections, holistic feedback, as well as rankings and percentages. This paper also discusses the hypothetical effects on the feedback delivery, including the timing and medium of feedback. We aim to help future investigations of the link between types of feedback and constructive or inhibitory student reactions. Future investigation and action research by international business educators could identify types of feedback and manners of feedback delivering with the most positive impacts to cope with cultural diversity. (For more information, please contact: Mark Tayar, Swinburne University of Technology, Australia: mtayar@swin.edu.au)

Design for Effective Learning - Making a Methodology Course the Backbone Platform for an IB Curriculum Valtteri Kaartemo, Turku School of Economics Peter Zettinig, Turku School of Economics Birgitta Sandberg, Turku School of Economics Zsuzsanna Vincze, Umeå University

In our presentation, we discuss the effective design for learning in an IB curriculum. The focus lies on a student-centered approach to develop their personal, professional and social capabilities. Particularly, we 
contribute to the track on "Developing an IB Curriculum: What Do Students Need to Know?" while at the same time suggesting a deviation toward 'What students need to do in order to transform'. We approach this question taking the classic '3P model of teaching and learning' (Dunkin \& Biddle 1974) as basis for our argumentation. We argue that in a Master of Science curriculum in IB, a research methods course can yield good results to obtain graduate attributes that fulfill the learning objectives at our Global Innovation Management Masters Degree Program. (For more information, please contact: Valtteri Kaartemo, Turku School of Economics, Finland: valtteri.kaartemo@utu.fi)

\section{Experiential Learning in the MBA Curriculum: The Use of Global Consultancy Projects James $\mathrm{P}$ Johnson, Rollins College \\ D. Michael Brown, Birmingham City Business School}

We examine the use of live consultancy projects in MBA programs and address the post-hoc evaluation of consultancy projects that are offered as part of the MBA curriculum. In doing so, we address two key areas of interest for business scholars: (1) the need to include practical training in the MBA curriculum; (2) bridging the academic/practice divide. We start with a brief review of the relevant literature on experiential learning in business programs and then we discuss the use of consultancy projects in a conventional MBA program offered at a leading business school in the south-east United States, and then we present the results of a two-phase study in which the post-hoc perceptions of both students and clients were sought. We believe that this is the first longitudinal study of the efficacy of consulting projects that form part of an MBA program. We conclude with a discussion of the findings of the study and their relevance for students, academics, and practitioners. (For more information, please contact: James P Johnson, Rollins College, USA: jpjohnson@rollins.edu)

\section{Commitment to the Study of International Business and Cultural Intelligence: A Multi-Level Model Jase Ramsey, University of Alabama Amine Abi Aad, University of Alabama Livia Lopes Barakat, Fundacao Dom Cabral}

Adopting a multilevel theoretical framework, we examined how metacognitive and motivational cultural intelligence influences an individual's commitment to the study of international business. Data from 292 undergraduate and graduate business students nested in 12 classrooms in the United States demonstrated that individuals' metacognitive and motivational cultural intelligence are positively related to their commitment to the study of international business. Furthermore, there is additional commitment when the classrooms' cultural intelligence capital is high. We discuss the theoretical and practical implications of these findings within a classroom context that involves cross-cultural interactions. (For more information, please contact: Jase Ramsey, University of Alabama, USA: jase@cba.ua.edu)

\section{Making of a "Global" Graduate \\ Massoud Saghafi, San Diego State University}

Are the international education programs successful in producing a new generation of internationally sophisticated American students with better and broader global understanding? The answer is positive based on a very limited prior research. Research on globalization of labor however primarily considers ethnocentrism and xenophobia as the main measure of globalization. Furthermore, these studies merely compare groups of students to determine if those with "international ..." are less ethnocentric than those without "international..." No academic study has addressed the ingredients for labeling a student a cross-culturally competent individual. This research will investigate the educational requirements to generate a cross-culturally competent cadre of globally human resources to join the workforce and addresses the following questions. What are the International Business Education programs doing in major US universities to prepare cross-culturally competent students? What is the state of global knowledge of current cadre of students? In which ways should US 
universities tweak their programs to meet international needs in practice? The results should help academia to gauge the international competence of current students and revise programmatic and other international learning activities to match the practitioners' needs. (For more information, please contact: Massoud Saghafi, San Diego State University, USA: msaghafi@mail.sdsu.edu)

\title{
Do Student Response Systems Cross Cultural Boundaries
}

Thomas Gene Drape, Azusa Pacific University

The pedagogical question is if the benefits from the use of Student Response System(s) (SRS) are universal and cross country and cultural boundaries. This session will share the results and feedback from the use of the SRS in a cross-cultural leadership course consisting of participants from eight different countries (Albania, Azerbaijan, Germany, Kenya, Romania, Slovenia, Ukraine, United States). Only one of the course participants was familiar with the SRS before attending the course. In addition, this session will demonstrate the use of SRS through an interactive session with AIB members using several of the same questions from the course. A comparison will be made between AIB member responses and course responses followed by a discussion of the responses and the use of the SRS. (For more information, please contact: Thomas Gene Drape, Azusa Pacific University, USA: tdrape@pugetsound.edu)

\section{Session: 3.2.13 - Interactive}

\section{Track: Track: 6 - Innovation and Knowledge Mgmt.}

\section{Performance, Knowledge, and Innovation}

Presented On: July 6, $2013-10: 55-12: 10$

Chair: Ronaldo Couto Parente, Florida International University

\author{
University-Industry Collaboration in R\&D: The Role of Business Sophistication and Labor Market Rigidity \\ Christopher Williams, University of Western Ontario \\ Gayle Allard, IE Business School \\ Candace A. Martinez, Saint Louis University
}

We investigate R\&D collaboration between universities and industry from a labor market rigidity perspective. While there is a well-established understanding of how business sophistication in the private sector in a country will induce R\&D collaboration between universities and industry, we argue that another aspect of these alliances has been under-researched: the role of labor market rigidity in a country, and, in particular, the difficulties employers face in hiring and firing people. We hypothesize that the presence of sophisticated business networks in a country will lead to more private sector R\&D collaborations with universities. Further, we hypothesize that the ease with which firms are legally allowed to hire and fire will directly and indirectly influence R\&D collaboration between universities and industry. Integrating data from various sources, we test our model on a sample of 137 countries. Results confirm the strong influence of developed and modern business networks in encouraging industry-university R\&D collaborations while also showing a direct impact of rigid hiring laws and an indirect impact of rigid firing laws. The findings have important implications for policy and theory of R\&D collaboration within a national innovation system. (For more information, please contact: Christopher Williams, University of Western Ontario, Canada: cwilliams@ivey.ca)

\section{Managing Learning Activities to Improve Performance of International Strategic Alliances Chia-Ling (Eunice) Liu, National Cheng Kung University Steven Lui, University of New South Wales John Liu, University of New South Wales}


Learning is important for firms to meet the objectives of their alliances. Firms implement various activities to learn about a partner, learn from a partner, and learn with a partner. Since small and big firms differ in their ability, motivation, and interactive dynamics to learn, the impacts of various types of alliance learning should be contingent on firm size. To examine this proposition, we develop a model that delineates the impacts of various learning activities on alliance performance of small and big firms, and test the model in a sample of 173 strategic alliances formed by high technology firms. We find that the relationship between learning and alliance performance is indeed different across small and big firms. Moreover, while partnership coordination for learning with a partner has the strongest positive relationship on alliance performance for small firms, activities to learn about a partner has the strongest positive relationship for large firms. Theoretical and managerial implications of our findings are discussed. (For more information, please contact: Chia-Ling (Eunice) Liu, National Cheng Kung University, Taiwan: clliu@mail.ncku.edu.tw)

\section{A Longitudinal Analysis of Blockbuster Drugs \\ Thomas J. Hannigan, Temple University \\ Ram Mudambi, Temple University \\ Andrew Sfekas, Temple University \\ Denise Dunlap, Northeastern University}

Blockbuster drugs represent a vital source of revenue for pharmaceutical firms. A relatively small number of drugs can make up approximately a third of the industry's global revenue. While blockbuster drugs represent a breakthrough innovation, we argue that the commercialization of these products allows them to attain significant global revenue. The capability to commercialize such a blockbuster may be a relative scarcity in the industry, with few firms possessing the wherewithal to maximize the revenue potential of a breakthrough innovation. In this paper, we analyze the full population of blockbuster drugs launched from 1980 to 2010, looking closely commercialization patterns and knowledge transfer mechanisms. We find that while many pharmaceutical players may discover blockbuster drugs, the majority of these breakthrough innovations are commercialized by so-called "major" firms. This finding is explored more deeply with three case studies of blockbuster drugs: Lipitor, Actos, and Plavix. These major firms with unique commercialization capabilities act as gatekeepers, selecting which innovations become the products with the greatest revenue. We argue that this process reflects those in creative industries, such as movie-making: movie studios are the "major" firms that exert control over other role players. (For more information, please contact: Thomas J. Hannigan, Temple University, USA: tj.hannigan@temple.edu)

\section{Patenting Abroad vs. Outsourcing Abroad: A Cross-Country Investigation of Innovation in Emerging Markets Hyunju Ahn, Korea University \\ Daeil Nam, Korea University}

In this study, by drawing on the concepts of exploration and exploitation, we examine: 1) whether the possession of a patent abroad strengthens the core competence of a firm, thus promoting exploration, and 2) whether outsourcing to foreign markets affects a firm's procurement of resources and knowledge, thus promoting exploitation. We test the moderating effects of product time scale (short-term and long-term orientation) to better explain the nature of innovation in emerging countries. To test our hypotheses, we use data from the World Bank Group, which includes 1555 firms in 11 developing nations. The results show strong support for the relationship between patenting abroad, outsourcing abroad, and innovation, but do not support the moderating variable involved in our analysis. Our post hoc analysis also reveals the importance of balancing exploration and exploitation in innovation. (For more information, please contact: Hyunju Ahn, Korea University, Korea, South: hjahn1122@korea.ac.kr) 


\section{Cross-Border Learning, Technological Turbulence and Firm Performance \\ Liliana Perez-Nordtvedt, University of Texas at Arlington \\ Debmalya Mukherjee, University of Akron \\ Ben Kedia, University of Memphis}

Using knowledge-based view (KBV) and contingency arguments, we develop and test a model that investigates the relationship between the effectiveness and efficiency of cross-border knowledge gained from international business affiliates (IBAs) and the focal (recipient) firm's performance. We argue that both of these dimensions of learning not only have a direct and positive effect on the performance of the focal firm but also a synergistic one. Also, we suggest that the direct and positive effect of both the effectiveness and the efficiency in learning from the IBA on focal firm performance is moderated by the technological turbulence of the context in which the focal firm operates. Results indicate that the effectiveness of learning, although not the efficiency in learning, positively impacts the focal firm's performance. However, cross-border learning efficiency and effectiveness jointly improve the focal firm's performance. Results show that the level of technological turbulence attenuates the positive effect of the effectiveness of learning on the focal firm's performance. In fact, under high technological turbulence conditions, effective learning becomes detrimental to the focal firm's performance. (For more information, please contact: Liliana Perez-Nordtvedt, University of Texas at Arlington, USA: Inordtvedt@uta.edu)

\section{Vicarious Learning in the Globalization of Venture Capital \\ David Lanier Major, Indiana University \\ David Waguespack, University of Maryland \\ Anil Gupta, University of Maryland at College Park}

In this paper we examine the differential impact of different types of vicarious learning on the seemingly imitative behavior of organizations. Vicarious learning is divided into two types: acquisition of declarative knowledge and acquisition of procedural knowledge. We argue that observation of the experiences of salient others results in the transfer of declarative knowledge; that learning from ties to experienced partners results in the transfer of declarative and procedural knowledge; and that the transfer of procedural knowledge fosters deeper decision commitment and higher quality decisions. Focusing on the globalization pattern of US venture capital firms, we examine the likelihood of an initial foreign investment, the extent of the investment commitment, and the quality of the investment decision. We find strong evidence of differences in behavior based on the type of knowledge transferred. The results suggest that vicarious learning transferred through network ties leads to deeper learning thereby setting the stage for superior performance. (For more information, please contact: David Lanier Major, Indiana University, USA: dlmajor@indiana.edu)

The Determinants of Innovative Performance in Virtual R\&D Teams in the 21st Century Frontier R\&D Program of Korea
Changsu Kim, Sogang University
Byungheon Lee, Kwangwoon University
Donseung Choi, Sogang University

This study examines the determinants of innovative performance in the 21st century frontier R\&D program of Korea. The empirical test was conducted for 73 R\&D teams, which are virtually organized, and which are funded by Korean government to help conduct frontier R\&D in the areas of basic science and technology. Drawing from the extant literature of social capital and organizational learning, this study examines the impact of team capability (measured by collective individual capabilities and team diversity) and social capital (measured by external linkages, prior ties, and adaptive learning) on innovative performance of R\&D teams. The innovative performance is captured by papers and patents produced by the R\&D teams. The results provide support for the effects of collective individual capabilities and adaptive learning on paper and patent publications. The team diversity was significant and positive only on paper publication while the external linkages significant and 
positive, albeit marginal, only on patent publication. The unexpected negative effect was found for prior ties in paper publication. We discuss theoretical and practical implications of the findings. (For more information, please contact: Changsu Kim, Sogang University, Korea, South: cskim@sogang.ac.kr)

\section{Session: 3.2.14 - Interactive}

\section{Novel Approaches in IB Research Methodology}

Presented On: July 6, $2013-10: 55-12: 10$

Chair: Haruo Hagiwara Horaguchi, Hosei University

Integrated Model to Analyze Brand Function for Products in the Global Marketplace

David Pan, Prince Sultan University

Alan Pan, Xiamen University

Frank Fu, Hong Kong Baptist University

Linda Clarke, University of Florida

Berna Mutlu, University of Florida

This study integrates the nuances of product and brand strategy research with socio-ethno considerations to derive a dynamic system of paradigms embracing the specific variable considerations in socio-cultural dimension. To connect current knowledge with future IB research, we have mapped out the pathway and signpost for brand function to bridge product with intended market segment with our proposed axioms in pair for Brand-in-Value for firms and Brand-in-Value for market in a cross-cultural setting that drive marketing strategies in a global environment. (For more information, please contact: Alan Pan, Xiamen University, China: apan@indiana.edu)

\section{Stepping through the Looking Glass: Reflections on Field Research \\ Christina Stringer, University of Auckland \\ Glenn Simmons, University of Auckland}

Methodological awareness around risks and challenges researchers can experience in the field is not widely addressed in management and international business literatures. Such challenges are more widely reported in, for example, anthropology and sociology. In the course of our research into labour and human rights abuses aboard South Korean foreign charter vessels fishing legally in New Zealand's waters, we encountered a number of incidents aimed at intimidating our participants, their families, our translators and ourselves. This paper details our experiences. From this we identify three levels of tangible risks and dangers: to researchers, participants, and third parties such as translators. We contend that researchers' awareness of risks and dangers they can encounter in the field needs further development, particularly in the international business literature. (For more information, please contact: Christina Stringer, University of Auckland, New Zealand:

c.stringer@auckland.ac.nz)

\section{Does Language Grammatical Structure Matter? Evidence from Gender Marking}

Amir Shoham, Temple university

Estefania Santacreu-Vasut, ESSEC and THEMA

Oded Shenkar, Ohio State University

We analyze the impact of female/male distinctions in the dominant language of countries on managerial and economic outcomes. Using four data sets, we find that countries whose dominant language marks gender more intensively have significantly lower female presence in the labor force and in senior managerial echelons. This 
suggests that language marking can offer an alternative to survey-based measures of culture. It may capture our ancestors culture, as grammar is inherited from the distant past. Further, language may influence cognition directly, reinforcing the need to view language design as a strategic tool of MNC's. (For more information, please contact: Amir Shoham, Temple university, USA: amir.shoham@temple.edu)

\section{(Self) Reflexivity as Method(ology): Advancing International Business Research David Sapto Adi Guttormsen, Coventry University}

In this polemic research note, the author argues that the application of (Self) Reflexivity as a method(ology) in International Business (IB) research remains alarmingly marginalised and falling short of advanced theoretical developments in the wider social sciences, with the negative implications of potentially distorting knowledge production and to lower the level of transparency, credibility, and trustworthiness of qualitative IB research - as well in the positivist embedded quantitative research realm. The paper reviews the current inclusion of (Self) Reflexivity in IB research and introduces advanced interdisciplinary developments on this topic by drawing upon sociologist Pierre Bourdieu. The arguably lack of sophisticated engagement with reflexivity in IB research will be showcased in numerous international business cases in a future version of this research-note. The paper aims to motivate for debating the present engagement, and the research practices of, reflexivity on the individual and disciplinary levels in IB research, in addition to identify avenues for further developing (Self) Reflexive engagement in IB. (For more information, please contact: David Sapto Adi Guttormsen, Coventry University, United Kingdom: david.guttormsen@coventry.ac.uk)

\section{The Polycontextualization of International Business Research: An Evolutionary Process Linda Clarke, University of Florida Debra Shapiro, University of Maryland Mary Ann Von Glinow, Florida International University Moriah Meyskens, University of San Diego}

Although international business scholars often describe the "context" in which their research takes place, it remains undemonstrated whether they capture the "polycontextuality," or multiple embedded contexts inherent in cultural phenomena. We examined 534 empirical articles in the Journal of International Business Studies (JIBS) from 1970-2007 using content analysis; coding each by various categories of context with which the study variables were operationalized. Our findings demonstrate that while the use of multiple, "polycontextual" variables has increased over time, most IB studies have yet to take into account the multiply embedded layers of context; including the macro-environment, cultural, or temporal-spatial considerations. (For more information, please contact: Linda Clarke, University of Florida, USA: clarkel@ufl.edu)

\section{Session: 3.2.15 - Interactive \\ Track: Track: 9 - Cross-Cultural Management and HRM}

\section{Cross-Cultural Management}

Presented On: July 6, $2013-10: 55-12: 10$

Chair: Romie Frederick Littrell, Auckland University of Technology 
Why the Institutional Distance Literature in Management is about Institutions, Not Distance

Robbert Maseland, University of Groningen

André van Hoorn, University of Groningen

We theoretically identify and empirically demonstrate a vital hitherto neglected design flaw that invalidates almost all of the distance research in management that has been done so far. The root of the flaw is that, when keeping the base (home or host) country fixed, differences in institutional distance between the various partner countries and the base country collapse into measures of the institutional profiles of these partner countries. Implication is that analyses examining the effect of institutional distance between one base country and various partner countries on such issues as foreign market entry mode or market selection conflate the effect of distance with the effect of these partner countries' institutions. Quantitatively, the problem is most severe for base countries with an institutional profile at the extreme of the distribution, notably the US. Empirical analyses of institutional distance and its effect on international management phenomena are only valid when they consider multiple base countries sampled from across the distribution of institutional profiles simultaneously. (For more information, please contact: Robbert Maseland, University of Groningen, Netherlands: r.k.j.maseland@rug.nl)

\author{
A Process-Based Explanation of the Psychic Distance Paradox - Evidence from Global Virtual Teams \\ Peter Magnusson, Florida International University \\ Anja Maria Schuster, University of St. Gallen \\ Vas Taras, University of North Carolina, Greensboro
}

This paper takes a new approach to the investigation of psychic distance's impact on performance, a relationship which has been associated with the 'psychic distance paradox' in international business literature. Studying the effect of team-level psychic distance on the performance of global virtual teams, we build on the input-process-outcome framework of team research to integrate process variables that might have been neglected in preceding work on psychic distance effects. We model a causal path which includes the mediators 'expectation of challenges' and 'level of team effort' as well as 'motivational cultural intelligence' as a moderating factor. The data supports our hypothesized causal path. With our findings we are able to start unveiling the psychic distance paradox through the integration of the literatures on psychic distance and global virtual teams. (For more information, please contact: Anja Maria Schuster, University of St. Gallen, Switzerland: anja.schuster@unisg.ch)

\author{
Rethinking Cultural Interaction: The Role of Cultural Attractiveness in International Business \\ Chengguang Li, University of Augsburg \\ Felix Brodbeck, University of Munich \\ Jan Hendrik Fisch, University of Augsburg
}

Prior research in international business has primarily paid attention to cultural distance when assessing the relationship between cultures. This approach suffers from several limitations, some of which can be attributed to the assumptions of discordance and symmetry. As a complement, we introduce the concept of cultural attractiveness, which overcomes these limitations and assesses how desirable a culture is. We examine the influence of cultural attractiveness on the level of MNE operations in 24 nations for the 1985-2010 period. Our results show that cultural attractiveness has significant explanatory power for the level of MNE operations in addition to the conventional construct of cultural distance. (For more information, please contact: Chengguang Li, University of Augsburg, Germany: chengguang.li@wiwi.uni-augsburg.de) 


\title{
Reconsidering the Borders of Culture: Regional Culture \\ Aycan Kara, Florida Atlantic University \\ Mark F. Peterson, Florida Atlantic University
}

The purpose of this paper is to consider the utility of analyzing within-nation regional culture. Culture as circumscribed by national boundaries has been a central concept in a variety of disciplines including international management and marketing. However, heterogeneity of individuals and groups within any nation that raises questions about utility of studying national culture has caught the attention of scholars in recent years. Most discussions about the pros and cons of studying national culture have been mainly empirical. We complement such work by considering three theoretical lenses (functional theory, neo-institutional theory, and complexity theory) that have been used to provide the basis for understanding the emergence of culture in general to suggest that within-nation regional cultures may develop as well. We use problems of social psychology from functional theory, the three pillars and the ideas of stable equilibrium in neo-institutional theory, and chaos of dynamic systems from complexity theory to consider the emergence of regional cultures and provide examples of regional cultural variations. We also review recent studies about within-nation regions and some suggestions for constructing regional value dimension scores. (For more information, please contact: Aycan Kara, Florida Atlantic University, USA: akara@fau.edu)

\author{
Ethically Questionable Negotiation Tactics: Evidence from Austria, China, Greece and Turkey \\ Abraham Stefanidis, St. John's University \\ Moshe Banai, City University of New York \\ Ahmet Erkus, Gedik University \\ Herbert Goelzner, Salzburg University of Applied Sciences and Technologies
}

This study identifies and compares the influence of vertical and horizontal individualism-collectivism, ethical idealism, and interpersonal trust on the endorsement of the pretending, deceiving and lying negotiation tactics in Austria, China, Greece and Turkey. A survey questionnaire was translated from English to the relevant four languages and administered to a sample of 1327 negotiators in those four countries. Individualism, and not collectivism, was found to be related to the endorsement of the use of the pretending negotiation tactic; horizontal individualism was universally related to the endorsement of the use of the deceiving negotiation tactic; and both vertical and horizontal collectivism were related to the endorsement of the lying negotiation tactic in the three European countries, but not in China. Ethical Ideology was negatively related to the endorsement of all three questionable negotiation tactics in all four countries. The results advance previous efforts towards the development of a universal negotiation theory. (For more information, please contact: Abraham Stefanidis, St. John's University, USA: stefania@stjohns.edu)

\section{The Effects of Acculturation on Psychological Proximity, Emotions and Their Consequences Majid Ghorbani, Renmin University of China \\ Masud Chand, Wichita University}

As immigration increases between countries all around the world and as second and later generations of immigrants from more collectivistic Eastern countries grow in the workforce of more individualistic Western countries, understanding the processes and consequences of acculturation has gained a new level of importance. At the heart of these emerging issues are the antecedents and consequences of morality and reparative behavior in Western versus Eastern cultures. We compare and contrast the effects of acculturation on the perception of psychological proximity (a gauge for the level of collectivism), the intensity of the two moral emotions of shame and guilt, and the degrees of compensation to victims of one's wrongdoing. Our sample is comprised of a collectivistic group from a shame culture, China, and an acculturated group of Chinese living in a much less collectivistic society from a guilt culture, Canada. Our results indicate that participants' perception of proximity to other people and the level of compensation offered to the victims of transgression are significantly different among the two samples. Furthermore, shame, but not guilt, mediates the relationship 
between perceived psychological proximity and the decision to compensate. (For more information, please contact: Majid Ghorbani, Renmin University of China, China: majidghorbani@rbs.org.cn)

\author{
Thou Shalt Not Steal: The Impact of Religion on Software Piracy \\ Amanda E.K. Budde-Sung, University of Sydney \\ Matthew C. Mitchell, Drake University
}

Software-producing companies occupy a prominent position in lists of the world's most profitable companies. However, their product, software, is routinely subject to unauthorized copying and distribution, costing billions of dollars per year in lost revenues. This multi-method study considers religion's impact upon software piracy. Quantitative results suggest that religion impacts the piracy rates of countries around the world. Interviews provide qualitative insight into this issue, offering suggested strategies for software-producing firms wishing to protect their products in global markets. (For more information, please contact: Amanda E.K. Budde-Sung, University of Sydney, Australia: amanda.budde-sung@sydney.edu.au)

\title{
Session: 3.3.1 - Panel
}

Track: Track: 6 - Innovation and Knowledge Mgmt.

\section{Sharing Knowledge at a Distance: Obstacles and Facilitators}

Presented On: July 6, 2013 - 13:20-14:35

Chair: Yves Doz, INSEAD

Discussant: Aya Chacar, Florida International University

Panelists:

Tina Ambos, University of Sussex

Aya Chacar, Florida International University

Thomas Hutzschenreuter, WHU Otto Beisheim School of Management

Yves Doz, INSEAD

Despite all the extensive research work on knowledge creation and sharing over the past decades the issue of sharing complex knowledge at a distance remains unresolved, or at least imperfectly settled. Most scholars, us included, argue that the very nature of complex knowledge (tacit, collective, context-dependent) rules out the possibility of sharing such knowledge at a distance. The socially situated nature of complex knowledge, and the fact that it can only be revealed and shown through co-practice in action, in particular perhaps infrequent circumstances (e.g., in unexpected crisis situations where explicit emergency procedures no longer apply) and locations (specific sites where action takes place) make sharing impossible. The "here, together, now" constraint applies. Although there is considerable support for that view, both theoretical and empirical, we propose to test its limits in the panel, by considering facilitators and obstacles to the sharing across distance, be it geographic, cultural, or other institutional distance type. The panellists will share their views in a Q\&A session that is based on their own knowledge and research in this area. (For more information, please contact: Yves Doz, INSEAD, France: yves.doz@insead.edu) 
Session: 3.3.2 - Panel

Track: Track: 9 - Cross-Cultural Management and HRM

\section{Indigenous and International Perspectives on Global Mindset; Implications for the 21st Century}

Presented On: July 6, $2013-13: 20-14: 35$

Chair: Rabi Bhagat, University of Memphis

Discussants: Nakiye A. Boyacigiller, Sabanci University and Mansour Javidan, Thunderbird School of Global Management

Panelists:

Rabi Bhagat, University of Memphis

Cordula Barzantny, Touluse Business School

Bharatendu Srivastava, Indian Institute of Management Calcutta

Subramanyam Raghunath, IIM Bangalore

This symposium is designed to address the topic of global mindset which is of growing importance in the field of International and Global business. We start off with a broad theoretical perspective on the country-specific and universal factors which foster the department of Global Mindset. Presentations from senior researchers in South Korea, India and U.S. discuss the nature of Global Mindset from the perspective of both universal and indigenous perpesctives. We have two outstanding scholars as discussants for the symposium and they are going to focus on the future of research on this topic which has become very crucial for the functioning of multinational and global businesses. Two hours of time (120 minutes) is requested for this symposium, and it is best suited as a cross-disciplinary symposium for tracks 9 and 5 . It focuses on topics and themes which are relavent from the perspective of cross-cultural management and also from the perspective of managing multi-national corporations. This symposium is going to be a major attraction for the participants of the AIB conference in Istanbul. (For more information, please contact: Rabi Bhagat, University of Memphis, USA:

rbhagat@memphis.edu)

\section{Session: 3.3.3 - Competitive}

Track: Track: 3 - IB Theory, FDI, and Entry Mode

\section{FDI Location}

Presented On: July 6, $2013-13: 20-14: 35$

Chair: Majid Eghbali-Zarch, University of Western Ontario

Strategic Hedging: Evidence from Brazilian Exporters

Dirk Michael Boehe, University Of Adelaide

How and why do exporters adjust their portfolios of destination countries in response to exchange rate movements and how do such geographic export diversification choices affect firm performance? Drawing on corporate strategy literature, we argue that firms enjoying low exchange rate competitiveness can increase their performance when they expand their exports over different world regions and vice-versa. Using a panel of Brazilian exporters for the period from 2001 to 2010 and simultaneous equation models with firm and period fixed effects, our results imply that unrelated geographic export diversification is more effective than related diversification for counteracting exchange rate pressures. (For more information, please contact: Dirk Michael Boehe, University Of Adelaide, Australia: dirkmichael.boehe@adelaide.edu.au) 
The Institutional Diversity of Regions and MNES' Internationalization

Jean-Luc Arregle, EMLyon Business School

Toyah L. Miller, Indiana University

Michael A. Hitt, Texas A\&M University

Paul W. Beamish, University of Western Ontario

Semi-globalization emphasizes the role of geographic regions in MNEs' internationalization due to the possibility of developing region-bound firm specific advantages and arbitrage. In this paper, we develop and test hypotheses about the effects of a region's institutional diversity on MNEs' propensity to internationalize in a country, as it influences the possibility and cost of RFSAs and arbitrage. We develop hypotheses about a Ushaped relationship between a region's institutional diversity, differentiating formal and informal institutions, and the propensity of a MNE to internationalize in a country in this region. We test these hypotheses on a sample of Japanese MNEs operating in 45 countries within eight regions. The results validate these U-shaped relationships showing that a region's diversity plays an important and complex role in semi-globalization. These results present a new perspective to understand how regions' characteristics explain semi-globalization and MNEs' internationalization. (For more information, please contact: Jean-Luc Arregle, EMLyon Business School, France: arregle@em-lyon.com)

\section{FDI in Tax Havens: Do Corporate Tax Rates Matter?}

Chris Jones, Aston University

Yama Temouri, Aston University

The purpose of this paper is to examine the determinants of a MNC's strategy to invest in countries classified as tax havens. To the best of our knowledge this has not been done before. We examine this using a standard empirical model of international business based on the OLI paradigm and linking it with certain financial specific advantages. The firm-level data used covers over 36,000 MNC's across the OECD between 2002 and 2011. We find that higher corporate taxes faced by MNC's at home increase the propensity of tax haven use. In addition, it is not only financial firms that undertake activity. Finally, there is also evidence that MNC's from countries with a more social market orientation are less likely to invest in tax havens. (For more information, please contact: Chris Jones, Aston University, United Kingdom: c.jones2@aston.ac.uk)

\section{Session: 3.3.4 - Panel}

\section{Hands-On, Minds-On: Experiential Learning Projects in International Business Education Part 1}

Presented On: July 6, 2013 - 13:20-14:35

Chair: Vas Taras, University of North Carolina, Greensboro

\section{Panelists:}

Vas Taras, University of North Carolina, Greensboro Miroslaw Jarosinski, Warsaw School of Economics Michel Librowicz, Université du Québec à Montréal Nick Robinson, North Island College Amanda E.K. Budde-Sung, University of Sydney Adam Johns, Rikkyo University Nobuya Takezawa, Rikkyo University Dirk Fischbach, Hochschule Harz Andres Velez-Calle, Universidad EAFIT Virginia Cathro, Otago University Maria Alejandra Gonzalez-Perez, Universidad EAFIT 
Alfredo Jiménez, University of Burgos

Anke Arnaud, Embry-Riddle Aeronautical University

Alfredo Behrens, Alfredo Behrens Consulting

Sebastián Steizel, Universidad Oberta de Catalunya

Michael Zisuh Ngoasong, Coventry University London Campus

Susan Forquer Gupta, Monmouth University

Terri Lituchy, University of the West Indies

Betty Jane Punnett, University of the West Indies

Toshiya Ozaki, Rikkyo University

Experiential learning is a "hot" topic in International Business and Cross-Cultural Management Education (IB$\mathrm{CCM}$ ), but the use of this approach is limited due to a lack of information and understanding on what tools are available and how to incorporate them in the curriculum. The proposed panel will bring together a 12 teams of academics who have developed and successfully used various experiential learning projects for IC-CCM education. The goals of the panel are to share information about the existing EL projects, share experiences, discuss challenges and best practices of using EL to improve IB-CCM education, provide a networking opportunity for academics interested in the EL approach, discuss how this panel/group can contribute to further discussion on the role of EL in IB-CCM education, possibly through special issues on EL in academic journals, targeted conferences and seminars, more and better research on the issue. (For more information, please contact: Vas Taras, University of North Carolina, Greensboro, USA: v_taras@uncg.edu)

\section{Session: 3.3.5 - Competitive}

\section{Track: Track: 7 - Emerging Economies}

\section{Diversification, Location, and Growth in Emerging Markets}

Presented On: July 6, $2013-13: 20-14: 35$

Chair: Andrei Panibratov, Saint-Petersburg State University

International Diversification and Growth Option Value: Growth Opportunities in Emerging and Advanced Markets Anju Seth, Virginia Tech

Todd Alessandri, Northeastern University

The growth opportunities afforded by international diversification represent an important goal for firms. We examine how the location of diversification influences growth option value. Based on real options theory, we argue that for U.S. firms, international diversification into emerging economies will likely increase growth option value given the associated higher endogenous and exogenous uncertainty. In contrast, expansion into advanced economies will likely negatively influence growth option value. Using data for 507 firms over 9 years, we find support for these arguments. We also argue for, and find modest support for, a moderating role for organizational slack in emerging markets. (For more information, please contact: Anju Seth, Virginia Tech, USA: aseth@vt.edu)

Revisiting the Curvilinear Relationship between Diversification and Performance: A Study of Business Groups in Emerging Economies

Qian Gu, Georgia State University

Jane Wenzhen Lu, University of Melbourne

Chi-Nien Chung, National University of Singapore

While extensive studies suggest an inverted U-shaped relationship between diversification and performance, most of these studies draw on firms in developed economies and it remains unclear whether this finding stays robust in emerging economies. This study investigates the performance implications of diversification pursued 
by business groups, a prevalent organizational form in emerging economies. Particularly, we examine the impacts of two important diversification decisions --- level of diversification and mode of diversification --- based on 89 Taiwanese business groups over a 20 -year time period. In contrast with the conventional argument for an inverted U-shaped relationship in previous studies, we find a U-shaped relationship between the level of diversification and business group performance. Furthermore, we find that the mode of diversification matters. For both related and unrelated diversification, business groups will gain better performance when using the new division approach rather than the new firm approach. (For more information, please contact: Qian Gu, Georgia State University, USA: qgu@gsu.edu)

\section{Location Determinants of R\&D Lab Closure in Emerging Economies \\ Grigorios Livanis, Northeastern University \\ Anna Lamin, Northeastern University}

While studies of R\&D activities have typically focused on firm entry, we focus on R\&D laboratory exit. Although potential benefits of agglomeration, particularly knowledge spillovers, may attract firms to a location, there is mixed evidence that firms' innovation outcomes improve. Given that firms may be sources, not just recipients, of knowledge spillovers, we examine whether collocation with other firms influences decisions to leave. This is especially important in emerging economies, characterized by weak intellectual property rights regimes and economic zones that encourage agglomeration. Using interviews with R\&D managers in India, we develop hypotheses on how agglomeration with other firms influences R\&D lab closure by Indian firms. Next drawing on a unique dataset of R\&D laboratories in India during 2003-2009, we examine how the presence of sameindustry and cross-industry R\&D labs affects the likelihood of lab closure. Our results highlight differences between the presence of foreign and domestic labs. The presence of domestic labs, but only from the same industry, increases the likelihood of closure, suggesting concerns over knowledge spillovers. However the presence of foreign labs from any industry increases the likelihood of closure, corroborating our interview information that foreign labs are causing labor market diversion. (For more information, please contact: Grigorios Livanis, Northeastern University, USA: g.livanis@neu.edu)

\section{Session: 3.3.6 - Competitive}

\section{Quantitative Methodology in IB}

Presented On: July 6, $2013-13: 20-14: 35$

Chair: Jose Ernesto Amoros, Universidad del Desarrollo

Modeling Complex Nesting Structures in International Business Research: An Illustration of Cross-Nested Multilevel Analysis of the Multinationality-Performance Relationship

Bo Bernhard Nielsen, Copenhagen Business School

Sabina Nielsen, Copenhagen Business School

International business (IB) phenomena often involve complex relationships between factors at different levels. Multinational corporations (MNCs) are influenced both by different country and industry environments which may have independent as well as interactive effects on MNC performance. While hierarchical random coefficient models (RCM) are often used for the analysis of multilevel phenomena, IB issues often result in more complex nested structures. This paper illustrates how cross-nested multilevel modeling allowing for predictor variables and cross-level interactions at multiple (crossed) levels of analysis may yield novel insights to IB research. The results have implications for IB research in its pursuit of an integrative approach to understanding the multilevel determinants of firm internationalization and performance. The paper further illustrates the importance of adequately modeling crossed-nested structures in multilevel models. (For more information, please contact: Bo Bernhard Nielsen, Copenhagen Business School, Denmark: bn.smg@cbs.dk) 
Avoiding Relegation - Diversity, Selectivity, Experience or Methodology?

Deepak Dhayanithy, Indian Institute of Management Kozhikode

Relegation is a serious concern for soccer clubs in the bottom echelons of a lucrative league such as the EPL. One key performance criterion for newly promoted clubs is to avoid relegation, and it is important for these clubs to invest in their squads and build up their international resource diversity. Previous International Business studies have studied the link between resource positions and firm performance in cross sectional data settings. However, a specific performance outcome such as relegation may not occur in one cross sectional snapshot, only to occur subsequently. This article explores resource and performance linkage in a time-to-event Cox regression framework, where the event is relegation; and compares the hypotheses testing results with results obtained from Logistic regression analysis. We find resources diversity is important in the Cox regressions whereas it is significant in only one particular definition of the observation time window in Logistic regressions, which is difficult to establish apriori. We call for a triangulation of cross sectional studies of performance events with an analysis in the time-to-event setting. This will help unravel explanatory variable effects on performance from the effects of the passage of time. (For more information, please contact: Deepak Dhayanithy, Indian Institute of Management Kozhikode, India: deepak@iimk.ac.in)

\title{
Exploratory Factor Analysis Use in International Business Research: Miscalculation of Factor Scores Tomasz Lenartowicz, Florida Atlantic University Tais Siqueira Barreto, Florida Atlantic University
}

Exploratory Factor Analysis (EFA) has been widely used in all fields of Social Sciences, but its use has never been investigated in International Business (IB) research. We reviewed articles published in the last five years in three top ranked IB journals. We found that most of the reviewed articles do not report basic information about the use of this technique, such as prerequisite tests, type of EFA, rotation, factor selection method, drop in variance, and score calculation method. Of the articles that provided some reports, the majority used Principal Component Analysis, Orthogonal Rotation, "Eigenvalue-Greater-Than 1" rule to select factors, had around $40 \%$ of drop in variance, and used an approximation method to calculate scores. The first four characteristics are not problematic, but the latter is critical. Based on statistics literature, we show the problems related to this flawed score calculation method and empirically illustrate them. Finally we address the risks of using such method. (For more information, please contact: Tais Siqueira Barreto, Florida Atlantic University, USA: tsiqueir@fau.edu)

\author{
Measuring Corporate Reputation: Testing Alternative Factor Structures and Nomological Validity \\ James Agarwal, University of Calgary \\ Oleksiy Osiyevskyy, University of Calgary \\ Percy M. Feldman, Pontificia Universidad Católica de Peru
}

In the past decade, management scholars have paid close attention to the construct of 'corporate reputation' which has been plagued by multiple definitions, conceptualizations, and operationalizations. The purpose of this article is to position corporate reputation as an 'assessment' construct that is modeled as a second-order reflective construct and to provide theoretical, methodological, and empirical support. Using survey data from a Latin American country, we empirically test the hypothesized second-order reflective model within a hierarchy of nested and non-nested models and compare its model fit and nomological validity with alternative conceptualizations of corporate reputation. While the second-order formative model provides the best model fit, it is the second-order reflective model that provides strong nomological validity and stability of parameter estimates. Modeling corporate reputation as a second-order reflective construct yields several methodological advantages including, estimating reliability at the item and first-order factor level, conceptual interpretability, and parsimony when tested within a nomological context. (For more information, please contact: James Agarwal, University of Calgary, Canada: james.agarwal@haskayne.ucalgary.ca) 


\section{Session: 3.3.7 - Competitive}

\section{Track: Track: 4 - Strategy, Alliances, and Competitiveness}

\section{IJV Stability and Duration}

Presented On: July 6, $2013-13: 20-14: 35$

Chair: Deepak K Datta, University of Texas at Arlington

The Effect of Asymmetrical Changes in Partners' Behavior and Attitudes on the Instability of International Joint Ventures

Rodrigo Isidor, University of Paderborn

Frank Hornung, University of Giessen

Rudy Kabst, University of Paderborn

The management of international joint ventures (IJVs) is an ongoing process that is affected by changes in partners' behaviors and attitudes towards the relationship over the course of collaboration. This study explores how these dynamic processes increase IJV instability. Drawing on social exchange theory, we show that the effect of changes in partners' interdependence, learning, relational norms, and trust on the stability of IJVs is mediated by asymmetrical changes in partners' commitments. Our analysis of $101 \mathrm{IJVs}$ generally validates this mediating model concluding that asymmetrical shifts in partners' commitments over the course of collaborations are detrimental to IJV stability. (For more information, please contact: Rodrigo Isidor, University of Paderborn, Germany: rodrigo.isidor@wirtschaft.uni-giessen.de)

The Impact of Business Relatedness on Mode Stability - An Empirical Investigation on International Joint Ventures

Michael Hunoldt, Friedrich Schiller University of Jena

The impact of business relatedness on the outcomes of interfirm collaboration has been frequently analyzed in management research over the last decades. In the context of IJVs, however, it has received only scant attention yet. Furthermore, existing studies in this field of research are characterized by both contradictory theoretical arguments and inconclusive empirical findings. Drawing on transaction cost economics and the resource based view this study aims to integrate different perspectives on the stability impact of business relatedness. Since theoretical arguments support a positive stability impact of both low and high business relatedness this paper suggests an U-shaped relationship. The study also examines the impact of an important contextual factor: the IJV governance structure. This factor is proposed to affect both the resource benefits and coordination costs resulting from partners' business relatedness. Empirical evidence supports the assumption of an U-shaped relationship between partners' business relatedness and IJV stability. Moreover, results show that the proposed moderator exerts an influence. (For more information, please contact: Michael Hunoldt, Friedrich Schiller University of Jena, Germany: michael.hunoldt@uni-jena.de)

\section{Knitting International Alliances: Cobweb of Cultural Distance, Joint Sensemaking, and Alliance Duration} Robin Pesch, University of Bayreuth Ricarda B. Bouncken, University of Bayreuth Jan Knocke, University of Bayreuth

This paper researches the influence of joint sensemaking on performance in international alliances and how it is affected by cultural distance and alliance duration. Prior studies have emphasized dangers of cultural distance and advantages by longer alliance duration. The results of our study - based on a survey of 163 German firms operating in different industries - show that joint sensemaking improves performance in international alliances. Joint sensemaking is associated with cognitive understanding and improved interrelation in international 
alliances. Cultural distance typically occurs between partnering individuals in international alliances. We explore the direct influence of three forms of cultural distance on joint sensemaking and how alliance duration interacts with the three forms. Our results show that two forms of cultural distance reduce sensemaking whereas one form improves joint sensemaking. Our findings on alliance duration are mixed, too. The interaction effects of alliance duration and cultural distance on joint sensemaking differ according to the form of cultural distance. (For more information, please contact: Robin Pesch, University of Bayreuth, Germany: robin.pesch@unibayreuth.de)

The Effects of Host Country Environment and Performance Risk on Ex Post Renegotiation in International Joint Ventures

Linda Hsiu-yun Hsieh, University of Birmingham

This paper draws on a risk-based view and institutional theory in order to develop a systematic understanding of the performance risk perceived by partners, and to investigate the implications of risk perception for ex post renegotiation in international joint ventures (IJVs). The key variables in this framework consist of three contextual factors which are considered as potential antecedents of perceived performance risk: environmental hostility, institutional satisfaction, cultural difference; perception of performance risk; and ex post renegotiation as a set of outcome variables. The framework is tested using a sample of 71 international joint ventures located in Taiwan. The findings show that environmental hostility, institutional satisfaction, and cultural difference are related to foreign partners' perception of performance risk. This study suggests that when foreign partners face likely performance risk after an IJV is established, they tend to resort to renegotiation in order to increase or maintain their confidence in their joint ventures. The survey findings also suggest that the decision to go for an extensive level of renegotiation to manage performance risk is primarily taken when the overall trustworthiness of one partner in the eyes of the other is low. (For more information, please contact: Linda Hsiu-yun Hsieh, University of Birmingham, United Kingdom: h.hsieh@bham.ac.uk)

\section{Session: 3.3.8 - Competitive}

\section{Track: Track: 8 - Developing Country MNCs}

\section{Corporate Governance in Developing Economy MNEs}

Presented On: July 6, $2013-13: 20-14: 35$

Chair: Huifen Cai, University of Huddersfield

Corporate Ownership Reform and Foreign Market Entry of Emerging Market Firms: Extending the Theoretical Lens of Firm-Specific Advantages

Ming Hua Li, Copenhagen Business School

Lin Cui, Australian National University

Jiangyong Lu, Guanghua School of Management

Few studies have examined the effects of corporate ownership reform on emerging market firms' advantages and capabilities for outward FDI. By linking the implications of such reform to the configuration of resources, organizational attributes, and strategic orientation of firms we advance an extended FSA framework incorporating the dynamics of organizational ambiguity to analyze EM firms' internationalization behavior. We validate our model by conducting an empirical study of 973 publicly listed Chinese firms spanning 8 years from 2002-2009. The findings suggest that various types of reformed shareholding have different effects on firms' proclivity for outward FDI. We also find evidence of synergies and misalignments between ownership derived FSAs and other firm specific organizational features including foreign managerial experience and business group affiliation. (For more information, please contact: Ming Hua Li, Copenhagen Business School, Denmark: mhl.int@cbs.dk) 
Foreign Ownership Strategies of Chinese MNCs: The Role of Institutional Factors

Diego Quer, University of Alicante

Enrique Claver, University of Alicante

Laura Rienda, University of Alicante

Drawing on the institutional theory, this paper examines the importance of decision specific experience and imitative behavior for Chinese multinationals (MNCs) foreign ownership structure decisions. From a sample of 189 outward foreign direct investment (FDI) decisions made by 35 large Chinese firms in 63 countries, we find strong evidence to support the hypothesis that Chinese firms tend to select ownership structures based on their experience with similar ownership structures in the past. Inter-organizational imitative behavior is also present, as later Chinese entrants tend to follow the entry mode patterns established by earlier Chinese entrants. Further, we investigate the potential moderating effects of cultural distance and firm's experience in the environment. (For more information, please contact: Diego Quer, University of Alicante, Spain: diego.quer@ua.es)

\title{
Do State-Owned Enterprises Pay More: Evidence from Outbound Chinese Cross-Border Mergers and Acquisitions Wenxin Guo, University of Illinois at Urbana-Champaign \\ Joseph Clougherty, University of Illinois at Urbana-Champaign \& CEPR-London Duesseldorf \\ Tomaso Duso, Deutsche Institut fuer Wirtschaftsforschung (DIW) and DICE, Heinrich-Heine University
}

While MNEs from emerging markets - and China in particular - tend to generate high acquisition premiums when they engage in cross-border merger activity, the determinants of this overbidding are not completely understood. We argue that state-ownership is a key factor in explaining the high acquisition premiums paid by emerging-market multinationals. Employing data on 450 Chinese outward cross-border M\&As over the 1990 to 2011 period, we find that Chinese state-owned MNEs pay higher acquisition premiums than do non-state-owned MNEs, and that state-owned MNEs pay even higher acquisition premiums when they act as parents and employ a privately-owned subsidiary to complete the cross-border M\&A. (For more information, please contact: Wenxin Guo, University of Illinois at Urbana-Champaign, USA: guo33@illinois.edu)

\author{
Institutional Distance and Cross-Border Acquisition Equity Participation by Emerging Market Multinationals \\ Nolan Gaffney, University of North Texas \\ Rusty Karst, University of North Texas \\ Robert Steinbauer, University of Memphis \\ Nicholas D. Rhew, University of Memphis
}

When pursuing cross-border acquisitions in economically distant locations, Emerging Market Multinational's equity participation follows an inverted $U$ relationship, in that equity share sought increases from low to moderate levels of economic distance, but then decreases sharply as the level of economic distance increases from moderate to high levels. Conversely, we find a $U$ shaped relationship between economic distance and equity participation in a representative sample of Developed Country Multinationals. Emerging Market

Multinationals also seek a larger equity share when pursuing cross-border acquisitions in countries with superior knowledge protecting institutions, counter to previous findings on MNE equity participation. Furthermore, when compared to a sample of Developed Country Multinationals cross-border acquisitions, knowledge distance has a larger effect on Emerging Market Multinational equity share pursuits. (For more information, please contact: Nolan Gaffney, University of North Texas, USA: nolan.gaffney@unt.edu) 


\title{
Session: 3.3.10 - Competitive
}

\section{Track: Track: 1 - Institutions, Governance, and CSR}

\section{CSR and Legitimacy}

Presented On: July 6, $2013-13: 20-14: 35$

Chair: Christof Miska, Vienna University of Economics and Business

\author{
Do All Firms Seek Legitimacy? An Examination of Firms in the Global Apparel Industry \\ Susan L Young, Seton Hall University \\ Mona Makhija, Ohio State University
}

Researchers have suggested that firms' corporate social responsibility (CSR) decisions are based on the desire to gain legitimacy in their institutional environment, which establishes societal norms and values related to CSR. This view implies convergence in firms' CSR behaviors within institutional contexts. We argue, however, that heterogeneity in firm attributes cause them to differ on their dependence on the goodwill of society at large, and therefore, their requirements for legitimacy. We suggest that firms' need for legitimacy will vary according to two factors, their transparency and economic vulnerability. Based on this argument, eight hypotheses are developed and tested using a unique database of firms' CSR responsiveness in the global apparel industry. Our findings indicate that, as predicted, CSR responsiveness does vary according to a firm's need for legitimacy. This research underscores the differential economic motivations that firms have for engaging in CSR and highlights the critical role of firm attributes in influencing its CSR behavior. (For more information, please contact: Susan $L$ Young, Seton Hall University, USA: susan.young@shu.edu)

\section{CSR, Attitude and Institutional Pressures \\ Rasi Kunapatarawong, Universidad Carlos III de Madrid}

A study about antecedents explaining why some firms succeed with their CSR initiatives while others fail would be fruitful as CSR provides many benefits to firms but failed CSR attempts damage firms. We believe (1) demands from various stakeholder groups to dedicate resources to CSR, (2) managers' attitude driving behavior as important to CSR implementation, and (3) disaggregation of CSR dimensions to be three important issues playing a role in creating robust CSR. We hypothesize that the kinds of CSR firms adopt are affected by different types of institutional pressures and are mediated by managers' CSR attitude. (For more information, please contact: Rasi Kunapatarawong, Universidad Carlos III de Madrid, Thailand: rasirasita@gmail.com)

\section{CSR Adoption and Involvement in Jus Cogens vs. No-Jus Cogens Human Rights Abuses. An Emperical Study on Large Global Corporations \\ Elisa Giuliani, University of Pisa \\ Davide Fiaschi, University of Pisa}

Management research on Corporate Social Responsibility (CSR) focuses almost exclusively on the impact of CSR on profitability or corporate value. A largely neglected question is whether CSR impacts positively on society. We address this gap in the literature by exploring the relationship between CSR adoption (as reflected by corporate declarations to adopt CSR policies) and corporate involvement in alleged human rights abuses. Using information on 140 large advanced country corporations, we find that there is a relationship between CSR and alleged human rights abuses, but that the nature of this relationship varies according to the type of abuse: firms that declare to be CSR-adopters appear less likely to be involved than non-adopters in the worst of the abuses (i.e. jus cogens abuses), but more likely than non-adopters to be involved in other types of "less serious" abuse (i.e. no-jus cogens abuses). Also, the adoption of CSR does not reduce corporate involvement in human rights abuses over time, displaying a lack of learning effects on this subject. Our analyses contribute to 
research on the impact of business on society and have some normative implications for corporate abuses of human rights. (For more information, please contact: Elisa Giuliani, University of Pisa, Italy: giulel@ec.unipi.it)

\author{
Legitimizing Export Expansion into Institutionally Distant Destinations \\ Ziliang Deng, Renmin University of China \\ Ruey-Jer Bryan Jean, National Chengchi University \\ Rudolf R. Sinkovics, University of Manchester
}

We examine the moderating effect of sub-national institutions in exporters' domestic market on the relationship between export expansion speed over institutional distance and firm performance. We propose that the development of market mechanism, as a form of formal institutions, can effectively offset the potential pressure and risks involved in rapid expansion over both formal and informal institutions. By employing data of more than 8,000 Chinese international new venture exporters during 2000-2006, we perform panel data regressions to test our theoretical hypotheses. The empirical findings generally corroborate the hypotheses. Our study contributes to the development of institutional theory in the area of international management in that it integrates the effects of both domestic and international institutions, and by doing so, it also conceptualises and empirically tests the joint effect of formal and informal dimensions of institutions on firm performance during internationalization. (For more information, please contact: Ziliang Deng, Renmin University of China, China: dengziliang@rbs.org.cn)

Session: 3.3.12 - Interactive

Track: Track: 5 - MNC Management and Organization

\title{
MNE Integration and Local Reach
}

Presented On: July 6, $2013-13: 20-14: 35$

Chair: Tom Roehl, Western Washington University

MNE Integration Processes: A Subsidiary Perspective

Birgitte Grogaard, University of Calgary

Helene Loe Colman, Norwegian Business School

Inger G. Stensaker, Norwegian School of Economics

It is widely recognized that multinational enterprises (MNEs) often struggle with the balancing act of global integration versus local adaptation. In this study, we identify how foreign subsidiaries make sense of their 'multiple embeddedness'. More specifically, we examine how foreign subsidiaries balance the dynamics of 'belonging' to the MNE vis-á-vis the external environment through a case study of three foreign subsidiaries in a multinational. We contribute to the MNE integration literature by identifying two key dimensions that impact the subsidiaries' interest in and focus on MNE integration: 1) The subsidiary's perceptions of the strategic importance of its local external environment, and 2) the subsidiary's perceived importance of contributing to the overall competence development in the MNE. (For more information, please contact: Birgitte Grogaard,

University of Calgary, Canada: bgrogaar@ucalgary.ca)

Balancing the Internationalization Process of a Multinational Corporation Expanding into in China - The Case of Acting Ambidextrously

Rian Drogendijk, Uppsala University

Henrik Dellestrand, Uppsala University

Mikael Eriksson, Uppsala University

Ulf Holm, Uppsala University 
This paper deals with the market expansion of Atlas Copco, a Swedish multinational corporation operating in the mining industry, into China. Entering and doing business in emerging markets such as China presents substantial challenges but also considerable opportunities for a western multinational corporation. The knowledge base of the organization may be geared towards high-end markets, and thus the routines for doing business are shaped in such a direction. However, emerging markets present new contextual demands. This will force the firm to act ambidextrously: to combine strategies of exploitation and exploration and thus to balance existing routines and internal pressures with the conflicting demands in the new market context. The paper illustrates this process by means of an in-depth phenomena-driven longitudinal case study of Atlas Copco. The case illuminates how a multinational corporation can balance and reconcile internal demands and conflicting contextual pressures for successful internationalization. Our paper contributes to the growing literature on ambidexterity in showing how companies can balance existing routines and strategies with novel ones in unknown market environments. (For more information, please contact: Rian Drogendijk, Uppsala University, Sweden: rian.drogendijk@fek.uu.se)

\section{The Liability of Intergroup Bias in Multicultural Teams: A Network Perspective \\ Pekka Johannes Vahtera, University of Leeds \\ Peter J Buckley, University of Leeds \\ Jeremy Clegg, University of Leeds \\ Adam Cross, University of Leeds}

Social capital is a key concept in explaining the success of network actors in multinational organizations. We argue that bridging and bonding social capital within multicultural and geographically dispersed teams can be a major source of intergroup bias. We elucidate the role of individuals' network position and group structure in creating negative intergroup bias within a sample of 160 intra-organizational actors, and considerable support for our hypotheses is found. Our findings indicate that high degree of bonding social capital underscores negative perceptions towards out-group members. At the same time, bridging ties within social identity groups but not between groups - entail more negative attitudes towards out-group members. Finally, higher geographical and cultural distance between team-members are both associated with negative relationships. In turn, these negative perceptions have a damaging effect on perceived individual performance. We conclude that identification with team-members plays an important role in creating social capital as well as negativity within multinational organizations, and that it has been given insufficient attention in the extant literature. (For more information, please contact: Pekka Johannes Vahtera, University of Leeds, United Kingdom: bnpjv@leeds.ac.uk)

Is It Local or Global, or Should We Care? A Look at Greenpeace International Support to Local Activities and Its Effect on Performance

Yves Plourde, University of Western Ontario

In this paper, I look at the effect of receiving support from the international network of the MNE on the performance of local projects. Adopting a situated performance perspective, I build on the notion of fit to suggest that while support from the international network is generally beneficial to performance, under certain circumstances, the absence of support from the network may be a more appropriate option. I empirically test this proposition by looking at 102 projects conducted by Greenpeace International in 5 different countries. My findings suggest that on average, a project for which there is a fit in terms of alignment between the scope of an issue and the scope of a project will have a greater probability of success than a project for which there is no fit. Future research is to explore the "grey zones", when there is no fit, to understand when international support will provide the best value to a subsidiary and the MNE. (For more information, please contact: Yves Plourde, University of Western Ontario, Canada: yplourde.phd@ivey.ca) 
International Joint Ventures Transformed: Foreign Buyouts, Firm Performance and Management Turnover

Jing-Lin Duanmu, University of Surrey

Neviana Petkova, University of Oregon

We study the effects of changes in organizational form on firm performance and management turnover. Our analysis sheds light on whether there are gains to be exploited by transitioning from product market relationships such as joint ventures to wholly owned subsidiaries through mergers and acqusitions (M\&As). Using a difference-in-differences propensity score matching estimator to control for selection bias, we show that performance is similar across both organizational forms. However, foreign buyout accompanied by management turnover leads to performance gains. Our findings suggest that the gains from M\&As are due both to synergies and management turnover. (For more information, please contact: Jing-Lin Duanmu, University of Surrey, United Kingdom: j.duanmu@surrey.ac.uk)

An Analysis of Entry Modes, Supply Networks and it Capabilities of EMNEs in the UK: The Case TATA, CEMEX $A N D K O C$

Gul Berna Ozcan, University of London

Adrian Coronado, University of London

G. Harindranath, University of London

This research project aims to analyse three Fortune 500 listed emerging market multi-nationals' (EMNE) expansion in the UK. We propose to study India's Tata Motors (Tata is the largest foreign investor in UK with 19 companies and a 45,000 workforce); Mexico's Cemex (with over $£ 1$ billion in annual sales and a UK supply network with over 500 locations) and Turkey's Koç (with 16.4 percent of UK's fitted kitchen market). We focus on three specific areas through an empirical investigation: i) The scope of entry modes in respective sectors vis-à-vis their competitors; ii) The structure of supply networks and logistical operations linking local activities to country of origin and others; iii) The nature of IT capabilities in management and control of operations in UK branches and their headquarters. (For more information, please contact: Gul Berna Ozcan, University of London, United Kingdom: g.ozcan@rhul.ac.uk)

\section{Session: 3.3.13 - Interactive}

Track: Track: 3 - IB Theory, FDI, and Entry Mode

\section{Interactions Between Foreign-Owned and Indigenous Firms}

Presented On: July 6, 2013 - 13:20-14:35

Chair: George Z. Peng, University of Regina

What Does FDI Bring to China? Competition Effect and Productivity Shock Analysis

Zhen Yang, Renmin University of China

Jiawen Yang, George Washington University

This paper investigates the compound impacts of Foreign Direct Investment (FDI) presence on China's manufacturing industries. We address two questions in this paper: First, does FDI improve competition or exert monopoly power in China's manufacturing industries? Second, how does FDI influence firm-level total factor productivity (TFP) of incumbent Chinese firms? We use firm-level data in China's manufacturing industries over 1998-2009 for the analysis. We extend the model developed by Roeger (1995) to test FDI presence on markups. Firm-level productivity is measured by adopting Levinsohn-Petrin (2003) semi-parametric methodology, which corrects the simultaneity and selection bias when estimating production function. The key finding is that FDI presence affects industries' markup in different ways. In general, FDI brings more monopoly power than competition to incumbent local firms, thus its anti-competition effect dominates. The relationship 
between FDI presence and markup is reverse U-shape. Furthermore, FDI presence increases local firm productivity, and this result is robust to different regression methods and different measurements of FDI presence. FDI has a short-term effect on productivity of export-oriented companies and a lagged effect on that of market-oriented companies. Finally, those companies controlled by central and provincial governments can benefit more from absorbing FDI. (For more information, please contact: Zhen Yang, Renmin University of China, China: yangzhen2010@ruc.edu.cn)

\section{FDI Spillovers and Relative Absorptive Capacity Yoo Jung $\mathrm{Ha}$, University of York Axele Giroud, UNCTAD}

This paper explores how far relative absorptive capacity is a condition of FDI spillovers. Relative absorptive capacity depends on the ability of the host country firm to search and acquire foreign technology from network relationships with MNE in which the local firm is embedded. Our empirical finding confirmed that a local firm needs strong relative absorptive capacity with regard to breadth of network relationships in order to benefit from technology spillovers from FDI. There is no evidence of how far strong relative absorptive capacity concerning depth of network relationship matters as a condition of successful FDI spillovers. Our key contribution lies in extended conceptualisation of absorptive capacity which has been widely considered as a key condition of successful FDI spillovers and generation of insights about FDI spillovers in developed host countries. (For more information, please contact: Yoo Jung Ha, University of York, United Kingdom: yoojung.ha@york.ac.uk)

\section{Survival Or Death When Foreigners Enter A Local Market? \\ Thi Song Hanh Pham, Sheffield Hallam University \\ Thang Tran, Central Institute for Economics and Management}

This paper examines the survival effect of foreign direct investment (FDI) on domestic private firms (DPF) in the context of existence of state owned enterprise (SOE) by using Hazard function and panel data of 86108 firms over the period from 2000- 2007 in Vietnam. We find that FDI inflow raise the hazard of exit, reduce the survival length of DPFs. In comparison with FDI, SOEs have higher crowding out effect to DPFs. Furthermore, state own enterprise exerts influence upon the extent to which FDI firms affect DPFs. We found that in sectors with high SOEs share, FDI firms have more effect. (For more information, please contact: Thi Song Hanh Pham, Sheffield Hallam University, United Kingdom: s.h.pham@shu.ac.uk)

\section{Barriers to Absorptive Capacity and Capability Upgrading in Developing Country Multinationals Alvaro Cuervo-Cazurra, Northeastern University Huaichuan Rui, University of London}

We study how barriers to absorptive capacity limit the upgrading of capabilities to international levels by developing country multinational companies (DMNCs). The case of the state-owned Chinese automobile Nanjing Automobile Group reveals additional barriers to absorptive capacity that limit the effective transformation of external knowledge into competitive advantage: not-invented-here biases, whereby employees dismiss external knowledge even if managers recognize its value; management preferences, whereby managers' biases and career prospects guide the selection of particular knowledge sources; and source relationships, whereby external sources limit the knowledge accessed by the company. These barriers complement other barriers to absorptive capacity discussed in the literature such as appropriability regimes, social integration mechanisms and activation triggers, and provide a more nuanced understanding of absorptive capacity. (For more information, please contact: Alvaro Cuervo-Cazurra, Northeastern University, USA: a.cuervocazurra@neu.edu) 
Transition economies have become a new frontier for multinational enterprises. This has introduced a highly dynamic competitive environment for domestic firms especially through the entry of multinational enterprises with superior capabilities. Drawing on the awareness-motivation-capability perspective, this paper develops a three-round framework that explores the dynamic capabilities of domestic and foreign firms and the related competitive dynamics between them in (and out of) transition economies. A set of theoretical propositions is developed and research and managerial contributions and implications are discussed. (For more information, please contact: Canan Mutlu, University of Texas at Dallas, USA: cxm101020@utdallas.edu)

\section{Session: 3.3.14 - Interactive}

\section{Track: Track: 9 - Cross-Cultural Management and HRM}

\section{Emerging Expatriation Issues}

Presented On: July 6, $2013-13: 20-14: 35$

Chair: Margaret Shaffer, University of Wisconsin-Milwaukee

The Perceived Impact of National Identity and Cross Border Business Focus among Expatriate and National Managers

Ramudu Bhanugopan, Charles Sturt University

Dianne Bealer, Charles Sturt University

This study examines the dimensions of national identity and cross border business focus for six different nationalities in a Middle Eastern work environment. Data were collected from 213 employees working in educational institutions, medical and oil related organisations in United Arab Emirates. Principal components factor analysis was used to determine the underlying factor structures of national identity and cross border business focus. The results demonstrated that the most significant demographic variables affecting national identity and cross border business focus were: age, level of education, industry and religion and the less significant were: gender, country of origin and nationality. This paper contributes to knowledge on national identity and augments explanation on cross border business focus of both expatriates and nationals. Implications, limitations and directions for future research are discussed. (For more information, please contact: Ramudu Bhanugopan, Charles Sturt University, Australia: bramudu@csu.edu.au)

\section{Linking IHRM and Crisis Management: Sectoral Contrasts in Managing Emergency Evacuation of Expatriates Anthony Fee, University of Technology Sydney \\ Susan McGrath-Champ, University of Sydney}

In volatile and uncertain times, pressure increases on firms to ensure that their operations and staff are secure and safe from threat of physical and psychological danger. Yet there is a noticeable absence of studies into how firms manage the evacuation of international staff in times of crisis, such as terrorist attacks, illness and injury, natural disasters, and what role international HRM function should play. This paper bridges the divide between international business/HRM literature and research into emergency response planning and crisis management. It presents the results of an empirical study exploring the international evacuation policies and procedures of 17 multinational organisations from three hazard-prone industries: international aid and development, mining and resources, and news media. Our results unveil starkly different approaches across the three industries. We label these approaches 'humanitarian' (international aid and development industry), 'regulatory' (mining and 
resources), and 'informal mentoring' (media). The study also highlights the relative minor, and primarily reactive, role played by international HRM in international crisis management. (For more information, please contact: Anthony Fee, University of Technology Sydney, Australia: anthony.fee@uts.edu.au)

\author{
When a Subsidiary Loses Its Status: Towards an Identification-based Model of Host Country Nationals' \\ Willingness to Share Knowledge with Expatriates \\ Sachiko Yamao, University of Melbourne \\ Toru Yoshikawa, Singapore Management University
}

We theorize how a foreign subsidiary's loss of its status relative to other units of the same multinational corporation (MNC) will influence host country national (HCN) managers' dual organizational identification and their willingness to share knowledge with expatriate managers. In particular, we examine the two contingent scenarios of subsidiary status loss (i.e., the parent-driven and subsidiary-engaged) that may lead HCN managers to develop different organizational identification (i.e., MNC-oriented and subsidiary-oriented). We then propose that different organizational identification of $\mathrm{HCN}$ managers will lead them to develop varying levels of willingness to share knowledge with expatriates. We further propose that HCN managers' generalized identification with expatriate managers moderates the relationship between HCN managers' organizational identification and their willingness to share knowledge with expatriates. (For more information, please contact: Sachiko Yamao, University of Melbourne, Australia: syamao@unimelb.edu.au)

\title{
Control and knowledge transfer through international transfer assignments - the case of offshore outsourcing Florence Duvivier, Solvay Brussels School of Economics and Management Carine Peeters, Universite libre de Bruxelles
}

In this paper we integrate two strands of research that have been developed independently: i) the extant knowledge of expatriation and inpatriation and, ii) the growing body of the liter-ature on offshoring strategies. We identify and describe the mechanisms through which dif-ferent types of international transfer assignees manage to exert control and transfer knowledge in service offshoring operations. Our study is based on the experience of a West-ern European country (Belgium). We conduct a qualitative research collected through 41 interviews and aimed at a managing director/CEO population. From our interview material, we identify three types of control systems ( i) through bureaucratic control, ii) through cul-tural control, and iii) through decreasing the information asymmetry) and three mechanisms to transfer knowledge between the parentcompany and the host company (i) through the information facilitator, ii) through training, and iii) through building trust) through which expatriates and inpatriates managed in services offshoring activities. Our findings support prior studies and extend the existing literature on the ability to exert control and to transfer knowledge through the use of international transfer assignments. In the discussion section, the risk of using expatriates to transfer the corporate culture and the preference to rely on inpatriates will be debated. (For more information, please contact: Florence Duvivier, Solvay Brussels School of Economics and Management, Belgium: florence.duvivier@ulb.ac.be)

\section{Ten Years of Repatriate Research: What We Have Learned Emmy van Esch, Hong Kong Baptist University Flora Chiang, Hong Kong Baptist University}

As boundary spanners, repatriates are essential for sustained organizational competitive advantages. However, the rapid growth of the repatriation literature over the past years has made it difficult to keep up with developments in this domain. This paper will therefore provide a coherent picture of its state of art, and in doing so, will critically assesses main challenges, gaps and limitations, and provide essential directions for future research. (For more information, please contact: Emmy van Esch, Hong Kong Baptist University, Hong Kong, SAR-PRC: 11467061@life.hkbu.edu.hk) 


\section{Substance Use in Expatriation \\ Olivier Wurtz, Vaasa University \\ Vesa Suutari, Vaasa University}

The only two expatriate studies on the consumption of alcohol and drugs showed that expatriates use more substances after their relocation than before their departure and indeed, more than a control group (Anderzen \& Arnetz, 1997; Truman, Sharar, \& Pompe, 2012). We explore, based on two studies, one of 205 expatriates, and one of 110 expatriate supervisor/colleague dyads, the antecedents and consequences of these behaviors. We show that stress at a medium to high level increases substance use for male, but not female expatriates. Experience of expatriation increases the risk of taking substance. Finally, we observe that consuming such substances harms professional adjustment. (For more information, please contact: Olivier Wurtz, Vaasa University, Finland: olwurtz@gmail.com)

\section{Scaling Back on International Assignments: Family Matters \\ Romila Singh, University of Wisconsin-Milwaukee \\ Janice R. W. Joplin, Southern Illinois University Edwardsville}

We develop and test a model that examines work-family conflict as the intervening mechanism by which the demands and resources associated with work and family roles influence expatriates' psychological withdrawal from work. Using multi-source data from 151 expatriates and their spouses, we examine the extent to which domain specific demands and resources trigger work-family conflict and the subsequent effect of work-family conflict on psychological work withdrawal. Results indicate that family interference with work fully mediates the relationship between family demands and resources and psychological work withdrawal. The results of this study suggest that family matters can lead expatriates to become negligent and neglectful of work responsibilities. (For more information, please contact: Romila Singh, University of Wisconsin-Milwaukee, USA: romila@uwm.edu)

\section{Expatriate Staffing in Foreign Greenfield Subsidiaries: The Role of Industry Christopher Williams, University of Western Ontario Michael Roberts, MacEwan University}

We investigate the role that industry type (manufacturing vs. services) plays in determining the use of expatriate managers deployed by MNEs in foreign subsidiaries at Greenfield start-up. This staffing decision is a major concern facing multinational enterprises (MNEs) as they internationalize, yet has not been studied comprehensively from an industry angle. Using knowledge-based theory (KBT), we develop and test a new model suggesting both a direct and indirect role for industry type. Drawing on a sample of 6,478 new subsidiaries established by Japanese MNEs over a 20 year period we find strong support for our hypotheses. We control for a broad range of salient factors, and use a hierarchical model to include a random effect for country. Industry type (services) has a direct positive effect on expatriate deployments, as well as significant moderating effects on the relationships between host country experience, ownership mode, and various forms of distance, and expatriate deployment. The findings have implications for international strategy and knowledge-based theories of the MNE, as well as for MNE managers. (For more information, please contact: Christopher Williams, University of Western Ontario, Canada: cwilliams@ivey.ca) 
Session: 3.3.15 - Interactive

Track: Track: 12 - Economic Geography and Value Chains

\section{Clusters, R\&D and Sustainability}

Presented On: July 6, $2013-13: 20-14: 35$

Chair: Bernard Wolf, York University

The Role of the International Joint Ventures (IJVs) in the Development and Upgrading of the Pakistani Automotive Clusters: The Impact of Relational Proximity

Zaheer Khan, University of Hull

John Nicholson, University of Hull

Svetla Trifonova Marinova, Aalborg University

While cluster development has been recognized for economic development, the role and the specific impact of the MNEs operating in a cluster on local firms has rarely been the major focus of scholarly attention. In this paper we empirically investigate how knowledge transfers from international joint ventures affect the movement up the value chain of local suppliers. In the context of the Pakistani automotive industry, we contribute to the developing literature examining relational proximity and conclude that relational proximity and its counter proposition of relational isolation have a significant impact on access to the international networks controlled by foreign investors in Pakistan and consequently the local suppliers' ability to learn and upgrade their operations. Our findings suggest that suppliers who have greater relational proximity with lead assemblers and their first tier global suppliers are in a better position to upgrade their products and processes. The findings indicate that global connections and associated relational proximity with lead assemblers at local level and their global tier 1 suppliers are important for lower tier suppliers based in the emerging economies. This connectivity is found to be essential to upgrade both their products and processes, as much of the codified and tacit knowledge resides across transnational value networks. (For more information, please contact: Zaheer Khan, University of Hull, United Kingdom: khan.zaheer@gmail.com)

\section{Where Do We Go from Here? Globalizing Subsidiaries Moving up the Value Chain Pavida Pananond, Thammasat University}

This paper studies the international expansion of local subsidiaries of multinational enterprises (MNEs). The main questions asked are why and how MNEs' local subsidiaries in developing economies undertake international expansion. Drawing from the global value chain perspective, I argue that local subsidiaries that were initially established to undertake low value-added production activities do not want to remain at there forever. The literature on initiative-taking subsidiary has predominantly focused on them becoming centers of excellence via R\&D and innovation in the host country. I propose a different route of local subsidiary upgrading via foreign direct investment. This strategy helps not only to gain internal prominence within the MNE network, but also to improve its external position within the global value chain. (For more information, please contact: Pavida Pananond, Thammasat University, Thailand: pavida@tbs.tu.ac.th)

Satellite Clusters, Flagship MNE Subsidiaries and Mothership Ecosystems: Specialization, Adaptation and Connectivity in Staving Off Technology Cluster Malaise

Johanna Clancy, National University of Ireland, Galway

Paul Ryan, National University of Ireland, Galway

Majella Giblin, Trinity College Dublin

David Collings, Dublin City University 
This paper links the literatures from international business and economic geography to explore how an industrial cluster extends its life-cycle and overcomes the vulnerabilities of technological specialization. The role of the MNE subsidiary and ancillary cluster members in this process is examined in a longitudinal study of the birth, growth and extended maturity of an Irish technological cluster. The case reveals the significance of interplays between the MNE subsidiary, the cluster that the subsidiary is a member of (the subsidiary's 'host' cluster) and the parent company that is often itself part of a cluster (the parent's 'home' cluster) in developing varied technological activity. Just as subsidiaries can 'tap into' the local cluster to source knowledge, local technological entrepreneurs can reverse the flow and access knowledge from the MNE parent's home cluster. (For more information, please contact: Johanna Clancy, National University of Ireland, Galway, Ireland: johanna.clancy@nuigalway.ie)

\section{Managing a Cross-border R\&D Team for Quality Innovation: The Moderating Role of Team Configuration Eunkwang Seo, Seoul National University Hyoseok Kang, Seoul National University Jaeyong Song, Seoul National University}

Although existing literatures have stressed the importance of establishing global R\&D centers across borders, few studies focused on the management of cross-border collaborations among them. We examine the effects of geographic diversity within a research team on innovation quality. We also investigate team-level factors that positively moderate the geographic effects. We conducted a negative binomial regression analysis of 461 research teams in top 20 pharmaceutical companies in 2004. The result shows that geographic diversity of individuals within a research team has an inverted-U shaped relationship with the innovation quality of the team. In terms of team configurations, asymmetrical locational arrangements of inventors with a centralized hub and capability gaps among researchers are shown to reinforce the positive effect of geographic diversity on innovation quality, respectively. Finally, the results show that a portion of repeated collaboration among researchers mitigates the quality decrease due to the excessive geographic diversity. (For more information, please contact: Jaeyong Song, Seoul National University, Korea, South: jsong@snu.ac.kr)

\section{Greening of International Business \\ John A. Mathews, Macquarie University \\ Hao Tan, University of Newcastle}

The greening of industry globally, led by entrepreneurial initiatives from multinational corporations, has yet to be reflected in the mainstream International Business literature. With some honourable exceptions, IB scholars have yet to engage with the opportunities and challenges presented to multinational enterprises (MNEs) by climate change, and have yet to apply lessons learnt over the past half century in the study of internationalization to the emergence of new MNEs engaged in building global green industries such as renewable energies. This paper explores these issues in the context of China's wind power industry, which has exploded onto the world stage and is now creating potentially powerful MNEs such as Goldwind, Sinovel and MingYang, whose accelerated impact calls for fresh explanatory frameworks. (For more information, please contact: John A. Mathews, Macquarie University, Australia: john.mathews@mgsm.edu.au)

\section{Drivers of Cross-national Variation in E-retail Spending \\ Nir Kshetri, University of North Carolina, Greensboro \\ Ralf Bebenroth, Kobe University \\ Nicholas C. Williamson, University of North Carolina, Greensboro}

The purpose of this paper is to examine the technological, market, industry and institutional sources associated with the cross-national heterogeneity in e-retail spending. We used time series cross sectional (TSCS) models linear in parameters for 10 year data from 43 countries to test our hypotheses. We found that how broadband Internet access, which has been identified as an important determinant of an economy's "e-readiness", explains 
international heterogeneity in e-retail spending. We also found various specific attributes of the traditional retail environment that produce enhancing effects as well as suppressing effects for e-retail spending. Finally, we also found regulatory forces' impacts on the development of the e-retail industry. (For more information, please contact: Nir Kshetri, University of North Carolina, Greensboro, USA: nbkshetr@uncg.edu)

\section{Global Value Chains and Interaction on Clusters: How Small and Medium Ornamental Rock Enterprises from Espírito Santo, Brazil, Co-Operate to Mitigate Export Risks from Their Global Operations Maria Laura Ferranty Maclennan, ESPM Ilan Avrichir, ESPM}

This article approaches the part played by the local interactions of small and medium businesses when competing on the global markets, from the theoretical perspective of clusters and global value chains. It states that, despite globalization, the local interactions among firms remain important. The basic research question is to examine the link between the global chain governance in which the cluster operates and the interactions within the cluster. To address this issue, this study applied quantitative and qualitative methods, from a survey of 32 firms associated with Centro Rochas. From the existing research on the ornamental rock cluster in Brazil, the main finding is that it operates in market type governance. This contradicts at least part of the conventional wisdom concerning governance of natural resources based global value chains. This governance of the cluster is embedded and pushes firms to prospect clients in different export markets, participate in fairs and to develop new distribution channels internationally. Also, the paper explains how co-operation plays an important role in the cluster, in mitigating credit risk from international buyers, promotion and branding, and also on protecting exotic raw materials from being available to Asian competitors. (For more information, please contact: Maria Laura Ferranty Maclennan, ESPM, Brazil: ferranty@hotmail.com)

\section{Session: 3.4.1 - Panel \\ Track: Track: 5 - MNC Management and Organization \\ Foreignness and Innovation: Advantage, Liability or Both?}

Presented On: July 6, 2013 - 14:50-16:05

Chair: Annique Un, Northeastern University

\section{Panelists:}

John Cantwell, Rutgers University

Jesper Edman, Hitotsubashi University

Annique Un, Northeastern University

Sri Zaheer, University of Minnesota

In this panel we will analyze whether foreignness provides an advantage or liability in the area of innovation. There is a debate on whether subsidiaries of foreign firms suffer a liability relative to domestic firms, because they are less embedded in the local social networks, or have an advantage, because they receive knowledge from the parent firm and its network of subsidiaries. Here we provide a focused analysis of the debate by analyzing innovation and will argue that subsidiaries tend to enjoy an advantage of foreignness in innovation over domestic firms for several reasons: receiving technology and innovations from the parent firm and its other subsidiaries, having an outsider status that enables wider exploration of new knowledge, and creating unique management practices that support innovation. However, we will also explain how the advantage of foreignness in innovation may not be translated into ultimate profitability relative to domestic firms because of the existence of other liabilities of foreignness. This does not mean that subsidiaries are financially operating at a loss abroad, but that they are merely less profitable than incumbent domestic firms. This provides a more nuanced and balanced analysis of the debate on the liability and advantage of foreignness. (For more information, please contact: Annique Un, Northeastern University, USA: a.un@neu.edu) 
Session: 3.4.2 - Panel

Track: Track: 11 - SMEs and Entrepreneurship

\section{Hidden Champions: The Innovative Companies from CEE and Turkey with a Global Reach}

Presented On: July 6, $2013-14: 50-16: 05$

Chair: Danica Purg, IEDC-Bled School of Management

Panelists:

Danica Purg, IEDC-Bled School of Management

Peter McKiernan, Murdoch University

Irina Skorobogatykh, Plekhanov Russian University of Economics

Dilek Cetindamar, Sabanci University

Based on the study of Prof. Hermann Simon (1996 and 2012) about the successful innovative and export based SMEs in Germany, labeled by Simon as "Hidden Champions", a research has been conducted on "Hidden Champions" in CEE, Kazakhstan and Turkey, by CEEMAN. The proposed panel will focus on Hidden Champions in Bosnia and Herzegovina, Russia, Slovenia and Turkey. The analyzed companies have a very low visibility profile in public domain in spite of their top position in the European and /or global market. A crosscultural analysis has been executed on a number of factors that could explain the success of these - mostly family owned - companies. In the panel, we shall present the cross-sectional findings on innovations, leadership, corporate culture and corporate governance. The attention will be given to bivalent character of these factors, as they can offer an explanation of success of these companies, and at the same time contain elements of concern for their future. (For more information, please contact: Danica Purg, IEDC-Bled School of Management, Slovenia: danica.purg@iedc.si)

\section{Session: 3.4.3 - Panel}

Track: Track: 5 - MNC Management and Organization

\section{Investigating and Reconciling the Roles of Formal Structure and Networks in MNCs}

Presented On: July 6, $2013-14: 50-16: 05$

Chair: Mikael Soendergaard, Aarhus University

Panelists:

Ulf Andersson, Copenhagen Business School

Francesco Ciabuschi, Uppsala University

Henrik Dellestrand, Uppsala University

William G. Egelhoff, Fordham University

Phillip Nell, Copenhagen Business School

Joachim Wolf, University of Kiel

The proposed panel employs six panelists and a moderator to conduct a roundtable discussion of the roles of formal structure and networks in MNCs. Both types of coordination are well developed subjects in the MNC literature, but generally as separate topics and often as opposing views about what kind of coordination is most important in MNCs. The purpose of the panel is to begin to reconcile and where possible integrate these two perspectives of coordination in MNCs. The session will use five questions to structure the discussion. The panelists tend to be familiar with each other's work and as a group have considerable experience in researching and conceptualizing the roles of both formal structure and networks in MNCs. (For more information, please contact: William G. Egelhoff, Fordham University, USA: egelhoff@fordham.edu) 
Session: 3.4.4 - Panel

Hands-On, Minds-On: Experiential Learning Projects in International Business Education Part 2

Presented On: July 6, $2013-14: 50-16: 05$

Chair: Vas Taras, University of North Carolina, Greensboro

Panelists:

Vas Taras, University of North Carolina, Greensboro

Miroslaw Jarosinski, Warsaw School of Economics

Michel Librowicz, Université du Québec à Montréal

Nick Robinson, North Island College

Amanda E.K. Budde-Sung, University of Sydney

Adam Johns, Rikkyo University

Nobuya Takezawa, Rikkyo University

Dirk Fischbach, Hochschule Harz

Andres Velez-Calle, Universidad EAFIT

Virginia Cathro, Otago University

Maria Alejandra Gonzalez-Perez, Universidad EAFIT

Alfredo Jiménez, University of Burgos

Anke Arnaud, Embry-Riddle Aeronautical University

Alfredo Behrens, Alfredo Behrens Consulting

Sebastián Steizel, Universidad Oberta de Catalunya

Michael Zisuh Ngoasong, Coventry University London Campus

Susan Forquer Gupta, Monmouth University

Terri Lituchy, University of the West Indies

Betty Jane Punnett, University of the West Indies

Toshiya Ozaki, Rikkyo University

Experiential learning is a "hot" topic in International Business and Cross-Cultural Management Education (IBCCM), but the use of this approach is limited due to a lack of information and understanding on what tools are available and how to incorporate them in the curriculum. The proposed panel will bring together a 12 teams of academics who have developed and successfully used various experiential learning projects for IC-CCM education. The goals of the panel are to share information about the existing EL projects, share experiences, discuss challenges and best practices of using EL to improve IB-CCM education, provide a networking opportunity for academics interested in the EL approach, discuss how this panel/group can contribute to further discussion on the role of EL in IB-CCM education, possibly through special issues on EL in academic journals, targeted conferences and seminars, more and better research on the issue. (For more information, please contact: Vas Taras, University of North Carolina, Greensboro, USA: v_taras@uncg.edu) 


\title{
Session: 3.4.5 - Competitive
}

Track: Track: 10 - Economics, Finance and Accounting

\section{Corporate Governance, Ownership Structures and Firm Performance}

Presented On: July 6, $2013-14: 50-16: 05$

Chair: Hussain Rammal, University of South Australia

Ownership Structure and Firm Performance: Evidence from EU an Subsidiary Panel Study in China Huifen Cai, University of Huddersfield

This paper adopts a multi-theoretic approach to investigate a phenomenon previously unexplored in extant literature, namely the differential impact of foreign resource, ownership type and institutions on its performance in China, using newly available official data on 329 wholly owned subsidiary (WOS) and 351 Joint Venture (JV) European Union (EU) manufacturing subsidiaries drawn from the State Statistical Bureau of China over a 10year period (1998-2007). A conceptual framework is developed that integrates the Resource-based theory, Agency theory, and Institutional theory of international business strategy, reflecting EU firm FDI ownership strategic choices (between JV and WOS). This study contributes to filling this gap by analysing relationship between ownership structure and performance of marketing seeking EU subsidiaries in China. The results showed that asset turnover, asset tangibility, and EU JV local private ownership has a significant negative relationship with subsidiary performance whereas among China's state-owned enterprises (SOEs), collectives (COE) , legal entities ( LPs), private enterprises ( POE), Hong Kong, Macau and Taiwan-funded (HMT) firms, WOS and JV show an insignificant relationship with performance. These findings highlight the importance of further studying the "transactions problems" that control ownership structure, especially in emerging markets. The findings have important implications for practitioners and policymaking (For more information, please contact: Huifen Cai, University of Huddersfield, United Kingdom: h.cai16888@hotmail.com)

\author{
Does Competition Matter for Corporate Governance? The Role of Country Characteristics \\ Jean-Claude Cosset, HEC Montreal \\ Hyacinthe Some, HEC Montreal \\ Pascale Valery, HEC Montreal
}

We investigate the empirical relation between competition and corporate governance and the effect of country characteristics on this relation. We find that competition is associated with lower corporate governance, but only in developed countries. In developing countries, firms from competitive industries have, on average, higher corporate governance than firms from less competitive industries. We next examine the impact of corporate governance on firm value given the level of competition. We find that corporate governance is positively associated with greater firm value, but only in less competitive industries, and only for developed countries. For developing countries, the evidence suggests that competition is valuable mostly in competitive industries. (For more information, please contact: Jean-Claude Cosset, HEC Montreal, Canada: jean-claude.cosset@hec.ca)

\section{The Impact of Foreign Directors on Board Compensation in Emerging African IPO Firms Bruce Hearn, University of Sussex}

This paper examines the effectiveness of monitoring of foreign nonexecutive with three different types of professional affiliation: foreign corporate partners, foreign venture capitalists and finally those that are independent. These are further differentiated to account for the impact of socio-cognitive institutions arising from nonexecutives emanating from either common law or civil code law countries. Using a unique comprehensive hand-collected sample of 190 IPO firms from across the developing region of Africa we find evidence that nonexecutives affiliated to foreign corporate partner have no significant impact on either of two measures of board compensation, namely aggregate fixed base salary and total remuneration, in line with their 
role on a subsidiary board which is commonly remunerated with a management fee. However increasing proportions of civil code law nonexecutives affiliated to foreign venture capitalists is associated with higher board compensation indicating weaker monitoring and less effective inhibiting of director's self-reward. Finally foreign independent nonexecutives are associated with elevated board total remuneration although this is particularly significant with common law independent directors. The weakness of their monitoring is likely a function of geographical, cultural and psychic distances and underscores the limitations of foreign nonexecutives as effective monitors in a developing context. (For more information, please contact: Bruce Hearn, University of Sussex, United Kingdom: b.a.hearn@sussex.ac.uk)

\section{Are Family Firms More Internationally Involved than Non-Family Firms: The Case of Italy Jean-Francois Hennart, Singapore Management University and University of Pavia Antonio Majocchi, University of Pavia}

There is limited research on the international strategies of family firms. Some have argued that because family owners tend to be relatively more risk averse and restrict their management to family members, who may be internationally inexperienced, family firms should be relatively less internationally involved than firms with dispersed ownership. On the other hand, family firms are said to have the longer time horizon needed for the significant investments required to penetrate international markets. Empirical studies tend to find that family firms are less internationalized, but they have mostly focused on SMEs and have used a less-than-optimal measure of internationalization. We look at the foreign sales of a panel of 263 Italian firms listed on the Milan stock exchange. Using a better measure of internationalization, we find that Italian family-managed firms have higher foreign sales than non-family firms, but that this is not true when the family does not participate in management. The positive impact that family management has on foreign sales is higher for regions at greater psychic distance from Italy. (For more information, please contact: Jean-Francois Hennart, Singapore Management University and University of Pavia, Italy: j.f.hennart@uvt.nl)

\section{Session: 3.4.6 - Competitive}

\section{Track: Track: 9 - Cross-Cultural Management and HRM}

\section{Women and Career Advancement Across Contexts}

Presented On: July 6, $2013-14: 50-16: 05$

Chair: Jane Salk, University of Texas at Dallas

The Role of Law, Networks, and Mentors in Overcoming Barriers to Organizational Leadership for Women with Children: A Multi-Country Study

Terry Morehead Dworkin, Indiana University and Seattle University

Aarti Ramaswami, ESSEC

Cindy A. Schipani, University of Michigan

This study is part of a larger project on career pathways for women to obtain organizational leadership. Based on data from 743-846 professionals graduating from leading business schools around the world, we examined the three-way interaction of gender $x$ dependents $x$ mentoring on networking outcomes such as benefiting from networks and dealing with network related challenges. Results suggest that having a mentor allows women with dependents to benefit most from networks compared to other groups (women without dependents, and men). However, mentoring seems more beneficial for women without dependents than for women with dependents to overcome network related challenges. In contrast, for men with dependents, their network challenges are reduced when they have mentors compared to when they do not. Thus, while mentored women with dependents may have access to a beneficial network, they continue to face network related challenges, despite having a mentor. We discuss study implications along with recommendations for potential governmental interventions. (For more information, please contact: Aarti Ramaswami, ESSEC, France: ramaswami@essec.edu) 
Family, Career Patterns, and the Notions of Success in Traditional-Collectivist Cultural Contexts

Fida Afiouni, American University of Beirut

Charlotte M. Karam, American University of Beirut

This paper aims to explore the role of family in the notions of success as well as the career patterns of women in traditional-collectivist cultural contexts, namely in the Arab Middle East. Semi-structured interviews were conducted with a sample of 32 middle-to-upper level socio-economic status women drawn from eight Arab countries. Our findings suggest the importance of family in the conceptualizations of success and in shaping the career patterns of women in our sample. Subjective, relational and interdependent criteria occupied a central place in the discourse of women when defining and describing their success. Moreover, our findings suggest that: (1) a unique career pattern is often adopted by married women (early marriage, childbearing, higher education, then career); and (2) family plays an important role in supporting and helping these women balance their work and their lives. (For more information, please contact: Fida Afiouni, American University of Beirut, Lebanon: fa16@aub.edu.Ib)

The Impacts of Gender Role Perceptions and Global Mindsets on Attitudes Towards Women's Career Advancement in Latin America

William Newburry, Florida International University

Marcelo J. Alvarado-Vargas, Florida International University

Armando Borda, Esan University

Carlos Miguel Ortiz Baldo, U. Fermin Toro and U. Pablo de Olavide

Edwin Duran-Zurita, Universidad Privada Boliviana

Jose Mauricio Geleilate, Florida International University

Massiel Guerra, Universidad del Desarrollo

Michel Hermans, IAE Business School - Austral University

Maria Virginia Lasio Morello, ESPOL

Sergio Madero, Tec de Monterrey

Miguel Olivas-Lujan, Clarion University

Anne Marie Zwerg-Villegas, EAFIT

In emerging markets such as those in Latin America, rapid economic growth has created a situation where qualified labor is often in short supply. As competition for human capital intensifies, ensuring all qualified employees have access to career advancement opportunities is critical to company success. This study examines attitudes towards the career advancement of one historically marginalized group in Latin America, women. We suggest that employee gender role perceptions and global mindsets influence these attitudes, which we examine within a sample of 940 respondents within the Latin American countries of Argentina, Bolivia, Brazil, Chile, Dominican Republic, Ecuador, Mexico, Panama, Peru, and Venezuela. Our findings suggest that both perceptions of women's roles and possessing a global mindset influence attitudes towards the career advancement of women in Latin America. (For more information, please contact: William Newburry, Florida International University, USA: newburry@fiu.edu) 


\section{Session: 3.4.7 - Competitive}

\section{Track: Track: 3 - IB Theory, FDI, and Entry Mode}

\section{Institutions and Institutional Distance}

Presented On: July 6, $2013-14: 50-16: 05$

Chair: Agnieszka Chidlow, Manchester Metropolitan University

Consumer Animosity, Market Legitimacy, and Foreign Entry-Mode Choice: An Institutional Perspective Cher-Min Fong, National Sun Yat-Sen University

Chun-Ling Lee, National Sun Yat-Sen University

Yunzhou Du, Anhui University of Finance \& Economics

This study draws on the institutional perspective to investigate consumer acceptance to various entry modes [i.e., greenfield, acquisition, and international joint venture (IJV)] adopted by multinational corporations (MNC) from a home county evoking animosity in the host country. This is the first empirical study that regards animosity as an institutional environment and explores the market legitimacy (consumer acceptance) of different entry modes. This cross-country study examines Chinese (with high animosity against Japan) and Taiwanese (with low animosity against Japan) consumer acceptance toward various entry modes adopted by Japanese MNCs when entering and launching products in these 2 markets. This study employs an experimental methodology using consumer data. The results indicate that, in an animosity context, local identity and local ownership of entry modes have significant effects on host country consumer acceptance. Chinese consumers exhibit different levels of acceptance to various entry modes adopted by Japanese MNCs. However, Taiwanese consumers do not display a preference for a specific entry mode. (For more information, please contact: ChunLing Lee, National Sun Yat-Sen University, Taiwan: d934010005@student.nsysu.edu.tw)

\section{Firm-Specific Intangible Assets and Subsidiary Profitability: The Moderating Role of Distance Farok J. Contractor, Rutgers University Yong Yang, University of Sussex Ajai Gaur, Rutgers University}

We draw on the literature on internalization advantages, and information asymmetry to argue that the benefits from parent firm's intangible assets can be best realized when the level of information asymmetry is low. We propose a richer conceptualization of distance, which affects the extent of information asymmetry that MNCs face in foreign subsidiaries. These distance measures include differences in technological capacity, intellectual property regimes language, economic development and geographic distance between the home and host countries. We test our theoretical arguments on a sample of 5,169 foreign affiliates belonging to 2,018 MNCs from 47 home and host countries over a 12-year time period from 1996 to 2007 . Our findings suggest difference measures of distance have different effects on subsidiary profitability. (For more information, please contact: Ajai Gaur, Rutgers University, USA: ajai@business.rutgers.edu)

Institutional Distance, Institutional Development, Institutional Instability and FDI: The Role of the Ability to Manage Institutional Idiosyncrasies

Kent Hui, Michigan State University

Shige Makino, Chinese University of Hong Kong

Tomas Hult, Michigan State University

Using institutional theory lens, the present study looks into the location decision of multinational enterprises (MNEs). More specifically, the present study, drawing on the theory of the ability to manage institutional idiosyncrasies, constructs the boundary conditions of the impact of institutional distance on a foreign market 
entry. In addition, the present study attempts to address the unresolved problems of distance research caused by its unconvincing assumptions. The impact of institutional dynamics - institutional instability on MNEs' learning and capability development is also explored. (For more information, please contact: Kent Hui, Michigan State University, USA: hui@bus.msu.edu)

\section{The Institutional Origins of Firm Capabilities and Global Expansion \\ Victor Zitian Chen, University of North Carolina, Charlotte \\ Jing Li, Simon Fraser University \\ Daniel M. Shapiro, Simon Fraser University}

In this paper, we posit that the characteristics of a firm's institutional environment at a sub-national level determine both its capabilities and the orientation of its global expansion. In the context of emerging-market (EM) outward FDI into developed markets (DMs), we theorize that home institutions have a twofold impact on outward FDI: first, they have a direct effect, because institutional differences between home and host regions are reduced when market-supporting institutions are developed at home, thus encouraging EM firms to have greater confidence in gaining a legitimate entry into DMs. Second, they have an indirect effect through the mediation of market-related firm capabilities: local market-supporting institutions create the conditions that induce an EM firm to develop skills in technology, branding and marketing, and management, which in turn enable the EM firm to expand into DMs. Additionally, home institutions in large EMs, such as China, vary significantly at sub-national levels. Consequently, firms from different regions of the same country may possess different capabilities and foreign expansion propensities. Using a firm-level survey of 553 Chinese firms headquartered in 68 different cities; measures of home institutional quality at the city level, we find very supportive empirical results for the arguments outlined. (For more information, please contact: Victor Zitian Chen, University of North Carolina, Charlotte, USA: emgp.editor@gmail.com)

\section{Session: 3.4.8 - Competitive}

\section{Track: Track: 1 - Institutions, Governance, and CSR}

\section{Multinational and Legal Institutions}

Presented On: July 6, $2013-14: 50-16: 05$

Chair: Michael-Jörg Oesterle, University of Stuttgart

The Disclosure Practices of Foreign Corporations in Australia: An Empirical Investigation

Zahid Riaz, University of Central Punjab

Sangeeta Ray, University of Sydney

Vikas Kumar, University of Sydney

Pradeep Kanta Ray, University of New South Wales

Firms are disadvantaged when they operate in foreign markets. Multinational corporations (MNCs) mitigate foreignness disadvantages by imitating domestic firms. This research examines whether MNC-subsidiaries are embedded into the local institutional context and the extent to which these subsidiaries respond to local laws and standards of disclosure of director and executive remuneration in host country. The empirical analysis examined the hypothesis that globalized firms are less likely to respond to increased disclosure requirements needed to address a higher level of information asymmetry. The analysis of disclosure level of MNC-subsidiaries vis-à-vis domestic firms suggests that, other things being equal, MNCs are less responsive to increased disclosure requirements than their local counterparts in Australia unless they have substantial interactions with domestic product-markets. These results demonstrate that MNCs are willing to incur the marginal cost of increasing disclosure if the benefits of increasing disclosure level justify it: that is, a clear presence of demand for information from product-markets. Another interesting finding relates to the negative association between 
legal system type (common law) of MNC's parent country and disclosure level of director and executive remuneration. (For more information, please contact: Pradeep Kanta Ray, University of New South Wales, Australia: pray@unsw.edu.au)

Timing Is Everything: Political Action and the Value of Experience over the Policy Life Cycle: The Case of Indian Anti-Dumping Law

Susan Elizabeth Feinberg, Temple University

Catherine Magelssen, Rutgers University

Matthew Grady Smith, United States Department of the Treasury

A significant component of firms' corporate political strategy formulation involves decisions about when to take action. We argue that the costs and benefits of taking political action vary over the life cycle of a policy. As an issue evolves from its earliest stages through to eventual legislative passage and rulemaking by administrative agencies, firms face different kinds of costs. We argue that different types of firm experience will lower the costs of taking political action at different times in the policy life cycle. We show that firms with greater experience dealing with administrative agencies are most likely to take political action at a time when agencies have the greatest influence on the outcomes of public policy, but these firms are less likely to take action at other times. We examine the timing of political action decisions in the context of Indian Antidumping law, which evolved from the signing of the WTO Agreement in 1994 through to domestic legislative changes and the eventual creation of a new agency to adjudicate antidumping claims. The first Anti-dumping case was filed in India in the early 1990s, during a period of steep IMF-imposed tariff reductions. (For more information, please contact: Susan Elizabeth Feinberg, Temple University, USA: feinberg@temple.edu)

What is my Neighbor Up To? The Effect of Local Density on the Diffusion of Certifiable Corporate Social Responsibility Practices among Multinational Enterprises and Domestic Firms

Bryan Husted, York University \& Tecnologico de Monterrey

Ivan Montiel, Loyola Marymount University

Petra Christmann, Rutgers University

This study adds new insights to the field of institutional theory and mimetic isomorphism by analyzing the impact of local density on the diffusion of certifiable corporate social responsibility (CSR) practices among both multinational enterprises and domestic firms In addition, the study analyzes the impact of unexpected adoptions on the diffusion of such practices. To study these effects, we use the case of certifiable CSR practices in Mexico and find substantial support for the effects of local density, multinationality, and unexpected adoption on ensuing adoptions. (For more information, please contact: Ivan Montiel, Loyola Marymount University, USA: ivanmontiel@gmail.com)

\section{Session: 3.4.9 - Competitive \\ Track: Track: 8 - Developing Country MNCs}

\section{Capability Learning and the External Environment of Developing Economy MNEs}

Presented On: July 6, $2013-14: 50-16: 05$

Chair: Maria Tereza Fleury, Fundacao Getulio Vargas

Upgrading Technological Capabilities by Developing Country Multinational Companies

Huaichuan Rui, University of London

Alvaro Cuervo-Cazurra, Northeastern University 
We study the process by which developing country multinationals (DMNCs) upgrade their technological capabilities to international levels. The case study of the Chinese telecommunication equipment manufacturer Huawei supports three conclusions. First, despite some views that DMNCs are technological laggards, we show that DMNCs can reach levels of technological sophistication of advanced economies in a compressed manner. Second, the way DMNCs improve their technological capabilities is by raising the mode of technology upgrading, moving across reengineering, replacing, refining, and recreating capabilities to reach the technological frontier. Third, managers at DMNCs raise the mode of technological upgrading by the continuous stretching of the skills and knowledge of engineers. These ideas extend the study of dynamism in capability life cycles and the creation of dynamic capabilities generated from studying 'Western' firms operating in countries with sophisticated innovation systems by explaining the development of technology in countries with weak innovation systems. (For more information, please contact: Alvaro Cuervo-Cazurra, Northeastern University, USA: a.cuervocazurra@neu.edu)

\section{Knowledge Will Set You Free: Enhancing the Firm's Responsiveness to Institutional Change Luis Alfonso Dau, Northeastern University}

This paper combines notions from the POST Model of Economic Geography and Learning Theory from International Business to study how firms may enhance their responsiveness to institutional processes and changes through different forms of international learning. Focusing on one form of institutional changes, namely pro-market reforms, we analyze how firms may boost the potential benefits from such changes through international strategies that increase their access to knowledge spillovers and absorptive capacity. These strategies include internationalizing through joint ventures, acquisitions, subsidiary network control, industry diversification, enhancing innovation capabilities, subsidiary network centralization, institutional exposure, and extant experiential knowledge. (For more information, please contact: Luis Alfonso Dau, Northeastern University, USA: I.dau@neu.edu)

\section{Managing Channels by Foreign Sales Subsidiaries: The Case of Firms from Developing Countries Operating in Developed Countries \\ Hsiao Wen Liu, National Chengchi University \\ Chow Ming Yu, National Chengchi University \\ Hui Yun Chiu, National Chengchi University}

Many firms from developing countries (LDCs) have engaged in foreign direct investment (FDI). Interestingly, some firms locate their investments in developed countries (DCs) (i.e., upstream FDI), instead of in countries economically similar to or less than their home countries (i.e., downstream FDI). However, only a few researchers have examined issues related to upstream FDI. Furthermore, when examining FDI, most studies have focused on manufacturing subsidiaries instead of sales subsidiaries. Using a case study approach by focusing on the behaviors of Taiwanese firms, we address two research questions: (1) what are the channel strategies adopted by sales subsidiaries of Taiwanese high-tech firms (i.e., LDCMNCs) in DCs? , and (2) how do these subsidiaries manage their channels in DCs? Our findings show that: (1) LDCMNCs tend to use multiple sales channels, to work with large national distributors, and to adopt high touch channels to market products; (2) to reduce channel conflict, less powerful LDCMNCs adopt multiple independent channel systems; and (3) due to limited resources, LDCMNCs try to design channel conflict prevention mechanisms, emphasize more on building distributors-relationships and use financial/high-power incentives to motivate distributors. (For more information, please contact: Hui Yun Chiu, National Chengchi University, Taiwan: 99355503@nccu.edu.tw)

Multi-dimensional Complementarities and the Growth Impact of Direct Investment from China on Host Countries Xiaolan Fu, Oxford University 
This paper examines the growth impact of developing country outward direct investment (OFDI) on the economic growth in host developing countries using a cross country panel dataset of Chinese OFDI over the 2003-2010 period. It finds that the growth effect of Chinese OFDI is determined by the multi-dimensional complementarities between the FDI flow and the host economies. Overall Chinese OFDI appears to have a positive and significant impact on their long-run economic growth in host economies despite a negative association with short-run growth as Chinese OFDI seeks a variety of objectives in addition to a high growth market. Chinese OFDI appears to have contributed positively to the economic growth not only in Africa but also in Europe and North America. However, its contribution in Asia and Latin America is insignificant. Although the illustration of Chinese OFDI being mainly resource-seeking is exaggerating and misleading, Chinese OFDI in resource-rich country cannot avoid the resource curse either. (For more information, please contact: Xiaolan Fu, Oxford University, United Kingdom: xiaolan.fu@qeh.ox.ac.uk)

\section{Session: 3.4.10 - Competitive}

Track: Track: 7 - Emerging Economies

\section{Network and Social Capital in Emerging Markets}

Presented On: July 6, $2013-14: 50-16: 05$

Chair: Qian Gu, Georgia State University

\section{How Do Managerial Ties Matter in Export Performance? The Contingency Role of Immigrant Effect and Institutional Environment}

Henry F. L. Chung, Massey University

Rosalie L. Tung, Simon Fraser University

Forrest Zhilin Yang, City University of Hong Kong

This study intends to address how and when managerial ties play a key role in improving export performance. We first decompose managerial ties into channel ties (CT) and non-channel ties (NCT). We then introduce the essential moderators: immigrant effect and institutional environment and examine their moderating effects of on the relationship between managerial ties and export performance. Based on the experiences of 245

Australasian firms exporting to Greater China (China, Taiwan and Hong Kong), we find that CT and NCT have a significant effect on export performance. Immigrant effect, both formal and informal institutional environments play a contingency role. Both immigrant effect and formal institutional environment positively moderate the effect of NCT on export performance while immigrant effect negatively moderates the impact of CT on export performance. We discuss these findings' implications for advancing the development of organizational networking, resource-based theory, and institutional theory. (For more information, please contact: Henry F. L. Chung, Massey University, New Zealand: h.chung@massey.ac.nz)

\section{Social Capital and Geographic SME Growth: An Emerging Market Perspective Natalya Totskaya, Concordia University}

Does a firm's stock of social capital influence the geographic scope of its activities? This study investigates the role played by bonding and bridging social capital of small and medium enterprises (SMEs) operating in Siberian region of Russia in making choices related to geographic growth strategies. The differences in social capital SMEs pursuing domestic and international growth are also tested. This study provides more insights on the knowledge of SME growth in the specific context of emerging markets. It emphasizes the role of horizontal bridging ties in SME development. This study adds support for prior research on the importance of environmental factors for the process of firm growth. The implications of this study include recommendations for SMEs on creating and maintaining their social capital to better assist in SMEs' geographic expansion. (For more information, please contact: Natalya Totskaya, Concordia University, Canada:

n_totska@jmsb.concordia.ca) 
Handling Pressures of Community Logic: The Impact of Clan Ties on Recruitment and Selection in Kazakhstan

Dana Minbaeva, Copenhagen Business School

Jasper Hotho, Copenhagen Business School

Maral Muratbekova-Touron, ESCP Paris

Larissa Rabbiosi, Copenhagen Business School

The paper aims at investigating how in pluralistic societies, such as emerging economies and countries in transition, organizational decision-makers respond to pressures of community logics in non-community settings, such as the work place. We theorize that in non-community settings, social relations and interactions with community members can act as social cues that induce and expose individuals to community logics. We subsequently propose that properties of these relations - immediacy and relatedness - will affect individual response strategies towards community logics. We test these ideas with an experimental vignette study of the effects of clan and kinship ties on recruitment and selection decisions in Kazakhstan, followed by qualitative interviews. (For more information, please contact: Dana Minbaeva, Copenhagen Business School, Denmark: dm.smg@cbs.dk)

\section{An Insideration Perspective on Relative Mode Advantage in Local Market Penetration Changhui Zhou, Peking University \\ Helen Liu, Industrial and Commercial Bank of China}

In this paper, we focus on foreign subsidiaries whose primary goal is to penetrate local market in the host country and examine whether and on what conditions international joint ventures (IJVs) and wholly-owned foreign subsidiary (WOFs) differ. We adopt a strategic insiders perspective (Luo, 2007) and suggest a notion of relative mode advantage. The insideration is an iterative, learning process, in which advantage of entry mode may exhibit relativity. That is, whether IJVs or WOFs has better ability to penetrate local market depends on how subsidiary and environmental conditions affect subsidiary learning and legitimacy. We test this idea by using a sample of 4531 foreign subsidiaries in China during 1998-2002. Results show that firstly, IJVs and WOFs do not differ significantly in terms of local market penetration; and secondly, the subsidiary conditions (i.e. subsidiary innovation and experience) and environmental conditions (i.e. FDI presence and institutional regime) significantly moderate the mode advantage in local market penetration. (For more information, please contact: Changhui Zhou, Peking University, China: czhou@gsm.pku.edu.cn)

\section{Session: 3.4.11 - Interactive}

\section{Track: Track: 6 - Innovation and Knowledge Mgmt.}

\section{Internationalization Process and Knowledge}

Presented On: July 6, $2013-14: 50-16: 05$

Chair: Sunil Venaik, University of Queensland

\section{Innovation Networks in Peripheral Economies}

Marcelo Cano Kollmann, Temple University

Ana Teresa Tavares-Lehmann, University of Porto

This paper explores a topic that has not received much attention in the literature - the global connectivity of peripheral economy innovation systems. Innovation networks encompass two different levels of analysis: the organizational level (e.g. firms and research organizations) and the individual level (i.e. inventors). Exploring the global connections of both firms and individual inventors based in Portugal, we disentangle both levels and challenge the assumption that innovation networks at the level of the organization and at the level of the individual have similar properties. Our results suggest that there are fundamental differences between these 
two networks, at least for peripheral economies. At the organizational level, we found that subsidiaries with parents in highly innovative core economies may provide the local economy with limited connectivity benefits. Conversely, at the personal level, innovative interactions with inventors based in highly innovative core economies may provide the local economy with substantial connectivity benefits to global innovation networks. We believe these insights may generalize to other peripheral contexts, and may shed light on the little known topic of how firms from peripheral economies source their knowledge and what are the collaborative patterns of local inventors. (For more information, please contact: Marcelo Cano Kollmann, Temple University, USA: mck@temple.edu)

\section{Complementarity or Damage of Structural Holes for Social Capital on Knowledge Creation Process Chih-Hsing Sam Liu, Ming Chuan University Ben Nanfeng Luo, University of New South Wales Bernard Gan, University of New South Wales}

Social capital has gained recent popularity as a means for established individuals to learn about new knowledge and enhance knowledge creation. However, the link between such social capital and the impact of network position on the knowledge creation process has not been examined in detail. Using panel data from scholars in academic fields, this paper investigated the conditions under which maintenance of social relations affects knowledge creation. Two regression models were used to test the hypotheses in a sample of 427 individuals and 3,356 observations of knowledge creation samples. The results showed that the number of social relations has an inverted U-shaped relationship with knowledge creation performance. Moreover, the moderating role of structural holes is recognized: it positively moderates with social relations and knowledge creation quality and negatively moderates with social relations and knowledge creation quantity. Managerial implications and future research directions are discussed. (For more information, please contact: Chih-Hsing Sam Liu, Ming Chuan University, Taiwan: phd20110909@gmail.com)

\section{Impact of Korean Market Reforms and Firm Heterogeneity on Innovation Investments \\ KwangWook Gang, Rensselaer Polytechnic Institute Jaya Dixit, Rensselaer Polytechnic Institute \\ Murali D.R. Chari, Rensselaer Polytechnic Institute}

A variety of countries have instituted pro-market reforms in order to develop their economies in the footsteps of the more developed countries. These institutional changes significantly influence firms' investment behavior. This paper investigates how market oriented reforms influence firms' incentives to innovate. We utilize market reforms in Korea after the Asian Financial Crisis as an empirical setting to show why institution based view is crucial to our understanding of factors that incentivize firms to innovate. Additionally, we draw on competitive strategy literature in investigating how market reforms combined with industry competition and resource attributes of the firms influence investments in innovation. Our findings suggest that market reforms positively influence R\&D investments. In addition, industry competition and a competitive resource portfolio positively influence innovation investments as reforms unfold. (For more information, please contact: KwangWook Gang, Rensselaer Polytechnic Institute, USA: gangk@rpi.edu)

\section{Co-evolution of Disseminative and Absorptive Capacities in HQ-Subsidiary Knowledge Flows: An Exploratory Case Study \\ Ieva Martinkenaite, BI Norwegian Business School \\ Randi Lunnan, Norwegian Business School}

In this paper we explore HQ-subsidiary knowledge flows in transition economies. Our case is a multinational company that has entered the Baltic States through market acquisitions. We focus on sender-receiver interaction in the knowledge transfer process between parent firm HQs and local subsidiary. Particular emphasis 
is placed on the dynamic interplay between their disseminative and absorptive capacities. We distinguish between motivation, ability and opportunity to 'teach' and 'learn' and explain co-evolutionary dynamics of these two organizational learning capabilities. Our longitudinal case study data shows that disseminative capacity of the parent firm and absorptive capacity of the subsidiary co-evolve through transfer "push" and absorptive "pull" processes in response to changing external (firm-specific) and internal (relationship-specific) opportunities. With this study we advance our understanding of joint learning in MNCs as opposed to absorptive learning of the recipient that is dominating current research on intra-MNC knowledge flows. (For more information, please contact: Ieva Martinkenaite, BI Norwegian Business School, Norway: ieva.martinkenaitepujanauskiene@bi.no)

\section{Management Knowledge Creation Process through Social Relationship Extension: The Multi-Stage Hypothesis Chih-Hsing Sam Liu, Ming Chuan University Wei-Long Lee, National Kaohsiung University of Applied Science Sheng-Fang Chou, National Taiwan Normal University Bernard Gan, University of New South Wales}

A proposed theoretical framework for the study of social relationships and knowledge creation includes both benefits and costs of social relations expansion over different phases of social capital accumulation and extension. In a sample of research hypothesis tests, the results strongly supported predictions of a horizontal, inversed S-shaped relationship between social capital and knowledge creation. Furthermore, individuals accumulating experience would achieve greater advantage from gaining in maintaining the social relations and improving the quality of knowledge. Further additional test robustness the findings. The novelty framework and findings highlight the complexity and temporal dynamics multi-countries social activities in academic field. (For more information, please contact: Chih-Hsing Sam Liu, Ming Chuan University, Taiwan:

phd20110909@gmail.com)

The Impact of International Knowledge Diversity on Innovation Quality Jan Hohberger, University of Technology, Sydney

Paul Almeida, Georgetown University

The opportunity to source knowledge from multiple locations and use it in innovative activities is one of the advantages of multinationality. This would suggest that using diverse international knowledge is important to innovation but existing empirical research gives us only a limited picture of this. Analyzing a sample of 35,000 patents from 346 US biotechnologies firms, we directly measure the extent of international knowledge diversity and relate it to quality of the associated innovation. Our results show that having internationally diverse knowledge inputs has a curvilinear (inverted- $U$ ) relationship to innovation quality. While having inventors from a diverse set of international locations does not directly impact quality, when knowledge is drawn from international locations where inventors reside, innovation quality increases. Additionally, the international diversity of prior co-inventor networks has a curvilinear (inverted-U) relationship with innovation quality. Thus, we develop a more complete picture of the effect of the international diversity of knowledge on innovativeness. (For more information, please contact: Jan Hohberger, University of Technology, Sydney, Australia: jan.hohberger@uts.edu.au)

Does Co-Location Accelerate Knowledge Outflows from FDI? The Role of MMC Subsidiaries' Technology Sourcing Strategies

Alessandra Perri, Ca' Foscari University of Venice

Raffaele Oriani, Luiss Guido Carli University of Rome

Francesco Rullani, Luiss Guido Carli University of Rome 
Despite the strategic importance of the knowledge outflows from FDI for local firms' competitiveness, no study has focused on the speed at which this phenomenon takes place. However, this issue is crucial since the speed at which firms absorb external knowledge influences the time they need to carry out subsequent innovations, their ability to adapt to external changes and enter new markets, thus ultimately affecting their chances to achieve a competitive advantage. This paper tries to fill this gap, by investigating the temporal patterns of knowledge outflows between foreign subsidiaries and firms located in host-regions. Combining International Business literature with insights on Innovation Strategy, we provide evidence on the timing of this phenomenon, and discuss the role played by multinational firms' technology sourcing strategies. (For more information, please contact: Alessandra Perri, Ca' Foscari University of Venice, Italy: alessandra.perri@unive.it)

\section{How is Knowledge Created and Shared in the MNE Network? Jongmin Lee, Yonsei University}

With the recent rise of the importance of foreign subsidiaries' roles in shaping the overall MNE strategy, many scholars perceive the foreign subsidiaries as sources of innovation. Naturally the headquarters' role of managing knowledge dispersed throughout the MNE network draws huge attentions. However, not many studies have examined the process of knowledge creation and sharing within the MNE network. This paper attempts to present an insight about how knowledge is created and shared within the MNE network, arguing that the process of knowledge creation and sharing in the MNE network constitutes an evolutionary cycle. The suggestions about future research are addressed as well. (For more information, please contact: Jongmin Lee, Yonsei University, Korea, South: jongmin731@hotmail.com)

\section{Session: 3.4.12 - Interactive \\ Track: Track: 1 - Institutions, Governance, and CSR}

\section{Effects of Country Environments}

Presented On: July 6, 2013 - 14:50-16:05

Chair: Jay Wu, Thammasat University

The Disciplinary Effect of Selling to Countries with Higher Levels of Press Freedom Estefania Amer Maistriau, University of Geneva

In countries where the press is free, firms are exposed to scrutiny by consumers, reporters, activists and other civil society members. In order to attract ethically-oriented consumers and avoid consumer awareness campaigns and boycotts, firms selling in these countries should have more incentives to exhibit high levels of CSR performance. Therefore, entering foreign markets with higher levels of press freedom than the home market should have a disciplinary effect on firms, leading to an increase in the level of CSR performance. According to the data, selling in China and the United States should lead a Chinese firm to have an environmental score, whose values are between 0 and 100, higher by 33 than if it was selling exclusively to China. The score in the society dimension of CSR and the probability of being ready to end a partnership with a supplier responsible for a human rights breach would also be higher. This disciplinary effect is absent as far as working conditions are concerned. Finally, I find that firm size and CSR performance are positively correlated, which is consistent with the fact that larger firms are more visible and, as a result, they are more exposed to scrutiny. (For more information, please contact: Estefania Amer Maistriau, University of Geneva, Switzerland: estefania.amer@unige.ch) 


\author{
Firm Transitioning during Environmental Transition: The Case of Promarket Reform in Eastern Europe \\ Vladislav Maksimov, University of Miami \\ Yadong Luo, University of Miami \\ Stephanie Wang, University of Miami
}

We advance an organization ecology model to examine the type and strength of forces influencing transition at the organizational level during periods of environmental transition. First, we examine the effects of inherited forces deriving from the imprinting influence of founding conditions, as well as their persistence. Second, we explore the effects of proactive forces manifested by firm initiatives to adapt to new conditions, as well as their conditionality. In a sample of 747 firms from six transition economies in Eastern Europe, we find that private ownership and large firm size at the founding promote domestic firm competitiveness during the period of transition, although the effect diminishes as firms operate longer under free market conditions. We also find that firm initiatives to upgrade brainpower and improve information flow efficiency bolster domestic firms' competitiveness, especially at low levels of market competition. Interestingly, the influence of the two sets of conditions is of relatively similar magnitude, suggesting that firm transitioning during periods of environmental transition depends equally on forces inherited from past environments and on forces purposefully employed by firms to adapt to present environments. (For more information, please contact: Vladislav Maksimov, University of Miami, USA: vmaksimov@bus.miami.edu)

\title{
The National Entrepreneurship Infrastructure: Can Nation-State Policies Accelerate the Transition from Wage Earner to Business Creator? \\ David Lanier Major, Indiana University
}

This cross-national study investigates the factors that encourage the development of homegrown businesses in nation-states around the world. Local factors contributing both to the recognition of entrepreneurial opportunities and the abundance of those opportunities together constitute the nation's entrepreneurial infrastructure. The condition of this infrastructure forecasts the nation's success at encouraging the conversion of wage workers to business founders. Extant research has already shown that urban policies play some role in constructing supportive infrastructures for business creation. This study begins to contribute to an understanding of the catalysts of entrepreneurship and to challenge some ideals of "limited government" by examining the relationship between national policy factors and the rate of domestic business creation. The proposed methodology includes both statistical and qualitative examination using components of established research methods. Ultimately, the final results of the study should yield policy prescriptions that leverage factors of the national entrepreneurial infrastructure to encourage local business creation. (For more information, please contact: David Lanier Major, Indiana University, USA: dImajor@indiana.edu)

\section{Legal Origins, Democracy, and Environmental Technology Innovation: A Cross-Country Examination W.G. Douglas Fernandez, Florida International University Jorge E. Rivera, George Washington University}

Environmental technology innovation has long been characterized as offering the best prospect for responding to environmental needs with minimal impact on business. Yet despite this potential "win-win" for businesses, the production of this innovation is far from even across countries. In order to shed light on the drivers of ET innovation, researchers have examined a variety of policy instruments. However, evidence regarding the effectiveness of specific policy instruments in stimulating ET innovation has been inconclusive. In this study, we propose that missing from this emerging body of policy literature has been sufficient attention to the broad legal and political institutional contexts from which ET innovation may also emanate. Specifically, we argue that the legal origins of a country affect its orientation toward ET innovation activity. Further, we argue that the level of democracy should positively moderate these relationships. Results from regression analyses performed using 1,276 wind energy patents provide support for our overarching theoretical arguments. (For more information, please contact: W.G. Douglas Fernandez, Florida International University, USA: wdoug002@fiu.edu) 
Delicate Diplomacy: Lessons from Libya about Balancing Political and Social Legitimacy

Izzet Sidki Darendeli, Temple University

T.L. Hill, Temple University

This paper broadens the literature on the political activities of MNEs by exploring whether and how strategies aiming non political actors complement political legitimacy strategies in rapidly changing socio-political environments. We use experience of multinational Turkish construction firms during the recent regime change in Libya as a natural experiment to explore the effectiveness of various kinds of political activities in times of transition. We find that MNEs need to assess the legitimacy of the government that they are operating in both before deciding upon their interactions with the government and also in choosing the strategies to complement their initial interactions decisions. Our analysis of the fates of these MNEs showed that while close ties to the Gaddafi regime were necessary to do business before the transitions, firms that invested in symbolic and actual relationships with non-state actors proved much more resilient than those that did not. These finding contributes a new variable to extant political strategy theory, suggest tactics for effective social strategy, and add to the recent discussion about how emerging country MNEs gain advantage in operating in risky and corrupt contexts. (For more information, please contact: Izzet Sidki Darendeli, Temple University, USA: izzetdarendeli@temple.edu)

\section{FDI and Public Opinion: Reaction toward Asian Inflows \\ Aljaz Kuncic, University of Ljubljana \\ Andreja Jaklic, University of Ljubljana}

This paper examines the role of formal and informal institutions in FDI dynamics. We examine the effects of public opinion (proxied with a summary index on attitudes on liberalization issues from the World Values Survey and European Values Survey) and institutional distance (in legal, political and economic institutions based on new database) on bilateral FDI inflows in OECD countries using a gravity specification for the period from 1990 to 2009. Reactions of FDI to public opinion is tested by looking into the interaction between the formal institutions and public pressure (informal institutions) and examined by the specifics of public opinion effect conditioned on FDI coming from emerging (and Asian) markets. Findings show that (1) the share of nonliberal population in the studied period is rising, (ii) that nonliberal attitude significantly reduce inward FDI and (iii) there are no identified differences in terms of the attitude toward emerging (Asian) markets inflows. Results are relevant for enterprises' investment strategies, consultancies influencing public opinion as well as for policy makers and governmental agencies involved in investment promotion programs. (For more information, please contact: Andreja Jaklic, University of Ljubljana, Slovenia: andreja.jaklic@fdv.uni-lj.si)

\section{Session: 3.4.13 - Interactive \\ Track: Track: 9 - Cross-Cultural Management and HRM}

\section{How to Achieve Successful Face-to-Face and Virtual Cross-Cultural Interactions}

Presented On: July 6, 2013 - 14:50-16:05

Chair: Nancy R. Buchan, University of South Carolina

When an Intercultural Business Negotiation Fails: Comparing the Emotions and Behavioral Tendencies of Individualistic and Collectivistic Negotiators

Rajesh Kumar, Nottingham University

Harri T. Luomala, University of Vaasa

J.D. Singh, International Management Institute, New Delhi 
The study explores the linkages between culture, emotions and behavioural intentions in unsuccessful intercultural business negotiations. The study tests the prediction that negotiators from individualistic cultures (Finland) will predominantly experience dejection-related emotions while negotiators from collectivistic cultures (India) will predominantly experience agitation-related emotions in an unsuccessful business negotiation. The study also examines if collectivistic negotiators demonstrate greater empathy compared to their individualistic counterparts. Thirdly, the idea that dejection and agitation lead to different behavioural tendencies in individualistic and collectivistic cultures is scrutinised. The paper concludes by outlining a set of novel theoretical and managerial implications. (For more information, please contact: Rajesh Kumar, Nottingham University, United Kingdom: rajesh.kumar@nottingham.ac.uk)

\author{
Joerg Dietz, University of Lausanne \\ Zeynep Aycan, Koc University \\ Stacey R. Fitzsimmons, Western Michigan University \\ Anne Marie Francesco, Hong Kong Baptist University \\ Karsten Jonsen, IMD \\ Hyun-Jung Lee, London School of Economics \\ Sonja A. Sackmann, University Bw Munich \\ Guenter K. Stahl, WU Vienna and INSEAD \\ Joyce Osland, San Jose State University
}

Designing a Relevant Cross-Cultural Management Course: $A$ View through the Lens of Scientific Mindfulness

Literature review and interviews with CCM instructors and former course participants who are now expatriates indicate that CCM courses could be made more relevant. To address this need, we advocate a new type of CCM course designed through the lens of scientific mindfulness, a holistic, cross-disciplinary, contextual, and reflexive approach to scholarship and practice with the intent to contribute to the betterment of society. In this essay, we discuss the course content and evaluation of a scientifically mindful course that recognizes that today's corporations are not purely economic but also social and political actors with a responsibility for global sustainability. (For more information, please contact: Anne Marie Francesco, Hong Kong Baptist University, Hong Kong, SAR-PRC: amfrancesco@yahoo.com)

\title{
Negotiating and Spanning Boundaries in Offshore Outsourcing: Indian and Danish Perceptions of Intercultural Collaboration
}

Anne-Marie Soederberg, Copenhagen Business School

Tine Sonne, Copenhagen Business School

Thomas Toeth, Copenhagen Business School

Much research of geographically distributed teams has taken its point of departure in Western MNCs and addressed headquarter concerns with managing employees at distant locations. In this single case study we look deeper into how vendor staff members in a virtual captive center perceive intercultural collaboration with managers and staff at the outsourcing client's site. We also raise the question: Who and what motivates them to stay at work in this Indian IT development context where many Western clients have experienced serious challenges with outsourcing due to high attrition rates? The single case is studied through company documents, semi-structured interviews with managers and employees from both the client and the vendor organizations, and participant observations of intercultural encounters. Since observations and interview accounts gave the impression that virtual team collaboration went fairly smooth, we further explored the specific conditions and organizational practices that seem to have fostered benefits: information and communication technologies, culture guidelines, and not least managers and staff who acts as boundary spanners and establishes common ground. Finally we highlight the power struggles between client and vendor about HR issues related to the chosen organizational setup of a virtual captive center. (For more information, please contact: Anne-Marie Soederberg, Copenhagen Business School, Denmark: ams.ikl@cbs.dk) 
Composing Teams to Optimize the Benefits of International Experience Diversity

Peder Greve, University of St. Gallen

Martin Engeler, University of St. Gallen

Winfried Ruigrok, University of St. Gallen

This paper contributes to a better understanding of the effects of experiential diversity in teams. Specifically, the link between diversity in team members' international experience backgrounds and team performance is examined from an information processing perspective. This paper addresses the conditions under which teams optimize the benefits of a greater pool of knowledge and perspectives at their disposal. A set of four hypotheses is developed and tested on a longitudinal dataset drawn from professional soccer teams in the German Bundesliga over a seven-year period. Findings show that a diverse international experience base is positively associated with team performance. This relationship is positively moderated by indicators of experiential team composition and managerial tenure. Specifically, performance gains of increasing international experience diversity are larger the more a team consists of members experienced in high quality settings, a team consists of members with narrow individual experience backgrounds, and a team is led by a longer tenured coach. Theoretical and practical implications of these findings are discussed together with future research directions. (For more information, please contact: Peder Greve, University of St. Gallen, Switzerland: pedermathias.greve@unisg.ch)

\section{Do Cross-Cultural Management Courses Affect Cultural Intelligence? It Depends on Students' Prior International Experience \\ Jacob Eisenberg, University College Dublin \\ Hyun-Jung Lee, London School of Economics \\ Barbara Brenner, Danube University \\ Frank Bruck, Bocconi University}

The rapid increase in cross-cultural management courses, brought about by economies' globalization and increased workforce mobility motivated us to examine the impact of such courses on cultural intelligence. Cultural Intelligence (CQ) refers to individual's abilities and skills to effectively manage interactions in crosscultural situations and, as such, is a crucial competence for successful international managers. In particular, we investigate how management students' prior international experience (living abroad for at least six months) affects their CQ before (time 1) and after (time 2) taking cross-cultural management courses (CCM). We use two different samples to test our hypotheses. Our results show that in time 1 prior international experience (IE) has a positive and significant effect on CQ and on all its sub-dimensions in both of our samples. In time 2 the positive correlations between international experience and CQ cease to be significant or greatly decrease in magnitude. We found that CCM courses have 'equalizing' effects on students since they affect more positively and strongly the CQ of low IE students than those with high IE. Lastly, we found that these CQ gains after the CCM course are more pronounced for the metacognitive and cognitive dimensions than for motivational and behavioral CQ. (For more information, please contact: Jacob Eisenberg, University College Dublin, Ireland: jacob.eisenberg@ucd.ie)

\section{Navigating New Horizons in Higher Education: Design-Inspired Leadership for 21st Century Universities} Fahri Karakas, University of East Anglia Mark Fenton-O'Creevy, Open University

This paper proposes that design-inspired leadership can be used to unleash creativity, lead innovation, and create effective change strategies; enabling higher educational institutions to survive and prosper among global competition. Design-inspired leadership is an exciting emerging paradigm at the intersection of leadership, creativity, design thinking, and innovation. The paper considers how educational leaders can use design-inspired 
leadership as an integral component of strategic innovation at the institutional level. (For more information, please contact: Fahri Karakas, University of East Anglia, United Kingdom: fahrikarakas@gmail.com)

\author{
International Work Environment Diversity and Emotional Labor \\ Watcharaphong Leartsurawat, Florida International University
}

This study examines the consequences of international services firms' expanded and complexes international work environments on their service employees' levels of emotional labor. Building upon the internationalization literatures, diversity and culture difference and the emotional labor concept, a proposed integrative conceptual framework is represented. The author articulates that service employees in firms with higher level of international work environment diversity will stumble upon higher level of emotional labor. The author then portrays a series of propositions as well as potential further opportunity for empirical research method and deduces with research discussion and conclusion. (For more information, please contact: Watcharaphong Leartsurawat, Florida International University, USA: wlear001@fiu.edu)

\title{
Session: 3.4.14 - Interactive \\ Track: Track: 3 - IB Theory, FDI, and Entry Mode \\ Entry Mode \& Ownership Strategy
}

Presented On: July 6, 2013 - 14:50-16:05

Chair: Paul W. Beamish, University of Western Ontario

Host Country Uncertainty and Level of Control in Cross-Border Acquisitions: A Comparison of Latin American and U.S. Multinationals

Shavin Malhotra, Ryerson University

Xiaohua Lin, Ryerson University

Carlyle Farrell, Ryerson University

This paper examines the impact of host country uncertainty on the level of control negotiated by Latin American and US multinational corporations (MNCs) in cross border acquisitions (CBAs). Our hypotheses are derived from transaction cost economics and are tested using a sample of 15,000 CBAs made by Latin American and U.S. MNCs over the period 2000 to 2008. Using Logit and Tobit regression models, we test the impact of three measures of host country uncertainty, i.e. cultural, geographic and institutional distance, on the level of control negotiated. Level of control is measured by share of equity. The study finds that MNCs' propensity to use shared as opposed to full ownership increases with host county uncertainty. However, we also find that the above relationship is significantly weaker for Latin American MNCs than for U.S. MNCs. Specifically, as host country uncertainty increases, Latin American firms show a greater propensity to opt for full ownership (high control), while U.S. MNCs opt for shared ownership (low control). We show that Latin American firms are less hindered by host country uncertainty than U.S. firms and prefer greater control even though such a strategy increases their exposure to risky host country environments. (For more information, please contact: Carlyle Farrell, Ryerson University, Canada: farrellc@ryerson.ca)

\section{Intra-Country and Intra-Region Cultural variation and Multinationals' Entry Mode Choice Arjen Slangen, Rotterdam School of Management}

Relaxing the often-made assumption that countries are culturally homogeneous, I examine how cultural variation within target countries and target subnational regions affects the entry mode chosen by multinational enterprises (MNEs). I argue that since a subsidiary's value-adding activities are often initially concentrated in a 
subnational region, entry mode decision makers will generally attach more importance to target-region than to target-country cultural variation. I therefore hypothesize that while both these institutional factors may increase the likelihood that MNEs choose joint ventures (JVs) over wholly-owned subsidiaries, target-region cultural variation will increase that likelihood more. I also hypothesize that the positive effect of target-region cultural variation on the likelihood of JV entry is stronger for subsidiaries with a relatively larger initial workforce and that the positive effect of target-country cultural variation on that likelihood is weaker for acquisitions than for greenfields. An analysis of 168 entries by Dutch MNEs into 94 regions of 37 countries lends substantial support to these hypotheses, suggesting that subnational regions are more relevant units than countries within institutions-based research on MNEs' investment strategies, and hence that such research needs to shift its focus from the national to the regional level. (For more information, please contact: Arjen Slangen, Rotterdam School of Management, Netherlands: aslangen@rsm.nl)

\section{Target Firm Control in International Acquisitions: Does Acquirer's Performance Matter? Sathyajit Gubbi, University of Groningen Mohan Subramaniam, Carroll School of Management}

Acquisitive entries in international markets are invariably linked to the transfer of ownership advantages and control. But when acquirers seek resources and capabilities embedded in the target, equity ownership alone may not help and certainly not when the acquirer hails from the developing economies. In this paper we investigate the conditions when these firms seek controlling equity in the foreign target. Analysis of a sample of cross-border acquisitions by Indian firms reveals that besides underlying motivations for the acquisition, past performance of the acquirer in the home market also matters. Firms seeking immediate gains in the form of new products and/or markets choose absolute ownership while those in search of long-term sources of sustainable gains (strategic assets) favor partial acquisitions. However, high performing acquirers from the developing countries behave very much like the advanced country counterparts; they opt for partial ownership for short-term gains and absolute ownership for long-term gains. (For more information, please contact: Sathyajit Gubbi, University of Groningen, Netherlands: s.r.gubbi@rug.nI)

\section{The Evolving Role of Japanese General Trading Companies in the Internationalization of Japanese Electronics MNCS \\ Jun Ito, National University of Singapore}

This study investigates the role of the Japanese Trading Companies (the Sogo Shosha) partnering with Japanese electronics MNCs when the electronics MNCs enter host countries and found subsidiaries and examines how the Sogo Shosha's equity ownership of these subsidiaries affects the subsidiaries' survival. Previous studies on Japanese MNCs and survival rates have not dealt with partnership with the Sogo Shosha. That is, the Sogo Shosha's unique standing and function have not been examined for a long time. This study focuses on this point and makes a significant contribution to our understanding of Japanese MNCs' ownership strategy when entering host countries with the Sogo Shosha. In this study, we investigate the relationship between the ownership strategies of the foreign subsidiaries of Japanese electronics MNCs partnering with the Sogo Shosha and these foreign subsidiaries' survival rates with samples of Japanese electronics MNCs. We test four hypotheses with a data set that includes foreign subsidiaries of Japanese electronics MNCs. The results present that too low ownership ratio and too high ownership ratio by the Sogo Shosha make survival rates lower. We also find that as the MNCs gain local experience, the effect of ownership by the Sogo Shosha becomes weaker. (For more information, please contact: Jun Ito, National University of Singapore, Singapore: junito@nus.edu.sg) 
Does Economic Freedom in the Host Country Moderate the Influences of Cultural Distance on FDI Establishment Mode Choice of MNEs? Evidence from FDIs in Asia and Latin America

Ahmad Arslan, University of Vaasa

Jorma Larimo, University of Vaasa

This paper analyzes the impacts of both cultural distance and economic freedom in the host country on establishment mode choice of multinational enterprises (MNEs). The current paper advances extant IB literature by being is one of the first studies to theoretically hypothesize as well as empirically analyze the moderating influences of level of economic freedom on the cultural distance impacts on FDI establishment mode choice. The empirical analysis based a sample of 301 FDIs in in Asia and Latin America by the 87 Finnish MNEs revealed that high cultural distance leads to preference of greenfield investments by the Finnish MNEs while high level of economic freedom in the host country results in preference of acquisitions. Finally, empirical results also revealed statistical support for moderating relationship between economic freedom and cultural distance in relation to cultural distance's impacts on FDI establishment mode choice of MNEs. (For more information, please contact: Ahmad Arslan, University of Vaasa, Finland: ahmad.arslan@uva.fi)

\title{
International Acquisitions by Developing Country Firms: Some Factors Influencing Acquired Equity in Target Sathyajit Gubbi, University of Groningen Mohan Subramaniam, Carroll School of Management
}

In this paper we explore an under researched but important aspect of the extent of ownership sought in the foreign targets of cross-border acquisitions by firms from the developing countries. Based on an analysis of 535 international acquisitions by Indian firms in the manufacturing sector over the period 2000-2010, we identify some antecedent factors that are likely to play a decisive role in the acquisition of a simple majority stake (greater than 50 percent) in the target. Our findings show that while financial resources of a firm have a significant impact in the case of standalone firms, they matter only when absolute control (greater than 95 percent) of the target is desired in the case of business group affiliated firms. We also find that standalone firms with greater internal financial resources are less inclined to acquire absolute control. (For more information, please contact: Sathyajit Gubbi, University of Groningen, Netherlands: s.r.gubbi@rug.nI)

\section{Session: 3.4.15 - Interactive}

\section{Track: Track: 11 - SMEs and Entrepreneurship}

\section{International New Ventures in High-Technology Industries}

Presented On: July 6, 2013 - 14:50-16:05

Chair: Jonathan Liebenau, London School of Economics

\author{
Determinants of Post-Entry Survival in New International Ventures \\ Zaheer Khan, University of Hull \\ Charles Dhanaraj, Indiana University
}

Recent research suggests that the capabilities needed for new ventures to survive international markets may be different from what got them into international markets (Mudambi and Zahra, 2007; Prashantham and Dhanaraj, 2010). Mortality of new international ventures (NIVs) is exacerbated by economic shocks in the environment, which elevates the liabilities of newness, smallness and foreignness in these firms. We investigate the key factors affecting the post-entry survival of these NIVs using in-depth analysis of seven 'born global' software firms originating from a peripheral economy-Pakistan. These 'born global' firms have survived through the dot-com crisis of 2000 and the global financial crisis of 2008 and continue to grow. We narrow our focus on four capabilities and six processes that drive growth in NIVs. Our study points to the important role of 
the founder/entrepreneur at the post-entry growth stage of these firms. We draw implications for entrepreneurship theory and practice. (For more information, please contact: Zaheer Khan, University of Hull, United Kingdom: khan.zaheer@gmail.com)

\author{
Extinction or Metamorphosis: Where the Typical Clusters Have Gone? \\ Vesna Sedoglavich, Australian National University \\ Gabriele Suder, SKEMA
}

The success of clusters of SMEs in promoting the growth of the companies within the cluster is largely due to the individual firms of the cluster collaborating and sharing resources. This paper investigates the facilitating role of a cluster in the process of internationalization. The research is grounded on the discussion of the three cluster models: pure agglomeration, industrial complex and social networks. In order to demonstrate the observed issues, we discuss three cluster examples from the science-based industry. SMEs behaviour exemplified through their organizational and strategic issues illustrate that the geographical location matters are much more complex than at first they appear. Different types of clusters have different advantages for different types of firms. Recognizing which type of firm will benefit from which particular cluster type requires consideration of organization, strategic objectives and institutional issues, many of which are neglected by the prior clusters literature. The research suggests that in the current reality there is a new type of cluster, which we refer to as a New Breed or Hybrid Clusters. (For more information, please contact: Gabriele Suder, SKEMA, France: gabriele.suder@skema.edu)

\title{
Resource Fungibility in Early Rapidly Internationalizing Small Firms: The More We Look the More We Learn Shingairai Masango, Sheffield Hallam University \\ Svetla Trifonova Marinova, Aalborg University
}

The paper applies the RBV to early rapidly internationalizing small firms (ERISFs). The objectives of the paper are firstly, to investigate the resource categories and characteristics which define the context specificity and fungibility of the ERISF resource base; secondly, to establish the conditions, which enhance the fungibility of the ERISFs resource base; thirdly, to explore the extent to which resource fungibility influences ERISFs internationalization. Applying case-study analysis, the authors find out that under the context specificity/resource fungibility dimension four resource types emerge: individual level resources (specialist technical knowledge, specialist international market knowledge, and knowledge based interpersonal network ties), the firm's product platform and organizational network ties. The fungibility of the ERISF's resource base is enhanced by the overlap between individual level resources and the entrepreneurial opportunities, the development of a product/technology platform, the interaction between the ERISFs product platform and network relationships, and the interaction between the ERISFs product platform, network relationships and individual level resources. These enable the firm to engage in a range of cross border activities (inward, outward, and collaborative) by enabling the 'redeployment' of the firm's product platform within multiple organizational and geographical settings. (For more information, please contact: Shingairai Masango, Sheffield Hallam University, United Kingdom: s.masango@shu.ac.uk)

\section{Cloud Computing, International Entrepreneurship and SMES \\ Peter Keith Ross, Griffith University \\ Michael Blumenstein, Griffith University}

Cloud computing strategies allow SMEs to access high-level information and communication technology (ICT) products and services which they may not have been able to afford under previous up-front capital investment ICT expenditure models (Ross 2011; Ross \& Blumenstein forthcoming). This research therefore finds that Cloud technologies are underpinning entrepreneurship by lowering the opportunity costs of potential new projects. Cloud technologies further support innovation through enhanced collaboration and foster an international 
entrepreneurial orientation by allowing SMEs to potentially sell goods and services to a global market. Cloudbased SMEs and start-up firms therefore have the capacity to be 'born-global' by their very nature. The Cloud computing paradigm however brings associated threats for SMEs, including potential challenges in adapting to Cloud-based business models and increased competition from globally based competitors. This research therefore suggests that while Cloud technologies offer a potential boon to SME entrepreneurship they are also ushering in a period of rationalisation, as some firms will inevitably fail to successfully adapt to a globally competitive Cloud environment. (For more information, please contact: Peter Keith Ross, Griffith University, Australia: p.ross@griffith.edu.au)

\section{Does Speed Matter? The Internationalisation of High-Tech SMES Reconsidered Lisa Hewerdine, University of Sydney Maria Rumyantseva, University of Sydney Catherine Welch, University of Sydney}

In this paper, we examine the association between the 'born global' and 'high tech' concepts. The assumption of the born global literature - that speed is the critical explanatory characteristic for high-tech SME internationalisation - is the focus of our paper. We use the emerging literature on concept formation to critically analyse the structure and adequacy of the born global concept. Then, in our qualitative case study, we track the internationalisation of high-tech start-up firms. With one exception, we found that even those firms which initially appeared to fit the 'born global' categorisation, on closer inspection were negative or at best marginal cases. Importantly, we found that the speed of their internationalisation was neither a significant explanatory factor nor a characteristic shared by all our high-tech case firms. In fact, the focus on speed limits the theoretical utility of the born global concept by not allowing for the lengthy technological innovation process high-tech firms may undergo. Our findings therefore suggest that the close association between 'high tech' and 'born global' requires re-examination. (For more information, please contact: Maria Rumyantseva, University of Sydney, Australia: maria.rumyantseva@sydney.edu.au)

\section{Internationalization of High-Tech Small and Medium Sized Enterprises: Open Innovation Perspective} Mahdi Tajeddin, Concordia University

Mehdi Farashahi, Concordia University

High-tech small and medium sized enterprises (SMEs) are often characterized as born-global, showing very rapid and intensive international growth enabled by the use of external resources such as partnerships and networks. Adopting an open innovation (OI) approach of the firm can be a useful way to increase speed and degree of internationalization of these high-tech SMEs. Although high-tech SMEs encounter different challenges to apply OI, they will benefit from aspects of knowledge and networking in relation to the OI approach. Therefore, this study is motivated by three research questions: What are the most important outcomes of the OI approach in the international high-tech SMEs? What is the effect of OI on the internationalization of hightech SMEs? How can we apply this approach in the international high-tech SMEs? This study highlights knowledge and networking as the most important drivers which will be improved by applying the OI approach in the international high-tech SMEs. This improvement will impact on degree and speed of internationalization of high-tech SMEs positively. Finally, we mention the limitations of small firm to apply OI. In this regard, we indicate two factors, intermediated network and manager characteristics, as moderator which modify the limitations and influence the link between OI and internationalization positively. (For more information, please contact: Mahdi Tajeddin, Concordia University, Canada: m_tajed@jmsb.concordia.ca)

Toward An Effectuation-Focused Interpretation of Small Firm Internationalization: A Case Study on Software Exporting Firms of Bangladesh

Nur Alam Md., Leipzig University

Utz Dornberger, Leipzig University 
As an emergent concept, effectuation is well established in literature. But, most of the discussion related to effectuation is based on theoretical arguments. Very few papers attempted to show the impact of effectuation on different aspect of business activities. This paper is one of endeavor to show the impact of effectuation on internationalization of small and medium firms particularly on speed of international entry, firm performance, international diversification (export diversification) and international alliance and partnership. The research sample is composed of 100 small and medium software exporters from Bangladesh. Software firms those who are exporting in at least one destination and are doing business for at least five years were only considered for interview. A Structural Equation Modeling (SEM) approach, particularly Partial Least Squares (PLS), was employed to evaluate the relationships between constructs and to measure the predictive power of the research model. The result shows that all hypotheses are proved by the model. So, it can be said that degree of effectuation has significant impact on firm performance, internationalization speed, international alliance and partnership, international diversification. (For more information, please contact: Utz Dornberger, Leipzig University, Germany: dornberger@uni-leipzig.de)

\section{International New Ventures - Developing a Competitive Advantage through Internationalization Roger Schweizer, University of Gothenburg}

Using a single-case study approach, this paper portrays and discusses the process of how a new venture develops a competitive advantage through instant and continuous internationalization. We find that this process consists of three interrelated phases: (1) conceptualization, (2) creation, and (3) leveraging of competitive advantage. Arguing that resources need to be managed adequately to create a competitive advantage, we study various resource management activities employed during the various phases. We show that whereas the new venture is expected to concentrate on identifying the appropriate (international) market during the first phase, the main focus in the second phase is on structuring and bundling of resources. During the final stage, the INV concentrates on leveraging, i.e. on applying its capabilities to create value for the firm and its customers. (For more information, please contact: Roger Schweizer, University of Gothenburg, Sweden: roger.schweizer@handels.gu.se)

\section{Session: 3.5.1 - Plenary}

\section{AIB Awards Ceremony and Business Meeting}

Presented On: July 6, $2013-16: 45-18: 00$

The session will start with the announcement of the winners for the various conference awards including the Buckley and Casson Dissertation Award, Best Paper Award, and the Haynes Prize.

Then, the business meeting will feature a presentation for the 2014 AIB Meeting in Vancouver and a review of the state of AIB and JIBS. The floor will then be open for questions and comments from the membership. 


\section{INDEX OF PROGRAM CONTRIBUTORS}




\section{INDEX OF PROGRAM CONTRIBUTORS}

\section{A}

Aafaqi, Rehana; University of Lethbridge, Canada (rehana.aafaqi@uleth.ca): 1.4.15

Abdi, Majid; University of Melbourne, Australia (majid.abdi@gmail.com): 1.4.5

Abi Aad, Amine; University of Alabama, USA (aaabiaad@crimson.ua.edu): 3.2.12

Achcaoucaou, Fariza; University of Barcelona, Spain (farizaa@ub.edu): 2.4.4

Adair, Wendi L.; University of Waterloo, Canada (wladair@uwaterloo.ca): 3.1.6

Adamuz, Mercedes; ITAM, Mexico (adamuz@itam.mx): 1.1.13

Afiouni, Fida; American University of Beirut, Lebanon (fa16@aub.edu.lb): 1.4.15, 3.4.6

Agarwal, James; University of Calgary, Canada (james.agarwal@haskayne.ucalgary.ca): 2.2.4, 3.3.6

Agca, Senay; George Washington University, USA (sagca@gwu.edu): 1.1.13

Aggarwal, Raj; University of Akron, USA (aggarwa@uakron.edu): 1.1.7

Aguilera, Ruth; University of Illinois at Urbana-Champaign, USA (ruth-agu@illinois.edu): 1.3.2, 2.4.12

Ahn, Hyunju; Korea University, Korea, South (hjahn1122@korea.ac.kr): 3.2.13

Ahsan, Faisal M; Indian Institute of Management Lucknow, India (fpm12005@iiml.ac.in): 3.1.14

Akaka, Melissa Archpru; University of Denver, USA (melissa.akaka@du.edu): 2.4 .15

Akbar, Mohammad; Indian Institute of Management Lucknow, India (ma@iiml.ac.in): 1.5.15, 3.1.14

Akbar, Yusaf; Central European University, Hungary (akbary@ceubusiness.org): 1.4.13

Akbari, Hamid; UOIT, Canada (hamid.akbari@uoit.ca): 1.4.6, 2.5.1

Akguc, Serkan; Temple University, USA (serkan.akguc@temple.edu): 2.4.7

Akin, Ahmet; Fatih University, Turkey (akin@fatih.edu.tr): 3.2 .9

Albareda, Laura; Deusto Business School, Spain (laura.albareda@deusto.es): 3.1.8

Alemdar, Sevkinaz; Procurement Coordinator, Erdemir Group, Turkey: 2.4.1

Alessandri, Todd; Northeastern University, USA (t.alessandri@neu.edu): 3.3.5

Alexander, Elizabeth; University of West England, United Kingdom (elizabeth2.alexander@uwe.ac.uk): 2.4.10

Alfoldi, Eva Andrea; University of Manchester, United Kingdom (eva.alfoldi@mbs.ac.uk): 1.4.12

Alhorr, Hadi; Saint Louis University, USA (halhorr@slu.edu): 2.5.11

Allard, Gayle; IE Business School, Spain (gayle.allard@ie.edu): 3.2.13

Allen, Matt; Babson College, USA (mallen4@babson.edu): 1.3.8

Alloughani, Mohammad E.; James Madison University, USA (allougme@jmu.edu): 2.5.13

Almeida, Paul; Georgetown University, USA (almeidap@georgetown.edu): 3.4.11

Alpustun, Aylin; Turk Ekonomi Bankasi, Turkey: 3.2.4

Alsharairi, Malek; German Jordanian University, Jordan (malek.alsharairi@gju.edu.jo): 1.3.15

Alvarado-Vargas, Marcelo J.; Florida International University, USA (malva043@fiu.edu): 1.1.4, 2.5.7, 3.4.6

Alvarez-Garrido, Elisa; Georgia State University, USA (ealvarezgarrido@gsu.edu): 3.1 .13

Amankwah-Amoah, Joseph; Bristol University, United Kingdom (joseph.amankwah-amoah@bristol.ac.uk): 1.5.13

Amann, Bruno; University of Toulouse, France (bruno.amann@club-internet.fr): 2.2.12

Ambos, Bjoern; University St. Gallen, Switzerland (bjoern.ambos@unisg.ch): 1.3.6, 2.1.8, 2.3.13

Ambos, Tina; University of Sussex, United Kingdom (tina.ambos@me.com): 1.4.4, 3.3.1

Amer Maistriau, Estefania; University of Geneva, Switzerland (estefania.amer@unige.ch): 3.4.12

Amoros, Jose Ernesto; Universidad del Desarrollo, Chile (eamoros@udd.cl): 1.1.10, 1.3.13, 3.3.6

Anderson, John; University of Northern lowa and Durham University, United Kingdom (j.r.anderson@durham.ac.uk):

1.3.9

Andersson, Ulf; Copenhagen Business School, Denmark (ua.smg@cbs.dk): 1.4.4, 2.1.4, 2.4.4, 3.4.3

Ando, Naoki; Hosei University, Japan (nando@hosei.ac.jp): 1.4.12, 3.2.5

Annavarjula, Madan; Bryant University, USA (mannavar@bryant.edu): 2.3.2

Ansari, Mahfooz A.; University of Lethbridge, Canada (mahfooz.ansari@uleth.ca): 1.4.15

Apaydin, Marina; American University of Beirut, Lebanon (ma266@aub.edu.lb): 3.1 .3

Ararat, Melsa; Sabanci University, Turkey (melsaararat@sabanciuniv.edu): 2.5.2 
Arnaud, Anke; Embry-Riddle Aeronautical University, USA (arnauda@erau.edu): 3.3.4, 3.4.4

Arnold, Denis; University of North Carolina, Charlotte, USA (denisarnold@uncc.edu): 1.3.14, 3.1.2

Arregle, Jean-Luc; EMLyon Business School, France (arregle@em-lyon.com): 1.3.2, 3.3.3

Arslan, Ahmad; University of Vaasa, Finland (ahmad.arslan@uva.fi): 2.1.11, 3.4.14

Asakawa, Kazuhiro; Keio University, Japan (asakawa@kbs.keio.ac.jp): 0.4, 1.3.6, 1.4.10, 2.1.4, 2.2.12

Ash, Steven; University of Akron, USA (ash@uakron.edu): 2.2.15

Asmussen, Christian; Copenhagen Business School, Denmark (cga.smg@cbs.dk): 1.3.2

Atamer, Tugrul; EMLyon Business School, France (atamer@em-lyon.com): 3.1.14

Athreye, Suma; Brunel University, United Kingdom (suma.athreye@brunel.ac.uk): 2.1.14

Atiyas, Izak; Director, TUSIAD-Sabanci University Competitiveness Forum, Turkey (izak@sabanciuniv.edu): 2.1.1

Audretsch, David; Indiana University, USA (daudrets@indiana.edu): 2.4.3

Aulakh, Preet S.; York University, Canada (paulakh@schulich.yorku.ca): 1.4.5, 3.2.6

Ault, Joshua K; University of Victoria, Canada (jault@uvic.ca): 1.1.11

Auster, Ellen R.; York University, Canada (eauster@schulich.yorku.ca): 1.4.6

Avlonitis, George J.; Athens University of Economics and Business, Greece (avlonitis@aueb.gr): 2.1.11

Avrichir, Ilan; ESPM, Brazil (iavrichir@espm.br): 3.3.15

Axinn, Catherine N.; Ohio University, USA (axinn@ohio.edu): 3.1 .5

Aybar, C. Bulent; Southern New Hampshire University, USA (c.aybar@snhu.edu): 1.3.15, 2.2.11

Aycan, Zeynep; Koc University, Turkey (zaycan@ku.edu.tr): 1.1.2, 3.4.13

B

Baack, Daniel W.; University of Denver, USA (dbaack@du.edu): 1.1.8, 2.4.15

Baack, Donald E.; Pittsburg State University, USA (debaack@pittstate.edu): 1.1.8

Bader, Benjamin; University of Hamburg, Germany (benjamin.bader@uni-hamburg.de): 2.2.10, 2.3.9

Bae, Zong-Tae; Kaist Business School, Korea, South (ztbae@business.kaist.ac.kr): 3.2.11

Bailey, Nicholas James; University of South Carolina, USA (nicholas.bailey@grad.moore.sc.edu): 2.1.12

Baker, David S.; University of Louisiana at Lafayette, USA (dbaker@louisiana.edu): 2.5.6

Balakrishnan, Melodena; University of Wollongong in Dubai, United Arab Emirates (melodenabalakrishnan@uowdubai.ac.ae): 0.4, 1.1.14, 1.5.1, 3.1.3

Baldegger, Rico; University of Applied Sciences Western Switzerland, Switzerland (rico.baldegger@hefr.ch): 2.4.11, 2.5.8

Ballesteros, Luis; University of Pennsylvania, USA (luisf@wharton.upenn.edu): 2.3.11

Bamiatzi, Vassiliki; Leeds University, United Kingdom (vb@lubs.leeds.ac.uk): 2.4 .5

Banai, Moshe; City University of New York, USA (moshe.banai@baruch.cuny.edu): 3.2.15

Banalieva, Elitsa; Northeastern University, USA (e.banalieva@neu.edu): 1.5.11

Bandeira-de-Mello, Rodrigo; Fundação Getúlio Vargas, Brazil (rodrigo.bandeira.demello@fgv.br): 2.4.12

Banerji, Kunal; Eastern Michigan University, USA: 1.4.10

Barakat, Livia Lopes; Fundacao Dom Cabral, Brazil (liviabarakat@fdc.org.br): 3.2.12

Barbero, Maria Ines; Universidad de San Andrés and Universidad de Buenos Aires, Argentina (mbarbero@udesa.edu.ar): 3.1.4

Barreto, Tais Siqueira; Florida Atlantic University, USA (tsiqueir@fau.edu): 3.3.6

Barzantny, Cordula; Touluse Business School, France (c.barzantny@esc-toulouse.fr): 3.3.2

Basti, Eyup; Fatih University, Turkey (ebasti@fatih.edu.tr): 3.2.9

Basuil, Dynah A; Asian Institute of Management, Philippines (dbasuil@aim.edu): 2.2.14, 3.2.7

Bathula, Hanoku; AIS St. Helens, New Zealand (hanokub@ais.ac.nz): 2.5.8

Baum, Matthias; University of Giessen, Germany (matthias.baum@wirtschaft.uni-giessen.de): 2.1.6

Bausch, Andreas; Justus Liebig University Giessen, Germany (andreas.bausch@wirtschaft.uni-giessen.de): 2.4.6

Bayraktar, Ahmet; Rutgers University, USA (ahmetbay@pegasus.rutgers.edu): 1.3.5

Bayyurt, Nizamettin; Fatih University, Turkey (bayyurt@fatih.edu.tr): 3.2.9

Bealer, Dianne; Charles Sturt University, Australia (dbealer@hct.ac.ae): 3.3.14

Beamish, Paul W.; University of Western Ontario, Canada (pbeamish@ivey.uwo.ca): 2.3.1, 3.3.3, 3.4.14

Bebenroth, Ralf; Kobe University, Japan (rbeben@rieb.kobe-u.ac.jp): 3.2.5, 3.3.15 
Beechler, Schon; INSEAD, USA (schon.beechler@gmail.com): 1.3.7

Behrens, Alfredo; Alfredo Behrens Consulting, Brazil (ab@alfredobehrens.com): 3.3.4, 3.4.4

Beleska-Spasova, Elena; University of Reading, United Kingdom (qb901446@reading.ac.uk): 1.3.8, 2.1.9

Belkhir, Mohamed; UAE University, United Arab Emirates (m.belkhir@uaeu.ac.ae): 1.1.13, 2.5.9

Bello, Daniel; Georgia State University, USA (dbello@gsu.edu): 1.4.2, 2.3.14

Bendickson, Joshua; Louisiana State University, USA (jbendi2@Isu.edu): 1.4.6, 2.5.14

Benischke, Mirko H.; University of Auckland, New Zealand (m.benischke@auckland.ac.nz): 1.4.8

Benito, Gabriel; BI Norwegian Business School , Norway (gabriel.r.g.benito@bi.no): 0.2

Bennasr, Hamdi; King Saud University, Saudi Arabia (hbennasr@ksu.edu.sa): 2.4.9, 2.5.9

Bensaou, Ben; INSEAD, France (ben.bensaou@insead.edu): 2.1.7

Berg, David M.; Hamline University, USA (dberg06@hamline.edu): 2.5.8

Berg, Nicola; University of Hamburg, Germany (nicola.berg@uni-hamburg.de): 2.2.10

Bernal Turnes, Paloma; Georgetown University, USA (pb737@georgetown.edu): 2.5.6, 3.2.12

Berry, Heather; George Washington University, USA (berryh@gwu.edu): 1.4.11, 2.4.4

Bertrand, Olivier; SKEMA, France (olivier.bertrand@skema.edu): 1.3.15, 1.4.9

Betancourt, Nathan Emil; University of Lugano, Switzerland (nathanbetancourt@gmail.com): 1.1.12

Betschinger, Marie-Ann; NRU Higher School of Economics, Russia (mbetschinger@hse.ru): 2.2.7

Beugelsdijk, Sjoerd; University of Groningen, Netherlands (s.beugelsdijk@rug.nl): 1.4.11, 2.1.8, 3.1.1

Bezerra, Maite; ESPM/SP, Brazil (maiteabezerra@gmail.com): 2.5.5

Bhagat, Rabi; University of Memphis, USA (rbhagat@memphis.edu): 3.3.2

Bhanugopan, Ramudu; Charles Sturt University, Australia (bramudu@csu.edu.au): 3.3.14

Bharati, Pratyush; University of Massachusetts Boston, USA (pratyush.bharati@umb.edu): 1.1.6

Bhaskarabhatla, Ajay; Erasmus University, Netherlands (bhaskarabhatla@ese.eur.nl): 2.2.9

Bi, Xiaogang; Nottingham University, China (x.bi@nottingham.edu.cn): 1.3.15

Bianchi, Constanza C.; Universidad Adolfo Ibanez, Chile (constanza.bianchi@uai.cl): 2.4.15, 3.1.15

Bianchi, Mattia; Stockholm School of Economics, Sweden (mattia.bianchi@hhs.se): 2.2.8

Bilgili, Tsvetomira Vladimirova; University of Memphis, USA (tvkaneva@memphis.edu): 1.5.6

Binder, Gregor; WU Vienna, Austria (gregor.binder@wu.ac.at): 2.5.9

Bird, Allan; Northeastern University, USA (a.bird@neu.edu): 1.3.8

Björkman , Ingmar ; Aalto University, Finland (ingmar.bjorkman@aalto.fi): 0.3, 3.2.1

Blake, Daniel; IE Business School, Spain (daniel.blake@ie.edu): 3.2.3

Blasi, Joseph; Rutgers University, USA (jbru@hotmail.com): 1.5.14

Blomkvist, Katarina; Uppsala University, Sweden (katarina.blomkvist@fek.uu.se): 2.2.12, 2.3.12

Blumenstein, Michael; Griffith University, Australia (m.blumenstein@griffith.edu.au): 3.4.15

Boateng, Agyenim; University of Nottingham, United Kingdom (agyenim.boateng@nottingham.edu.cn): 1.3.15, 2.2.14

Boddewyn, Jean; Baruch College, USA (jean.boddewyn@baruch.cuny.edu): 1.1.11, 1.3.14

Bode, Alexander; Technische Universität Darmstadt, Germany (bode@bwl.tu-darmstadt.de): 2.5.8

Boehe, Dirk Michael; University Of Adelaide, Australia (dirkmichael.boehe@adelaide.edu.au): 1.5.12, 2.5.5, 3.3.3

Bolzani, Daniela; University of Bologna, Italy (daniela.bolzani2@unibo.it): 1.3.11

Borda, Armando; Esan University, Peru (abord001@fiu.edu): 3.4.6

Borini, Felipe Mendes; ESPM/SP, Brazil (fborini@espm.br): 2.5.5

Bort, Suleika; University of Mannheim, Germany (sbort@rumms.uni-mannheim.de): 2.1.6

Bortoluzzi, Guido; University of Trieste, Italy (guido.bortoluzzi@econ.units.it): 1.4.13, 2.3.5

Boschma, Ron; Utrecht University, Netherlands (r.boschma@geo.uu.nl): 2.5.3

Bosma, Niels; Utrecht University, Netherlands (n.s.bosma@uu.nl): 1.3.13

Boso, Nathaniel; University of Leeds, United Kingdom (n.boso@leeds.ac.uk): 2.4 .5

Bouncken, Ricarda B.; University of Bayreuth, Germany (bouncken@uni-bayreuth.de): 3.3.7

Bounfour, Ahmed; Université de Paris Sud 11, France (a.bounfour@wanadoo.fr): 1.1.13

Boussebaa, Mehdi; University of Bath, United Kingdom (m.boussebaa@bath.ac.uk): 3.1.13

Bowe, Michael; University of Manchester, United Kingdom (michael.bowe@mbs.ac.uk): 3.1.12

Bowen, Harry P.; Queens University of Charlotte, USA (bowenh@queens.edu): 1.4.1 
Boyacigiller, Nakiye A.; Sabanci University, Turkey (nakiye@sabanciuniv.edu): 0.3, 1.3.7, 1.4.3, 2.1.1, 3.3.2

Bozos, Konstantinos; Leeds University, United Kingdom (kb@lubs.leeds.ac.uk): 1.3.15, 2.4.5

Bradlow, Ann; Northwestern University, USA (abradlow@northwestern.edu): 1.3.7

Brandl, Kristin; Copenhagen Business School, Denmark (kbr.int@cbs.dk): 1.5.4

Brannen, Mary Yoko; University of Victoria, Canada (maryyoko@uvic.ca): 0.5, 1.5.9, 2.4.2

Braun, Daniel; University of Goettingen, Germany (daniel.braun@wiwi.uni-goettingen.de): 1.1.13

Brenner, Barbara; Danube University, Austria (barbara.brenner@donau-uni.ac.at): 2.3.13, 3.4.13

Brewer, Paul; University of Queensland, Australia (p.brewer@business.uq.edu.au): 2.5.15

Brewster, Chris; University of Reading, United Kingdom (c.j.brewster@reading.ac.uk): 1.3.8

Brodbeck, Felix; University of Munich, Germany (brodbeck@psy.Imu.de): 3.2.15

Brown, D. Michael; Birmingham City Business School, United Kingdom (mike.brown@bcu.ac.uk): 3.2.12

Bruck, Frank; Bocconi University, Italy (frank.brueck@wu.ac.at): 3.4.13

Bruining, Hans; Erasmus University, Netherlands (jbruining@rsm.nl): 2.3.12

Bryant, Chuck; Cleveland State University, USA (chuckbryant@mac.com): 1.4.14

Buchan, Nancy R.; University of South Carolina, USA (nancy.buchan@moore.sc.edu): 3.1.6, 3.4.13

Buckley, Peter J; University of Leeds, United Kingdom (pjb@lubs.leeds.ac.uk): 0.2, 1.2, 1.5.1, 2.2.4, 2.3.6, 2.3.7, 3.3.12

Budde-Sung, Amanda E.K.; University of Sydney, Australia (amanda.budde-sung@sydney.edu.au): 1.3.12, 2.3.10, 3.2.15, 3.3.4, 3.4.4

Budhwar, Pawan; Aston University, United Kingdom (p.s.budhwar@aston.ac.uk): 2.4 .13

Bugador, Roderick; University of Reading, United Kingdom (r.bugador@pgr.reading.ac.uk): 2.5.11

Bullough, Amanda; Thunderbird School of Global Management, USA (amanda.bullough@thunderbird.edu): 2.1.15

Burmester, Brent; Auckland University, New Zealand (b.burmester@auckland.ac.nz): 1.1.8, 2.1.9

Butler, Kirt C.; Michigan State University, USA (butler@msu.edu): 1.1.13, 2.5.8

C

Cadogan, John W.; Loughborough University, United Kingdom (j.w.cadogan@lboro.ac.uk): 2.4 .5

Cai, Huifen; University of Huddersfield, United Kingdom (h.cai16888@hotmail.com): 3.3.8, 3.4.5

Cai, Yingdan; University of Groningen, Netherlands (y.cai@rug.nl): 1.5.6

Caligiuri, Paula; Northeastern University, USA (p.caligiuri@neu.edu): 3.2.1

Calixto, Cyntia Vilasboas; Fundação Getulio Vargas (FGV - EAESP), Brazil (cyntiacalixto@gmail.com): 2.5.12

Camilo, Silvio Parodi; UNESC, Brazil (parodi@parodiconsultoria.com.br): 2.4.12

Cannizzaro, Anthony; George Washington University, USA (tony_c@gwmail.gwu.edu): 1.4.7

Cannon, Hugh M.; Wayne State University, USA (hugh.cannon@wayne.edu): 2.4.15

Cano Kollmann, Marcelo; Temple University, USA (mck@temple.edu): 3.4.11

Cantwell, John; Rutgers University, USA (cantwell@business.rutgers.edu): 0.1, 1.5.1, 2.1.13, 2.5.4, 3.2.1, 3.4.1

Cao, Lan; Leuphana University Lueneburg, Germany (lan.cao@leuphana.de): 2.2.10

Caprar, Dan; University of New South Wales, Australia (dan.caprar@unsw.edu.au): 2.5.8

Carlsson, Kjell; Wilson Perumal \& Company, USA (kcarlsson@wilsonperumal.com): 3.2.8

Carneiro, Jorge; Pontifical Catholic University of Rio de Janeiro, Brazil (jorgemtc@iag.puc-rio.br): 2.1.11, 2.4.13

Carney, Michael; Concordia University, Canada (mcarney@jmsb.concordia.ca): 1.1.14, 2.4.9

Carnovale, Steven; Rutgers University, USA (scarnova@pegasus.rutgers.edu): 3.1 .9

Caroli, Matteo Giuliano; Luiss Guido Carli University, Italy (mcaroli@luiss.it): 2.2.13

Carreira, Helder Costa; - Instituto Politecnico de Leiria \& globADVANTAGE, Portugal (h.costacarreira@gmail.com): 2.2.7

Carvalho, Sergio; University of Manitoba, Canada (sergio.carvalho@umanitoba.ca): 2.4.15

Casap, Lucia; Magenta Consulting, Moldova (I.casap@consulting.md): 2.3.4

Castañer, Xavier; Université de Lausanne, Switzerland (xavier.castaner@unil.ch): 2.2.14

Castro, luri; Caixa Economica Federal - Brazil, Brazil (iuri.castro@caixa.gov.br): 2.3.13

Casulli, Lucrezia; University of Strathclyde, United Kingdom (eziacasulli@hotmail.com): 1.4.13

Cathro, Virginia; Otago University, New Zealand (virginia.cathro@otago.ac.nz): 3.3.4, 3.4.4

Cavazos, David E.; University of New Mexico, USA (dcavazos@unm.edu): 1.3.12 
Cavusgil, Tamer; Georgia State University, USA (cavusgil@gsu.edu): 1.4.3, 2.2.1, 2.3.1, 2.4 .5

Caykoylu, Sinan; Simon Fraser University, Canada (scaykoyl@sfu.ca): 1.3.4, 1.5.14

Celo, Sokol; Suffolk University, USA (scelo@suffolk.edu): 2.3.6, 2.4.6

Cemalcilar, Zeynep; Koc University, Turkey (zcemalcilar@ku.edu.tr): 1.1.2

Cerdin, Jean Luc; ESSEC, France (cerdin@essec.fr): 2.1.15

Cerit, A. Guldem; Dokuz Eylul University, Turkey: 3.2.4

Cetindamar, Dilek; Sabanci University, Turkey (dilek@sabanciuniv.edu): 2.4.13, 3.4.2

Chacar, Aya; Florida International University, USA (chacara@fiu.edu): 0.2, 2.2.2, 2.5.14, 3.3.1

Chai, Dominic; Seoul National University, Korea, South (dchai@snu.ac.kr): 2.4.7

Chan, Tsang-Sing; Lingnan University, Hong Kong, SAR-PRC (chants@In.edu.hk): 0.4, 2.2.13

Chand, Masud; Wichita University, USA (masud.chand@wichita.edu): 1.3.4, 3.2.15

Chandra, Ramdas; Nova Southeastern University, USA (cramdas@nova.edu): 2.5 .5

Chang, Frances Y.M.; Macquarie University, Australia (frances.chang@mq.edu.au): 1.5.5

Chang, Song; Chinese University of Hong Kong, Hong Kong, SAR-PRC (changsong@baf.msmail.cuhk.edu.hk): 1.4.15

Chang, Sungyong; Columbia University, USA (sc3339@columbia.edu): 2.3 .5

Chapman, Malcolm Kenneth; University of Leeds, United Kingdom (mkc@lubs.leeds.ac.uk): 2.2.4

Chari, Murali D.R.; Rensselaer Polytechnic Institute, USA (charim2@rpi.edu): 3.4.11

Chatterjee, Chirantan; Indian Institute of Management Bangalore, India (chirantan.chatterjee@iimb.ernet.in): 2.2.9

Chaudhry, Peggy E.; Villanova University, USA (peggy.chaudhry@villanova.edu): 2.4.15

Cheglakova, Liudmila M.; NRU Higher School of Economics, Russia (Icheglakova@mail.ru): 1.3.13

Chelliah, Shankar; Universiti Sains Malaysia, Malaysia (shankar@usm.my): 1.4.7, 2.1.14

Chen, Alex Xin; University of Hong Kong, Hong Kong, SAR-PRC (alexchen@hku.hk): 2.3.5

Chen, Homin; National Taiwan University, Taiwan (hmchen@ntu.edu.tw): 1.1.8, 2.2.8

Chen, Hsiu-Li; Ming Chuan University, Taiwan (hlchen@mail.mcu.edu.tw): 2.4.15

Chen, Ke; University of International Business and Economics, China (chenke@uibe.edu.cn): 1.3.5

Chen, Pi-Chi; University of London, United Kingdom (pi-chi.chen@bbk.ac.uk): 2.2.9

Chen, Po-Yuan; National Chengchi University, Taiwan (95355512@nccu.edu.tw): 2.1.14

Chen, Roger; University of San Francisco, USA (chenr@usfca.edu): 2.2.8

Chen, Shoshi; Tel Aviv University, Israel (shos@post.tau.ac.il): 2.3.9

Chen, Victor Zitian; University of North Carolina, Charlotte, USA (emgp.editor@gmail.com): 2.5.4, 3.2.11, 3.4.7

Chen, Wein-Hong; National Dong Hwa University, Taiwan (weinhong@mail.ndhu.edu.tw): 2.1.14

Chen, Wenjie; George Washington University, USA (chenw@gwu.edu): 1.1.13, 2.4.7

Chen, Xiaoping; University of Washington, USA (xpchen@u.washington.edu): 3.1.6

Chen, Xiaoyun; University of Macau, Macau (xychen@umac.mo): 2.3.5, 2.4.13

Chen, Yin-Ru; National Taiwan University, Taiwan (jubikevin@yahoo.com.tw): 1.1.8, 2.2.8

Chen, Yu-Chen; National Taiwan Normal University, Taiwan (60056011o@ntnu.edu.tw): 3.1.13

Cheng, Dazhong; Fudan University, China (chengdz@fudan.edu.cn): 2.4.7

Cheng, Hsiang-Lin; National Chung-Cheng University, Taiwan (cheng5689@gmail.com): 2.3.13, 2.4.4

Chetty, Sylvie K.; University of Otago, New Zealand (sylvie.chetty@otago.ac.nz): 1.4.13, 2.1.5, 3.2.2

Chi, Tailan; University of Kansas, USA (chi@ku.edu): 1.3.7

Chiang, Flora; Hong Kong Baptist University, Hong Kong, SAR-PRC (fchiang@hkbu.edu.hk): 3.3.14

Chiang, Yun-Hwa; Ming Chuan University, Taiwan (hui3789@yahoo.com.tw): 3.1.13

Chiao, Yu-Ching; National Chung Hsing University, Taiwan (chiaoy@dragon.nchu.edu.tw): 1.1.4

Chiarvesio, Maria; University of Udine, Italy (chiarvesio@uniud.it): 2.3.5

Chidlow, Agnieszka; Manchester Metropolitan University, United Kingdom (a.chidlow@mmu.ac.uk): 2.2.4, 3.4.7

Chintakananda, Asda; Nanyang Technological University, Singapore (asda@ntu.edu.sg): 2.5.10

Chittoor, Raveendra; Indian School of Business, India (raveendra_chittoor@isb.edu): 3.2.6

Chiu, Chi-yue; Nanyang Technological University \& The Chinese Academy of Social Sciences, Singapore

(cy.cychiu@gmail.com): 2.4.15

Chiu, Hsin Hui; California State University, USA (hsinhui.chiu@csun.edu): 3.2.7

Chiu, Hui Yun; National Chengchi University, Taiwan (99355503@nccu.edu.tw): 1.5.14, 3.4.9

Chiu, Yaping; Chung Yuan Christian University, Taiwan (yaping@cycu.edu.tw): 2.3.13 
Chng, Daniel Han Ming; China Europe International Business School, China (dchng@ceibs.edu): 3.1.13

Cho, Hyejin; Korea University, Korea, South (hyejinstory@korea.ac.kr): 3.1.7

Cho, Youngsam; Korea University, Korea, South (zegal82@korea.ac.kr): 2.5.13

Choe, Soonkyoo; Yonsei University, Korea, South (skychoe@yonsei.ac.kr): 1.3.6, 2.3.13, 3.1.10

Choi, Donseung; Sogang University, Korea, South (youngrevival@naver.com): 3.2.13

Choi, Jongmoo Jay; Temple University, USA (jjchoi@temple.edu): 2.4.7

Choi, Yonghoon; Doshisha University, Japan (ychoi@mail.doshisha.ac.jp): 2.3.14

Chou, Sheng-Fang; National Taiwan Normal University, Taiwan (dodo.chou@gmail.com): 2.2.12, 3.4.11

Chowdhury, Imran; Pace University, USA (ichowdhury@pace.edu): 1.5.12

Christmann, Petra; Rutgers University, USA (christmann@business.rutgers.edu): 0.1, 3.4.8

Chuang, Fu-Mei; Loughborough University, United Kingdom (f.chuang@lboro.ac.uk): 3.1 .5

Chung, Chi-Nien; National University of Singapore, Singapore (bizccn@nus.edu.sg): 3.3 .5

Chung, Chris Changwha; Korea University, Korea, South (chungc@korea.ac.kr): 3.2.7

Chung, Henry F. L.; Massey University, New Zealand (h.chung@massey.ac.nz): 3.4.10

Chung, Jaiho; Korea University, Korea, South (jhochung@korea.ac.kr): 3.1.7

Chung, Leanne; Cardiff University, United Kingdom (chungl1@cf.ac.uk): 2.2.12

Ciabuschi, Francesco; Uppsala University, Sweden (francesco.ciabuschi@fek.uu.se): 3.4.3

Ciravegna, Luciano; University of London, United Kingdom (luciano.ciravegna@rhul.ac.uk): 2.1.10, 3.1.8

Cirjevskis, Andrejs; Riga International School of Economics and Business Administration (RISEBA), Latvia (andrejs.cirjevskis@riseba.lv): 3.1.15

Ciszewska-Milinaric, Mariola; Kozminski Academy, Poland (mariolac@kozminski.edu.pl): 1.4.13

Clancy, Johanna; National University of Ireland, Galway, Ireland (johanna.clancy@nuigalway.ie): 3.3.15

Clarke, Linda; University of Florida, USA (clarkel@ufl.edu): 3.2.14

Clarke, Ruth; Nova Southeastern University, USA (rclarke@nova.edu): 2.5.5

Claver, Enrique; University of Alicante, Spain (enrique.claver@ua.es): 3.3.8

Clegg, Jeremy; University of Leeds, United Kingdom (ljc@lubs.leeds.ac.uk): 0.5, 1.4.12, 3.3.12

Click, Reid; George Washington University, USA (rclick@gwu.edu): 2.5.10

Clougherty, Joseph; University of Illinois at Urbana-Champaign \& CEPR-London, USA (jaclough@illinois.edu): 3.3.8

Coduras, Alicia; Universidad Antonio de Nebrija/ Global Entrepreneurship Research Association, Spain

(acoduras@gemconsortium.org): 1.3.13

Colakoglu, Saba; Berry College and Koc University, USA (scolakoglu@berry.edu): 1.3.8, 1.5.14

Colli, Andrea; Bocconi University, Italy (andrea.colli@unibocconi.it): 2.1.12

Collings, David; Dublin City University, Ireland (david.collings@dcu.ie): 3.3.15

Colman, Helene Loe; Norwegian Business School, Norway (helene.I.colman@bi.no): 3.3.12

Colpan, Asli M.; Harvard University, USA (colpan@fas.harvard.edu): 3.1.4

Comma, Jeannine ; University of the West Indies, Barbados (jeannine.comma@cavehill.uwi.edu): 2.1.12

Contractor, Farok J.; Rutgers University, USA (farok@andromeda.rutgers.edu): 1.4.1, 2.3.1, 2.5.4, 3.4.7

Cordeiro-Nilsson, Cheryl; University of Gothenburg, Sweden (cheryl.cordeiro@handels.gu.se): 2.2.3

Coronado, Adrian; University of London, United Kingdom (adrian.coronado@rhul.ac.uk): 3.3.12

Cosset, Jean-Claude; HEC Montreal, Canada (jean-claude.cosset@hec.ca): 3.1.12, 3.4.5

Costa, Sidney; USP, Brazil (sidneycosta@gmail.com): 2.5.5

Couper, Carole; University of Glasgow, United Kingdom (c.couper.1@research.gla.ac.uk): 1.5.5

Crespi, Rafel; Universidad de las Islas Baleares, Spain (rafel.crespi@uib.es): 2.4.12

Crespo, Nuno; Advance, ISEG, Technical University of Lisbon, Portugal (ncrespo@iseg.utl.pt): 2.4.11

Cross, Adam; University of Leeds, United Kingdom (arc@lubs.leeds.ac.uk): 2.2.4, 3.3.12

Crotty, Jo; University of Salford Manchester, United Kingdom (j.crotty@salford.ac.uk): 2.3.11

Cuervo-Cazurra, Alvaro; Northeastern University, USA (a.cuervocazurra@neu.edu): 2.1.3, 2.5.5, 3.3.13, 3.4.9

Cui, Geng; Lingnan University, Hong Kong, SAR-PRC (gcui@In.edu.hk): 2.2.13

Cui, Lin; Australian National University, Australia (lin.cui@anu.edu.au): 3.1.14, 3.2.6, 3.3.8

Cunha, Reynaldo Dannecker; ESPM, Brazil (rcunha@espm.br): 2.1.11

Curci, Roberto; Butler University, USA (rcurci@butler.edu): 2.1.6

Cuypers, llya; Singapore Management University, Singapore (ilyacuypers@smu.edu.sg): 1.5.6, 2.1.7 
Dagnino, Giovanni Battista; University of Catania, Italy (dagnino@unict.it): 2.5.12

Dai, Li; Loyola Marymount University, USA (li.dai@Imu.edu): 2.4.11

Daly, Paula S.; James Madison University, USA (dalyps@jmu.edu): 1.3.12, 2.5.13

Darendeli, Izzet Sidki; Temple University, USA (izzetdarendeli@temple.edu): 3.4.12

Dasi, Angels; Universitat de Valencia, Spain (angels.dasi@uv.es): 1.5.9, 2.4.11

Dastmalchian, Ali; University of Victoria, Canada (dastmal@uvic.ca): 1.1.5

Datta, Deepak K; University of Texas at Arlington, USA (ddatta@uta.edu): 2.2.14, 3.2.7, 3.3.7

Dau, Luis Alfonso; Northeastern University, USA (I.dau@neu.edu): 2.2.13, 3.4.9

Dauth, Tobias; Rouen Business School, France (tdt@rouenbs.fr): 1.5.14

Davidkov, Tzvetan; Sofia University "St. Kliment Ohridski", Bulgaria (tzvetandavidkov@feb.uni-sofia.bg): 1.1.14

Davis, Jeff; Orabrush, USA (jeffdavis@orabrush.com): 3.1.6

Davis, Susannah M.; Nottingham University, China (susannah.davis@nottingham.edu.cn): 2.1.12

Davison, Matt; University of Western Ontario, Canada (mdavison@uwo.ca): 2.2.11

Davis-Sramek, Elizabeth; University of Louisville, USA (beth.davis@louisville.edu): 2.5.6

De Beule, Filip; University of Leuven, Belgium (filip.debeule@kuleuven.be): 2.2.14

de Faria, Pedro; University of Groningen, Netherlands (p.m.m.de.faria@rug.nl): 2.2.6

de Jong, Gjalt; University of Groningen, Netherlands (g.de.jong@rug.nl): 1.4.11

De Prijcker, Sofie; Ghent University, Belgium (sofie.deprijcker@ugent.be): 2.2.5

Deakin, Simon; University of Cambridge, United Kingdom (s.deakin@cbr.cam.ac.uk): 2.4.7

Debrah, Yaw A.; Swansea University, United Kingdom (y.a.debrah@swansea.ac.uk): 1.5.13

Dehon, Catherine; ULB - Solvay Brussels School of Economics and Management, Belgium (cdehon@ulb.ac.be): 3.2.8

Dejoux, Cecile; CNAM Paris, France (dejoux.cecile@gmail.com): 2.3.9

Delios, Andrew; National University of Singapore, Canada (andrew@nus.edu.sg): 0.2, 1.4.12

Deller, Juergen; Leuphana University Lueneburg, Germany (deller@leuphana.de): 2.2.10

Dellestrand, Henrik; Uppsala University, Sweden (henrik.dellestrand@fek.uu.se): 1.5.7, 3.3.12, 3.4.3

Demir, Robert; The Ratio Institute and Stockholm University, Sweden (rd@fek.su.se): 3.1.13

Demirbag, Mehmet; University of Sheffield, United Kingdom (m.demirbag@sheffield.ac.uk): 1.4.6, 3.2.9

Demirer, Petek; Koc University, Turkey (pdemirer@ku.edu.tr): 1.1.2

Deng, Ziliang; Renmin University of China, China (dengziliang@rbs.org.cn): 2.1.6, 3.3.10

Denicolai, Stefano; University of Pavia, Italy (stefano.denicolai@unipv.it): 2.1.5

Desender, Kurt; University Carlos III, Madrid, Spain (kdesende@emp.uc3m.es): 2.4 .12

Devinney, Timothy Michael; University of Technology, Sydney, Australia (timothy.devinney@gmail.com): 2.5.12, 3.1.2

Dhanaraj, Charles; Indiana University, USA (dhanaraj@iupui.edu): 1.1.12, 3.4.15

Dhandapani, Karthik; Indian Institute of Management Ahmedabad, India (dkarthik@iimahd.ernet.in): 2.5.7

Dhayanithy, Deepak; Indian Institute of Management Kozhikode, India (deepak@iimk.ac.in): 3.3.6

Di Maria, Eleonora; University of Padova, Italy (eleonora.dimaria@unipd.it): 2.3.5

Dibble, Rebekah; University of San Fransisco, USA (rdibble@usfca.edu): 2.1.15

Dietz, Joerg; University of Lausanne, Switzerland (jorg.dietz@unil.ch): 3.4.13

Dikova, Desislava; Vienna University of Economics and Business, Austria (desislava.dikova@wu.ac.at): 3.1.9

Dimitratos, Pavlos; University of Glasgow, United Kingdom (pavlos.dimitratos@glasgow.ac.uk): 1.1.9, 1.3.13

Dimitriadi, Nikolay; Rostov State Economic University (RINH), Russia (ndimitriadi@yandex.ru): 1.1.5

Dimitrova, Mihaela; University of Wisconsin-Milwaukee, USA (mihaela@uwm.edu): 1.1.14, 2.3.9

Dinh, Trung Quang; University of Akureyri, Vietnam (quangtrung38@gmail.com): 3.1.15

Dixit, Jaya; Rensselaer Polytechnic Institute, USA (dixitj@rpi.edu): 3.4.11

Doh, Jonathan; Villanova University, USA (jonathan.doh@villanova.edu): 1.3.1, 2.4.10, 3.2.3

Dolles, Harald; Molde University College, Norway (harald.dolles@himolde.no): 3.1.15

Donaldson, Lex; University of New South Wales, Australia (lexd@agsm.edu.au): 2.2.12

Dornberger, Utz; Leipzig University, Germany (dornberger@uni-leipzig.de): 3.4.15

Dorobantu, Sinziana; New York University, USA (sdoroban@stern.nyu.edu): 2.4.12 
Douglas Fernandez, W.G.; Florida International University, USA (wdoug002@fiu.edu): 1.1.12, 3.4.12

Dow, Douglas; Melbourne Business School, Australia (d.dow@mbs.edu): 2.4.6

Dow, Sandra; Monterey Institute of International Studies, USA (sandra.dow@miis.edu): 2.4.12

Dowling, Peter John; La Trobe University, Australia (p.dowling@latrobe.edu.au): 3.2 .5

Doz, Yves; INSEAD, France (yves.doz@insead.edu): 3.3.1

Dragon, Katharina Agnes; Fundacao Getulio Vargas, Brazil (katha_dragon@gmx.de): 1.1.15

Drape, Thomas Gene; Azusa Pacific University, USA (tdrape@pugetsound.edu): 3.2.12

Dreyer, Laetitia; ESC Rennes Business School, France (laetitiadreyer@numericable.fr): 2.3.14

Driffield, Nigel; Aston University, United Kingdom (n.I.driffield@aston.ac.uk): 2.3.11, 3.2.10

Drogendijk, Rian; Uppsala University, Sweden (rian.drogendijk@fek.uu.se): 0.2, 1.3.2, 2.2.9, 3.3.12

Drost, Ellen A.; California State University Los Angeles, USA (edrost@calstatela.edu): 1.1.14

Du, Guochen; Peking University, China (dugch@gsm.pku.edu.cn): 1.4.14

Du, Min; University of Nottingham, United Kingdom (lixmd10@nottingham.ac.uk): 2.2.14

Du, Yunzhou; Anhui University of Finance \& Economics, China (duyunzhou_2000@sina.com): 3.4.7

Duanmu, Jing-Lin; University of Surrey, United Kingdom (j.duanmu@surrey.ac.uk): 3.3.12

DuBois, Frank; American University, USA (jetfrank@yahoo.com): 2.4.15

Dunlap, Denise; Northeastern University, USA (d.dunlap@neu.edu): 3.2.13

Duran, Patricio; University of South Carolina, USA (patricio.duran@grad.moore.sc.edu): 2.5.10

Durand, Aurelia; HEC Montreal, Canada (aurelia.durand@hec.ca): 1.1.6

Duran-Zurita, Edwin; Universidad Privada Boliviana, Bolivia (eduran@upb.edu): 3.4.6

Duso, Tomaso; Deutsche Institut fuer Wirtschaftsforschung (DIW) and DICE, Heinrich-Heine University Duesseldorf, Germany (duso@dice.hhu.de): 3.3.8

Duvivier, Florence; Solvay Brussels School of Economics and Management, Belgium (florence.duvivier@ulb.ac.be): 3.3.14

\section{E}

Eager, Wendy; Eastern Washington University, USA (weager@ewu.edu): 1.4.8

Eddleston, Kimberly; Northeastern University, USA (k.eddleston@neu.edu): 1.5.11

Eden, Lorraine; Texas A\&M University, USA (leden@tamu.edu): 0.2, 2.1.9, 2.2.2, 2.3.7, 2.5.10

Edman, Jesper; Hitotsubashi University, Japan (jedman@ics.hit-u.ac.jp): 3.4.1

Egelhoff, William G.; Fordham University, USA (egelhoff@fordham.edu): 2.3.10, 3.4.3

Eghbali-Zarch, Majid; University of Western Ontario, Canada (meghbali-zarch.phd@ivey.ca): 1.4.10, 3.3.3

Eisenberg, Jacob; University College Dublin, Ireland (jacob.eisenberg@ucd.ie): 2.5.13, 3.4.13

Elahee, Mohammad Niamat; Quinnipiac University, USA (mohammad.elahee@quinnipiac.edu): 0.4, 2.3.14

Elango, B.; Illinois State University, USA (elango@ilstu.edu): 3.1.14

Elbanna, Said; UAEU, United Arab Emirates (selbanna@uaeu.ac.ae): 1.1.9

Elia, Stefano; Politecnico di Milano, Italy (stefano.elia@polimi.it): 1.4.9, 2.2.14

Enderwick, Peter; Auckland Technical university, New Zealand (peter.enderwick@aut.ac.nz): 2.3.6

Engeler, Martin; University of St. Gallen, Switzerland (martin.engeler@unisg.ch): 3.4.13

Engle, Robert L.; Quinnipiac University, USA (robert.engle@quinnipiac.edu): 1.1.5

Erarslan, Ayse Burcin; Koc University, Turkey (aerarslan@ku.edu.tr): 2.2.3

Eriksson, Kent; Royal Institute of Technology, Sweden (kent.eriksson@abe.kth.se): 2.3.13, 2.4.10

Eriksson, Mikael; Uppsala University, Sweden (mikael.eriksson@fek.uu.se): 3.3 .12

Erkus, Ahmet; Gedik University, Turkey (ahmet.erkus@gedik.edu.tr): 3.2.15

Ernst, Ricardo; Georgetown University, USA (ernstr@georgetown.edu): 2.5.6

Ertug, Gokhan; Singapore Management University, Singapore (gokhanertug@smu.edu.sg): 1.5.6, 2.1.7

Eskinazi, Hande; Director of Corporate Strategy and Business, Pfizer, Turkey: 2.5.2

Ettlie, John E.; Rochester Institute of Technology, USA (jettlie@saunders.rit.edu): 1.1.9

Evirgen, Cuneyt; Sabanci University, Turkey (cuneyt-edu@sabanciuniv.edu): 1.4.3

\section{$\mathbf{F}$}

Fabian, Frances; University of Memphis, USA (ffabian@memphis.edu): 1.3.14 
Fan, Bo; Southern New Hampshire University, USA (bo.fan@snhu.edu): 1.3.15

Fan, Di; Victoria University, Australia (di.fan@vu.edu.au): 2.3.9

Fan, Qingli; St. Andrews University, United Kingdom (lilifan1994@hotmail.com): 2.5.11

Fan, Terence Ping-Ching; Singapore Management University, Singapore (terencefan@smu.edu.sg): 1.1.9

Fan, Yang; Erasmus University, Netherlands (yfan@rsm.nl): 2.3.12

Fan, Zheng; Shanghai International Studies University, China (fanzh280@shisu.edu.cn): 2.5.11

Fang, Tony; Stockholm University, Sweden (tony.fang@fek.su.se): 2.2.3

Farashahi, Mehdi; Concordia University, Canada (mfarashahi@jmsb.concordia.ca): 3.4.15

Farndale, Elaine; Pennsylvania State University / Tilburg University, USA (euf3@psu.edu): 1.3.8, 2.5.13

Farrell, Carlyle; Ryerson University, Canada (farrellc@ryerson.ca): 3.4.14

Fastoso, Fernando; Bradford University, United Kingdom (f.fastoso@bradford.ac.uk): 1.4.14

Fathallah, Ramzi; University of Western Ontario, Canada (rfathallah.phd@ivey.ca): 1.1.14

Fazekas, Balazs; Kyoto University, Japan (baraju77@gmail.com): 1.3.15

Fee, Anthony; University of Technology Sydney, Australia (anthony.fee@uts.edu.au): 3.3.14

Feinberg, Susan Elizabeth; Temple University, USA (feinberg@temple.edu): 2.3.11, 3.4.8

Feldman, Percy M.; Pontificia Universidad Católica de Peru, Peru (percy.marquina@pucp.pe): 3.3.6

Felicio, Wilson; Insper, Brazil (zelucaco@hotmail.com): 2.2.11

Felzensztein, Christian; Universidad Adolfo Ibanez, Chile (c.felzensztein@uai.cl): 2.1.10, 2.4.15

Feng, Jing Betty; Georgia State University, USA (jfeng4@gsu.edu): 2.1.8, 2.2.9

Feng, Liyun; A.P. Moller Maersk Group A/S, Denmark (liyun.feng@maersk.dk): 2.5.11

Feng, Taiwen; Northwestern Polytechnical University, China (typhoonfeng@gmail.com): 2.3.9

Fenton-O'Creevy, Mark; Open University, United Kingdom (m.p.fenton-ocreevy@open.ac.uk): 3.4.13

Fernhaber, Stephanie A.; Butler University, USA (sfernhab@butler.edu): 2.4.11

Ferreira, Manuel Portugal; Uninove - Universidade Nove de Julho, Brazil (manuel.portugal.ferreira@gmail.com): 2.1.7, 2.2.7, 3.1.14

Fey, Carl Felix; Nottingham University, China (carl.fey@hhs.se): 0.5, 2.1.8, 3.2.1

Fiaschi, Davide; University of Pisa, Italy (dfiaschi@ec.unipi.it): 3.3.10

Ficici, Aysun; Southern New Hampshire University, USA (a.ficici@snhu.edu): 1.3.15

Figueira-de-Lemos, Francisco; Uppsala University, Sweden (francisco.lemos@fek.uu.se): 1.1.12

Figueiredo, Paulo N.; Brazilian School of Public and Business Administration (EBAPE), Brazil (pnf@fgv.br): 2.3.12

Filatotchev, Igor; City University London, United Kingdom (igor.filatotchev@city.ac.uk): 2.5.9

Filip, Andrei; ESSEC, France (filip@essec.fr): 3.1.12

Filipi, Gjergji; AGENDA Institute, Albania (gjergji.filipi@gmail.com): 2.3.4

Filippov, Sergey; Delft University of Technology, Netherlands (s.filippov@tudelft.nl): 2.2.1, 2.5.7

Fini, Riccardo; University of Bologna, Italy (riccardo.fini@unibo.it): 1.3.11

Fisch, Jan Hendrik; University of Augsburg, Germany (fisch@wiwi.uni-augsburg.de): 1.1.4, 3.1.10, 3.2.15

Fischbach, Dirk; Hochschule Harz, Germany (dfischbach@hs-harz.de): 3.3.4, 3.4.4

Fitzsimmons, Stacey R.; Western Michigan University, USA (stacey.fitzsimmons@wmich.edu): 3.4.13

Fletcher, Margaret; University of Glasgow, United Kingdom (margaret.fletcher@glasgow.ac.uk): 2.3.8

Fleury, Afonso; University of São Paulo, Brazil (acfleury@usp.br): 1.1.3, 1.4.6, 2.4.13

Fleury, Maria Tereza; Fundacao Getulio Vargas, Brazil (mtereza.fleury@fgv.br): 1.1.3, 2.4.13, 3.4.9

Flores, Ricardo; University of New South Wales, Australia (r.flores@unsw.edu.au): 1.3.2

Floyd, Steven W.; University of Massachusetts Amherst, USA (sfloyd@isenberg.umass.edu): 1.4.13

Fong, Cher-Min; National Sun Yat-Sen University, Taiwan (cmfong@bm.nsysu.edu.tw): 1.3.15, 3.4.7

Fontes, Margarida; UMOSE - Laboratório Nacional de Engenharia e Geologia and Dinamia, Portugal (margarida.fontes@lneg.pt): 2.4.11

Ford, John B.; Old Dominion University, USA (jbford@odu.edu): 3.1.15

Forquer Gupta, Susan; Monmouth University, USA (sgupta@monmouth.edu): 3.3.4, 3.4.4

Forsans, Nicolas; University of Leeds, United Kingdom (nf@lubs.leeds.ac.uk): 2.3.6

Forte, Rosa Portela; University of Porto, Portugal (rforte@fep.up.pt): 2.5.5

Francesco, Anne Marie; Hong Kong Baptist University, Hong Kong, SAR-PRC (amfrancesco@yahoo.com): 2.5.13, 3.4.13

Freeman, Susan; University of Adelaide, Australia (susan.freeman@adelaide.edu.au): 2.1.11, 2.3.9 
Freiling, Jörg; University of Bremen, Germany (freiling@uni-bremen.de): 3.1.5

Friesen, Daniel; Wayne State University, USA (dy8750@wayne.edu): 2.4.15

Froese, Fabian Jintae; Georg-August-University Goettingen, Germany (fabian.froese@gmail.com): 2.5.8, 3.2.5

Fu, Frank; Hong Kong Baptist University, Hong Kong, SAR-PRC (frankfu@hkbu.edu.hk): 3.2.14

Fu, Pingping; Chinese University of Hong Kong, Hong Kong, SAR-PRC (ppfu@cuhk.edu.hk): 1.4.15

Fu, Xiaolan; Oxford University, United Kingdom (xiaolan.fu@qeh.ox.ac.uk): 2.4.10, 3.4.9

Fuchs, Manfred; University of Graz, Austria (manfred.fuchs@uni-graz.at): 2.2.12

Fuerst, Sascha; Universidad EAFIT, Colombia (sfuerst@eafit.edu.co): 1.3.3

G

Gaffney, Nolan; University of North Texas, USA (nolan.gaffney@unt.edu): 1.3.14, 3.3.8

Galperin, Bella; University of Tampa, USA (bgalperin@ut.edu): 2.3.3

Gammelgaard, Jens; Copenhagen Business School, Denmark (jg.int@cbs.dk): 2.5.11

Gan, Bernard; University of New South Wales, Australia (leo0869@gmail.com): 2.2.12, 3.4.11

Gang, KwangWook; Rensselaer Polytechnic Institute, USA (gangk@rpi.edu): 3.4.11

Gao, Gerald Yong; University of Missouri-St. Louis, USA (gaogy@umsl.edu): 3.1.9

Gao, Zhaojun; Peking University, China (thinking4ever2009@pku.edu.cn): 2.5.15

Garcia, P. Roberto; Indiana University, USA (prgarcia@indiana.edu): 2.5.8

Garcia-Prieto, Patricia; ULB - Solvay Brussels School of Economics and Management, Belgium

(pgarciap@ulb.ac.be):3.2.8

Gardberg, Naomi A.; Baruch College-CUNY, USA (naomi.gardberg@baruch.cuny.edu): 2.5.14, 3.1.14

Garrido, Ivan Lapuente; UNISINOS - Universidade do Vale do Rio dos Sinos, Brazil (igarrido@unisinos.br): 2.5.12

Gatignon, Aline; INSEAD, France (aline.gatignon@insead.edu): 1.5.10

Gaur, Ajai; Rutgers University, USA (ajai@business.rutgers.edu): 3.4.7

Gedajlovic, Eric R.; Simon Fraser University, Canada (erg@sfu.ca): 2.4.9

Geleilate, Jose Mauricio; Florida International University, USA (jgele002@fiu.edu): 2.5.7, 3.4.6

Genc, Mehmet Erdem; Montclair State University, USA (memogenc@gmail.com): 3.1.14

Genc, Omer Faruk; Temple University, USA (omer.genc@temple.edu): 1.3.15

Geng, Xuesong; Singapore Management University, Singapore (xsgeng@smu.edu.sg): 1.4.9, 2.1.5

George, Suresh; Coventry University, United Kingdom (s.george@coventry.ac.uk): 2.4 .8

Germain, Richard; University of Louisville, USA (richard.germain@louisville.edu): 2.5.6

Geyikdagi, Necla V.; Yeditepe University, Turkey (geyikdagi@superonline.com): 1.3.15

Ghauri, Pervez N.; King's College London, United Kingdom (pervez.ghauri@kcl.ac.uk): 1.4.3, 2.2.7, 2.3.11, 3.1.2

Ghemawat, Pankaj; IESE, Spain (pghemawat@iese.edu): 0.7, 1.2, 2.2.2

Ghorbani, Majid; Renmin University of China, China (majidghorbani@rbs.org.cn): 1.3.4, 3.2.15

Giachetti, Claudio; Ca' Foscari University of Venice, Italy (claudio.giachetti@unive.it): 2.3.10, 2.5.12

Giblin, Majella; Trinity College Dublin, Ireland (giblinma@tcd.ie): 3.3.15

Gilbert, Brett; Rutgers University, USA (bagilbert@business.rutgers.edu): 2.4.11

Girma, Sourafel; University of Nottingham, United Kingdom (sourafel.girma@nottingham.ac.uk): 2.2.14

Giroud, Axele; UNCTAD, Switzerland (axele.giroud@unctad.org): 2.3.7, 3.3.13

Giuliani, Elisa; University of Pisa, Italy (giulel@ec.unipi.it): 2.4.3, 3.3.10

Gleason, Kimberly; University of Pittsburgh, USA (gleasonk@pitt.edu): 2.2.11

Gleich, Wolfgang M.; University of Augsburg, Germany (wolfgang.gleich@wiwi.uni-augsburg.de): 1.1.4, 2.2.15

Godinez, Jose; University of Edinburgh, United Kingdom (j.r.godinez@sms.ed.ac.uk): 1.3.14

Goelzner, Herbert; Salzburg University of Applied Sciences and Technologies, Austria (herbert.goelzner@fhsalzburg.ac.at): 3.2 .15

Goerzen, Anthony; Queen's University, Canada (agoerzen@business.queensu.ca): 2.1.7, 2.3.14

Gokalp, Omer Nesim; University of Texas at Dallas, USA (omer.gokalp@utdallas.edu): 1.5.13

Golgeci, Ismail; University of Vaasa, Finland (igolgeci@uva.fi): 2.1.11

Gong, Yuan-yuan; Kyoto University, Japan (lillian.gong@gmail.com): 1.3.15

Gong, Yundan; Aston University, United Kingdom (y.gong1@aston.ac.uk): 2.2.14

Gonzalez-Perez, Maria Alejandra; Universidad EAFIT, Colombia (mgonza40@eafit.edu.co): 3.2.7, 3.3.4, 3.4.4

AIB 2013 Conference Proceedings

Page 305 
Goodell, John W.; University of Akron, USA (johngoo@uakron.edu): 1.1.7, 2.5.9

Gooris, Julien; Université libre de Bruxelles, Belgium (jgooris@ulb.ac.be): 1.1.12

Görg, Holger; University of Kiel and CEPR, Germany (holger.goerg@ifw-kiel.de): 2.2.14

Gray, Sidney John; University of Sydney, Australia (sid.gray@sydney.edu.au): 1.5.15, 2.4.14

Greve, Peder; University of St. Gallen, Switzerland (peder-mathias.greve@unisg.ch): 2.3.4, 2.5.14, 3.1.14, 3.4.13

Grimpe, Christoph; Copenhagen Business School, Denmark (cg.ino@cbs.dk): 2.4.10

Grogaard, Birgitte; University of Calgary, Canada (bgrogaar@ucalgary.ca): 2.3.10, 3.3.12

Grosse, Robert; George Mason University, USA (rgrosse@gmu.edu): 0.6, 0.7, 1.5.1, 2.1.10

Gu, Qian; Georgia State University, USA (qgu@gsu.edu): 1.1.15, 3.3.5, 3.4.10

Gubbi, Sathyajit; University of Groningen, Netherlands (s.r.gubbi@rug.nl): 1.5.6, 3.4.14

Guedri, Zied; EMLyon Business School, France (guedri@em-lyon.com): 3.1.14

Guenther, Christina; WHU, Germany (christina.guenther@whu.edu): 1.5.15

Guerra, Massiel; Universidad del Desarrollo, Chile (mguerra@udd.cl): 3.4.6

Guillaumon, Siegrid; Catholic University of Brasilia, Brazil (ziggybahia@yahoo.com.br): 2.2.3

Gunkel, Marjaana; Leuphana University Lueneburg, Germany (mgunkel@inkubator.leuphana.de): 1.1 .5

Guo, Liang; Rouen Business School, France (Igg@rouenbs.fr): 2.4 .9

Guo, Wenxin; University of Illinois at Urbana-Champaign, USA (guo33@illinois.edu): 3.3.8

Gupta, Anil; University of Maryland at College Park, USA (agupta@rhsmith.umd.edu): 3.2.13

Gupta, Roopak Kumar; Indian Institute of Management Indore, India (f11roopakk@iimidr.ac.in): 2.5.9

Gur, Furkan Amil; Louisiana State University, USA (fgur1@Isu.edu): 2.5.14

Guttormsen, David Sapto Adi; Coventry University, United Kingdom (david.guttormsen@coventry.ac.uk): 1.3.13, 3.2.14

$\mathbf{H}$

Ha, Yoo Jung; University of York, United Kingdom (yoojung.ha@york.ac.uk): 3.3.13

Hadjikhani, Amjad; Uppsala University, Sweden (amjad.hadjikhani@fek.uu.se): 2.2.7

Hagen, Birgit; University of Pavia, Italy (bhagen@eco.unipv.it): 1.4.13

Hagen, James M.; Hamline University, USA (jhagen05@hamline.edu): 1.3.9, 2.5.8

Hamblin Wang, Sophia; Australian National University, Australia (sophia.hamblinwang@anu.edu.au): 1.3.9, 3.1.14

Hammes, Martin; WHU, Germany (martin.hammes@whu.edu): 1.5.15

Han, I; Feng Chia University, Taiwan (ihan@fcu.edu.tw): 1.5.15

Han, Jian; China Europe International Business School, China (jianhan@ceibs.edu): 3.1.13

Han, Yi; Peking University, China: 1.4.14

Hannigan, Thomas J.; Temple University, USA (tj.hannigan@temple.edu): 3.2.13

Hansen, Michael W; Copenhagen Business School, Denmark (mwh.ikl@cbs.dk): 3.2.11

Harindranath, G.; University of London, United Kingdom (g.harindranath@rhul.ac.uk): 3.3.12

Harris, Simon; University of Edinburgh, United Kingdom (simon.harris@ed.ac.uk): 1.5.8, 2.3.8

Harvey, Michael; University of Mississippi, USA (mharvey@bus.olemiss.edu): 2.3.13

Harzing, Anne-Wil; Melbourne University, Australia (anne@harzing.com): 1.3.8

Hatem , Tarek; American University in Cairo, Egypt (tarekha@aucegypt.edu): 1.5.5

Hawkins, Matthew Allen; ESADE Business School, Spain (matthewallen.hawkins@esade.edu): 2.4.6

Haxhi, Ilir; University of Amsterdam, Netherlands (i.haxhi@uva.nl): 3.1.8

He, Wei; Purdue University North Central, USA (weihe@pnc.edu): 2.3.15

Hearn, Bruce; University of Sussex, United Kingdom (b.a.hearn@sussex.ac.uk): 3.4.5

Hebert, Louis; HEC Montreal, Canada (louis.hebert@hec.ca): 2.2.13

Hemmert, Martin; Korea University, Korea, South (mhemmert@korea.ac.kr): 2.2.14

Hendrix, Maurice; Coventry University, United Kingdom (maurice.hendrix@coventry.ac.uk): 2.4.8

Henisz, Witold; University of Pennsylvania, USA (henisz@wharton.upenn.edu): 2.4.12

Hennart, Jean-Francois; Singapore Management University and University of Pavia, Italy (j.f.hennart@uvt.nl): 1.5.6, 2.1.3, 2.3.1, 3.2.11, 3.4.5

Hermann, Charles ; Texas A\&M University, USA (hermann@tamu.edu): 2.2.7

Hermans, Michel; IAE Business School - Austral University, Argentina (mhermans@iae.edu.ar): 3.4.6 
Heugens, Pursey P.M.A.R.; Erasmus University, Netherlands (pheugens@rsm.nl): 2.4.9, 2.5.10

Hewerdine, Lisa; University of Sydney, Australia (lisa.hewerdine@sydney.edu.au): 3.4.15

Hikino, Takashi; Kyoto University, Japan (hikino@econ.kyoto-u.ac.jp): 3.1.4

Hill, T.L.; Temple University, USA (t1201@temple.edu): 3.4.12

Hilmarsson, Hilmar Thor; University of Akureyri, Iceland (hilmar@unak.is): 3.1.15

Hilmersson, Mikael; Linnaeus University, Sweden (mikael.hilmersson@Inu.se): 2.4.14

Hirschi, Andreas; University of Lausanne, Switzerland (andreas.hirschi@unil.ch): 2.2.10

Hitotsuyanagi, Azusa; Georg-August-Universitaet Goettingen, Germany (azusa.hitotsuyanagi@wiwi.unigoettingen.de): 3.2 .5

Hitt, Michael A.; Texas A\&M University, USA (mhitt@mays.tamu.edu): 3.3.3

Ho, Mia Hsiao-Wen; Soochow University, Taiwan (mia.hohw@gmail.com): 2.3.15

Ho, Yi-Hui; Chang Jung Christian University, Taiwan (vicky@mail.cjcu.edu.tw): 1.1.15, 1.5.14

Hoenen, Anne Kristin; WU Vienna, Austria (anne.hoenen@wu.ac.at): 2.5.11, 3.2.11

Hofer, Katharina Maria; Johannes Kepler University Linz, Austria (katharina.hofer@jku.at): 2.4.5

Hofstede, Geert; University of Maastricht, Netherlands (hofstede@bart.nl): 3.1.1

Hohberger, Jan; University of Technology, Sydney, Australia (jan.hohberger@uts.edu.au): 3.4.11

Holm, Ulf; Uppsala University, Sweden (ulf.holm@fek.uu.se): 3.3.12

Holtbruegge, Dirk; University of Erlangen-Nuernberg, Germany (dirk.holtbruegge@wiso.uni-erlangen.de): 2.2.10

Hong, Eunsuk; SOAS, University of London, United Kingdom (e.hong@soas.ac.uk): 2.2.6

Hong, Gahye; Korea University, Korea, South (gahye.hong@gmail.com): 2.5.13

Hong, Hae-Jung; Rouen Business School, France (hjh@rouenbs.fr): 2.1.15

Hong, Jung-Kee; University of South Australia, Australia (honjk002@live.com): 1.1.7

Hong, Sungjin J.; Queen's University Belfast, United Kingdom (s.hong@qub.ac.uk): 2.1.7

Horaguchi, Haruo Hagiwara; Hosei University, Japan (horaguch@hosei.ac.jp): 2.2.11, 3.2.14

Horn, Sierk; University of Leeds, United Kingdom (s.horn@leeds.ac.uk): 2.2.4

Hornung, Frank; University of Giessen, Germany: 3.3.7

Hoskisson, Robert; Rice University, USA (robert.hoskisson@rice.edu): 2.5.12

Hosseini, Hamid S.; King's College, USA (hamidhosseini@kings.edu): 3.1.7

Hotho, Jasper; Copenhagen Business School, Denmark (jjh.smg@cbs.dk): 2.2.13, 3.4.10

Howe, David C.; University of Utah, USA (dave.c.howe@gmail.com): 1.3.13

Hruby, Jörg; Hochschule für Unternehmensführung, Germany (hruby@bwl-studieren-ohne-grenzen.de): 1.4.15,

2.1.15

Hsieh, Chueh-An; National Sun Yat-Sen University, Taiwan (chsieh@mail.nsysu.edu): 3.1.5

Hsieh, Linda Hsiu-yun; University of Birmingham, United Kingdom (h.hsieh@bham.ac.uk): 3.3.7

Hsieh, Ru-Mei; National Pingtung University of Science and Technology, Taiwan (giordanoisu@yahoo.com.tw): 1.5.5

Hsu, Chia-Wen; National Chung Cheng University, Taiwan (cwhsu@ccu.edu.tw): 1.1.8, 2.2.8

Hsu, Wen Chung; National Chi Nan University, Taiwan (wchsu25@gmail.com): 2.3.5

Hu, Helen; University of Melbourne, Australia (hehu@unimelb.edu.au): 3.2.6

Hu, Tianyou; National University of Singapore, Singapore (ht@nus.edu.sg): 2.3.6

Huang, Chun Ju; National Chung Hsing University, Taiwan (scar133@gmail.com): 1.1.4

Huang, Kenneth; Singapore Management University, Singapore (kennethhuang@smu.edu.sg): 1.4.9, 2.1.5

Huang, Ming-Chang; Providence Univesity, Taiwan (mchuang@pu.edu.tw): 2.3.13, 2.4.4

Huang, Victor Zengyu; Zayed University, United Arab Emirates (victor.huang@zu.ac.ae): 2.5.8

Huang, Ying; University of Massachusetts Lowell, USA (ying_huang1@uml.edu): 2.3.14

Hudson, Monika; University of San Francisco, USA (mgtgrp@hotmail.com): 1.5.3

Hughes, Douglas E.; Michigan State University, USA (dhughes@msu.edu): 2.5.6

Hui, Kent; Michigan State University, USA (hui@bus.msu.edu): 3.4.7

Hult, Tomas; Michigan State University, USA (hult@msu.edu): 1.1.1, 2.4.5, 3.4.7

Hunoldt, Michael; Friedrich Schiller University of Jena, Germany (michael.hunoldt@uni-jena.de): 3.3.7

Husted, Bryan; York University \& Tecnologico de Monterrey, Canada (bryanhusted@gmail.com): 3.4.8

Hutzschenreuter, Thomas; WHU Otto Beisheim School of Management, Germany (th@whu.edu): 1.3.3, 1.5.15,

2.4.1, 3.3.1 
Iborra, Maria; Universitat de Valencia, Spain (miborra@uv.es): 2.4.11

Igan, Deniz; International Monetary Fund, USA (digan@imf.org): 1.1.13

Imbach, Mathias; University of St. Gallen, Switzerland (mathias.imbach@unisg.ch): 2.5.14

Ireneo-Manalo, Teresita; Acsenda School of Management, Canada (tessi@Acsenda.com): 1.4.5

Isidor, Rodrigo; University of Paderborn, Germany (rodrigo.isidor@wirtschaft.uni-giessen.de): 3.3.7

Ito, Jun; National University of Singapore, Singapore (junito@nus.edu.sg): 3.4.14

Ito, Kiyohiko; University of Hawaii at Manoa, USA (k.ito@hawaii.edu): 2.3.10

\section{$J$}

Jain, Arun Kumar; Indian Institute of Management Lucknow, India (arunjain@iiml.ac.in): 3.1.14

Jain, Naveen Kumar; University of Akron, USA (njain@uakron.edu): 2.2.13

Jaklic, Andreja; University of Ljubljana, Slovenia (andreja.jaklic@fdv.uni-lj.si): 3.4.12

James, Barclay E.; Louisiana State University, USA (bjames@lsu.edu): 1.4.6

Janavaras, Basil; Minnesota State University, USA (basil.janavaras@mnsu.edu): 2.4 .8

Janczak, Sergio; Kings University College at Western University, Canada (sjanczak@uwo.ca): 1.5.7

Jandhyala, Srividya; George Washington University, USA (srividya@gwu.edu): 2.5.12, 3.2.3

Janne, Odile E.M.; University of London, United Kingdom (o.janne@bbk.ac.uk): 2.2.9

Järlström, Maria; University of Vaasa, Finland (maria.jarlstrom@uva.fi): 1.5.9

Jarosinski, Miroslaw; Warsaw School of Economics, Poland (mjaros@sgh.waw.pl): 3.3.4, 3.4.4

Jaussaud, Jacques; University of Pau UPPA, France (jacques.jaussaud@univ-pau.fr): 2.2.12

Javalgi, Rajshekhar (Raj) G.; Cleveland State University, USA (r.javalgi@csuohio.edu): 1.4.14, 2.3.8

Javidan, Mansour; Thunderbird School of Global Management, USA (mansour.javidan@thunderbird.edu): 2.1.15, 3.3.2

Jaw, Bih-Shiaw; National Sun Yat-Sen University, Taiwan (bsjaw510@mail.nsysu.edu.tw): 2.5.14

Jaw, Y.L.; National Taiwan University, Taiwan (yljaw@management.ntu.edu.tw): 2.3.14

Jean, Ruey-Jer Bryan; National Chengchi University, Taiwan (bryan@nccu.edu.tw): 2.1.6, 2.5.15, 3.3.10

Jena, Deepak; Indian School of Business, India (deepak_jena@isb.edu): 3.2.6

Jensen, Karina R.; Reims Management School, France (karina.jensen@reims-ms.fr): 2.3.12

Jensen, Peter D. Oerberg; Copenhagen Business School, Denmark (poe.smg@cbs.dk): 2.2.15

Jeong, Insik; Korea University, Korea, South (ijeong@korea.ac.kr): 1.5.8

Jeong, Sophia; Chinese University of Hong Kong, Hong Kong, SAR-PRC (sophia@baf.msmail.cuhk.edu.hk): 1.4.15

Jeong, Yujin; American University, USA (yjeong@american.edu): 2.5.10

Ji, Fiona Xiaoying; Ohio University, USA (jix@ohio.edu): 3.1.5

Jiang, Fuming; Curtin University, Australia (fuming.jiang@curtin.edu.au): 1.4.6

Jiang, Ruihua Joy; Oakland University, USA (jiang@oakland.edu): 1.5.11

Jiang, Yuan; Indiana University - Purdue University Fort Wayne, USA (jiangy@ipfw.edu): 1.5.14

Jiménez, Alfredo; University of Burgos, Spain (ajimenez@ubu.es): 2.5.8, 3.3.4, 3.4.4

Jindra, Björn; Halle Institute for Economic Research, Germany (bjoern.jindra@iwh-halle.de): 1.4.11

Joardar, Arpita; Clark University, USA (ajoardar@clarku.edu): 2.1.6, 2.4.11

Joebstl, Beatrix; University of Graz, Austria (beatrix.joebstl@edu.uni-graz.at): 2.2.12

Joh, Gun-Ho; San Diego State University, USA (gun.joh@sdsu.edu): 3.1.12

Johansson, Johny K.; Georgetown University, USA (johanssj@georgetown.edu): 1.3.5, 2.4.15, 3.1.9

Johns, Adam; Rikkyo University, Japan (johns@rikkyo.ac.jp): 3.3.4, 3.4.4

Johnson, James P; Rollins College, USA (jpjohnson@rollins.edu): 2.5.7, 3.2.12

Jokinen, Tiina; University of Vaasa, Finland (tiina.jokinen@uva.fi): 1.5.9

Jones, Chris; Aston University, United Kingdom (c.jones2@aston.ac.uk): 2.3.11, 3.3.3

Jones, Geoffrey; Harvard Business School, USA (gjones@hbs.edu): 2.5.4, 3.1.4

Jones, Marian Vanessa; University of Glasgow, United Kingdom (marian.v.jones@glasgow.ac.uk): 1.3.3, 1.4.13

Jones, Stephen; University of Minnesota, USA (jone2093@umn.edu): 2.4.10

Jonsen, Karsten; IMD, Switzerland (karsten.jonsen@imd.org): 1.5.14, 2.1.15, 3.4.13

Joplin, Janice R. W.; Southern Illinois University Edwardsville, USA (jjoplin@siue.edu): 2.5.13, 3.3.14 
Ju, Min; University of Missouri - St Louis, USA (jum@umsl.edu): 1.5.8

Jun, Jongkun; Hankuk University of Foreign Studies, Korea, South (jkjun@hufs.ac.kr): 1.5.9

K

Kaartemo, Valtteri; Turku School of Economics, Finland (valtteri.kaartemo@utu.fi): 3.2.12

Kabst, Rudy; University of Paderborn, Germany (kabst@upb.de): 3.3.7

Kalafatoglu, Tugba; ESADE Business School, Spain (tugba@tugbakalafatoglu.com): 3.2.2, 3.2.4

Kalinic, Igor; University of Groningen, Netherlands (i.kalinic@rug.nl): 1.1.14

Kalinowski, Margarete; ESADE Business School, Spain (margarete.kalinowski@esade.edu): 3.2.6

Kamal, Fariha; US Census, USA (fkamal@maxwell.syr.edu): 1.1.13

Kandogan, Yener; University of Michigan-Flint, USA (yener@umflint.edu): 2.2.15

Kang, Haiying; University of South Australia, Australia (haiying.kang@mymail.unisa.edu.au): 3.2.5

Kang, Hyoseok; Seoul National University, Korea, South (hyokang@live.com): 3.3.15

Kang, Min-Ping; National Taiwan Normal University, Taiwan (mpkang@ntnu.edu.tw): 2.1.14

Kang, Shin Hyung; KAIST Business School, Korea, South (davidkang@business.kaist.ac.kr): 3.2.11

Kannan, Yezen; Duquesne University, USA (kannany@duq.edu): 2.2.11

Kano, Liena; University of Calgary, Canada (liena.kano@haskayne.ucalgary.ca): 2.2.14

Kappen, Philip; Uppsala University, Sweden (philip.kappen@fek.uu.se): 2.2.12, 2.3.12

Kara, Aycan; Florida Atlantic University, USA (akara@fau.edu): 3.1.1, 3.2.15

Karadeniz, Esra; Yeditepe University, Turkey (ekaradeniz@yeditepe.edu.tr): 1.1.10

Karaevli, Ayse; Sabanci University, Turkey (akaraevli@sabanciuniv.edu): 1.1.3

Karakas, Fahri; University of East Anglia, United Kingdom (fahrikarakas@gmail.com): 3.4.13

Karam, Charlotte M.; American University of Beirut, Lebanon (ck16@aub.edu.lb): 3.4.6

Karaman, Filiz; Yildiz Technical University, Turkey (filiz_karaman@hotmail.com): 1.3.15

Karamustafa, Guldem; University of Geneva, Switzerland (guldem.karamustafa@unige.ch): 3.1.6

Karmali, Shazia; University of Victoria, Canada (skarmali@uvic.ca): 1.1.14

Karna, Amit; EBS Business School, Germany (amit.karna@ebs.edu): 1.4.9, 2.5.7

Karst, Rusty; University of North Texas, USA (rusty.karst@unt.edu): 3.3.8

Katsikea, Evangelia; Athens University of Economics and Business, Greece (ekt@aueb.gr): 2.1.11

Katsikeas, Constantine; Leeds University, United Kingdom (csk@lubs.leeds.ac.uk): 1.4.2

Kavadis, Nikolaos; Erasmus University, Netherlands (nkavadis@rsm.nl): 2.2.14

Kawai, Norifumi; University of Sussex, United Kingdom (n.kawai@sussex.ac.uk): 2.2.10

Kedia, Ben; University of Memphis, USA (bkedia@memphis.edu): 1.5.6, 3.2.13

Keller, Josh; Nanyang Technological University, Singapore (jwkeller@ntu.edu.sg): 1.5.5

Kelley, Donna; Babson College, USA (dkelley@babson.edu): 1.1.10, 1.4.13

Kelley, Keith James; Florida International University, USA (kkelley@fiu.edu): 1.1.4

Kent, Muhtar; Chairman and CEO of The Coca-Cola Company, USA: 0.7

Keyman, E. Fuat; Director, International Policy Center, Turkey (fuat@sabanciuniv.edu): 2.1.1

Khammash, Marv; University of Sussex, United Kingdom (m.khammash@sussex.ac.uk): 2.2.10

Khan, Omar J.; Morgan State University, USA (omar.khan@morgan.edu): 2.2.8

Khan, Zaheer; University of Hull, United Kingdom (khan.zaheer@gmail.com): 3.3.15, 3.4.15

Khurram, Shahzad; University Paris Sud, France (khurramjah2002@yahoo.co.uk): 1.5.12

Kiessling, Timothy; Bond University, Australia (tkiessli@bond.edu.au): 2.3.13

Kim, Boine; Sung Kyun Kwan University, Korea, South (bk130@naver.com): 2.5.13

Kim, Changsu; Sogang University, Korea, South (cskim@sogang.ac.kr): 3.1.13, 3.2.13

Kim, Eun Woo; Yonsei University, Korea, South (kimeunwoo@hotmail.com): 3.1.10

Kim, Eun-Hee; George Washington University, USA (eunheek@gwu.edu): 3.2.3

Kim, Eunmi; Korea University, Korea, South (giveme428@korea.ac.kr): 1.5.8

Kim, Heechun; Georgia State University, USA (heechunkim@gsu.edu): 2.5.12

Kim, Hicheon; Korea University, Korea, South (hkim01@korea.ac.kr): 3.1.4

Kim, Hyunseob; Ohio State University, USA (kim_4218@fisher.osu.edu): 2.3.5

Kim, Jin Uk; University of Illinois at Urbana-Champaign, USA (jkim198@illinois.edu): 1.3.2, 1.5.15, 2.4.14

AIB 2013 Conference Proceedings

Page 309 
Kim, Jongsoo; University of Illinois at Urbana-Champaign, USA (jkim456@uiuc.edu): 2.2.14

Kim, Juhee; Korea University, Korea, South (juhee421@gmail.com): 1.4.14

Kim, Midam; University of Kansas and Northwestern University, USA (midamkim@ku.edu): 1.3.7

Kim, Min Jung; Korea University, Korea, South (kmjsechkie@korea.ac.kr): 1.5.15

Kim, Minyoung; University of Kansas, USA (mkim@ku.edu): 1.3.7

Kim, Nahyun; Korea University, Korea, South (buffy14@korea.ac.kr): 1.1.15

Kim, Sang Ji; Seoul National University, Korea, South (sangji.kim@gmail.com): 2.4.14

Kim, Sylvia; Fresno Pacific University, USA (Sylvia.Kim@fresno.edu): 2.2.5

Kim, Youngok; University of New South Wales, Australia (y.kim@unsw.edu.au): 1.5.15

King, Lawrence; Cambridge University, United Kingdom (Iguorbs@gmail.com): 2.4 .9

Kirca, Ahmet H; Michigan State University, USA (kirca@msu.edu): 1.3.5, 1.4.3, 2.1.3

Klaas, Brian; University of South Carolina, USA (klaasb@moore.sc.edu): 3.1.6

Kleindienst, Ingo; WHU, Germany (ingo.kleindienst@whu.edu): 1.5.15

Knight, Gary; Willamette University, USA (gknight@willamette.edu): 1.3.6, 1.4.10, 3.2.2

Knocke, Jan; University of Bayreuth, Germany (jan.knocke@gmx.eu): 3.3.7

Kobrin, Steve; University of Pennsylvania, USA (kobrins@wharton.upenn.edu): 3.2.3

Kogut, Bruce; Columbia University, USA (bruce.kogut@columbia.edu): 2.4.1

Kontinen, Tanja; University of Jyvaskyla, Finland (tanja.kontinen@jyu.fi): 1.4.13

Kostova, Tatiana; University of South Carolina, USA (kostova@moore.sc.edu): 2.2.2, 2.4.1, 2.5.10

Kotabe, Masaaki; Temple University, USA (mkotabe@temple.edu): 3.1.9

Kothari, Tanvi H.; San Jose State University, USA (kothari.t@gmail.com): 3.1.3

Kotosaka, Masahiro; Ritsumeikan University, Japan (kotosaka@gmail.com): 2.3.8

Koveshnikov, Alexei; Hanken School of Economics, Finland (alexei.koveshnikov@hanken.fi): 2.3.9

Krammer, Sorin M.S.; Groningen University, Netherlands (m.s.s.krammer@rug.nl): 1.3.14, 2.3.15

Krishnan, Thilagavathi; Universiti Sains Malaysia, Malaysia (taniperumkarunai@gmail.com): 2.1.14

Krivogorsky, Victoria; San Diego State University, USA (vkrivogo@mail.sdsu.edu): 3.1.12

Kruse, Doug; Rutgers University, USA (dkruse@smlr.rutgers.edu): 1.5.14

Kshetri, Nir; University of North Carolina, Greensboro, USA (nbkshetr@uncg.edu): 3.3.15

Kuivalainen, Olli; Lappeenranta University of Technology, Finland (olli.kuivalainen@lut.fi): 3.2.2

Kulatilaka, Nalin; Boston University, USA (nalink@bu.edu): 2.1.2

Kulchina, Elena; Duke University, USA (elena.kulchina@duke.edu): 2.5.1

Kumar, Maya; Western University, Canada (mkumar@ivey.ca): 2.4.13

Kumar, Nishant; Stockholm University, Sweden (nku@fek.su.se): 2.4.11

Kumar, Rajesh; Nottingham University, United Kingdom (rajesh.kumar@nottingham.ac.uk): 3.4.13

Kumar, Vikas; University of Sydney, Australia (vikas.kumar@sydney.edu.au): 3.1.14, 3.2.6, 3.4.8

Kunapatarawong, Rasi; Universidad Carlos III de Madrid, Thailand (rasirasita@gmail.com): 3.3.10

Kuncic, Aljaz; University of Ljubljana, Slovenia (aljaz.kuncic@fdv.uni-lj.si): 3.4.12

Kundu, Sumit; Florida International University, USA (kundus@fiu.edu): 1.1.12, 2.1.3, 2.5.7

Kunz, Werner; University of Massachusetts Boston, USA (werner.kunz@umb.edu): 2.4.6

Kurt, Yusuf; University of Manchester, United Kingdom (yusuf.kurt@postgrad.mbs.ac.uk): 3.2.2

Kurtulus, Aylin Kalafatoglu ; Co-chairman, Ant Group Corporation, Turkey: 2.5.2

Kuskova, Valentina V.; NRU Higher School of Economics, Russia (vkuskova@hotmail.com): 1.3.13

Kuznetsov, Andrei; Lancashire Business School, United Kingdom (akuznetsov@uclan.ac.uk): 1.3.9, 2.1.13

Kuznetsova, Olga; Manchester Metropolitan University, United Kingdom (o.kuznetsova@mmu.ac.uk): 1.3.9, 2.1.13

Kwak, Jooyoung; Yonsei University, Korea, South (jooyoung.kwak@yonsei.ac.kr): 3.1.10

$\mathbf{L}$

La Rocca, Maurizio; University of Calabria, Italy (m.larocca@unical.it): 2.5.12

Lahiri, Somnath; Illinois State University, USA (slahiri@ilstu.edu): 2.1.14, 2.2.13

Lamin, Anna; Northeastern University, USA (a.lamin@neu.edu): 3.2.9, 3.3.5

Lancheros, Sandra; Aston University, United Kingdom (s.lancheros@aston.ac.uk): 2.2.14

Larimo, Jorma; University of Vaasa, Finland (jorma.larimo@uwasa.fi): 3.4.14 
Larsen, Marcus M.; Copenhagen Business School, Denmark (mml.smg@cbs.dk): 1.1.6, 3.2.8

Lasio Morello, Maria Virginia; ESPOL, Ecuador (mlasio@espol.edu.ec): 3.4 .6

Latukha, Marina; Saint-Petersburg State University, Russia (marina.latuha@gsom.pu.ru): 1.5.14

Lau, Theresa; Hong Kong Polytechnic University, Hong Kong, SAR-PRC (theresa.lau@polyu.edu.hk): 2.5.13

Lau, Victor P.; Hang Seng Management College, Hong Kong, SAR-PRC (victorlau@hsmc.edu.hk): 1.1.14

Lawrence, Martyn; Emerald Group Publishing, United Kingdom (MLawrence@emeraldinsight.com): 1.1.7

Lazarova, Mila; Simon Fraser University, Canada (mbl@sfu.ca): 2.1.15, 2.3.9

Lazzarini, Sergio Giovanetti; Insper Institute of Education and Research, Brazil (sergiogl1@insper.edu.br): 1.5.12, 2.5 .12

Le Cottier, Aude; IE Business School, Spain (ale.phd2013@student.ie.edu): 2.1.12

Leartsurawat, Watcharaphong; Florida International University, USA (wlear001@fiu.edu): 3.4.13

Leclair, Dan; AACSB International, USA (dan@aacsb.edu): 0.7, 1.1.1

Lee, Ahreum; Temple University, USA (ahreum.lee@temple.edu): 2.4.11

Lee, Byungheon; Kwangwoon University, Korea, South (bhlee@kw.ac.kr): 3.2.13

Lee, Cheng-Yu; Southern Taiwan University, Taiwan (cylee@mail.stust.edu.tw): 1.1.14

Lee, Chia-Jung; National Taiwan University, Taiwan (Icj73828@gmail.com): 1.1.14

Lee, Chol; Sogang University, Korea, South (chollee@sogang.ac.kr): 1.3.5

Lee, Chun-Ling; National Sun Yat-Sen University, Taiwan (d934010005@student.nsysu.edu.tw): 1.3.15, 3.4.7

Lee, Hyun-Jung; London School of Economics, United Kingdom (h.lee@lse.ac.uk): 3.4 .13

Lee, In Hyeock (Ian); Loyola University Chicago, USA (ilee1@luc.edu): 1.3.11, 2.2.6

Lee, Jae-Eun; Sunchon National University, Korea, South (sky2u@sunchon.ac.kr): 2.3.13

Lee, Jongmin; Yonsei University, Korea, South (jongmin731@hotmail.com): 1.5.9, 3.4.11

Lee, Jun Ho; University of Illinois at Urbana-Champaign, USA (lee453@illinois.edu): 2.4.12

Lee, Meng-Hsiu; National Sun Yat-Sen University, Taiwan (st873217@yahoo.com.tw): 2.5.14

Lee, Seung-Hyun; University of Texas at Dallas, USA (lee.1085@utdallas.edu): 1.3.14, 2.1.7

Lee, Wei-Long; National Kaohsiung University of Applied Science, Taiwan (papersubmition13@gmail.com): 3.4.11

Lee, You-il; University of South Australia, Australia (you-il.lee@unisa.edu.au): 1.1.7

Lee, Youngwoo; Korea University, Korea, South (lee3992@korea.ac.kr): 2.2.14

Lehmberg, Derek G.; North Dakota State University, USA (derek.lehmberg@ndsu.edu): 2.2.11

Lei, Clara; Institute for Tourism Studies, Macau (clara.lei@yahoo.com.hk): 2.5.8

Lejarraga, Jose; IE University, Spain (jose.lejarraga@ie.edu): 2.2.8

Lenartowicz, Tomasz; Florida Atlantic University, USA (lenartow@fau.edu): 3.3.6

Lenway, Stefanie; Michigan State University, USA (lenway@bus.msu.edu): 1.1.1, 2.3.15

Leon-Darder, Fidel; University of Valencia, Spain (fidel.leon@uv.es): 2.4.4

Lepak, Dave; Rutgers University, USA (lepak@smlr.rutgers.edu): 1.5.14

Levie, Jonathan; Strathclyde University, United Kingdom (j.levie@strath.ac.uk): 1.3.13

Levy, Orly; IMD Business School, Switzerland (orly.levy@imd.ch): 1.3.7

Lew, Yong Kyu; University of Manchester, United Kingdom (yongkyu.lew@mbs.ac.uk): 2.3.12

Li, Brian; GP Electronics (Hong Kong) Ltd., Hong Kong, SAR-PRC (brian.li@gpe-hkg.com): 2.4.13

Li, Chenchen; Nanyang Technological University, Singapore (lich0017@e.ntu.edu.sg): 2.4.15

Li, Chengguang; University of Augsburg, Germany (chengguang.li@wiwi.uni-augsburg.de): 3.2.15

Li, Dan; Indiana University, USA (lid@indiana.edu): 2.2.7, 2.4.4

Li, Dongmei; Nanyang Technological University, Singapore (c080007@e.ntu.edu.sg): 2.4 .15

Li, Jiatao; HKUST, Hong Kong, SAR-PRC (mnjtli@ust.hk): 1.3.6, 1.4.5

Li, Jing; Simon Fraser University, Canada (jingli@sfu.ca): 3.4.7

Li, Jizhong; Curtin University, Australia (forestapache@hotmail.com): 1.4.6

Li, Lee; York University, Canada (leeli@yorku.ca): 2.3.10

Li, Lei; Nottingham University, China (lei.li@nottingham.edu.cn): 1.1.11, 1.3.15, 3.1.2

Li, Ling-Yee (Esther); Lingnan University, Hong Kong, SAR-PRC (estherli@In.edu.hk): 2.1.6

Li, Ming Hua; Copenhagen Business School, Denmark (mhl.int@cbs.dk): 3.3.8

Li, Peter; Copenhagen Business School, Denmark (pli.int@cbs.dk): 1.1.9, 2.3.8

Li, Quan; Texas A\&M University, USA (quanli@tamu.edu): 2.3.6 
Li, Xiaolin; Guangdong University of Business Studies, China (Ilix|@163.com): 2.2.13

Li, Xiaoying; Brunel University, United Kingdom (xiaoying.li@brunel.ac.uk): 1.4.12

Li, Xin; Copenhagen Business School, Denmark (xl.int@cbs.dk): 2.5.11

Li, Yi; Australian National University, Australia (yi.li@anu.edu.au): 1.3.9, 1.5.13, 3.1.14

Li, Yingxia; Beijing Union University, China (lyingxia@gmail.com): 2.5.11

Li, Yuanxu; Fudan University, China (liyuanxu08@gmail.com): 3.2.11

Li, Zijie; University of International Business and Economics, China (lizijie@126.com): 1.5.13

Liang, Hsiao-Yun; National Cheng Kung University, Taiwan (ireneyun0916@gmail.com): 3.1.13

Liang, Hsin-Yu; Feng Chia University, Taiwan (lianghy@fcu.edu.tw): 1.5.15

Liang, Xueji; National University of Singapore, Singapore (g0900955@nus.edu.sg): 2.4.14

Liang, Yuting; University of International Business and Economics, China (liangyuting6688@163.com): 1.5.13

Liao, Yuan; University of New South Wales, Australia (yuan.liao@unsw.edu.au): 1.3.4, 2.1.15

Librowicz, Michel; Université du Québec à Montréal, Canada (librowicz.michel@uqam.ca): 3.3.4, 3.4 .4

Liebenau, Jonathan; London School of Economics, United Kingdom (j.m.liebenau@lse.ac.uk): 3.4.15

Lien, Yung-Chih; National Taiwan University, Taiwan (lienyc@ntu.edu.tw): 2.2.8

Liesch, Peter Wayne; University of Queensland, Australia (p.liesch@uq.edu.au): 1.3.3, 2.3.6, 2.4 .6

Li'HC, Leo; King's College London, United Kingdom (leo.hc.li@gmail.com): 2.4.14

Lim, Jongha; University of Missouri, USA (limjong@missouri.edu): 2.2.5

Lin, Carol Yeh-Yun; National Chengchi University, Taiwan (yehyunln@nccu.edu.tw): 1.1.13

Lin, Cheng-Chen (Timothy); National Pingtung University of Science and Technology, Taiwan (timothy@mail.npust.edu.tw): 2.5.13

Lin, Chieh-Yu; Chang Jung Christian University, Taiwan (jylin@mail.cjcu.edu.tw): 1.1.15, 1.5.14

Lin, Howard; Ryerson University, Canada (hlin@ryerson.ca): 0.4

Lin, Hsin-Mei; National Chi Nan University, Taiwan (hmlin@ncnu.edu.tw): 3.1.14

Lin, Shu-Jou; National Taiwan Normal University, Taiwan (lin.sj@ntnu.edu.tw): 3.1.13

Lin, Wen-Ting; National Chung-Cheng University, Taiwan (wentinglin@ccu.edu.tw): 2.4.4

Lin, Xiaohua; Ryerson University, Canada (hlin@ryerson.ca): 2.5.14, 3.4.14

Lindahl, Frederick; George Washington University, USA (lindahl@gwu.edu): 1.1.7

Lindahl, Olof; Uppsala University, Sweden (olof.lindahl@fek.uu.se): 1.5.7

Lindsay, Val; University of Wollongong in Dubai, United Arab Emirates (valerielindsay@uowdubai.ac.ae): 1.1.14

Lindstrand, Angelika; Stockholm School of Economics, Sweden (angelika.lindstrand@hhs.se): 2.2.8, 2.4.10

LiPuma, Joseph A.; EMLyon Business School, France (lipuma@em-lyon.com): 3.1.5

Litovsky, Yana; Global Entrepreneurship Research Association, USA (ylitovsky@gemconsortium.org): 1.3.13

Littrell, Romie Frederick; Auckland University of Technology, New Zealand (romie.littrell@aut.ac.nz): 1.5.3, 3.2.15

Lituchy, Terri; University of the West Indies, Barbados (terrilituchy@yahoo.com): 2.3.3, 3.3.4, 3.4.4

Liu, Chang; University of International Business and Economics, China (szs_Ic1992@163.com): 1.5.13

Liu, Chia-Ling (Eunice); National Cheng Kung University, Taiwan (clliu@mail.ncku.edu.tw): 2.5.15, 3.2.13

Liu, Chih-Hsing Sam; Ming Chuan University, Taiwan (phd20110909@gmail.com):2.2.12, 3.4.11

Liu, Dong; Georgia Institute of Technology, USA (dong.liu@scheller.gatech.edu): 3.1.6

Liu, Helen; Industrial and Commercial Bank of China, China (helenliu.gsm@gmail.com): 3.4.10

Liu, Hsiao Wen; National Chengchi University, Taiwan (96355506@nccu.edu.tw): 1.5.14, 3.4.9

Liu, John; University of New South Wales, Australia (wliu@unsw.edu.au): 3.2.13

Liu, Leigh Anne; Georgia State University, USA (laliu@gsu.edu): 2.2.9

Liu, Ling; University of Edinburgh, United Kingdom (ling.liu@ed.ac.uk): 1.3.14

Liu, Xiaming; University of London, United Kingdom (Xiaming.liu@bbk.ac.uk): 1.4.12

Liu, Xiaohui; Loughborough University, United Kingdom (X.Liu2@Iboro.ac.uk): 1.1.15

Liu, Xiyou; Peking University, China (jhqsIxy@163.com): 1.1.15, 2.4.5

Liu, Ying; Florida International University, USA (lilianeliuying@gmail.com): 2.5.11

Livanis, Grigorios; Northeastern University, USA (g.livanis@neu.edu): 3.3.5

Ljubica, Jasenko; University of Split, Croatia (jasenko01@net.hr): 1.1.5

Ljung, Anna; Uppsala University, Sweden (anna.ljung@fek.uu.se): 1.5.10

Lo, Shihmin; National Chi Nan University, Taiwan (smlo@ncnu.edu.tw): 2.3.13 
Loewenthal, Neli; George Washington University, USA (nelik@gwmail.gwu.edu): 1.4.7

Loohuis, Raymond; University of Twente, Netherlands (r.p.a.loohuis@utwente.nl): 1.3.7

Lopez, Luis E.; INCAE Business School, Costa Rica (luis.lopez@incae.edu): 2.1.10, 3.1.8

Lopez-Puertas Lamy, Monica; Unicredit Banca - University of Bologna, Italy (monica.lopezpuertas@unibo.it): 2.4.12

Lora, Mayra Ivanoff; FGV-EESP, Brazil (mayra.lora@fgv.br): 3.1.12

Lorenzen, Mark; Copenhagen Business School and Editor, Industry and Innovation, Denmark (ml.ino@cbs.dk): 2.5.3

Love, James; Aston University, United Kingdom (j.h.love@aston.ac.uk): 3.2.10

Low, Yi Jia Kathleen; WU Vienna, Austria (ylow@wu.ac.at): 1.3.1, 2.4.10

Lu, Jane Wenzhen; University of Melbourne, Australia (jane.lu@unimelb.edu.au): 2.4.14, 3.1.10, 3.2.6, 3.3.5

Lu, Jiangyong; Guanghua School of Management, China (lujiangyong@gsm.pku.edu.cn): 3.3.8

Lu, Lin-Hua; National Cheng Kung University, Taiwan (r4895111@mail.ncku.edu.tw): 1.1.15

Lu, Ting-Chun; National Chengchi University, Taiwan (96355502@nccu.edu.tw): 2.3.13

Lu, Vinh Nhat; Australian National University, Australia (vinh.lu@anu.edu.au): 1.3.5, 3.1.3

Lucea, Rafael; George Washington University, USA (rafel@gwu.edu): 1.3.1

Lui, Steven; University of New South Wales, Australia (steven.lui@unsw.edu.au): 3.2.13

Luiz, John Manuel; University of Cape Town, South Africa (john.luiz@gsb.uct.ac.za): 1.3.14

Lukoianova, Tatiana; University of Western Ontario, Canada (vshchlk@gmail.com): 2.3.6

Lundan, Sarianna; University of Bremen, Germany (s.lundan@uni-bremen.de): 0.2, 2.1.2

Lunnan, Randi; Norwegian Business School, Norway (randi.lunnan@bi.no): 1.1.6, 3.4.11

Luo, Ben Nanfeng; University of New South Wales, Australia (nanfeng.luo@unsw.edu.au): 3.4.11

Luo, Yadong; University of Miami, USA (yadong@miami.edu): 1.5.11, 3.4.12

Luomala, Harri T.; University of Vaasa, Finland (harri.luomala@uwasa.fi): 3.4.13

Lyles, Marjorie A.; Indiana University, USA (mlyles@iupui.edu): 0.2

M

Ma, Jieqiong; Saint Louis University, USA (jma17@slu.edu): 3.2.11

Ma, Xufei; Chinese University of Hong Kong, Hong Kong, SAR-PRC (xufei@cuhk.edu.hk): 2.2.6

Machado, Marcilio; Fucape - Fundação Capixaba de Pesquisa, Brazil (marcilio@famex.com.br): 2.5 .5

Machikita, Tomohiro; Institute of Developing Economies, Japan (tomohiro_machikita@ide.go.jp): 3.1.13

Maclennan, Maria Laura Ferranty; ESPM, Brazil (ferranty@hotmail.com): 3.3.15

Madero, Sergio; Tec de Monterrey, Mexico (smadero@itesm.mx): 3.4.6

Madhavan, Ravi; University of Pittsburgh, USA (rmadhavan@katz.pitt.edu): 3.2.11

Magelssen, Catherine; Rutgers University, USA (catiem@pegasus.rutgers.edu): 2.2.14, 3.4.8

Magnusson, Peter; Florida International University, USA (peter.magnusson@fiu.edu): 1.5.8, 3.2.15

Mahnke, Volker; University of Edinburgh, United Kingdom (volker.mahnke@ed.ac.uk): 2.3.8

Maitland, Elizabeth; University of New South Wales, Australia (e.maitland@unsw.edu.au): 1.1.8

Majerbi, Basma; University of Victoria, Canada (majerbi@uvic.ca): 1.1.7

Majocchi, Antonio; University of Pavia, Italy (antonio.majocchi@unipv.it): 2.4.12, 3.4.5

Major, David Lanier; Indiana University, USA (dlmajor@indiana.edu): 3.2.13, 3.4.12

Makhija, Anil K.; Ohio State University, USA (makhija_1@fisher.osu.edu): 2.2.5

Makhija, Mona; Ohio State University, USA (makhija_2@cob.osu.edu): 3.1.14, 3.3.10

Makino, Shige; Chinese University of Hong Kong, Hong Kong, SAR-PRC (makino@baf.msmail.cuhk.edu.hk): 0.2, 3.4.7

Makri, Katerina; Athens University of Economics and Business, Greece (katerinamakri83@gmail.com): 2.1.11

Maksimov, Vladislav; University of Miami, USA (vmaksimov@bus.miami.edu): 1.5.11, 2.4.11, 3.4.12

Maley, Jane; Jane Maley Consulting, Australia (jane@janemaleyconsulting.com): 2.3.13

Malhotra, Shavin; Ryerson University, Canada (shavinm@gmail.com): 3.4.14

Malik, Tariq Hussain; Dongbei University of Finance \& Economics, China (tmalik@dufe.edu.cn): 1.1.11, 2.5.14

Mallick, Sushanta; Queen Mary University of London, United Kingdom (s.k.mallick@qmul.ac.uk): 2.2.5

Manigart, Sophie; Vlerick Business School, Belgium (sophie.manigart@vlerick.com): 2.2.5

Manning, Stephan; University of Massachusetts Boston, USA (stephan.manning@umb.edu): 1.1.6, 2.4.6

Mao, Jina; Skidmore College, USA (jmao@skidmore.edu): 2.1.15

AIB 2013 Conference Proceedings

Page 313 
Marcon, Rosilene; UNIVALI, Brazil (rmarcon@univali.br): 2.4.12

Marinov, Marin Alexandrov; University of Gloucestershire, United Kingdom (m_a_marinov@hotmail.com): 3.1.15 Marinova, Svetla Trifonova; Aalborg University, Denmark (svetla@id.aau.dk): 3.1.15, 3.3.15, 3.4.15

Mariotti, Sergio; Politecnico di Milano, Italy (sergio.mariotti@polimi.it): 1.4.9, 2.1.12

Martin, Silvia; Lynchburg College, USA (sylvie.Izn@gmail.com): 2.3.8

Martin, Xavier; Tilburg University, Netherlands (x.martin@tilburguniversity.edu): 2.2.12

Martinet, Alain Charles; University of Lyon 3, France (alain.martinet@univ-lyon3.fr): 3.1.14

Martinez, Candace A.; Saint Louis University, USA (cmarti58@slu.edu): 3.2.13

Martinkenaite, leva; BI Norwegian Business School, Norway (ieva.martinkenaite-pujanauskiene@bi.no): 3.4.11

Marzocchi, Gian Luca; University of Bologna, Italy (gianluca.marzocchi@unibo.it): 1.3.11

Masango, Shingairai; Sheffield Hallam University, United Kingdom (s.masango@shu.ac.uk): 3.4.15

Maseland, Robbert; University of Groningen, Netherlands (r.k.j.maseland@rug.nl): 2.1.8, 3.2.15

Maskell, Peter; Copenhagen Business School, Denmark (pm.ino@cbs.dk): 2.5.3

Mathews, John A.; Macquarie University, Australia (john.mathews@mgsm.edu.au): 3.3.15

Mathews, Shane; Queensland University of Technology, Australia (sw.mathews@qut.edu.au): 2.4.15

Matos, Fatima Regina Ney; University of Fortaleza, Brazil (fneymatos@unifor.br): 1.5.12

Matsue, Regina Yoshie; University of Fortaleza, Brazil (rymatsue08@unifor.br): 1.5.12

Matysiak, Lars; Justus Liebig University Giessen, Germany (research@matysiak.com): 1.4.5, 2.4.6

Maurer, Noel; Harvard Business School, USA (nmaurer@hbs.edu): 3.2.3

Maznevski, Martha; IMD, Switzerland (martha.maznevski@imd.org): 1.5.2

McCarthy, Daniel; Northeastern University, USA (da.mccarthy@neu.edu): 3.2.9

McDaniel, Dana M.; California State University, Long Beach, USA (dana.mcdaniel@csulb.edu): 3.1.6

McDermott, Gerald Andrew; University of South Carolina, USA (gerald.mcdermott@moore.sc.edu): 1.3.1, 2.4.3

McDougall-Covin, Patricia; Indiana University, USA (kiyaktun@msu.edu): 2.3.12

McGaughey, Sara; Griffith University, Australia (s.mcgaughey@griffith.edu.au): 1.1.6, 1.4.12

McGrath-Champ, Susan; University of Sydney, Australia (susan.mcgrathchamp@sydney.edu.au): 3.3.14

McGuinness, Martina; University of Sheffield, United Kingdom (m.mcguinness@sheffield.ac.uk): 3.2.9

McGuire, Jean B.; Louisiana State University, USA (mcguire@Isu.edu): 2.4.12

McKern, Bruce; China Europe International Business School, China (bmckern@ceibs.edu): 2.2.1, 2.3.5

McKiernan, Peter; Murdoch University, Australia (peter.mckiernan@strath.ac.uk): 3.4.2

Md., Nur Alam; Leipzig University, Bangladesh (kinku_ku@yahoo.com): 3.4.15

Mei, Hsiao-Chen; National Chi Nan University, Taiwan (katiemhc@gmail.com): 2.3.13

Mellahi, Kamel; Warwick University, United Kingdom (kamel.mellahi@wbs.ac.uk): 1.4 .6

Mendoza, Xavier; ESADE Business School, Spain (xavier.mendoza@esade.edu): 2.4 .6

Meraz, Juan; Missouri State University, USA (JuanMeraz@missouristate.edu): 0.4

Merchant, Hemant; University of South Florida - St. Pete, USA (hmerchant@usfsp.edu): 2.5.11

Mersland, Roy; University of Agder, Norway (roy.mersland@uia.no): 1.5.14

Meuer, Johannes; University of Zurich, Switzerland (johannes.meuer@business.uzh.ch): 1.1.12

Meyer, Klaus E.; China Europe International Business School, China (kmeyer@ceibs.edu): 1.4.5, 2.2.2, 2.3.1, 3.2.10

Meyskens, Moriah; University of San Diego, USA (mmeyskens@sandiego.edu): 3.2.14

Mezias, John; University of Miami, USA (jmezias@miami.edu): 0.5

Miah, Khasro; North South University, Bangladesh (khasro@northsouth.edu): 1.3.8

Midgley, David; INSEAD, France (david.midgley@insead.edu):3.1.9

Mihailova, Irina; Aalto University, Finland (irina.mihailova@aalto.fi): 1.5.7

Mileski, Joan P; Texas A\&M University at Galveston, USA (mileskij@tamug.edu): 3.2.4

Miller, Toyah L.; Indiana University, USA (milleto2@indiana.edu): 3.3.3

Minbaeva, Dana; Copenhagen Business School, Denmark (dm.smg@cbs.dk): 0.3, 1.3.8, 1.5.7, 3.2.1, 3.4.10

Minkov, Michael; International University College, Bulgaria (minkov@iuc.bg): 3.1.1

Minor, Michael; University of Texas Pan American, USA (msminor@hotmail.com): 2.5.8

Miocevic, Dario; University of Split, Croatia (dmiocevi@efst.hr): 2.3.8

Miravitlles, Paloma; University of Barcelona, Spain (paloma.miravitlles@ub.edu): 2.4.4

Mirkin, Yakov; Institute of World Economy and International Relations, Russia (yakov.mirkin@gmail.com): 1.3.9 
Mirza, Hafiz; Division on Investment and Enterprise, UNCTAD, Switzerland (Hafiz.Mirza@unctad.org): 2.3.7

Miska, Christof; Vienna University of Economics and Business, Austria (christof.miska@wu.ac.at): 3.1.2, 3.3.10

Mitchell, Matthew C.; Drake University, USA (matthew.mitchell@drake.edu): 3.2.15

Mockaitis, Audra I.; Monash University, Australia (audra.mockaitis@monash.edu): 2.1.13

Moeller, Miriam; University of Queensland, Australia (m.moeller@uq.edu.au): 1.4.15, 2.3.13

Mohr, Alex; University of Kent, United Kingdom (a.t.mohr@kent.ac.uk): 1.4.14, 2.1.7, 2.2.10

Mol, Michael J; University of Warwick, United Kingdom (michael.mol@wbs.ac.uk): 1.4.9, 1.5.4

Monteiro, Felipe; INSEAD, France (felipe.monteiro@insead.edu): 1.3.7, 1.4.4, 3.2.10

Monticelli, Jefferson Marlon; UNISINOS - Universidade do Vale do Rio dos Sinos, Brazil (jeffmarlon@hotmail.com): 2.5 .12

Montiel, Ivan; Loyola Marymount University, USA (ivanmontiel@gmail.com): 3.4.8

Moon, Jon Jungbien; Korea University, Korea, South (jonjmoon@korea.ac.kr): 1.1.15, 1.5.15, 3.1.7

Moore, Fiona; University of London, United Kingdom (fiona.moore@rhul.ac.uk): 3.1.8

Moore, Karl; McGill University, Canada (karl.moore@mcgill.ca): 2.5.4

Moosmayer, Dirk C.; Nottingham University, China (dirk.moosmayer@nottingham.edu.cn): 1.3.12, 2.1.12

Moraes, Daniel Gomes de; FGV-EESP, Brazil (daniel.moraes@citi.com): 3.1.12

Morehead Dworkin, Terry; Indiana University and Seattle University, USA (dworkint@indiana.edu): 3.4.6

Morgan, Glenn; University of Cardiff, United Kingdom (morgangd1@cardiff.ac.uk): 3.1.13

Mori, Neema; University of Dar es Salaam, Tanzania (neema@imedtz.org): 1.5.14

Morris, Shad; Ohio State University, USA (morris@fisher.osu.edu): 3.1.13

Motohashi, Kazuyuki; University of Tokyo, Japan (motohashi@tmi.t.u-tokyo.ac.jp): 2.1.5

Mudambi, Ram; Temple University, USA (rmudambi@temple.edu): 0.5, 1.4.4, 2.3.7, 2.5.3, 3.2.13

Mudambi, Susan M.; Temple University, USA (susan.mudambi@temple.edu): 2.4.15

Muehlfeld, Katrin; Utrecht University, Netherlands (k.muehlfeld@uu.nl): 1.1.4, 1.3.7

Mughan, Terry; University of Victoria, Canada (terry.mughan@googlemail.com): 2.4.2

Mukherjee, Debmalya; University of Akron, USA (dmukher@uakron.edu): 2.2.15, 3.2.13

Müllner, Jakob; Vienna University of Economics and Business, Austria (jakob.muellner@wu.ac.at): 3.1.12

Munjal, Surender; University of Leeds, United Kingdom (smu@lubs.leeds.ac.uk): 2.3.6

Murad, Wahid; University of Adelaide, Australia (wahid.murad@adelaide.edu.au): 2.1.11

Muratbekova-Touron, Maral; ESCP Paris, France (mmuratbekova@escpeurope.eu): 3.4.10

Murphy, William; University of Saskatchewan, Canada (wmurphy@edwards.usask.ca): 2.1.11

Murray, Janet Y.; University of Missouri-St. Louis, USA (murrayjan@umsl.edu): 2.5.2, 3.1.9

Murtha, Thomas P.; Michigan State University, USA (murtha@bus.msu.edu): 2.3.15

Mutlu, Berna; University of Florida, USA (berna.mutlu@warrington.ufl.edu): 3.2.14

Mutlu, Canan; University of Texas at Dallas, USA (cxm101020@utdallas.edu): 1.3.14, 3.3.13

$\mathbf{N}$

Nair, Smitha; University of Sheffield, United Kingdom (ecp09srn@sheffield.ac.uk): 1.4.6

Nakamura, Hiroshi; Keio University, Japan: 1.4.10

Nakos, George; Clayton State University, USA (georgenakos@clayton.edu): 1.1.9

Nam, Daeil; Korea University, Korea, South (namdaeil@korea.ac.kr): 1.4.14, 3.2.13

Napier, Nancy K; Boise State University, USA (nnapier@boisestate.edu): 1.4.15

Nartey, Lite; University of South Carolina, USA (lite.nartey@moore.sc.edu): 2.4.12, 2.5.1

Narula, Rajneesh; University of Reading, United Kingdom (r.narula@reading.ac.uk): 1.3.1

Nebus, James; Suffolk University, USA (jnebus@suffolk.edu): 3.1.8

Nekhili, Medhi; Rouen Business School, France (guoliang@live.com): 2.4 .9

Nell, Phillip; Copenhagen Business School, Denmark (pcn.smg@cbs.dk): 2.2.13, 3.4.3

Nemeth, Alexander; Freiberg University, Germany (nemeth.alexander@gmail.com): 2.5.11

Newburry, William; Florida International University, USA (newburry@fiu.edu): 0.4, 2.4.5, 3.1.14, 3.4.6

Newenham-Kahindi, Aloysius; University of Saskatchewan, Canada (newenham-kahindi@edwards.usask.ca): 2.3.11

Ngoasong, Michael Zisuh; Coventry University London Campus, United Kingdom

(michael.ngoasong@culc.coventry.ac.uk): 3.3.4, 3.4.4

AIB 2013 Conference Proceedings

Page 315 
Nguyen, Pham Hoanh Son; France Business School, France (pham.nguyen@esc-clermont.fr): 3.1.14

Nguyen, Quyen; University of Reading, United Kingdom (t.k.q.nguyen@henley.ac.uk): 1.4.12, 2.4.14, 2.5 .1

Nicholson, John; University of Hull, United Kingdom (j.d.nicholson@hull.ac.uk): 3.3 .15

Nielsen, Bo Bernhard; Copenhagen Business School, Denmark (bn.smg@cbs.dk): 1.3.2, 3.3.6

Nielsen, Sabina; Copenhagen Business School, Denmark (sta.int@cbs.dk): 3.3.6

Nigam, Roli; Laval University, Canada (rolinigam@gmail.com): 2.2.13

Nikitenko, Gleb; University of San Francisco, USA (nikitenko@usfca.edu): 1.5.3

Nippa, Michael C.; Freiberg University, Germany (nippa@bwl.tu-freiberg.de): 1.4.11, 2.5.11

Nkongolo-Bakenda, Jean-Marie; University of Regina, Canada (jean-marie.nkongolo-bakenda@uregina.ca): 3.2.11

Noack, Sebastian; Quadriga Deutschland GmbH, Germany (mba.noack@hotmail.com): 1.4.15

Nonis, Sarath A.; Arkansas State University, USA (snonis@astate.edu): 1.3.12

Nummela, Niina; Turku School of Economics, Finland (niina.nummela@utu.fi): 1.1.14, 1.3.3

0

Obloj, Krzysztof; Kozminski University and University of Warsaw, Poland (kobloj@kozminski.edu.pl): 1.4.13, 2.4.13

Odorici, Vincenza; Univesity of Bologna, Italy (vincenza.odorici@unibo.it): 2.4.12

Oehme, Marie; University of Mannheim, Germany (oehme@bwl.uni-mannheim.de): 2.1.6

Oehmichen, Jana; Georg-August-University Goettingen, Germany (jana.oehmichen@wiwi.uni-goettingen.de): 1.1.13, 1.4.8

Oesterle, Michael-Jörg; University of Stuttgart, Germany (michael-joerg.oesterle@bwi.uni-stuttgart.de): 2.5.14, 3.1.10, 3.4.8

Oetzel, Jennifer; American University, USA (oetzelj@american.edu): 1.4.8, 2.1.2, 2.5.10

Ogasavara, Mario Henrique; University of Fortaleza, Brazil (marioga@unifor.br): 1.5.12

Oh, Chang Hoon; Simon Fraser University, Canada (coh@sfu.edu.ca): 1.4.1, 2.3.11, 2.5.10

Ojala, Arto; University of Jyvaskyla, Finland (arto.k.ojala@jyu.fi): 1.4.13

Olivas-Lujan, Miguel; Clarion University, USA (molivas@clarion.edu): 3.4.6

Oliveira Junior, Moacir de Miranda; USP, Brazil (mirandaoliveira@usp.br): 2.5.5

Omar, Ayman; American University, USA (omar@american.edu): 2.5.6

Onrust, Marjolijn; University of Groningen, Netherlands (m.onrust@rug.nl): 2.1.8

Ordenana, Xavier; ESPAE Graduate School of Management, Ecuador (xordenan@espol.edu.ec): 2.5.8

O'Reilly, Daragh; University of Sheffield, United Kingdom (d.t.oreilly@sheffield.ac.uk): 3.1.15

Oriani, Raffaele; Luiss Guido Carli University of Rome, Italy (roriani@luiss.it): 3.4.11

Orly, Levy; IMD, Switzerland (orly.levy@imd.org): 2.1.15

Ortiz Baldo, Carlos Miguel; U. Fermin Toro and U. Pablo de Olavide, Venezuela: 3.4.6

Osegowitsch, Tom; University of Melbourne, Australia (ot@unimelb.edu.au): 1.3.2

Osiyevskyy, Oleksiy; University of Calgary, Canada (oleksiy.osiyevskyy@haskayne.ucalgary.ca): 3.3.6

Osland, Joyce; San Jose State University, USA (joyce.osland@sjsu.edu): 1.5.2, 2.3.3, 3.4.13

Oxelheim, Lars; Lund University, Sweden (lars.oxelheim@fek.lu.se): 3.2.7

Ozaki, Toshiya; Rikkyo University, Japan (ozakitt@gmail.com): 3.3.4, 3.4.4

Ozbek, Nurgul; Stockholm School of Economics, Sweden (nurgul.ozbek@hhs.se): 2.4.10

Ozcan, Gul Berna; University of London, United Kingdom (g.ozcan@rhul.ac.uk): 1.4.14, 3.3.12

Ozcan, Pinar; Warwick Business School, United Kingdom (pinar.ozcan@wbs.ac.uk): 3.2.2

Ozcan, Seval; Blanchard International Turkey, Turkey: 3.2.4

Ozel, Soli; Kadir Has University, Turkey (soli@khas.edu.tr): 2.1.1

Ozer, Muammer; City University of Hong Kong, Hong Kong, SAR-PRC (mgozer@cityu.edu.hk): 1.1.9

$\mathbf{P}$

Pahlberg, Cecilia; Uppsala University, Sweden (cecilia.pahlberg@fek.uu.se): 2.2.7

Paik, Yongsun; Loyola Marymount University, USA (yspaik@Imu.edu): 1.3.11, 3.2.5

Pak, Yong Suhk; Yonsei University, Korea, South (yspak@yonsei.ac.kr): 1.5.9, 3.2.5

Pan, Alan; Xiamen University, China (apan@indiana.edu): 3.2.14

Pan, David; Prince Sultan University, Saudi Arabia (david.pan@ufl.edu): 3.2.14 
Pananond, Pavida; Thammasat University, Thailand (pavida@tbs.tu.ac.th): 2.4.13, 3.3.15

Pangarkar, Nitin; National University of Singapore, Singapore (bizpn@nus.edu.sg): 1.5.11, 2.3.14

Panibratov, Andrei; Saint-Petersburg State University, Russia (panibratov@mail.ru): 1.5.14, 3.3.5

Parente, Ronaldo Couto; Florida International University, USA (rcparent@fiu.edu): 1.1.4, 1.4.4, 2.5.7, 3.2.13

Park, Byung II; Hankuk University of Foreign Studies, Korea, South (leedspark@hufs.ac.kr): 2.3.11

Park, Chansoo; Memorial University of Newfoundland, Canada (chansoo.park@ubc.ca): 1.4.5, 1.5.7

Park , Hyeon ; Georgia State University, USA (hjpark@gsu.edu): 3.2.1

Park, Sarah; EMLyon Business School, France (park@em-lyon.com): 3.1.5

Park, Seung Ho; Moscow School of Management SKOLKOVO, Russia (shpark99@me.com): 1.5.10, 3.2.9

Park, Yeonjin; Seoul National University, Korea, South (chris81@snu.ac.kr): 2.4.14

Pascal, Daudi; University of Agder, Norway (daudi.p.ndaki@uia.no): 1.5.14

Pater, Max; University of Groningen, Netherlands (maxpater@gmail.com): 1.1.14

Patnaik, Sanjay; George Washington University, USA (spatnaik@gwu.edu): 1.1.4, 1.4.7, 2.5.1

Pauluzzo, Rubens; University of Udine, Italy (rubenspauluzzo@gmail.com): 2.2.3

Peacock, Tanya; University of Hawaii at Manoa, USA (peacock4@hawaii.edu): 2.3.10

Pedersen, Torben; Copenhagen Business School, Denmark (tp.smg@cbs.dk): 1.4.1, 1.5.9, 2.4.6, 3.2.1

Peerally, Jahan Ara; HEC Montréal, Canada (jahan.peerally@hec.ca): 2.3.12

Peeters, Carine; Universite libre de Bruxelles, Belgium (carine.peeters@ulb.ac.be): 1.4.9, 1.5.4, 3.2.8, 3.3.14

Peiperl, Maury; IMD, Switzerland (maury.peiperl@imd.org): 1.3.7, 2.1.15

Peiter, Bjoern; Hochschule für Unternehmensführung, Germany (bjoern.peiter@t-systems.com): 2.1.15

Pekerti, Andre Anugerah; University of Queensland, Australia (a.pekerti@business.uq.edu.au): 1.4.15

Peltokorpi, Vesa; Japan Advanced Institute of Science and Technology, Japan (vesap@jaist.ac.jp): 2.2.10

Peng, George Z.; University of Regina, Canada (george.peng@uregina.ca): 3.2.11, 3.3.13

Peng, Mike W.; University of Texas at Dallas, USA (mikepeng@utdallas.edu): 1.5.13, 3.3.13

Peng, Siqing; Peking University, China (pengsq@gsm.pku.edu.cn): 2.4.15

Peng, T.K.; I-Shou University, Taiwan (tkpeng@isu.edu.tw): 2.5.13

Pereira, Luís Henrique; Fundação Getulio Vargas, Brazil (Luis.Pereira@fgv.br): 1.1.15

Pereira, Vinicius Silva; Fundacao Getulio Vargas, Brazil (viniciuss56@gmail.com): 2.5.7

Perez-Nordtvedt, Liliana; University of Texas at Arlington, USA (Inordtvedt@uta.edu): 3.2.13

Perri, Alessandra; Ca' Foscari University of Venice, Italy (alessandra.perri@unive.it): 3.4.11

Perri, Alessandro; University of South Carolina, USA (alessandro.perri@grad.moore.sc.edu): 2.2.15

Pesch, Robin; University of Bayreuth, Germany (robin.pesch@uni-bayreuth.de): 3.3.7

Pestre, Florent; University Paris Sud, France (florent.pestre@u-psud.fr): 1.5.12

Petersen, Bent; Copenhagen Business School, Denmark (bp.smg@cbs.dk): 1.5.4, 2.2.15

Peterson, Mark F.; Florida Atlantic University, USA (mpeterso@fau.edu): 3.1.1, 3.2.15

Petkova, Neviana; University of Oregon, USA (npetkova@Icbmail.uoregon.edu): 3.3 .12

Petrovici, Dan Alex; University of Kent, United Kingdom (d.a.petrovici@kent.ac.uk): 3.1.15

Pham, Thi Song Hanh; Sheffield Hallam University, United Kingdom (s.h.pham@shu.ac.uk): 3.3.13

Phene, Anupama; George Washington University, USA (anuphene@gwu.edu): 1.4.11, 2.1.4, 2.5.12

Phillips, Margaret; Pepperdine University, USA (margaret.phillips@pepperdine.edu): 2.3.3

Phusavat, Kongkiti; Kasetsart University, Thailand (fengkkp@ku.ac.th): 3.2.9

Picone, Pasquale Massimo; University of Catania, Italy (pmpicone@unict.it): 2.5.12

Piekkari, Rebecca; Aalto University, Finland (rebecca.piekkari@aalto.fi): 1.5.9, 2.4.2

Pisani, Niccolo; IESE, Spain (npisani@iese.edu): 3.2.8

Piscitello, Lucia; Politecnico di Milano, Italy (lucia.piscitello@polimi.it): 1.4.9, 2.1.12, 2.2.14

Pitelis, Christos; Cambridge University, United Kingdom (c.pitelis@jbs.cam.ac.uk): 2.3.7

Pla-Barber, Jose; University of Valencia, Spain (jose.pla@uv.es): 0.4

Plakoyiannaki, Emmanuella; Aristotle University of Thessaloniki, Greece (emplak@econ.auth.gr): 2.2.4, 3.1.8

Plourde, Yves; University of Western Ontario, Canada (yplourde.phd@ivey.ca): 3.3.12

Polat, Cigdem; University of Augsburg, Germany (cigdem2208@hotmail.com): 3.1.10

Pongeluppe, Leandro; Insper Institute of Education and Research, Brazil (leandro.pongeluppe@gmail.com): 1.5.12

Popli, Manish; Indian Institute of Management Lucknow, India (fpm12004@iiml.ac.in): 1.5.6, 3.1.14 
Pourmand, Firouze; Linnaeus University, Sweden (firouze.pourmand@Inu.se): 2.4.14

Prange, Christiane; EMLyon Business School, France (prange@em-lyon.com): 3.1.5

Prashantham, Shameen; Nottingham University, China (shameen.prashantham@nottingham.edu.cn): 1.4.13, 1.5.5

Presutti, Manuela; Univesity of Bologna, Italy (manuela.presutti@unibo.it): 2.4.12

Procher, Vivien; University of Wuppertal, Germany (procher@uni-wuppertal.de): 1.3.7

Pu, Xiaoyu; SUNY Oswego, USA (xiaoyu.pu@oswego.edu): 2.1.13

Puck, Jonas F.; WU Vienna, Austria (jonas.puck@wu.ac.at): 2.1.15, 2.5.9

Pudelko, Markus; Tuebingen University, Germany (markus.pudelko@uni-tuebingen.de): 1.3.8, 1.5.9

Puffer, Sheila; Northeastern University, USA (s.puffer@neu.edu): 3.2.9

Punnett, Betty Jane; University of the West Indies, Barbados (eureka@caribsurf.com): 2.3.3, 3.3.4, 3.4.4

Punton-Li, Emma L.; Department for Work and Pensions, United Kingdom (emma.punton-li@dwp.gsi.gov.uk): 2.4.14

Puranam, Phanish; INSEAD, Singapore (phanish.puranam@insead.edu): 1.5.7

Purg, Danica; IEDC-Bled School of Management, Slovenia (danica.purg@iedc.si): 3.4 .2

Puri, Manas; University of Rome Tor Vergata, Italy (puri@economia.uniroma2.it): 2.1.13

Purkayastha, Saptarshi; Indian Institute of Management Kozhikode, India (spurkayastha1@gmail.com): 2.1.14, 3.2.6

Q

Qian, Chunxiang; Hankuk University of Foreign Studies, Korea, South (chyan415@hotmail.com): 1.5.15

Qian, Gongming; Chinese University of Hong Kong, Hong Kong, SAR-PRC (qian@baf.msmail.cuhk.edu.hk): 2.3.10, 2.5.5

Qian, Xiaolin; University of Macau, Macau (xiaolinqian@umac.mo): 1.5.11

Qiang, Cuili; City University of Hong Kong, Hong Kong, SAR-PRC (cuilqian@cityu.edu.hk): 2.2.6

Qin, Cindy; University of San Francisco, USA (dqin3@usfca.edu): 2.2.8

Qu, Jane; University of New South Wales, Australia (janeq@unsw.edu.au): 2.2.12

Quer, Diego; University of Alicante, Spain (diego.quer@ua.es): 3.3.8

$\mathbf{R}$

Ra, Wonchan; Hankuk University of Foreign Studies, Korea, South (wonra1@gmail.com): 1.5.15

Rabbiosi, Larissa; Copenhagen Business School, Denmark (Ir.int@cbs.dk): 1.3.8, 3.4.10

Rachdi, Houssem; University of Jendouba, Tunisia (rachdihoussem@gmail.com): 1.1.7

Rademaker, Linda; Tilburg University, Netherlands (c.h.a.rademaker@tilburguniversity.edu): 2.2.12

Raghunath, Subramanyam; IIM Bangalore, India (srnathiimb@gmail.com): 0.4, 3.3.2

Rahman, Abdur; Lahore University of Management Sciences, Pakistan (abdur.malik@lums.edu.pk): 3.2.9

Rahman, Noushi; Pace University, USA (nrahman@pace.edu): 1.3.14

Ramamurti, Ravi; Northeastern University, USA (r.ramamurti@neu.edu): 0.2, 1.1.3, 2.2.1, 3.1.4

Ramanantsoa, Bernard; Dean, HEC Paris, France: 0.7

Ramaswami, Aarti; ESSEC, France (ramaswami@essec.edu): 3.4.6

Ramirez, Andres; Bryant University, USA (aramirez@bryant.edu): 2.3.2

Rammal, Hussain; University of South Australia, Australia (hussain.rammal@unisa.edu.au): 3.4.5

Ramsey, Jase; University of Alabama, USA (jase@cba.ua.edu): 3.2.12

Randrianasolo, Arilova A; Saint Louis University, USA (randriaa@slu.edu): 1.5.8, 2.3.11

Rathert, Nikolas; Freie Universitaet Berlin, Germany (nikolas.rathert@fu-berlin.de): 1.4.8

Ratnaike, Yasanji; Leeds University, United Kingdom (ycr@live.co.uk): 1.3.15

Raupp, Julia Ines; WU Vienna, Austria (julia.raupp@wu.ac.at): 2.1.15

Ravlin, Elizabeth; University of South Carolina, USA (ravlin@moore.sc.edu): 3.1.6

Ray, Pradeep Kanta; University of New South Wales, Australia (pray@unsw.edu.au): 1.5.12, 3.4.8

Ray, Sangeeta; University of Sydney, Australia (s.ray@econ.usyd.edu.au): 1.5.12, 3.4.8

Ray, Sougata; Indian Institute of Management Calcutta, India (sougata@iimcal.ac.in): 3.2.6

Reiche, Sebastian; IESE, Spain (sreiche@iese.edu): 1.3.8, 2.3.9

Reis, Germano Glufke; FGV/EAESP, Brazil (germanoglufkereis@yahoo.com.br): 2.4.13

Relyea, Clint; Arkansas State University, USA (crelyea@astate.edu): 1.3.12

Remhof, Stefan; University of Magdeburg, Germany (stefan.remhof@gmx.de): 1.1.5 
Ren, Hong; University of Wisconsin-Milwaukee, USA (renh@uwm.edu): 2.2.10

Ren, Zhaoyi; Shanghai International Studies University, China (dddn2008@126.com): 2.5.11

Renata, Miyamoto; FGV, Brazil (r.miyamoto@yahoo.com.br): 3.1.7

Reuer, Jeffrey; Purdue Universtiy, USA (jreuer@purdue.edu): 2.1.7

Rhew, Nicholas D.; University of Memphis, USA (ndrhew@memphis.edu): 1.3.14, 3.3.8

Rialp, Josep; Autonomous University of Barcelona, Spain (josep.rialp@uab.cat): 2.3.13

Riaz, Zahid; University of Central Punjab, Australia (zahid.riaz@ucp.edu.pk): 3.4.8

Ricart, Joan Enric; IESE, Spain (jericart@iese.edu): 3.2.8

Rich, Loren Haynes; Brigham Young University, USA (loren.rich@gmail.com): 1.3.13

Richey, Glenn; University of Alabama, USA (grichey@cba.ua.edu): 1.5.8

Richter, Ulf Henning; Nottingham University, China (ulf.richter@nottingham.edu.cn): 2.3.11, 2.5.12

Riddle, Liesl; George Washington University, USA (Iriddle@gwu.edu): 1.4.3

Rienda, Laura; University of Alicante, Spain (laura.rienda@ua.es): 3.3.8

Ritvala, Tiina; Aalto University, Finland (tiina.ritvala@aalto.fi): 3.1.8

Rivas, Jose Luis; ITAM, Mexico (jose.rivas@itam.mx): 1.1.13

Rivera, Jorge E.; George Washington University, USA (jrivera@gwu.edu): 3.4 .12

Riviere, Monica lleana; SKEMA, France (monicaileana.rogoz@skema.edu): 1.4.11

Roath, Anthony; University of Bath, United Kingdom (asr40@management.bath.ac.uk): 1.4.12, 2.3.14, 3.2.10

Roberts, Michael; MacEwan University, Canada (robertsm35@macewan.ca): 3.3.14

Robins, James; WU Vienna, Austria (jrobins@wu.ac.at): 2.4.10

Robinson, Nick; North Island College, Canada (nick.robinson@nic.bc.ca): 3.3.4, 3.4.4

Rocha, Thelma Valeria; ESPM, Brazil (tvrocha@espm.br): 2.1.11

Rodriguez, Carlos Adrian; McGill University, Costa Rica (carlos.rodriguez@mail.mcgill.ca): 1.4.12

Roehl, Tom; Western Washington University, USA (Tom.Roehl@wwu.edu): 3.3.12

Rose, Elizabeth; Aalto University and University of Otago, New Zealand (elizabeth.rose@aalto.fi): 0.4, 1.5.10, 2.3.10, 2.4.7

Ross, Peter Keith; Griffith University, Australia (p.ross@griffith.edu.au): 3.4.15

Rossi Junior, Jose Luiz; Insper, Brazil (joselrj1@insper.edu.br): 2.2.11

Roth, Kendall; University of South Carolina, USA (kroth@moore.sc.edu): 1.5 .1

Rottig, Daniel; Florida Gulf Coast University, USA (drottig@fgcu.edu): 0.4, 1.5.13, 2.5.8

Ruel, Huub; Windesheim University of Applied Sciences/University of Twente, Netherlands (huubruel@hotmail.com): 1.3.7

Rufin, Carlos; Suffolk University, USA (crufin@suffolk.edu): 1.5.12, 3.1.8

Rugman, Alan M.; University of Reading, United Kingdom (a.rugman@henley.ac.uk): 0.3, 0.7, 1.2, 1.3.2, 2.1.3, 2.4.6, 2.5.1

Rui, Huaichuan; University of London, United Kingdom (huaichuan.rui@rhul.ac.uk): 3.1.2, 3.3.13, 3.4.9

Ruigrok, Winfried; University of St. Gallen, Switzerland (winfried.ruigrok@unisg.ch): 2.3.4, 2.5.14, 3.2.5, 3.4.13

Rullani, Francesco; Luiss Guido Carli University of Rome, Italy (frullani@luiss.it): 3.4.11

Rumyantseva, Maria; University of Sydney, Australia (maria.rumyantseva@sydney.edu.au): 3.4.15

Rungsithong, Rapeeporn; University of Bath, United Kingdom (rr257@bath.ac.uk): 1.4.12, 2.3.14, 3.2.10

Rupf, Corinna; University of Stuttgart, Germany (corinna.rupf@bwi.uni-stuttgart.de): 2.5.14

Ryan, Paul; National University of Ireland, Galway, Ireland (paul.a.ryan@nuigalway.ie): 3.3.15

\section{S}

Sackmann, Sonja A.; University Bw Munich, Germany (sonja.sackmann@unibw.de): 3.4.13

Saeed, Abubakr; Brunel University, United Kingdom (abubakr.sd@gmail.com): 2.1.14

Safon, Vicente; Universitat de Valencia, Spain (vicente.safon@uv.es): 2.4.11

Saghafi, Massoud; San Diego State University, USA (msaghafi@mail.sdsu.edu): 3.2.12

Sahbaz, Ussal; The Economic Policy Research Foundation of Turkey (TEPAV), Turkey (ussal@tepav.org.tr): 1.1.10

Sakakibara, Mariko; UCLA, USA (mariko.sakakibara@anderson.ucla.edu): 2.1.4

Sakawa, Hideaki; Nagoya City University, Japan (sakawa@econ.nagoya-cu.ac.jp): 3.1.12

Sakikawa, Takashi; Niigata University, Japan (saki@mot.niigata-u.ac.jp): 1.5.14 
Saleem, Ashar; Lahore University of Management Sciences, Pakistan (asharsIm@gmail.com): 3.2.9

Salk, Jane; University of Texas at Dallas, USA (Jane.Salk@utdallas.edu): 3.4.6

Sambharya, Rakesh; Rutgers University, USA (sambhary@camden.rutgers.edu): 1.4.10, 2.4.9

Samiee, Saeed; University of Tulsa, USA (samiee@utulsa.edu): 1.4.2

Sammartino, Andre; University of Melbourne, Australia (samma@unimelb.edu.au): 1.1.8, 1.3.2, 1.5.6

Sampath, Vijay Sai; Pace University, USA (vs31371n@pace.edu): 1.3.14

Sampson, Scott E.; Brigham Young University, USA (drsampson3@gmail.com): 1.3.13

Sandberg, Birgitta; Turku School of Economics, Finland (birgitta.sandberg@utu.fi): 3.2.12

Sandberg, Susanne; Linnaeus University, Sweden (susanne.sandberg@lnu.se): 2.4.14

Santacreu-Vasut, Estefania; ESSEC and THEMA, France (santacreuvasut@essec.edu): 3.2.14

Santalo, Juan; IE Business School, Spain (juan.santalo@ie.edu): 2.1.12

Santangelo, Grazia; University of Catania, Italy (grsanta@unict.it): 0.2, 2.4.4

Santoro, Michael D.; Lehigh University, USA (mds8@lehigh.edu): 1.5.11

Santos, Nancy; University of Porto, Portugal (nancy_santos@hotmail.com): 2.5.5

Sapienza, Harry; University of Minnesota, USA (sapienza@umn.edu): 1.3.11

Sarfati, Gilberto; FGV-EAESP, Brazil (gilberto.sarfati@fgv.br): 1.5.13

Sarkar, Prabirjit; Jadavpur University, United Kingdom (prabirjit@gmail.com): 2.4.7

Sartor, Michael A.; University of Western Ontario, Canada (msartor.phd@ivey.ca): 1.1.12, 1.5.13, 2.2 .7

Sauerwald, Steve; University of Texas at Dallas, USA (steve.sauerwald@utdallas.edu): 2.4.9

Sauvant, Karl P.; Columbia University, USA (ksauva@law.columbia.edu): 3.2.11

Sawada, Naohiro; Aoyama Gakuin University, Japan: 1.4.10

Sawant, Rajeev J.; Baruch College, USA (rajeev.sawant@baruch.cuny.edu): 1.4.6

Schaaper, Johannes; Bordeaux Management School, France (jan.schaaper@bem.edu): 2.2.12

Schadewitz, Hannu; University of Turku, Finland (hannu.schadewitz@tse.fi): 1.1.7

Schiozer, Rafael; FGV - Brazil, Brazil (rafael.schiozer@fgv.br): 3.1.7

Schipani, Cindy A. ; University of Michigan, USA (schipani@umich.edu): 3.4.6

Schlaegel, Christopher; Otto von Guericke University Magdeburg, Germany (cschlaeg@ovgu.de): 1.1.5, 2.1.11

Schlegelmilch, Bodo B.; Wirtschaftsuniversität-Wien, Austria (bodo.schlegelmilch@wu.ac.at): 1.4.2

Schmeisser, Bjoern R.; University of Augsburg, Germany (bjoern.schmeisser@wiwi.uni-augsburg.de): 2.2.15

Schmid, Stefan; ESCP Europe, Germany (sschmid@escpeurope.eu): 1.5.14

Schmidt, Flavia de Holanda; IPEA, Brazil (flhschmidt@yahoo.com.br): 2.1.11

Schmidt, Mareike; University of Bremen, Germany (mareike.schmidt@uni-bremen.de): 3.1.5

Schneider, Susan Carol; University of Geneva, Switzerland (susan.schneider@unige.ch): 3.1.6

Schneper, William D.; Franklin \& Marshall College, USA (william.schneper@fandm.edu): 1.4.8

Scholz, Brett David; University of Canberra, Australia (brett.scholz@adelaide.edu.au): 3.1.3

Schotter, Andreas; Western University, Canada (aschotter@ivey.ca): 1.5.3, 2.5.15

Schrapp, Sebastian; University of Goettingen, Germany (sebastian.schrapp@wiwi.uni-goettingen.de): 1.4.8

Schrock, Wyatt Austin; Michigan State University, USA (schrockw@msu.edu): 2.5.6

Schueffel, Patrick; University of Applied Sciences Western Switzerland, Switzerland (patrick@schueffel.de): 2.4.11

Schuster, Anja Maria; University of St. Gallen, Switzerland (anja.schuster@unisg.ch): 2.1.8, 2.5.8, 3.2.15

Schweizer, Roger; University of Gothenburg, Sweden (roger.schweizer@handels.gu.se): 1.3.12, 3.4.15

Scott, Andrea; Pepperdine University, USA (andrea.scott@pepperdine.edu): 2.3.3

Seawright, Kristie Kay; Brigham Young University, USA (kseawright@byu.edu): 1.3.13

Sedoglavich, Vesna; Australian National University, Australia (vesna.sedoglavich@anu.edu.au): 3.4.15

Sekiguchi, Tomoki; Osaka University, Japan (tomoki@econ.osaka-u.ac.jp): 2.5.13

Selmier, II, W. Travis; Indiana University, USA (wselmier@indiana.edu): 2.3.11

Semeniuk, Monica J.; Simon Fraser University, Canada (monica_semeniuk@sfu.ca): 1.1.5

Semenov, Alexey V.; Saint Louis University, USA (asemenov@slu.edu): 1.5.8, 2.5.11

Seo, Eunkwang; Seoul National University, Korea, South (eunkwang.seo@gmail.com): 3.3.15

Serra, Fernando Ribeiro; Uninove - Universidade Nove de Julho \& globADVANTAGE, Brazil (fernandoars@uninove.br): 2.2.7, 3.1.14

Seth, Anju; Virginia Tech, USA (aseth@vt.edu): 3.3.5 
Sethi, S. Prakash; City University of New York, USA (prakash.sethi@baruch.cuny.edu): 3.1.10

Sfekas, Andrew; Temple University, USA (sfekas@temple.edu): 3.2.13

Shadnam, Masoud; Rouen Business School, France (msh@rouenbs.fr): 1.3.4

Shaffer, Margaret; University of Wisconsin-Milwaukee, USA (shafferm@uwm.edu): 1.1.14, 2.3.9, 2.5.13, 3.3.14

Shan, Mengmeng; Fudan University, China (tosmm1988@163.com): 3.2.11

Shane, Elyse Renee; University of Adelaide, Australia (elyse.shane@adelaide.edu.au): 2.1.11

Shapiro, Daniel M.; Simon Fraser University, Canada (dshapiro@sfu.ca): 3.4.7

Shapiro, Debra; University of Maryland, USA (dshapiro@rhsmith.umd.edu): 3.2.14

Sharmelly, Rifat; University of New South Wales, Australia (rifat.sharmelly@unsw.edu.au): 1.5.12

Shen, Jie; University of South Australia, Australia (shen1000@hotmail.com): 3.2.5

Shen, Xiaobai; University of Edinburgh, United Kingdom (Xiaobai.Shen@ed.ac.uk): 3.2.10

Shen, Yan; University of Victoria, Canada (yanshen@uvic.ca): 2.1.15

Sheng, Hsia Hua; FGV-EAESP, Brazil (hsia.sheng@fgv.br): 2.5.7, 3.1.12

Shenkar, Oded; Ohio State University, USA (shenkar_1@fisher.osu.edu): 2.4.2, 2.5.4, 3.2.14

Sher, Peter J.; National Chi Nan University, Taiwan (sher@ncnu.edu.tw): 2.3.13

Shi, Linda Hui; University of Victoria, Canada (Ishi@uvic.ca): 2.3.14

Shi, Weilei (Stone); Baruch College-CUNY, USA (weilei.shi@baruch.cuny.edu): 3.2.11

Shih, Hsi-An; National Cheng Kung University, Taiwan (hashih1224@gmail.com): 3.1.13

Shin, Jiyoung; Korea University, Korea, South (alfolike@gmail.com): 3.1.7

Shin, Joon-Ho; ESADE Business School, Spain (joonho.shin@esade.edu): 2.4.6

Shin, Mannsoo; Korea University, Korea, South (shinms@korea.ac.kr): 2.5.13

Shirodkar, Vikrant; University of Sussex, United Kingdom (v.shirodkar@sussex.ac.uk): 1.5.10

Shoham, Amir; Temple university, USA (amir.shoham@temple.edu): 3.2.14

Sikarwar, Ekta; Indian Institute of Management Indore, India (f10ektas@iimidr.ac.in): 2.5.9

Silenskyte, Ausrine; University of Vaasa, Finland (ausrine.silenskyte@uva.fi): 2.1.11

Silva, Jorge Ferreira da; PUC-Rio, Brazil (shopshop@iag.puc-rio.br): 2.1.11

Simmons, Glenn; University of Auckland, New Zealand (g.simmons@auckland.ac.nz): 3.2.14

Simoes, Luis Ganhao; ESTG - Instituto Politecnico de Leiria, Portugal (luis.ganhao@portucelsoporcel.com): 3.1.14

Simoes, Vitor Corado; Advance, ISEG, Technical University of Lisbon, Portugal (vcs@doc.iseg.utl.pt): 2.4.11

Simons, Ben; University of Auckland, New Zealand (b.r.simons18@gmail.com): 2.1.9

Simpson, Dayna; Monash University, Australia (dayna.simpson@monash.edu): 2.1.13

Singal, Ajay Kumar; Indian Institute of Management Lucknow, India (ajay.singal@iiml.ac.in): 3.1.14

Singh, Ajit; University of Cambridge, United Kingdom (as14@cam.ac.uk): 2.4.7

Singh, Deeksha; Temple University, USA (deeksha@temple.edu): 2.4.11

Singh, J.D.; International Management Institute, New Delhi, India (jdsingh@jimnoida.ac.in): 3.4.13

Singh, Romila; University of Wisconsin-Milwaukee, USA (romila@uwm.edu): 3.3.14

Sinha, Ashutosh Kumar; IIM Lucknow, India (ashutosh@iiml.ac.in): 1.5.6

Sinkovics, Rudolf R.; University of Manchester, United Kingdom (rudolf.sinkovics@manchester.ac.uk): 2.3.12, 2.5.15, 3.3.10

Siqueira, Ana; Duquesne University, USA (siqueiraa@duq.edu): 1.4.4

Skorobogatykh, Irina; Plekhanov Russian University of Economics, Russia (skorobogatyhkii@rea.ru): 3.4.2

Slangen, Arjen; Rotterdam School of Management, Netherlands (aslangen@rsm.nl): 2.1.8, 3.1.9, 3.4.14

Slonovschi, Dumitru; Magenta Consulting, Moldova (d.slonovschi@consulting.md): 2.3 .4

Smeets, Roger; Rutgers University, USA (rsmeets@business.rutgers.edu): 3.2.10

Smith, Matthew Grady; United States Department of the Treasury, USA (matthewgradysmith@gmail.com): 3.4.8

Smith, Natalya; University of Liverpool, ULMS, United Kingdom (natalya.smith@liv.ac.uk): 1.3.9

Soderman, Sten; Stockholm University, Sweden (sod@fek.su.se): 3.1.15

Soederberg, Anne-Marie; Copenhagen Business School, Denmark (ams.ikl@cbs.dk): 3.4.13

Soendergaard, Mikael; Aarhus University, Denmark (msoendergaard@econ.au.dk): 3.1.1, 3.4.3

Sofka, Wolfgang; Copenhagen Business School, Denmark (ws.smg@cbs.dk): 2.2.6, 2.4.10

Soleimani, M. Abrahim; Eastern Washington University, USA (absoleimani@gmail.com): 1.4.8

Some, Hyacinthe; HEC Montreal, Canada (yirlier-hyacinthe.some@hec.ca): 3.4.5 
Sonderegger, Petra; , India (pcs2002@columbia.edu): 1.4.9

Song, Chang; Renmin University of China, China (changsong22@sina.com): 1.1.15

Song, Jaeyong; Seoul National University, Korea, South (jsong@snu.ac.kr): 2.1.13, 2.3.5, 2.4.14, 3.2.1, 3.3.15

Song, Sangcheol; Saint Joseph's University, USA (ssong@sju.edu): 1.3.6

Sonne, Tine; Copenhagen Business School, Denmark (tine_sonne@hotmail.com): 3.4 .13

Spadafora, Ettore; University of South Carolina, USA (ettore.spadafora@grad.moore.sc.edu): 2.3.10

Spencer, Jennfier; George Washington University, USA (spencer.jennifer@gmail.com): 0.5, 2.2.2

Spicer, Andrew; University of South Carolina, USA (aspicer@moore.sc.edu): 1.5.13

Spigarelli, Francesca; University of Macerata, Italy (spigarelli@unimc.it): 1.5.13

Srikanth, Kannan; Indian School of Business, India (kannan_srikanth@isb.edu): 1.5.7

Srivastava, Bharatendu; Indian Institute of Management Calcutta, India (bnsri@iimcal.ac.in): 3.3.2

Stahl, Guenter K.; WU Vienna and INSEAD, France (guenter.stahl@wu.ac.at): 1.3.8, 3.1.2, 3.4.13

Stefanidis, Abraham; St. John's University, USA (stefania@stjohns.edu): 3.2.15

Stein, Christina Marion; University of Stuttgart, Germany (christina.stein@bwi.uni-stuttgart.de): 2.5.14

Steinbauer, Robert; University of Memphis, USA (rstnbuer@memphis.edu): 1.3.14, 3.3.8

Steizel, Sebastián; Universidad Oberta de Catalunya, Argentina (ssteizel@udesa.edu.ar): 3.3.4, 3.4.4

Stensaker, Inger G.; Norwegian School of Economics, Norway (inger.stensaker@nhh.no): 3.3.12

Sternquist, Brenda; Michigan State University, USA (sternqui@msu.edu): 2.3.14, 2.5.6

Stewart, Callum; University of Cape Town, South Africa (johnluiz@telkomsa.net): 1.3.14

Stoian, Cristina; Brunel University, United Kingdom (cristina.stoian@brunel.ac.uk): 2.3.13

Storper, Michael; UCLA and London School of Economics, United Kingdom (m.storper@lse.ac.uk): 2.5.3

Story, Vicky M.; University of Nottingham, United Kingdom (vicky.story@nottingham.ac.uk): 2.4 .5

Stoyanov, Stoyan Petrov; University of Edinburgh, United Kingdom (s.p.stoyanov@sms.ed.ac.uk): 2.2.9

Strange, Roger; University of Sussex, United Kingdom (R.N.Strange@sussex.ac.uk): 2.1.5

Stringer, Christina; University of Auckland, New Zealand (c.stringer@auckland.ac.nz): 3.2.14

Sturdy, Andrew; University of Bristol, United Kingdom (andrew.sturdy@bristol.ac.uk): 3.1.13

Su, Zhan; Laval University, Canada (zhan.su@fsa.ulaval.ca): 2.2.13

Suban, Robert; University of Manchester, United Kingdom (robert.suban@postgrad.mbs.ac.uk): 3.1.12

Subramaniam, Mohan; Carroll School of Management, USA (mohan.subramaniam@bc.edu): 3.4.14

Suder, Gabriele; SKEMA, France (gabriele.suder@skema.edu): 1.3.12, 1.4.11, 1.5.1, 2.5.2, 3.4.15

Suh, Taewon; Texas State University, USA (ts21@txstate.edu): 2.2 .8

Sui, Sui; Ryerson University, Canada (sui.sui.66@gmail.com): 1.3.11, 2.1.6

Sun, Laixiang; SOAS, University of London, United Kingdom (Is28@soas.ac.uk): 2.2.6

Sun, Meijui; Ming Chuan University, Taiwan (mjsun@mail.mcu.edu.tw): 3.1.10

Sutherland, Dylan; Durham University, United Kingdom (dylan.sutherlan@durham.ac.uk): 1.3.9

Suutari, Vesa; Vaasa University, Finland (vesa.suutari@uwasa.fi): 3.3.14

Swiatowiec-Szczepanska, Justyna; Poznan University of Economics, Poland (justyna.swiatowiecszczepanska@ue.poznan.pl): 2.3.15

\section{$\mathbf{T}$}

Tabacco, Raffaella; University of Udine, Italy (tabacco@uniud.it): 2.3.5

Taeube, Florian; EBS Business School, Germany (florian.taeube@ebs.edu): 1.4 .9

Tajeddin, Mahdi; Concordia University, Canada (m_tajed@jmsb.concordia.ca): 3.4.15

Takchi, Caroline; PricewaterhouseCoopers, Australia (caroline.takchi@au.pwc.com): 2.4.14

Takezawa, Nobuya; Rikkyo University, Japan (takezawa@rikkyo.ac.jp): 3.3.4, 3.4.4

Taleb, Ali; MacEwan University, Canada (taleba@macewan.ca): 2.2.13, 2.3.15

Tallman, Stephen; University of Richmond, USA (stallman@richmond.edu): 0.2, 1.2, 1.4.11, 1.5 .4

Tan, Alex Tai Loong; University of Western Australia, Australia (a.tan@uwa.edu.au): 1.1.9

Tan, Brian Roy; Nanyang Technological University, Singapore (abrtan@ntu.edu.sg): 2.5.10

Tan, Danchi; National Chengchi University, Taiwan (dctan@nccu.edu.tw): 2.2.12

Tan, Hao; University of Newcastle, Australia (hao.tan@newcastle.edu.au): 2.2.15, 3.3.15

Tang, Jing'an; Sacred Heart University, USA (tangj3@sacredheart.edu): 2.1.9 
Tang, Linghui; College of New Jersey, USA (tang@tcnj.edu): 1.3.13

Tang, Yi; Hong Kong Polytechnic University, Hong Kong, SAR-PRC (msytang@polyu.edu.hk): 2.2.6

Taras, Vas; University of North Carolina, Greensboro, USA (v_taras@uncg.edu): 2.5.8, 3.2.15, 3.3.4, 3.4.4

Tatoglu, Ekrem; Bahcesehir University, Turkey (ekrem.tatoglu@bahcesehir.edu.tr): 1.1.5, 3.1.15

Taussig, Markus D.; National University of Singapore, Singapore (markus@nus.edu.sg): 2.3.15, 3.2.8

Tavares-Lehmann, Ana Teresa; University of Porto, Portugal (atavares@fep.up.pt): 3.4.11

Tavoletti, Ernesto; University of Macerata, Italy (ernesto.tavoletti@unimc.it): 2.1.13

Tayar, Mark; Swinburne University of Technology, Australia (mtayar@swin.edu.au): 3.2.12

Taylor, Marilyn; University of Missouri at Kansas City, USA (taylorm@umkc.edu): 1.5.3, 3.1.2

Taylor, Sully; Portland State University, USA (sullyt@sba.pdx.edu): 1.3.7

Teagarden, Mary Boyden; Thunderbird School of Global Management, USA (mary.teagarden@thunderbird.edu): 1.5.3, 2.5.15

Teece, David; University of California - Berkeley, USA (teece@haas.berkeley.edu): 1.2

Teegen, Hildy; University of South Carolina, USA (teegen@moore.sc.edu): 1.1.1, 2.1.2

Temouri, Yama; Aston University, United Kingdom (y.temouri1@aston.ac.uk): 3.3.3

Tenzer, Helene; Tübingen University, Germany (helene.tenzer@uni-tuebingen.de): 1.5.9

Thakur, Pooja; Virginia Tech, USA (pthakur@vt.edu): 1.4.12, 3.2.9

Thams, Yannick; Florida International University, USA (thamsy@fiu.edu): 1.1.4, 2.5.11

Theodosiou, Marios; University of Cyprus, Cyprus (mariosth@ucy.ac.cy): 2.1.11

Thite, Mohan; Griffith University, Australia (m.thite@griffith.edu.au): 2.4.13

Thomas, David Clinton; University of New South Wales, Australia (d.thomas@unsw.edu.au): 1.4.15

Thomas, Ekaterina; Staffordshire University, United Kingdom (ekaterina.thomas@staffs.ac.uk): 1.3.9

Thun, Eric; Oxford University, United Kingdom (eric.thun@sbs.ox.ac.uk): 2.4.3

Tietze, Susanne; Sheffield Hallam University, United Kingdom (s.tietze@shu.ac.uk): 2.4 .2

Toeth, Thomas; Copenhagen Business School, Denmark (tto.ikl@cbs.dk): 3.4.13

Tolentino, Paz Estrella; University of London, United Kingdom (p.tolentino@bbk.ac.uk): 2.2.9, 3.2.6

Toppin, Brian; University of the West Indies, Barbados (brian.toppin@cavehill.uwi.edu): 2.1.15

Torres Preto, Miguel; Technical University of Lisbon and Center for Innovation, Technology and Policy Research, Portugal (miguel.preto@ist.utl.pt): 2.2.6

Totskaya, Natalya; Concordia University, Canada (n_totska@jmsb.concordia.ca): 3.4.10

Toulan, Omar; McGill University, Canada (omar.toulan@mail.mcgill.ca): 1.4.12

Toygar, Gulfem; Otto Group, Turkey: 3.2.4

Tracogna, Andrea; University of Trieste, Italy (andrea.tracogna@econ.units.it): 1.4.13

Tran, Thang; Central Institute for Economics and Management, Vietnam (ttran@essex.ac.uk): 3.3.13

Trevino, Len; Loyola University New Orleans, USA (lentrevino@cba.loyno.edu): 2.5.11

Tu, Winnie; Nottingham University, China (winnie.tu@nottingham.edu.cn): 1.3.15

Tuncer, Guneri; Koc University, Turkey (gunerituncer@yahoo.com): 1.1.2

Tung, Rosalie L.; Simon Fraser University, Canada (tung@sfu.ca): 3.4.10

Türktan, Gülden; President, KAGIDER, Turkey: 2.5.2

Tzou, Joseph Shyh-Yeu; National Taiwan University, Taiwan (joesytzou@gmail.com): 2.3.14

\section{U}

Ubukata, Masato; Kushiro Public University, Japan (ubukata@kushiro-pu.ac.jp): 3.1.12

Ueki, Yasushi; Institute of Developing Economies, Japan (yasushi_ueki@ide.go.jp):3.1.13

Ulusemre, Tolga; University of South Carolina, USA (tolga.ulusemre@grad.moore.sc.edu): 1.1.11

Un, Annique; Northeastern University, USA (a.un@neu.edu): 2.1.4, 3.4.1

Upadhyayula, Rajesh; Indian Institute of Management Kozhikode, India (rajesh@iimk.ac.in): 2.5.7

Urbig, Diemo; University of Wuppertal, Germany (urbig@uni-wuppertal.de): 1.3.7

Utete, Gwinyai; Louisiana State University, USA (gutete@Isu.edu): 1.1.13 


\section{V}

Vachani, Sushil; Boston University, USA (sushilvachani@gmail.com): 2.1.2

Vadi, Maaja; University of Tartu, Estonia (maaja.vadi@ut.ee): 3.1.1

Vahlne, Jan-Erik; University of Gothenburg, Sweden (jan-erik.vahlne@handels.gu.se): 1.3.3

Vahtera, Pekka Johannes; University of Leeds, United Kingdom (bnpjv@leeds.ac.uk): 3.3.12

Vaiginiene, Erika; Vilnius University, Lithuania (erika.vaiginiene@ef.vu.It): 2.5.8

Valentino, Alfredo; Luiss Guido Carli University, Italy (valentinoa@luiss.it): 2.2.13

Valery, Pascale; HEC Montreal, Canada (pascale.valery@hec.ca): 3.4.5

Valk, Reimara; Avans University of Applied Sciences, Netherlands (reimara.valk@gmail.com): 2.2.3

Vambery, Robert; Pace University, USA (rvambery@pace.edu): 1.3.14

van Ees, Hans; University of Groningen, Netherlands (h.van.ees@rug.nl): 1.5.6

van Esch, Emmy; Hong Kong Baptist University, Hong Kong, SAR-PRC (11467061@life.hkbu.edu.hk): 3.3.14

van Essen, Marc; University of South Carolina, USA (marc.vanessen@moore.sc.edu): 2.4.9

van Hoorn, André; University of Groningen, Netherlands (vanhoorn@gmail.com): 2.1.8, 2.2.4, 3.2.15

van Kranenburg, Hans; Radboud Universiteit Nijmegen, Netherlands (h.vankranenburg@fm.ru.nl): 1.1.12

van Oosterhout, J. (Hans); Erasmus University, Netherlands (joosterhout@rsm.nl): 1.1.12, 2.4.9

van Veen, Kees; University of Groningen, Netherlands (k.van.veen@rug.nl): 1.5.6

van Witteloostuijn, Arjen; Tilburg University, Netherlands (a.vanwitteloostuijn@uvt.nl): 1.3.7

Varnali, Renin; Bogazici University, Turkey (reninvarnali@gmail.com): 2.5.2

Vasconcellos, Silvio Luis de; IENH - Instituicao Evangelica de Novo Hamburgo, Brazil (silvio.v@ienh.com.br): 2.5.12

Vasudeva, Gurneeta; University of Minnesota, USA (gurneeta@umn.edu): 2.2.2, 2.4.10

Veale, Roberta; University of Adelaide, Australia (roberta.veale@adelaide.edu.au): 1.3.5

Velez-Calle, Andres; Universidad EAFIT, Colombia (avelezca@eafit.edu.co): 3.3.4, 3.4.4

Venaik, Sunil; University of Queensland, Australia (svenaik@business.uq.edu.au):3.1.9, 3.4.11

Venzin, Markus; Università Bocconi, Italy (markus.venzin@unibocconi.it): 2.1.12

Verbeke, Alain; University of Calgary, Canada (alain.verbeke@haskayne.ucalgary.ca): 1.2, 1.4.1

Vertinsky, Ilan; University of British Columbia, Canada (ilan.vertinsky@ubc.ca): 1.4.5, 1.5.7

Vincze, Zsuzsanna; Umeå University, Sweden (zsuzsanna.vincze@usbe.umu.se): 3.2.12

Vives, Luis; ESADE Business School, Spain (luis.vives@esade.edu): 3.2.6

Vo, Dut Van; University of Groningen, Netherlands (d.v.vo@rug.nl): 1.4.11

Voinea, Cosmina Lelia; Radboud Universiteit Nijmegen, Netherlands (c.voinea@fm.ru.nl): 1.1.12, 2.1.14

Voinea, Liviu; Academy of Economic Studies Bucharest, Romania (liviu.voinea@mfinante.ro): 2.3.4

Vojvodic, Goran; George Washington University, USA (goranv@gwu.edu): 1.4.7

Volkov, Mikhail; National Research University Higher School of Economics, Russia (mvolkov1203@gmail.com): 2.2.3

Volkova, Irina O.; NRU Higher School of Economics, Russia (iovolkova@hse.ru): 1.3.13

Von Glinow, Mary Ann; Florida International University, USA (vonglino@fiu.edu): 2.4.13, 2.5.11, 3.2.14

Vora, Davina; SUNY New Paltz, USA (vorad@newpaltz.edu): 1.5.2, 3.1.6

Voss, Hinrich; University of Leeds, United Kingdom (hv@lubs.leeds.ac.uk): 3.1.14

Vural, Metin Onal; IE Business School, Spain (onalv@yahoo.com): 2.1.5

W

Waguespack, David; University of Maryland, USA (waguespack@rhsmith.umd.edu): 3.2.13

Walker, James; University of Reading, United Kingdom (j.t.walker@reading.ac.uk): 1.3.8

Wan, William P.; City University of Hong Kong, Hong Kong, SAR-PRC (william.wan@cityu.edu.hk): 1.1.15

Wang, Chengang; University of Bradford, United Kingdom (c.wang9@bradford.ac.uk): 1.4.14, 2.1.7

Wang, Christina Yu-Ping; National Sun Yat-Sen University, Taiwan (cwang@mail.nsysu.edu.tw): 2.5.14

Wang, Dan; Monash University, Australia (stella8497@gmail.com): 2.3.9

Wang, Fatima; King's College London, United Kingdom (fatima.wang@kcl.ac.uk): 1.5.8, 2.4.14

Wang, Fenghua; Shanghai International Studies University, China (wangfenghua@shisu.edu.cn): 2.5.11

Wang, Heli; Singapore Management University, Singapore (hlwang@smu.edu.sg): 1.4.9, 2.1.5, 3.1.10

Wang, Hui; Peking University, China (wanghui@gsm.pku.edu.cn): 2.4.11

Wang, Hui-Wen; National Sun Yat-Sen University, Taiwan (d984010008@student.nsysu.edu.tw): 1.3.15

AIB 2013 Conference Proceedings

Page 324 
Wang, Lingling; Southern New Hampshire University, USA (lingling.wang@snhu.edu): 1.3.15

Wang, Qian; Zhejiang University, China (fionawq2010@gmail.com): 3.1.10

Wang, Rui; Peking University, China (rwang@gsm.pku.edu.cn): 1.4.14

Wang, Shiye; Peking University, China (wangshiye@pku.edu.cn): 1.4.14

Wang, Stephanie; University of Miami, USA (slu@bus.miami.edu): 1.5.11, 3.4.12

Wang, Tiedong; University of International Business \& Economics, China (tiedongwang@yahoo.com.cn): 1.5.8

Wang, Xinran; Hong Kong Baptist University, China (wangxinranwang@gmail.com): 1.5.13

Wang, Yu-Kai; Soochow University, Taiwan (ywang012@scu.edu.tw): 2.5.14, 3.2.7

Wang, Zhenning; Nottingham University, China (zhenning.wang@nottingham.edu.cn): 1.3.15

Wankel, Charles; St. John's University, USA (wankelc@verizon.net): 2.5.8

Ward, Anna-Katherine; Virginia Tech, USA (annakward@hotmail.com): 3.1.6

Wasowska, Aleksandra; University of Warsaw, Poland (awasowska@mail.wz.uw.edu.pl): 1.4.13, 2.4.13

Watanabel, Naoki; Ritsumeikan University, Japan (n-wata@fc.ritsumei.ac.jp): 3.1.12

Webster, Cynthia M.; Macquarie University, Australia (cynthia.webster@mq.edu.au): 1.5.5

Wechtler, Heidi Marie; Macquarie University, Australia (heidi_wechtler@yahoo.fr): 2.3.9

Weiner, Robert; George Washington University, USA (rweiner@gwu.edu): 1.4.7, 2.5.10, 3.2.3

Weitzel, Utz; Radboud Universiteit Nijmegen, Netherlands (u.weitzel@fm.ru.nl): 1.1.4

Welch, Catherine; University of Sydney, Australia (catherine.welch@sydney.edu.au): 1.3.3, 2.2.4, 3.4.15

Welch, Lawrence; Melbourne Business School, Australia (I.welch@mbs.edu): 2.4.6

Weng, David H.; City University of Hong Kong, Hong Kong, SAR-PRC (d.weng@cityu.edu.hk): 2.2.13

Wernz, Christian; Virginia Tech, USA (cwernz@vt.edu): 3.2.9

Westbrock, Bastian; Utrecht University, Netherlands (b.westbrock@uu.nl): 1.1.4

Westman, Mina; Tel Aviv University, Israel (westman@post.tau.ac.il): 2.3.9

Westney, Eleanor; York University, Canada (ewestney@schulich.yorku.ca): 0.3, 2.4.1

White, Marion Owyar-Hosseini; James Madison University, USA (owyarhmm@jmu.edu): 1.3.12, 2.5.13

Wihlborg, Clas; Chapman University, USA (wihlborg@chapman.edu): 3.2.7

Wilkins, Mira; Florida International University, USA (wilkinsm@fiu.edu): 2.5.4, 3.1.4

Wilkinson, Adrian; Griffith University, Australia (adrian.wilkinson@griffith.edu.au): 2.4 .13

Williams, Christopher; University of Western Ontario, Canada (cwilliams@ivey.ca): 3.2.13, 3.3.14

Williamson, Nicholas C.; University of North Carolina, Greensboro, USA (ncwillia@uncg.edu): 3.3.15

Williamson, Peter; University of Cambridge, United Kingdom (p.williamson@jbs.cam.ac.uk): 1.1.3, 1.4.4

Wills, Jim; University of Hawai'i at Manoa, USA (jwills@hawaii.edu): 1.1.9

Wilson, Rick T.; Hofstra University, USA (rick.t.wilson@hofstra.edu): 1.1.8

Wolf, Bernard; York University , Canada (bwolf@schulich.yorku.ca): 2.5.8, 3.3.15

Wolf, Joachim; University of Kiel, Germany (wolf@bwl.uni-kiel.de): 2.3.10, 3.4.3

Wolff, Michael; Georg-August-University Goettingen, Germany (michael.wolff@wiwi.uni-goettingen.de): 1.1.13, 1.4.8

Wolters, Tim; University of Twente, Netherlands: 1.3.7

Wong, Loong; Murdoch University, Australia (I.wong@muirdoch.edu.au): 1.5.3

Wood, Donald; Eureka Mgmt. Consultants, Saint Vincent and the Grenadines (eurekadw@caribsurf.com): 2.3.3

Wood, Geoffrey; University of Warwick, United Kingdom (geoffrey.wood@wbs.ac.uk): 1.3.8

Woodward, Richard; University of Edinburgh, United Kingdom (rick.woodward@ed.ac.uk): 2.2.9

Wright, Mike; Imperial College, United Kingdom (mike.wright@imperial.ac.uk): 2.2.5

Wrigley, Neil; University of Southampton and Editor, Journal of Economic Geography, United Kingdom: 2.5.3

Wu, Changqi; Peking University, China (topdog@gsm.pku.edu.cn): 0.4, 2.5.15

Wu, Hsueh Liang; National Taiwan University, Taiwan (hlwu@ntu.edu.tw): 1.1.14

Wu, Jay; Thammasat University, Thailand (jwuinbkk@gmail.com): 1.1.13, 3.4.12

Wu, Jie; University of Macau, Macau (jiewu@umac.mo): 2.3.14, 2.4.13

Wu, Sibin; University of Texas-Pan American, USA (sibinwu@utpa.edu): 2.4.11

Wu, Zhan; University of Sydney, Australia (w.zhan@econ.usyd.edu.au): 3.3.13

Wurster, Dennis; ESCP Europe, Germany (dwurster@escpeurope.eu): 1.5.14

Wurtz, Olivier; Vaasa University, Finland (olwurtz@gmail.com): 2.3.9, 3.3.14

Wymbs, Clifford; Baruch College-CUNY, USA (clifford.wymbs@baruch.cuny.edu): 2.3.15 


\section{$\mathbf{X}$}

Xavier, Wlamir Goncalves; FGV/EAESP e UNISUL, Brazil (wlamirxavier@gmail.com): 2.1.14, 2.4.12

Xia, Weidong; Florida International University, USA (xiaw@fiu.edu): 2.5.11

Xie, Zhenzhen; Hong Kong University of Science \& Technology, Hong Kong, SAR-PRC (xiezz@ust.hk): 1.3.6, 1.4.5

Xu, Kai; Texas A\&M University, China (kaixu@mays.tamu.edu): 2.1.9

Xu, Yue; Hull University Business School, United Kingdom (tina.yuexu@gmail.com): 2.4.11

Xu, Yuehua; Sun Yat-Sen University, China (xuyueh@mail.sysu.edu.cn): 1.1.15

\section{Y}

Yajnik, Nilay; Narsee Monjee Institute of Management Studies, India (nilayy@nmims.edu): 2.5.8

Yamanoi, Junichi; Chuo University, Japan (yamanoi@fps.chuo-u.ac.jp): 1.4.15, 2.2.6

Yamao, Sachiko; University of Melbourne, Australia (syamao@unimelb.edu.au): 2.5.13, 3.3.14

Yamazaki, Yoshitaka; International University of Japan, Japan (yyama@iuj.ac.jp): 2.5.13

Yamin, Mo; University of Manchester, United Kingdom (mo.yamin@mbs.ac.uk): 1.5.11, 3.1.12

Yan, Zheng; National University of Singapore, Singapore (zhengyan@nus.edu.sg): 1.4.14

Yang, Forrest Zhilin; City University of Hong Kong, Hong Kong, SAR-PRC (mkzyang@cityu.edu.hk): 3.4.10

Yang, Haibin; City University of Hong Kong, Hong Kong, SAR-PRC (haibin@cityu.edu.hk): 2.2.6

Yang, Jiawen; George Washington University, USA (jwyang@gwu.edu): 2.4.5, 3.3.13

Yang, Jing Yu (Gracy); University of Sydney, Australia (gracy.yang@sydney.edu.au): 1.4.15

Yang, Jun; Florida International University, USA (jyang007@fiu.edu): 2.5.11

Yang, Kuo-Pin; National Dong Hwa University, Taiwan (kpyang@mail.ndhu.edu.tw): 1.1.4

Yang, Xiaohua; University of San Francisco, USA (xyang14@usfca.edu): 1.5.3, 2.2.8, 3.1.2

Yang, Xiyan; Shanghai University of International Business and Economics, China (yangxiyan@shift.edu.cn): 2.5.14

Yang, Yong; University of Sussex, United Kingdom (yong.yang@sussex.ac.uk): 2.2.5, 3.2.10, 3.4.7

Yang, Zhen; Renmin University of China, China (yangzhen2010@ruc.edu.cn): 3.3.13

Yao, Fiona Kun; University of California, Berkeley, USA (fionayao@haas.berkeley.edu): 1.4.15

Yao, Xin; University of Colorado, USA (xinevayao@gmail.com): 1.5.5

Yao, Ying Sheng; National Chi Nan University, Taiwan (michelle_hsu@weichuan.com.tw): 2.5.15

Yaprak, Attila; Wayne State University, USA (attila.yaprak@wayne.edu): 2.4.15

Yavuz, R. Isil; Ozyegin University, Turkey (isil.yavuz@ozyegin.edu.tr): 1.3.11, 3.1.5

Yen, I-Fan; National Chi Nan University, Taiwan (ifan0715@gmail.com): 3.1.14

Yeniyurt, Sengun; Rutgers University, USA (yeniyurt@business.rutgers.edu): 3.1.9

Yeung, Bernard; National University of Singapore, Singapore (bizdean@nus.edu.sg): 0.7

Yi, EunJoo; University of Pittsburgh, USA (eunjoo8@gmail.com): 3.2.11

Yildiz, Harun Emre; Stockholm School of Economics, Sweden (emre.yildiz@hhs.se): 2.1.8

Yin, Haitao; Shanghai Jiao Tong University, China (haitao.yin@gmail.com): 1.1.15

Yin, Jason Z.; Seton Hall University, USA (yinjason@shu.edu): 2.1.13

Yip, George; China Europe International Business School, China (gyip@ceibs.edu): 2.2.1

Yiu, Daphne W.; Chinese University of Hong Kong, Hong Kong, SAR-PRC (dyiu@cuhk.edu.hk): 1.1.15

Yordanova, Desislava I.; Sofia University "St. Kliment Ohridski", Bulgaria (d_yordanova@abv.bg): 1.1.14

Yoshikawa, Toru; Singapore Management University, Singapore (toru@smu.edu.sg): 1.1.13, 3.3.14

Yosun, Türkan; Sabanci University, Turkey (turkanyosun@sabanciuniv.edu): 2.4.13

Young, Michael N.; Hong Kong Baptist University, Hong Kong, SAR-PRC (michaely@hkbu.edu.hk): 1.5.13

Young, Stephen; University of Glasgow, United Kingdom (stephen.young@glasgow.ac.uk): 1.5.5

Young, Susan L; Seton Hall University, USA (susan.young@shu.edu): 3.3.10

Yu, Chow Ming; National Chengchi University, Taiwan (yu54@nccu.edu.tw): 1.5.14, 3.4.9

Yu, Pei-Li; National Cheng Kung University, Taiwan (h4584933@ms24.hinet.net): 2.3 .15

Yu, Shu; National University of Singapore, China (yushu@nus.edu.sg): 1.4.12, 2.2.14

Yuan, Lin; University of Macau, Macau (linyuan@umac.mo): 1.5.11

Yunlu, Dilek Gulistan; University of Wisconsin-Milwaukee, USA (dgyunlu@uwm.edu): 1.1.14, 2.2.10 


\section{Z}

Zaheer, Aks; University of Minnesota, USA (azaheer@umn.edu): 0.5, 3.2.8

Zaheer, Sri; University of Minnesota, USA (szaheer@umn.edu): 0.3, 1.1.1, 1.3.11, 3.4.1

Zakaria, Norhayati; University of Wollongong in Dubai, United Arab Emirates (yatizakaria11@gmail.com): 2.5.8

Zambaldi, Felipe; FGV/EAESP, Brazil (felipe.zambaldi@fgv.br): 2.4.13

Zander, Ivo; Uppsala University, Sweden (ivo.zander@fek.uu.se): 2.2.12

Zander, Udo; Stockholm School of Economics, Sweden (udo.zander@hhs.se): 0.3

Zdravkovic, Srdan; Bryant University, USA (szdravko@bryant.edu): 1.3.5, 2.3.2

Zdziarski, Michal; Warsaw University, Poland (m.zdziarski@uw.edu.pl): 1.4.13, 3.1.3

Zeidan, Rodrigo; Fundação Dom Cabral and Nottingham University, China (rodrigo.zeidan@fdc.org.br): 3.1.12

Zeng, Yuping; Southern Illinois University Edwardsville, USA (yzeng@siue.edu): 1.3.6

Zettinig, Peter; Turku School of Economics, Finland (peter.zettinig@utu.fi): 3.2.12

Zhan, James; Division on Investment and Enterprise, UNCTAD, Switzerland: 2.3.7

Zhang, Jianhua; University of Gothenburg, Sweden (jianhua.zhang@economics.gu.se): 3.2.7

Zhang, Jie; Peking University \& China HuaRong Asset Management Co., Ltd., China (jacqueline35@sina.com): 1.1.15, 2.4.5

Zhang, Lian; Yonsei University, Korea, South (summer906@hotmail.com): 2.3.13

Zhang, Ling Eleanor; Hanken School of Economics, Finland (ling.zhang@hanken.fi): 1.3.13

Zhang, Min (Megan); University of Western Ontario, Canada (mzhang.phd@ivey.ca): 1.1.6

Zhang, Qiyuan; University of Hong Kong, Hong Kong, SAR-PRC (bessieqy@hku.hk): 2.1.14

Zhang, Wen; City University of Hong Kong, Hong Kong, SAR-PRC (mgwendy@cityu.edu.hk): 1.1.9

Zhang, Yuqian; Macquarie University, Australia (yuqian.zhang@students.mq.edu.au): 3.2.12

Zhao, Fang; American University of Sharjah, United Arab Emirates (fzhao@aus.edu): 2.5.8

Zhao, Hongxin; Saint Louis University, USA (zhaox@slu.edu): 1.5.8, 3.2.11

Zhao, Jinlin; Florida International University, USA (zhaoj@fiu.edu): 2.3.15

Zhao, Meng; Moscow School of Management SKOLKOVO, Russia (meng_zhao@skolkovo.ru): 1.5.10

Zhao, Yanhui; Michigan State University, USA (zhaoyanh@msu.edu): 2.3.15

Zhelyazkov, Pavel; Harvard Business School, USA (pzhelyazkov@hbs.edu): 2.3.15

Zheng, Connie Shao-mei; Deakin University, Australia (connie.zheng@deakin.edu.au): 1.1.15, 1.3.12

Zhong, Bijuan; Ohio State University, USA (zhong_40@fisher.osu.edu): 3.1.13

Zhou, Changhui; Peking University, China (czhou@gsm.pku.edu.cn): 3.4.10

Zhou, Kevin Zheng; University of Hong Kong, Hong Kong, SAR-PRC (kevinzhou@business.hku.hk): 2.1.14, 2.3.5

Zhou, Nan; Moscow School of Management SKOLKOVO, Russia (nan_zhou@skolkovo.ru): 1.5.10

Zhou, Xiaoyu; Peking University, China (aptxyu@gmail.com): 1.4.14

Zhu, Cherrie Jiuhua; Monash University, Australia (cherrie.zhu@monash.edu): 2.1.13, 2.3.9

Zhu, Hui; Cape Bretton University, Canada (julia_zhu@cbu.ca): 2.5.11

Zhu, Jinwei; Jiangnan University, China (jinweizhu@jiangnan.edu.cn): 2.1.13

Zhu, Renhong; Sun Yat-Sen University, China (mnszrh@zsu.edu.cn): 1.5.5

Zimmerman, Alan S.; City University of New York-College of Staten Island, USA (alan.zimmerman@csi.cuny.edu):

2.4.15, 3.1.7

Zimmermann, Jörg; European Commission - Joint Research Center, Spain (jzi@gmx.de): 1.1.8, 2.2.6

Zisk, Daniel S.; James Madison University, USA (ziskds@jmu.edu): 1.3.12

Zucchella, Antonella; University of Pavia, Italy (antonella.zucchella@unipv.it): 1.4.13, 2.1.5, 2.4.11

Zwerg-Villegas, Anne Marie; EAFIT, Colombia (annezwevil@gmail.com): 3.4.6 
(This page is intentionally blank) 



\section{Proceedings of the $55^{\text {th }}$ Annual Meeting of the Academy of International Business \\ "Bridging the Divide: Linking IB to Complementary Disciplines and Practice" Istanbul, Turkey July 3-6, 2013}

ISSN: 2078-0435

(C) 2013 Academy of International Business

For more information, please contact:

AIB Executive Secretariat

G. Tomas M. Hult, Executive Director, or

Tunga Kiyak, Managing Director

$$
\text { Eppley Center }
$$

465 N. Shaw Ln Rm 7

Michigan State University

East Lansing, MI 48824, USA

Phone: +1 (517) 432-1452 • Fax: +1 (517) 432-1009

E-mail: aib@aib.msu.edu•Web: http://aib.msu.edu/ 